\title{
Complex Systems: Nonlinearity and Structural Complexity in spatially extended and discrete systems
}

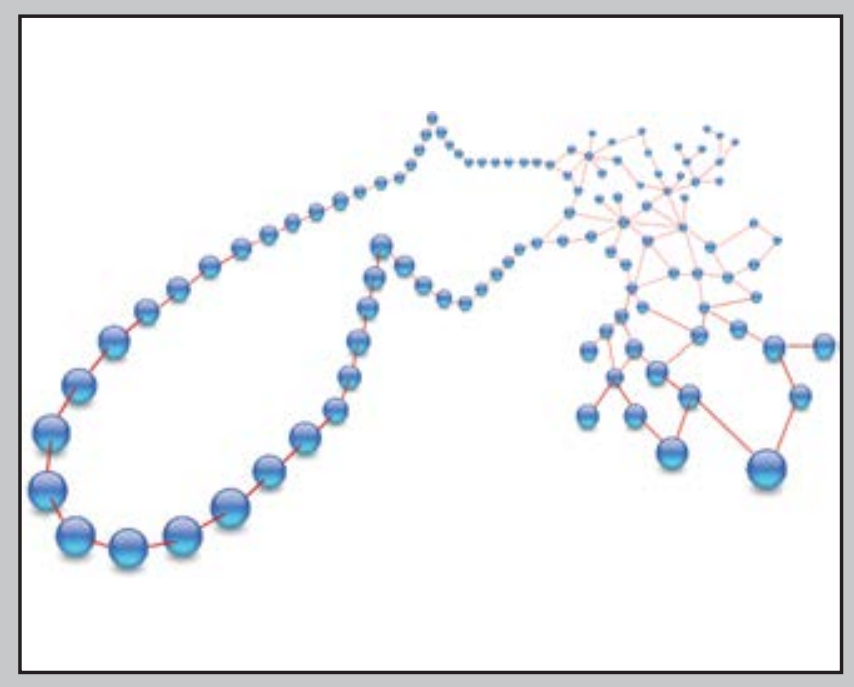

Jesús Gómez Gardeñes 

Complex Systems:

Nonlinearity and Structural Complexity in spatially extended and discrete systems 
Colección de Estudios de Física

Vol. 67

Esta colección recoge las tesis presentadas en el Departamento de Fúsica de la Materia Condensada de la Universidad de Zaragoza desde su constitución en $198 \%$. 


\author{
Complex Systems" \\ Nonlinearity and Structural Complexity \\ in spatially extended and discrete systems
}

Jesús Gómez Gardeñes

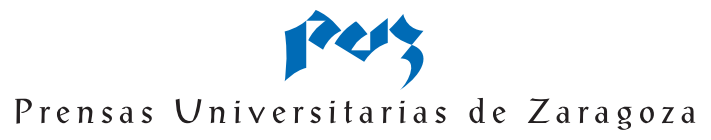




\section{GÓMEZ GARDEÑES, Jesús}

Complex Systems : nonlinearity and structural complexity in spatially extended and discrete systems / Jesús Gómez Gardeñes. — Zaragoza : Prensas Universitarias de Zaragoza, 2007

XII, 307 p. ; 25 cm. — (Colección de Estudios de Física ; 67)

Tesis-Universidad de Zaragoza

ISBN 978-84-7733-906-9

1. Materia condensada-Tesis doctorales. I. Universidad de Zaragoza. II. Título. III. Serie: Colección de Estudios de Física (Prensas Universitarias de Zaragoza) ; 67 $538.9(043.2)$

No está permitida la reproducción total o parcial de este libro, ni su tratamiento informático, ni la transmisión de ninguna forma o por cualquier medio, ya sea electrónico, mecánico, por fotocopia, por registro u otros métodos, ni su préstamo, alquiler o cualquier forma de cesión de uso del ejemplar, sin el permiso previo y por escrito de los titulares del Copyright.

(C) Jesús Gómez Gardeñes

(C) De la presente edición, Prensas Universitarias de Zaragoza

1. ${ }^{\text {a }}$ edición, 2007

Prensas Universitarias de Zaragoza. Edificio de Ciencias Geológicas, c/ Pedro Cerbuna, 12, 50009 Zaragoza, España. Tel.: 976761 330. Fax: 976761063 puz@unizar.es http://puz.unizar.es

Impreso en España

Imprime: Servicio de Publicaciones. Universidad de Zaragoza

D.L.: Z-2180/2007 
A mis padres,

Jesús y Lola. 



\section{Contents}

I Intrinsic localization in nonlinear Schrödinger lattices 9

2 Discrete Breathers and Nonlinear Schrödinger lattices $\quad 15$

2.1 The Salerno Model . . . . . . . . . . . . . . . . . . . . 17

2.2 Discrete space-time symmetries: $(p, q)$ resonant states $\ldots \ldots .22$

2.3 Discrete Breathers numerics . . . . . . . . . . . . . . . . . . 24

2.3.1 Newton continuation . . . . . . . . . . . . . 24

2.3.2 Floquet stability analysis . . . . . . . . . 26

3 Discrete Breathers in 1D Nonlinear Schrödinger lattices $\quad 31$

3.1 DB's in the standard Salerno Model . . . . . . . . . . . . . 32

3.1.1 The structure of the solution $\ldots \ldots \ldots \ldots$

3.1.2 The background amplitude . . . . . . . . . . . . 40

3.1 .3 Floquet analysis . . . . . . . . . . . . . . . 41

3.2 Particle perspective on DB's . . . . . . . . . . . . . 47

3.2.1 Collective variables theory. . . . . . . . . . . 47

3.2.2 Energy balance governs mobility. . . . . . . . . . 50

3.2.3 Oscillating breathers . . . . . . . . . . . . . 55

3.2.4 Validity and limitations of particle perspective . . . . 57

3.3 DB's in the Salerno Model with competing nonlinearities . . . . 58

3.3 .1 Continuum limit . . . . . . . . . . . . 60

3.3.2 Pinned discrete breathers . . . . . . . . . . . 61

3.3.3 Moving discrete breathers . . . . . . . . . . . . . . 68

3.4 Conclusions and Prospective Remarks . . . . . . . . . . . . . 72

4 Discrete Breathers in 2D Nonlinear Schrödinger lattices $\quad 79$

4.1 The Salerno model in two dimensions . . . . . . . . . . . . . 81

4.2 Pinned discrete breathers . . . . . . . . . . . . 85

4.2.1 Pinned DB's in the standard Salerno model . . . . . . . 85 
4.2.2 Pinned DB's in the Salerno model with competing non-

linearities . . . . . . . . . . . . . . 90

4.3 Discrete vortex breathers . . . . . . . . . . . . . . . 96

$4.3 .1 \quad$ Vortex crosses . . . . . . . . . . . . . . . . . 97

4.3 .2 Vortex squares . . . . . . . . . . . . . . . 101

4.3.3 Bound states of discrete vortex crosses . . . . . . . . 104

4.4 Mobile discrete breathers . . . . . . . . . . . . . . . 106

4.4.1 Structure and stability of $(1,0,1)$ fixed points. . . . . . 106

4.4.2 Unstable manifold behaviour and ubiquity of pulson

states. . . . . . . . . . . . . . . 110

4.5 Conclusions and Prospective Remarks . . . . . . . . . . . . 113

\section{Structure and Dynamics of Complex Networks 115}

5 Network Structure and Generation $\quad 121$

5.1 Describing Complex Networks . . . . . . . . . . . . . . . 122

5.1 .1 Basic definitions . . . . . . . . . . . . . . 122

$5.1 .2 \quad$ Single nodes properties . . . . . . . . . . . . . . . . 123

5.1 .3 Network properties . . . . . . . . . . . . . . . . 125

5.1.4 Looking at Networks Mesoscale . . . . . . . . . . . . 130

5.2 Overview of network generation models . . . . . . . . 133

5.2 .1 Random graphs . . . . . . . . . . . . . . . . . . . 133

5.2 .2 Small-world networks . . . . . . . . . . . . . . . . 134

5.2 .3 Scale-Free networks . . . . . . . . . . . . . 136

5.3 Global versus local knowledge . . . . . . . . . . . . . . . 139

5.3 .1 The model . . . . . . . . . . . . . . . . . . . . 140

5.3 .2 Network properties . . . . . . . . . . . . . . . . 141

5.4 Interpolation between Random and Scale-Free networks . . . 146

5.4 .1 The model . . . . . . . . . . . . . . . . . . . 147

5.4.2 Network growth and degree evolution . . . . . . . . 148

5.4 .3 Network properties . . . . . . . . . . . . . . . 152

6 Propagation through Complex Networks 157

6.1 Epidemic spreading and Immunization . . . . . . . . . . . 158

6.1.1 Modeling epidemic spreading . . . . . . . . . . . . 159

6.1.2 Epidemic spreading in general complex networks . . . . 162

6.1 .3 Immunization strategies . . . . . . . . . . . . . . 165

6.1.4 Covering based Immunization . . . . . . . . . . . . . 169

6.2 Information transmission and Jamming . . . . . . . . . . . . . . 180

6.2.1 Information dynamics on Networks . . . . . . . . . 181

6.2 .2 Shortest path routing . . . . . . . . . . . . 186 
6.2.3 Congestion-aware routing . . . . . . . . . . . . . . . . 191

6.3 Conclusions . . . . . . . . . . . . . . . . . . . . . . . . 204

III Nonlinear Dynamics of Complex Networks 207

7 Activatory-Inhibitory interactions in Networks 213

7.1 Modeling biological networks . . . . . . . . . . . . . . . . . . 214

7.1.1 Structure . . . . . . . . . . . . . . . . 215

7.1 .2 Dynamics . . . . . . . . . . . . . . . . . . . 217

7.2 Regulatory dynamics in scale-free networks . . . . . . . . . 225

7.2.1 The model: basic dynamical features . . . . . . . . . 226

7.2.2 Statistical characterization of island's dynamics and structure . . . . . . . . . . . . . . . 240

7.2.3 Structure inside dynamical islands . . . . . . . . . . . 247

7.3 Conclusions . . . . . . . . . . . . . . . . . . . 251

8 Synchronization on Complex Networks 253

8.1 The Kuramoto model . . . . . . . . . . . . . . . . . . . . . . . 254

8.1.1 Solution to the Kuramoto model . . . . . . . . . . . 256

8.1.2 Synchronization in complex networks . . . . . . . . . 259

8.2 Synchronization in local scale-free networks . . . . . . . . . 263

8.3 Homogeneous versus heterogeneous topologies . . . . . . . . . 267

8.4 Synchronization in structured networks . . . . . . . . . . . . 279

8.5 Conclusions . . . . . . . . . . . . . . . . . . . 284

9 Conclusions 285

$\begin{array}{ll}\text { Appendices } & 291\end{array}$

A Computation of Periodic Orbits . . . . . . . . . . . . . . 291

B Linear Stability of Periodic Orbits . . . . . . . . . . . . . . 295

$\begin{array}{ll}\text { Bibliography } & 300\end{array}$ 



\title{
Chapter 1
}

\section{Complex Systems?}

\begin{abstract}
The greatest challenge today, not just in cell biology and ecology but in all of science, is the accurate and complete description of complex systems. Scientist have broken down many kinds of systems. They think they know most of the elements and forces. The next task is to reassemble them, at least in mathematical models that capture the key properties of the entire ensembles.
\end{abstract}

Edward O. Wilson [1].

This thesis covers the analysis of two fundamental ingredients for the correct modeling of real macroscopic systems: Nonlinearity and Structural Complexity. The study of systems where these ingredients are present is systematically related to the field of "Physics of Complex Systems". It is not easy however to find a formal definition of what a complex system is and most books on the matter submit the reader from the very beginning to some illustrative examples of complex phenomena rather than establishing the general principles and characteristics of a complex system.

The standard classification of the wide range of studied natural systems into physical fields is mainly based on the energy range involved for their description, namely, (ranging from higher to lower energy) particle physics; nuclear physics; molecular atomic and optical physics; soft and condensed matter. With this classification in mind the question about what field the Physics of Complex System belongs to naturally arises. However, complex systems are common to a number of physical disciplines belonging to different "energy ranges" so it is difficult to place them into a single physical compartment. In addition, one can find lots of examples of systems called "complex" outside these traditional branches of physics in chemistry, biology, ecology, and social and 
economical sciences. Then, rather than becoming a particular physical field, the physics of complex systems has emerged as an interdisciplinary subject. What are the unifying characteristics of complexity in the phenomena studied by such a diverse branch of scientific disciplines? One can state that the fingerprint of a complex system is reflected by the display of organization without a central organizing principle. This collective organizational behaviour is not usually explained by decomposing a complex system into its parts and analyzing their isolated properties. In this sense, the Physics of Complex Systems is a new way for analyzing systems where complex phenomena are displayed rather than a new physical field.

One of the first attempts to show the need of a Physics of Complex Systems is due to Philip W. Anderson in his celebrated article "More is different" [2]. In this article, Anderson tells about the concept of broken symmetry reflected when one moves from a small system to a macroscopic one. In this transition it may happen that some symmetries of the single systems, that determine their physical behaviour, are lost when embedded in a bulk of many systems and unexpected phenomena occur (Emergence). In this latter situation the knowledge of the physical laws governing each single building block is in many cases not enough to explain the collective behaviour of the big system.

\section{Emergentism versus Reductionism}

This new way of thinking in physics is strongly related with the Emergentism philosophy. The advent of Emergentism philosophy constituted a punch at the old-fashioned Reductionist movement that led the theory of science for decades and, in particular, the way of physics during the XIX century and the first half of the last century.

Emergentism states that the observed phenomena are classified into different levels of description and that each one of these levels is independent in the sense that each has its own laws. The emergence of such levels is the result of the increase of the problem's complexity. On the other hand, reductionists assume the unity of science so that a hierarchical organization is established among disciplines:

- Chemistry is based on Physics.

- Fundamental Biology is based on Chemistry.

- Psychology is based on Biology.

- Sociology is based on Psychology. 
- Political science and Anthropology are both based on Sociology.

Whereas the first two of these reductions were commonly accepted, it was not the case for the last ones yielding a big controversy. For example, aspects of evolutionary psychology and socio-biology are rejected by those who claim that complex systems are inherently irreducible. On the contrary, strong reductionists claim that the behavioural sciences should become truly scientific disciplines by being based on genetic biology arguments. Examples of this long-standing controversy can be found in several scientific forums. Perhaps, the most fruitful ones, in the sense of the number of rationales on the subject, are found in the context of life sciences: the mind-body problem, the explanation of Darwinian evolution theory, etc...

As the theory of science evolved, the Reductionism-Emergentism controversy progressively affected each stage of the above chain of reductions, arriving up to the very first level. In fact, it was Karl Popper, one of the godfathers of the modern theory of science, who stated that Chemistry was not reducible to Physics [3].

The big controversy arrives to Physics under the term "Complex Systems" questioning the possibility of explaining every physical system only in terms of the properties of its constituents (Constructionist hypothesis). As mentioned above, a great deal of physical systems is said to display complex behaviour, i.e. they display some new phenomena that cannot be predicted by only looking at their parts. This impossibility goes beyond the limitations related to the large amount of elements that are involved; there are cases where the properties of the isolated elements seem to be violated by the physical description of the macroscopic behaviour. The concept of symmetry breaking is thus central for a proper (in physical terms) definition of what is called emergent phenomena in Physics. The aforementioned article by P. W. Anderson [2] introduced this new concept in "one of the early manifestos for this infinitely quiet revolution" leading the new way of looking at physical phenomena. Let us review the most salient examples of the so-called complex behaviour.

Symmetry breaking arguments have been recursively invoked to explain unexpected experimental discoveries in the field of condensed matter physics. The most representative examples are superconductivity, superfluidity, liquid crystals and antiferromagnetism. The physical explanation of each of these collective phenomena has contributed to the growth of conceptually new frameworks in many-body physics. It was Landau $[4,5]$ the first to formulate phase transitions as processes where symmetry reductions occurred. This point of view allowed him, after the theoretical explanation of ferromagnetism, to predict antiferromagnetism [6, 7] by generalizing the idea of spin rotation symmetry breaking. Subsequently, ideas on gauge symmetry breaking led to the 
explanation of superfluidity [8,9] and superconductivity [10]. Anderson [2] thus claimed that symmetry breaking in many-body systems is a generalized phenomenon yielding different emergent collective behaviours depending on the particular type of broken symmetry. Therefore, it seems reasonable that, since the principles that govern the behaviour of a macroscopic system of particles appear defined by the whole system, these phenomena should be studied separately to those found for more elementary levels of description.

The success of the holistic description of simple (in terms of the knowledge of the governing physical laws) systems where collective behaviour shows up, paved the way for the search of new conceptual schemes to explain other emergent phenomena at higher complexity levels. This search was carried out by the study of mathematical models that account for different emergent behaviours such as the appearance of dynamically coherent states, spontaneous localization or pattern formation in extended systems. A second set of motivating phenomena is revealed by the observation of self-similar (fractal) patterns in natural systems, that is viewed as the emergence of self-organization behaviour. These examples are different forms of the dynamical or spatio-temporal order that appears in seemingly different systems of a large number of interacting elements in nature.

The breakthroughs in the description of the first class of phenomena is closely related to the advances in the studies of nonlinear dynamical systems. From the very first soliton theory [11] in continuous nonlinear systems and the discovery of localized states in nonlinear chains [12], we have seen that coherent structures emerge from large scale nonlinear models, possess their own entity (particle-like behaviour, well-defined life times, characteristic interaction patterns, etc...). In fact, no matter the complexity of the underlying equations, spatial or temporal coherent structures are many times described with the help of a low dimensional phase space.

The concept of self-organized criticality, introduced by Per Bak, Kurt Wiessenfeld and Chao Tang $[13,14]$, constitutes one of the best explanation of nature complexity and, perhaps, it represents one of the major conceptual achievements of the physics of complexity. Self-organized criticality tries to capture the essential ingredients to explain the critical-like behaviour (manifested by observations of fractal structure and power-laws) of many natural systems without a central controller unit. The original idea was to describe the dynamics of sandpiles, accounting for the avalanches that may happen when grains are progressively incorporated, by means of a simple cellular automaton model. The success of this simple model was seized to relate the model to a variety of phenomena where criticality was already observed, like earthquakes, forest fires, epidemics and, indeed, evolution theory (relating it to the 
the theory of punctuated-equilibrium [15]).

Reading again Anderson in [16] (written nearly 20 years after his treatise on complexity) and reviewing the most relevant examples of complex behaviour up to now, it is evident that emergence is progressively being accepted as a necessary ingredient to face all the new phenomena that has appeared in the last decades under the name of Complexity. A number of scientific institutes and groups are nowadays contributing to the growth of complexity physics which is still in its infancy. Centers with a long history tackling complexity such as the "Sante Fe Institute", the "Max Plank Institute for Physics of Complex Systems" at Dresden, the "Complex Systems group and the Center for Nonlinear Studies" at Los Alamos, the "New England Complex Systems Institute" at Boston, etc... and those of much recent creation like our "Institute of Biocomputation and Physics of Complex Systems" at Zaragoza are actively contributing to this growth.

In summary, the physics of complex systems tries to explain emergent phenomena without losing the sight of the whole system (unlike the fully reductionist way of doing, which destroys the systemic level). It is then important to keep in mind that, although physical systems have a clear hierarchical ordering (nobody doubts that a system is composed by its parts) "each level can require a whole new conceptual structure" [2] (at least for our limited way of thinking), making thus impossible bridging the gaps by the systematic use of a bottom-up approaches.

\section{What are the essential ingredients of a Complex System?}

All the examples listed above are labeled as complex phenomena due to the impossibility to explain them by studying in isolation the parts of the systems where they occur. The behaviour of these systems is thus intrinsically new with respect to the properties of the single elements of the system.

It is clear that a complex system is composed of a large number of elements. However, a big ensemble of building blocks is not enough by itself to guarantee the emergence of unexpected phenomena such as those described above (long range correlations from short-range interactions, localization in extended systems, self-organization and adaptability, etc...). Then, it is important to distinguish the attribute complex from complicated. Airplanes, computers or swiss clocks are examples of complicated systems made up of a large number of pieces. They all conform a directed cause-effect chain so that the malfunctioning of a single piece stops the whole system. They are also designed by an external agent (humans) for a unique function. 
What are then the key ingredients of a system for the observation of complex phenomena? Before being tempted to answer this question, it comes the doubt about whether it is reasonable to expect complex systems to have a well defined number of characteristic properties. Unavoidably, our mind tends naturally to overuse classification, which is the natural (hardwired) way of thinking we manage for everyday's life. However, the holistic roots of emergent phenomena makes it intrinsically difficult to have well defined boundaries between what is a real complex system and what is not. (In fact, we have up to now defined a complex system by their behavioural rather than structural properties.) On the other hand, the number of complex phenomena observed numerically and experimentally gives us some hints for unveiling some recurrent structural ingredients. Let us briefly summarize the most relevant ones:

- Nonlinearity: It is clear that only a few natural systems can be described by means of linear relations. The need for a nonlinear modeling of the interactions is clearly seen by the nonlinear response of many real systems to perturbations. In fact, nonlinearity also appears when first principles equations are obtained for very simple systems.

- Non regular structure of the interactions: The network of interactions is of utmost importance. It is revealed a high diversity in the amount of connections that a single element of the system has with combination of short-range and long-range links. In addition, loops are very usual in real systems.

- Surroundings do matter: Many complex systems are open. They may share a balance between dissipated energy and incoming energy flux with the surroundings in order to achieve dynamical stability.

Although neither complete nor precise (they can be presented alone or all together), the properties listed above are shared by a number of systems where complex phenomena is observed. Evidently, natural complex systems, like e.g. a protein, incorporate all these complexity levels, but, on the other hand, synthetic models incorporating only one of them are able to reproduce the relevant phenomena. It is seen that one of these ingredients in synthetic models, specially in the case of nonlinear systems with many degrees of freedom, can lead the system towards complexity. It is also worth stressing that, as well as the difficulties for explaining complex phenomena, all the above properties incorporate additional mathematical and computational difficulties.

Despite the efforts for unveiling the attributes of a complex system, the question about what are the essential ingredients that generate complexity remains open, specially when it is clear that a variety of dynamical mechanisms can produce self-similar structures. 


\section{Our "Complex Systems"}

The association of several ingredients of complexity in synthetic models is even more interesting than the study of systems where only one source of complexity exists. One would expect the observation of new emergent phenomena, different from those related to any of the sources of complexity. This expectation motivates our studies in this Thesis. In particular, we will focus on extended systems of interacting elements where two sources of complexity are present, namely, nonlinearity and/or structural complexity.

As explained above, the emergence of coherent structures in extended nonlinear systems has been studied since decades. Our concern in this Thesis is to dedicate a first part to the study of localization in nonlinear homogeneous lattices. In particular, we will address the study of localized states in one and two-dimensional nonlinear Schödinger lattices. These states, usually termed intrinsic localized modes or discrete breathers are time periodic, spatially localized and are seen as ubiquitous solutions to a number of homogeneous nonlinear lattices (for it the attribute "intrinsic" in their denomination). Besides, nonlinear Schödinger lattices are seen as paradigmatic equations of importance for several branches of physics like Bose-Einstein condensates or nonlinear optics. In this first part we will be specially interested in the mobility of such coherent structures. The main difference with classical solitons in continuum equations relies in the absence of continuum space translational symmetry, that makes the finding of such solutions non trivial. Besides, we will study other types of coherent states, like discrete vortices or oscillating discrete breathers, in order to have a complete description of the behaviour of localized solutions in this important class of lattices.

The second part of this Thesis will concern the study of complex networks, i.e. extended systems of interacting elements where the patterns of connections between them is random. The study of this class of systems has been traditionally ascribed to graph theory. However, the recent discoveries on the self-similar character of the structure of connections in many real (social, biological, technological, etc...) systems have led to a burst in the activity of the so-called physics of complex networks. There is a lot of important consequences of the scale-free behaviour of real networks, like robustness under random perturbations, the "small-world" effect, absence of threshold for epidemics spreading and a complete new behaviour for most dynamical processes that take place on top of them. The self-similar patterns of connections and its ubiquity in nature lead to the conclusion that a large amount of systems share the same self-organizational principles. However, the question about the mechanism that drives the evolution of networks to these common structural patterns is still unsolved. We will focus on both the modeling of network 
growth and the study of several simple dynamics of interest in human-made (technological) scale-free networks.

The study of complex network structure and the analysis of simple dynamics on top of scale free graphs try to unveil the improvements that a heterogeneous pattern of connections provides to the deployment of the network's function. However, these two elements, function (dynamics) and structure, are many times presented to us entangled. That is, the growth and time evolution of the network of interactions (that determines its scale-free feature) is performed at the same time the system develops its function. Therefore, the structure is the result of a kind of selective process that drives to the most efficient architecture. In this case, the study of how network grows is similar to the problem of finding those network architectures that are the most efficient for its functioning. Besides, most of the dynamics of real systems are seen to be nonlinear and, therefore, the analysis of systems of elements with both nonlinear and random interactions comes as necessary. Our main purpose in the third part of the Thesis is to analyze two systems of this kind and, therefore, approach to the problem on the Structure-Function relation. We will analyze this relation in two biologically relevant systems, namely, a scale-free network with activatory-inhibitory (Michaelis-Menten type) interactions and the Kuramoto model of phase oscillators on top of different network architectures. In these two studies we do not pretend to find the definite answer to the Structure-Function problem, but to discuss new tools and discover new phenomena that could lead to a better understanding of this relation.

The studies on this Thesis are thus separated in three parts depending on the sources of complexity involved in their description: nonlinearity (Part I), structural complexity (Part II) and (finally Part III) both. Along this thesis we will face problems associated to the emergence of coherent structures, selfsimilar structural patterns and finally self-organization of dynamical patterns. Therefore, the concept of emergence will be the recurrent idea behind our studies. 


\section{Part I}

\section{Intrinsic localization in nonlinear Schrödinger lattices}





\title{
Presentation of Part I
}

\begin{abstract}
In this first part of the Thesis we will analyze the phenomenon of intrinsic localization in nonlinear lattices. In particular, we will focus on the study of intrinsic localized states solutions to nonlinear Schrödinger lattices. Let us first motivate this study by reviewing the birth and growth of the physics of intrinsic localized modes.

Localized states in condensed matter physics has been usually ascribed to the presence of inhomogeneities or disorder that, due to the breaking of the discrete translational symmetry of the system, lead to the observation of phonon modes which are localized around these inhomogeneities or to the socalled Anderson localization[17]. This conception of localization in discrete systems has been recently extended with the discovery $[18,19]$ of the so-called intrinsic localization in completely homogeneous periodic structures. The term intrinsic localization refers to the possibility of having spatially localized and time periodic excitations due to the presence of both discreteness and strong nonlinearity in homogeneous lattices. These two ingredients, discreteness and nonlinearity, are seen as the essential ingredients for the observation of intrinsic localized modes.

Intrinsic localized modes were also termed as discrete breathers in the literature. These two names reflect, as reported in [20], the convergence of two different ways of interpreting this kind of solutions. On one hand, the view of intrinsic localization in lattices as a result of the presence of nonlinearity instead of impurities, and on the other hand, as a result of the stabilization of the localized periodic oscillation due to discreteness, that avoids the decay of the excitation into radiation. The former interpretation led to term these solutions as intrinsic localized modes pointing out that localization of the periodic state is a result of the self-trapping effects caused by the nonlinear terms in contrast to the localization around extrinsic defects or impurities in linear lattices. The latter point of view tries to resemble these states to that particular soliton solution (called breather due to its time periodicity) obtained for the continuous sine-Gordon system [21]. The finding of this breathing solution to the sine-Gordon model remained as a very exceptional feature for continuous models related to its integrable character. While physicists concerned with continuous models tried to reproduce these solutions in other continuous models they realized that the discretization performed for the numerical simulations provided an apparent robustness to localized breathing solutions. These observations constituted the hint for the existence of discrete breathers as true solutions of nonlinear lattices.
\end{abstract}


The above two approaches to intrinsic localization in nonlinear lattices (one going from linear to nonlinear discrete systems and the second from continuous to discrete nonlinear models) finally converged to conclude that intrinsic localized modes or discrete breathers should be generic solutions to nonlinear extended lattices. The heuristic explanation argued was that in nonlinear lattices the plane wave spectra are bounded due to discreteness, thus making possible the absence of multi-harmonic resonances of the exact discrete breather solution with extended modes. Therefore, the combination of nonlinearity and discreteness is sufficient for the physical existence of discrete breathers resulting in its generality and broad interest. This extreme was further supported by the development of the theory for the existence of intrinsic localized modes in nonlinear discrete systems [22] and nowadays the study of nonlinear lattices have become the subject of a considerable multidisciplinary interest. The broad range of applications in physics subdisciplines covers fields as diverse as biophysics (myelinated nerve fibers [23], DNA [24, 25], biopolymer chains [26]), nonlinear optical devices (photonic crystals [27] and waveguides [28, 29]), and Josephson effect [30] (superconducting devices [31, 32], Bose-Einstein condensates [33-35]), among others [36-38]. From a theoretical perspective nonlinear lattices have been progressively recognized not as mere discretizations (unavoidable for numerical computations) of nonlinear continuous field equations, but as a target of interest in their own right, due to the distinctive features associated with discreteness, whose relevance to experimental features have been largely established.

Though many of the properties of discrete breathers are today well characterized $^{1}$, several questions remain unsolved and their most promising applications are yet to be developed. From the theoretical point of view, the question on their mobility has been a constant source of controversy. The heuristic arguments tell about the impossibility of having moving localized structures in lattices due to the radiative losses unavoidably associated to the translational motion of the localized pulse in generic systems. Our main concern in this part will be to analyze this problem for nonlinear Schrödinger lattices where important applications are found.

The purpose of this first part of the Thesis is to characterize these mobile solutions along with the well known pinned ones and, besides, more exotic types of localized structures (like bound states of intrinsic localized modes, oscillating discrete breathers, pulsons, or discrete vortices) by a systematic study of their existence, stability and dynamics. For these purposes, we have divided this part into three chapters. First, in chapter 2, we will briefly present the nonlinear Schrödinger lattice equations and the essential techniques to be

\footnotetext{
${ }^{1}$ Interesting reviews and tutorials on the subject are found in [20, 39-43].
} 
employed in the following chapters. Secondly, in chapter 3, we will analyze the one-dimensional case, where we will mainly focus on the mobility of localized structures and its physical interpretation. In this chapter, we will also analyze a new type of pinned solutions, termed cuspons, that arise when competing (self-focusing and self-defocusing) nonlinearities come into play. Finally, the two-dimensional version of the model equations is studied in chapter 4. Here, besides generic two-dimensional solutions like discrete vortices, two important issues are of importance, namely: $(i)$ the quasi-collapse instability of discrete breathers (that turns localized solutions into pulsonic structures), and (ii) the extension of the one-dimensional mobile solutions to their two-dimensional counterparts.

The discussion of the results in chapters 3 and 4 tries to unify different approaches to and studies of the problem in order to provide a coherent enough picture about the behaviour of discrete breathers in nonlinear Schrödinger lattices. We hope that the results shown would provide a path for studying similar problems in other interesting models. 



\title{
Chapter 2
}

\section{Discrete Breathers and Nonlinear Schr̈odinger lattices}

\begin{abstract}
I was observing the motion of a boat which was rapidly drawn along a narrow channel by a pair of horses, when the boat suddenly stopped but not so the mass of water in the channel which it had put in motion; it accumulated round the prow of the vessel in a state of violent agitation, then suddenly leaving it behind, rolled forward with great velocity, assuming the form of a large solitary elevation, a round, smooth and well-defined heap of water, which continued its course along the channel apparently without change of form or diminution of speed. I followed it on a horseback, and overtook it still rolling on at a rate of some eight or nine miles an hour, preserving its original figure some thirty feet long and a foot to a foot and a half in height.
\end{abstract}

Observation of a solitary wave formation in 1834 by John Scott Russel [44].

Discrete breathers, also called intrinsic localized modes, are ubiquitous solutions $\left\{\Phi_{n}(t)\right\}$, with $n=-\infty, \ldots, 0, \ldots, \infty$, to extended anharmonic lattices. These states are time periodic, with a well defined frequency $\omega_{b}$, solutions where the energy is confined on a few number of sites. A general form of this solutions can be expressed as

$$
\Phi_{n}(t)=f\left(n-x_{0}\right) \exp \left(\mathrm{i} \omega_{b} t\right),
$$

with $f\left(n-x_{0}\right) \sim \exp \left[-\Gamma\left|n-x_{0}\right|\right]$ when $n \rightarrow \pm \infty$. The parameter $x_{0}$ is the localization center and $\Gamma(>0)$ accounts for the decay rate and hence quantifies the degree of energy localization.

The first observations of energy localization in anharmonic lattices where accidentally found by E. Fermi, J.R. Pasta, S.M. Ulam and M. Tsingou [12] in 
1955. This "little discovery" were followed by the burst of nonlinear science led by theoretical advances in soliton theory or integrable systems. The growth of computer processing power turned again the view to nonlinear lattices and, after the theoretical work of A.J. Sievers, S. Takeno and K. Kisoda [18, 19] in 1988 discovering a new localized mode for pure anharmonic lattices, several numerical observations of these kind of solutions were reported by several authors for different nonlinear lattices [45-48]. It is worth mentioning that the observation of these localized states was found to be generic of homogeneous nonlinear lattices and therefore different from that due to presence of Anderson modes as a consequence of the existence of any inhomogeneity (defect or impurity) of the harmonic lattice. Observations of genuine discrete breather solutions were mainly based on the numerical simulations of the nonlinear dynamics.

The question on their existence as true solutions of the nonlinear lattice remained unsolved until R.S. Mackay and S. Aubry [22] established the theorem for the existence of discrete breather solutions. This theorem is based on the concept of anti-integrability (developed by S. Aubry for studying the Frenkel-Kontorova model $[49,50])$ or, applied to general lattices, the anticontinuum limit. This concept refers to the limiting case when there is no coupling between adjacent sites of the lattice so that the system is composed of a set of independent oscillators whose dynamics is governed by their corresponding on-site potentials. Then, considering the state where a single oscillator evolves following an orbit of frequency $\omega_{b}$ while the remaining sites are in the rest state one can ask whether this state of energy confinement would remain when the coupling between sites is adiabatically incorporated. The continuability of discrete breathers from the uncoupled limit implies two conditions

- Non-resonance condition: The oscillation frequency and its harmonics must rely outside the phonon band of the lattice at the rest state

$$
n \omega_{b} \neq \omega(q) \quad \forall q \in[-\pi / 2, \pi / 2] \quad(n=1,2, \ldots)
$$

- Anharmonicity condition: The on-site potentials governing the dynamics of the isolated sites must be nonlinear so that the frequencies, $\omega_{b}$, of their orbits fulfills $\partial \omega_{b} / \partial I \neq 0$, where $I$ is the action.

The proof of the existence theorem is based on the implicit function theorem and provides a practical way for constructing localized solutions of the type (2.1).

After the rigorous formulation of the existence conditions of discrete breathers several questions arised. From one hand, its stability and robustness in noisy environments has been studied in detail [51] since their experimental observation and potential applications to real systems implies relative large life times. 
Another hot topic is the issue of their mobility. Taking into account the general form (2.1) of a pinned discrete breather one would expect their mobile counterparts to have the form

$$
\Phi_{n}(t)=f\left(n-v_{b}-x_{0}\right) \exp \left(\mathrm{i} \omega_{b} t\right),
$$

with $f\left(n-v_{b}-x_{0}\right) \sim \exp \left[\Gamma\left|n-v_{b} t-x_{0}\right|\right]$ when $n \rightarrow \pm \infty$. The possibility of transferring energy packets across lattices opens the door to a wide range of applications in nonlinear optics, solid state and soft matter physics. However, since the continuous translational invariance is broken due to discreteness, the computation of pinned discrete breathers of the form (2.1) does not guarantee the success in constructing mobile localized states like (2.3) by means of a change of the reference system. Different approaches have been used for studying this problem ranging from the "kicking" method [52-55] (where a static solution is perturbed with the so-called pinning or marginal mode in order to make it move) to analytical approximations were continuous variables (collective coordinates) accounting for the localization center are introduced [56-60]. Our approach to this problem tries to generalize the method of continuation for pinned breathers to obtain mobile solutions. For this purpose we start with make use of the concept of $(p, q)$ resonant states that will allow us to unify the problem of finding both mobile and pinned discrete breathers.

In this chapter we introduce the set of nonlinear Schrödinger equations that we study along the two forthcoming chapters as well as to summarize the basic definitions and techniques used for characterizing breather solutions to these equations. We will start in section 2.1 describing the Salerno model [61] which provides a two-parametric family of nonlinear Schrödinger lattices. In section 2.2 we address the definition of the concept of $(p, q)$ resonant solutions to which general discrete breather solutions belong. Finally, in section2.3 the basic technique to obtain and characterize discrete breathers are summarized.

\subsection{The Salerno Model}

The continuous nonlinear Schrödidinger equation (NLS) constitutes a key tool for a number of fields as diverse as the study of Bose-Einstein condensates (where the mean field approximation is of the NLS-type, the Gross-Pittaevskii equation), the study of nonlinear (Kerr type) optical fibers, molecular chains (where Davydov solitons are studied), etc... Besides, the NLS equation is specially interesting for nonlinear physics since it appears when considering the lowest order of nonlinearity for any dynamical equation on a dispersive medium where energy is conserved. The most general form of this equation is

$$
\mathrm{i} \dot{\Phi}(x, t)=-\nabla^{2} \Phi(x, t)-\gamma|\Phi(x, t)|^{2} \Phi(x, t),
$$


where $\Phi(x, t)$ is a complex field which, in the context of Bose-Einstein condensates, accounts for the macroscopic wave-function of the condensate. The parameter $\gamma$ accounts of the competence between the dispersive (Laplacian term) and the nonlinear parts. This cubic nonlinear equation posses the singular property of being integrable. The integrability was probed by means of the Inverse Scattering Method (ISM) technique [11, 62, 63] in [64] providing a family of nonlinear waves.

\section{The Discrete Nonlinear Schrödinger equation}

The physical relevance of the NLS equation along with its integrable character make it one of the most studied models by nonlinear physicists during the last decades. Besides, discretizations of this equation are also of great interest. The natural the discretization of eq. (2.4) yields the so-called standard discrete nonlinear Schrödinger equation (DNLS) [23],

$$
\mathrm{i} \dot{\Phi}_{n}=-C\left(\Phi_{n+1}+\Phi_{n-1}\right)-\gamma\left|\Phi_{n}\right|^{2} \Phi_{n},
$$

where $\Phi_{n}$ is now a complex variable, the parameter $C$ amounts the nearest neighbor coupling, and $\gamma$ is the strength of the nonlinearity. The above discretization does not conserve the integrability of the continuous model (2.4) although the wide applicability to physical fields is preserved. In particular, the DNLS equation is particularly relevant for

- Dynamical description of Bose-Einstein condensates trapped in a periodic potential well (optical trap) [33-35, 65-68].

- Pulse dynamics in nonlinear waveguide arrays [28, 29, 69-72].

- Adiabatic approximation of the Holstein polaron [23].

- Excitation dynamics in biopolymers lattices [26].

The dynamics governed by the DNLS equation (2.5) is derived from the Hamiltonian

$$
\mathcal{H}=-C \sum_{n}\left(\Phi_{n} \bar{\Phi}_{n+1}+\bar{\Phi}_{n} \Phi_{n+1}\right)-\frac{\gamma}{2} \sum_{n}\left|\Phi_{n}\right|^{4}
$$

where $\bar{\Phi}_{n}$ denotes the complex conjugate of $\Phi_{n}$. Both variables, $\Phi_{n}$ and $\bar{\Phi}_{n}$, are canonically conjugated with the usual Poisson structure

$$
\{U, V\}=\sum_{n}\left[\frac{U}{\Phi_{n}} \frac{V}{\bar{\Phi}_{n}}-\frac{V}{\Phi_{n}} \frac{U}{\bar{\Phi}_{n}}\right] .
$$


Beside, the DNLS equation has a second integral of the dynamics, namely the norm,

$$
N=\sum_{n}\left|\Phi_{n}\right|^{2},
$$

that in the context of Bose-Einstein condensates accounts for the total number of bosons whereas for waveguides arrays it is the total power of the beam.

\section{The Ablowitz-Ladik equation}

Another important discretization of the continuous NLS equation is the socalled Ablowitz-Ladik equation (AL). Although this lattice is not so physically relevant as the usual discretization, DNLS equation (2.5), the AL model preserves the integrability of its continuous counterpart (2.4). In fact, the AL model is an extremely exceptional example of an integrable nonlinear lattice that was discovered by M.J. Ablowitz and J.F. Ladik in $1976[73,74]$ by means of the ISM in its discrete version $[75,76]$. The AL model reads as follows

$$
\mathrm{i} \dot{\Phi}_{n}=-\left(\Phi_{n+1}+\Phi_{n-1}\right)\left[C+\frac{\gamma}{2}\left|\Phi_{n}\right|^{2}\right],
$$

where again $\Phi_{n}(t)$ is a complex probability amplitude, the parameter $C$ amounts the nearest neighbor coupling, and $\gamma$ is the strength of the nonlinearity. The nonlinear term in the $\mathrm{AL}$ equation is of the intersite type and hence differs with its counterpart in the DNLS model which is an onsite nonlinearity.

The AL model (2.9) has a deformed Poisson structure defined by

$$
\{U, V\}=\sum_{n}\left[\frac{U}{\Phi_{n}} \frac{V}{\bar{\Phi}_{n}}-\frac{V}{\Phi_{n}} \frac{U}{\bar{\Phi}_{n}}\right]\left(1+\frac{\gamma}{2}\left|\Phi_{n}\right|^{2}\right),
$$

and the conserved Hamiltonian is

$$
\mathcal{H}=-C \sum_{n}\left(\Phi_{n} \bar{\Phi}_{n+1}+\bar{\Phi}_{n} \Phi_{n+1}\right)
$$

The integrability of the AL equation results in an infinite number of conserved quantities. Along with the Hamiltonian the two conserved magnitudes of lowest order in $\left\{\Phi_{n}\right\}$ are

$$
\begin{aligned}
N & =\frac{2}{\gamma} \sum_{n} \ln \left(1+\frac{\gamma}{2}\left|\Phi_{n}\right|^{2}\right), \\
P & =\mathrm{i} \sum_{n}\left(\Phi_{n} \bar{\Phi}_{n+1}-\bar{\Phi}_{n} \Phi_{n+1}\right),
\end{aligned}
$$

which are the norm and the momentum respectively. 
The integrable AL equation possesses a two-parameter family of exact breather solutions of the form

$$
\begin{gathered}
\Phi_{n}(t)=\quad \sqrt{\frac{2}{\gamma}} \sinh \beta \operatorname{sech}\left[\beta\left(n-x_{0}(t)\right)\right] \times \\
\exp \quad\left[\mathrm{i}\left(\alpha\left(n-x_{0}(t)\right)+\Omega(t)\right)\right] .
\end{gathered}
$$

As can be observed the solutions possess the continuous spatial symmetry $x_{0} \rightarrow x_{0}+\epsilon$ and hence analytic mobile breather solutions with a similar form to eq. (2.3) are available for this exceptional lattice. The two parameters of this breather family can be chosen to be the breather frequency $\omega_{b}$ and velocity $v_{b}$,

$$
\begin{aligned}
v_{b} & =\dot{x}_{0}=\frac{2 \sinh \beta \sin \alpha}{\beta} \\
\omega_{b} & =\dot{\Omega}=2 \cosh \beta \cos \alpha+\alpha v_{b},
\end{aligned}
$$

where $-\pi \leq \alpha \leq \pi$ and $0<\beta<\infty$. The AL moving breather (instantaneous) profile interpolates between the rest state $\Phi_{n}=0$ of the lattice (at $n \rightarrow \pm \infty$ ) in an exponentially localized region around $x_{0}(t)$, while traveling with velocity $v_{b}$.

\section{The Salerno model}

In the above two equations, DNLS (2.5) and AL (2.9), the self-focussing effect of local nonlinearity balanced by the opposite effect of the dispersive coupling makes possible the existence of localized periodic solutions (breathers) of the discrete field, where the profile of $\left|\Phi_{n}\right|$ decays exponentially away from the localization center:

$$
\left.\Phi_{n}(t)=\left|\Phi_{n}\right| \exp \left[i \omega_{b}(t)\right)\right]
$$

In the uncoupled limit $C \rightarrow 0$ of the DNLS equation, also known as the antiintegrable or anti-continuous limit, discrete breathers can be easily constructed by selecting a periodic oscillation $\Phi_{n_{0}}(t)$ of frequency $\omega_{b}=\gamma\left|\Phi_{n_{0}}\right|^{2}$ at site $n_{0}$ and $\Phi_{n}=0$ for $n \neq n_{0}$. These solutions can be uniquely continued (we will see the procedure below) to nonzero values of the coupling $C$, and constitute the one-parameter family of immobile on-site breathers of the DNLS equation.

Unfortunately, the continuation from the uncoupled limit does not provide solutions where the localization center moves along the lattice with velocity $v_{b}$ (as for the $\mathrm{AL}$ case), i.e, mobile discrete breathers. On the other hand, the connection between the integrable (though physically limited) AL equation 
and the physically relevant (though nonintegrable) DNLS equation is provided by the model originally introduced by M. Salerno in [77],

$$
\mathrm{i} \dot{\Phi}_{n}=-\left(\Phi_{n+1}+\Phi_{n-1}\right)\left[C+\mu\left|\Phi_{n}\right|^{2}\right]-2 \nu \Phi_{n}\left|\Phi_{n}\right|^{2} .
$$

The above lattice provides a Hamiltonian interpolation between the standard DNLS equation (2.5), for $\mu=0$ and $\nu=\gamma / 2$, and the integrable AL lattice (2.9) when $\mu=\gamma / 2$ and $\nu=0$. In the following we will set the value of $\gamma=2$. The Hamiltonian of the Salerno equation is given by

$$
\begin{aligned}
\mathcal{H}= & -C \sum_{n}\left(\Phi_{n} \bar{\Phi}_{n+1}+\bar{\Phi}_{n} \Phi_{n+1}\right)-2 \frac{\nu}{\mu} \sum_{n}\left|\Phi_{n}\right|^{2} \\
& +2 \frac{\nu}{\mu^{2}} \sum_{n} \ln \left(1+\mu\left|\Phi_{n}\right|^{2}\right),
\end{aligned}
$$

which contains the AL and DNLS Hamiltonian for the above limits. The Poisson structure of the Salerno model takes the form

$$
\{U, V\}=\sum_{n}\left[\frac{U}{\Phi_{n}} \frac{V}{\bar{\Phi}_{n}}-\frac{V}{\Phi_{n}} \frac{U}{\bar{\Phi}_{n}}\right]\left(1+\mu\left|\Phi_{n}\right|^{2}\right),
$$

which, for $\mu \neq 0$, takes the same functional form as that of the Ablowitz-Ladik equation, eq. (2.10), and in the limit $\mu=0$ it becomes the standard Poisson structure according to that of the DNLS limit, eq. (2.7). In addition to the Hamiltonian, this equation possesses, for any value of the parameters $\mu$ and $\nu$, the following conserved norm

$$
N=\frac{1}{\mu} \sum_{n} \ln \left(1+\mu\left|\Phi_{n}\right|^{2}\right) .
$$

While the SM was originally introduced in a rather abstract context, it has recently found direct physical realization, as an asymptotic form of the GrossPitaevskii equation describing a Bose-Einstein condensate of bosonic atoms with magnetic momentum trapped in a deep optical lattice [78]. In that case, the onsite nonlinearity is generated, as usual, by collisions between atoms, while the intersite nonlinear terms account for the long-range dipole-dipole interactions. This latter interaction may be attractive $(\mu>0)$ or repulsive $(\mu<0)$, if the external magnetic field polarizes the atomic momentum along the lattice or perpendicular to it, respectively.

The continuation of the family (both pinned and mobile) discrete breathers from the AL integrable limit allows numerical observations of the interplay between the integrable term, weighted by the parameter $\mu$, and the nonintegrability, weighted by $\nu$. We will inspect the effects that the combination of these two nonlinearities, with both similar and opposite signs, has on discrete breathers dynamics. 


\subsection{Discrete space-time symmetries: $(p, q)$ resonant states}

In order to unify the problem of finding pinned and mobile discrete breathers by means of continuation methods we start defining the concept of $(p, q)$ resonant states. Suppose that a frequency $\omega_{b}=2 \pi / T_{b}$ is given, we will say that a solution $\Phi=\left\{\Phi_{n}(t)\right\}$ is $(p, q)$ resonant with respect to the reference frequency $\omega_{b}$, if the following condition holds, for all $n$ and $t$ :

$$
\Phi_{n}(t)=\Phi_{n+p}\left(t+q T_{b}\right) .
$$

After $q T_{b}$-periods, these solutions repeat the same profile but displaced by $p$ lattice sites. In more technical terms, these $(p, q)$ resonant solutions are fixed points $\Phi$ of the operator

$$
\begin{aligned}
\mathcal{L}^{p} \mathcal{T}^{q} & =\mathcal{M} \\
(\mathcal{M}-\mathcal{I}) \Phi & =0
\end{aligned}
$$

where $\mathcal{L}$ and $\mathcal{T}$ are, respectively, the lattice translation and the $T_{b}$-time evolution operator

$$
\begin{aligned}
\mathcal{L}\left\{\Phi_{n}(t)\right\} & =\left\{\Phi_{n+1}(t)\right\} \\
\mathcal{T}\left\{\Phi_{n}(t)\right\} & =\left\{\Phi_{n}\left(t+T_{b}\right)\right\} .
\end{aligned}
$$

We now consider some examples of $(p, q)$ resonant solutions with respect to the frequency $\omega_{b}$; the first example is simply provided by the family of plane wave solutions of eq. (2.18):

$$
\Phi_{n}(t)=A \exp [\mathrm{i}(k n-\omega t)] .
$$

It is easily seen, by inserting (2.27) in eq. (2.18), that the values of $\omega, k$ and $|A|$ define a surface in the three-dimensional space, the nonlinear dispersion relation surface $\omega(k, A)$ (see figure 2.1 ):

$$
\omega=-2\left[1+\mu|A|^{2}\right] \cos k-2 \nu|A|^{2} .
$$

Note that due to the nonlinear character of the eq. (2.18), the frequency $\omega$ depends on both wave number $k$ and amplitude $|A|$ of the plane wave.

One can easily determine those plane waves that are $(p, q)$ resonant with respect to $\omega_{b}$ : the eq. (2.22) imposes the following condition on $\omega$ and $k$

$$
\frac{\omega}{\omega_{b}}=\frac{1}{q}\left(\frac{p}{2 \pi} k-m\right),
$$


where $m$ is any arbitrary integer. These planes in the 3 -d space $(\omega,|A|, k)$ intersect the dispersion relation surface at (in general) several one-parameter families (branches) $k_{j}(|A|)$, in the first Brillouin zone $(-\pi \leq k \leq \pi)$.

If we are not interested in unreasonably large (and not interesting) amplitude values $|A|$ of the plane waves, the number of branches is finite: one can see that for fixed values of all the parameters $\left(p, q, \omega_{b}, \nu, \mu\right)$, there is a finite number of branches in the limit $|A| \rightarrow 0$; there is also a well defined (parameter dependent) threshold value of the amplitude at which a pair of new branches (tangent bifurcation) appear (i.e. these plane waves can only resonate with $\omega_{b}$ for amplitudes above some threshold value).

Thus, by a suitable bounding of the amplitude, for each couple $(p, q)$ one finds a finite number, $s$, of branches of $(p, q)$ resonant plane waves. (Note also that this number diverges when $p / q$ tends to an irrational).

A different, and highly nontrivial, example of $(p, q)$ resonant solutions is provided by the solitary waves (2.14) of the AL lattice. From eq. (2.16) it is clear that the choice $2 \pi v_{b} / \omega_{b}=p / q$ selects a $(p, q)$ resonant solitary wave with respect to the frequency $\omega_{b}$, i.e. a breather solution where the two time scales involved, given by its frequency $\omega_{b}$ and velocity $v_{b}$, are commensurate. The set of velocity values of resonant AL breathers is dense and any AL moving breather is a limit of some sequence of resonant ones. Note also that immobile breathers are $(0,1)$ resonant with respect to the frequency $\omega_{b}$.

In the integrable limit, the plane waves and the $\mathrm{AL}$ breathers are both exact independent solutions. Integrability makes possible that the initial localization of energy is maintained with time evolution, without decaying away by exciting radiation. It is a well established result that (even far away from this integrable limit) immobile discrete breathers remain exact solutions of the lattice dynamics. Our concern in the next sections is the question of moving

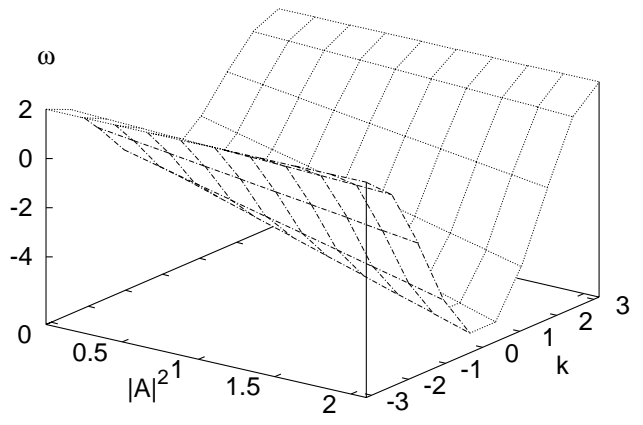

Figure 2.1: Plot of the nonlinear dispersion relation surface of nonlinear plane waves, eq. (2.28), as a function of the amplitude $A$ and the wave number $k$ of the plane wave. The values of $\mu$ and $\nu$ are fixed to 0.5 . 
discrete breathers away from integrability in eq. (2.18). In order to study them, we will focus on $(p, q)$ resonant solutions. The motivating of this restriction comes from its accessibility to numerics. First we will motivate the numerical (Newton) method that allow us to study these solutions with an adequately high precision.

\subsection{Discrete Breathers numerics}

We introduce here the numerical techniques that we have used. As a whole, one could refer to them as the (SVD-) regularized Newton method. They do not constitute a novel method in "discrete Breather numerics", as they have been already used, e.g. in [79] to refine moving breathers of Klein-Gordon lattices obtained by other numerical means (see, by contrast, [80]). From the methodological side, what is novel here is the systematic use of them in the investigation of the family of moving Schrödinger breathers reported below in 3.1 .

To some extent, the presentation here is self-contained but for further details on these techniques we refer to the Appendices and the proposed bibliography. First, in 2.2 we introduce the notion of $(p, q)$ resonant solution, providing some illustrative examples. The (SVD) regularized Newton algorithm is presented in 2.3.1, and finally in 2.3 .2 we briefly explain the basics of Floquet stability analysis.

\subsubsection{Newton continuation}

A well-known numerical procedure to obtain exact periodic solutions of nonlinear lattices is the Newton continuation $[22,53,79,81]$. The different practical implementations of this procedure work very successfully when, for example, one obtains numerically exact immobile discrete breathers of eq. (2.18), from the uncoupled limit $\mu=0$ and $C=0$, where exact periodic discrete breathers are trivially constructed.

The iteration of the Newton operator $\mathcal{T}$ converges rapidly to its fixed point (i.e. the solution to be computed) provided the starting point, $\hat{\Phi}^{0}$, is close enough, and the solution of the following system of linear equations is a wellposed problem:

$$
(D \mathcal{T}-1)\left(\Phi^{n}-\Phi^{n+1}\right)=[\mathcal{T}-\mathcal{I}] \Phi^{n},
$$

where $D \mathcal{T}$ is the Jacobian matrix of the Newton operator, and $\Phi^{n}$ (the $n$-th iteration solution of (2.30)) converges quadratically to the fixed point solution. By adiabatic change of a model parameter, one constructs a uniquely continued 
exact fixed point solution for each parameter value, using each time, as starting point of the Newton iteration, the solution previously computed.

The matrix $(D \mathcal{T}-1)$ must be invertible, in order to uniquely compute $\Phi^{n+1}$. Degeneracies associated with the +1 eigenvalues of $D \mathcal{T}$, if any, have to be removed in order to obtain a unique fixed point solution. When continuing immobile (time periodic) discrete breathers of eq. (2.18), a convenient prescription is commonly used, namely to restrict the operator action to the subspace of time-reversible solutions (see Appendix A and [53, 81]). This provides a practical way of removing degeneracies, allowing unique continuation of immobile discrete breathers.

However, for the continuation of general $(p, q)$ resonant solutions (of which periodic solutions are only the particular case $p=0$ and $q=1$ ), one has to use $\mathcal{M}=\mathcal{L}^{p} \mathcal{T}^{q}$ as the Newton operator. One has also to deal with the degeneracies of $\mathcal{M}$, and imposing time-reversibility could, in this case, be too restrictive, since in general $(p, q)$ resonant solutions are not time-reversible.

A well-known solution to the problem of removing degeneracies when no clear restrictions are available, is provided by the so-called singular value decomposition (SVD) $[53,79,82,83]$ of the matrix $\left(D \mathcal{L}^{p} \mathcal{T}^{q}-1\right)$ :

$$
\left(D \mathcal{L}^{p} \mathcal{T}^{q}-1\right)=J=P V Q
$$

where $P, V$ and $Q$ are $2 N \times 2 N$ square matrices. $P$ and $Q$ are orthogonal matrices and $V$ is diagonal $\left(v_{j} \delta_{i j}\right)$ with possibly null (zero) elements, called singular values, associated with the null space of $J$ (the subspace that is mapped to zero $J x=0)$. The columns of $P$ whose same-numbered elements $v_{j}$ are nonzero are an orthonormal set of basis vectors that span the range of $J$ (the subspace reached by this matrix). The rows of $Q$ whose same-numbered elements $v_{j}$ are zero are an orthonormal basis for the null space of $J$. One can numerically use this SVD decomposition, checking the (numerical) vectors spanning the null space to identify degeneracies, and using at iteration steps the pseudoinverse matrix

$$
Q^{*} \hat{V}^{-1} P^{*},
$$

where $\hat{V}^{-1}$ is diagonal with elements $1 / v_{j}$ for $v_{j} \neq 0$ and 0 for $v_{j}=0$.

The convergence criterion for the fixed point solution is that

$$
\sum_{j}\left|\left([\mathcal{T}-\mathcal{I}] \Phi^{n+1}\right)_{j}\right|<N \cdot 10^{-16}
$$

where $\mathrm{N}$ is the size of the lattice, i.e. the solutions obtained along the two forthcoming chapters can be regarded as exact up to machine precision. As a judicious test of our numerical codes, we have used both procedures (reduction 
to time-reversible subspace and SVD decomposition) to obtain immobile discrete breathers (for which both methods are valid) of the Salerno model. Both agree, up to the highest possible accuracy, from the uncoupled limit up to the A-L limit (and viceversa).

\subsubsection{Floquet stability analysis}

A very useful outcome of the numerical Newton method of computing solutions of eq. (2.18) is the Jacobian matrix of the Newton operator, usually called the Floquet matrix $F$. This matrix is the linear operator associated with the linear stability problem (see Appendix B and [84]) of the fixed point solution.

Indeed, the Jacobian $F$ of the Newton operator $\mathcal{M}$

$$
F=D \mathcal{M}
$$

maps vectors in the tangent space of the solution (small initial perturbations $\vec{\epsilon}(0)$ of the fixed point solution) into their $T_{\mathcal{M}}$-evolved vectors, i.e. $\vec{\epsilon}\left(T_{\mathcal{M}}\right)$, after a period of $\mathcal{M}$. That is:

$$
\vec{\epsilon}\left(T_{\mathcal{M}}\right)=\mathcal{F} \vec{\epsilon}(0)
$$

The Floquet matrix of a Hamiltonian system is real and symplectic, so the Floquet eigenvalues $\lambda$ come in quadruplets, $\lambda, 1 / \lambda, \bar{\lambda}, 1 / \bar{\lambda}$. The necessary condition for the stability of the solution is that all the eigenvalues lie on the unit circle of the complex plane, $|\lambda|=1$.

To illustrate the Floquet analysis of $(p, q)$ resonant solutions of the NLS lattice (2.18), we now obtain the Floquet spectrum of modulational instabilities of a $(p, q)$ resonant plane wave,

$$
\Phi_{n}(t)=A \exp i(k n-\omega t) .
$$

One has to investigate the evolution of small perturbations, in both amplitude and phase, of the plane wave

$$
\Phi_{n}(t)=\left(A+I_{n}\right) \operatorname{expi}\left(k n-\omega t+\varphi_{n}\right),
$$

where we assume that the perturbation parameters are small compared with those of the plane wave solution. Introducing expression (2.37) in (2.18) and considering the following form for the perturbations $\left\{I_{n}, \varphi_{n}\right\}$ :

$$
\begin{aligned}
I_{n}(t) & =I \operatorname{expi}(Q n-\Omega t) \\
\varphi_{n}(t) & =\varphi \operatorname{expi}(Q n-\Omega t)
\end{aligned}
$$




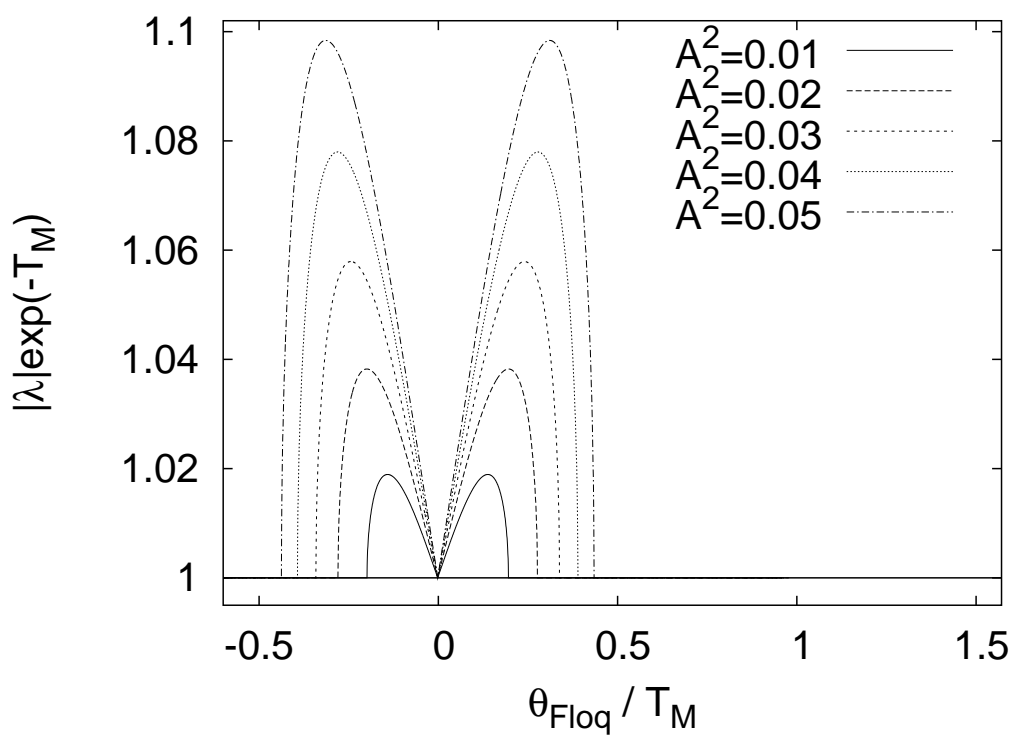

Figure 2.2: Plot of the modulus of the unstable Floquet eigenvalues $|\lambda|$ (corresponding to the positive values of $\Im(\Omega)$ in eqs. (2.44) and (2.45)), versus the Floquet angle, $\theta_{\text {Floq }}$. Both quantities are conveniently normalized to the period of the map $T_{\mathcal{M}}$. The amplitude of the excursion of $|\lambda|$ and the range of values of $\theta_{\text {Floq }}$ for which $|\lambda|>1$ grow as the amplitude $A$ of the plane wave is increased. The parameters in eq. (2.18) are $\mu=\nu=0.5$ and the wave number of the plane wave is $k=0.5$.

we obtain the dispersion relation for the perturbation parameter $\Omega$ :

$$
\begin{aligned}
{\left[\Omega-2\left(1+\mu A^{2}\right) \sin k \sin Q\right]^{2} } & =16\left(1+\mu A^{2}\right) \times \\
\sin ^{2} Q / 2 \cos k[(1 & \left.+\mu A^{2}\right) \sin ^{2} Q / 2 \cos k \\
& \left.-\mu A^{2} \cos k-\nu A^{2}\right],
\end{aligned}
$$

as obtained in $[85,86]$. From the above expression one derives the values of $\Omega(A, Q, k ; \nu, \mu)$ for the modulational perturbations. When the parameter $\Omega$ has a nonzero imaginary part, i.e. the right-hand side of (2.39) is negative, the plane wave $(A, k)$ becomes unstable under the corresponding modulational $(Q)$ perturbation, whose amplitude will grow exponentially fast in the linear regime (tangent space).

Modulational perturbations (2.38) correspond to eigenvectors $\left\{I_{n}, \varphi_{n}\right\}$ of the Floquet matrix:

$$
\begin{array}{r}
I_{n}\left(t+T_{\mathcal{M}}\right)=\exp \left(-\mathrm{i} \Omega T_{\mathcal{M}}\right) I_{n}(t) \\
\varphi_{n}\left(t+T_{\mathcal{M}}\right)=\exp \left(-\mathrm{i} \Omega T_{\mathcal{M}}\right) \varphi_{n}(t)
\end{array}
$$

with associated Floquet eigenvalues $\exp \left(-\mathrm{i} \Omega T_{\mathcal{M}}\right)$. The real part of $\Omega$ gives the 


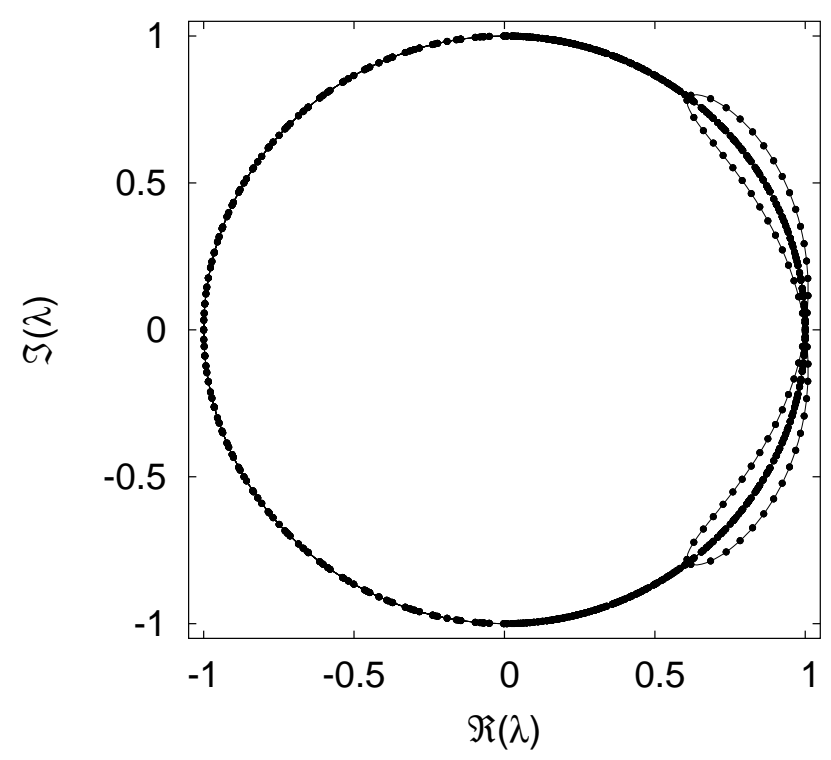

Figure 2.3: Plot of the Floquet spectra of a plane wave with modulational instability (circles) and the theoretical prediction (lines) for the distribution of the Floquet eigenvalues in the complex plane given by eqs. (2.44) and (2.45). The amplitude and wave number of the plane wave are $A=0.1$ and $k=0.1 \cdot 2 \pi$; the nonintegrable parameter value is $\nu=0.1$ and the lattice size is of 400 sites.

angle in the complex plane,

$$
\theta_{\text {Floq }}=-\Re(\Omega) T_{\mathcal{M}}
$$

while the imaginary part $\Im(\Omega)$ gives the modulus of the Floquet eigenvalue,

$$
|\lambda|=\exp \left(\Im(\Omega) T_{\mathcal{M}}\right),
$$

thus providing the information about the linear stability of the solution.

The distribution of angles and moduli in the Floquet spectrum of the modulational instability can be obtained from eq. (2.39) by taking the real and imaginary parts of $\Omega$ :

$$
\begin{aligned}
\Re(\Omega) & =2\left(1+\mu A^{2}\right) \sin k \sin Q \\
\Im(\Omega)^{2} & =-16\left(1+\mu A^{2}\right) \sin ^{2} Q / 2 \cos k \times \\
& \times\left[\left(1+\mu A^{2}\right) \sin ^{2} Q / 2 \cos k\right. \\
& \left.-\mu A^{2} \cos k-\nu A^{2}\right] .
\end{aligned}
$$

In figure 2.2 we represent the modulus of the unstable eigenvalues as a function of the Floquet angle for the spectrum of a $(p, q)$ resonant plane 
wave, taken as an example to visualize the non-point-like character of the instability in the Floquet spectrum in the thermodynamic limit. Note that there is no plane wave harmonic instability $\left(\theta_{F l o q}=0\right)$ due to this mechanism of modulational instabilities.

A numerical computation of the Floquet spectrum of a plane wave (with arbitrary wave number) of a lattice of $N=400$ sites, with periodic boundary conditions is shown in the complex plane representation of figure 2.3. The instability globes, at angles symmetrically placed around zero in this figure, nicely fit the theoretical (thermodynamic limit) values obtained from eqs. (2.44) and (2.45). 



\title{
Chapter 3
}

\section{Discrete Breathers in one-dimensional Nonlinear Schrödinger lattices}

\begin{abstract}
A one-dimensional dynamical system of 64 particles with forces between neighbors containing nonlinear terms has been studied on the Los Alamos computer MANIAC I (...) The results show very little, if any, tendency toward equipartition of energy among the degrees of freedom.
\end{abstract}

First insights on intrinsic localization by E. Fermi, J.R. Pasta, S.M. Ulam and M. Tsingou in 1955 (Extracted from [12]).

In this chapter we report on the most salient findings on discrete breather solutions to the Salerno model (2.18). The generalized continuation scheme based on the $(p, q)$ resonance condition allows to find both pinned and mobile discrete breathers continuing those analytical solutions found for the integrable Ablowitz-Ladik equation.

In the light of the obtained results we can distinguish two regions of the Salerno model. First, the so-called standard Salerno model where $\mu>0$. In this case pinned discrete breathers are feasible states of the dynamics and their characterization for the interesting DNLS limit $[87,88]$ and the SM $[58,59,89,90]$ has been deeply studied. Then, our primary concern in this region is the issue of discrete breather mobility. The main numerical facts about mobile discrete breathers are shown in section 3.1. The numerical solutions are found to be (up to numerical precision) the superposition of a traveling exponentially localized oscillation (the core), and an extended background, which is a 
linear superposition of finite amplitude nonlinear plane waves. Then, contrary to the exact immobile breather solution (space-homoclinic and time-periodic orbit), which asymptotically connects the rest state (vacuum or ground state) of the lattice with itself, each exact mobile localized solution is instead homoclinic to a specific lattice state of extended radiation. In other words, exact stationary mobility of discrete breathers requires an extended excited state of the lattice. In section 3.2 we analyze the numerical results in the light of collective variable theories, correlating them with the main theoretical predictions of this successful (however incomplete) physical perspective. In particular, the existence of Peierls-Nabarro barriers to translational core motion is confirmed, and its subtle relation to the background amplitude is discussed. We present also numerical confirmation of the existence of another type of localized states: exact oscillating anchored breathers. Along with the discussion in this section, a physical interpretation of the role of the interaction background-core in the energy balance emerges, paving the way to a satisfactory integration of the results into a collective variable theory.

The second region of interest is the Salerno model with competing nonlinearities $(\mu<0)$ that we study in section 3.3. In this case the Salerno Model combines onsite self-focusing and intersite self-defocusing cubic terms, which turns to be physically meaningful for describing a Bose-Einstein condensate of dipolar atoms trapped in a strong periodic potential. The analytical results using the continuum approximation predicts a threshold value $\mu_{c}<0$ so that for $\mu<\mu_{c}$ pinned discrete breather solutions do not exist. On the other hand, the numerical continuation of exact discrete breathers shows that the pinned breather family continues beyond this threshold in the form of a novel solution: cuspon states. In-phase and out-of-phase bound states of discrete breathers are also constructed in order to shed light on the new transition found. This results makes the Salerno model with competing nonlinearities also interesting for what concerns pinned discrete breathers. Mobile discrete breathers are finally studied in this region revealing the same results as in the standard Salerno model: they are composed of a moving core and an extended background.

\subsection{Discrete breathers in the standard Salerno model}

The computation of $(p, q)$ resonant discrete breathers in the standard Salerno lattice $(\mu>0)$ is performed following the path $\nu+\mu=1$ (see Figure 3.1) from the AL integrable lattice $(\nu=0, \mu=1)$ to the standard DNLS equation $(\nu=1, \mu=0)$. The choice of the path does not affect the generality of the 


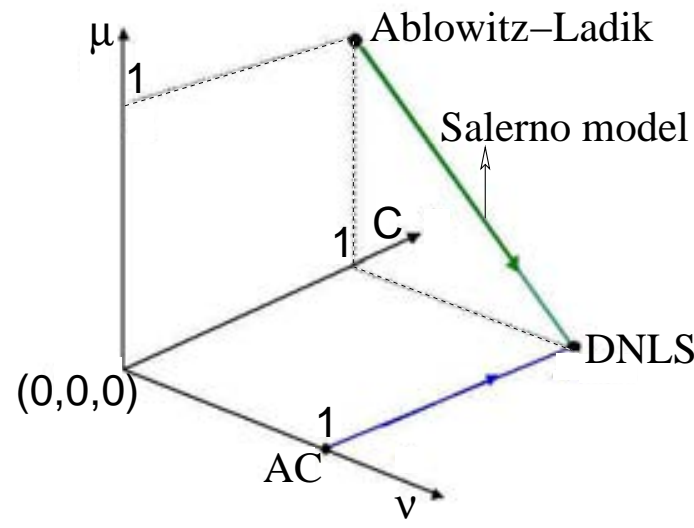

Figure 3.1: Two different paths to reach the DNLS limit in the $(C, \nu, \mu)$ space. The standard Salerno path, $\nu+\mu=1$, is used for continuing the $\mathrm{AL}(p, q)$ resonant breathers to the DNLS limit. The continuation from the anticontinuum limit can be used only for immobile $((0,1)$ resonant) breathers.

results since for every solution for the nonlinear parameters $(\nu, \mu)$ one can easily obtain the corresponding one for other set $\left(\nu^{\prime}, \mu^{\prime}=\mu \nu^{\prime} / \nu\right)$ by means of the rescaling $\Phi_{n}^{\prime}(t)=\sqrt{\nu^{\prime} / \nu} \Phi_{n}(t)$.

We have computed pinned discrete breathers $((0,1)$ resonant solutions) to the DNLS equation (2.5) by $(i)$ continuing those analytical pinned solutions of the AL lattice $(\alpha=0)$ following the Salerno path and (ii) starting from the anticontinuum limit $C=0$ of the DNLS equation and changing the coupling $C$. These two approaches yields the same solutions when compared at the DNLS limit. However, the continuation from the uncoupled limit does not offer the possibility of continuing a second family of pinned, $(0,1)$ resonant, discrete breathers present at the AL limit: Looking at eq. (2.15) one realize that for $(\alpha=\pi)$ a set of pinned solutions with phase difference betweeen adjacent sites equal to $\pi$ and $\omega_{b}<0$ is also available. This type of pinned solutions are usually termed staggered discrete breathers and, as we will see below, their behaviour in the standard Salerno model is far from trivial. One can study this new type of pinned solutions by considering the case $\nu<0$ for standard (unstaggered) pinned breathers, corresponding to $\alpha=0$ in the AL lattice, since the change $\nu^{\prime}=-\nu, t^{\prime}=-t$ corresponds to a staggering transformation of the solution $\Phi_{n}^{\prime}=(-1)^{n} \Phi_{n}$. Then, the study of $(p, q)$ resonant solutions along the path $\mu-\nu=1$ of the standard Salerno model $(\mu>0)$ is also of interest. The Salerno continuation of standard pinned breathers also provides further confirmation of an important and well-known theoretical result. At the integrable AL lattice, one-site and two-site immobile breathers are but two particular choices of the continuous one-parameter $\left(x_{0}\right.$, the localization center) family of immobile solitary waves, i.e. constant $x_{0}(t)=n$ or $n+1 / 2$ respectively, in eq. (2.14). The well-known result, confirmed by our numerics, is that away from the AL limit only these (one-site and two-site) immobile 

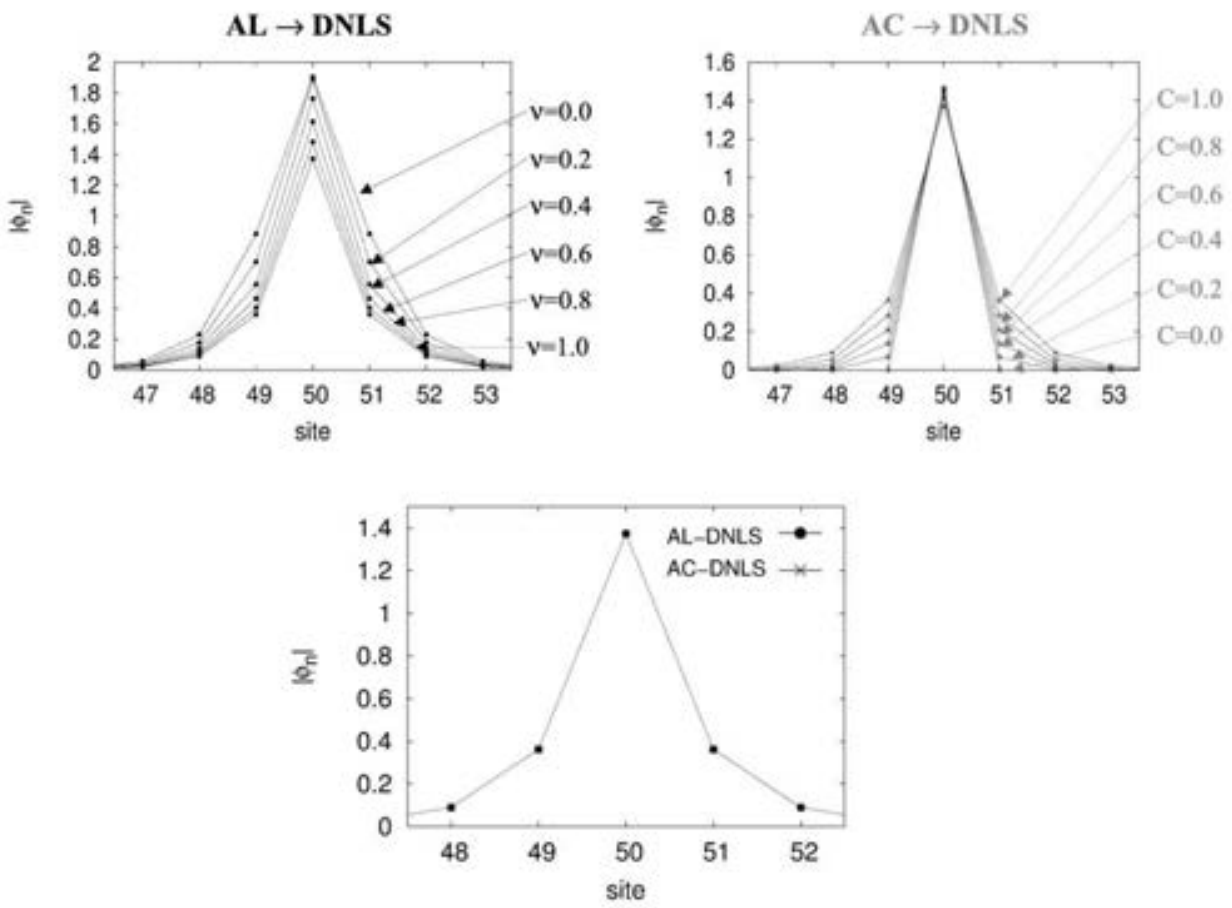

Figure 3.2: (Bottom) Convergence of the two solutions found at the DNLS limit $(\nu=1, \mu=0)$ when one is obtained by continuation from the $\mathrm{AL}(\nu=0, \mu=1)$ following the Salerno path (Top-left) and the other by adiabatically increasing the coupling $C$ from the anticontinuum limit of the DNLS equation (Top-right). The frequency of the solutions is set to $\omega_{b}=4.3$.

discrete breathers persist under adiabatic continuation. No immobile breather centered in between exists. For positive values of the parameter $\nu$, the one-site immobile one has a lower value of energy $\mathcal{H}$, and it is a linearly stable solution, while the energy of the two-site breather is higher and it is linearly unstable. The relative situation is reversed for negative values of $\nu$. This result can be interpreted as the emergence of a (Peierls-Nabarro) potential function of the breather center $x_{0}$, which destroys the continuous degeneracy of immobile breathers, leaving only two of them per lattice unit, namely those localized at maxima and minima of the Peierls potential. This interpretation, which is captured in the theoretical framework of collective variable approaches, turns out to play a central role in building up the physical interpretation of the numerical results on mobile discrete Schrödinger breathers as we will see in section 3.2 .

As introduced previously the translational motion of discrete breathers introduces a new time scale (the inverse velocity) so generically a moving breather 
excites resonances with plane wave band spectra. This fact poses no problem to the persistence of localization when the lattice dynamics is governed by power balance (forced and damped lattices [91-93]): the emitted power is exactly compensated by the input from the homogeneous external force field, during stationary breather motion. However, for generic (nonintegrable) Hamiltonian lattices one would expect that the radiative losses would tend to delocalize energy and some energy compensating mechanism is needed in order to sustain exact stationary states of breather translational motion. From the (particle) perspective of collective variables theory it is well known that the localized breather experiences a periodic Peierls-Nabarro potential function of its position, so that the motion of the localized field oscillation over this landscape should be expected to induce the emission of radiation at the expense of translational (and/or internal) breather kinetic energy, which thus would unavoidably decay on time.

In this section, we focus on the numerical results on mobile discrete Schrödinger breathers in the NLS lattice (2.18). These numerics are computed using the tools explained in the previous chapter. The Newton fixed point continuation requires a good initial guess (meaning that the starting initial conditions have to be in a small neighborhood of the fixed point). Very close to $\nu=0$, the AL solitary traveling waves (exact solutions at $\nu=0$ ) provide good starting points. After convergence to the fixed point, we increase adiabatically the value of the parameter $\left(\Delta \nu=10^{-3}\right)$, and start iteration from the previous fixed point.

An important step in the numerical method used here, is obtaining a basis for the subspace of (tangent space) vectors with Floquet eigenvalue +1 . These are associated to those degeneracies (symmetries) that one has to eliminate in order to regularize the linear system at each (Newton) iteration step when numerically converging to the fixed point solution.

Away from the AL limit, it is known (as reported e.g. in [94]) that only two conserved quantities remain generically as dynamical invariants, the Hamiltonian (2.19) and the norm (2.21). They are respectively associated to the continuous time translation and gauge (global phase rotation) invariance. Using the notation $u_{i}=\Re\left(\Phi_{i}\right)$ and $v_{i}=\Im\left(\Phi_{i}\right)$, one easily obtains that

$$
\begin{aligned}
& \delta u_{i}(t)=\dot{u}_{i}(t) \\
& \delta v_{i}(t)=\dot{v}_{i}(t),
\end{aligned}
$$

is the perturbation associated with time translational invariance, while

$$
\begin{aligned}
\delta u_{i}(t) & =v_{i}(t) \\
\delta v_{i}(t) & =-u_{i}(t),
\end{aligned}
$$


is the one with gauge invariance. These are, consequently, Floquet eigenvectors with associated eigenvalue +1 , and we can easily check that they coincide with the (two) basis vectors provided generically (i.e. except at special bifurcation values of the parameter, see below in 3.1.3) by the numerical Singular Value Decomposition (2.32) explained in the previous chapter.

In subsection 3.1.1 we summarize our findings on the generic structure of mobile Schrödinger discrete breathers. For this, as explained earlier, we have explored particular values for the integers $(p, q)$ and performed continuation of $(p, q)$ resonant AL traveling waves. The variation of the main structural characteristics of the fixed points along the continuation parameter $\nu$ is examined in detail in 3.1.2, for both signs of this parameter. Of particular interest are the observed drastic changes in the structure for $\nu \simeq-0.3$ and $\nu \simeq-0.39$. Then, in 3.1.3, we show the main conclusions on the stability analysis of the mobile Schrödinger discrete breathers, in a sector of the breather parameter space.

\subsubsection{The structure of the solution}

In figure 3.3 we plot the spatial profile of a $(1,1)$ mobile Schrödinger discrete breather for nonintegrability parameter value $\nu=1.0$, and $\omega_{b}=2.678$.

A quick inspection of this figure provides a first glance of the general structure of the computed $(p, q)$ resonant solutions: The fixed point $\hat{\Phi}$ is the superposition of an (exponentially) localized oscillation (the core) moving on top of an extended background.

$$
\hat{\Phi}=\hat{\Phi}_{\text {core }}+\hat{\Phi}_{\text {backg }} .
$$

In other terms, far away from the core localization site $n_{0}$, the solution does not tend to the rest state $\hat{\Phi}_{n}=0$, but to an extended excited state of the lattice, i.e. for $\left|n-n_{0}\right| \gg 1$

$$
\hat{\Phi}_{n}(t)=\left(\hat{\Phi}_{\mathrm{backg}}\right)_{n}(t) \neq 0 .
$$

One easily realizes (for example, consider a site very far from $n_{0}$ ) that the background has to be itself $(p, q)$ resonant. This can be quickly checked in our numerics: Indeed, the power spectrum,

$$
S(\omega)=\left|\int_{-\infty}^{\infty} \Re\left[\hat{\Phi}_{n}(t)\right] \exp (\mathrm{i} \omega t) d t\right|^{2}
$$

at a site $n$ far from $n_{0}$ reveals a finite number of $s$ peaks $\omega_{j}, j=0, \ldots, s-1$; one can check that each $\omega_{j}$ numerically fits to a branch of $(p, q)$ resonant plane 

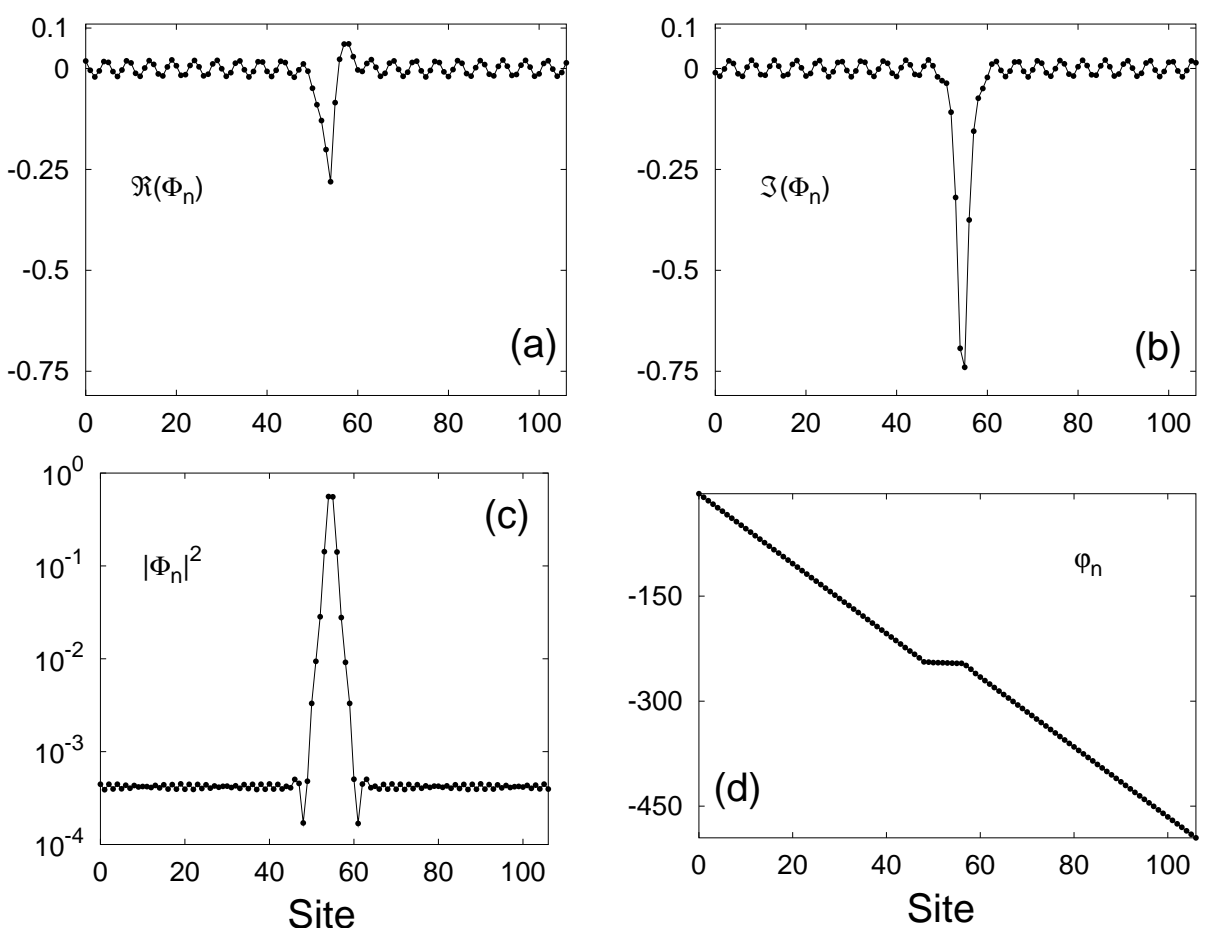

Figure 3.3: Instantaneous profile of a $(1,1)$ resonant breather with $\omega_{b}=2.678$ and $v_{b}=0.426$; the nonintegrable parameter is $\nu=1.0$ (standard DNLS equation). (a) Real part, (b) imaginary part, (c) modulus and (d) phase. The resonant condition for the harmonic composition of the background gives the contribution of three plane waves. The existence of these plane waves is revealed by the modulation of the extended tail in the modulus profile (c).

waves (see eq. (2.27)); this provides a set of amplitudes $A_{j}$, and finally one confirms that the superposition of the $\left(A_{j}, \omega_{j}\right)$ plane waves fits the numerical solution $\hat{\Phi}_{n}(t)$.

While immobile discrete breathers can be described as a sort of homoclinic (and time periodic) connection on the rest state, the mobile localized core instead connects a specific linear superposition of low amplitude nonlinear plane waves. One could say that the localized core needs for its motion to "surf over" a specific extended state of radiation (see figure 3.4 ):

$$
\left(\hat{\Phi}_{\text {backg }}\right)_{n}(t)=\sum_{j=0}^{s-1} A_{j} \operatorname{expi}\left(k n-\omega_{j} t\right) .
$$

We note that among the members of the (s-parameter) continuous family of $(p, q)$ resonant plane waves (see Section I), the fixed point solution contains only a particular member $\left(A_{j}, \omega_{j}\right)$ from each branch (see figure 3.5.a). This 
selection varies smoothly with the (adiabatic) continuation parameter $\nu$. In particular, the amplitude modulus $\left|A_{j}\right|$ selected increases smoothly from its zero value at the integrable limit $(\nu=0)$, for both signs of $\nu$.

If the bare core of a fixed point solution (i.e. after subtraction of the background) is taken as initial condition for a direct numerical integration of the equations of motion, one observes radiative losses, along with the corresponding changes in shape, velocity, etc. of the localized moving core. The motion of the bare localized core (not anymore a solution) excites extended states of the lattice. Thus, regarding the exact fixed point solution, one could say that radiative losses of the running core are exactly canceled out when the localized core runs, with specific velocity, on top of the specific linear combination of $\left(A_{j}, \omega_{j}\right)$ resonant plane waves $(3.6)$.

A complementary numerical observation is the following: Taking as initial condition for a direct integration of the equations of motion (2.18), a superposition of an immobile discrete breather and the background of a $(p, q)$ resonant mobile breather, it evolves into a moving discrete breather, with approximate velocity $v_{b}=\left(p \omega_{b}\right) /(2 \pi q)$. One thus would say that the background promotes breather translational motion with adequate velocity. In the next section 3.1.2, a connection between background characteristics and the particle perspective (i.e. the Peierls-Nabarro barrier of collective variable theories), will be established in order to further illuminate the physical description of discrete breather mobility.

Whatever physical perspective one may prefer, the numerical fact is that the generic structure of the fixed point solution is given by the superposition (3.3). Not too far from $\nu \simeq 0$, where the amplitudes $A_{j}$ of the fixed point background have small values, one can carefully check that if the bare core is

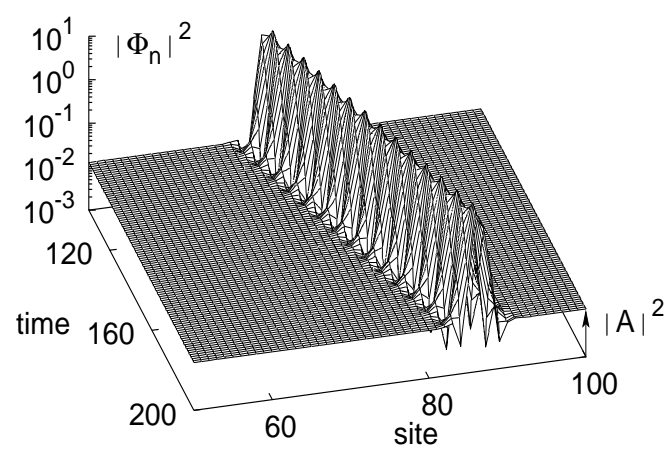

Figure 3.4: Time evolution of $\left|\Phi_{n}\right|^{2}$ profile of a mobile discrete Schrödinger breather. The frequency of the solution is $\omega_{b}=$ 5.050 and the velocity is $v_{b}=$ 0.804 . Note that the background is composed by a single plane wave with amplitude $A$. The nonintegrable parameter of eq. (2.18) is $\nu=0.2$. 

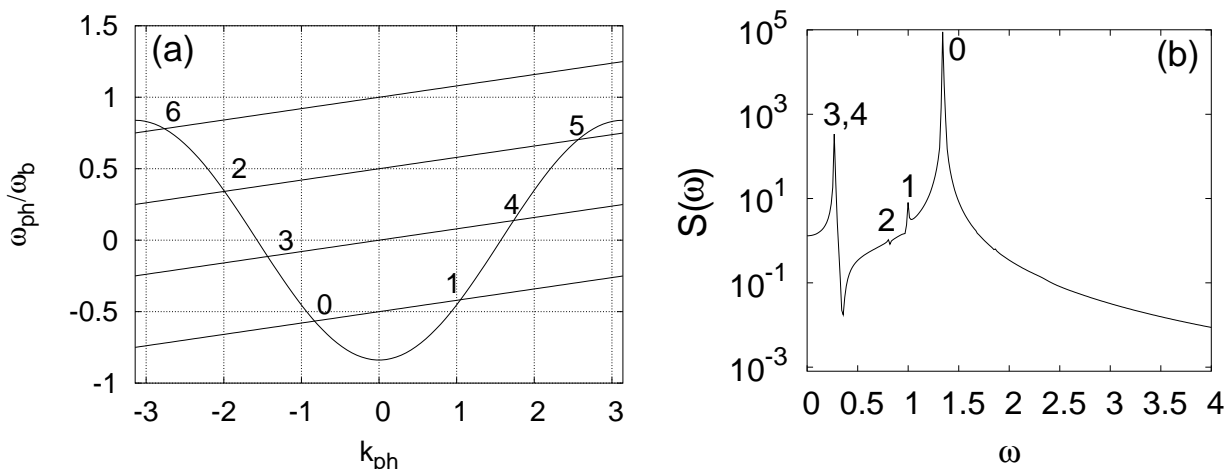

Figure 3.5: (a) Plot of the graphical solving of the resonant condition (in the $A_{j} \rightarrow 0$ limit) for a $(1,2)$ resonant breather with $\omega_{b}=2.384$ and $v_{b}=0.189$. (b) Power Spectrum $S(\omega)$ of the background of this solution at $\nu=1.0$. From (a) eq. (2.29) gives the contribution of seven plane waves $(j=0, \ldots, 6)$ but only five $(j=0, \ldots, 4)$ of them are visible due to the difference of orders of magnitude between the amplitudes $\left|A_{j}\right|$. The agreement between the resonant condition equation (for the fitted value of $A_{j}$ ) and the frequencies observed in $S(\omega)$ is up to machine accuracy.

given as a starting guess for Newton iteration, this converges well to the exact complete solution (core + background), by developing the specific selection of $A_{j}$ amplitudes. This confirms the robustness of the numerics.

Though previous observations of nondecaying tails of numerically accurate mobile discrete breathers in Klein-Gordon lattices [53] and/or (solitary) traveling waves [95] in self-focusing equations had been reported (see also the interesting discussions on this issue in [80] and [96]), no systematic study on those tails and their role is available. However we clearly see that they are an essential part of the exact solution. As argued in the introductory section, the translational motion of a discrete breather introduces a new time scale. In a nonintegrable context, this fact unavoidably implies resonances with plane wave band spectra, and an exact self-sustained moving DB solution could only exist on top of a developed resonant background. This seems to have been (with a few exemptions) not fully appreciated in most of current literature on mobile breathers, where the background is most often either ignored or deliberately suppressed.

A notable feature of the plane wave content of the background $\hat{\Phi}_{\text {backg }}$ is that the amplitude modulus $\left|A_{j}\right|$ in (3.6) differ by orders of magnitude, i.e. $\left|A_{1}\right| \gg\left|A_{2}\right| \gg\left|A_{3}\right| \ldots$, so that only a few frequencies are dominant for most practical purposes (see figure 3.5.b). In other words, the extended background associated to a spatially localized moving core is, in turn, strongly localized in the reciprocal ( $k$-space) lattice. The possible relevance of this observation is further discussed below in the concluding section. 


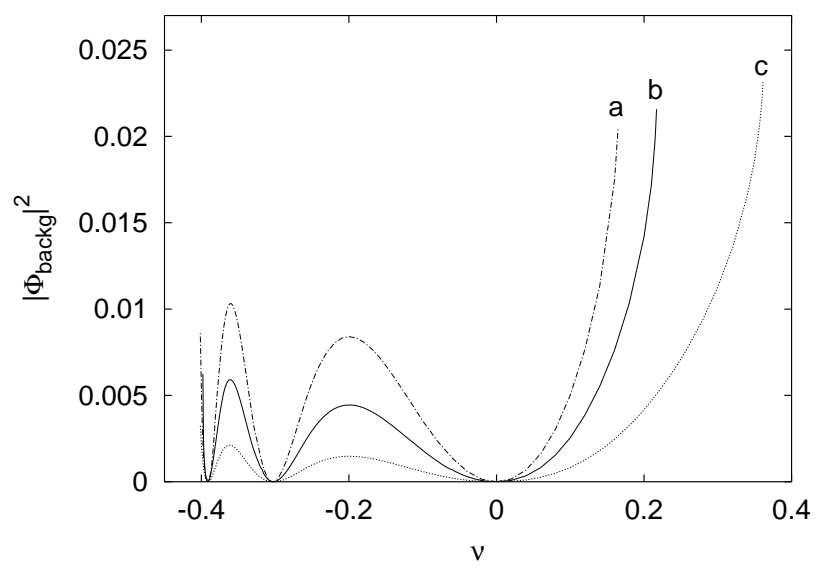

Figure 3.6: Background amplitude versus $\nu$ for three different $(1 / 1)$ resonant breathers with frequencies: (a) $\omega_{b}=5.65$, (b) $\omega_{b}=4.91$, (c) $\omega_{b}=4.34$. Note the two different behaviours: for positive values of $\nu\left|\Phi_{b a c k g}\right|^{2}$ is a monotonous increasing function of $\nu$ while for the negative part it shows smooth rises and falls.

\subsubsection{The background amplitude}

In order to characterize the specific features of the nonintegrable motion of discrete breathers, we focus here on the (perhaps) most remarkable among those features: the background amplitude of the uniquely continued fixed point. How does it evolve along the continuation path in parameter space?

For positive values of $\nu$ we have followed the line in parameter space (figure 3.1) $\mu+\nu=1$ (see equation (2.18)), while for negative values, we took the path $\mu-\nu=1$. Note that taking this latter path is similar to studying staggered breathers in the former one due to the staggering transformation reported above. We do not expect other paths to make important differences. As stated earlier, near $\nu \simeq 0$, the amplitude grows from its zero value (at the integrable limit) for both signs of this parameter, for it is a nonintegrable effect. However, for larger values of nonintegrability $|\nu|$ the background amplitude evolution shows some important differences for the two signs of $\nu$.

In figure 3.6 we plot the background amplitude (modulus) of the $(1,1)$ resonant fixed point, versus the continuation parameter $\nu$, for three different values of the breather frequency $\omega_{b}$. For $\nu>0$, one observes that the amplitude steadily increases with $\nu$ before continuation stops (i.e. Newton iteration ceases to converge beyond a certain maximum $\nu$ value). Note that the amplitude grows faster for higher values of the frequency, and that the continuation stops (correspondingly) at a smaller value of $\nu$. This may suggest that the failure of fixed point continuation is related to a somewhat excessive growth of the 
background amplitude, an issue that will be discussed later.

For $\nu<0$, after an initial growth the background amplitude decreases down to almost negligible values around $\nu \simeq-0.3$, then grows and again decreases close to zero at $\nu \simeq-0.39$, and so on, in progressively narrower intervals with larger peak amplitude, until continuation stops. Most noticeable is the fact that the intervals neither depend on the breather frequency $\omega_{b}$ nor on the breather velocity $v_{b}$. Why do background amplitudes decay so dramatically at those regions in parameter space? An important hint is presented in the next section, where the Floquet stability analysis of immobile discrete breathers will show a coincident situation of mirror-symmetry breaking (and its absence for positive $\nu$ values). For other values of $p$ and $q$ that we have numerically investigated, the same features of the background amplitude variation as shown in figure 3.6 are qualitatively reproduced.

\subsubsection{Floquet analysis}

On the basis of the general arguments given in [84, 97], the Floquet spectra of immobile DB in the thermodynamic limit, $N \rightarrow \infty$, consists of two components: the (continuous) Floquet spectrum of the asymptotic state of the solution (rest state), and a discrete part associated with spatially localized eigenvectors. The continuous part is composed by small amplitude (linear) plane waves, the so-called phonons. However, for mobile DB the asymptotic state of a $(p, q)$ resonant fixed point solution is a superposition of plane waves, the background $\hat{\Phi}_{\text {backg }}$. From this, one should expect the Floquet spectrum of a $(p, q)$ resonant DB being composed of two components: the discrete (spatially localized eigenvectors) and a continuous part associated with the linear stability of the background plane waves. The continuous part of the Floquet spectrum should reflect the same results of the modulational instability analysis of section 2.3.2. In particular, this means that any modulational instability a plane wave may suffer will be also an instability of a fixed point solution whose background contains this plane wave. In the future we will refer to any instability of the continuous part of the Floquet spectrum as background instability. Any instability from the discrete part is a core instability.

First we focus on core instabilities. For this we turn attention to the continuation of mobile $(p, q)$ resonant breathers. Figure 3.7 shows in the $\nu-\omega_{b}$ plane (dotted line), the values $\nu_{\max }\left(\omega_{b}\right)$ where the numerical continuations stop due to non convergence of Newton iteration for $p=1, q=1$ and $\nu>0$. As it was remarked above, the continuation stop is associated with the rapid increase of the background amplitude shown in figure 3.5. Only low frequency breathers, for which the background amplitude increases more slowly, can be 


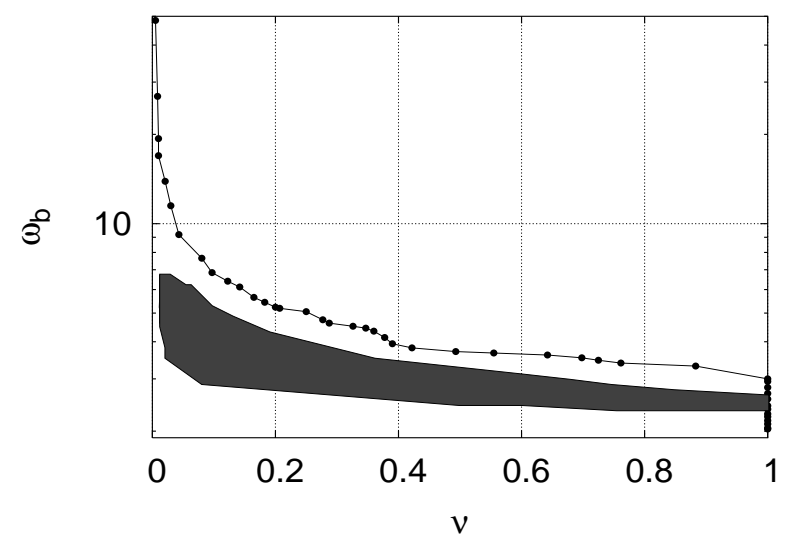

Figure 3.7: Continuation diagram of $(1,1)$ resonant breathers as a function of the frequency $\omega_{b}$. The end of the numerical continuation, $\nu_{\max }\left(\omega_{b}\right)$, is represented by the line with dots. The region where mobile breathers suffer from core instabilities is limited by the shaded area.

numerically continued all the way to the standard DNLS equation. The linear stability analysis of $(p, q)$ resonant breathers yields a well defined region in the $\nu-\omega_{b}$ diagram where core instabilities appear. There is an island inside the continuation region of figure 3.7, where the Floquet spectra contain a real eigenvalue $\lambda>1$. We observe the evolution of this Floquet eigenvalue (and its complex conjugate) as the parameter $\nu$ is increased in figure 3.8.a, for a (1, 1 ) breather of frequency $\omega_{b}=2.678$. Here the angle $\left(\theta_{\text {Floq }}\right)$ in the complex plane is plotted versus $\nu$. The interval of constant zero angle corresponds to the section (constant $\omega_{b}$ ) of the instability island in figure 3.7.

Along the whole continuation path, the profile of the corresponding unstable eigenvector is localized. An example of this profile inside the instability island is shown in figures 3.8.b and 3.8.c, where one observes that the localized instability shows a decaying background along the direction opposite to the motion. The decay rate increases as the modulus of the eigenvalue grows and decreases again when $\lambda$ returns to the unit circle. On the other hand, the stable Floquet eigenvector associated with $1 / \lambda$ shows a wing decaying along the mirror symmetric direction. The direct integration of the equation of motion reveals that the unstable solution experiences a pinning after a transient of regular motion with velocity $v_{b}=p /\left(q T_{b}\right)$. After the solution pins at site $n$, its core center oscillates around this site. The trapping of the unstable MB could be interpreted as a result of the energy losses that the growth of the linearly unstable perturbation induces on the solution.

Returning to the instability island shown in the diagram of figure 3.7, some final observations are worth summarizing: $(i)$ there is a range of frequencies 
(a)

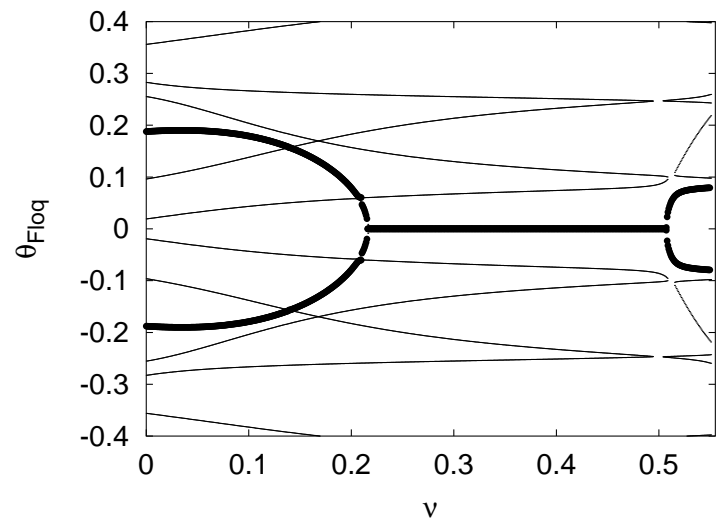

(b)

(c)
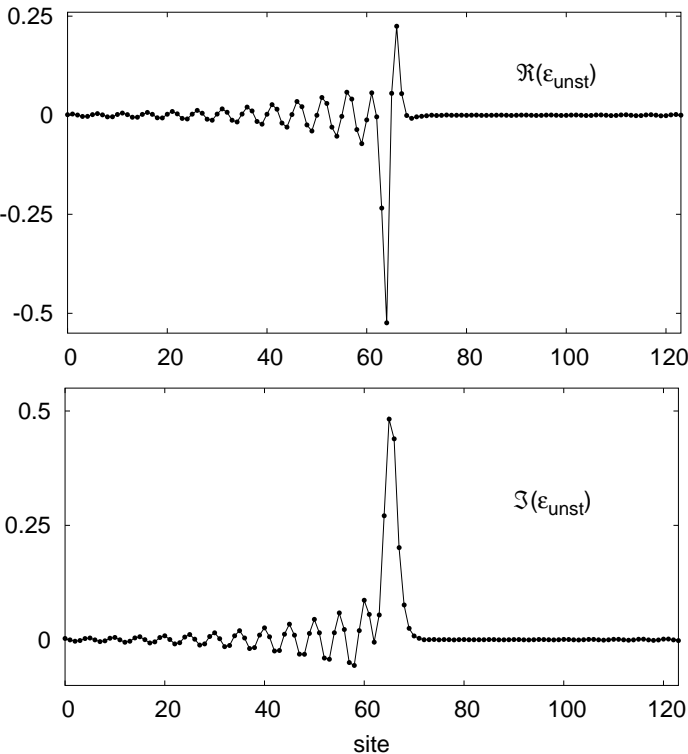

Figure 3.8: (a) Floquet angle evolution of the spectra of a $(1,1)$ resonant breather with $\omega_{b}=2.678$. The thick trajectory corresponds to the localized eigenvector that becomes unstable $\left(\theta_{\text {Floq }}=0\right.$ interval). Instantaneous profile of the real $(\mathbf{b})$ and imaginary (c) part of the Floquet unstable eigenvector of a $(1,1)$ breather with $\omega_{b}=$ 3.207 and $\nu=0.26$. The decaying tails along the direction opposite to the motion reveals the energy loss that the unstable eigenvector causes to the solution.

where mobile breathers of the standard DNLS equation $(\nu=1)$ suffer from this instability; ( $i i)$ very high frequency breathers do not experience this instability (in the short range where they can be continued); (iii) very low frequency breathers are stable all the way up to $\nu=1$.

We turn now to background instabilities. Once we know the plane wave content $\left(k_{0}, k_{1}, ..\right)$ of a $(p / q)$-resonant fixed point, we can know whether the 


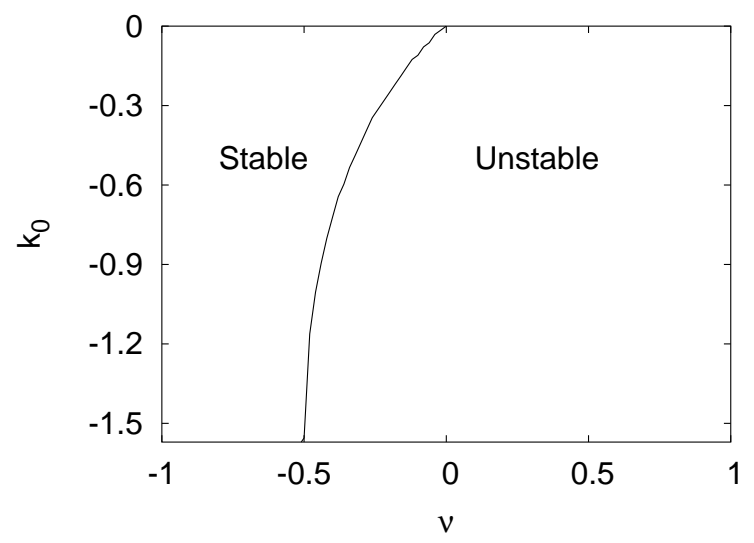

Figure 3.9: Modulational instability existence diagram for a plane wave with wave number $k_{0} \in[-\pi / 2,0]$. This diagram fixes the region where mobile discrete breathers with a background composed of only one plane wave do not suffer from background instability.

solution is subject to MI or not and, if it is unstable, what are the harmful perturbations $(Q)$. This problem is not so simple because we cannot know $a$ priori the plane wave content if we do not have the amplitudes of each one (2.29). However, we can derive a necessary condition for not having MI if we consider that, from (2.29), the background is always composed of at least one plane wave $(m=0)$ with $k_{0}$ between $[-\pi / 2,0]$. From this we can simplify the analysis of the background stability to the $k_{0}$ plane wave stability as a necessary condition for the MB stability. For this we calculate, for each $\nu$ and $k$, the value of the right-hand side of (2.39) for all the range of $Q([-\pi, \pi])$ and $A$. If this value is always positive the plane wave with this $k_{0}$ is free from modulational instabilities at this point of the model (2.18) with parameter $\nu$. From this extensive exploration we obtain, see figure 3.9, the region in the $k-\nu$ plane where MI is present.

In the range of $\nu$ between $[-1,-0.5]$ there is no modulational instability for single plane waves of any value of $k$ between $[-\pi / 2,0]$, and in particular for $k_{0}$. However, this does not guarantee that moving breathers are free from these instabilities in this region, unless the background has only one plane wave (as is sometimes the case). On the contrary, in the region $\nu>0$ any moving breather suffers such instabilities. The transition area in the region $\nu \in[-0.5,0]$ presents MI depending on which $k_{0}$ we have. For the range where no plane-wave with $k$ between $[-\pi / 2,0]$ is subject to MI we can assure that if there is only one contribution, $k_{0}$, to the background the corresponding MB solution is stable. For example, this is the case for $(1 / 1)$ resonant breathers if $\omega_{b}>4$ and for $(1 / 2)$ resonant breathers if $\omega_{b}>8.46$. The Floquet spectra of 
(a)

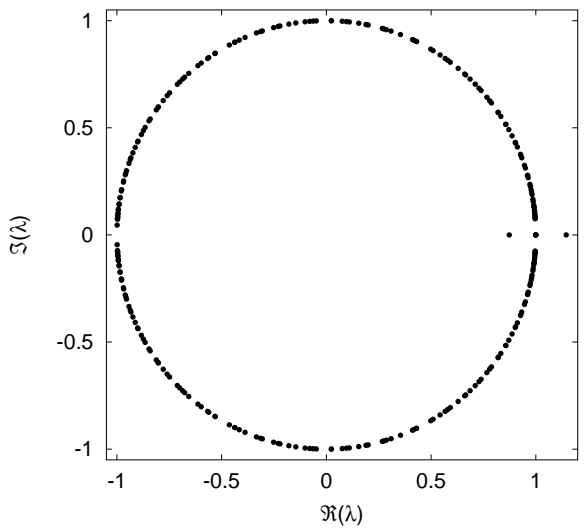

(b)

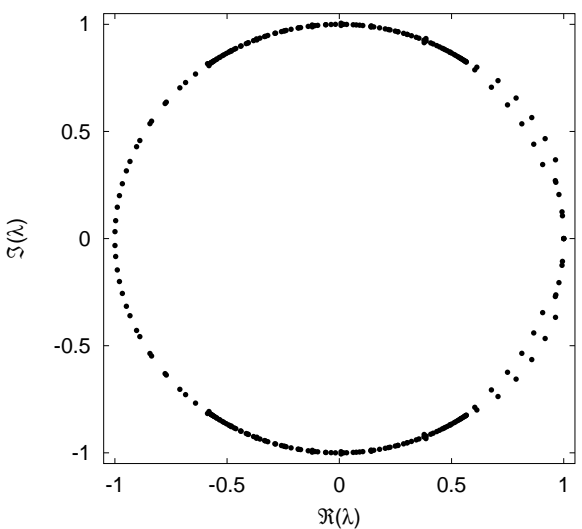

(c)

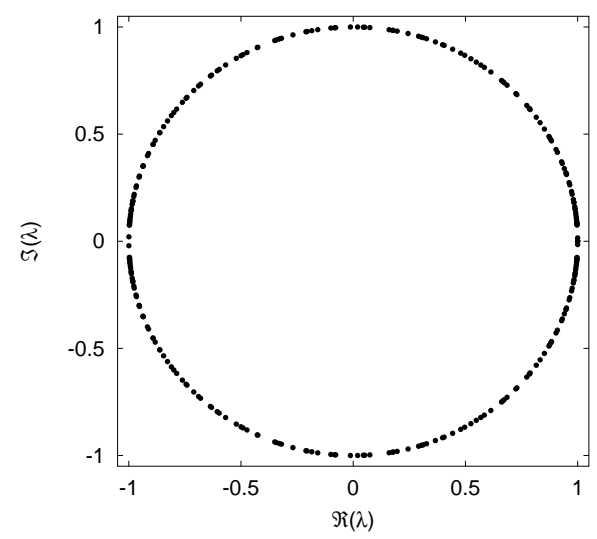

Figure 3.10: Floquet spectra of $(1,1)$ resonant breathers: (a) for $\omega_{b}=4.348, v_{b}=$ 0.692 and $\nu=0.08$ the spectra shows the core (localized) instability; (b) for $\omega_{b}=$ $6.610, v_{b}=1.052$ and $\nu=0.07$ the spectra shows the background (modulational) instability (also present but not visible in (a)); (c) for $\omega_{b}=4.348, v_{b}=0.692$ and $\nu=-0.39$ the solution is linearly stable. 


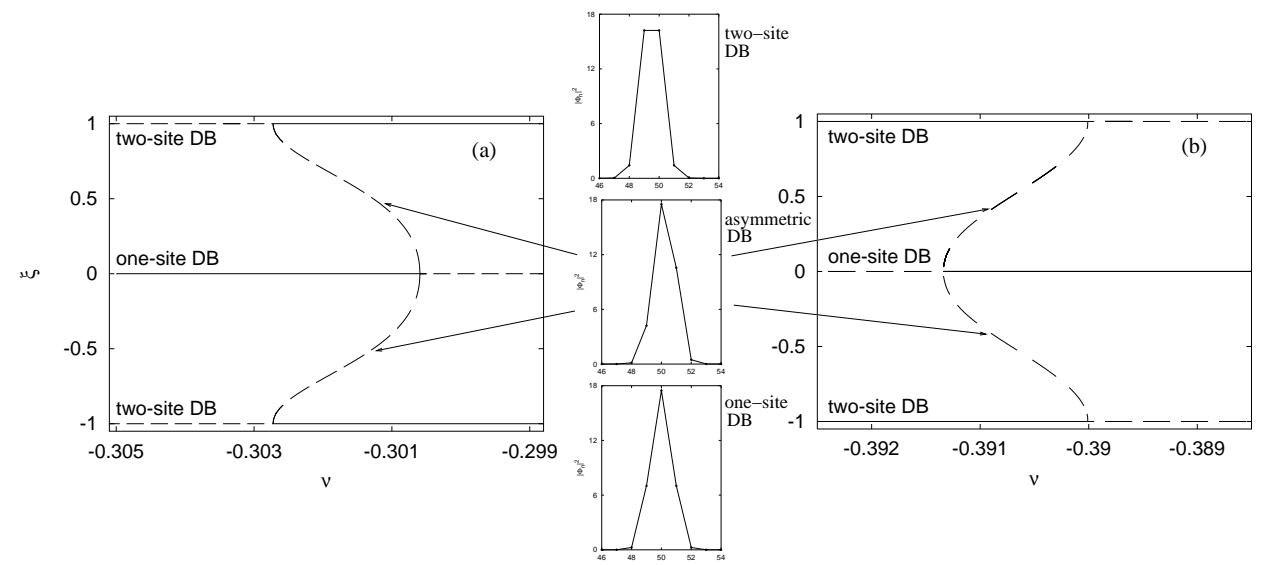

Figure 3.11: Graphical representation of the two first symmetry breaking bifurcations for $\nu<0$. The quantity $\xi$ in the vertical axes of both figures is defined, referred to the one-site breather, as the difference between the modulus $|\Phi|$ of the two sites adjacent to the maximum $\left(\left|\Phi_{\max }\right|\right)$, i.e. $\xi \sim\left|\Phi_{\max -1}\right|-\left|\Phi_{\max +1}\right|$. For the one-site DB $\xi=0$ and for the two-site $\mathrm{DB} \xi=1$, for this $\xi$ is conveniently normalized with the the difference between $\Phi_{\max }$ and $\Phi_{\max \pm 1}$. The continuous lines represent the regions where the static solutions are linearly stable while the discontinuous ones represent the unstable regions. The modulus profile of the three immobile coexisting solutions are plotted in the central insets for $\omega_{b}=6.215$ and $\nu=-0.3012$.

a moving breather satisfying these requirements is plotted in figure 3.10.c.

After the analysis of both types of instabilities eventually experienced by moving Schrödinger breathers, we finally report on a most relevant numerical fact revealed by the Floquet analysis of the family of standard immobile discrete breathers for $\nu<0$ (or, similarly, the family of staggered immobile discrete breathers for $\nu>0)$. Near $\nu \simeq-0.3$ an immobile two-site DB experiences a mirror symmetry-breaking (pitchfork) bifurcation becoming linearly unstable. When approaching the bifurcation point, two conjugate Floquet eigenvalues quickly approach +1 , where they meet, and then separate along the real axis. The eigenvector associated to the unstable $\lambda>1$ Floquet eigenvalue is localized and odd-symmetric, and is termed the symmetry-breaking or depinning mode $\phi^{d e p}$. We recall here that the background of an immobile breather is the rest state $\hat{\Phi}=0$, whose continuous spectrum consists of small amplitude (linear) plane waves. The depinning mode, on the other hand, is a localized core instability of the immobile breather, favoring a translation of the core center. For a smaller value of $\nu \simeq-0.39$ there is another symmetry-breaking bifurcation where the two-site breather becomes stable, again interchanging the stable character with the one-site. The corresponding bifurcation diagram for these two symmetry breaking transitions is plotted in figure 3.11. 
In the first symmetry breaking bifurcation, two unstable mirror-asymmetric immobile breathers emerge from the bifurcation point, progressively evolve toward the (stable) two-site breather, and finally collide in a new pitchfork bifurcation from where a unstable two-site breather emerges. The net result is an inversion of stability between one-site and two-site immobile breathers. Around the narrow interval of $\nu$ values where these two bifurcations occur, the energies of the three types of breathers involved (one-site, two-site, and asymmetric) have very small differences. From a particle perspective, this should make the breather motion easier. It is precisely in this same narrow interval where (see 3.1.2) we observe that the background amplitude of moving breathers becomes negligible. This is not a coincidence as we will argue in 3.2.

\subsection{Particle perspective on discrete breathers}

The appealing framework and success of collective variable approaches (see e.g. $[56-60,98])$ to the problem of nonintegrable motion of discrete breathers relies on the fidelity of a particle-like description of these field excitations that they provide. In these approaches, the effective dynamics of only a few degrees of freedom (e.g. the localization center, and the spatial width of the state, etc...in some instances $[65,68])$ replaces the whole description of the moving localized state.

Though unable to account for all the nonintegrable features, perturbative collective variable theories of NLS lattices provide a sensible physical characterization of important features of the nonintegrable mobility of localized solutions, like the emergence [99] of a Peierls-Nabarro barrier to motion. Here we summarize the main results of this particle-like description and compare them with the behaviour of numerically exact $(p, q)$ resonant moving breathers. Our goal is twofold: to acquire a correct physical understanding of the numerical facts, and then to make an assessment of validity and intrinsic limitations of collective variable approaches.

\subsubsection{Collective variables theory.}

A presentation of the particle perspective on moving Schrödinger breathers near the AL integrable limit can be found in $[58,59]$ (see also [56, 57, 60, 98]), where the interested reader will find the relevant formal aspects of the theory.

Using the integrable solitary wave (2.14) as an ansatz for the moving breather solution in the perturbed AL lattice, $\nu \neq 0$ and small in (2.18), one considers the parameters $\alpha, \beta, x_{0}$ and $\Omega$ as dynamical variables (variation 
of constants). The time evolution of these parameters in the perturbed lattice is governed by:

$$
\begin{aligned}
\dot{x}_{0} & =2 \sin \alpha \frac{\sinh \beta}{\beta} \\
\dot{\Omega} & =2 \cos \alpha \cosh \beta+\alpha \dot{x}_{0}+g(\beta) \\
\dot{\beta} & =0 \\
\dot{\alpha} & =-\nu \sum_{s=0}^{\infty} \frac{8 \pi^{3} \sinh ^{2} \beta}{\beta^{3} \sinh \left(\pi^{2} s / \beta\right)} \sin \left(2 \pi s x_{0}\right)
\end{aligned}
$$

where

$$
\begin{array}{r}
g(\beta)=2 \nu\left[\frac{2 \sinh \beta \cosh \beta}{\beta}-\frac{\sinh ^{2} \beta}{\beta}-1\right]+\nu \sum_{s=1}^{\infty} 4 \pi^{2} \cos \left(2 \pi s x_{0}\right) \times \\
{\left[\frac{\sinh ^{2} \beta \cosh \left(\pi^{2} s / \beta\right) \pi^{2} s}{\beta^{4} \sinh ^{2}\left(\pi^{2} s / \beta\right)}-\frac{2 \sinh ^{2} \beta}{\beta^{3} \sinh \left(\pi^{2} s / \beta\right)}+\frac{2 \sinh \beta \cosh \beta}{\beta^{2} \sinh \left(\pi^{2} s / \beta\right)}\right] .(3.11)}
\end{array}
$$

These relations can be viewed as the Euler-Lagrange equations of the collective variable Lagrangian obtained in $[58,59]$. The variation of the breather parameters give the evolution of solution (2.14) for the perturbed AL equation. Furthermore, one can regard eqs. (3.7) and (3.10), as the Hamilton equations for the canonical conjugate variables $x_{0}$ and $\alpha$ of the following effective Hamiltonian:

$$
\mathcal{H}_{\text {eff }}=\mathcal{T}_{\text {eff }}+\mathcal{V}_{\text {eff }}=-2 \cos \alpha \frac{\sinh \beta}{\beta}-\nu \sum_{s=1}^{\infty} \frac{4 \pi^{2} \sinh ^{2} \beta}{\beta^{3} \sinh \left(\pi^{2} s / \beta\right)} \cos \left(2 \pi s x_{0}\right)
$$

This effective Hamiltonian dictates the dynamics of the position of the solitary wave. Note that the (collective) variable $\beta$ is an invariant of motion, so it enters as a parameter into the effective Hamiltonian, and that the timeaverage value of $\dot{\Omega}$ (the parameter $\omega_{b}$ of the integrable solitary wave, now a function of time) is an increasing function of this parameter $\beta$. The effective potential $\mathcal{V}_{\text {eff }}$ acts as a barrier to the displacement motion ( $x_{0}$ variations $)$ and is naturally related to the Peierls-Nabarro potential. The amplitude of this barrier is an increasing function of both the nonintegrability parameter $|\nu|$ and $\beta$. The equilibrium points (representing immobile breathers) of this potential are $x_{0}=n$ and $n \pm 1 / 2$ with $n$ an integer. For $\alpha=0$, the former are stable (centers) one-site breathers, while the latter are unstable (saddle) two-site breathers; for the case $\alpha=\pi$ (staggered breathers) the stability is reversed.

A remarkable further consequence is the following [57]: there are no perturbative traveling wave solutions, for values of $\nu$ larger than certain critical value $\nu_{c r}(\beta)$. In particular, for $\beta>\beta_{c} \simeq 3.6862$, one cannot continue AL mobile 
(a)

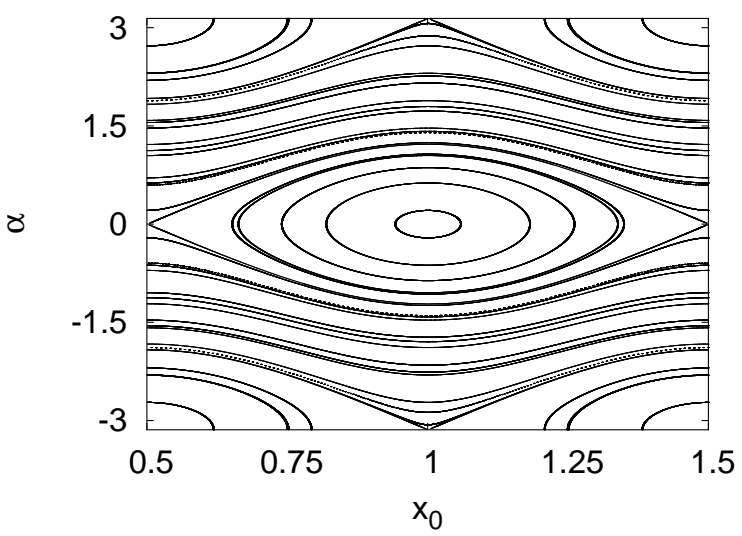

(b)

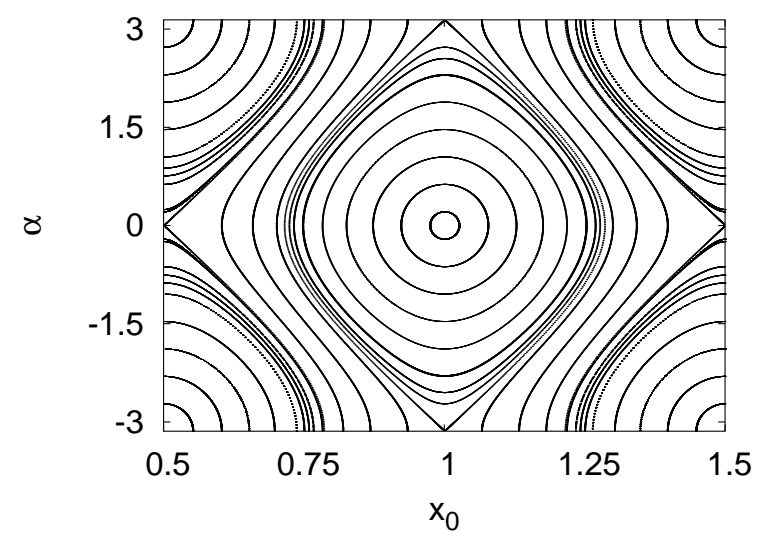

Figure 3.12: Collective variable $\left(\alpha, x_{0}\right)$ phase portrait transition for a value of $\beta=$ 3.0. (a) Shows the phase portrait for $\nu=0.2 \quad\left(<\nu_{c r}\right)$, there are $x_{0}$-unbounded trajectories (mobile breathers) coexisting with bounded ones (oscillating breathers). When $\nu=1.0 \quad\left(>\nu_{c r}\right)(\mathbf{b})$ we only have $x_{0}$-bounded trajectories: there are no mobile solutions.

breathers (i.e. $\nu_{c r}=0$ (see also important remarks in [60]). This consequence could be also (qualitatively) expected for a class of nonintegrable Schrödinger lattices (for some qualified perturbations of the integrable limit) with on-site nonlinearity. One expects also that lattices with purely inter-site (FPU-like) nonlinearity do not show this kind of transition.

In figure 3.12 we plot typical phase portraits at both sides of $\nu_{c r}$. figure 3.12.a shows the dynamics for $\nu$ smaller than the threshold value (given by $\beta$ ): there are open trajectories in $x_{0}$ corresponding to mobile breathers and closed orbits between the separatrix manifolds corresponding to breathers which oscillate around the equilibrium position of $\mathcal{V}_{\text {eff }}$. figure 3.12 .b is the phase portrait after the transition: there are no longer mobile solutions and (besides the os- 
cillating breathers) there are instead open trajectories in $\alpha$. The transition point, for a given $\beta$, occurs when trajectories with rotating $\alpha$ appear, and moving breathers disappear as the effect of separatrix line rearrangement on the cylinder $\left(x_{0}, \alpha\right.$ (modulo $\left.\left.2 \pi\right)\right)$ phase portrait.

Note that the existence of oscillating breathers is a consequence of the existence of a Peierls-Nabarro potential. These breather solutions do not perturbatively continue from the integrable limit. In 3.2 .3 we will investigate them and provide further numerical confirmation of the existence of these genuinely nonperturbative solutions, predicted by the collective variables theory.

\subsubsection{Energy balance governs mobility.}

In order to correlate collective variable predictions with the numerical results presented in section 3.3.3 one should first realize that our direct numerical approach computes breathers with fixed values of $\omega_{b}$ and $v_{b}$ and that these parameters are not tied to any specific ansatz. In particular, the connection of these two parameters with the collective variables is given by eq. (2.16) in the integrable limit. For the perturbed (near-integrable) lattice, $\omega_{b}$ and $v_{b}$ are identified as the time averages of $\dot{\Omega}$ and $\dot{x}_{0}$, respectively.

The Peierls-Nabarro (PN for short) barrier is naturally identified as the energy difference (given by the Hamiltonian (2.19)) between the two immobile breathers of the same frequency $\omega_{b}$, one centered at a site $n$ and the other (two-site) at a bond $n \pm 1 / 2$ :

$$
E_{P N}\left(\nu, \omega_{b}\right)=H\left(\nu, \omega_{b}, n\right)-H\left(\nu, \omega_{b}, n \pm \frac{1}{2}\right)
$$

In the integrable AL limit this barrier is zero due to the degeneracy (continuous translation invariance) of the breather family solution, but for $\nu \neq 0$ this invariance is broken and only these two isolated solutions persist. The energy difference of the two pinned solutions is thus viewed as the minimal extra "kinetic energy" of center of mass translation that a mobile breather must incorporate for overcoming the barriers to its motion.

We have studied the behaviour of the PN barrier in the Salerno model by continuing immobile breathers, both centered at a site and at a bond, while computing their energy difference. The computations of the barrier are made for a grid of values of $\omega_{b}$. figure 3.13 shows the "equipotential" lines of the PN barrier in the $\left(\nu, \omega_{b}\right)$ plane. The results show different behaviours depending on the sign of $\nu$ :

- $\nu<0$.- Here one observes the effects of the symmetry-breaking bifurcations cascade described in 3.1.3. The successive stability inversions 


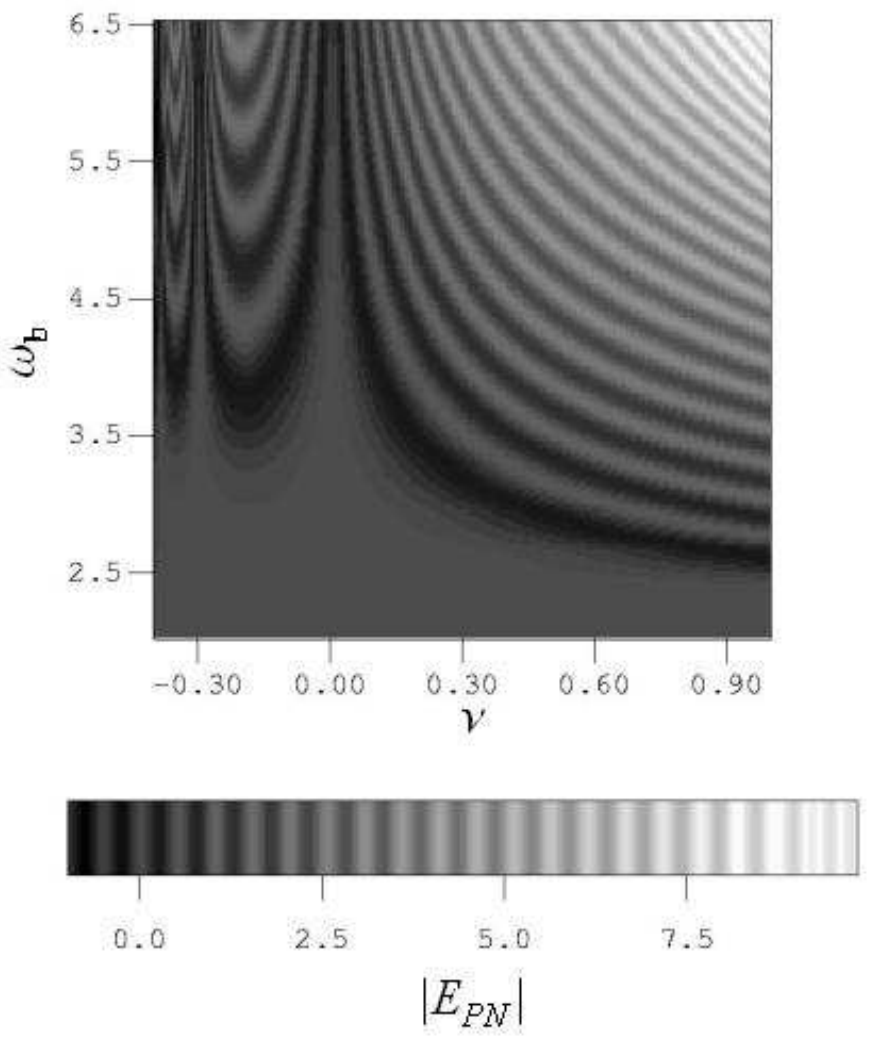

Figure 3.13: Density Plot of the absolute value of the Peierls Nabarro barrier, $\left|E_{P N}\right|$, as a function of $\omega_{b}$ and $\nu$. For positive values of $\nu,\left|E_{P N}\right|$ is a monotonous increasing function of $\nu$ and $\omega$. For negative values the plot reveals the oscillating behaviour of $\left|E_{P N}\right|$ as a function of $\nu$ (for a given value of $\omega_{b}$ ).

between site and bond centered breathers involve a substantial decrease of the Peierls barrier. The appearance of asymmetric solutions in these bifurcations introduces a new energy and, correspondingly, the Peierls barrier is computed as the maximum energy difference between the three pinned solutions: the two symmetric (site and bond centered) and the asymmetric breather.

- $\nu>0$.- In this case the behaviour of the Peierls barrier follows qualitatively the collective variable predictions on the effective potential experienced by the particle. The increasing character, with $\nu$ and $\omega_{b}$, of the numerical barrier is qualitatively the same as that predicted from $V_{\text {eff }}$ (as a function of $\nu$ and $\beta$ ) by the theory. 
The PN barrier of $\left(\omega_{b}\right)$ immobile breathers and the background amplitude of $\left(\omega_{b}, v_{b}=\frac{p \omega_{b}}{2 \pi q}\right)$ mobile breathers are in fact strongly correlated. This correlation is obtained considering the functions $\left|E_{P N}\right|(\nu)$ and $\left|\hat{\Phi}_{\text {backg }}\right|^{2}(\nu)$. Both functions are plotted for a fixed value of $\omega_{b}=4.34$ in figure 3.14.a. The behaviour of $\left|E_{P N}\right|(\nu)$ for negative $\nu$ (revealing the cascade of bifurcations explained before in 3.1.3) is closely followed by $\left|\hat{\Phi}_{\text {backg }}\right|^{2}(\nu)$ with the corresponding sequence of growths and decays. The strong correlation holds also for positive values of $\nu$, where numerical PN barrier data are available for a larger interval of $\nu$ values (due to the absence of the symmetry-breaking cascade of bifurcations). Indeed, the correlation is so strong that one is tempted to view the PN barrier and the background amplitude as complementary aspects of a single phenomenon: the breaking of the continuous translational invariance, and the associated lack of core momentum conservation. Indeed, the background amplitude of moving breathers is a monotone increasing function of the PN barrier of pinned breathers of the same frequency, as shown in figure 3.14.b, where $\left|\hat{\Phi}_{b a c k g}\right|^{2}\left(\left|E_{P N}\right|\right)$ is plotted.

However, we also observe clearly in figure 3.14.a that, when the continuation end is approached, the rate of growth of $\left|\hat{\Phi}_{\text {backg }}\right|^{2}(\nu)$ increases dramatically (the concavity of the curve in log scale turns upwards), while the PN barrier does not increase much faster than before. This is reflected in figure 3.14.b, where the slope approaches verticality, indicating that, in this range of $E_{P N}$ values, the background grows rapidly.

This numerical observation suggests taking a closer look at the precise influence of the background amplitude on the core energy variations associated with the existence of PN barriers. To this end, we use the conservation of the Hamiltonian (2.19) and insert this equation into the form (3.3) of the $(p, q)$ resonant fixed point. The energy of the solution can be decomposed in the following terms:

$$
\mathcal{H}[\hat{\Phi}]=\mathcal{H}\left[\hat{\Phi}_{\text {core }}\right]+\mathcal{H}\left[\hat{\Phi}_{\text {backg }}\right]+\mathcal{H}^{\text {int }},
$$

where $\mathcal{H}^{\text {int }}$ is the interaction energy, i.e. the crossed terms of $\hat{\Phi}_{\text {core }}$ and $\hat{\Phi}_{\text {backg }}$. Let us now consider the simplest case in which the background has a single resonant plane wave. Along with the total energy, also the energy of the plane wave is a constant in time so that

$$
\frac{\partial \mathcal{H}\left[\hat{\Phi}_{\text {core }}\right]}{\partial t}=-\frac{\partial \mathcal{H}^{i n t}}{\partial t} .
$$

In other words, the variations of the core energy during the motion are exactly compensated by those of the interaction term.

If one takes, as an ansatz for $\hat{\Phi}_{\text {core }}$, the AL solution, one formally obtains for $\mathcal{H}^{\text {core }} \equiv \mathcal{H}\left[\hat{\Phi}_{\text {core }}\right]$ the collective variables Hamiltonian (3.12). But note that 
(a)

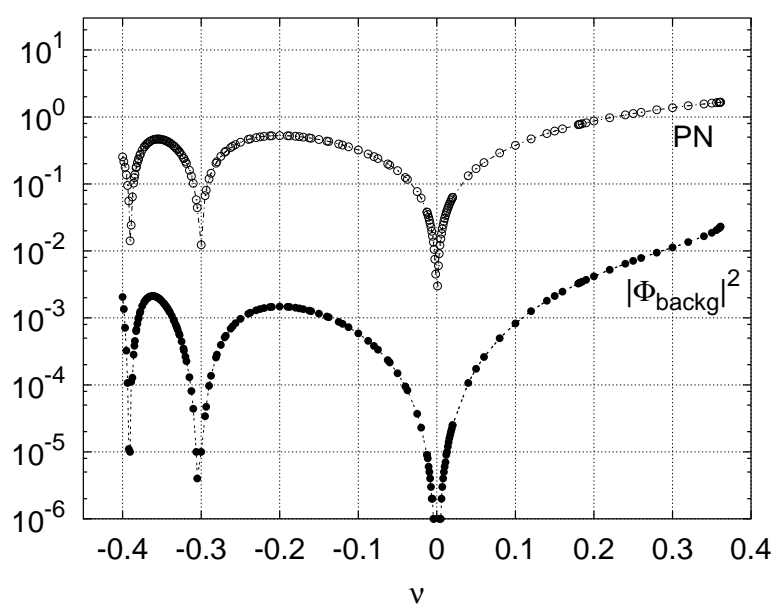

(b)

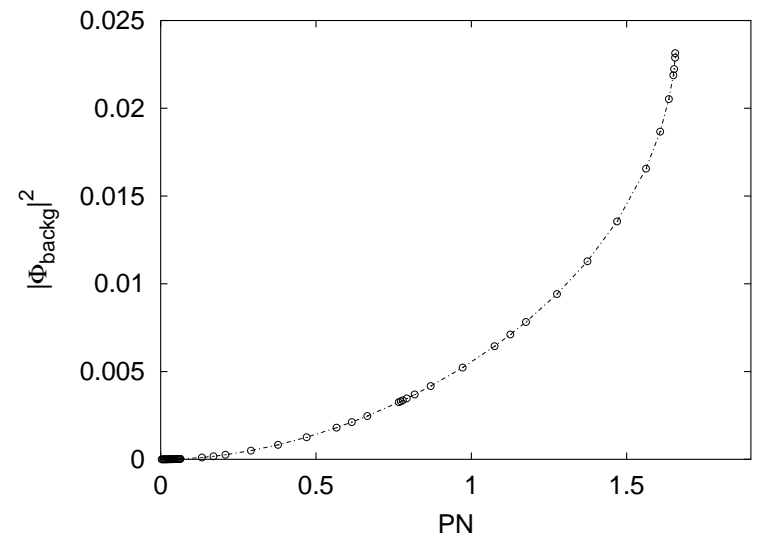

Figure 3.14: Peierls Nabarro barrier $\left|E_{P N}\right|$ from immobile breather with $\omega_{b}=4.34$ and background square amplitude $\left|\Phi_{\text {backg }}\right|^{2}$ for a $(1,1)$ resonant breather of the same frequency $\left(v_{b}=0.691\right)$. In (a) we show both quantities in semi-log scale as functions of $\nu$, illustrating the strong correlation between them for both signs of $\nu$. Figure (b) shows for positive values of $\nu$, the nonlinear relation between $\left|\hat{\Phi}_{\text {backg }}\right|^{2}$ and $\left|E_{P N}\right|$. Note the sudden increase of the slope close to the end of numerical continuation.

here it would not anymore be a constant of motion, due to the interaction with the background. Instead, we directly compute numerically the evolution of the core energy $\mathcal{H}^{\text {core }}$, which in turn determines $\mathcal{H}^{\text {int }}$ up to an additive constant.

For this we take a fixed point solution with a single plane wave in its background, and then subtract off the plane wave to obtain $\hat{\Phi}_{\text {core }}$, from where $\mathcal{H}^{\text {core }}(t)$ is computed. In figure 3.15 we have plotted the evolution of the core energy as a function of the localization position (center) of the breather core. The localization center of a lattice function $\Phi_{n}$ is defined using the conserved 


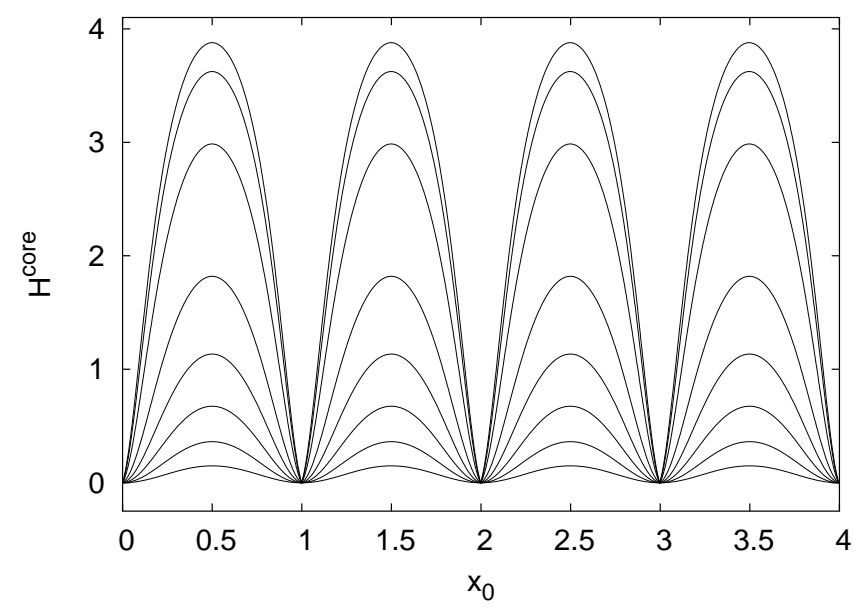

Figure 3.15: Plot of $\mathcal{H}^{\text {core }}$ of a $(1,1)$ resonant breather as a function of the localization center $x_{0}$ for different values of $\nu$. The parameter of the solution are $\omega_{b}=5.056$ and $v_{b}=0.805$. The values of $\nu$ are $0.04,0.08,0.12,0.16,0.20,0.24,0.25$ and 0.2512 (end of the continuation), the amplitude of the oscillation of $\mathcal{H}^{\text {int }}$ grows with $\nu$. (The minimum value of $\mathcal{H}^{\text {int }}$ has been set to zero in order to compare the different functions.)

norm (2.21):

$$
x_{0}=\frac{\sum_{n} n \ln \left(1+\mu\left|\Phi_{n}\right|^{2}\right)}{\mu \mathcal{N}}
$$

As expected, the core has extracted the maximum available from the interaction energy (with the background) when the core passes at $n \pm 1 / 2$ (maxima of the PN barrier) and has returned it to the interaction term when at $n$ (minima of the PN barrier).

Another interesting feature of these numerically obtained functions is seen from the variations in the form of the oscillation of $\mathcal{H}^{\text {core }}$ as the nonintegrable parameter $\nu$ is increased. At the same time, as the energy difference between $n$ and $n \pm 1 / 2$ increases the modulus of the derivative $\partial \mathcal{H}^{\text {core }} / \partial x_{0}$ in the neighborhood of $x_{0}=n$ also increases. These variations become faster when the end of the continuation is approached, reaching a cuspidal point for the last $\nu$ reached. The background amplitude is included in $\mathcal{H}^{i n t}$, and of course in $\partial \mathcal{H}^{i n t} / \partial t$; the dramatic variation of it at the end of the continuation could be interpreted in terms of this derivative variation in $x_{0}=n$. 


\subsubsection{Oscillating breathers}

The emergence of the Peierls barrier and the behaviour of the background amplitude illustrate the physical interpretation of this background as a (p/q)resonant energy support to overcome the barrier to motion. We now confirm this statement searching for another kind of solution: oscillating breathers. These solutions are predicted by collective coordinates approaches and are a consequence of the loss of translational invariance out of the integrable limit. Following the above interpretation of the background role one can imagine certain solutions with a background amplitude not high enough for surpassing the Peierls barrier and allowing travel along the lattice. In terms of a well defined potential, considering the particle perspective, the center of these localized solutions would be oscillating between $(n-1 / 2)$ and $(n+1 / 2)$ for the unstaggered ones or between $n$ and $(n \pm 1)$ for the staggered ones.

From our perspective, the oscillating breathers are solutions with two frequencies: the internal one of the breather $\left(\omega_{b}\right)$ and the one corresponding to the oscillatory motion $\left(\omega_{o s c}\right)$. Once again, we have a problem dealing with two time scales and consequently we have to impose that the two frequencies are commensurate $p \omega_{b}=q \omega_{\text {osc }}$. The fixed point problem is now associated with the map:

$$
\mathcal{T}_{q T_{b}} \Phi_{n}(t)=\Phi_{n}(t)
$$

We cannot, however, develop the Newton iteration scheme in a similar way as for mobile breathers. There is no longer any family of oscillating breathers providing a good start point for the continuation (they are intrinsic solutions of the nonintegrable regime because they appear as the Peierls barrier emerges). The way to obtain a good ansantz (as Cretegny and Aubry already used to find mobile breathers in Klein-Gordon lattices [53]) is to perform a small perturbation of the static solution (pinned at a site $n$ ) with the depinning internal mode:

$$
\Phi_{n}^{\text {ansantz }}=\Phi_{n}^{\text {static }}\left(\omega_{b}\right)+\epsilon \delta \phi_{n}^{\text {dep }}
$$

The dynamics of the perturbed solution for small enough values of $\epsilon$ shows the oscillating behaviour expected and for large enough values of $\epsilon$ the breather starts to move. Obviously in both cases the motion finishes after a transient due to radiation (they are not exact solutions). Tuning the parameter $\epsilon$ we search for those oscillatory transients whose $\omega_{o s c}$ is resonant with the breather frequency $\omega_{b}$. The transient is much more stable when the nonintegrable parameter $\nu$ is very small, close to the AL limit. We first search here for a good initial guess for the method and then obtain the exact solution of the map (3.17). Once the exact solution is obtained for a small $\nu$, we can perform the continuation to higher values in the same way as we did for mobile solutions. In figure 3.16. a we show the evolution of the amplitude of oscillation as $\nu$ is 


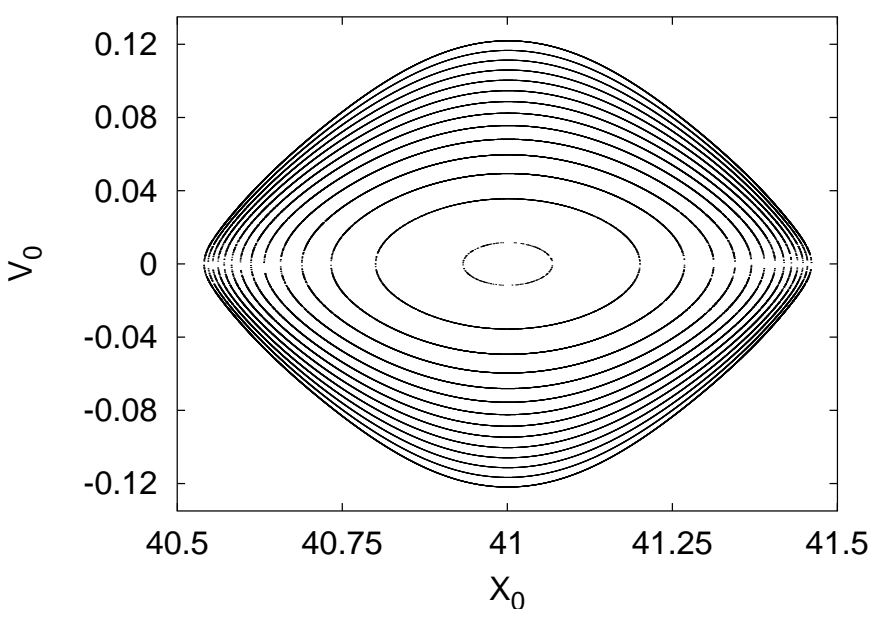

(a)

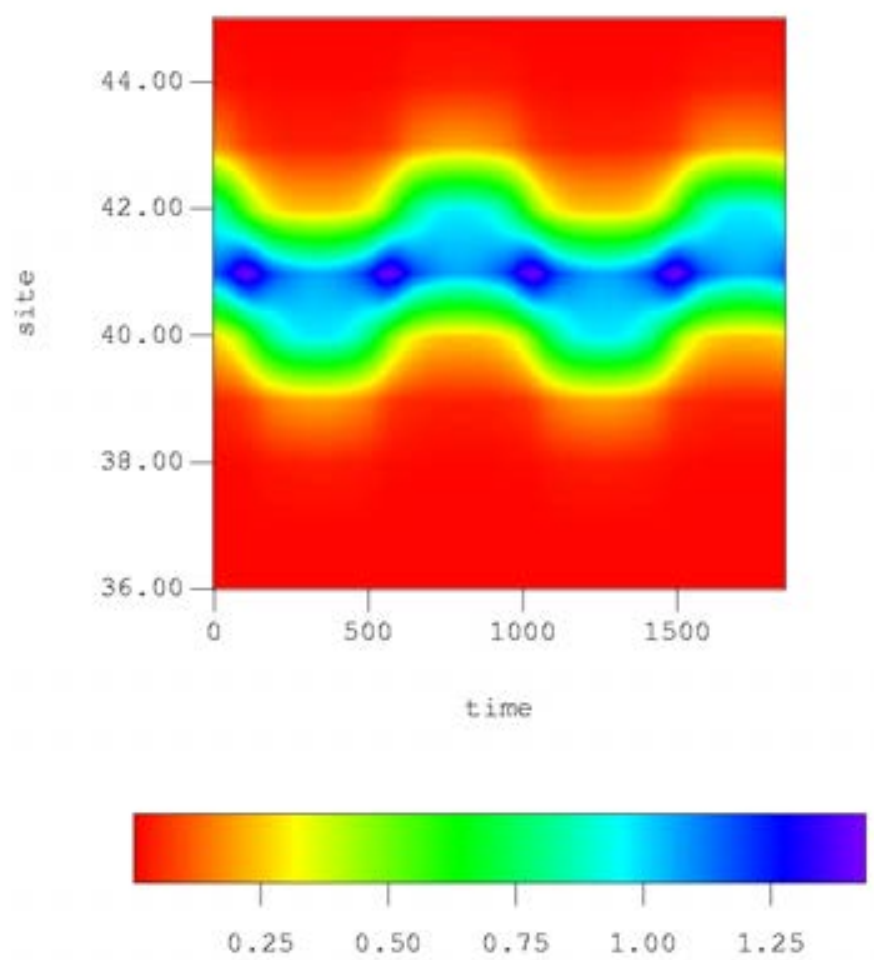

(b)

Figure 3.16: (a) Evolution of the localization center $x_{0}$ of an exact (1/18)-oscillating breather for different values of $\nu: 0.05,0.06, . ., 0.18$. The internal frequency is $\omega_{b}=$ 3.086. The amplitude of the oscillation of $x_{0}$ increases with $\nu$ revealing the nonlinear character of the motion for the highest values of $\nu$. (b) Density plot of the time evolution of $\left|\hat{\Phi}_{n}\right|^{2}$ for the above oscillating breather. 
increased from 0.05 to 0.18 . The amplitude of the oscillation is represented by the phase portrait of the localization center of the breather defined as in (3.16). The continuation reflects that the amplitude of the oscillation, for a fixed value of $\omega_{\text {osc }}$, grows with $\nu$. In figure 3.16.b the density plot of $\left|\hat{\Phi}_{n}\right|^{2}$ is shown as a function of time, revealing the oscillating pattern of the solution.

The existence of exact oscillating breathers is a consequence of the existence of a Peierls barrier. The structure of these solutions reveals the existence of a background (resonant with the map) whose amplitude grows as $\nu$ (and consequently the amplitude of oscillation) is increased. This is the picture we expected from the role played by the interaction background-core in the energy balance during motion. The monotonous growing behaviour of the background versus the oscillation amplitude, strongly suggests that if the amplitude of the former is increased the solution will be able to translate steadily. This has been checked by direct numerical integration, because no exact solutions connecting the oscillating with the mobile ones can be obtained due to the different maps employed to obtain both types of solutions. However, the existence of a background in the exact oscillating breather solutions and its behaviour with the amplitude of the breather oscillations are fully consistent with the interpretation of the results obtained for the mobile solutions.

\subsubsection{Validity and limitations of particle perspective}

The most basic result of the perturbative collective variable theories away from the integrable regime is the existence of a Peierls-Nabarro potential function of the core (collective variable) center. It expresses (in particle-like terms) that the breather position is no longer indifferent because the continuous translational invariance has been broken. From this also naturally comes the existence of oscillating breathers. We have seen how our numerics fully confirm the qualitative validity of these predictions.

A further prediction concerns the phase portrait's transition studied in [57]. Despite the fact that our end of continuation is correlated with the equipotential lines profile of the numerical PN barriers, and the phase portrait transition is also related to their sudden growth, no clear connection (between transition and end of continuation) can be established. The end of continuation is itself sensibly interpreted as a numerical consequence of the sudden increases of the amplitude background, and does not imply neccesarily the existence of any global phase portrait transition.

However, in some respects the perturbative collective variable theory is clearly incomplete: For example, it is unable to predict the observed localized (core) instability bifurcation and the observed symmetry breaking transitions 
for $\nu<0$. These bifurcations could easily appear in a theory with (at least) two variables (a dimer) experiencing the Peierls-Nabarro potential, which would demand an improved perturbative ansatz. This improved ansatz must coincide in the integrable limit with the AL solution. One can use the numerical results to guide the construction of such an improved ansatz. In this respect, the following observation may be relevant. The parameter $\beta$ of the AL solution determines both the amplitude and the width of the localized pulse. However, our numerical estimates of these breather characteristics for immobile breathers show clearly that, for fixed value of $\omega_{b}$, the breather width is independent of $\nu$, while the amplitude varies with it. In other words, away from integrability, width and amplitude of the (immobile) breather are no longer a single collective variable.

Beyond any other limitation of the perturbative collective variable theory, the background (an indispensable part of the exact solution) is absent in the perturbative ansatz, and it cannot appear later in that context. A complete theory of (nonlinear Schrödinger) breather motion should somehow incorporate the background in the ansatz itself. If correct, it should then predict that the background amplitude grows from zero with the nonintegrability parameter $\nu$, and (ideally) so on with all the numerically observed behaviours. One possible way to develop the analytical approach could be to use the method presented in [100]. In this scheme, eq. (3.15) may play an important role, for it provides the energy balance governing the translational motion of the breather core. In other words, our results show that the core energy is not an invariant of motion and this requires the existence of a finely tuned background whose nonlinear interaction with the core compensate the core energy variations.

\subsection{Discrete breathers in the Salerno model with com- peting nonlinearities}

In the above sections we have mainly focused on the study of mobile discrete breathers. In fact, the characterization of usual (non-staggered) pinned discrete breathers along the standard $(\mu>0$ Salerno path was already considered in previous works $[58,59,89,90]$ concluding that eq. (2.18) gives rise to pinned discrete breathers at all values of the DNLS parameter $\nu$, and all positive values of the $\mathrm{AL}$ coefficient, $\mu$. As already mentioned above if $\nu$ is negative one can make it positive by means of the staggering transformation, and hence study those staggered pinned discrete breather along the SM with $\nu>0$ (and hence finding the symmetry breaking bifurcation reported in section 3.1.3). However, the sign of $\mu$ cannot be altered. In particular, the proper AL model $(\nu=0)$ with $\mu<0$ does not give rise to localized solutions. The latter 
circumstance suggests considering soliton dynamics in the SM with $\mu<0$, i.e., with competing nonlinearities, which is the subject of the present section ${ }^{1}$.

In order to study the SM with competing nonlinearities, it is necessary to redefine the conserved norm (2.21) and Hamiltonian (2.19) by

$$
\begin{gathered}
N=\frac{1}{\mu} \sum_{n} \ln \left(\left.|1+\mu| \Phi_{n}\right|^{2} \mid\right) \\
\mathcal{H}=\sum_{n}\left[-\left(\Phi_{n} \Phi_{n+1}^{*}+\Phi_{n+1} \Phi_{n}^{*}\right)-2 \frac{\nu}{\mu}\left|\Phi_{n}\right|^{2}\right. \\
\left.+2 \frac{\nu}{\mu^{2}} \ln \left(\left.|1+\mu| \Phi_{n}\right|^{2} \mid\right)\right] .
\end{gathered}
$$

Whereas the Poisson structure of the standard Salerno model (eq. 2.20) remains valid. The above redefinitions of the norm (3.19) and Hamiltonian (3.20) are introduced in order to remain valid when $\left[1+\mu\left|\Phi_{n}\right|^{2}\right]$ takes negative values at some sites, due to the use of $\mu<0$.

In this section we will study the existence and characterization of both pinned and mobile discrete breathers when these two competing (on-site selffocusing and inter-site self-defocusing) nonlinearities coexist in the Salerno model. In 3.3 .1 a continuum approximation (CA) of the Salerno model is used in order to investigate the behaviour of the discrete breathers when $\mu<0$ in an analytical form. It is found that, although they might exist in a semiinfinite band of frequencies (as occurs for the above studied case $\mu>0$ ), they actually occupy a finite band, with an solution (peakon) at the edge of the band. After this calculations a family of discrete breathers is constructed for $\mu<0$ in section 3.3.2 by means of a continuation of these pinned solutions from the standard DNLS limit $(\mu=0)$ where they are easily obtained. The continuation results show that they form a family of regular pinned discrete breathers, including a peakon-like one, similar to what was found in the CA, but discrete breathers extend beyond the peakon in the form of a novel solution termed cuspon that we will characterize in this part. In section 3.3.2, the pinned breather stability is explored by means of both standard Floquet analysis and direct simulations, with the conclusion that only a small part of the family is unstable. Two-breathers bound states are reported in 3.3.2, where it is demonstrated that stability exchange between in-phase and out-of-phase states occurs at a point where the bound breathers are peakons. For what concerns to mobile breathers we show in section 3.3.3 that they can be continued up to a critical strength of the inter-site self-defocusing nonlinearity.

\footnotetext{
${ }^{1}$ Remind that the SM with $\mu<0$ is also physically relevant for it describes the repulsive case for the long-range dipole-dipole interactions in a Bose-Einstein condensate of bosonic atoms with magnetic momentum trapped in a deep optical lattice as introduced in section 2.1 .
} 


\subsubsection{Continuum limit}

To introduce the continuum approximation (CA) in eq. (2.18), we define $\Phi(x, t) \equiv e^{2 i t} \Psi(x, t)$, and expand $\Psi_{n \pm 1} \approx \Psi \pm \Psi_{x}+(1 / 2) \Psi_{x x}$, where $\Psi$ is now treated as a function of the continuous coordinate $x$, which coincides with $n$ when it takes integer values. After that, the continuum counterpart of eq. (2.18) is derived,

$$
i \Psi_{t}=-2(1-|\mu|)|\Psi|^{2} \Psi-\left(1-|\mu||\Psi|^{2}\right) \Psi_{x x},
$$

where we have set $\nu=+1$ and $\mu<0$, in order to inspect the interesting region. Equation (3.21 ) conserves the norm and Hamiltonian, which are straightforward counterparts of expressions (3.19) and (3.20),

$$
\begin{aligned}
N_{\text {cont }} & =\frac{1}{\mu} \int_{-\infty}^{+\infty} d x \ln \left(\left.|1-| \mu|| \Psi\right|^{2} \mid\right) \\
\mathcal{H}_{\text {cont }} & =\int_{-\infty}^{+\infty}\left[\left|\Psi_{x}\right|^{2}+2\left(\frac{1}{|\mu|}-1\right)|\Psi|^{2}+\frac{2}{\mu^{2}} \ln \left(\left.|1-| \mu|| \Psi\right|^{2} \mid\right)\right]
\end{aligned}
$$

Localized solutions to eq. (3.21) are sought as $\Psi(x, t)=U(x) e^{i \omega t}$, with a real function $U(x)$, this solutions are usually referred to as envelope solitons in the continuum context [101]. The localized envelope $U(x)$ obeys the equation

$$
\frac{d^{2} U}{d x^{2}}=\frac{\omega-2(1-|\mu|) U^{2}}{1-|\mu| U^{2}} U
$$

which may give rise to solitons, provided that $\omega>0$ and $|\mu|<1$. The absence of soliton solutions for $|\mu|>1$ implies that if the intersite self-defocusing, accounted for by $\mu<0$, is stronger than the onsite self-focusing, the selftrapping of solitons is impossible in the CA. Equation (3.24) can be cast in the form

$$
\frac{U_{x x}^{\prime \prime}}{\left(|\mu|^{-1}-1\right)}=-W^{\prime}(U)
$$

where the effective potential $W(U)$ is

$$
W=-\frac{1}{2} U^{2}-\frac{1-\Omega}{2|\mu|} \ln \left(1-|\mu| U^{2}\right), \text { with } \Omega \equiv \frac{|\mu| \omega}{1-|\mu|}
$$

the expansion of the potential (3.26) for $U^{2} \rightarrow 0$ yields

$$
W \approx \frac{\left[-\Omega U^{2}+|\mu|(1-\Omega) U^{4}\right]}{2} .
$$

This form of the equation shows that solitons exist in a finite band of frequencies, $0<\Omega<1$, rather than in the entire semi-infinite band, $\Omega>0$, where 
the linearization of equation (3.24) produces exponentially decaying solutions that could serve as the solitons' tails. The reduction of the semi-infinite band to a finite one is typical for soliton families in models with competing nonlinearities, such as the cubic-quintic NLS equation [102]. Further, it follows from the divergence of potential (3.26) at $U^{2}=1 /|\mu|$ that the solitons's amplitude $A$, which is a monotonously increasing function of $\Omega$, is smaller than $1 / \sqrt{|\mu|}$ for $0<\Omega<1$, and $A=1 / \sqrt{|\mu|}$ at $\Omega=1$.

Solitons can be found in an explicit form near the edges of the existence band: at small $\omega$ (i.e., small $\Omega$ ),

$$
U(x) \approx \sqrt{\omega(1-|\mu|)} \operatorname{sech}(\sqrt{2 \omega} x),
$$

while precisely at the opposite edge of the band, $\Omega=1$, the exact solution is a peakon,

$$
U_{\text {peakon }}(x)=(1 / \sqrt{|\mu|}) \exp (-\sqrt{(1 /|\mu|)-1}|x|) .
$$

In other words, at a given frequency $\omega$, the peakon solution is found at

$$
|\mu|=\left|\mu_{p}\right| \equiv 1 /(1+\omega) .
$$

Note that norm (2.21) of the peakon is

$$
N_{\text {peakon }}=\pi^{2} /[6 \sqrt{|\mu|(1-|\mu|)}]
$$

and its energy is also finite. Close to this point, i.e., for $0<1-\Omega \ll 1$, the solution is different from the limiting form (3.29) in a narrow interval $|x| \lesssim \sqrt{|\mu| /(1-|\mu|)}(1-\Omega)$, where the peak is smoothed.

Finally let us remark that the CA based on eq. (3.21) is valid if the intrinsic scale of all continuum solutions, that may be estimated through the curvature of the soliton's profile at $x=0$ as $l \sim 1 / \sqrt{\left|U_{x x}^{\prime \prime} / U\right|}$, is large, $l \gg 1$ (recall the lattice spacing is $1 \mathrm{in}$ the present notation). According to eq. (3.29), the latter condition implies $(1 /|\mu|)-1 \ll 1$ (i.e., strictly speaking, the CA applies in the case when the competing nonlinearities in the SM nearly cancel each other).

It is relevant to note that, in the standard version of the SM (previously studied in sections 3.1 and 3.2), with $\mu>0$, the CA presented here give rise to pinned envelope solitons in the entire semi-infinite band, $\omega>0$ and then consistent with the exact solutions obtained for the discrete model in the above sections and earlier works [58, 59, 89, 90].

\subsubsection{Pinned discrete breathers}

In order to find exact pinned $((0,1)$ resonant) discrete breather solutions in a numerical form, we look for solutions to eq. (2.18) which are localized and 
time periodic with frequency $\omega_{b}=2 \pi / T_{b}$ (that is related to $\omega$ in the continuum equation (3.21) by $\left.\omega_{b} \equiv \omega-2\right)$. Pinned solutions are widely known for the DNLS limit $(\mu=0)$ since they can be obtained both continuing the analytical AL pinned beathers along the standard SM (as previously done in sections 3.1 and 3.2) and by the continuation from the anticontinuum limit, $C=0$, of the DNLS equation (2.5). It is then possible to make a numerical continuation of such solutions for $\mu<0$ by adiabatic changes of the model parameter $\mu$ and successive applications of the shooting methods in order to obtain the numerically exact pinned discrete breather for a given frequency $\omega_{b}$ and $\mu$. In general all the pinned solutions were computed starting from the DNLS limit, $\mu=0$, and increasing $|\mu|$ at a fixed value of $\omega_{b}$. The continuations were performed using an increment $\delta(|\mu|)=10^{-2}$ at each step, or smaller if higher accuracy was needed.

As shown in the previous section, the breather family in the continuum equation (3.21) ends with the peakon solution (3.29). To compare the numerically determined shape of the discrete breathers with the feasible peakon limit, we fitted the breathers' tails to the asymptotic form

$$
\left|\Phi_{n}\right|=A \exp \left[-\Gamma\left(\left|n-n_{0}\right|\right)\right],
$$

with constant $A, \Gamma$, and $n_{0}$, which follows from the linearized equation (2.18) for large $|n|$. This procedure yielded the decay rate, $\Gamma=\Gamma\left(\mu, \omega_{b}\right)$, amplitude, $A=A\left(\mu, \omega_{b}\right)$ (and the center's position $n_{0}$ ), as functions of parameters $\mu$ and $\omega_{b}$ of the pinned breather family. Once $A\left(\mu, \omega_{b}\right)$ and $n_{0}$ were found, we defined $\gamma\left(\mu, \omega_{b}\right) \equiv A-\left|\Phi_{n_{0}}\right|$ to measure a deviation of the true discrete soliton from a conjectured peakon shape obtained by formal extension of the tail inward.

In figure 3.17.a we show the evolution of $\gamma$ produced by several continuations of the discrete breather solutions (at different frequencies $\omega_{b}$ ). We define $\mu_{p}\left(\omega_{b}\right)$ as a value of $\mu$ at which an exact discrete peakon of internal frequency $\omega_{b}$ is found, that we realize as vanishing of $\gamma\left(\mu, \omega_{b}\right)$ at $\mu=\mu_{p}$. In figure 3.17.b we plot the evolution of the breather's amplitude as the continuation is performed. It is observed that the amplitude increases with $|\mu|$, reaching the predicted value, $1 / \sqrt{|\mu|}$, at the exact peakon solution.

A noteworthy result, evident from figure 3.17 , is the persistence of discrete breathers beyond the peakon limit (which means continuability of the solutions to $\gamma<0$ ). The apparent intersection of different curves at one point in figure 3.17.a is a spurious feature (see the inset in the figure): an accurate consideration shows that the curves actually intersect at close but different points. In contrast, the intersection of the curves in figure 3.17.b indeed happens at a single point, which corresponds to discrete breathers taking the peakon shape.

Figure 3.18 displays typical examples of the numerically found discrete 

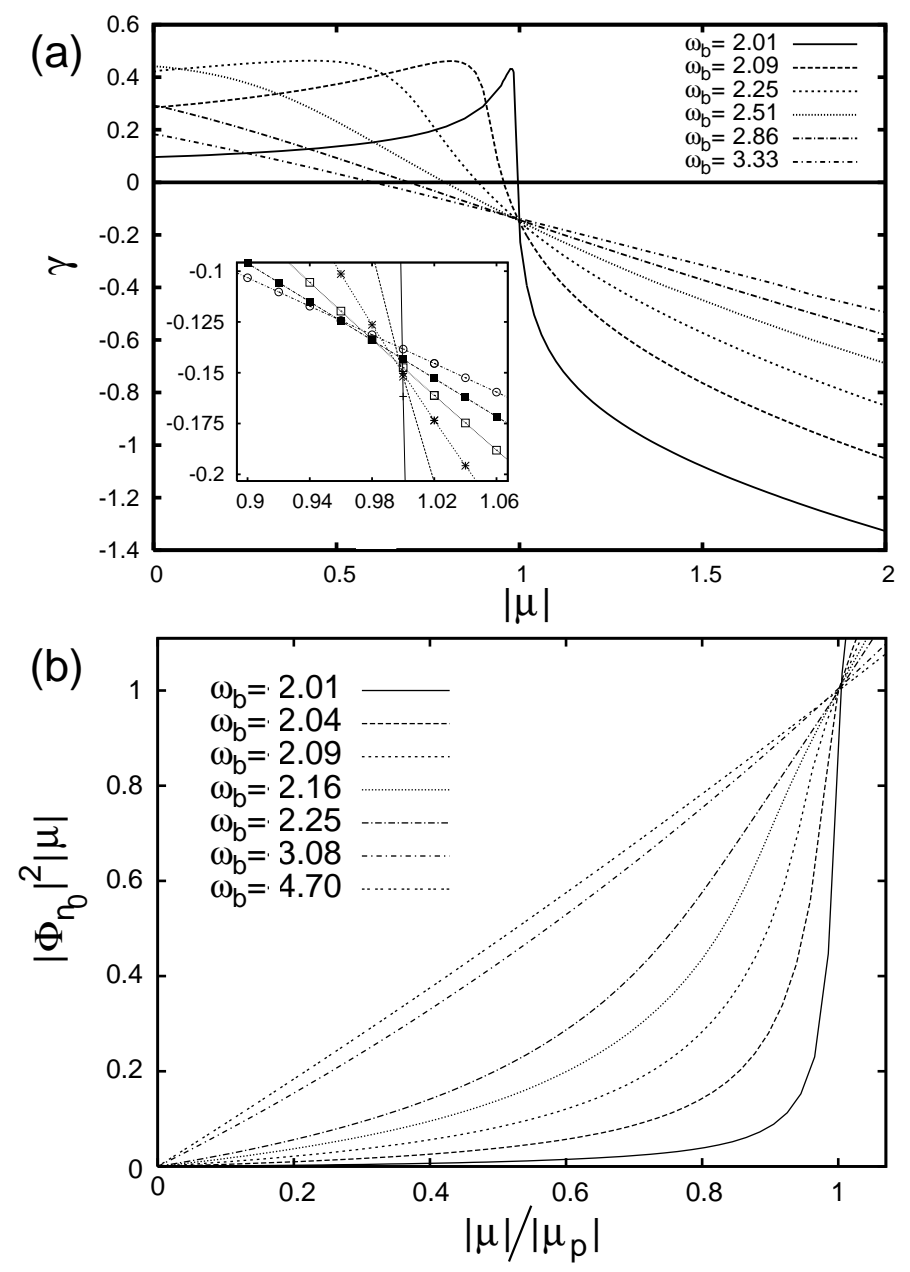

Figure 3.17: (a) The mismatch with the peakon shape, $\gamma$, as a function of $|\mu|$, for discrete breathers found at different frequencies $\omega_{b}$. (Note in the inset that there is no common intersection of all the curves). (b) The breather's amplitude vs. $|\mu|$. The axes are rescaled to show that the amplitude of the peakon solutions (attained at $\left.|\mu|=\left|\mu_{p}\right|\right)$ are equal to $1 / \sqrt{|\mu|}$, as predicted by the continuum approximation.

breathers. It demonstrates that the solutions corresponding to $\gamma<0$ are cuspons, with a super-exponential shape, that do not exist in the continuum equation (3.21). The discrete character of the SM with the competing nonlinearities allows this new type of solution, as happens with the quasi-collapsing states in the standard DNLS equation in two dimensions (see next chapter). Cuspon solutions continue into the region of $|\mu|>1$, where the CA yields no breathers, but, due to the sharp change of the solution with the increase of $|\mu|$, finding numerical solutions at larger values of $|\mu|$ becomes increasingly more 


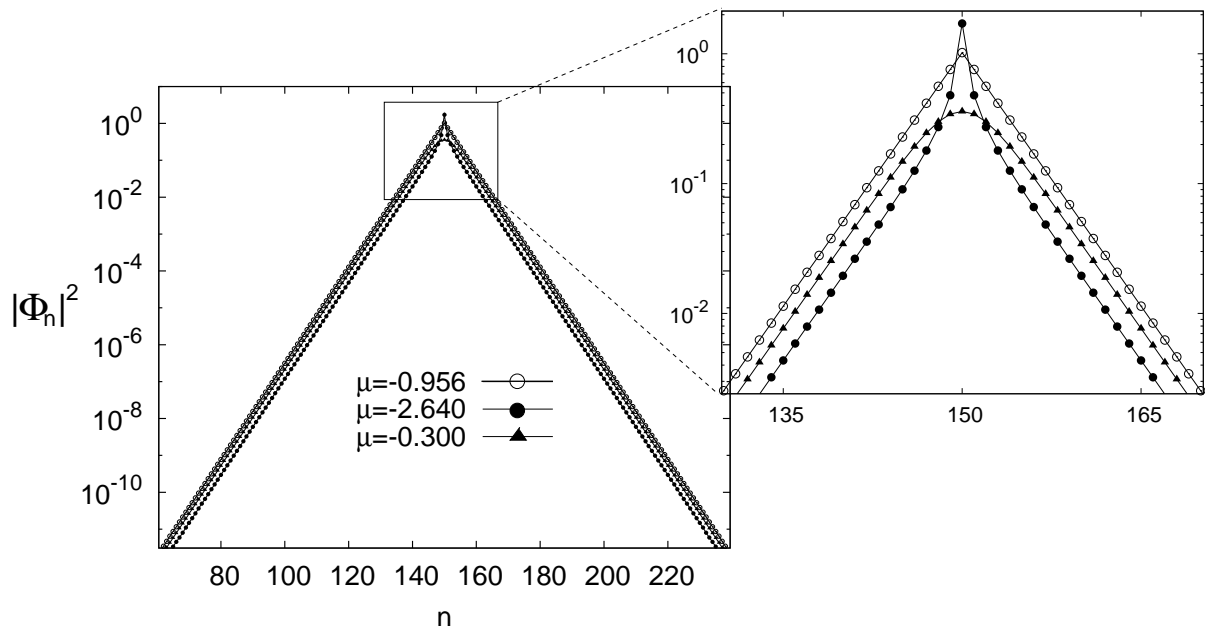

Figure 3.18: Generic examples of three different types of discrete breathers of frequency $\omega_{b}=2.091$ : a quasi-continuous sech-like solution at $|\mu|=0.3$, an exact peakon at $|\mu|=0.956$, and a cuspon at $|\mu|=2.64$.

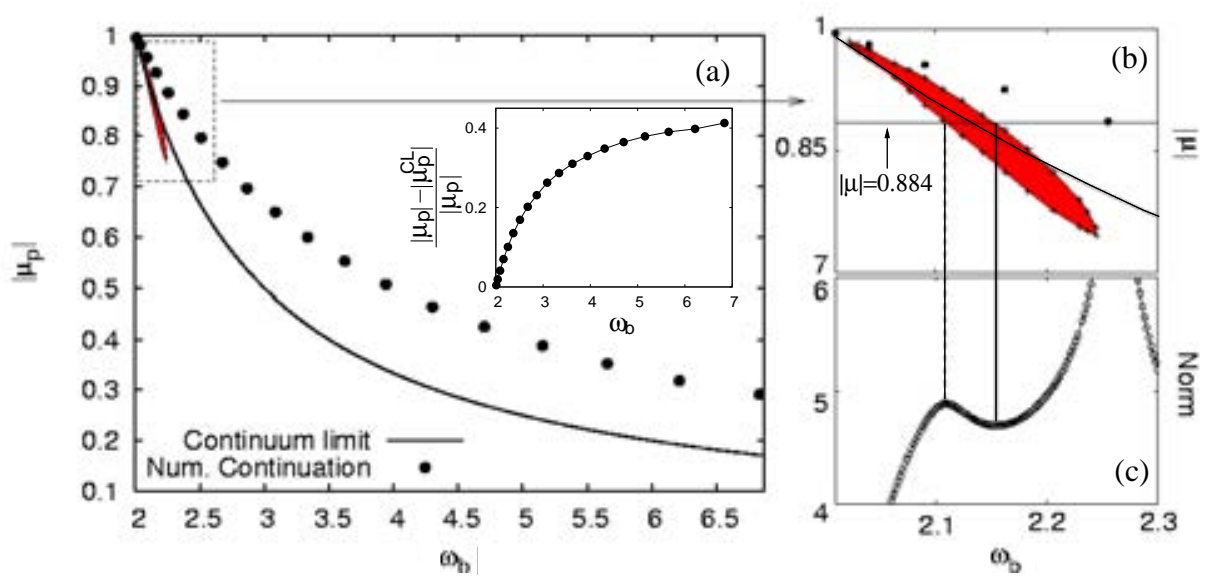

Figure 3.19: (a) The value of $\left|\mu_{p}\right|$, at which the soliton assumes the peakon shape: the prediction of the continuum approximation, equation (3.30) (solid curve), and numerical results for discrete breathers (dots). The small region where the pinned discrete breathers are found to be unstable (for that purpose, the vertical axis shows $|\mu|$, rather than $\left.\left|\mu_{p}\right|\right)$ is also shown. The inset displays the relative difference between the numerically found values of $\left|\mu_{p}\right|$ and the predicted ones, $\left|\mu_{p}^{C L}\right|$, provided by the continuum approximation. (b) Zoom of the area in the $\left(\omega_{b},\left|\mu_{p}\right|\right)$ plane where the instability island is located. (c) Norm of the discrete breathers vs. their frequency for $|\mu|=0.884$.

difficult. 
In figure 3.19.a we compare the line of the existence of the peakons in the continuum limit, and the actual location of discrete peakons. It is seen that the agreement between the CA and numerical findings is good for smaller $\left|\omega_{b}\right|$ (in this case, the discrete breathers are broad), while at larger $\left|\omega_{b}\right|$ the discrete breathers are narrow, hence the agreement with the CA deteriorates.

\section{Floquet analysis}

Performing the linear stability analyses of pinned breathers it is found that these solutions are linearly stable along the whole $\mu$-continuation, except for a relatively small region, as shown in figure 3.20.a. The entire instability island in the $\left(\omega_{b},|\mu|\right)$ plane is displayed in figure 3.19.b. The instability displayed is revealed by a Floquet multiplier leaving the unit circle at +1 (harmonic bifurcation). The eigenvector associated to this multiplier show a localized profile around the pinned solution. Note, in particular, that the peakon and cuspon solutions are stable. The stability of the discrete breathers was also checked by direct simulations of perturbed (along the unstable direction given by the Floquet eigenvector whose Floquet multiplier is $\lambda>1$ ) solutions, using the full equation (2.18). The results of these simulations corroborated the
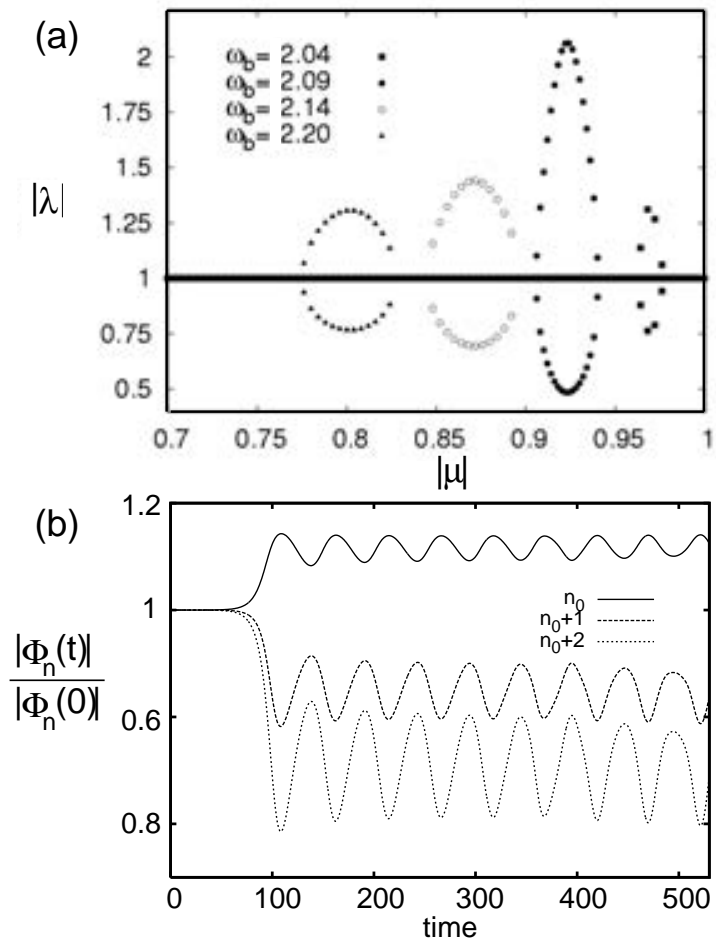

Figure 3.20: (a) The absolute value of the Floquet multipliers, $\{|\lambda|\}$, for the linearization of perturbations around discrete solitons, is shown vs. $|\mu|$, at several fixed values of the frequency (which are chosen so as to make the instability intervals well separated). (b) A robust pulson generated from an unstable breather at $|\mu|=0.922$. 


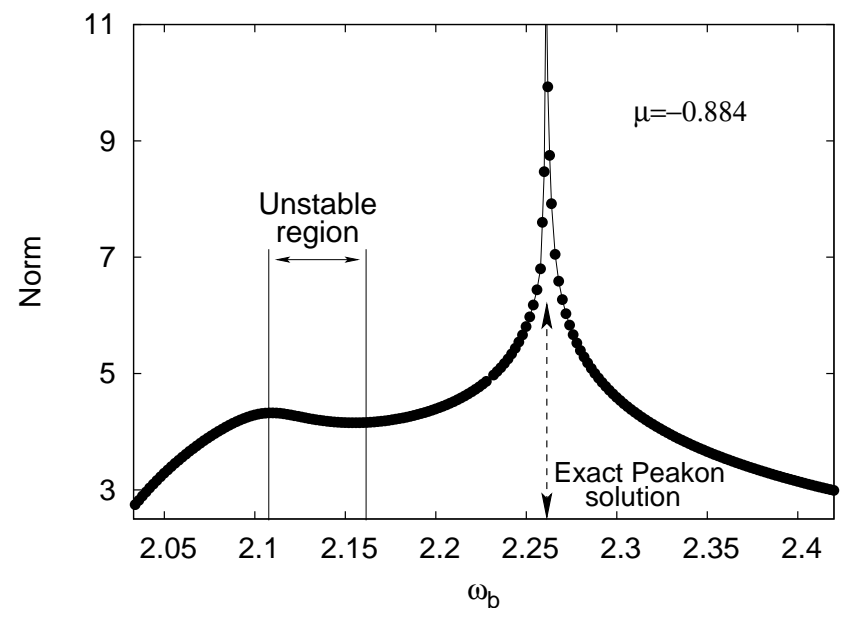

Figure 3.21: The norm of the discrete breathers vs. the frequency, for $|\mu|=0.884$.

predictions of the linear stability analysis.

Direct simulations of the evolution of perturbed unstable breathers, a typical example of which is displayed in figure 3.20.b, show that, after a transient stage, a localized pulson (showing simultaneous width and amplitude oscillations) is formed. The pulsons are (quasi-)periodic in time, and persist indefinitely. This behaviour resembles that found in the ordinary two-dimensional DNLS equation in quasi-collapsing states that will be described in next chapter.

A necessary stability condition for soliton families in models of the NLS type may be provided by the Vakhitov-Kolokolov (VK) criterion [103]: if the norm $N$ of the breather is known as a function of its frequency $\omega_{b}$, the breathers can be stable against small perturbations with real eigenvalues, provided that $d N / d \omega_{b}<0$. Although the applicability of the VK criterion to the present model has not been proven (and counter-examples are known, when solutions predicted by the criterion to be unstable are actually stable [104]), it is relevant to test the criterion here, numerically computing $N\left(\omega_{b}\right)$ according to eq. (3.19). The result is that the VK criterion precisely explains the stability and instability of the discrete breathers, except for the cuspons (see below), as shown in figure 3.19.c.

A noteworthy feature of the $N\left(\omega_{b}\right)$ dependence is a divergence of the total norm due to the infinite contribution of the central site to expression (3.19) in the case of the exact peakon solution, with $\left|\Phi_{n_{0}}\right|^{2}=1 /|\mu|$. An example of the $N\left(\omega_{b}\right)$ dependence showing the divergence is plotted in figure 3.21. As concerns the cuspons, whose amplitude exceeds the critical value, $1 / \sqrt{|\mu|}$, the 
norm (3.19) converges for them, and features a positive slope (see figure 3.21), $d N / d \omega_{b}>0$. The VK criterion predicts instability in this case; however, the direct computation of the Floquet multipliers, as well as direct simulations, reveal no instability of the cuspons. Thus, while the VK criterion is perfectly correct for regular pinned breathers and peakons in the present model, it is irrelevant for cuspons, cf. the situation in [104].

\section{Bound states of breathers and their stability}

The above findings on the characterization and stability of pinned discrete breathers suggest looking at the behaviour of more exotic $(0,1)$ resonant localized structures. For this purpose, we have also explored bound states of pinned discrete breathers solutions to the SM with competing nonlinearities. For this purpose, we performed numerical continuation in $\mu$, starting with the well known bound states of the standard DNLS equation (constructed by continuation from the anticontinuum DNLS limit) at $\mu=0$. In that limit, two different types of bound states are known, in-phase and $\pi$-out-of-phase ones, which are represented, respectively, by even and odd solutions. It is well known that only the states of the latter type are stable [105, 106].

The numerical continuation of breather bound states was performed for pairs of identical discrete breathers of a given frequency $\omega_{b}$ and different dis-
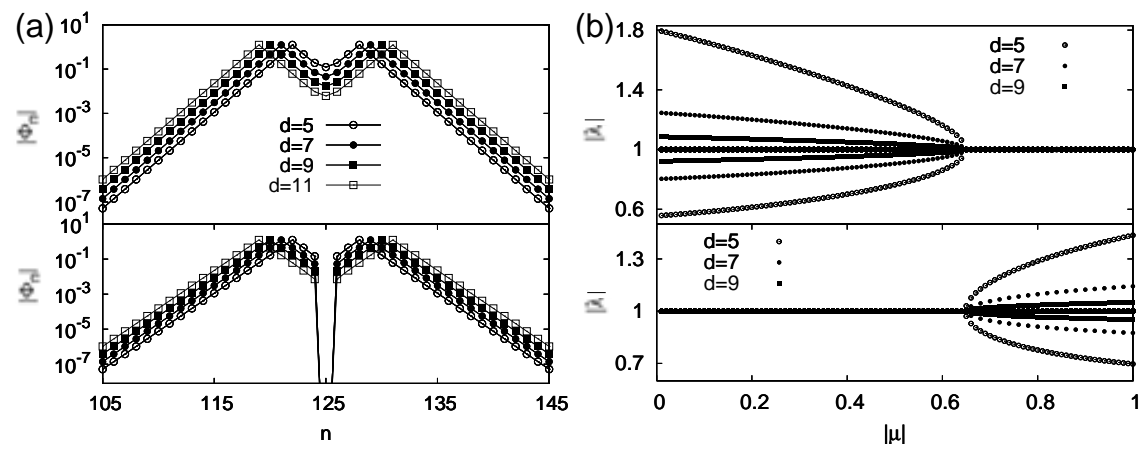

Figure 3.22: (a) Profiles of typical in-phase (top) and out-of-phase (bottom) bound states of two peakons, with different distances between their centers, at $\omega_{b}=3.086$ and $|\mu|=\left|\mu_{p}\right|=0.645$. (b) Absolute values of the Floquet multiplies that determine the stability of three bound states, with the same fixed frequency, $\omega_{b}=3.086$, and different separations between the breathers. The in-phase (top) and out-of-phase (bottom) bound states are stabilized and destabilized, respectively, at the point where the bound breathers are peakons, see panel (a). Unstable states are less unstable (with smaller absolute values of the Floquet multipliers accounting for the instability) if the distance between the breathers is larger. 
tances between them. The continuation of in- and out-of-phase bound states gives bound states of peakons, see figure. 3.22.a. The latter solution is found at exactly the same value, $\mu=\mu_{p}\left(\omega_{b}\right)$, which gives rise to the single peakon. We have also examined the linear stability for the computed solutions. A remarkable feature of the bound states observed with increase of $|\mu|$ is the stability interchange between the in-phase and out-of-phase states, as shown in figure 3.22.b. This stability interchange occurs precisely at $\mu=\mu_{p}\left(\omega_{b}\right)$, regardless of the separation between the bound breathers.

\subsubsection{Moving discrete breathers}

In order to complete the full picture on the characterization of discrete breather solutions in the SM with competing nonlinearities we turn our attention to mobile breathers. We have proceeded in the same way as for the standard SM and used the generic method for the continuation of $(p, q)$ resonant solutions. In order to explore the behaviour of these solutions we have numerically continued them from the DNLS limit where, in turn, they were earlier obtained in previous sections ${ }^{2}$. Among those mobile solutions we have chosen for the continuation into the SM with competing nonlinearities only those that were linearly stable at the DNLS limit.

As in the standard SM when $\nu \neq 0$ the obtained states are composed of a traveling localized core and an extended background, $\Phi_{n}=\left(\Phi_{n}\right)_{\text {core }}+$ $\left(\Phi_{n}\right)_{\mathrm{bckg}}$, see figure 3.23 which is a superposition of nonlinear plane waves whose amplitude is related to the height of the corresponding Peierls-Nabarro barrier.

The important result obtained along the continuation by the SM with $\mu<0$ is that the mobile breathers can only be continued up to a certain critical value, $\mu=\mu_{c}\left(\omega_{b}\right)$, close to, but smaller in absolute value than, $\mu_{p}\left(\omega_{b}\right)$ at which the pinned discrete breather becomes a peakon. The Floquet stability analysis reveals that the extended background of the mobile breathers is subjected to modulational instability. (However, this is too weak to manifest itself in the simulations and it is only noticeable by looking at the Floquet spectra when the amplitude of the background is very high). On the the other hand we do not observe any localized eigenvector with eigenvalue $|\lambda|>1$ and thus the core is not affected by any unstable perturbation. The stability of mobile solutions is corroborated when simulations of the dynamics are performed, allowing for interesting numerical experiments (see below). The background amplitude is

\footnotetext{
${ }^{2}$ Remind that only a subset of those mobile solutions (those with small enough frequency) continued from the AL limit $(\mu=1, \nu=0)$ remains along the whole standard SM path $(\mu>0)$ to the DNLS $(\mu=0, \nu=1)$. Then, only those states that could be obtained at the DNLS limit are, in principle, continuable into the SM with competing nonlinearities $(\mu<0)$.
} 


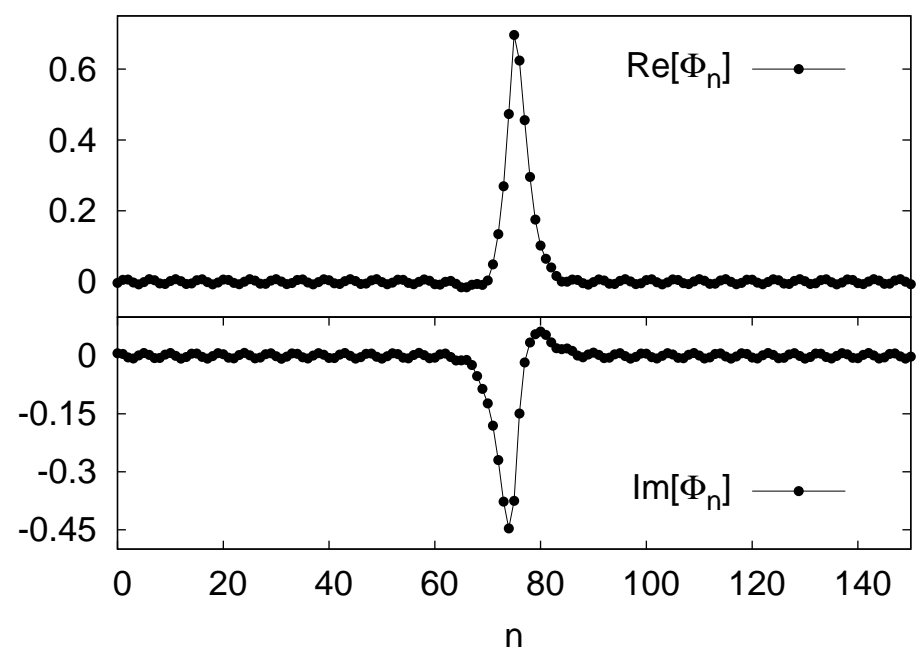

Figure 3.23: The real and imaginary parts of the lattice wave field in a moving discrete breather, for $\omega_{b}=2.24$ and $\mu=-0.7$.

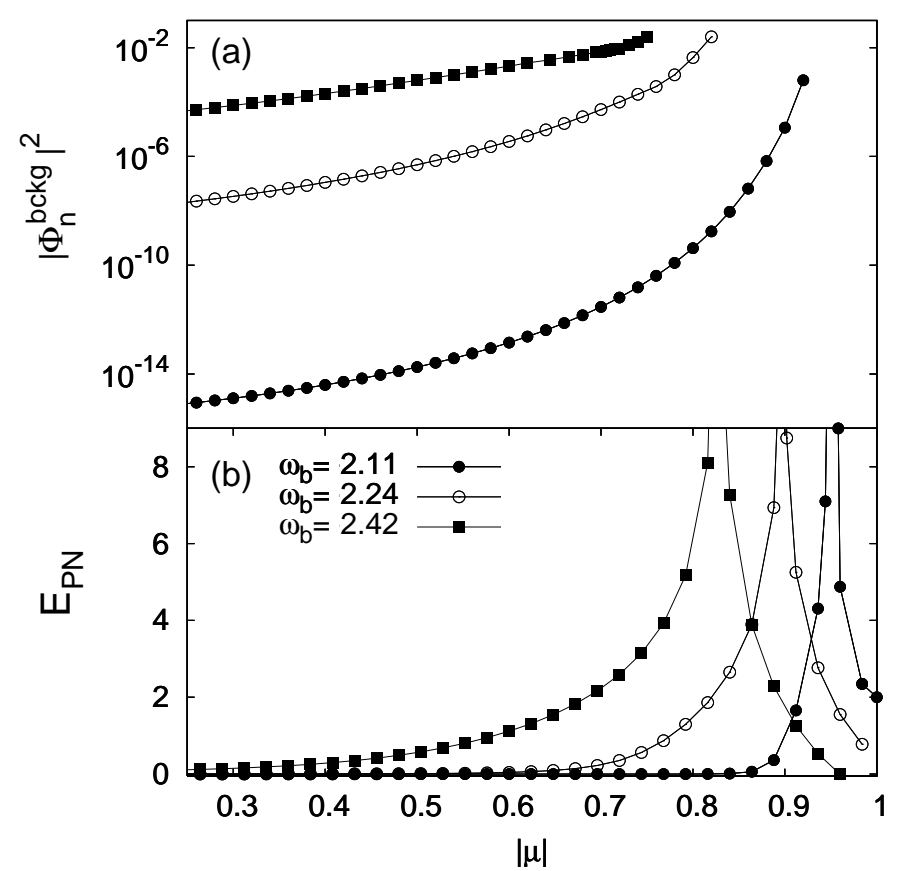

Figure 3.24: The background amplitude (a) and the height of the Peierls-Nabarro barrier (b), as functions of $|\mu|$, for three mobile discrete breathers. 

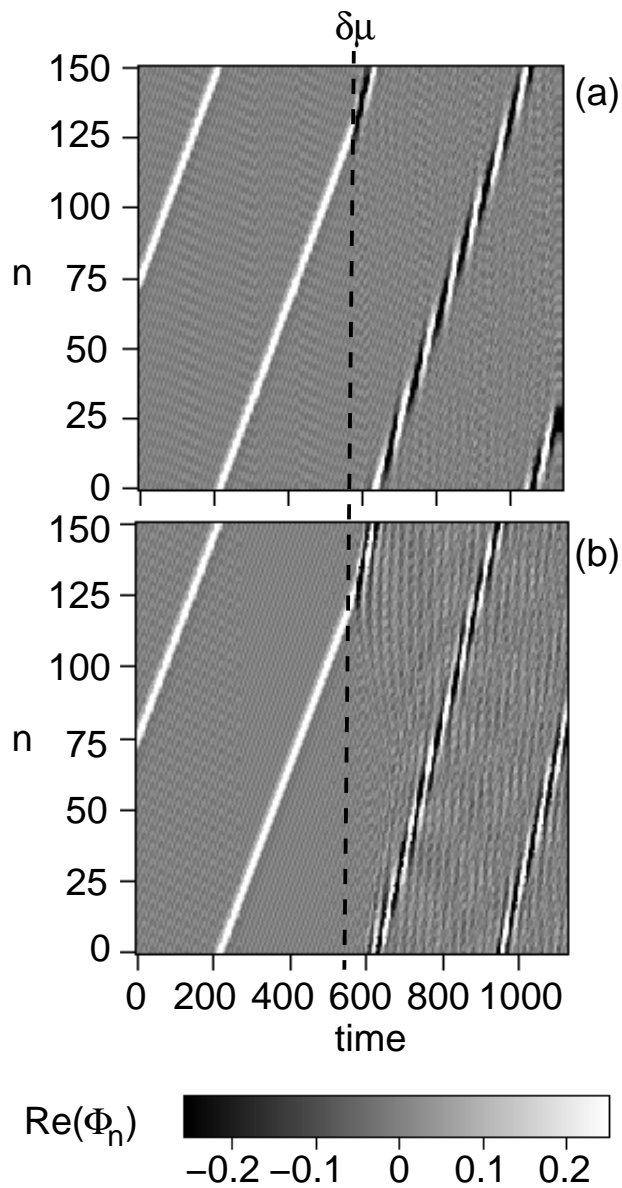

Figure 3.25: The time evolution of the real part of the lattice wave field in a mobile state. Initially, a moving soliton is created corresponding to $|\mu|=0.8$ and $\omega_{b}=-2.24$. Then, $\mu$ jumps instantaneously to $\mu^{\prime}=\mu+$ $\delta \mu$. After the jump, followed by emission of some transient radiation, the moving core becomes broader and starts to move faster. In (a) $\left|\mu^{\prime}\right|=0.84$, and in (b) $\left|\mu^{\prime}\right|=0.89$.

a growing function of $|\mu|$ having a very sharp increase when $|\mu|$ approaches $\mu=\mu_{c}\left(\omega_{b}\right)$, see figure 3.24.a. This behaviour of the background amplitude suggests that the PN barrier also grows with $|\mu|$ and becomes very high near the critical point. To check this expectation, we have computed the height of the PN barrier for the same frequencies $\omega_{b}$ for which the mobile breathers were numerically calculated, using the energy definition as in equation (3.20). Figure 3.24.b confirms that the PN barrier dramatically increases when the continuation approaches the critical point, $\mu=\mu_{c}\left(\omega_{b}\right)$, although the PN barrier diverges not exactly at this point, but rather at $\mu=\mu_{p}\left(\omega_{b}\right)$, where the pinned breather assumes the peakon shape.

The strong dependence of the PN barrier on $\mu$ suggests a numerical experiment to test the behaviour of mobile breathers when the lattice's pinning force suddenly changes. To this end, we took an initial mobile breather at values of $\mu$ and $\omega_{b}$ for which the PN barrier is low. Then we monitored the evolution 


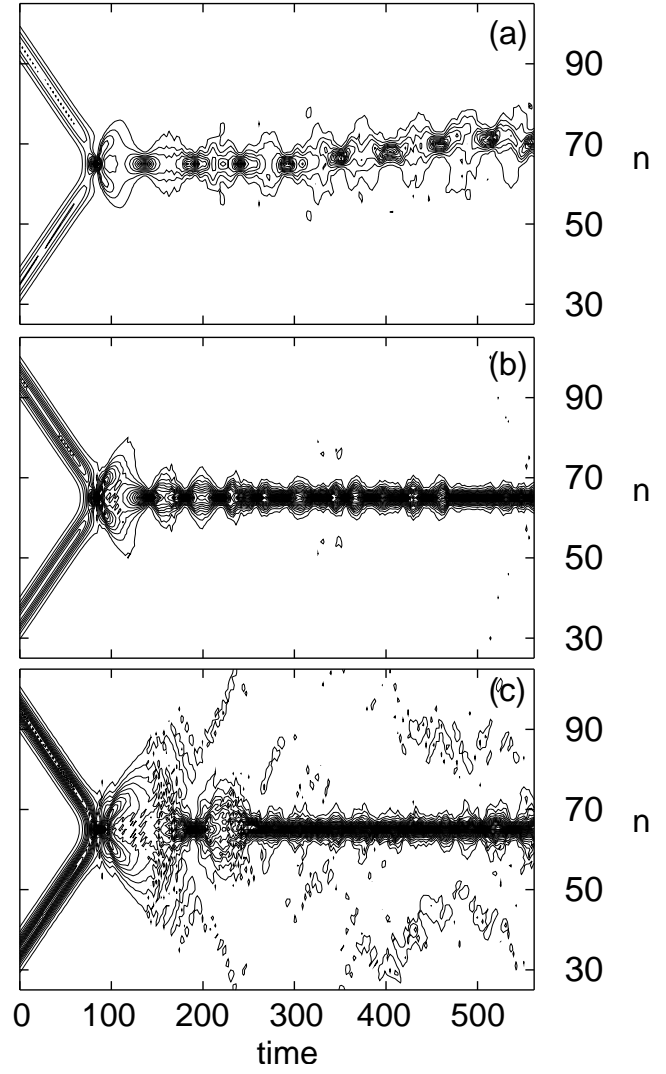

Figure 3.26: Contour plots showing the evolution of the lattice field $\left|\Phi_{n}\right|$ in three cases of collisions between identical breathers moving in opposite directions. The breather's frequency is $\omega_{b}=-2.11$, and $|\mu|=0.6$ (a), $|\mu|=0.8$ (b), and $|\mu|=0.9$ (c).

of the moving solution following an instantaneous change in the nonlinearity, $\mu \rightarrow \mu+\delta \mu \equiv \mu^{\prime}$, which makes the PN barrier essentially higher than experienced by the original soliton. The numerical experiments are illustrated by figure 3.25. We observe that the core of the mobile breather does not become pinned due to the increase of the PN barrier, but rather accommodates itself, with some radiation loss, into a broader state with a smaller amplitude, so that the PN barrier, as experienced by the new state for $\mu=\mu^{\prime}$, is low enough to allow the breather to remain mobile. Besides that, we observe an increment in the core's velocity, so that the larger the jump of the PN-barrier's height the faster is the new moving state. The fact that the sudden increase of the PN barrier does not prevent the motion of the breather solution reveals, on one hand, that the relation between PN barrier and mobility is far from trivial, and on the other hand, that mobility is quite a robust feature.

Finally, we simulated collisions between identical lattice breathers moving in opposite directions. The results show that the colliding breathers always merge into a single localized state, which subsequently features intrinsic pulsations. If the PN barrier is low, the emerging pulse can itself move in a chaotic 
way, due to interaction with the lattice phonon field (radiation) generated in the course of the collision. On the contrary, for values of $\mu$ and $\omega_{b}$ at which the original breathers experience a high PN barrier, the finally generated single soliton is always strongly pinned.

The most notable and generic feature of the collision manifests itself in the merger scenario. When the cores of the mobile breathers collide, sudden delocalization is first observed, with transfer of energy from the collision point to adjacent lattice sites. Then, almost all the energy is collected back at the collision spot, and thus a single localized state emerges. An example of the collision is shown in figure 3.26. This scenario was observed in all simulations of the collisions. The appearance of pulsons as the product of soliton collisions, as well as the fact that they also appear as asymptotic states of the evolution of perturbed unstable breathers (see section 3.3.2), shows the ubiquity of this type of localized excitations in the present model.

\subsection{Conclusions and Prospective Remarks}

In this chapter we have studied numerically several features about discrete breathers solutions to the one-dimensional Salerno lattice (2.18). It is then convenient to summarize the results and obtain a global picture of the work. A schematic picture of the results obtained in this chapter can be found in figure 3.27 .

Pinned Breathers.- This class of solutions have been extensively studied by earlier works for the standard Salerno model $(\mu>0)$ with positive values of the nonintegrability parameter, $\nu$. In this region we have confirmed that the continued (along the SM path) breathers coincide in the DNLS limit with those obtained by a continuation from the uncoupled (anticontinuum) limit of the latter equation. We have extended the computation of numerically exact pinned breathers in the standard SM $(\mu>0)$ for negative values of $\nu$. In this case there exist narrow regions where the immobile breathers experience mirror symmetry-breaking bifurcations.

The analysis of the pinned discrete system in the Salerno model with competing nonlinearities $(\mu<0)$ yields a family of breathers, which includes a peakon as the continuum counterpart predicts. However, the family continues beyond the peakon, in the form of special pinned discrete breathers termed cuspons. Stability analysis of the pinned breathers in the SM with competing nonlinearities reveals that only a small part of the soliton family is unstable; the evolution of the unstable breathers leads asymptotically to pulsons, i.e. localized solutions where the width oscillates. In this part of the SM bound 
states of identical breathers were also investigated, revealing a stability exchange: the in-phase and out-of-phase bound states, which are unstable and stable, respectively, in the DNLS limit, exchange their stability character exactly at the point where the bound breathers are peakons.

The computation of pinned breathers both on-site and inter-site centered has served for computing the Peierls-Nabarro barrier and thus provide a useful tool to analyze the results obtained for mobile breathers.

Mobile breathers.- Using a regularized Newton method we have continued the family of mobile Ablowitz-Ladik discrete breathers into the nonintegrable domain of model parameters. The continuation was then performed for a fine grid of frequencies belonging to the family of $(p=1, q=1)$ resonant discrete breathers. We find that these solutions decay asymptotically, in space, to an excited lattice extended state (the background), whose amplitude vanishes at the integrable Ablowitz-Ladik limit. This component of the solution is unambiguously found to be a linear combination of nonlinear resonant plane waves whose amplitudes decay typically, in $k$-space, exponentially. The exponentially localized oscillation (the core) of the amplitude probability rides over this extended radiation state.

$$
\hat{\Phi}=\hat{\Phi} \text { core }+\hat{\Phi}_{\text {backg }}
$$

This expression defines the purely localized component $\hat{\Phi}_{\text {core }}$ of the solution. The background is a finite linear combination of nonlinear plane waves,

$$
\left(\hat{\Phi}_{\mathrm{bckg}}\right)_{n}(t)=\sum_{j=1}^{s} A_{j} \exp \left[\mathrm{i}\left(k_{j} n-\omega\left(k_{j}, A_{j}\right) t\right)\right] .
$$

These plane waves are exact solutions of the Salerno model (2.18). The results concerning the characterization of the background can be summarized as follows:

(i) The set of " $s$ " plane waves which take part in the background of a $(p, q)$-resonant discrete breather with internal frequency $\omega_{b}$ is derived by the simple selection rule for the wave-numbers $k_{j}$

$$
\frac{\omega\left(k_{j}, A_{j}\right)}{\omega_{b}}=\frac{1}{q}\left(\frac{p}{2 \pi} k_{j}-m\right),
$$

i.e. only the plane waves which are $(p, q)$-resonant with the internal period of the breather can be components of $\left\{\Phi_{n}^{\mathrm{bckg}}(t)\right\}$. The number of solutions of (3.35) fixes " $s$ ".

(ii) The amplitudes $\left\{A_{j}\right\}$ of the nonlinear plane waves differ by orders of magnitude yielding a localization in the $k$-space. 
(iii) There exist a strong positive correlation between the amplitude of the background and the strength of the Peierls-Nabarro barrier arising from the periodic lattice. This correlation is particularly clear when symmetry breaking transitions occur for the also studied case of $\nu<0$ and $\mu>0$, and reflects the link between non-integrability and the existence of the background dressing of the mobile core. Another interesting effect is obtained for the SM with competing nonlinearities. In this case the continuation of mobile breathers of a given frequency stops near the divergence of the Peierls-Nabarro barrier for pinned breathers with the same frequency.

(iv) Finally, the interpretation of the correlation described in (iii) is reinforced from a study of the energy evolution of the mobile core: There is an energy balance brought by the background when the core moves along the lattice. In particular, it can be observed how the core energy oscillates periodically so that it takes the maximum energy value when the core visits the inter-site configuration. This extra energy periodically obtained by the core is provided by the interaction background-core, with the energy maximum clearly related to the background amplitude.

It is worth stressing that the most relevant predictions of perturbative collective variable theory are confirmed by our numerical results, which show the existence of Peierls-Nabarro barriers to breather translational motion. Furthermore, the existence of exact oscillating breather solutions for the standard SM is numerically confirmed. They are found to contain an extended background whose amplitude is typically much smaller than for mobile breathers.

The correlation between the Peierls-Nabarro barrier $E_{P N}$ (computed from immobile breathers) and the amplitude background of moving breathers correctly suggests that the background has a role in the energy balance required to overcome the barriers to translational motion. The interpretation is also fully consistent with the observations on the background amplitude behaviour of spatially oscillating anchored breathers in the standard SM. Currently used effective particle (collective variable) theories are thus seen as intrinsically incomplete, because core energy is not an invariant of motion. Any sensible improved approach must adopt equation (3.33) as starting point for improved perturbative ansatzes, and we hope that our work will stimulate further studies along these lines.

Numerically exact moving discrete breathers with an infinitely extended tail of small amplitude were already observed in some cases for Klein-Gordon lattices with Morse potential by Cretegny and Aubry [53], however no investigation of the background of these exact solutions is reported, so they were able to "..suggest that generally a strictly localized breather cannot propagate 


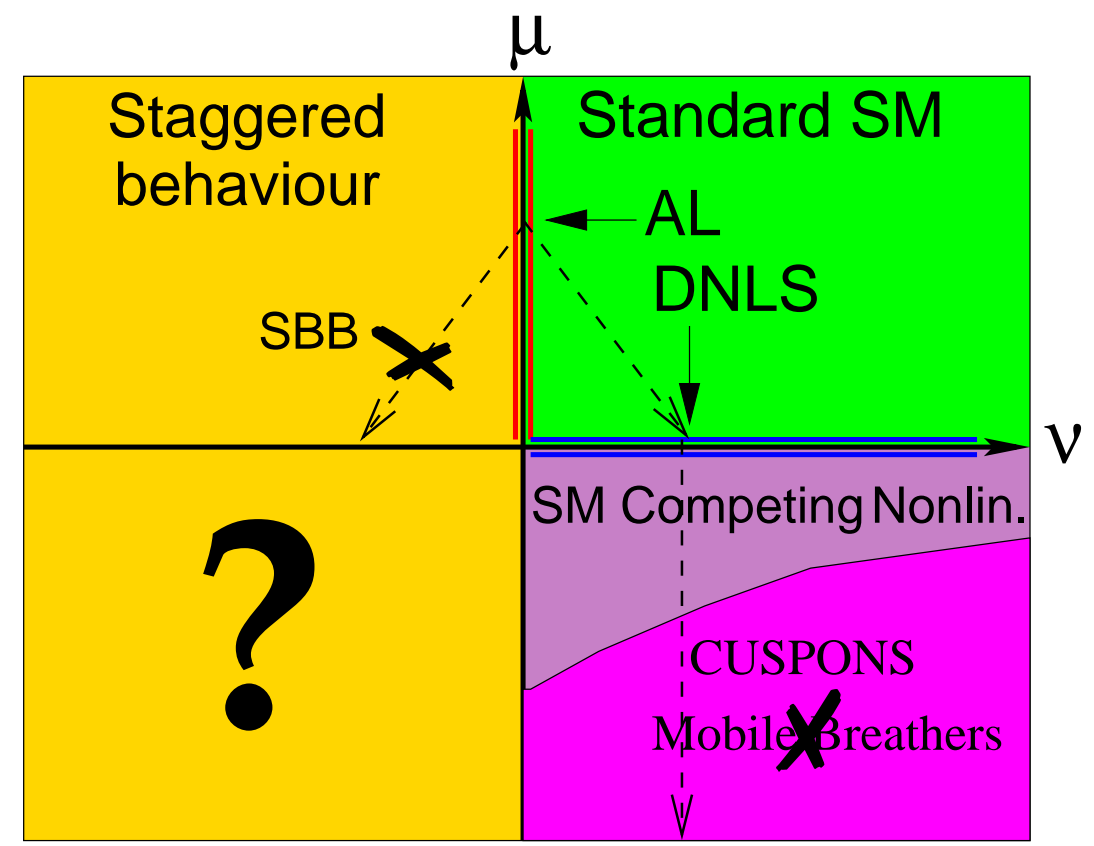

Figure 3.27: Schematic plot of discrete breather's existence diagram in the $(\nu, \mu)$ plane. For the standard Salerno model $\mu>0$ we have continued pinned breathers along the path $\mu+\nu=1$ for $(\nu>0)$ from the Ablowitz-Ladik integrable lattice to the DNLS limit. Mobile breathers can be continued all the way (from AL to DNLS eqs.) along this path provided their frequencies are small enough. For the standard Salerno model with $\nu<0$ we have performed the continuation along the path $\mu-\nu=1$ finding a symmetry breaking bifurcation for pinned breathers at $\nu \simeq-0.3$ that prevents continuing both pinned and mobile solutions far beyond this point and then excluding the possibility of reaching the DNLS equation with $\nu<0$. The staggering transformation between the two regions $(\nu>0$ and $\nu<0)$ of the standard Salerno model implies that staggered breathers cannot be continued to the DNLS limit with $\nu>0$ because of a symmetry breaking bifurcation. For the Salerno model with competing nonlinearities $(\mu<0)$ we find a transition from smooth peaked pinned breathers to cuspon states where the energy is hyperlocalized around the breather center. Cuspon breathers appear as stable solutions of the dynamics. Mobile breathers in the competing Salerno mobile cannot be continued beyond this transition point since the Peierls-Nabarro energy diverges at this point. The staggering transformation implies that these latter results applies for staggered breathers when $\mu<0$ and $\nu<0$. However, this region could not be explored for typical breather states since, on one hand, the purely AL lattices with $\mu<0$ does not admit localized states as solutions (preventing the continuation from $\nu>0$ and $\mu<0$ ) and, on the other hand, the continuation from $\nu<0$ and $\mu<0$ stops near the symmetry breaking bifurcation as reported above. Then, this region of the Salerno model with competing nonlinearities remains unexplored and apparently forbidden for our continuation methods. 
without radiating energy". Our systematic study of the NLS lattices allows us to go further by showing that the extended background (here fully characterized) plays an important and subtle role in the translational motion of the localized core. Indeed, it is an indispensable part of the exact solution in the nonintegrable regime. Exact mobile localization only exists over finely tuned extended states of the nonlinear lattice. Mobile "pure" (i.e. rest state background) localization must be regarded as very exceptional [96].

Before concluding this chapter, it is worth commenting on some of the differences between the Newton continuation of fixed points that we use in this chapter, and other important recent approaches to breather numerics. The work by Ablowitz et al [107] uses discrete Fourier analysis to obtain a nonlinear nonlocal integral equation, from where the "... soliton is thus viewed as a fixed point of a nonlinear functional" (sic) in the Fourier transformed space of functions. Following these authors, their results seem to differ from those of early pioneering work [108] (nowadays textbook material [23]) " in which a continuous traveling solitary waves were reported using Fourier series expansions with finite period $L$ while assuming convergence as $L \rightarrow \infty$ " (sic). Ablowitz et al term continuous a solution that can be defined off the lattice points, which they see as "necessary when discussing traveling waves in lattices" (sic), and disagree with some conclusions reported in the earlier works.

The ("orthodoxy matters") discussion above helps us to clarify how differently our numerical approaches "sees" the discrete Schrödinger breather problem: The very concept of a variable defined off the lattice points is intrinsically alien to our discrete approach, which neither needs of it nor excludes its eventual consideration. In contrast to those views (but not at all in logical opposition), we consistently view the thermodynamical limit $(N \rightarrow \infty)$ in lattice space, much in the sense used e.g. by Serge Aubry in his celebrated work on the Frenkel-Kontorova ground state problem [49]: The infinite size limit is built up from a subsequence of PBC (finite) lattices for which the limit is well defined. This will make the Fourier-transformed $k$-space continuum.

Closer to our approach in some respects, though technically different in many others, is the formal approach purposed recently by James and collaborators $[109,110]$. It is also worth mentioning that these results have been reproduced recently for other kind of solutions (dark breathers) [111] and have constituted [112] a "(negative) result" about the impossibility of constructing "exponentially localized fundamental (single-humped) moving discrete solitons" in the nonintegrable part of the Salerno model.

There are, at very different levels, several open questions to further research. From a technical point of view, it is important to analyze carefully the irrational limit $p / q \rightarrow \sigma$, of the solutions. In particular, in this limit the 
number of resonant plane wave branches tends to a continuum and one could (or not) expect that exponential localization in the reciprocal lattice persists in that limit. This can be addressed numerically, though systematic investigations may require some efforts in optimizing the time efficiency of current numerical schemes.

An important issue regarding applications is the phenomenology of multibreather states. In particular, studies on collisions of a pair of breathers may find in this study of exact mobility a useful reference in order to deal with the complexities that emerge from the many time-length scales involved in these physically relevant phenomena. Much simpler multibreather states, e.g. trainlike chains of (moderately) separated moving breathers could also be investigated. Not least, the perspective and results presented here may be of some interest to studies of the effects of coupling to (nonthermal and/or thermal) radiation baths in the breather and multibreather states of nonlinear lattices [51] and the practical manipulation and patterning of localized "hot spots" by external fields [113]. 



\section{Chapter 4}

\section{Discrete Breathers in two-dimensional Nonlinear Schrödinger lattices}

Given the ubiquity of such breathers in discrete nonlinear physical systems (which exist on essentially all length scales), these nonlinear excitations are likely to be important in many physical phenomena, including melting, fracture, and the buckling and folding of biopolymers. They may also prove useful in technologies ranging from 'smart' materials with tunable collective responses to light-induced, all-optical switches and networks. With the acquisition of this new animal, the nonlinear 'zoo' has become an altogether more interesting place.

David K. Campbell in [114].

The study of two-dimensional nonlinear Schrödinger lattices has attracted much attention $[115,116]$ in recent years due to the new phenomena emerging when the dimensionality of the lattice is increased. Some examples of these new features are the existence of vortex-breathers [117] which supports energy flux, the appearance of an energy threshold for the creation of discrete breathers [118-122] and the ubiquity of an instability (the quasi-collapse) of some discrete breather solutions leading to a highly localized pulson state [123128]. These theoretical efforts have their counterpart in recent advances in the field of nonlinear optics. The studies of two-dimensional arrays of coupled nonlinear waveguides allow the experimental observation of those effects studied theoretically. Specially relevant is the recent experimental breakthrough (theoretically designed in [129]) by Fleisher et al [72, 130], where a two-dimensional array of nonlinear waveguides is induced in a photosensitive material. This 
technique provides a clear experimental verification of the two-dimensional discrete breather existence in this system. In particular, besides the observation of standard discrete breathers, these works reported the first observations of staggered discrete breathers.

Our study in this chapter focus on the computation of numerically exact discrete breathers in two-dimensional anisotropic nonlinear Schrödinger lattices, i.e. where the couplings in the two spatial directions are not necessarily equal. The use of the shooting methods introduced in section 2.3.1, and redefined here for the two-dimensional case in section 4.1, allow us to find these solutions and analyze their structural and stability properties. Both pinned and mobile discrete breathers are studied. In the latter case we will study only the ones whose motion is along one axis of the lattice. The analysis of the numerically exact solutions help to shed light on some features of the properties and stability of localized solutions reported in previous works.

After introducing in section 4.1 the two-dimensional anisotropic Salerno lattice and provide explanations on the implementation of the numerical procedures used to study the dynamics of $2 \mathrm{D}$ discrete breathers, we will focus on pinned ones. The analysis of the results on pinned discrete breathers for anisotropic nonlinear Schrödinger lattices is reported in section 4.2 for both the standard version of the SM (section 4.2.1) and that with competing nonlinearities (section 4.2.2). In both studies we present the numerical computations of the fixed point norm, as a function of three parameters: breather frequency, transversal coupling, and nonlinearity (see below). They show, as anticipated, the so-called quasi-collapse transition. In these studies we present numerically computed sectors of the bifurcation surface and take a brief look at the nonlinear dynamics on the unstable manifold, whose typical trajectories have been called pulson states. Early numerical work on the 2D quasi-collapse phenomena in isotropic lattices was reported in [127, 128] and [125]. A three-year-old account of the "state of knowledge" on 2D Schrödinger lattices can be found in Section six of [131]. Interestingly, for the case of competing nonlinearities a transition to 2D cuspon states is also found. In this region of the Salerno model we have also studied the existence and stability of in-phase and out-ofphase bound states of pinned breathers motivated by the results obtained in the previous chapter for the $1 \mathrm{D}$ case (section 3.3.2).

As introduced above, a new class of breathing solutions are possible in the 2D model: discrete vortices [117]. We investigate vortex breathers of two types, vortex crosses and vortex squares, in section 4.3 (in the framework of the isotropic model). The analysis of their linear stability reveals parametric stability regions (which turn out to be rather narrow) for the vortices, and helps to identify various bifurcations (including a generic Hamiltonian Hopf bifur- 
cation) responsible for their destabilization. Direct simulations demonstrate that the instability transforms the vortices into ordinary breathers in the case of the standard Salerno model, and into vortex pulsons, that keep the vortical topology, in the most interesting case of competing nonlinearities. Finally, we have also introduced bound states of vortex crosses and analyze their stability.

Mobile solutions are finally reported in section 4.4. For this type of solutions we have focused on a single type of mobile breather, namely those moving along the direction of stronger lattice coupling constant. The structure of each of these mobile exact discrete breathers is that of a localized moving core superimposed on a specific extended state of resonant small amplitude radiation, the background. An extensive Floquet stability analysis of this type of solutions is performed in two sectors of the three-dimensional parameter space, revealing the existence of two different transitions. The tangent space eigenvectors associated to each of the transitions are presented, and the relation of the unstable manifold trajectories to pulson states is analyzed afterwards.

\subsection{The Salerno model in two dimensions}

Motivated by the results reported in the last chapter our aim here focus on extending the continuation scheme for calculating exact discrete breathers in higher dimensional systems. In particular we focus on the following twodimensional nonlinear Schrödinger lattice

$$
\begin{aligned}
\mathrm{i} \dot{\Phi}_{n m}= & -\left[C_{1}\left(\Phi_{n+1, m}+\Phi_{n-1, m}\right)+C_{2}\left(\Phi_{n, m+1}+\Phi_{n, m-1}\right)\right]\left(1+\mu\left|\Phi_{n, m}\right|^{2}\right) \\
& -2 \nu \Phi_{n, m}\left|\Phi_{n, m}\right|^{2}
\end{aligned}
$$

This lattice can be viewed as the two-dimensional Salerno model. The two coupling parameters $C_{1}$ and $C_{2}$ provide a technical advantage for numerics (see below), but they are also introduced for theoretical and experimental interest. The possibility of controlling the ratio between the two linear couplings of the two transversal directions has been studied in various works as a way of analyzing how the intrinsic $2 \mathrm{D}$ phenomena (such as the quasi-collapse) emerge. In fact, for $C_{1}<<C_{2}, \mu=0$ and $\nu \neq 0$ equation (4.1) describes a set of weakly coupled nonlinear waveguide arrays and can be considered as a case of "intermediate dimensionality". This extreme has been studied experimentally in [132] and using perturbative methods in [133]. On the other hand, this equation incorporates, as two particular limits, the physically relevant standard two-dimensional DNLS equation $(\mu=0, \nu \neq 0)$ and the two-dimensional counterpart of the AL lattice $(\mu>0, \nu=0)$ which is not integrable. The continuation between these two limits provides a useful tool for studying the interplay between the on-site and inter-site nonlinearities in the 2D case. More- 
over, the anisotropy (or freedom in the values of the coupling parameters $C_{1}$ and $C_{2}$ ) allows to include an integrable model among the members of the family of nonlinear lattices described by eq. (4.1). That is, for $\nu=0, C_{i}=0$ and $C_{j} \neq 0$ one obtains a set of integrable AL 1D chains. In this sense, every 2D model included by eq. (4.1) is connected with this integrable model where analytic discrete breathers are available.

The SM (4.1) may find a straightforward physical application as a discrete model for the BEC of dipolar atoms trapped in a deep two-dimensional optical lattice [78]; in that case, as stated for the 1D Salerno model, assuming that a strong magnetic field aligns the momenta parallel (perpendicular) to the lattice plane, and the condensate is strongly confined in the vertical direction, one will again deal with the dipole-dipole attraction (repulsion), i.e. $\mu>0(\mu<0)$ in eq. (4.1).

Similarly to the 1D version of the Salerno model eq. (4.1) has two dynamical invariants, the Hamiltonian

$$
\begin{aligned}
\mathcal{H}= & -C_{1} \sum_{n, m}\left(\Phi_{n, m} \bar{\Phi}_{n+1, m}+\Phi_{n+1, m} \bar{\Phi}_{n, m}\right) \\
& -C_{2} \sum_{n, m}\left(\Phi_{n, m} \bar{\Phi}_{n, m+1}+\Phi_{n, m+1} \bar{\Phi}_{n, m}\right) \\
& -\frac{2 \nu}{\mu} \sum_{n, m}\left|\Phi_{n, m}\right|^{2}+\frac{2 \nu}{\mu^{2}} \sum_{n, m} \ln \left(\left.|1+\mu| \Phi_{n, m}\right|^{2} \mid\right),
\end{aligned}
$$

and, due to the phase invariance of the equations of motion, the following norm $(4.1)$

$$
N=\frac{1}{\mu} \sum_{n, m} \ln \left(\left.|1+\mu| \Phi_{n, m}\right|^{2} \mid\right)
$$

Note that we have included here the needed redefinition in the logarithmic terms of both quantities in order to manage with a correct description of the dynamical invariants within the Salerno model with competing nonlinearities.

In the same manner as in the 1D case we will focus on a special set of 2D discrete breathers. For this, we have to generalize the definition (2.22) introduced in section 2.2 for a $(p, q)$ resonant solution in the 1D model to the $2 \mathrm{D}$ case. In this context, discrete breathers solutions are characterized by three time scales. Namely, one associated with the internal oscillation $\omega_{b}$ and the other two derived from the translation of the localization center, i.e. its velocity $\vec{v}_{b}=\left(v_{x}, v_{y}\right)$. The subset of 3 -tuples $\left(\omega_{b}, \vec{v}_{b}\right)$ that fulfill the $\left(p_{x}, p_{y}, q\right)$ resonance condition

$$
\begin{aligned}
& v_{x} \frac{2 \pi}{\omega_{b}}=\frac{p_{x}}{q} \\
& v_{y} \frac{2 \pi}{\omega_{b}}=\frac{p_{y}}{q},
\end{aligned}
$$


(where $p_{x}, p_{y}$ and $q$ are integers) denote the breather solutions that can be obtained with our continuation method. These solutions are those that after $q$ periods of the internal frequency, $\hat{\Phi}\left(t_{0}+q T_{b}\right)$, translates $p_{x}$ and $p_{y}$ lattice sites in the $x$ and $y$ direction of the square lattice, respectively, i.e.

$$
\hat{\Phi}_{n, m}\left(t_{0}\right)=\hat{\Phi}_{n+p_{x}, m+p_{y}}\left(t_{0}+q T_{b}\right),
$$

where, again, PBC are applied $\Phi_{N_{x}+1, m}=\Phi_{1, m}, \Phi_{0, m}=\Phi_{N_{x}, m}, \Phi_{n, N_{y}+1}=$ $\Phi_{n, 1}$ and $\Phi_{n, 0}=\Phi_{n, N_{y}}$ (with $N_{x}$ and $N_{y}$ being the lattice size in the $x$ and $y$ direction respectively). Consequently, a $\left(p_{x}, p_{y}, q\right)$-resonant state will be a solution of the following set of equations

$$
\begin{aligned}
F_{\left(p_{x}, p_{y}, q, \omega_{b}, \nu, C_{1}, C_{2}\right)}\left[\left\{\hat{\Phi}_{n, m}\left(t_{0}\right)\right\}\right] & =L_{y}^{p_{y}} L_{x}^{p_{x}} T_{\left(\omega_{b}, \nu, C_{1}, C_{2}\right)}^{q}\left[\left\{\hat{\Phi}_{n, m}\left(t_{0}\right)\right\}\right]= \\
& =\left\{\hat{\Phi}_{n, m}(t)\right\}
\end{aligned}
$$

where the operators $L_{i}$ are the lattice translation in the $i$-direction,

$$
\begin{aligned}
& L_{x}\left[\left\{\Phi_{n, m}\left(t_{0}\right)\right\}\right]=\left\{\Phi_{n+1, m}\left(t_{0}\right)\right\}, \\
& L_{y}\left[\left\{\Phi_{n, m}\left(t_{0}\right)\right\}\right]=\left\{\Phi_{n, m+1}\left(t_{0}\right)\right\} .
\end{aligned}
$$

Besides, $T_{\left(\omega_{b}, \nu, C_{1}, C_{2}\right)}$ is the time evolution operator given by equation (4.1) over one period $T_{b}=2 \pi / \omega_{b}$,

$$
T_{\left(\omega_{b}, \nu, C_{1}, C_{2}\right)}\left[\left\{\Phi_{n, m}\left(t_{0}\right)\right\}\right]=\left\{\Phi_{n, m}\left(t_{0}+T_{b}\right)\right\} .
$$

In order to illustrate the $2 \mathrm{D}$ time scales resonance let us to consider the plane wave solutions of equation (4.1): $\Phi_{n, m}(t)=A \exp \left[\mathrm{i}\left(k_{x} n+k_{y} m-\omega t\right)\right]$. These solutions possess the following nonlinear dispersion relation

$$
\omega(\vec{k}, A)=2\left(C_{1} \cos k_{x}+C_{2} \cos k_{y}\right)\left(1+\mu A^{2}\right)-2 \nu A^{2} .
$$

Hence, we can obtain the subset of plane waves which are $\left(p_{x}, p_{y}, q\right)$-resonant with some time scale $\tau$ (i.e. after a time $q \tau$ they have translated $p_{x}$ and $p_{y}$ sites in the $x$ and $y$ direction, respectively). Each member of these subsets will be labeled by the pair $\vec{k}=\left(k_{x}, k_{y}\right)$ and from the condition (4.7) it follows that the corresponding set of values of $\vec{k}$ for each family will satisfy the relation

$$
\omega(\vec{k}, A)=\frac{1}{q \tau}\left(\vec{p} \cdot \vec{k}-\frac{m}{2 \pi}\right),
$$

where $m$ is an integer and $\vec{p}=\left(p_{x}, p_{y}\right)$. In figure 4.1 the corresponding values of $\vec{k}$ are represented for two resonances of type $\left(p_{x}=1, p_{y}=0, q=1\right)$ and $\left(p_{x}=1, p_{y}=1, q=1\right)$.

The method used for solving equation (4.7) for each resonant 3 -tuple $\left(\omega_{b}, \vec{v}_{b}\right)$ is the same as in the 1D case, already described in section 2.3.1. Then, the 

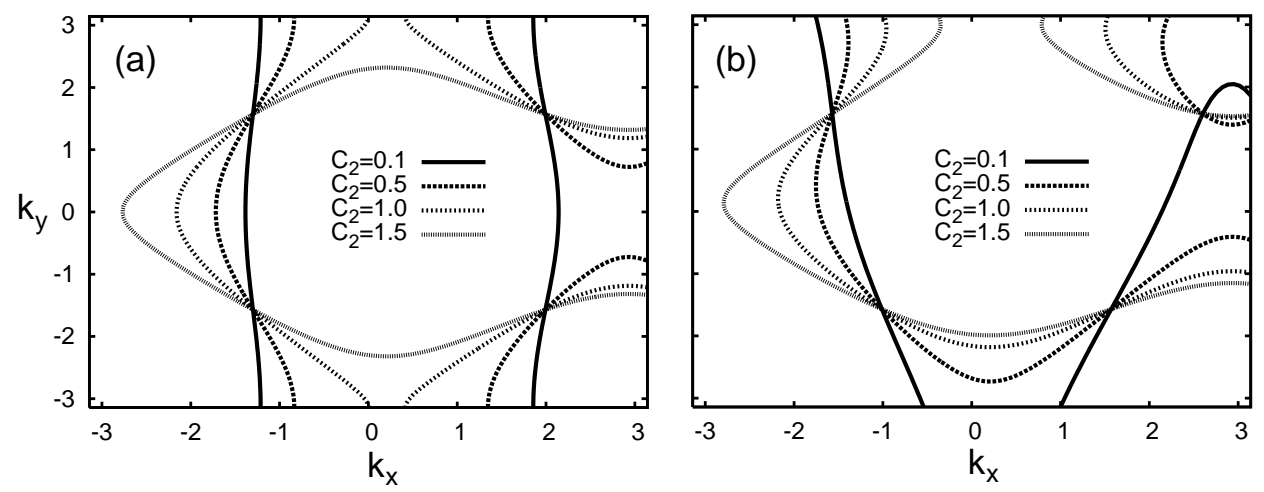

Figure 4.1: Wave numbers, $\vec{k}=\left(k_{x}, k_{y}\right)$, of the $(1,0,1)$ (a) and $(1,1,1)$ (b) resonant plane waves for $m=0$ (see equation (4.12)). Different values of $C_{2}$, while $C_{1}$ is fixed $\left(C_{1}=1\right)$, are shown. The reference time scale for the resonance is set to $\tau=2.4315$ $(\omega=2.584)$.

implicit function theorem assures that a fixed point solution of a map (4.7) given by $\vec{\xi}=\left(p_{x}, p_{y}, q, \omega_{b}, \nu, C_{1}, C_{2}\right)$ can be obtained provided that (i) the Jacobian of the operator $F_{\vec{\xi}}\left[\left\{\Phi_{n, m}\left(t_{0}\right)\right\}\right]-I$ is invertible, and (ii) we know a fixed point of a map corresponding to an infinitesimally close set of parameters, $\vec{\xi}-\delta \vec{\xi}=\left(p_{x}, p_{y}, q, \omega_{b}-\delta \omega_{b}, \nu-\delta \nu, C_{1}-\delta C_{1}, C_{2}-\delta C_{2}\right)$. As explained in section 2.3.1 the first demand can be satisfied using a singular value decomposition (SVD) of the Jacobian in order to obtain the pseudo-inverse operator. On the other hand, when the second condition is fulfilled convergence of the NewtonRaphson iterative scheme is guaranteed. For this, we start with a sufficiently good trial solution, $\left\{\Phi_{n, m}^{0}\left(t_{0}\right)\right\}$ and solve the equation

$$
\left\{\delta \Phi_{n, m}^{0}\left(t_{0}\right)\right\}=-D F_{\vec{\xi}}\left[\left\{\Phi_{n, m}^{0}\left(t_{0}\right)\right\}\right]^{-1} \cdot F_{\vec{\xi}}\left[\left\{\Phi_{n, m}^{0}\left(t_{0}\right)\right\}\right],
$$

in order to obtain $\left\{\Phi_{n, m}^{1}\left(t_{0}\right)\right\}=\left\{\Phi_{n, m}^{0}\left(t_{0}\right)\right\}+\left\{\delta \Phi_{n, m}^{0}\left(t_{0}\right)\right\}$. We iterate these calculations to the desired convergence, and then the solution, $\left\{\hat{\Phi}_{n, m}\left(t_{0}\right)\right\}$, is obtained. In our numerics this is the case when

$$
F_{\vec{\xi}}\left[\left\{\Phi_{n, m}^{i}\left(t_{0}\right)\right\}\right]<N \cdot 10^{-16},
$$

(where $N$ is the total number of sites in the square lattice) is fulfilled. Once the solution is found we use it as the following trial solution, $\left\{\Phi_{n, m}^{0}\left(t_{0}\right)\right\}$, for solving the map (4.7) corresponding to the next set of parameters $\vec{\xi}^{\prime}=\vec{\xi}+\delta \vec{\xi}$.

There are two possible paths for developing the continuation method depending on the choice of the starting point of the continuation. One possibility is to start from the full anti-continuum limit, $C_{1}=C_{2}=0$, where a pinned 
breather solution of frequency $\omega_{b}$ is written as

$$
\hat{\Phi}_{n, m}(t)=\delta_{n, n_{0}} \delta_{m, m_{0}} \sqrt{\frac{\omega_{b}}{2 \nu}} \exp \left(\mathrm{i} \omega_{b} t\right) .
$$

Starting from the above solution, we can perform the continuation increasing the parameters $C_{1}$ and $C_{2}$ as usual, and so obtain the whole family of ( $p_{x}=$ $\left.0, p_{y}=0, q=1\right)$ resonant discrete breathers. An alternative path starts from the one-dimensional limit, $C_{2}=0$. The choice of this second limit (which implies taking as the very initial trial solution of the continuation the whole set of 1D solutions obtained in the previous chapter) is justified when seeking mobile solutions. As stated above, this limit offers the possibility of studying strongly anisotropic lattices as a controlled interpolating situation between one and two dimensions. On the other hand, employing this strategy we can only obtain those solutions which are $\left(p_{x}=p, p_{y}=0, q\right)$-resonant, i.e. the two-dimensional continuation of those one-dimensional $\left(p=p_{x}, q\right)$-resonant discrete breathers. Hence, the solution from which we start is

$$
\hat{\Phi}_{n, m}(t)=\delta_{m, m_{0}} \hat{\Phi}_{n}^{1 D}(t),
$$

where $\hat{\Phi}_{n}^{1 D}(t)$ is the corresponding $\left(p=p_{x}, q\right)$-resonant one-dimensional solution.

In what follows we will employ both continuation paths when we study the case of pinned breathers (section 4.2), and we will show that the results obtained are the same when approaching the same limit (the standard twodimensional DNLS).

\subsection{Pinned discrete breathers}

We first focus on the characterization of pinned $((0,0,1)$-resonant) discrete breathers for the standard Salerno model (with special attention to the DNLS equation) in section 4.2.1 and for the SM with self-defocusing inter-site nonlinearity in section 4.2.2.

\subsubsection{Pinned discrete breathers in the standard Salerno model}

As we have discussed, we can choose two different starting points for the continuation of $(0,0,1)$-resonant fixed points (pinned breathers) of equation (4.7): (i) the full anti-continuum (AC) limit $\left(C_{1}=C_{2}=0\right)$, or (ii) the (one-dimensional, 1D) limit of uncoupled chains $\left(C_{1} \neq 0, C_{2}=0\right)$, where they were obtained in 
the previous chapter from continuation along the standard 1D Salerno model by increasing values of the parameter $\nu$ from the one-dimensional AL lattice (2.9). As a test for our codes, we have checked that both paths arrive to the same solution. In fact, unique continuations can proceed along any path on the plane of parameters $\left(C_{2}, \nu\right)$ that we have explored.

Early works [123-125] on the isotropic two-dimensional standard DNLS equation analyzed the so-called quasi-collapse instability of pinned discrete breathers, i.e. the condensation of all the energy into a few modes in discrete nonlinear systems, which corresponds to the onset of a singularity (wave collapse) [126] in multidimensional continuum models. Subsequent numerical works [127] extended these studies to the isotropic 2D Salerno lattice and addressed the question of how the instability is affected by the presence of impurity lattice sites.

As expected, our results further corroborate the existence of quasi-collapse instabilities in the anisotropic case: The phase diagram in parameter space $\left(\omega_{b}, C_{2}, \nu\right)$ consists of two regions (stable and unstable) separated by the surface of transition. As we perform the continuation of breather solutions across the parameter space we scan the Floquet stability of the computed solution. In figure 4.2 we present the two stability transition curves in the plane $\left(\omega_{b}, C_{2}, \nu=1\right)$, i.e. the function $C_{2}^{t h}\left(\omega_{b}\right)$, corresponding to the two different continuation starts. The continuation from the $\mathrm{AC}$ limit is made through the path $C_{1}=C_{2}$ and the one from the $1 \mathrm{D}$ limit is made at $C_{1}=1$. The convergence of the two paths at $C_{2}=1$ is clearly seen.

The Vakhitov-Kolokolov criterion [103] for stability of the pinned discrete breather solution derived and used for the 2D DNLS in [124, 125],

$$
\left(\frac{\partial N}{\partial \omega_{b}}\right)_{C_{2}, \nu}>0
$$

is of a very general character and our numerics illustrate it clearly. On the other hand, the Floquet stability analysis detects the dimensionality (and a basis in tangent space) of the unstable linear manifold associated with the quasicollapse instability that these exact discrete breathers experience for some parameter values. We have computed numerically, for a fine grid of $\omega_{b}$ values and a coarser grid of $C_{2}$ and $\nu$, the function $N\left(\omega_{b}, C_{2}, \nu\right)$, from which we show some sectors in figures 4.3 and 4.4 .

In figure 4.3 we show the numerically computed norm (4.3) as a function of the breather frequency $N\left(\omega_{b}\right)$, for three different values of the transversal coupling $C_{2}$, and a fixed value of $\nu=1$ (anisotropic DNLS limit). We observe the existence of a minimum value, $\min N\left(\omega_{b}\right)=N^{t h} \neq 0$, which is thus seen as an excitation threshold for the creation of these solutions. The position of 


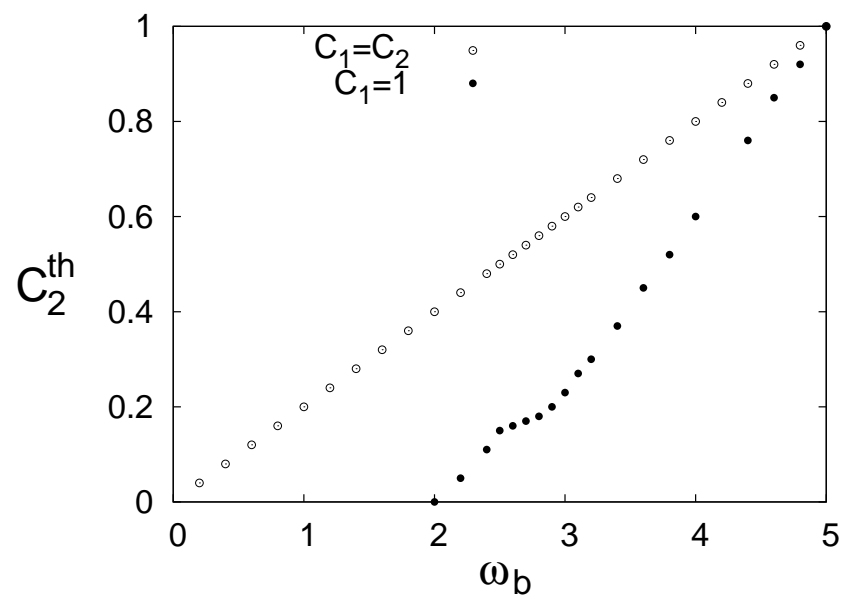

Figure 4.2: Evolution of the threshold value of the coupling parameter, $C_{2}^{t h}$, as a function of the frequency, $\omega_{b}$, for two different continuations starts. The values of $C_{2}^{\text {th }}$ limit the region where pinned discrete breathers are linearly stable (unstable for $C_{2}>C_{2}^{t h}$ ). The instability yields a hyper-localized state (quasi-collapse). The continuation from the fully uncoupled limit $\left(C_{1}=C_{2}=0\right)$ (filled circles) is performed using the path $C_{1}=C_{2}$. For the continuation (bold circles) from the 1-dimensional limit $\left(C_{1}=1, C_{2}=0\right)$ the coupling in the new direction $C_{2}$ is progressively increased.

the minimum $\omega_{b}^{t h}\left(C_{2}\right)$, which naturally increases with $C_{2}$, separates the stable and unstable branches of pinned breathers. Breathers corresponding to values of $\omega_{b}$ where $N\left(\omega_{b}\right)$ has a negative slope are unstable: This is shown in the insets, where the Floquet spectra of two representative examples of pinned discrete Schrödinger breathers are plotted in the complex plane. Note that the high accuracy of the numerical solution allows an unprecedented detailed Floquet analysis of the instability, paving the way to rigorous analytical characterizations of the quasi-collapse unstable manifold. This is a one-dimensional manifold, as our numerical results unambiguously confirm. Then, in the regime of small time scales, the unstable manifold is fully characterized by a single Floquet eigenvector.

Figure 4.4 shows the (surface) function $N\left(\omega_{b}, \nu\right)$ for the volume sector of constant $C_{2}(=0.5)$. Most noticeably, the critical (threshold) line of bifurcation points $\left(\frac{\partial N}{\partial \omega_{b}}=0\right)$, as seen in the inset, does not define a monotone function $\omega_{b}^{t h}(\nu)$. In fact, in the whole interval of $0 \leq \nu \leq 1$ values, the range of values of $\omega_{b}^{t h}$ is quite short, indicating the insensitivity of the gross features of the quasicollapse transition to the value of $\nu$. However, considering finer details, one sees that the threshold curve $\omega_{b}^{t h}(\nu)$ smoothly reaches its slightly larger values around midway between the DNLS and the AL limits. In other words, intermediate values of the interpolating (Salerno) parameter $\nu$ somewhat favour 


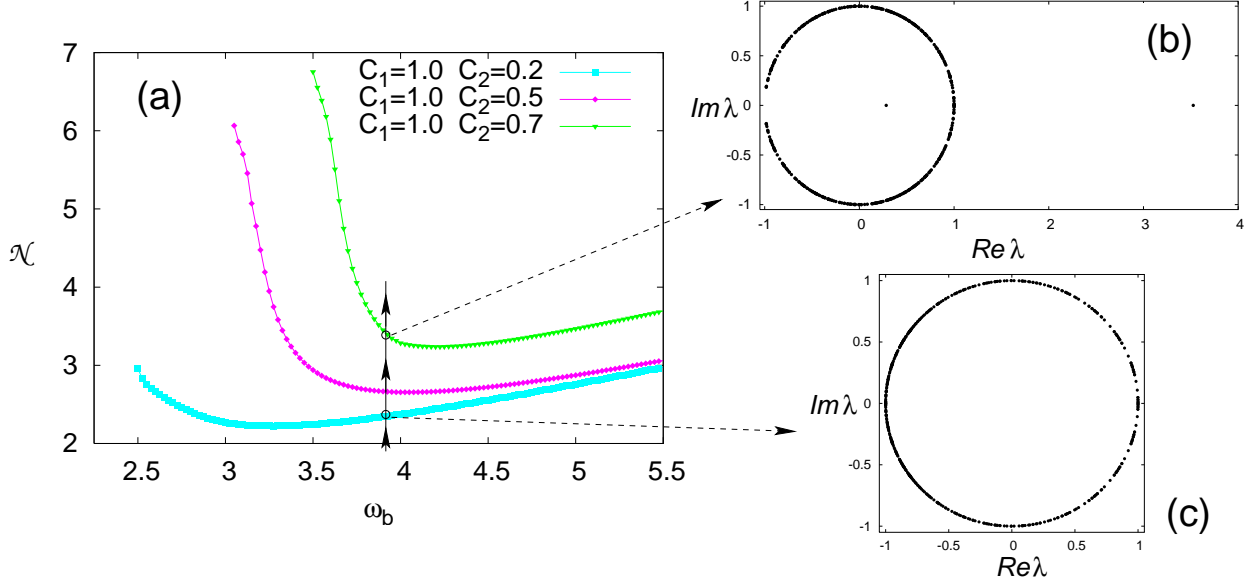

Figure 4.3: (a) Plot of the Norm, $N$, of the computed solutions as a function of their frequency $\omega_{b}$ for different values of the coupling parameters. The continuations have been made starting from the one-dimensional limit $\left(C_{1}=1\right)$. For the regions where $\partial N / \partial \omega_{b}$ is positive (negative) the continued solutions are stable (unstable). We can monitor the change of the linear stability of a solution of a given frequency during its continuation in $C_{2}$ looking at the Floquet spectra. Figures (b) and (c) show the Floquet spectra of a discrete breather of frequency $\omega_{b}=3.93$ at $C_{1}=1, C_{2}=0.7$ (where $\partial N / \partial \omega_{b}<0$ ) and at $C_{1}=1, C_{2}=0.2$ (where $\partial N / \partial \omega_{b}>0$ ), respectively.

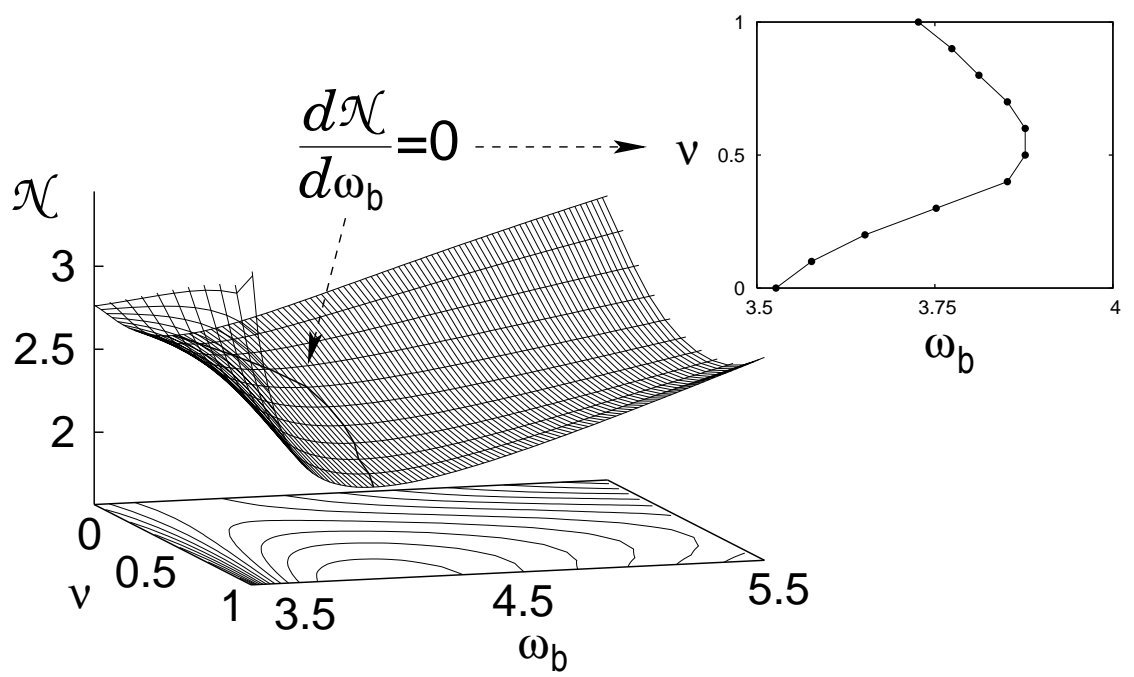

Figure 4.4: Surface $N\left(\omega_{b}, \nu\right)$ for the case $C_{2}=0.5$. The inset shows the curve $\nu\left(\omega_{b}\right)$ corresponding to $\partial N / \partial \omega_{b}=0$. This curve gives the transition points where the discrete breather changes its stability character. 

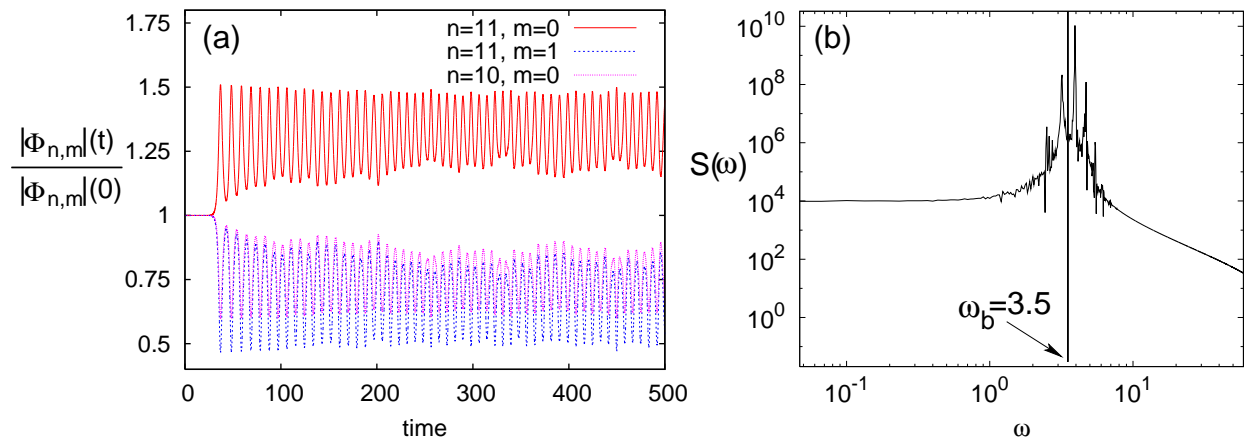

Figure 4.5: (a) Time evolution of the amplitude $\left|\Phi_{n, m}\right|$ for the localization center $(n=11, m=0)$ and two adjacent sites $(n=10, m=0)$ and $(n=11, m=1)$. Each amplitude is normalized to its initial value, so that it can be seen how the quasi-collapse instability is developed. The parameters of equation (4.1) are $C_{1}=1$, $C_{2}=0.5, \nu=1.0(\mu=0)$ and the frequency of the pinned breather is $\omega_{b}=3.50$. When the instability is fully developed, we analyze the final state by means of the Power Spectrum $S(\omega)$ of the time evolution of $\Re\left[\Phi_{10,0}(t)\right]$ (the real part of localization center). As can be observed in (b) the internal frequency of the breather (highest peak in the spectrum) shifts to a higher value $\left(\omega_{b}^{*}=4.03\right)$ and the other peaks are located at the frequencies of the harmonics resulting from the combination of the internal frequency with the frequency $\left(\omega_{q c}=0.78\right)$ associated with the amplitude $\left|\Phi_{n, m}\right|$ oscillations shown in (a).

the enhancement of the quasi-collapse unstable region. These conclusions are in contrast with the stated conclusion (for isotropic lattices) in [127] that the Ablowitz-Ladik term increases the stability regime.

When instability is allowed to develop beyond the fixed point tangent space into the nonlinear realm of perturbations, the trajectory obtained by direct integration of the equations of motion invariably ends after a transient (of time scale given by the real Floquet exponent larger than 1) in a localized solution with complex dynamics, the pulson states, that we have already observed in the one-dimensional Salerno model with competing nonlinearities for a narrow region in the $\left(\mu, \omega_{b}\right)$-plane (see section 3.3.2). In the two-dimensional context these states were characterized in [131] in the following terms "... where the peak intensity $\left|\Phi_{m, n}\right|^{2}$ oscillates between the central site and its four nearest neighbours (...) it is not known whether these pulson states represent true quasiperiodic solutions to the DNLS equation". What makes these trajectories on the unstable nonlinear quasi-collapse manifold of much practical relevance and interest is their ubiquity: They appear as persistent localized states in the Hamiltonian dynamical evolution from a wide variety of initial conditions. Their description requires at least two frequencies, namely the internal (genuine breather-like frequency) and the frequency of the oscillations of the breather 
width around a mean width value, which turns out to be less than the width of the unstable exact discrete breather. Second and outer shells of neighbours (in both lattice axes) also participate in the width oscillations.

Though a more detailed characterization of the pulson states would be required, it is illustrative to consider (figure 4.5) the power spectrum $S(\omega)$ (eq. 3.5 ) of the field at the central site of a typical trajectory on the unstable nonlinear manifold of a quasi-collapsing pinned discrete breather. This shows peaks at the combinations $\omega_{b}^{*}+j \omega_{q c}(j=0, \pm 1, \pm 2 \ldots)$, where $\omega_{q c}$ is the frequency of the width oscillations characterizing the pulson state, while $\omega_{b}^{*}>\omega_{b}$ is a frequency higher than the (initial condition) fixed point frequency $\omega_{b}$. The new frequency $\omega_{b}^{*}$ turns out to be very close to the breather frequency of the same (initial) norm on the stable branch. In other words, the instability drives a shift of breathing frequency towards the stable branch, while the excess energy is transferred to the oscillatory motion of the observable width. This behavior seems to be the essence of the physical characterization of the nonlinear quasi-collapse manifold dynamics.

The numerical observation of a two-frequency power spectrum for a typical pulson state points towards an eventual positive answer to the question (on true quasiperiodicity) arised in [131]. This point serves to illustrate how the high accuracy of the fixed point numerical solution provides detailed clues on many still unsolved (from a mathematical and physical point of view) questions on two-dimensional Schrödinger localization, which are of prospective experimental interest in nonlinear (photonic, Josephson, ...) physics technologies.

\subsubsection{Pinned discrete breathers in the Salerno model with competing nonlinearities}

Let us now analyze the effects that competing on-site self-focusing and intersite self-defocusing nonlinearities have on pinned discrete breathers. For this purpose we start from the above computed pinned breather solutions for the 2D DNLS equation, corresponding to $\mu=0$, and parameterized by the corresponding values of $\omega_{b}, C_{1}$ and $C_{2}$. In paticular we will keep fixed the value $C_{1}=1$ and let vary $C_{2}=C$. We will also keep fixed the value of $\nu=1$ (due to the scaling invariance of the model (4.1)). Then, our continuation seeds is a set of solutions at different frequencies and several different values of $C$, which are subsequently continued in $\mu<0$. With these continuations we are able to construct families of the breather solutions (labeled by their internal frequency $\omega_{b}$ ) for different values of $\mu$ and $C$. In this way, we scanned the family of discrete breathers in the entire $\left(C, \mu<0, \omega_{b}\right)$-space of the Salerno 

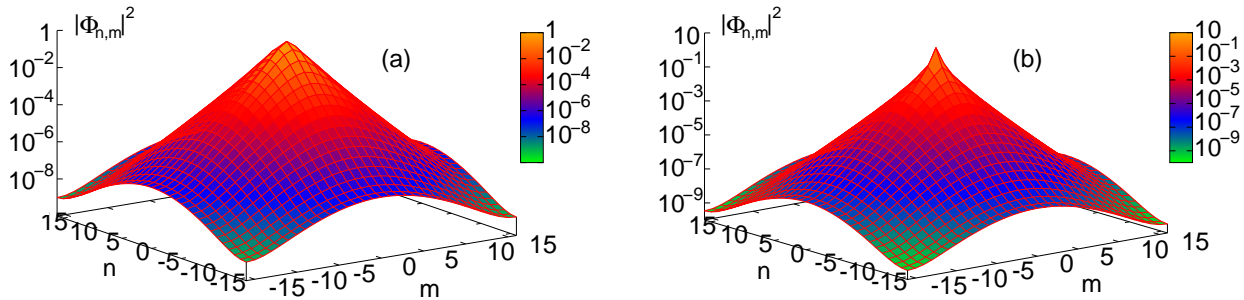

Figure 4.6: Intensity profiles, $\left|\Phi_{n, m}\right|^{2}$, of two discrete breathers found for $C=1$ (isotropic case) and frequency $\omega_{b}=4.22$ : (a) $\mu=-0.2$; (b) $\mu=-0.88$. The latter solution is a cuspon, which features stronger localization at its center than in the tails.

model with competing nonlinearities ${ }^{1}$.

As noted above, varying $C$ from 0 to 1 allows one to observe the transition from one- to two-dimensional lattice and to study the strong anisotropic limit $(C<<1)$. We will concentrate on the existence of $2 \mathrm{D}$ cuspons and their stability. Let us remind that in the $1 \mathrm{D}$ case, cuspons exist for $\mu<0$, when the on-site and inter-site nonlinearities are competing (see section 3.3.2). These solutions present highly localized profiles, with the decay rate around the localization center higher than in tails of the solutions. Another relevant result of the 1D case is that small-frequency breathers are unstable in a narrow interval of negative values of $\mu$. The instability observed in the 1D case transforms the discrete breather into a pulsonic state. We have seen in the previous section 4.2.1 that the standard Salerno model (and in particular the 2D DNLS lattice) features a similar unstable behavior for small-frequency breathers. It is then interesting to find a link between the breather instability in the 2D standard Salerno model (4.1) with $\mu>0$ and the above-reported instability of breathers in the 1D Salerno model when $\mu<0$.

First, we consider the shape of solutions produced by the continuation. As expected, cuspons appear when $\mu$ is negative and of sufficiently large absolute value. In figure 4.6 we display discrete breathers found at $C=1$ (in the isotropic lattice) and $\omega_{b}=4.22$, for two different values of $\mu$. As seen in figure 4.6.b, the cuspon indeed features a higher spatial decay rate around its center, $\left(n_{0}, m_{0}\right)$, than far from it.

To characterize the transition from usual discrete breathers to the cuspons, we fitted the decaying tail of the breather along the vertical and horizontal directions on the lattice, $\left(n_{0}, m \rightarrow \pm \infty\right)$ and $\left(n \rightarrow \pm \infty, m_{0}\right)$, to the expected

\footnotetext{
${ }^{1}$ Typically, the difference between the breather frequencies used for each $C$ was $\delta \omega_{b}=$ $8 \cdot 10^{-2}$ (which is also the lowest frequency taken close to the edge of the phonon band), and the continuation step in $\mu$ was $\delta \mu=2 \cdot 10^{-2}$.
} 


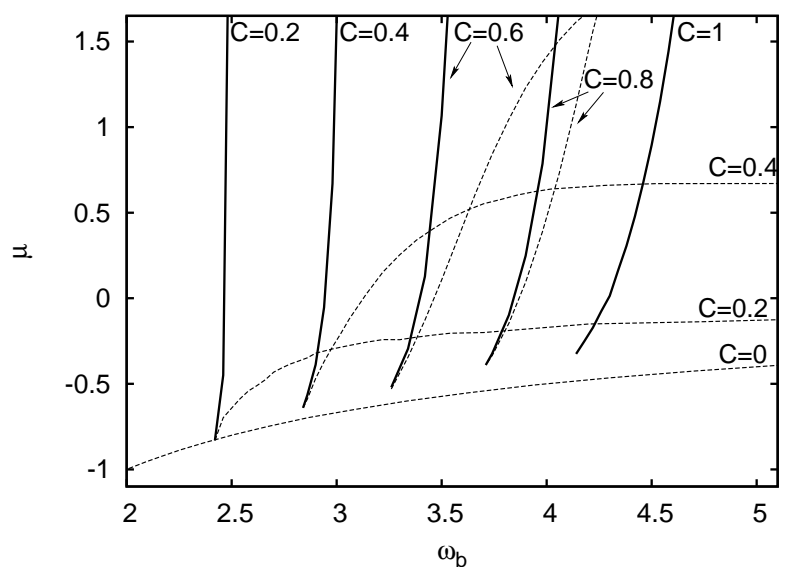

Figure 4.7: Solid and dashed curves show, respectively, the critical values of the intersite nonlinearity coefficient, $\mu_{y}$ and $\mu_{x}=0$, as a function of the breather's frequency for several values of the anisotropy parameter $C$.

asymptotic forms, $\left|\Phi_{n_{0}, m \rightarrow \pm \infty}^{\text {(asymp) }}\right|=A_{y} \exp \left(-\Gamma_{y}\left|m-m_{0}\right|\right)$ and $\left|\Phi_{n \rightarrow \pm \infty, m_{0}}^{(\text {asymp }}\right|=$ $A_{x} \exp \left(-\Gamma_{x}\left|n-n_{0}\right|\right)$, respectively. Once two pairs of parameters $\left(A_{x}, \Gamma_{x}\right)$ and $\left(A_{y}, \Gamma_{y}\right)$ are found, one can determine whether the decay rate (localization degree) around the breather's center is higher or lower than in the tails, by defining two sharpness parameters (this is a similar definition to that adopted in section 3.3.2 for the $1 \mathrm{D}$ case), $\gamma_{x} \equiv A_{x}-\left|\Phi_{n_{0}, m_{0}}\right|$, and $\gamma_{y} \equiv A_{y}-\left|\Phi_{n_{0}, m_{0}}\right|$. Obviously, $\gamma_{x} \equiv \gamma_{y}$ in the isotropic model $(C=1)$; however, $\gamma_{x}$ and $\gamma_{y}$ are different in anisotropic lattices. We have computed both quantities as the continuation in $\mu$ was performed for each breather at frequency $\omega_{b}$. For a given pair of parameters $C$ and $\omega_{b}$, it was found that, for higher (in particular, less negative) values of $\mu$, both $\gamma_{x}$ and $\gamma_{y}$ are positive, thus pointing out that the localized states are ordinary discrete breathers (not cuspons). Decreasing $\mu$, one finds a critical value, $\mu=\mu_{y}$, at which $\gamma_{y}=0$, which corresponds to a peakon profile in the (vertical) direction of weak coupling, $\left(n_{0}, m\right)$. If $\mu$ is further decreased, we then have $\gamma_{y}<0$, while $\gamma_{x}$ is still positive (i.e., the breather is a semi-cuspon), until the second critical point is reached, $\mu=\mu_{x}<$ $\mu_{y}$, where $\gamma_{x}=0$, and the breather assumes a peakon profile in the (horizontal) direction of strong coupling, $\left(n, m_{0}\right)$. Finally, at $\mu<\mu_{x}$, both $\gamma_{x}$ and $\gamma_{y}$ are negative, and the discrete breather is a cuspon in both directions. Figure 4.7 shows the critical values, $\mu_{x}$ and $\mu_{y}$, versus $\omega_{b}$ for several fixed values of $C$. As noted above, $\mu_{x}=\mu_{y}$ when $C=1$, while for $C=0$ (the 1D limit), only $\mu_{x}$ exists. 


\section{Floquet analysis}

Concomitant with the continuation of the breather solutions in $\mu$, we examined their linear stability. Performing the Floquet analysis for every computed solution, we have generated a full stability diagram in the $\left(\omega_{b}, \mu\right)$-plane for several values of $C$ (including both positive and negative $\mu$ in order to link the unstable region of the $1 \mathrm{D}$ SM at $\mu<0$ and those found for the standard 2D $\mathrm{SM})$. This is shown in figure 4.8. At the isotropic case, $C=1$, and for $\mu>0$ we observe, as expected from the results obtained in the previous section, an unstable region corresponding to the low-frequency breathers. As previously reported, the development of this instability yields to the development of a pulsonic state (similar to what was found in in the 1D version of the model when $\mu<0$ ). On the other hand, for $\mu<0$, all cuspons are found to be linearly stable. This is a new result concerning $2 \mathrm{D}$ nonlinear lattices of the Schrödinger type. Taking a close look at the evolution of the stability diagrams as $C$ decreases, one can monitor a transition from the $2 \mathrm{D}$ isotropic model to its 1D counterpart. We thus observe (see contour plots in figure 4.8) that there is a subregion in the $\left(\omega_{b}, \mu\right)$ plane, for each value of $C$, where the largest values of $\left|\lambda_{j}\right|$ are much higher than in the rest of the unstable region. This subregion continuously deforms as $C$ varies, and, as $C \rightarrow 0$, it approaches the unstable region found in the 1D Salerno model.

We have also checked the validity of the Vakhitov-Kolokolov (VK) criterion for the stability of breathers in the Salerno model with competing nonlinearities. For this we have computed the norm of the solutions as per eq. (4.3), to generate surfaces $N\left(\omega_{b}, \mu\right)$ for several values of $C$. These surfaces are plotted in figure 4.8. In the figure, we have also plotted curves at which $\partial N \partial \omega_{b}$ changes sign, thus separating the predicted stable and unstable regions. Comparison with the rigorous results produced by the Floquet analysis confirms again the validity of the VK criterion for predicting the unstable region found for pinned breathers at $\mu<0$. A noteworthy feature of surfaces $N\left(\omega_{b}, \mu\right)$ is the divergence when the breather's amplitude attains the value $\left|\Phi_{n_{0}, m_{0}}\right|^{2}=1 /|\mu|$. In the 1D model $(C=0)$, this happens for an exact peakon solution, whereas for $C>0$ we observe that the divergence curve in the $\left(\omega_{b}, \mu\right)$ plane is located below the curve of $\mu=\mu_{y}\left(\omega_{b}\right)$, i.e. it happens for cuspon states. Examining the norm for cuspons with the amplitude exceeding $1 / \sqrt{|\mu|}$, we conclude that $\partial N \partial \omega_{b}<0$ for all $\omega_{b}$ in this region (after the divergence of the norm occurs). Then, the VK criterion predicts that cuspon breathers are unstable contrary to the results of the Floquet analysis. Hence, the VK criterion does not apply to the cuspons with $\left|\Phi_{n_{0}, m_{0}}\right|>1 / \sqrt{|\mu|}$. The stability of perturbed cuspons was also confirmed by direct simulations of the dynamics (4.1) revealing not only that they are linearly stable solutions but also high robust. 


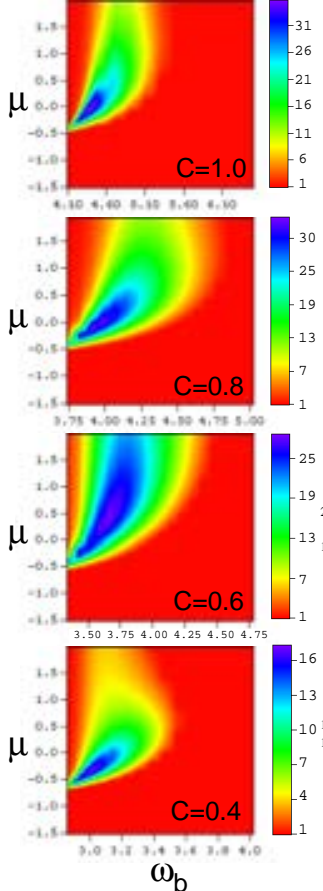

$\omega_{\mathrm{b}}$
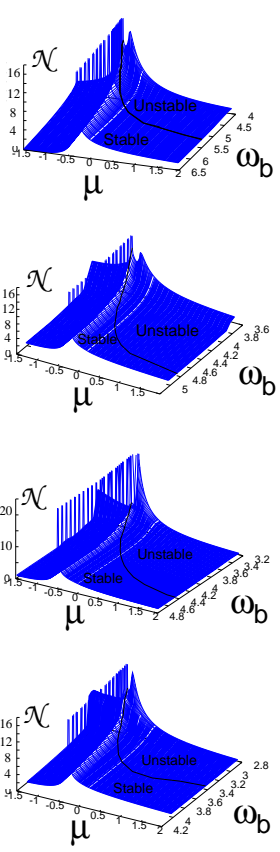
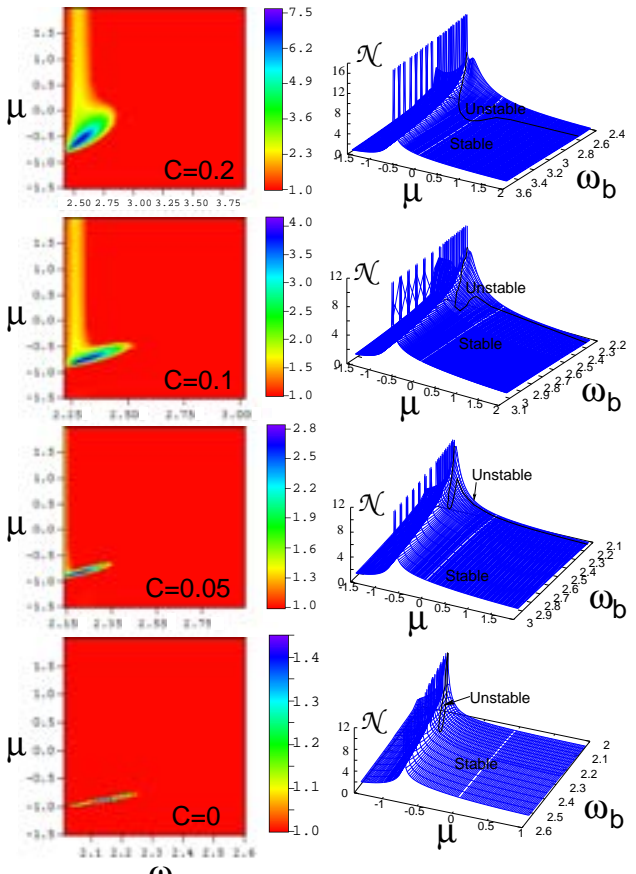

$\omega_{\mathrm{b}}$

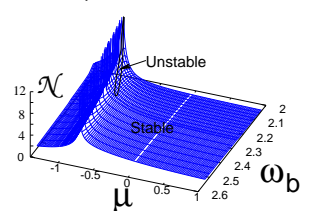

Figure 4.8: Stability diagrams in the $\left(\mu, \omega_{b}\right)$-plane corresponding to different values of the anisotropy parameter $C$. Contour plots show maximum absolute values of the Floquet multipliers, $\max \left\{\left|\lambda_{j}\right|\right\}$. Beside each contour plot, norm surfaces, $N\left(\mu, \omega_{b}\right)$, are plotted for the corresponding values of $C$. Curves at which the sign of slope $\partial \mathcal{N} / \partial \omega_{b}$ changes are plotted on top of the surfaces. According to the VakhitovKolokolov criterion, these curves separate stability and instability regions.

\section{Bound states of discrete breathers}

In addition to isolated pinned breathers, we have studied their bound states when $\mu<0$. Two types, in-phase and $\pi$-out-of-phase, of pairs of identical breathers, with the same frequency $\omega_{b}$ and different distances between them, has been analyzed. For this purpose, we first continued these solutions, at $\mu=$ 0 , from the anti-continuum limit up to the 2D DNLS equation $(C=\nu=\alpha=$ 1 ), and then decreased the value of $\mu$ into the region of competing nonlinearities $(\mu<0)$. At the same time, the linear stability analysis of these periodic solutions was performed by the numerical computation of their Floquet spectra.

We have computed two different patterns of bound states of breathers. The first type consists of two discrete breathers with their centers, $\left(n_{0}^{(j)}, m_{0}^{(j)}\right)$, with $j=1,2$, lying on the same lattice axis (so that $n_{0}^{(1)}=n_{0}^{(2)}$ or $m_{0}^{(1)}=m_{0}^{(2)}$ ), 


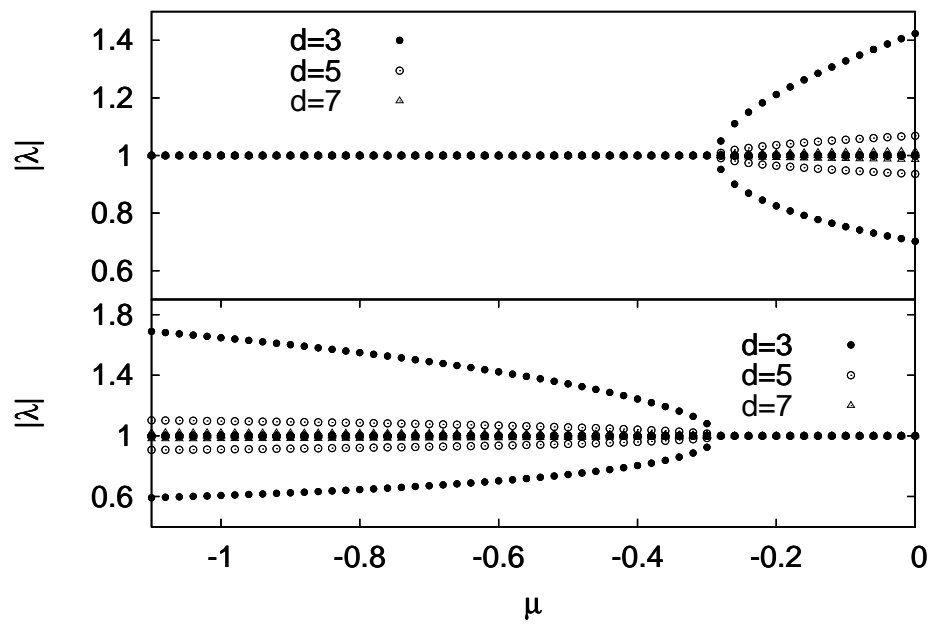

Figure 4.9: The absolute value of the Floquet multipliers as a function of $\mu$ for inphase (top) and out-of-phase (bottom) axis-aligned bound states of breathers with $\omega_{b}=7(C=1)$. The figure shows cases when the two breather centers are separated by $d=3,5$ and 7 . It can be observed that, irrespective of the value of $d$, the stability interchange occurs at $\mu=-0.3$.

whereas for the second type of bound states the centers are related by $n_{0}^{(1)}=$ $n_{0}^{(2)} \pm d$ and $m_{0}^{(1)}=m_{0}^{(2)} \mp d$, i.e. they are aligned along a diagonal of the lattice.

In figure 4.9 we show the absolute value of the Floquet multipliers as a function of $\mu$ for in-phase and out-of-phase bound states, aligned along a lattice axis for the case of $\omega_{b}=7.0$, with three different values of the distance between breather centers in the pair. Results of similar computations for the diagonalaligned bound states with $\omega_{b}=8.0$ are shown in figure 4.10. As in the $1 \mathrm{D}$ version of the model (see section3.3.2), for $\mu=0$ in-phase bound states are linearly unstable (the more unstable the closer breathers are in the pair), while out-of-phase pairs are stable. As observed in figures 4.9 and 4.10 , at $\mu=-0.3$ for the pattern of the first type $\left(\omega_{b}=7.0\right)$, and at $\mu=-0.25$ for the second one $\left(\omega_{b}=8.0\right)$, the in-phase bound states become stable regardless of the distance between breathers. Simultaneously, out-of-phase states become unstable, also regardless of the separation between breather centers.

The same stability exchange between in- and out-of-phase states was observed in the 1D case, where it occurs at the value of $\mu$ at which the discrete breather solution is a peakon. However, here in the 2D case the discrete breathers in the pair are cuspons on both sides of the stability-exchange point. 


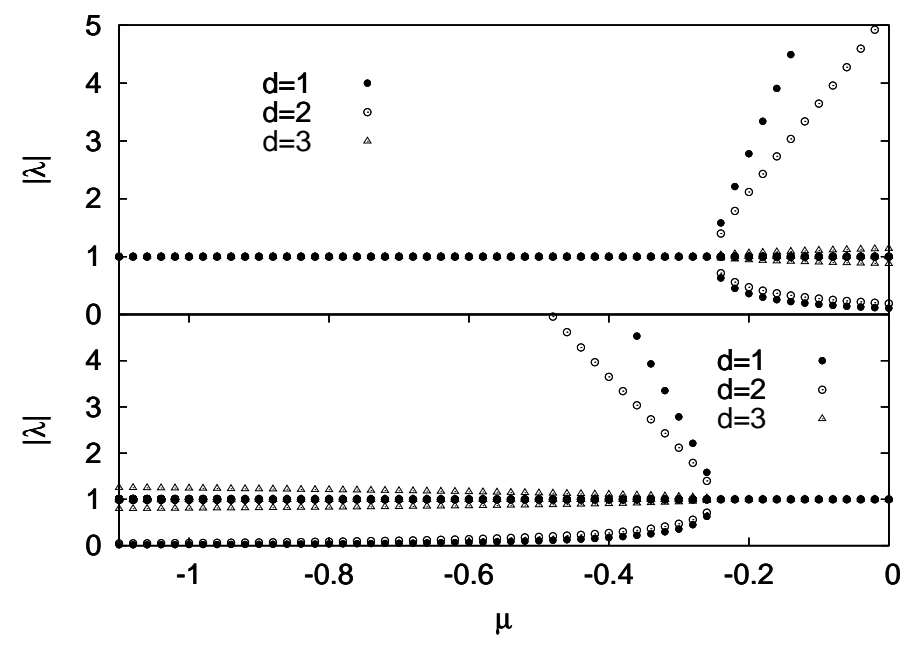

Figure 4.10: The same as in the previous figure for in-phase (top) and out-of-phase (bottom) diagonal-aligned bound states of breathers with $\omega_{b}=8(C=1)$. Shown are the results for the states with the separation between the breather centers $d=1,2$ and 3 . It can be observed that for all these states undergo the stability exchange at $\mu=-0.25$.

Nevertheless, we find, for both types of the bound states, that the values of $\mu$ at this point is exactly the same at which the cuspon's norm, $N\left(\omega_{b}, \mu\right)$, diverges (see the previous subsection). In other words, the stability interchange between in- and out-of-phase bound states is associated with the divergence of the breather norm $N\left(\omega_{b}, \mu\right)$, rather than to the appearance of a peakon (contrary to the $1 \mathrm{D}$ case, where the emergence of a peakon and norm divergence occur simultaneously).

As a conclusion, although the divergence of the norm does not switch the stability of single pinned discrete breathers, it marks the stability border of bound states of breathers, regardless of their size and orientation relative to the lattice.

\subsection{Discrete vortex breathers}

A natural generalization of the fundamental discrete breathers are discrete vortices, which are well-known solutions of the ordinary 2D DNLS model [117]. A vortex is characterized by the phase circulation around its center, $\Delta \theta$, that must be a multiple of $2 \pi$. Hence they may be labeled by an integer number (vorticity, or topological charge), $S \equiv \Delta \theta /(2 \pi)$. 

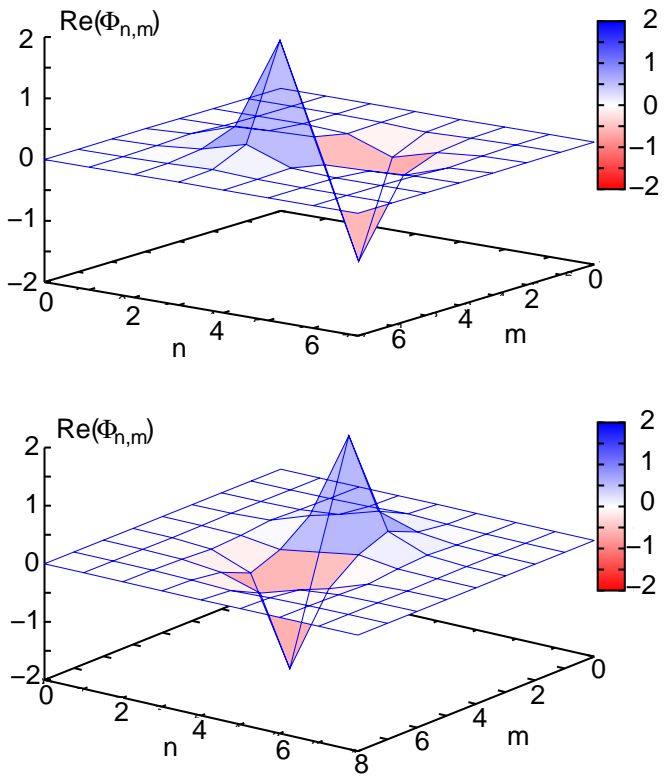

Figure 4.11: Two examples of fundamental $(|S|=1)$ discrete vortices. Profiles of the real part of the square vortex with $M=1$ and vortex cross are shown in the top and bottom panels, respectively. Both solutions are found for $\mu=-0.4$ and $\omega_{b}=7.0$ (as noted in the text, we fix $C=1$ for the vortex solutions).

In this section, we consider vortices only in the isotropic model $(C=1)$, with the purpose of analyzing their behaviour at both the standard SM $(\mu>0)$ and at the SM with competing nonlinearities $(\mu<0)$. In the framework of the 2D DNLS model, influence of the lattice anisotropy on fundamental and vortical discrete breathers was studied in [134]).

We will construct two types of vortices, on-site- and off-site-centered ones (alias vortex crosses and vortex squares), both with $|S|=1$. Vortex squares are characterized by the number of lattice bonds, $M$, that each side of the square comprises; in this section, we only deal with $M=1$. Two examples of these two species of the solutions are plotted in figure 4.11.

\subsubsection{Vortex crosses}

In order to construct fundamental $(|S|=1)$ vortex crosses centered around the lattice site $\left(n_{0}, m_{0}\right)$, we start with the anticontinuum $\left(C_{1}=C_{2}=0\right)$ DNLS $(\mu=0)$ limit. The corresponding seed pattern includes nonzero fields

$$
\Phi_{n_{0}, m_{0}+1}=-i \Phi_{n_{0}+1, m_{0}}=-\Phi_{n_{0}, m_{0}-1}=i \Phi_{n_{0}-1, m_{0}}=\sqrt{\omega_{b} / 2} .
$$



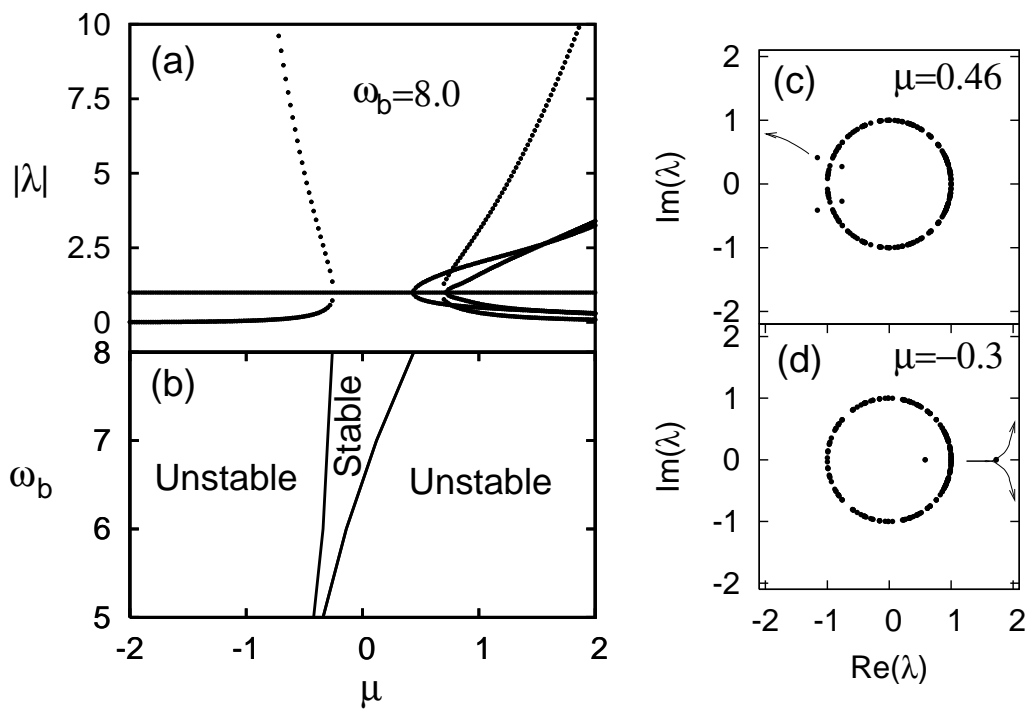

Figure 4.12: (a) The absolute value of the Floquet multipliers as a function of $\mu$ for a vortex cross with $\omega_{b}=8$. Two bifurcations can be inferred from the Floquet distributions in panels (c) and (d): a Hamiltonian Hopf bifurcation at $\mu=0.46$, and a harmonic bifurcation at $\mu=-0.3$. A similar set of two bifurcations is found at other frequencies. The entire stability diagram is displayed in panel (b), showing a narrow stability region. (As noted in the text, we fix $C=1$ for the vortex solutions).

Then, by adiabatically increasing the inter-site coupling (Newton continuation in $C$ using $C_{1}=C_{2}=C$ ), we reach the isotropic DNLS model, and start the continuation to positive values of the inter-site nonlinearity, $\mu$. Performing the continuation in $C$ at $\mu=0$, we have found that, for low-frequency vortex solutions, there is a critical value, $C_{c}$, that depends on frequency $\omega_{b}$, at which a Hamiltonian Hopf bifurcation (HHB) [135] occurs and the vortex solution turns unstable for $C>C_{c}\left(\omega_{b}\right)$. This phenomenon was already reported in previous works $[117,134]$.

Higher-frequency vortex solutions, which are stable in the DNLS equation in the considered range of parameters, undergo destabilization through a bifurcation of the same type as a result of the continuation in $\mu$, at $C=1$. The Hamiltonian-Hopf character of the bifurcation can be seen in figure 4.12.c, which shows the Floquet spectrum after the bifurcation: it is seen that a quadruplet of complex eigenvalues $\lambda_{j}$ exit the unit circle. After this (first) bifurcation, further bifurcations of the same type occur at increasing values of $\mu$, as observed in the right part of figure 4.12.a. Similar to what was reported in Ref. [117] for the DNLS model, in direct simulations unstable vortex crosses evolve into on-site-centered fundamental discrete breathers (with $S=0$ ) by 
transferring almost all the energy to one of the sites which originally formed the cross. The corresponding instability border (for $C=1$ ) in the $\left(\mu, \omega_{b}\right)$ plane is depicted by the right curve of figure 4.12.b.

More interesting is the case of $\mu<0$. In this regime, we have found that fundamental vortex crosses experience another bifurcation, with a quadruplet of Floquet eigenvalues leaving the unit circle at $\lambda=+1$ (the so-called harmonic bifurcation). With the decrease of $\mu$, the corresponding two pairs of the eigenvalues move along the real axis in the opposite direction, until each pair breaks up, as shown in figure 4.12.d. The unstable eigenvectors, $\delta \Phi^{*}$ and $\delta \Phi^{* *}$, associated with this bifurcation are plotted in figure 4.13.a and 4.13.b (in this notation, ${ }^{*}$ does not stand for complex conjugation). The shape of each eigenvector reveals strong localization at two opposite sites of the vortex cross, each one separately breaking the spatial symmetry (2D isotropy) of the original solution. Adding a small perturbation to the solution along one unstable direction causes oscillations of the amplitudes around the vortex center, as shown in figure 4.13.c. Such behaviour persists at longer times; in fact, the vortex pattern does not disappear but rather suffers irregular modulations of its local amplitudes.

This picture of the instability development supplements the stability diagram for the fundamental vortex crosses, which is displayed in figure 4.12.b in the $\left(\mu, \omega_{b}\right)$ plane (as noted above, for the isotropic model, with $C=1$ ). Note that the border of the instability which transforms the vortex cross into its oscillatory counterpart (the left curve in the figure) stays in the $\mu<0$ region, even for large frequencies. Therefore, unlike the HHB described above, this instability is dominated by the competition between the self-defocusing intersite and self-focusing on-site nonlinearities. A further insight into the nature of this bifurcation is provided by the observation that it coincides exactly with the divergence of norm $N\left(\omega_{b}, \mu\right)$ of the discrete breather (and of the vortex cross solution), and thus it coincides with the stability interchange between in-phase and out-of-phase bound states analyzed above in section 4.2.2.

Regarding the vortex cross as made up of two (perpendicular) out-ofphase bound states of breathers (say, left-right and top-bottom), one would be tempted to interpret the quadruplet of eigenvalues leaving the unit circle at +1 as the two pairs of eigenvalues that signal the simultaneous instability of both out-of-phase bound states. At least, this interpretation would explain the fact that a quadruplet of eigenvalues simultaneously leave the unit circle at +1 , and it is fully consistent with the shape of the Floquet eigenvectors in figure 4.13. This interpretation suggests that the bifurcation of vortex crosses occurring in the left part of figure 4.12.b is the same one experienced by out-ofphase bound pairs of breathers in figure 4.9 (for separation $d=1$ ). In any case, 
(a)

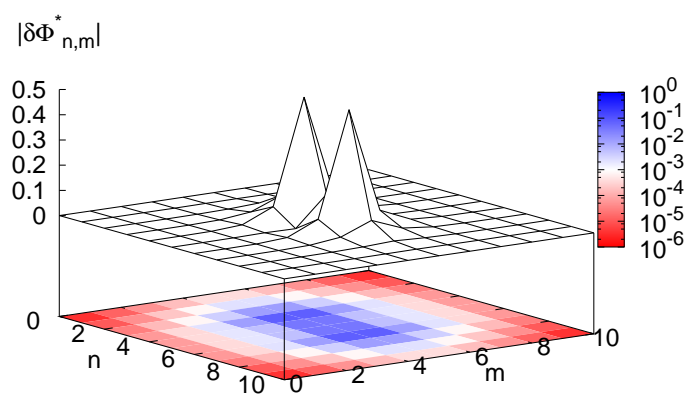

(b)
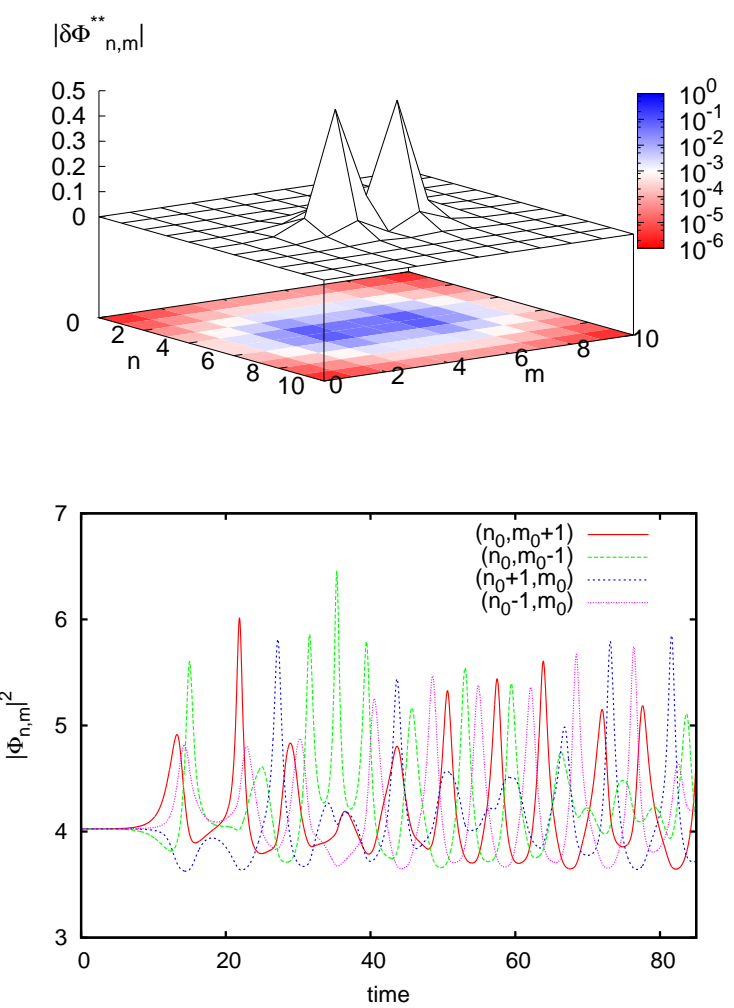

(c)

Figure 4.13: (a) and (b) Intensity profiles of the unstable Floquet eigenvectors, $\delta \Phi^{*}$ $\delta \Phi^{* *}$, corresponding to the bifurcation at $\mu=-0.3$ (for $C=1$ ) of the vortex cross with $\omega_{b}=8$, see figure 4.11.d. (c) Time evolution of the lattice field at sites around the center of the same unstable vortex solution. Pulsonic dynamics of the amplitudes is observed, without decay of the vortex pattern. 
a noteworthy numerical finding is that these bifurcations (of bound states and vortex crosses) not only coincide but are also characterized by the divergence of the breather norm.

\subsubsection{Vortex squares}

We have also studied the smallest $(M=1)$ vortex squares carrying $S=1$ vorticity. For this purpose, we have performed the continuation of the corresponding solution family, starting from a configuration with nonzero components $\Phi_{n_{0}, m_{0}}=-i \Phi_{n_{0}, m_{0}+1}=-\Phi_{n_{0}+1, m_{0}+1}=i \Phi_{n_{0}+1, m_{0}}=\sqrt{\omega_{b} / 2}$ in the anticontinuum limit, eq. (4.18). As in the case of the vortex cross, we have first performed the continuation in the coupling constant $C$ to obtain the corresponding solutions for the DNLS model $(C=1, \mu=0)$. Again, for lowfrequency vortex squares, we have observed an HHB at some critical value of $C$. For high-frequency solutions, a bifurcation of the same type is observed when the continuation is performed from the DNLS model to values $\mu>0$. In figure 4.14.a, one can observe this bifurcation for the vortex square with $\omega_{b}=8$. The corresponding HHB (see figure 4.14.c) occurs with a quadruplet of the Floquet eigenvalues leaving the unit circle. The behavior of the unstable solution is the same as for the vortex cross, and, after a transient, a regular breather with $S=0$ emerges at one of corner sites of the former vortex square, while the field at three other corners nearly vanishes (i.e. the energy mainly concentrates at a single site of the initial vortex structure).

With the continuation of the vortex square to $\mu<0$, we have again (as in the case of vortex crosses) found that the solutions suffer a destabilizing bifurcation different from that at $\mu>0$. However, the bifurcation for $\mu<0$ (see figure 4.14.d) is also different from its counterpart for the vortex cross (which was displayed above in figure 4.12.d). At some value $\mu<0$, a quadruplet of Floquet multipliers leave the unit circle, to return to it at +1 . After this brief excursion, they immediately leave the unit circle again, and instability grows with $|\mu|$. Unlike its counterpart for the vortex cross, this bifurcation does not correspond to the interchange of stability for the bound state of breathers analyzed in 4.2.2, which actually occurs at a lower value of $\mu$, where the vortex square is already unstable. However, it is remarkable that precisely at this value of $\mu$ the quadruplet of eigenvalues outside the unit circle meet instantaneously at +1 , so that the vortex square is marginally stable at that point.

Profiles of unstable eigenvectors, $\delta \Phi^{*}$ and $\delta \Phi^{* *}$, are shown in figure 4.15.a and 4.15.b. Each one is localized at two non-adjacent corners of the plaquette where the vortex square is located. The dynamics triggered by the original 

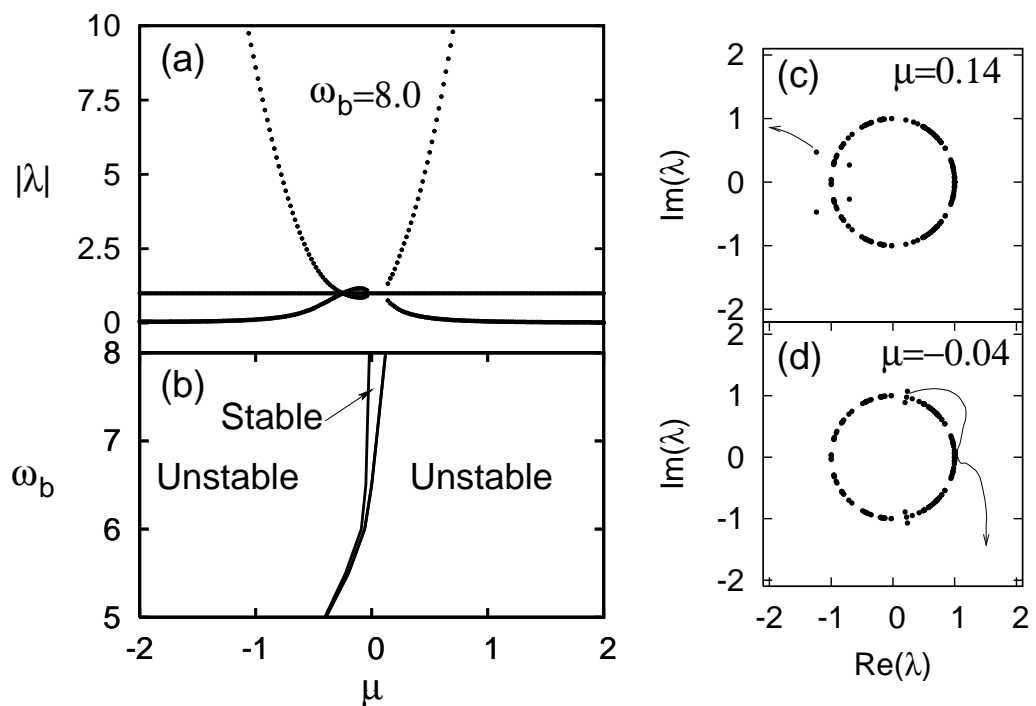

Figure 4.14: (a) The absolute value of the Floquet multipliers as a function of $\mu$ for a vortex square of minimum size $(M=1)$ with $\omega_{b}=8(C=1)$. Two bifurcations are revealed by Floquet distributions in panels (c) and (d). At $\mu=0.14$, we find a Hamiltonian Hopf bifurcation, whereas at $\mu=-0.04$ a quadruplet of eigenvalues leave the unit circle and start a short trip to +1 , from where they leave the unit circle again. The entire stability diagram is represented in panel (b), showing a narrow stability region.

solution being perturbed by this $\delta \Phi^{*}$ (or equivalently $\delta \Phi^{* *}$ ) is displayed in figure 4.15.c. Again (as in the case of the vortex cross), the vortex pattern is not destroyed (in contrast with the unstable behavior at $\mu>0$ ). Instead, the lattice field at the vortex-square sites develops a periodic pulsonic behavior, in which at least two frequencies can be identified. One of the frequencies accounts for periodic transfer of energy between four corners of the square vortex, following the same path as the flux current:

$$
\left(n_{0}, m_{0}\right) \rightarrow\left(n_{0}, m_{0}+1\right) \rightarrow\left(n_{0}+1, m_{0}+1\right) \rightarrow\left(n_{0}+1, m_{0}\right) \rightarrow\left(n_{0}, m_{0}\right) \rightarrow \ldots
$$

Another noteworthy feature of the dynamics in this case is that the total amount of energy that is periodically transferred between neighboring sites varies, also in a regular periodic fashion, thus giving rise to the second frequency. Again (as happened for the vortex cross), the instability observed at $\mu<0$ induces a pulsonic dynamics of the lattice amplitudes but, in the present case, the dynamics is much more regular. An intriguing numerical observation is that the value of $\mu$ at which the quadruplet of eigenvalues meet at +1 (so that the vortex-square solution momentarily becomes marginally stable) occurs 
(a)

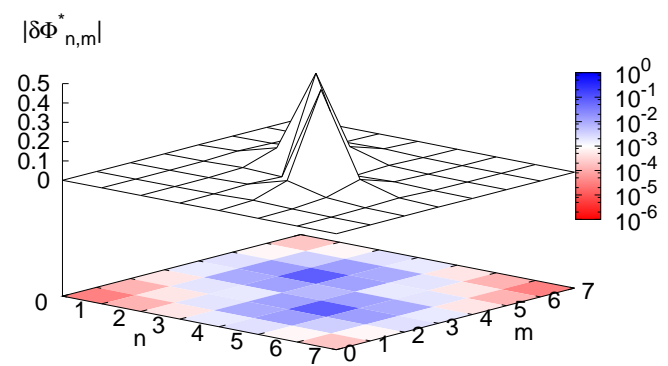

(b)

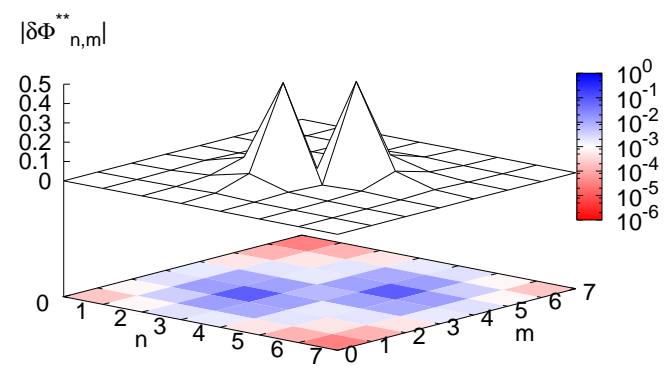

(c)

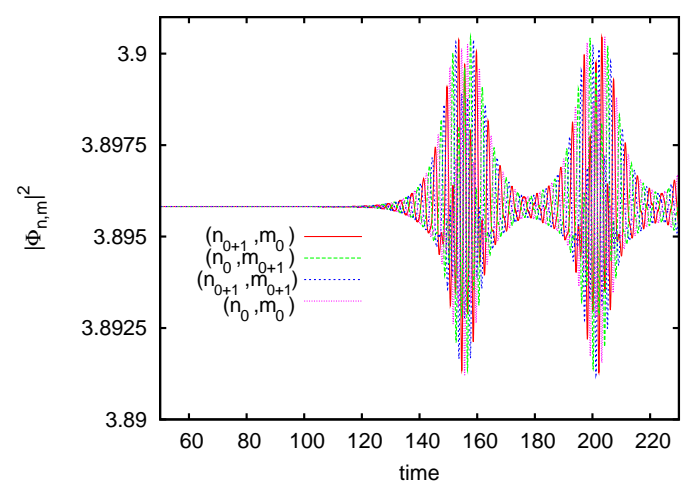

Figure 4.15: (a) and (b) Intensity profiles of the unstable Floquet eigenvectors, $\delta \Phi^{*}$ and $\delta \Phi^{* *}$, corresponding to the bifurcation, at $\mu=-0.04$ (for $C=1$ ), of the vortex square with $\omega_{b}=8$, shown in figure 4.13.d. (c) The time evolution of the lattice field at the vortex-square's corners for the same unstable solution. The simulations reveal periodic evolution of the amplitudes with a clear sequence of energy transfer between the adjacent sites following the same pattern as the current flux in the original vortex solution. 
exactly when the breather norm diverges.

The entire stability diagram for the fundamental vortex squares is presented in figure 4.14.b. Again, we find a narrow stability region for low-frequency vortex squares that expands as the frequency increases.

\subsubsection{Bound states of discrete vortex crosses}

As a first step towards the characterization of the stability of more complex 2D arrangements of vortices, we have studied two types of bound states of vortex crosses, with the vortex centers aligned along a lattice axis (say, the $x$-direction). In the two types of the bound state, the vortices have equal or opposite vorticities, see figures 4.16.a and 4.16.b. Both types of solutions were studied on the isotropic Salerno lattice with competing nonlinearities $(C=\nu=1$ and $\mu<0)$, and were numerically obtained by the continuation at $\mu=0$ from the anticontinuum limit $(C=0)$, followed by the a second continuation in the direction of negative inter-site nonlinearity $\mu$. The Floquet spectrum of the solution was also numerically computed along the continuation path.

At $\mu=0$, bound states of vortices with equal vorticities are stable, while those with opposite vorticities are unstable. To explain this numerical observation, one has to realize that the right-most member of the breather set forming the left vortex, and its left-most counterpart in the right vortex are out-of-phase (in-phase) in the former (latter) case, see figures 4.16.a and figures 4.16.b. Then, the stability analysis of bound states of breathers reported above in section 4.2.2 suggests that the stability of the bound states of vortices is actually dominated by the stability of the local bound state of the two constituent breathers (one from each vortex) that are in the closest proximity. This analysis is further validated by comparison of unstable Floquet eigenvalues for the bound state of vortices with opposite vorticities and those for the bound state of in-phase breathers (for the corresponding values of the frequency and separation between the centers).

When $\mu$ decreases, a destabilizing bifurcation occurs, as expected, in the equal-vorticity bound state, precisely at the same value of $\mu$ where the simultaneous instability of the vortex cross (in section 4.3.1) and the out-of-phase bound state of ordinary breathers occurs. By inspection of the Floquet spectrum for the bound state of vortices, one can clearly identify pairs of eigenvalues associated with each of these instabilities that take place simultaneously at this bifurcation point. It clear that the stability of bound states of discrete vortex and that of single vortices in the SM with $\mu<0$ is related to the behaviour found for bound states of two pinned breather solutions. The decomposition 
(a)

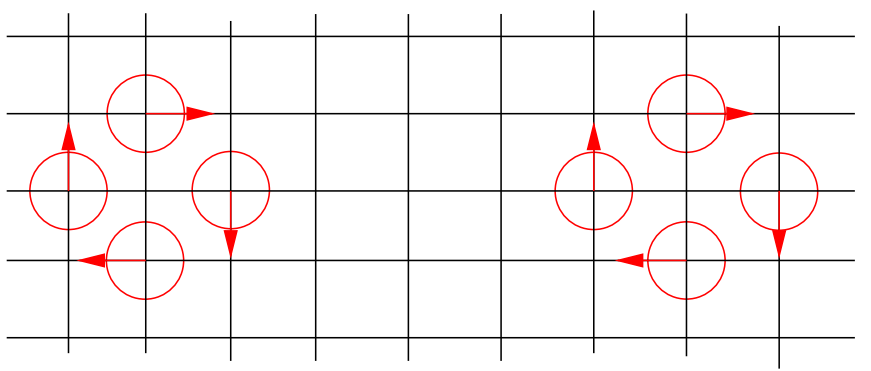

(b)
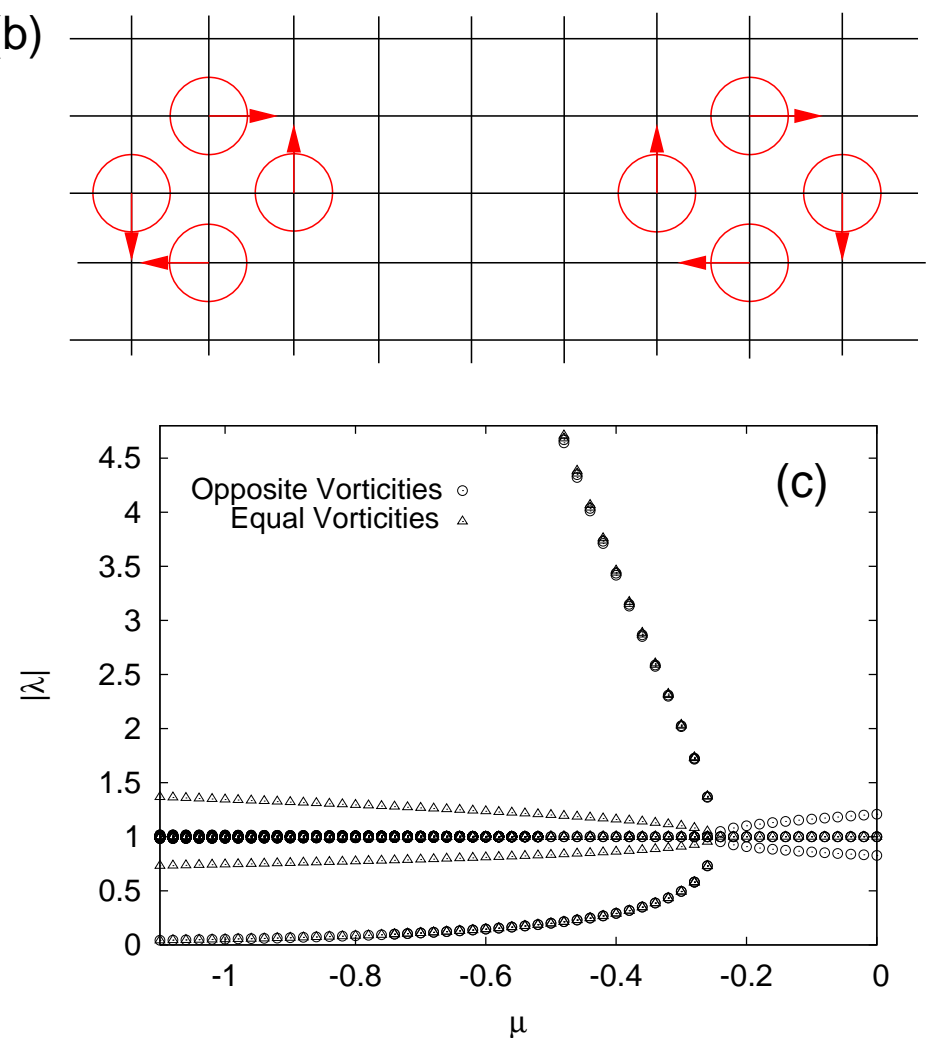

Figure 4.16: A schematic representation of the in-phase (a) and out-of-phase (b) bound states of vortices with $S=1$, in the limit of $C=0, \nu=1, \mu=0$. Vectors stand for the instantaneous values of $\Phi_{n, m}$ in the complex plane, with $\left|\Phi_{n, m}\right|=\sqrt{\omega_{b} / 2}$. These solutions are continued in $C$ up to $C=1$, and then continued in $\mu$. Panel (c) shows the evolution of the Floquet multipliers as a function of $\mu$ when $\mu<0$. The results correspond to $\omega_{b}=8$ and the distance between the two vortex centers is set to be $d=5$ (as seen in (a) and (b)).

of any complex solution in terms of this latter building blocks is clearly of importance. 


\subsection{Mobile discrete breathers}

Early and current attempts to explore straightaway discrete breather mobility in isotropic 2D Schrödinger lattices seem to agree that "kicking" procedures meet huge difficulties in delivering good mobile solutions, contrary to the numerical experiences in 1D lattices. We note here that the formal basis for those methods [52] takes advantage of the Floquet spectra analysis of exact pinned breathers, where the so-called depinning (symmetry-breaking) mode is identified. This allows, provided Peierls-Nabarro barriers are small enough, to obtain nice numerical 1D mobile discrete breathers, by computing trajectories from perturbations of the exact pinned breather along the tangent space direction specified by the depinning eigenvector. The presence of symmetry-breaking instabilities leading to exchange of stability between one-site and two-site centered pinned breathers [91] and the associated lowering of the Peierls-Nabarro barriers to breather displacements (as we observed for the 1D standard SM when $\nu<0$ in section 3.1.3), hugely facilitates the success of these procedures when applied to (both Hamiltonian and dissipative) one-dimensional lattices $[92,93]$.

In contrast, our "anisotropic lattice" continuation approach takes advantage of the availability of exact $1 \mathrm{D}$ mobile solutions by monitoring the parameter $C_{2}$ of transversal coupling, and then does not rely on how easily one promotes clean mobility from pinned localization. In this way we obtain accurate numerical $\left(p_{x}, p_{y}=0, q\right)$ fixed points, that is Schrödinger discrete breathers moving along the strong coupling direction. We will leave open the question for arbitrary direction of motion which would imply more sophisticated (and possibly more unclean) methods of construction.

In this section we will focus on the behaviour of the 1D mobile breathers found for the standard SM (then we set $C_{1}=1$ ) when the coupling in the transverse direction (accounted by $C_{2}$ ) is adiabatically incorporated and hence the dimensionality of the solution is increased.

\subsubsection{Structure and stability of $(1,0,1)$ fixed points.}

In figure 4.17 we visualize the instantaneous real and imaginary components of the 2D discrete field profile of a typical $(1,0,1)$ Schrödinger breather. Its structure can be seen as the natural extension to two-dimensional lattices of the structure of mobile Schrödinger breathers analyzed in the previous chapter. The numerical solution is spatially asymptotic to a finely tuned small-amplitude extended (delocalized) radiation state $\left(\Phi_{\mathrm{bckg}}\right)_{m, n}(t)$ when $m, n \rightarrow \infty$. The 
fixed point solution can be thus decomposed as

$$
\Phi_{m, n}(t)=\left(\Phi_{\mathrm{core}}\right)_{m, n}(t)+\left(\Phi_{\mathrm{bckg}}\right)_{m, n}(t),
$$

which defines $\left(\Phi_{\text {core }}\right)_{m, n}(t)$, the spatially localized component of the solution. It turns out that the spatially delocalized component is a highly localized state in the (continuum, in the thermodynamic limit) $k$-space of wavevectors. More precisely, $\left(\Phi_{\mathrm{bckg}}\right)_{m, n}(t)$ is a finite linear combination of $(1,0,1)$-resonant nonlinear (i.e. amplitude-dependent frequency $\omega$ ) 2D planewaves. It can be said that, as might be expected, 1D Schrödinger breather mobility smoothly persists when (strong $C_{1}$-coupling) $1 \mathrm{D}$ chains are coupled transversally. Importantly, the numerical continuation for increasing values of the transversal coupling $C_{2}$ proceeds far from the weak coupling regime into where the genuine twodimensional effects start to be manifest, as we will see below.

Most noticeable, the SVD-regularized Newton procedure invariably selects the values $k_{y}= \pm \pi / 2$ for all values of $C_{2}$ and $\nu$, and thus the values of $k_{x}$ for the $2 \mathrm{D}$ resonant planewave are independent of $C_{2}$ (so it remains equal to the $k$ values of the $1 \mathrm{D}(1,1)$ fixed point for the uncoupled chain). The appearance of an extended background modulation in the transversal direction of $k_{y}= \pm \pi / 2$ appears naturally as the best choice to take advantage of approximately 1D breather propagation along strong coupling direction, for it keeps the value of $k_{x}$ favoured by the strong coupling $C_{1}$ value: Any other value of $k_{y}$ would entail a different $k_{x}$ value. Note however that this provides only a plausibility argument for the interpretation of the numerical observation $\left(k_{y}= \pm \pi / 2\right)$.

The high accuracy of the computed solutions allows a detailed analysis of many issues concerning 2D Schrödinger breather exact mobility along the strong coupling direction. We will focus here on how the existence of quasi-collapse instabilities of pinned Schrödinger breathers, for increasing $C_{2}$ coupling values, influences the stability properties of moving $(1,0,1)$ breathers in the standard SM. In other words, we search here for genuine $2 \mathrm{D}$ effects on these "strong-coupling-direction" (quasi-1D) moving breathers.

We have performed an exhaustive exploration of two sectors of the parameter space $\left(C_{2}, \omega_{b}, \nu\right)$, corresponding to the breather frequency values $\omega_{b}=$ 2.5843 , and $\omega_{b}=2.712$, by computing the continued $(1,0,1)$ fixed point along the standard Salerno model. These values of $\omega_{b}$ were chosen low enough to allow the analysis of pinned breather quasi-collapse effects on mobility, which occurs at relatively low values of $C_{2}^{t h}(\nu)$ for these values of $\omega$ as seen in section 4.2.1 (see e.g. figure 4.2 for the case $\nu=1, \mu=0$ ).

The Floquet analysis of the computed solutions provides the stability diagrams represented in figures 4.18. Both show no qualitative differences: There are two regions in the $\left(C_{2}, \nu\right)$ plane where the $(1,0,1)$ mobile breather is lin- 
(a)

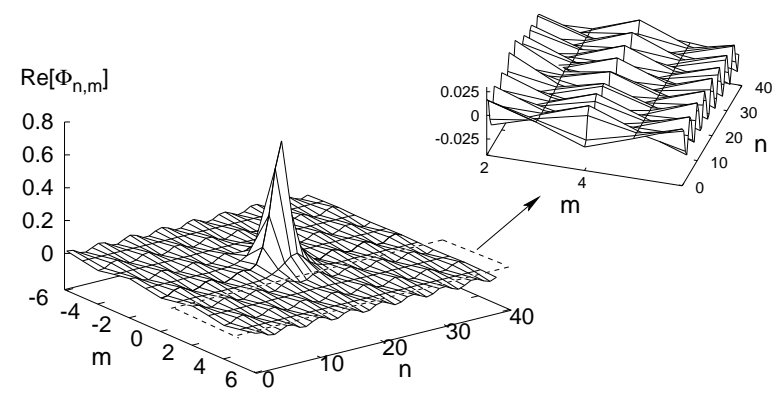

(c)

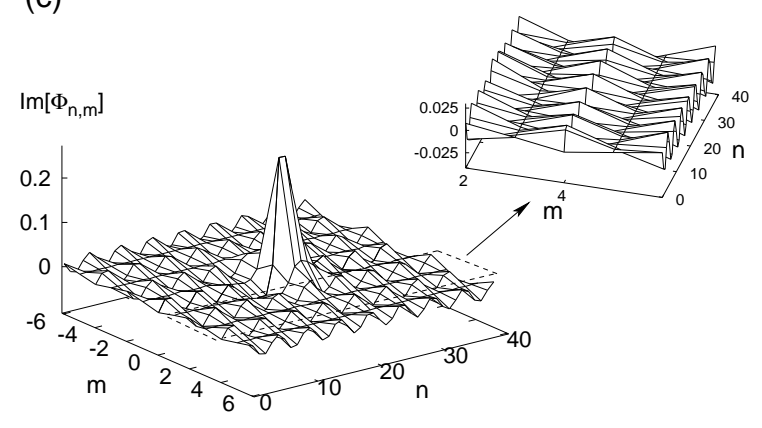

(b)

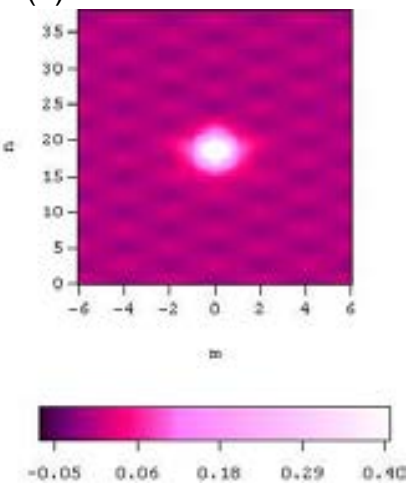

(d)

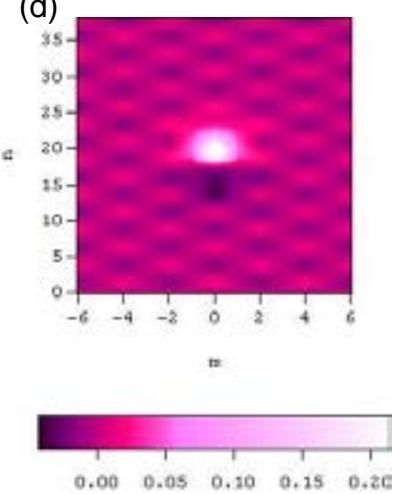

Figure 4.17: Real, (a) and (b), and imaginary, (c) and (d), of a mobile (1,0,1)discrete breather of frequency $\omega_{b}=2.712$. The parameters of equation (4.1) are $C_{1}=1, C_{2}=0.14$ and $\nu=0.95(\mu=0.05)$. The insets in (a) and (c) show the background far from the moving core. It can be observed that the wavenumbers in the transversal direction are $k_{y}= \pm \pi / 2$. (b) and (d) show the contour plot for both real and imaginary parts.

early unstable. The figures are not "schematic": Every point of the plane in a fine grid of values of $C_{2}$ and $\nu$ has been analyzed, i.e. the Floquet spectrum of the computed $(1,0,1)$ fixed point is scrutinized, as shown in figures $4.18 . \mathrm{b}$ and 4.18.d, where the modulus of the Floquet eigenvalues is shown as a function of either $\nu$ (figure 4.18.b) or $C_{2}$ (figure 4.18.d).

The first unstable region appears at low values of $C_{2}$ and intermediate to high values of the Salerno parameter $\nu$, i.e. it does not occur close to the AL limit. This unstable region is also bounded above in the direction of $C_{2}$ : The variation of the modulus of the unstable Floquet eigenvalue versus the transversal coupling parameter $C_{2}$ shows that the mobile breather becomes stable again at larger values of $C_{2}$, before the second instability at even higher coupling takes place. An important observation is that the pinned discrete breather of the same frequency is linearly stable at the points in this unstable 

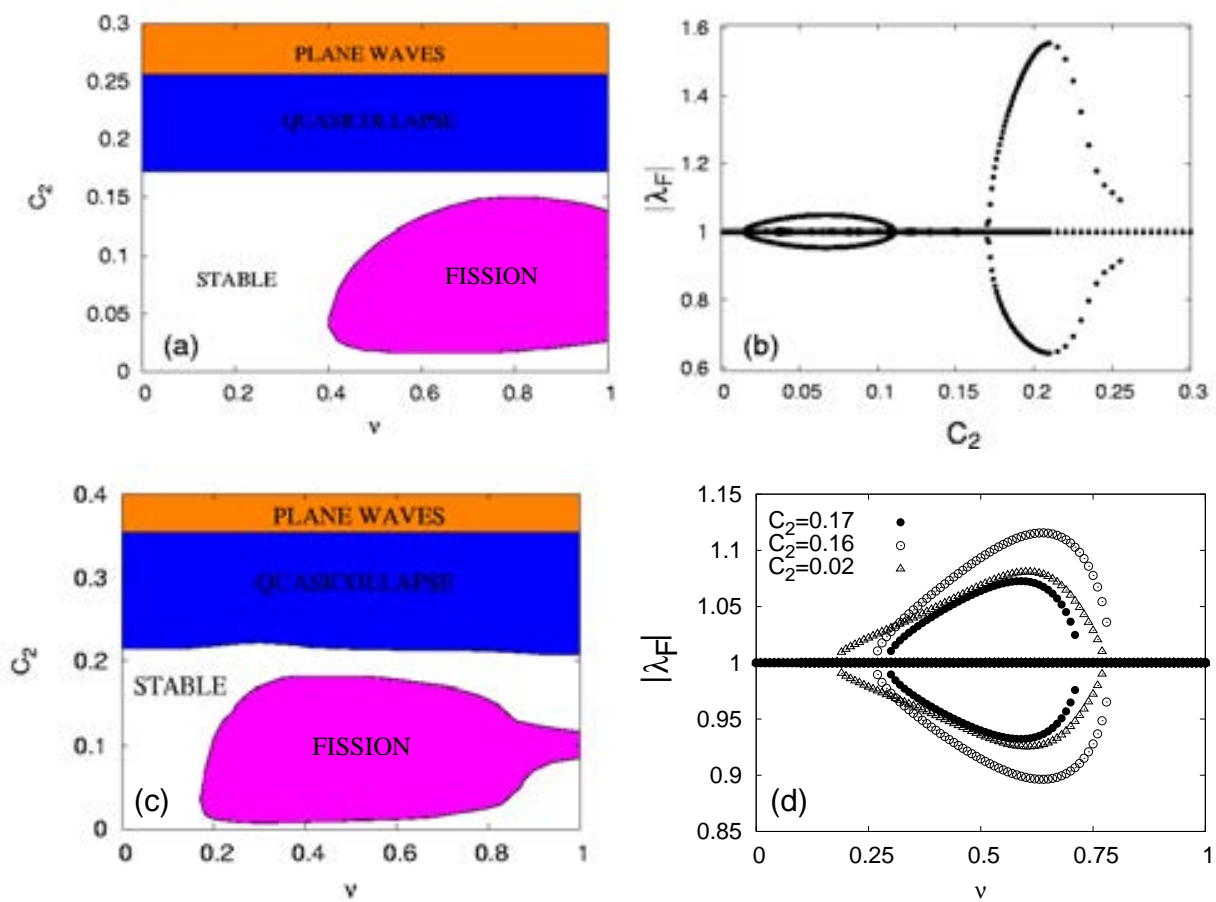

Figure 4.18: Stability diagram and evolution of the modulus of the Floquet eigenvalues for two $(1,0,1)$-discrete breather of frequencies $\omega_{b}=2.584(\mathbf{a})$ and (b), and $\omega_{b}=$ 2.712 (c) and (d) $\left(C_{1}=1\right)$. The stability diagram (a) and (c) show two regions where the mobile discrete breather becomes unstable. For low values of the coupling $C_{2}$ there is a subset of values of $\nu$ where the breather suffers from fission (see text and figures (4.19.a) and (4.19.b). On the other hand for higher values of $C_{2}$ there is a second region (quasi independent of $\nu$ ) where the unstable breather yields a traveling quasi-collapsing state (see text and figures (4.19.c) and (4.19.d)). The evolution of the modulus of the Floquet eigenvalues along different paths $\nu=0.50(\mathbf{b})$ and $C_{2}=0.17$, $0.16,0.02(\mathbf{c})$ is shown.

region for $(1,0,1)$ mobile breathers. Thus this instability cannot be ascribed to pinned quasi-collapse effects.

The second transition occurs for values of $C_{2}$ close to, but slightly higher than, the values $C_{2}^{t h}$ of the quasi-collapse of the pinned breather of the same frequency. We had already seen in the previous section that the quasi-collapse transition $C_{2}^{\text {th }}(\nu)$ is only very weakly dependent on the value of $\nu$, and note that the same is true for this mobile breather bifurcation. These results suggest that this second transition is related to quasi-collapsing phenomena. Significantly, the stability of the $(1,0,1)$ mobile breather persists for a small interval of coupling values above the pinned breather quasi-collapse. This should be regarded as natural, for the mobile breather is a different solution. Note in figure 4.18.b that the modulus of the unstable Floquet eigenvalue, in the inte- 
rior of the unstable region, reaches much higher values than those typical for the first type of instability, and decreases for larger values of $C_{2}$, before the breather solution ceases to exist and only plane wave solutions are obtained by our numerical method. Note that this behaviour of the unstable Floquet eigenvalue also fits well to the main features of the pinned quasi-collapse instability strength, as described by the slope $\partial N / \partial \omega_{b}$. From now on we will refer to this instability of mobile breathers as the quasi-collapse instability.

In the next section we characterize both generic types of instability, by looking at the details of the unstable manifold associated with each type. As we will see, pulson states turn out to play a role in the description of typical trajectories on the unstable nonlinear manifolds.

\subsubsection{Unstable manifold behaviour and ubiquity of pulson states.}

First, we analyze the quasi-collapse instability of $(1,0,1)$ mobile breathers. The unstable linear subspace in the tangent space of the fixed point is onedimensional. The typical instantaneous profile of the (modulus) unstable Floquet eigenvector driving the instability is shown in figure 4.19.d. It is an exponentially localized 2D profile which decays asymptotically to zero as $m, n \rightarrow \infty$, i.e. it does not excite radiation. These characteristics are shared by the quasicollapse unstable eigenvector of the pinned breathers, which further reinforce the previous considerations leading us to consider this instability as the mobile counterpart of the pinned quasi-collapse transition.

In figure 4.19.c we have visualized the time evolution of the field modulus contour plot for a typical trajectory on the unstable manifold. This is obtained by direct numerical integration of the equations of motion, from an initial condition in which a small perturbation along the quasi-collapse eigenvector has been added to the unstable fixed point solution. One sees that the breather translational motion slows down, and the energy is transferred to width oscillations. These oscillations turn out to be more irregular, see figure 4.20, than those observed in section 4.2 when we inspected typical trajectories on the unstable nonlinear manifold of pinned breathers.

The difference in the character of the width oscillations in both (pinned and mobile) cases may be ascribed to the presence of an extended background component in the mobile breather solution, which naturally enters into the energy transfer taking place during temporal evolution. The slowing down of the translational motion continues and eventually the breather pins into a convulsive pulson state surrounded by the remaining radiation.

Now we pay attention to the "low $C_{2}$ " instability of $(1,0,1)$ mobile breathers. 

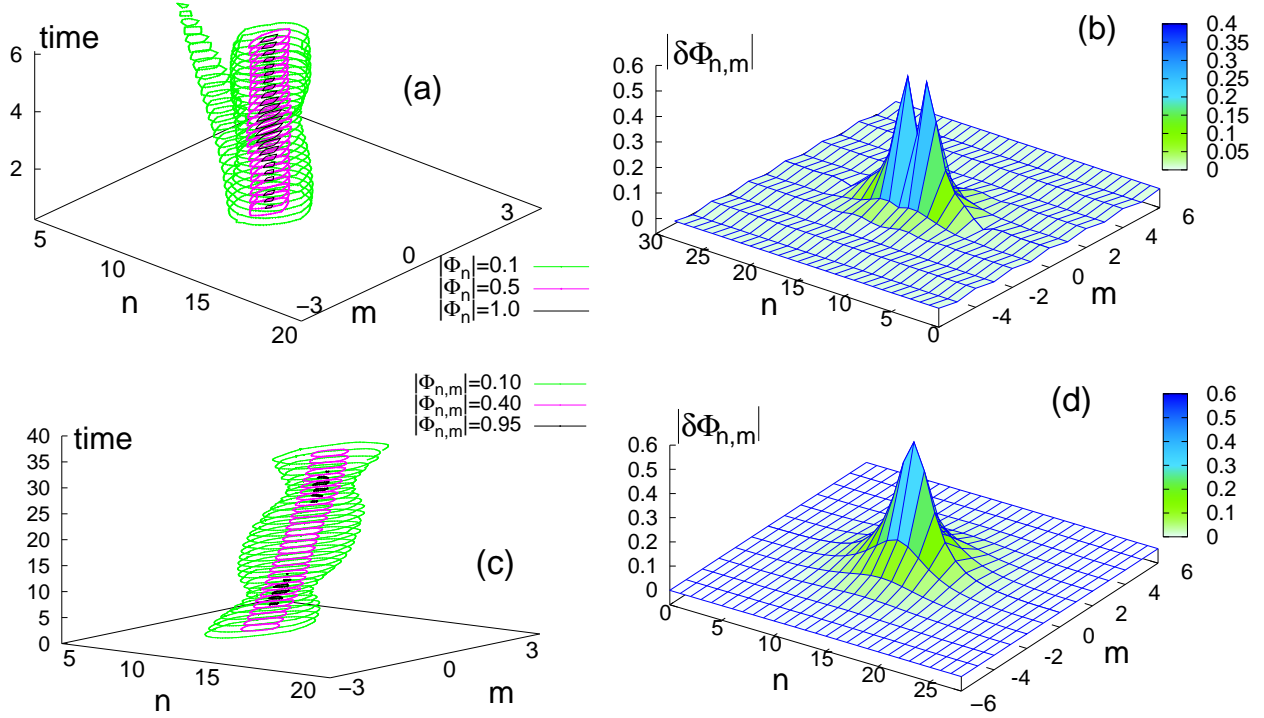

Figure 4.19: Time evolution of two unstable solutions, (a) and (c), of frequency omega $_{b}=2.584$ and the associated unstable Floquet eigenvector, (b) and (d) respectively $\left(C_{1}=1\right)$. Figures $(\mathbf{a})$ and $(\mathbf{c})$ show the time evolution of the contour lines corresponding to three different values of $\left|\Phi_{n, m}\right|$, in order to visualize the 4dimensional functions $\left|\Phi_{n, m}\right|(t)$. Figures (a) and (b) shows the fission of the breather solution when perturbed along the unstable "M-shaped" Floquet eigenvector plotted in (b). It can be seen how a low amplitude pulse emerges and the mobile breather becomes pinned. After this transient this low amplitude pulse decays into radiation. The parameter of equation (4.1) are $C_{1}=1, C_{2}=0.08$ and $\nu=0.5(\mu=0.5)$. In the case of figures (c) and (d) the parameters are the same except for $C_{2}=0.19$. In this case the solution is in the "quasi-collapse" unstable region shown in figure (4.18.a). The final state when perturbed along the unstable eigenvector $(\mathbf{d})$ is a traveling breather whose amplitude oscillates in the same fashion as that of the pinned quasi-collapsing breathers, i.e the localization center oscillates out of phase with respect to all the other sites on the lattice.

The modulus profile of the unstable Floquet eigenvector that drives this instability is M-shaped (bimodal), as shown in figure 4.19.b, and is asymptotic to an extended planewave-like profile as $m, n \rightarrow \infty$, i.e. it is not a purely localized perturbation. It is indeed rather different from the quasi-collapse unstable eigenvector analyzed above, which is consistent with the fact that the pinned breather of the same frequency is linearly stable in this region of parameter space. As argued above, this instability is not related to quasi-collapse phenomena, and it does not appear in the region of small values of the Salerno parameter $\nu$, close to the AL limit.

A typical trajectory on the unstable manifold associated with this instability is shown in figure 4.19.a, where we have plotted the time evolution of 

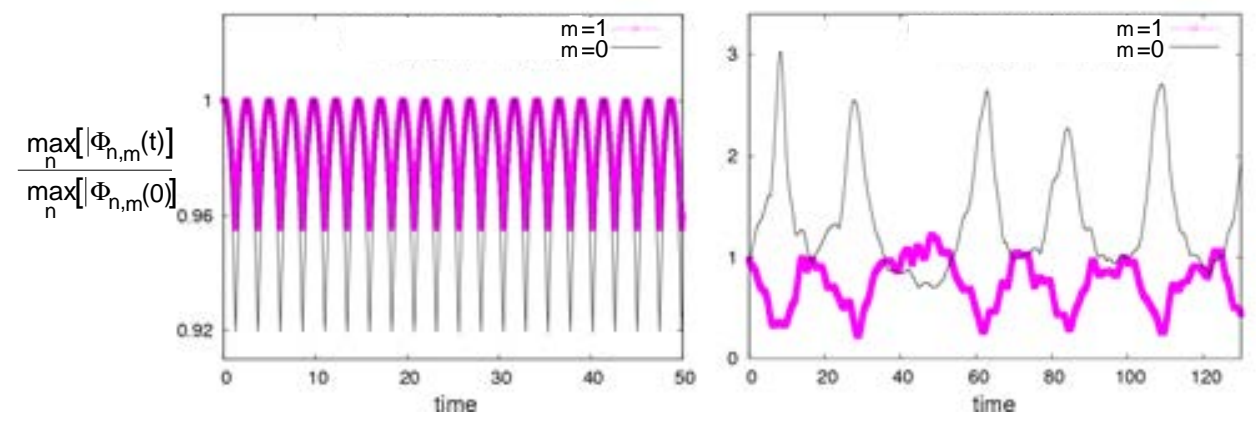

Figure 4.20: Time evolution of the maximum value of the modulus $\left|\Phi_{n, m}\right|(t)$ along the central $(m=0)$ chain and the adjacent $(m=1)$ one for a mobile $(1,0,1)$ breather with frequency $\omega_{b}=2.584$. This magnitude is normalized to the initial value $\left|\Phi_{n, m}\right|\left(t_{0}\right)$. Figure(a) shows this evolution for an stable situation $\left(C_{2}=0.15, \nu=0.5\right)$. It can be observed how the localization center $(m=0)$ and is neighbour in the transversal direction $(m=1)$ follows two in-phase periodic trajectories in their modulus due to the Peierls-Nabarro barrier surpassed during the motion. In contrast, figure (b), shows the case when the breather is unstable $\left(C_{2}=0.19, \nu=0.5\right)$. Here the quasicollapse dynamics is manifested while the localization center moves across the lattice. As can be observed, the oscillations of the two amplitudes are out of phase and the amplitudes of these oscillations are one order of magnitude higher than those of figure (a).

the field modulus contour plot. We can see there that the mobile breather pins quickly while a small pulse moving backwards is ejected, which spreads and finally mixes with the remaining delocalized background. However some energy is transferred to width oscillations of the pinned breather so that also in this case we observe the formation of pulson states surrounded by the remaining radiation. As the main difference of this behaviour, with respect to the evolution observed on the quasi-collapse unstable manifold, is the ejection of the small moving pulse, we refer to this instability as fission.

By increasing the strength of the initial perturbation along the direction of the unstable eigenvector, one observes that the size of the ejected pulse increases. This observation is consistent with the results reported in [127], where the evolution of initial moving Gaussian pulses in isotropic 2D Schrödinger lattices was studied. These numerical experiences lead the authors to conclude that "the characteristic feature of the discrete quasi-collapse of a moving pulse is the splitting of the initially moving broad pulse into a track of the standing narrow structures ..." (sic). However, we see from our study of the stability of exact moving discrete breathers that the fission and the quasicollapse instabilities have different origins and they appear in different regions of parameter space. On the other hand, the ubiquitous phenomenon of width oscillations of pinned localized structures (pulson states) cannot be ascribed 
to quasi-collapse. They also appear as the preferred way to allocate excess of (localization) energy in regions of parameter space far from the quasi-collapse unstable region.

\subsection{Conclusions and Prospective Remarks}

We have studied here the dynamics of exact numerical discrete breathers (pinned, vortical and mobile ones) in a two-dimensional anisotropic nonlinear Schrödinger lattices. These solutions are computed from a set of uncoupled 1D chains into increasing non-zero values of the coupling in the transversal direction in order to reach the $2 \mathrm{D}$ limit. It is convenient review the most salient results in order to have a compact picture of the $2 \mathrm{D}$ behavior of discrete breathers.

Pinned breathers.- We have performed an extensive exploration in the parameter space $\left(\omega_{b}, C_{2}, \nu\right)$ of breather frequency, transversal coupling and Salerno parameter, by computing the Floquet spectra of the numerical solutions. Both the 1D solutions of the standard and the competing SM have been continued into the 2D regime in order to see the effects of the dimensional increment. In particular we have found the link between the unstable behaviour found for certain breather frequencies in the 1D competing Salerno model (whose pulsonic character resembled those of the well known 2D unstable solutions) and the quasi-collapse instability that appears for low frequency breathers when the coupling in the transverse direction is incorporated. Furthermore, we have analyzed the dynamics on the quasi-collapse unstable manifold, where the unstable breather experiences a shift in frequency towards the (higher) value of the stable breather with the same norm. The excess of energy is coherently transferred to oscillations of the breather width, so that the resulting pulson state is characterized by two frequencies. We have also recovered the 2D counterpart of the 1D cuspons and peakons for the 2D SM with competing nonlinearities. Again these hyperlocalized states are stable Finally, the stability analysis of in-phase and out-of-phase bound states of breathers in the isotropic lattice reveals that there is a stability interchange between both types of bound states, precisely at the same value of the intersite-nonlinearity parameter $(\mu)$ where the breather norm diverges as happened for the 1D model.

Vortex breathers.- In addition to fundamental breathers, discrete vortices of two types, cross- and square-shaped ones, have also been constructed, and their stability regions identified. In direct simulations, unstable vortices in the standard 2D Salerno model of the ordinary type transform into regular breathers, while in the model with the competing nonlinearities the instability turns vortices into localized vortical pulsons, without destroying their topo- 
logical character. It is then worth mentioning the ubiquity of this pulsonic attractors of the dynamics in the model. Regarding the stability of bound states of vortex crosses, we have shown that it is determined by the stability of the local bound state of two constituent breathers (forming the two vortices) which are in the closest proximity.

Mobile breathers.- We have studied discrete breathers moving along the strong coupling direction for the standard 2D SM. These solutions are composed of an exponentially localized core on top of an extended background which is itself the finite sum of a finite set of nonlinear 2D plane waves. The time scales associated with these plane waves are resonant with the core internal frequency as happens in the 1D case. In particular, the background chooses a finite set of plane waves from a continuous family of resonant solutions. The Floquet analysis of these mobile discrete breathers reveals the existence of two distinct types of instability. One is the counterpart, for mobile breathers, of the quasi-collapse experienced by pinned breathers. The other instability occurs in a region of parameter space where pinned breathers are linearly stable. The analysis of the dynamics on the unstable manifold show that the excess of energy is partly transferred to a small moving pulse, ejected from the center of localization, which justifies the designation of a fission instability. However, part of the energy excess is also transferred to width oscillations. The appearance of pulson states far from the quasi-collapse regime indicates that the tendency to allocate energy in the form of width oscillations is a general 2D feature, not exclusively associated to quasi-collapse instabilities.

We leave the question on mobility of $2 \mathrm{D}$ discrete breathers in an arbitrary lattice direction. The results obtained here shed light about how this mobility can be obtained. In fact, our experiences show that mobility of pinned breathers can be induced based on the existence of the extended background in the numerically exact mobile solution. On the other hand, the results obtained here and the aforementioned future work may help to design and better understand recent numerical experiments reported in [136], concerning the interaction between high amplitude pinned breathers and mobile ones. These experiments provides a possible way for routing and blocking mobile discrete breathers via the interaction with the high amplitude pinned ones, resulting in a plausible implementation of logical functions. 


\section{Part II}

\section{Structure and Dynamics of Complex Networks}





\section{Presentation of Part II}

T

he second part of the Thesis is devoted to the study of the structure of complex networks. Traditionally, physics has focused on systems where the underlying topology of elements' interactions is described by regular lattices such as those studied in the preceeding part. However, in the recent years, physicists have started to look to those systems where the interactions among constituents reflect the abstract relations between pairs of elements rather than being determined by the proximity in a physical space. These relations can be determined by the existence of monetary transactions between banks in economic networks, or cooperative and friendship relations between individuals in social networks, or assemblies of different molecules working together to develop cellular tasks in biological networks, etc... From the highest to the lowest level of description we find complex networks of interactive elements that cannot be described by regular patterns of connections. The growing interest in the characterization of the above systems has led to the emergence of the so-called network science [137]. Let us review the development of this new interdisciplinary field.

One can settle the first steps of network science with the works on graph theory $[138,139]$ in the middle of last century. The most remarkable result is the theoretical analysis of a random network by the mathematicians Paul Erdös and Alfréd Rényi [140, 141]. However, networks where interactions among elements are completely random are a coarse-grained approach to real networked systems, assuming a homogeneous disorder in what concerns the patterns of connections. The burst in the study of complex networks came with the advent of the XXI century along with the development of the Internet and the World Wide Web. This development has provided a large amount of data-sets for unveiling the relations established among industrial companies, institutions, scientists, etc... Besides, the explosion of human mobility (provided by the increase of accessible infrastructures and transportation companies) and the boom of new telecommunications tools (mobile phones, instant messaging services, etc...) has hugely facilitated the stablishment of new agent networks with a high global character. These two ingredients, the emergence of new networked systems and the high accessibility to data-sets describing them, constituted an unprecedented opportunity for scientist to analyse their topological features.

The analysis of real complex networks revealed that seemingly different systems share a common property when looking to the distribution of the number of connections that the elements of the networks have. It is found 
[142-154] that most of the networks present a power law functional shape for this statistical quantity and, therefore, they differ from that accounted by Paul Erdös and Alfréd Rényi, where all the elements present a similar number of neighbours. Besides the surprising fact that real networks (accounting for many different types of interactions) share the scale-free character, understanding the (common or not) origin of this internal organization has become a challenging question for many researchers.

The above astonishing findings lead physicists to construct simple models of network growth in order to reproduce the "universal" properties found for real networks. In this sense, the models developed by Duncan J. Watts and Steven H. Strogatz [155], and Albert-László Barabási and Réka Albert [145] deserve special mention. Another direction of research has been focused by the search for statistical measures of network topology in order to handle efficiently the large amount of available data-sets and characterize those networks they represent with a few meaningful indicators. The purpose of these two types of studies differ strongly from those of concern of traditional graph theory (where rigorous theorems of difficult real application are proved) and, at the same time, are methodologically far from the meticulous system characterization performed by biologists (who tend to overpay attention to single element details to catalogue systems so that a unitary analysis of different systems becomes difficult). The statistical point of view and the unitary approach to the problem of network characterization performed by physicists have clearly taken advantage over other disciplines under the name of statistical physics of complex networks ${ }^{2}$. In chapter 5 we will briefly review the most important tools for characterizing network structure and present two model of synthetic network generation.

The next step of network science has been to look to network dynamics. Most networks are not composed of mere static objects but, on the contrary, their elements develop a function. This function can be as simple as being routers for the transfer of entities among their elements, or as complicated as being regulatory agents of some internal dynamical processes performed at each network node.

It is important to difference two kind of studies to the problem of network dynamics. On one hand, given that real networks can be described by a set of statistical measures and that one can stablish subsets of networks which are qualitatively similar in terms of these quantities, it is therefore interesting to find how to implement dynamical processes on top of the network in order to take advantage of these topological characteristics. This search for efficient algorithms is not only motivated for practical purposes but it is also interest-

\footnotetext{
${ }^{2}$ Interesting reviews and tutorials on the subject are found in [156-163].
} 
ing for studying the interplay between dynamics and the underlying networked substrate. In this sense, the models developed for constructing synthetic networks are a useful benchmark for studying this interplay before applying the results to real networks. This first kind of studies are therefore interesting for networks whose dynamics can be modeled or modified. This is the case of technological, logistic and certain social networks, e.g. information and transportation networks are susceptible targets of this kind of studies. In chapter 6 we will deal with two related problems, namely the interplay between network structure and, first, the performance of immunization strategies aimed at stopping the spread of epidemics and, second, the routing policies for information dynamics.

As we mentioned above there is a second kind of studies on network dynamics. Instead of varying the dynamical properties assuming a fixed substrate in this second class of studies both structure and dynamics are asummed to co-evolve towards a stable state where system's performance is optimal. We will leave the discussions on this interesting problem for part III and now let us focus on the study of the structure of networks and its influence over the dynamical performance. 



\title{
Chapter 5
}

\section{Network Structure and Generation}

\begin{abstract}
There may well be no useful parallel to be drawn between the way in which complexity appears in the simplest cases of many-body theory and chemistry and the way it appears in the truly complex cultural and biological ones, except perhaps to say that, in general, the relationship between the system and its parts is intelectually a one-way street.
\end{abstract}

- Philip W. Anderson in More is Different [2].

This chapter is devoted to the description of the structure of networks and the modelling of their growth and evolution. We have seen in the preceding pages that a great amount of empirical data about the patterns of connections among the constituents of social, technological, logistic and biological networks is nowadays available. At this point several questions arise such as How similar real networks are? or Is there any common feature (regularity) between networks with the same function? In order to answer (if possible) these kind of questions we have to define some properties that would allow us to give a quantitative and qualitative description of the architecture of networks. After defining these magnitudes we will briefly describe some important models of synthetic networks that try to capture some of the ingredients observed when analysing the "native" ones. We will round off the chapter with a deep analysis of two models of network design that will be employed along the forthcoming parts of this Thesis. 


\subsection{Describing Complex Networks}

Before defining the magnitudes employed for describing the networks we give a brief account of some formal definitions and notations inherited from classical graph theory. Afterwards, we list and explain the most used quantities for characterizing networks' structure at local, global and mesoscopic levels.

\subsubsection{Basic definitions}

We start with the formal definition of a network (or, in mathematicals terms, a graph) $\mathcal{G}(V, E)$ as an ordered pair of set of sets: a non null set $V$ of elements called nodes (or vertices) and another set $E$ of pairs $(i, j)$, with $i, j \in V$, called links (or edges, arcs) that denote that nodes $i$ and $j$ are connected. Normally one imposes that $i \neq j$ so that self-connections are avoided. We will denote by $N$ and $\mathcal{L}$ the cardinalities of the sets $V$ and $E$ respectively. Along with this definition we can also consider that the elements in $E$ are ordered pairs $(i, j) \neq(j, i)$. In this case we will talk of a directed network (or digraph). It is also very common to assign weigths (numbers) to the edges so that we have a weighted (or valued) network. The cardinality of $V$ and $E$ can tell us about the nature of the graph. Taking into account that the maximal cardinality of $E$ is $\left(\begin{array}{c}N \\ 2\end{array}\right)$ we will talk about a sparse network when $\mathcal{L} \ll N^{2}$ and a dense one when $\mathcal{L} \sim N^{2}$.

A subgraph $\mathcal{G}^{\prime}\left(V^{\prime}, E^{\prime}\right)$ of $\mathcal{G}(V, E)$ is a graph such that $V^{\prime} \subseteq V$ and $E^{\prime} \subseteq E$. A subgraph is said to be maximal with respect to a given property if it cannot be extended by adding elements either to $V^{\prime}$ or $E^{\prime}$ without loosing its property. Aditionally, we say that a subgraph $\mathcal{G}^{\prime}$ is induced by $\mathcal{G}$ if $E^{\prime}$ contains all the pairs $(i, j) \in E$ with $i, j \in V^{\prime}$.

The complement of a graph $\mathcal{G}(V, E)$ is the graph $G^{\prime}\left(V^{\prime}, E^{\prime}\right)$ (sometimes denoted $\bar{G}$ ) so that $V=V^{\prime}$ but whose edge set $E^{\prime}$ consists of the edges not present in $E$. Then a graph $\mathcal{G}\left(V, E \cup E^{\prime}\right)$ will be a complete graph, i.e. every node will be connected with the rest of the $N-1$ nodes.

In order to manage with a graph one usually label with natural numbers the elements of $V$ so that $i=1, \ldots, N$. Different assignation of labels to the elements of $V$ yield isomorphic networks and the topological properties are not affected. Formally, two graphs $\mathcal{G}_{1}\left(V_{1}, E_{1}\right)$ and $\mathcal{G}_{2}\left(V_{2}, E_{2}\right)$ are said isomorphic when one can stablish a bijective relation $\phi: V_{1} \rightarrow V_{2}$ that preserve the connections, i.e. if $(i, j) \in E_{1}$ then $(\phi(i), \phi(j)) \in E_{2}$.

One can represent the graph with the so-called adjacency matrix $\mathcal{A}$ whose elements are $a_{i j}=1$ if $(i, j) \in E$ and $a_{i j}=0$ otherwise. This matrix will 
be symmetric for undirected graphs but, in general, this is not the case for digraphs. In the case of weighted networks one can replace the non zero elements of $\mathcal{A}$ by the weights of the corresponding links in order to obtain a complete representation of the graph.

The analysis of the adjacency matrix will give the topological characterization of the networks. At present, there is a large amount of different magnitudes used for characterizing the networks architecture. However, the more specific field we study the more properties we will find for describing these complex topologies. Then, we will only emphasize on those quantities which are of general use and therefore will be used along the works described in this Thesis. We will divide the magnitudes depending on the scale involved for their definition. From our point of view this is a useful definition since local (microscopic) or global (macroscopic) properties play a key role depending on the kind of dynamics placed on top of the network.

\subsubsection{Single nodes properties}

\section{Local Magnitudes}

We will refer to local quantities when one takes into account a node $i$ and its neighbours, $\Gamma_{i}$. Obviously, the first local property is the degree of a node $i, k_{i}$, which is the cardinal of the set $\Gamma_{i}$, i.e. the number of nodes which $i$ is linked to or, in terms of the adjacency matrix

$$
k_{i}=\sum_{j=1}^{N} a_{i j} .
$$

If one is considering a directed network one will talk about the "in-degre" of a node $i, k_{i}^{\text {in }}$, and its "out degree", $k_{i}^{\text {out }}$, which are the number of incoming and outgoing links that a node shares with its neighbours. Again, we can obtain such quantities from the adjacency matrix by

$$
k_{i}^{\text {in }}=\sum_{j=1}^{N} a_{i j} \quad \text { and } \quad k_{i}^{\text {out }}=\sum_{i=1}^{N} a_{i j} .
$$

Another interesting local measure is the so-called clustering coefficient of a node which measures the number of connections among the neighbours of a node, $e_{i}$. This quantity is usually normalized to one by dividing by its maximum value $\left(\begin{array}{c}k_{i} \\ 2\end{array}\right)$ so that it measures the probability that two neighbours $j$ and $m$ of a node $i\left(a_{i j}=a_{i m}=1\right)$ are also linked to each other $\left(a_{j m}=1\right)$. 
A formal expression of the clustering of a node $i$ with $k_{i}$ neighbours is

$$
c_{i}=\frac{2 \sum_{j, m=1}^{N} a_{i j} a_{i m} a_{j m}}{k_{i}\left(k_{i}-1\right)} .
$$

A third local property arise when looking at the degree of the neighbours of a given node (here we assumme that this information is on the local horizon of the nodes) so that we can define the average nearest neighbours degree. We can write this quantity as

$$
k_{n n_{i}}=\frac{\sum_{j=1}^{N} a_{i j}\left(\sum_{m=1}^{N} a_{j m}\right)}{\sum_{j=1}^{N} a_{i j}} .
$$

\section{Global Magnitudes}

Now we will define two properties that are defined taken into account pairs of nodes that are not necessarily linked and are then influenced by the topology of the whole graph. These measures are the closeness centrality and the betweeness centrality. As we will see in section 6.2 these two magnitudes will play a key role when dealing with problems of propagation through networks.

First of all we define the distance between two elements of the network, $d_{i j}$, as the length of the geodesic that goes from node $i$ to $j$. In principle, one can observe more than one geodesic for a pair of nodes. One can then construct the distance matrix $\mathcal{D}$ so that the element $\mathcal{D}_{i j}=d_{i j}$. This matrix is symmetric in the case of undirected graphs but, in principle, this is not the case in directed ones. Once we have constructed $\mathcal{D}$ one can compute the closeness centrality of a node, $D_{i}$, as the inverse of the average distance between it and the rest of the nodes

$$
D_{i}=\frac{N-1}{\sum_{j \in V i} d_{i, j}} .
$$

The purpose of measuring this property is to know whether a node holds a central or a peripheral position in the network.

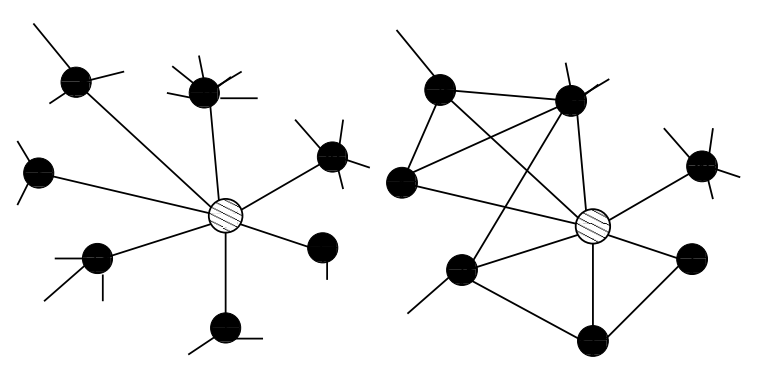

Figure 5.1: Two different situation in terms of the clustering of the striped node. In the first case (left) clustering is 0 while for the second example (right) the probability of finding two connected neighbours raises to $2 / 7$. 
Closely related with the above magnitude but rather more sophisticated is the betweeness centrality of a node, $b_{i}$. The betweeness tell us how many geodesics between any pair of nodes $(j, m)$ go through node $i$. Then, measuring the betweenes of a node implies not only knowing $\mathcal{D}$ but the different sequences of nodes (of minimal cardinality) we have to cover for going from one node to another. If we express by $\sigma_{j, m}$ the number of geodesics for going from $j$ to $m$ we can construct a $\sigma_{j, m} \times N$ matrix $G(j, m)$ whose elements are either 1 or 0 so that row $k$ tell us the sequence of nodes that are contained in geodesic $k$ by $G(j, m)_{k, l}=1$ if node $l$ is part of the geodesic sequence and 0 if not. Then we can express the betweenes of node $i$ by

$$
b_{i}=\sum_{\{j, m \in V \mid j \neq m\}} \frac{\sum_{k=1}^{\sigma_{j, m}} G(j, m)_{k, i}}{\sigma_{j, m}} .
$$

It is easy to realize that this magnitude will be important whenever there is a flow of information across the network since it allows us to unveil which nodes will support the highest traffic levels.

\subsubsection{Network properties}

Up to now, we have defined quantities that only made reference to the properties of single nodes. However we need a statistical characterization of these magnitudes in order to have a proper description of the networks. These statistical indicators will involve averages of the single-node quantities over the whole set $V$ of nodes. This implies that the validity of the information provided by the statistics will be only justified when the cardinality of $V$, is very large $N \rightarrow \infty$. This assumption is not always fulfilled when looking at real networks, e.g. the largest studied network (the WWW) has $10^{9}$ nodes which is orders of magnitude smaller than the number of particles of the systems studied by statistical and condensed matter physics. However, the use of a statistical description has turned out to be very convenient when studying networks and the existence of finite size effects do not prevent physicist from considering networks as macroscopic objects (although in terms of statistical mechanics they are mesoscopic ones).

\section{Degree Distribution}

The degree distribution, $P(k)$, gives the probability that a given node is connected to $k$ neighbours. The construction of $P(k)$ is obviously easy when one knows the connectivity $k_{i}$ of every node $i$ and then one can compute the number of nodes with a given connectivity $k, N_{k}=N P(k)$. With the degree 

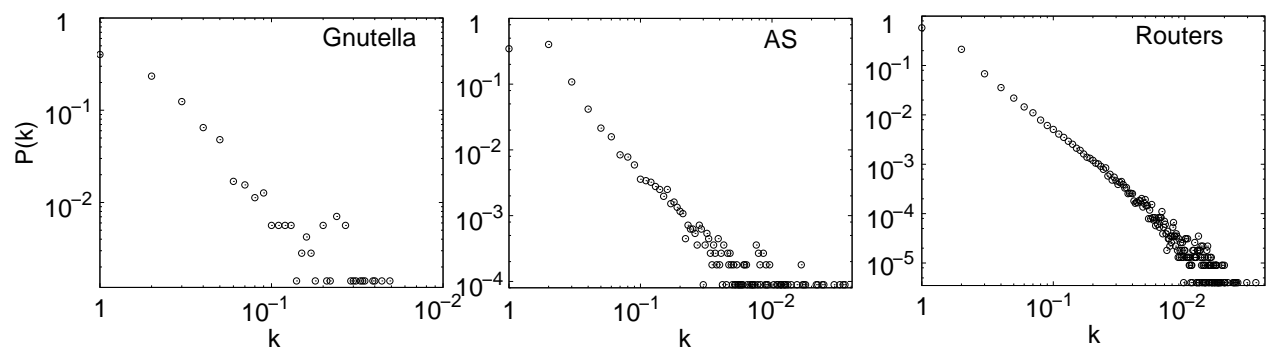

Figure 5.2: Degree distribution of the three real networks: Gnutella peer-to-peer network [164], the Internet network at the Autonomous Systems representation [165] and at the Router level [166]. The network sizes are $N=711, N=11174$ and $N=228263$ respectively.

distribution one can measure the average degree of the nodes

$$
\langle k\rangle=\sum_{k} k P(k) .
$$

Analogously, we can define the in-degree and out-degree distribution of a directed graph, $P^{\text {in }}\left(k^{\text {in }}\right)$ and $P^{\text {out }}\left(k^{\text {out }}\right)$. In this case we will obtain that $\left\langle k^{\text {in }}\right\rangle=\left\langle k^{\text {out }}\right\rangle=\langle k\rangle / 2$. In order to obtain information of the fluctuations on the degree distribution one can compute the second moments of $P(k)$,

$$
\left\langle k^{2}\right\rangle=\sum_{k} k^{2} P(k)
$$

The behaviour of $\left\langle k^{2}\right\rangle$ turns out to be very important for studying dynamical process as we will see in chapter 6 .

As mentioned above, the measurement of the degree distribution in real networks lead to an astonishing result. It was found that lots of networks share a common pattern for the shape of $P(k)$ : a power law behaviour $P(k) \sim k^{-\gamma}$. This result is far from the expected result when considering a fully random graph (one would expect to obtain a Poisson distribution as we will see below) and, as a plus, the form of a power law pointed out that there was a kind of sef-organization in real networks. Power laws appear in the context of phase transitions when a system goes from a disordered to an ordered phase. It is well known that at the transition the system posseses a self-similar character, that is, no matter the re-scaling we employ to analyse the system we will always observe that the system looks the same. This effect is reflected by a power law since it is the unique function that after changing the scale remains with the same functional form: if $k \rightarrow a k$ then $P(k) \rightarrow a^{-\gamma} P(k)$. Because of this feature this class of networks are ususally termed "Scale-free" networks in the 
literature. In figure 5.2 we show different degree distributions observed when analysing real networks. We will analyse the emergence of such behaviour for the degree distribution in section 5.2.3. Let us remark here that the term scale-free could be in principle misleading because the absence of a typical scale in the degree distribution does not imply that we cannot observe scales when looking at other topological magnitudes.

\section{Degre-Degree Correlations}

The knowledge of the degree distribution fully characterize the network topology when the statistical independence of the nodes is fulfilled. However this is not the case for many real networks. Then, it is convenient to compute the conditional probability $P\left(k^{\prime} \mid k\right)$, i.e the probability that a node of degree $k$ is linked to another one with degree $k^{\prime}$. This probability satisfy

$$
\sum_{k^{\prime}} P\left(k^{\prime} \mid k\right)=1
$$

and

$$
k P\left(k^{\prime} \mid k\right) P(k)=k^{\prime} P\left(k \mid k^{\prime}\right) P\left(k^{\prime}\right),
$$

which are the normalization and the detailed balance ${ }^{1}$ respectively. As we stated above, for uncorrelated graphs $P\left(k^{\prime} \mid k\right)$ is given by the degree distribution. From (5.9) and (5.10) we obtain in this case $P\left(k^{\prime} \mid k\right)=k^{\prime} P\left(k^{\prime}\right) /\langle k\rangle$.

In order to measure the behaviour of $P\left(k^{\prime} \mid k\right)$, it is convenient to compute the average degree of the neighbours of a node with connectivity $k, k_{n n}(k)$. In general, the computation of the matrix, $P_{k^{\prime}, k}=P\left(k^{\prime} \mid k\right)$, yields very noisy results since the finite size effects do not allow a proper statistical treatment. Then, we can compute the function $k_{n n}(k)$,

$$
k_{n n}(k)=\sum_{k^{\prime}} k^{\prime} P\left(k^{\prime} \mid k\right),
$$

by simply computing the average neigbours degree, $k_{n n_{i}}$ introduced in section 5.1 .2 and averaging in every subset of nodes with identical degree

$$
k_{n n}(k)=\frac{\sum_{\left\{i \in V \mid k_{i}=k\right\}} k_{n n_{i}}}{k P(k)} .
$$

When degree correlations are absent eq. (5.12) yields $k_{n n}(k)=\left\langle k^{2}\right\rangle /\langle k\rangle$ and thus $k_{n n}(k)$ does not depend on $k$. As noted above this is not the case

\footnotetext{
${ }^{1}$ This equation is constructed by introducing the probability of finding a link connecting two nodes with degree $k$ and $k^{\prime}, P\left(k, k^{\prime}\right)=2 k P(k) P\left(k^{\prime} \mid k\right) /\langle k\rangle$, and imposing its simmetry property $P\left(k, k^{\prime}\right)=P\left(k^{\prime}, k\right)$.
} 


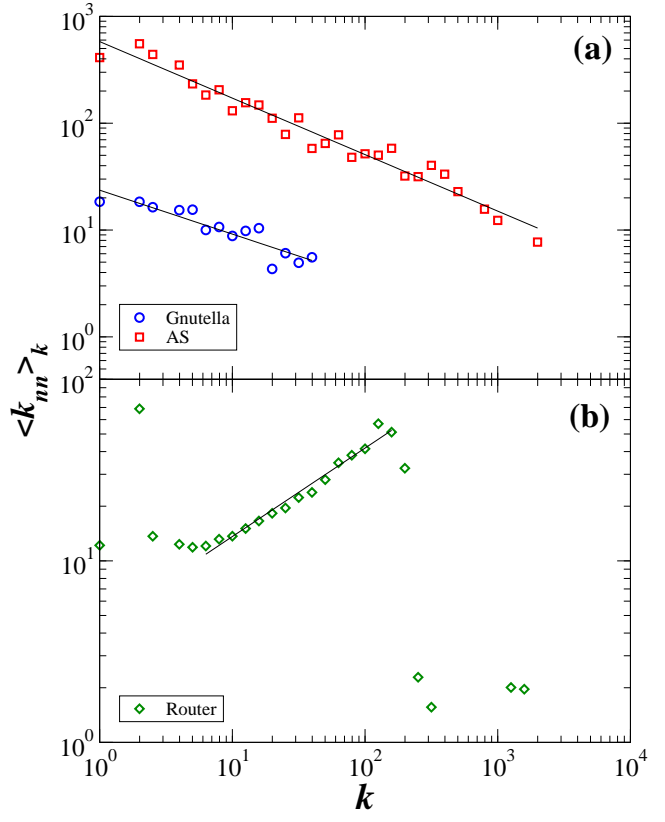

Figure 5.3: Degree-degree correlations of some of the real networks used in this thesis. (a) Average connectivity of a vertex with degree $k$, $k_{n n}(k)$, for the Gnutella peer-to-peer network [164] and the Internet (at the Autonomous System level, AS [165]) graphs. These networks display disassortative degree correlations between connected vertices, where two neighboring vertices likely have dissimilar degrees. (b) $k_{n n}(k)$ for the Internet (at the Router level [166]) graph. This network displays assortative degree correlations between connected vertices, where connected vertices tend to have similar degrees. The continuous lines are the best fits to the power law $k_{n n}(k)=A k^{\nu}$.

of real growing networks and one can usually find that $k_{n n}(k) \sim k^{\nu}$. When $k_{n n}(k)$ is a increasing (decreasing) function of $k, \nu>0(\nu<0)$, we say that the network is assortative (dissasortative). The assortativity denote the tendency of nodes of similar degree to connect with each other while in dissasortative networks higly connected nodes tend to be surrounded by low degree ones. There is a clear difference in the behaviour of $k_{n n}(k)$ depending on the type of the network. In particular, it has been observed that social network tend to be assortative while the rest ones (like technological and biological networks) show dissasortative trends. In figure 5.3 we show the analysis for $k_{n n}(k)$ on two real networks.

\section{Average Shortest Path Length}

We can condense the information of the distance matrix $\mathcal{D}$ by computing the mean of all the geodesic lengths between the nodes of the network. This gives the average shortest path length, $L$

$$
L=\frac{1}{N(N-1)} \sum_{\{i, j \in V \mid i \neq j\}} d_{i j} .
$$

Along with $L$ we can define the diameter of a network as the maximum value of the distances between nodes, $D=\max \left\{d_{i j} \mid i, j \in V\right\}$. The analysis of this quantity tells us whether the graph is composed by one or several components. 


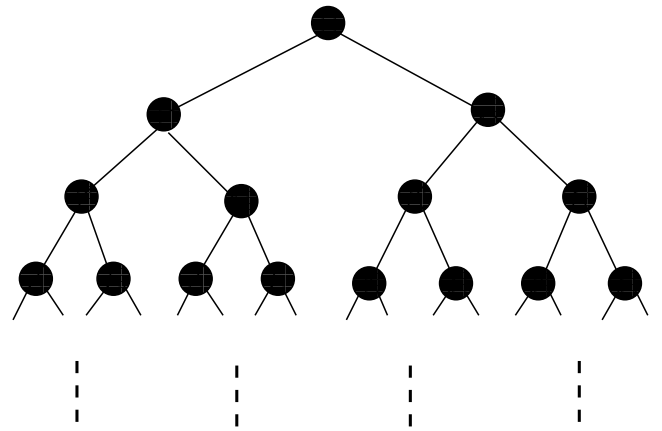

Figure 5.4: An example of a treelike graph with coordination number 2 .

We term a component of a graph as a subset of nodes $V^{\prime} \subseteq V$ so that the distance between any node $i \in V^{\prime}$ and the rest of nodes $j \in V \backslash V^{\prime}$ diverges while the distance with the nodes $j \in V^{\prime}$ remains finite. Obviously both $L$ and $D$ will diverge if the graph is partitioned in two or more components. In real graphs this situation is commonly found and one usually restrict the analysis of the network to the component with maximal cardinality which is the so-called "giant component".

Formaly, a network whose $L$ grows logaritmically with the number of nodes, $L \sim \log N$, or even slower, is called a "small-world". This term comes from the famous experiment by Milgram in the 60's [167]. This experiment consisted in asking a group of people to deliver some documents to a person characterized by his name, place of residence and his proffesion. Then people were asked to send the documents to those of their acquaintances that they think of being closer (either physically or socially according to the initial parameters given) to the final recipient. The results pointed out that every pair of people in the world are in average connected by only 6 steps in the global network of social acquaintances. This results is broadly known as the "six degrees of separation". Other experiments performed using the e-mail networks [168] confirmed the small-world character of the global society.

From the mathematical point of view the small-world effect is nothing but the outcome of an exponential increase of the nodes with the distance from a central element. A simple proof of the small-world phenomena can be performed using one of the simplest networked structure: a tree-like graph. A tree-like graph (see figure 5.4) is composed of a hierarchical structure where every node is connected to $d$ descendants and its ancestor ( $d$ is usually termed coordination number). In this case the number of nodes which are in the $i$ shell (or generation) from a given node is $N_{i}=d^{i}$. Then, the whole set of nodes are $N=d^{L}$ so that the average path length of the network is given by $L=\log N / \log d$ so that $L$ remains small even for large tree-like graphs. 


\section{Clustering}

In section 5.1.2 we introduced the concept of the clustering coefficient of a node, $c_{i}$. One can measure the average clustering coefficient of the nodes of a network, $c$, and consider it as a measure of the density of small loops of length 3 in the network. Then, $c$ will tell us how similar is our network to a tree-like networked structure (see figure 5.4) (if $c \rightarrow 1$ one can say that the network is far from being a tree graph). This measure was introduced by Watts and Strogatz [155] but there are, however, another possible formulation for measuring the density of short circuits in a network that is called transitiviy, $T$. Trasitivity is defined by

$$
T=\frac{3 \times \operatorname{card}[\{(i, j, m) \mid(i, j),(i, m),(j, m) \in E\}]}{\operatorname{card}[\{(i, j, m) \mid(i, j),(i, m) \in E\}]} .
$$

While clustering was introduced recently in the physical literature the concept of transitivity was largely employed mainly by sociologist. These two magnitudes turn out to be meaningfull when looking at networks of different fields. It is well known that social networks of acquaintances present a high clustering denoting the fact that two friends of a person are very likely to know each other. That is the case of scientific coautorship networks [148, 169]. On the other hand, technological networks like peer-to-peer networks [170] or power grids [155] are very poor in length 3 loops since they are redundant in what refers to resources supply.

The existence of a high clustering together with the small-world charater lead to very efficient communication structures. This is because the increase of networks' clustering leads to a growth of the number of geodesics between the pairs of nodes.

\subsubsection{Looking at Networks Mesoscale}

The statistical description of the networks considered above can be extended by looking at the kind of internal organization of subsets of nodes (if there is any). This implies that neither single nodes nor the whole ensemble of vertices are studied, but groups of them whose cardinals may vary a lot depending on the kind of network we deal with. The scale involved in such studies neither can be called "micro" nor "macro" and thus we term it the network mesoscale. Two concepts are central in this picture of the networks: communities and motifs. 


\section{Communities}

The concept of community ${ }^{2}$ has to do with the existence a cohesive subset of nodes. This cohesion is achieved by the existence of large number of links among the members of this subset compared to the typical number of links that they share with the rest of the network. More formally we can say that, given a graph $\mathcal{G}(U, V)$ a community is a subgraph $\mathcal{G}^{\prime}\left(U^{\prime}, V^{\prime}\right)$ so that $L^{\prime} \lesssim$ card $\left\{i \in U^{\prime} \mid(i, j) \in V, j \in U\right\}$. Obviously, we need a quantitative description on how cohesive a subset of nodes is in order to decide whether or not they form a community.

Several ways for quantifying community structures have been proposed. However we can distinguish to kinds of definitions depending of the conditions imposed. The first type imposes certain constrains to the number of links within the members of the community. In this sense, the strongest definition states that a community is a subgraph $\mathcal{G}^{\prime}\left(U^{\prime}, V^{\prime}\right)$ so that if $i, j \in U^{\prime}$ then $\left.(i, j) \in V^{\prime}\right)$, i.e. all pairs of community members are linked to each other. This definition is too restrictive and then far from being useful. In fact, the maximal subgraph $\mathcal{G}^{\prime}\left(U^{\prime}, V^{\prime}\right)$ that fulfills the above requirement is termed a clique. One can relax the conditions and considering that communities as the $n$-clique of the graph. N-cliques are maximal subgraphs in which the largest geodesic distance between any two nodes is no greater than $n$. Other way for relaxing the former condition is to reduce the number of other nodes in the subgraph to which each node must be connected, the concept of $k$-plex is then applied. A $k$-plex is a maximal subgraph containing $n$ nodes, in which each node is adjacent to no fewer than $n-k$ nodes in the subgraph.

The above definitions have to do only with the links within the elements of the subgraphs and there is no mention to the ratio between these inner-links and those going to the rest of the network. From our point of view this kind of arguments have to be incorporated to our criterion in order to get closer to the cohesive picture. Then, we have to look to communities as groups of nodes within which connections are dense, and between which connections are sparser or, more formally, we can say that, given a graph $\mathcal{G}(U, V)$ a community is a subgraph $\mathcal{G}^{\prime}\left(U^{\prime}, V^{\prime}\right)$ so that $L^{\prime} \lesssim$ card $\left\{i \in U^{\prime} \mid(i, j) \in V, j \in U\right\}$. An example of this consideration is plotted in figure 5.5.a. There are several definitions with this philosophy (see [171]). One definition is the following: $\mathcal{G}^{\prime}\left(U^{\prime}, V^{\prime}\right)$ is a community if the sum of all degrees within $G^{\prime}$ is larger than the sum of all degrees toward the rest of the graph [172].

\footnotetext{
${ }^{2}$ The first network description in terms of communities appear in the context of social science [171]
} 


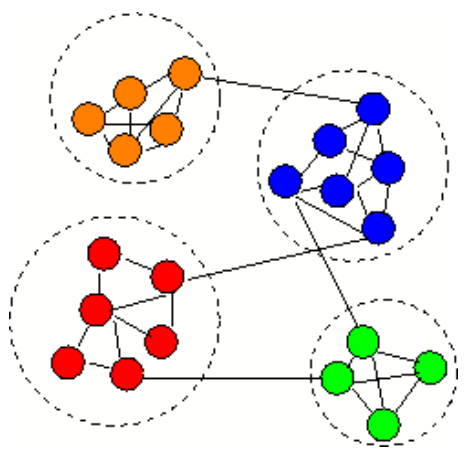

(a)

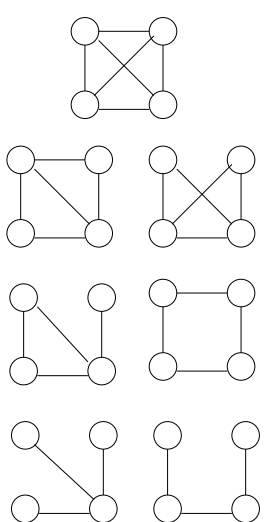

(b)

Figure 5.5: (a) Schematic picture of a set of 4 communities (surrounded by dashed circles). The density of inner-links between nodes of the same community is much larger than that of the links with members of the rest of the network. (b) The 7 possible 4-nodes motifs.

\section{Motifs}

We define a motif $M$ as a pattern of interconnections so that its occurrence in a graph is significantly higher than in randomized versions of the graph, i.e. graphs with the same number of nodes, links and degree distribution as the original one, but where the links are distributed at random. More formally, $M$ is usually considered as a $n$-node connected graph which is a subgraph of $\mathcal{G}$. An example of all the possible 4-node connected graphs is illustrated in figure 5.5.b. The concept of motifs (originally introduced by Uri Alon and coworkers [173-177]) was employed for studying the finding of recurrent patterns of interconnections between a small number of nodes in biological and other networks.

In order to obtain a quantitative description for the appearance of the significant motifs in a graph $\mathcal{G}$, one makes use of matching algorithms for counting the total number of occurrences of each $n$-node subgraph $M$ in the original graph and in the randomized ones. Then, one can define the statistical significance of a given motif $M$ by some score function, like the so-called $Z$ score $[174,176]$

$$
Z_{M}=\frac{n_{M}-\left\langle n_{M}^{\text {rand }}\right\rangle}{\sigma_{n_{M}}^{\text {rand }}}
$$

where $n_{M}$ and $n_{M}^{\text {rand }}$ are the number of times the subgraph $M$ appears in $\mathcal{G}$ and in its randomized counterpart repectively. $\sigma_{n_{M}}^{\text {rand }}$ is the standard deviation of the number of appearances in the randomized network ensemble. 


\subsection{Overview of network generation models}

In this section we briefly account for several important network models. Nowadays there is a huge number of ways for generating complex networks that try to capture the properties of real graphs. Many of them are variations of the models we present below since they represent seminal works on the matter. For a complete review on currents trends in network modeling we refer the reader to $[156,158,159,163]$.

\subsubsection{Random graphs}

We call random graphs to those network where the links between nodes are randomly distributed ${ }^{3}$. In their seminal work in the subject, Erdös and Rényi [140] (ER for short) proposed a method for the construction of random graphs with $N$ nodes and $\mathcal{L}$ links: Starting from $N$ isolated nodes, pairs of randomly chosen nodes are linked avoiding self and multiple connections. This process is stoped when $\mathcal{L}$ links have been stablished. A single graph obtained using the above recipe is one of the $\left.\left(\begin{array}{c}N \\ 2 \\ \mathcal{L}\end{array}\right)\right)$ possible equiprobable realizations. The set of all these possible realizations is called the set of uniform random graphs with $N$ nodes and $\mathcal{L}$ links, $\mathcal{G}_{N, \mathcal{L}}^{E R}$. In a random graph the probability that two given nodes are linked is $\mathcal{L} /\left(\begin{array}{c}N \\ 2\end{array}\right)$.

Another possible strategy for constructing random graphs is to sample every pair of nodes and with probability $0<p<1$ link them. This procedure defines a different set called random binomial graphs, $\mathcal{G}_{N, p}^{E R}$, that contains graphs with different number of total links $\mathcal{L}$ being

$$
p^{\mathcal{L}}(1-p)\left(\begin{array}{c}
N \\
2
\end{array}\right)-\mathcal{L}
$$

the probability that a graph belonging to $\mathcal{G}_{N, p}^{E R}$ has $\mathcal{L}$ links. Then the average number of links of a graphs in this set is $p\left(\begin{array}{c}N \\ 2\end{array}\right)$.

The two sets (uniform and binomial) of random graphs are tightly related to the canonical and grand canonical ensembles of the equilibrium statistical mechanics when one looks at the number of edges as the number of particles in the system. Both ensembles converge to the same set in the thermodynamic limit $N \rightarrow \infty$ when approached keeping $\langle k\rangle$ fixed (wich is equivalent to fix $2 \mathcal{L} / N$ and $p(N-1)$ in the uniform and the binomial sets repectively).

\footnotetext{
${ }^{3}$ In fact, all the network models analysed in this chapter are strictly random in the sense of the mechanism adopted for their construction. However, the term random graph is overused in the literature for calling Erdös-Rényi networks.
} 
The structural properties of the ER graphs vary as a function of $p$, showing a dramatic change at the critical probability $p_{c}=1 / N$ that corresponds to $\langle k\rangle=1$. In particular:

- If $p<p_{c}$, the size of the giant component of the graph is of the order $O(\ln N)$ graph and there is no graph component with more than one loop.

- If $p=p_{c}$, the size of the giant component goes with $O\left(N^{2 / 3}\right)$.

- If $p>p_{c}$, the graph has a giant component with a number of loops that scales as $O(N)$ and there is no other graph component with more than $O(\ln N)$ elements neither with more than one loop.

This transition (characterized by Erdös and Rényi in [141]) is strongly related with the percolation transition studied in the theory of critical phenomena [178].

In ER graphs the probability that a node has $k$ neighbours follows the binomial distribution

$$
P(k)=\left(\begin{array}{c}
N-1 \\
k
\end{array}\right) p^{k}(1-p)^{N-1-k},
$$

that for $\langle k\rangle$ fixed and $N \rightarrow \infty$ tends to the Poisson distribution

$$
P(k)=\frac{\langle k\rangle^{k}}{k !} \exp (-\langle k\rangle) .
$$

Erdös and Rényi graphs are uncorrelated since the links are launched at random independently of the degree of the nodes. As a consequence, $P\left(k^{\prime} \mid k\right)$ and $k_{n n}(k)$ are independent of $k$. Concerning to the connectivity properties of an ER graph, when $p>\ln N / N$ nearly all the generated graphs are composed of one single component and the average path length takes values around $\langle k\rangle=$ $\ln N / \ln (p N)=\ln N /\langle k\rangle$ because locally the ER topology is viewed as a tree like structure where a single node has $\langle k\rangle$ neighbours, $\langle k\rangle^{2}$ nodes at distance $2, \ldots$ Finally, since $p$ is the probability of two nodes sharing a link there will be $p k(k-1) / 2$ links among the neighbours of a node of degree $k$ so that the clustering coefficient goes as $c=p=\langle k\rangle / N$, and then it vanishes in the thermodynamic limit.

\subsubsection{Small-world networks}

In 1998 Watts and Strogats (WS) proposed a method of graph constrution that allows to obtain networks with a high clustering coefficient and small average 


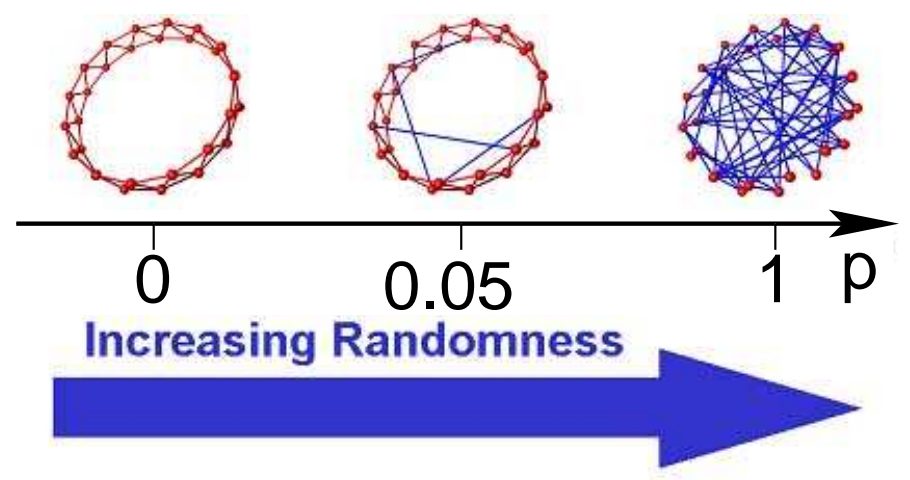

Figure 5.6: Three kind of networks obtained used the Watts-Strogatz method starting from a regular one-diemnsional network where every node is linked to its 4 nearest neighbours. For $p=0$ we obtain the regular network. For small values of $p \ll 1$ we have "Small World" networks from the small amount of reassigned links. Finally, for $p=1$ random networks are obtained.

path leghth [155]. We have seen that ER graphs present a small value of $L$ and that $c$ vanishes when $N \rightarrow \infty$. On the other hand, a regular network with connections to first, second, third,... next nearest neighbours presents a high value of the clustering coefficient joined with large values of $L$. In some sense, the WS model interpolates smoothly between these two topologies.

The WS procedure starts from a ring (see figure 5.6) where every node is symmetrically linked to its $2 m$ next nearest nodes so that there is $L=m N$ links. Then, every link is considered and with probability $p$ it is substituted by another link that connects one of the original nodes with a new one chosen at random. Note that for $p=0$ we maintain the original regular topology whereas for $p=1$ an ER random graph is generated. In figure 5.7 it is shown how for a range of $p$ values the WS model generates networks with both the small world property (due to shortcuts added when $p \neq 0$ ) and high clustering coefficient (inherited from the regular topology), two characteristics shared by a number of real networks. This result reveals that the clustering coefficient is very robust under link reasignation whereas $L$ rapidly decreases when a few shortcuts are incorporated.

Analytical calculations on the transition observed in the WS model are found in [179-182]. It has been shown that the appearance of the small world character as $p$ increases is not a phase transition but a crossover phenomenon. The characteristic length satisfy the scaling relation $L(N, p)=N f(N p)$ where

$$
f(x) \sim \begin{cases}c & \text { if } x \ll 1 \\ \frac{\ln x}{x} & \text { if } x \gg 1\end{cases}
$$


Besides, in [181] the authors found the analytical expressions for the clustering and the degree distribution as a function of the control parameter $p$

$$
\begin{aligned}
c(p) & =\frac{3(m-1)}{2(2 m-1)}(1-p)^{3} \\
P(k ; p) & =\sum_{i=0}^{\min (k-m, m)}\left(\begin{array}{c}
m \\
i
\end{array}\right)(1-p)^{i} p^{m-i} \frac{(p m)^{k-m-i}}{(k-m-i) !} \exp (-p m)(5.21)
\end{aligned}
$$

the last equation (5.21) is valid provided $k \geq m$ otherwise $P(k<m ; p)=0$. The WS model was later modified by Newman and Watts in order to solve the possible formation of disconnected graphs of the network as shortcuts were incorporated. Then, they proposed to add new links between randomly chosen nodes instead of making the rewiring process [183]. They considered every node and with probaibility $p$ a link was stablished with any other node of the network so that the average number of shortcuts added is $p N$.

\subsubsection{Scale-Free networks}

There are a large number of models that reproduce the power law functional form for the degree distribution. However, we will focus here on those models that incorporates the growing character present in real networks, where the amount of nodes grows with time, to the formulation of the model. These models usually consist of an initial small subset of nodes to which new nodes are sequentially incorporated by launching new links over those nodes that already take part of the network (see figure 5.8). In particular, the work by Barabási and Albert (BA) in 1999 [145] suposed an important breakthrough to the problem of finding the roots of the SF behaviour of real networks and had the growing process as a key ingredient of their formulation.

The BA models works starting from an initial core of $m_{0}$ isolated nodes.

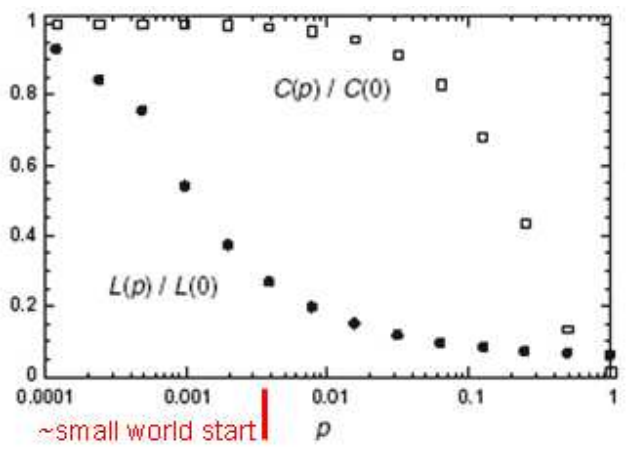

Figure 5.7: Evolucion of the clustering coefficient and the average shortest path length as a function of $p$. Note that near $p=4 \cdot 10^{-3}$ the clustering remains comparable to the values of the regular network whereas $L$ has decreased significatively. 
At each time step a new node is incorporated to the network by launching $m \leq m_{0}$ links over the already existing ones so that the network core grows linearly in time. The probability that one of the core nodes, $i$, receives a link from the new node is proportional to its degree, $k_{i}$,

$$
\Pi_{i}^{\mathrm{pa}}=\frac{k_{i}}{\sum_{j=1}^{N(t)} k_{j}},
$$

where $N(t)$ is the number of nodes that form the network core at time $t$, $N(t)=m_{0}+t-1$. Besides, the total number of links at time $t$ evolves as $L(t)=m t$. The above rule for node selection was termed preferential attachment and favours that a node with more links than others will increase its connectivity at a higher rate (this is usually referred to as richer gets richer). Obviously, the soonest a node is incorporated to the network core the most connected it will be at larger times.

The solution of the BA model was found by the same authors by means of a mean field approximation ${ }^{4}[145,187]$. In this formulation the connectivity of a node $i, k_{i}$, is considered as a real continuous and time-derivable variable. Considering that new nodes are uniformly incorporated in time and that they attach $m$ new links, we can write the evolution equation of $k_{i}$ as

$$
\frac{\partial k_{i}}{\partial t}=m \Pi_{i}^{\mathrm{pa}}\left(k_{i}\right)=m \frac{k_{i}}{\sum_{j=1}^{N(t)} k_{j}}=\frac{k_{i}}{2 t},
$$

with the initial condition $k_{i}\left(t_{i}\right)=m$, and $t_{i}$ being the time when node $i$ was added to the network core. The solution to eq. (5.23) is

$$
k_{i}(t)=m\left(\frac{t}{t_{i}}\right)^{1 / 2}
$$

\footnotetext{
${ }^{4}$ Other solutions to this important model have been found solving the rate equation for the connectivity distribution [184-186].
}

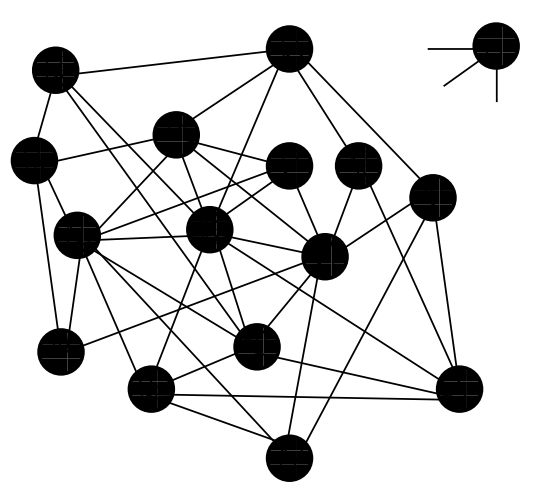

Figure 5.8: Schematic representation of the grwoth process. At each time steps a new node is incorporated to the network core linking to $m=3$ nodes that already belong to the core. 


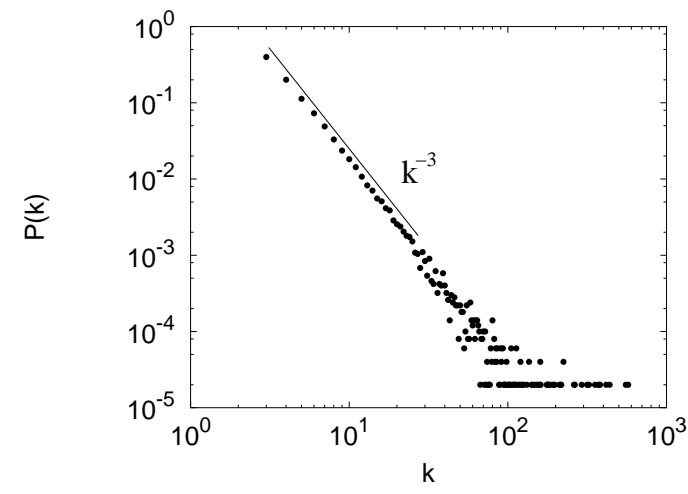

Figure 5.9: Degree distribution, $P(k)$, for a BA network with $N=$ $5 \cdot 10^{4}$. The linear fit of the data in the log-log plot yield an exponent for the power law of $\gamma=3$.

In order to obtain the degree distribution we first take the cumulative distribution, $P\left(k_{i}<k\right)$. From (5.24) one obtains

$$
P\left(k_{i}<k\right)=P\left(t_{i} \geq \frac{m^{2}}{k^{2}} t\right) .
$$

Finally, since the adition of new nodes is performed uniformly, the probability of finding at time $t$ a node that was incorporated to the network at time $t_{i}$ is $P\left(t_{i} ; t\right)=1 /\left(m_{0}+t\right)$. Then, the above probability (5.25) can be written as

$$
P\left(k_{i}<k\right)=1-\frac{m^{2} t}{k^{2}\left(m_{0}+t\right)},
$$

so that the degree distribution yiels

$$
P(k)=\frac{\partial P\left(k_{i}<k\right)}{\partial k}=\frac{2 m^{2} t}{m_{0}+t} k^{-3} .
$$

Taking the limit when $t \rightarrow \infty$ we obtain the power law $P(k)=2 m^{2} k^{-3}$ with the exponent $\gamma=3$. In figure 5.9 we show the numerical results for the degree distribution when the BA is implemented.

Analytical calculations accounting for other magnitudes have been performed. For example in [188] the authors showed that the shortest path length is smaller than that observed for ER graphs. In particular $L \sim \log N / \log (\log N)$. Besides, the clustering coefficient in BA networks vanishes in the thermodynamic limit as happened for ER graphs. However, although the clustering decay is seen to be slower, $c \sim N^{-3 / 4}$, than in the case of ER networks, it represents a major weakness of the BA model.

Variations of the preferential attachment rule (5.22) has been broadly studied after the BA model appeared. These variations try to obtain more flexible models in order to grow networks with other characteristics similar to those 
found in real network for which the BA fails to reproduce (existence of high clustering, the presence of degree corelations, the variety of exponents found for the ower law distribution, etc ...) while keeping the SF character induced by the preferential attachment. Some examples of these variations can be found in [186, 189-199].

\subsection{Global versus local knowledge}

In this section, we revisit one of the main assumptions of the Barabási-Albert model: the preferential attachment rule. We study a model in which the PA rule is applied to a neighborhood of newly created nodes and thus no global knowledge of the network is assumed. We numerically show that global properties of the BA model such as the connectivity distribution and the average shortest path length are quite robust when there is some degree of local knowledge. In contrast, other properties such as the clustering coefficient and degree-degree correlations differ and approach the values measured for realworld networks.

As explained in Sec. 5.2.3 the first scale-free network model, introduced by Barabási and Albert, postulated that there are two fundamental ingredients of many real networks $[145,187]$ : their growing character and the preferential attachment (PA) rule. The preferential attachment rule considers that the probability that an old node links to newly added nodes is proportional to its degree $k$ (see eq. 5.22). However, the BA model assumes that one knows the connectivity of all nodes when a new node links to the network. This is clearly an unrealistic assumption. This drawback of the model construction has not passed unnoticed and many models have been introduced to produce scale-free networks and to test whether or not the basic assumptions of the BA recipe are necessary conditions to build up these networks $[156,158]$. There are some models in which the PA rule is limited to a neighborhood due to geographic constraints [200], or where its linear character is investigated [201].

In the model decribed here, we adopt a different perspective. Our aim is to test to what extend the global character of the PA rule in the original BA model is important. We introduce a model in which the PA is applied only to a neighborhood of the newly added node depending on the value of a variable which measures the affinity between different nodes. By going down from the BA limit of the model to the the limit where all nodes are distinct, we test to what extend the global knowledge of each node's connectivity is fundamental to get a scale-free graph. Through numerical simulations we find that in a wide range of the model parameters, average quantities such as the connectivity distribution and the shortest path length are not affected by the use of local 
knowledge of the network whereas other properties like the clustering coefficient are more sensitive to local details.

\subsubsection{The model}

The model is defined in two layers. The first discriminates among all the nodes by assigning to each node at the moment of its creation a parameter $a_{i}$ which measures how close or distinct a given node is from the rest of the elements that compose the network. Then, we apply the preferential attachment rule in the neighborhood defined by nodes with common affinities. Specifically, the network is constructed by repeated iteration of the following rules:

(i) Start from a small core of nodes, $m_{o}$, linked together. Assign to each of these $m_{o}$ nodes a random affinity $a_{i}$ taken from a probability distribution, $P(a)$. In what follows, we will use for simplicity a form for $P(a)$ uniformly distributed between $(0,1)$.

(ii) At each time step, a new node $j$ with a random affinity $a_{j}$ is introduced and linked to $m$ nodes already present in the network according to the rules specified below.

(iii) Search through all nodes of the network verifying whether or not the condition $a_{i}-\mu \leq a_{j} \leq a_{i}+\mu$ is fulfilled, where $\mu$ is a parameter that controls the affinity tolerance of the nodes. The nodes that satisfy the affinity condition are grouped in a set $A$ as potential candidates to gain new links.

(iv) Apply the preferential attachment rule to the set $A^{5}$, i.e., when choosing the nodes to which the new vertex links, we impose that the probability that vertex $i$ connects to the new node depends on its connectivity such that

$$
\Pi\left(k_{i}\right)=\frac{k_{i}}{\sum_{s \in A} k_{s}} .
$$

(v) Finally, repeat steps (ii)-(iv) $t$ times such that the final size of the network is $N=m_{o}+t$.

It is worth mentioning that the inclusion of the affinity parameter $a$ is not a mere artifact. Indeed, most real systems are formed by non-identical elements and thus it is natural to assume that although a given node could have a

\footnotetext{
${ }^{5}$ In case that the number of elements in the set $A$ is smaller than $m$ we just add a link to all nodes in $A$ without applying the PA rule.
} 
large connectivity a newly created element will not link to that node because they have very little in common. This feature is clearly manifested in social networks like the WWW - where individuals bookmark different web pages accordingly to their "affinity" - or the scientist citation network [142]. In this way, it is very unlikely to find a citation in a condensed matter paper referring to a paper wrote by a psychologist. Additionally, the same argument can be translated to biological networks such as predator-prey webs or protein-protein interaction networks.

Obviously, when $\mu$ is large enough as to dilute the first layer of the model, we recover the BA model. The problem then consists of determining to what extend the local preferential attachment will give the same results, or in other words, does the knowledge of the entire network substantially contribute to the properties observed in the BA networks?

\subsubsection{Network properties}

We have performed extensive numerical simulations of the model described in the preceding section. In all cases, the numerical results have been obtained after averaging over at least 500 iterations varying the system size from $10^{3} \mathrm{up}$ to $1.2 \times 10^{4}$ nodes. We first generate the BA network by setting the parameter $\mu$ to its maximum value such that the preferential attachment applies to the entire set of nodes and then tune $\mu$ in order to systematically reduce its value and therefore the size of the set $A$ to which the second choice eq. (5.28) is applied.

Figure 5.10 shows the number of nodes with connectivity $k$ for several values of $\mu$. It turns out that irrespective of the range to which the preferential attachment is applied the stationary probability of having a node with connectivity $k$ is the same as for the BA model, namely, $P_{k} \sim k^{-\gamma}$ with $\gamma \approx 3$. This result could be intuitively understood by noting that although the rules for the network generation has been changed at a local level, from a global perspective the average properties should not change radically. To realize this point, think of the network as being made up of different small components, as given by the affinity constraint, each of which is constructed following the BA algorithm. It is then clear that for large system sizes, each graph will follow the power law distribution $P_{k} \sim k^{-3}$ and so will be for the entire network.

The above argument applies only to average global properties, but there is nothing that guarantees a priori that the components of the network will link together in such a way that other properties will not be affected. This is the case of the average shortest path length $L$. As already introduced, complex networks show the noticeable property, known as small-world property, that the 


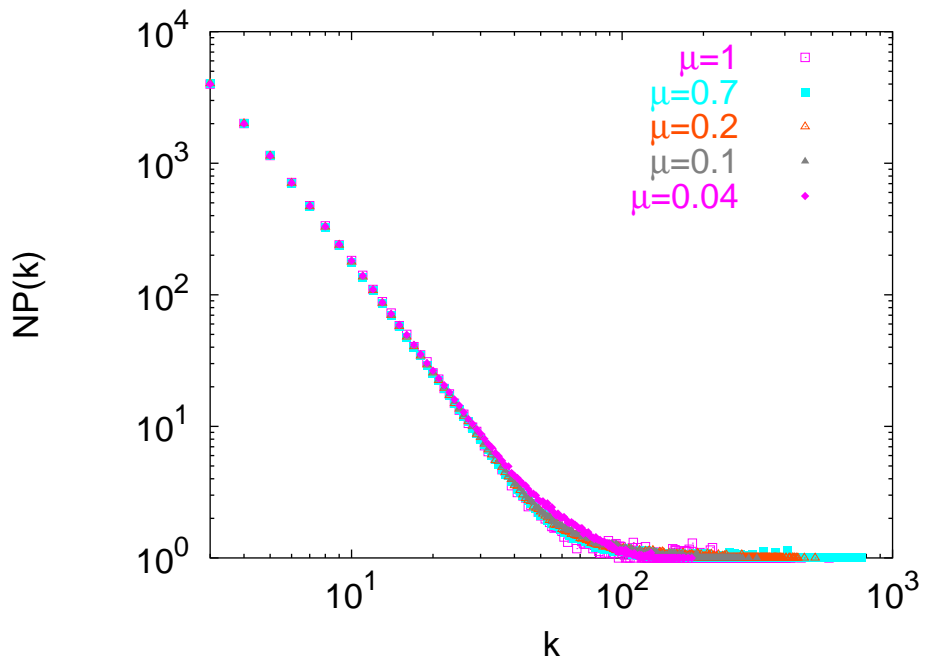

Figure 5.10: Number of nodes with connectivity $k$ for different values of $\mu$. The size of the network is $N=10^{4}$ nodes and $m_{o}=m=3$. The power-law distribution has an exponent equal to 3 . Note that the BA limit corresponds to $\mu=1$.

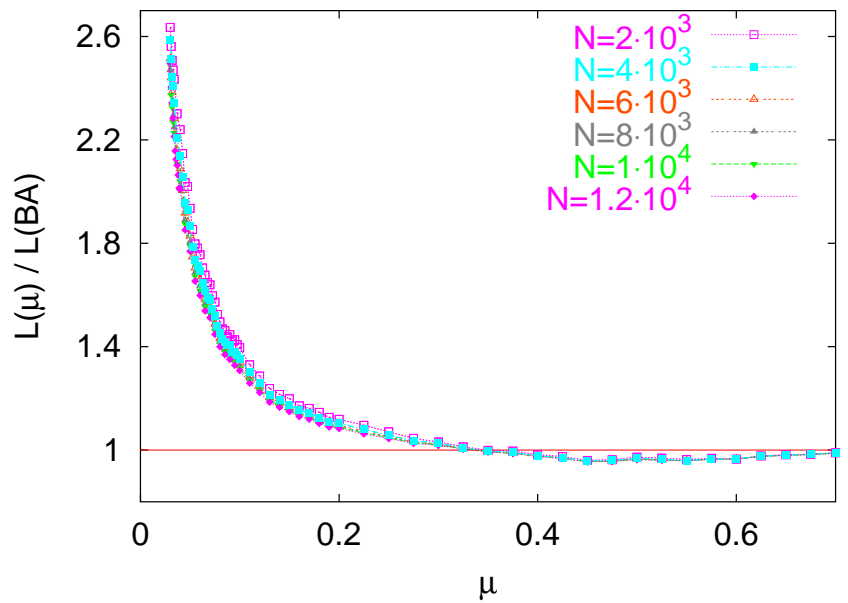

Figure 5.11: Ratio between the average shortest path length for different $\mu$ values, $L(\mu)$, and that of the BA network $(L(1))$ for several system sizes. The horizontal line marks the BA limit. A transition from graphs fulfilling the small-world property to a regime in which networks break down in many small pieces rising the value of $L(\mu)$ is observed. See the text for further details. 
(a)

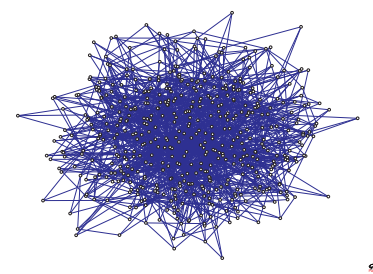

(c)

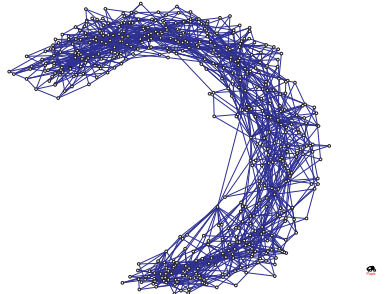

(b)

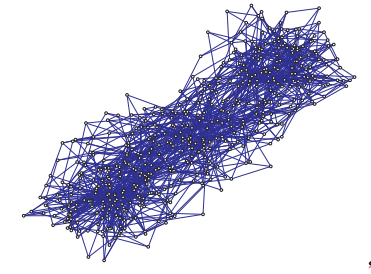

(d)

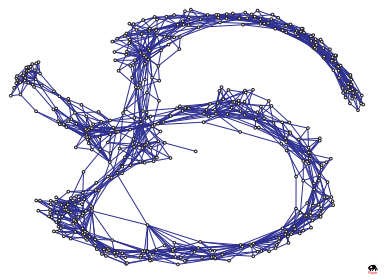

Figure 5.12: Graph representations of four networks produced with different values of $\mu$. The values of $\mu$ correspond to (a) $\mu=1$, (b) $\mu=0.2$, (c) $\mu=0.1$, (d) $\mu=0.04$. Each network is made up of $N=500$ nodes.

average path length increases at most with the logarithm of its size. We expect that for high values of $\mu$ the network is composed by a unique giant component and no fragmentation arises. When the range to which the affinity criterion is applied decreases, the network will gradually loose its compactness and will stretch approaching a one-dimensional structure with some small components. Further reduction of $\mu$ provokes the break down of the network in many isolated clusters.

Figures 5.11 and 5.12 substantiate this picture. Figure 5.11 represents the ratio between the average path length obtained for different values of $\mu$ and that of the BA network, for several system sizes. As $\mu$ restricts the PA range, the network undergoes a transition characterized by a growth of $L(\mu)$ an eventually becomes fragmented giving rise to an infinite shortest path length. We note here that although the results shown in the figure have been obtained for a uniform distribution of affinity values $a_{i}$, the qualitative behavior does not change for other probability distributions and only the value at which the transition is observed slightly shifts to the right. The shape of the network as the parameter $\mu$ is varied can be observed in figure 5.12, where we have represented how the network looks like for the limiting values of $\mu$. It is clear that when the PA range reduces too much the structure of the network radically changes while keeping the same degree distribution.

We now focus our attention on other properties with a local character. This is the case of the clustering coefficient of a node $c_{i}$. The clustering coefficient is of local character as it gives the probability that two nodes with a common 

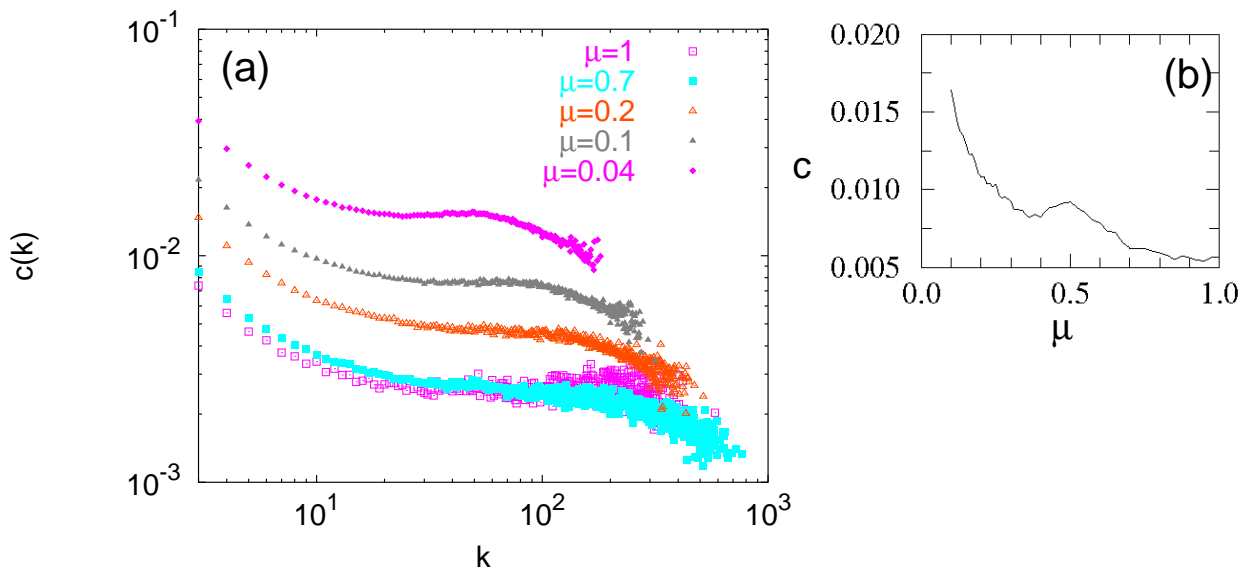

Figure 5.13: (a) Average clustering coefficient $c_{k}$ of nodes with degree $k$ for five different values of the parameter $\mu$. Note that as $\mu$ decreases, the clustering coefficient departs from the BA limit $(\mu=1)$. (b) The average clustering $c$ as a function of $\mu$ confirms this result. The parameters used for the generation of the networks are as of figure 5.10 .

neighbor are also linked together. Thus, it is expected that this magnitude, in our model, depends on the affinity of each node and the range of preferential attachment given by $\mu$. Figure 5.13.a shows the average clustering coefficient of nodes with a given connectivity $k$, for different values of the parameter $\mu$. The BA limit exhibits almost no correlations with the degree $k$ of the vertices and the smallest value for the clustering coefficient. As $\mu$ is reduced, the first selection of nodes by their affinity values plays a more dominant role contributing to the rising of $c_{i}$ for small and large connectivities. Near the transition, $\mu \sim 0.04$, the average coefficient is about one order of magnitude greater than that of the BA network. In order to see this growth of the clustering as the locality of the PA is increased we show in figure 5.13.b the mean clustering coefficient (averaged over all the elements of the network), $c$, as a function of $\mu$. The results reveals that the clustering grows in a rather regular fashion when $\mu$ decreases so that finally $c$ is incremented by a factor 3 at $\mu=0.1$ respect to the value at the $\mathrm{BA}$ limit.

These results are important for what concerns to the existence of cycles of small length in the network (triangles and rectangular loops are among these graph components). They are important because they express the degree of redundancy and multiplicity of paths among nodes in the topology of the network. The results obtained for $c_{k}$ indicate that as the region where the PA applies is reduced, the number of cycles increases and non-random correlations arise. This is illustrated in figure 5.14, where the average nearest neighbor 


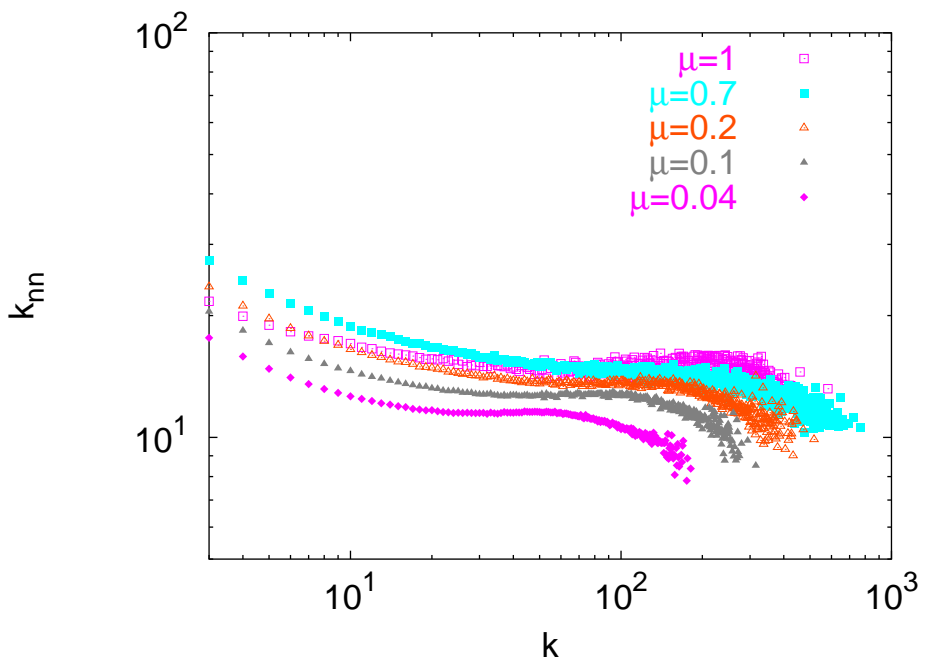

Figure 5.14: Average nearest neighbor connectivity $k_{n n}$ against $k$ for several values of $\mu$. Results are averaged over 100 network realizations for each $\mu$ value. Other parameters are as of figure 5.10.

degree, $k_{n n}(k)$ of a node with connectivity $k$ is depicted. While the BA model exhibits no correlations, it is manifested the tendency that networks generated with small values of $\mu$ display disassortative mixing at both ends of the connectivity range.

Finally, let us point out that although the values found for several magnitudes can not be directly associated with real data, there are some regions of the parameter space $\mu$ where non-trivial properties arise. In this sense, it would be interesting to perform the same analysis in more realistic growing network models looking for more similarities with real-world networks. For example, the exponent of the connectivity distribution can be tuned to small values by incorporating the first level of selection of the present model in the generalized BA model [158], which is known to give arbitrary $\gamma$ values in the interval $(2,3)$. As a plus, this model can be used to test the dependence of the network functioning with its topological structure (when the degree distribution is fixed). In particular, we will make use of it for analysing the performance of different processes placed on top of the network [communication between nodes (section 6.2.2) and sinchronizability of Kuramoto networks (section 8.2)] when clustering and average path length are varied. 


\subsection{Interpolation between Random and Scale-Free networks}

The seminal paper by Barabási and Albert [145, 187], showed that many real world networks can not be described by Erdös-Rényi type graphs where the connectivity distribution follows a Poisson-like distribution. While today we have recognized that preferential attachment is not a necessary condition for the formation of scale-free networks [202], it seems to be clear that it is an important mechanism. Indeed, most of the existing models intrinsically incorporate a preferential attachment like rule. On the other hand, uniform random linking of nodes on growing networks gives rise to networks where the degree distribution decays exponentially fast with the degree $k$, thus producing homogeneous networks with a well defined (and meaningful) average value for $k[155,181]$. The combination of the two rules, i.e, uniform and preferential linking, have been also analyzed in several models for interpolating between scale-free and exponential networks. For instance, Liu et al [203] have studied a model in which the probability of establishing new links goes as a linear combination of both in such a way that a new link is established between a node $i$ and a new one proportionally to $(1-p) k_{i}+p$, where $p$ weights the contribution of the two mechanisms. However, in previous models of this sort, there is an assumption that does not apply always. It has to do with the fact that the network always grows around a single component of connected nodes and uniform or preferential links from the emerging nodes are always made with elements belonging to this unique cluster. This single component grows linearly in time until it reaches the size of the network. Since there are no clusters of nodes other than the giant component, the models can not account for phenomena such as the coalescence of small networks into a larger one, nor for situations in which more than one node is added to a preexisting structure at each time step, features that may be relevant in social, economic and other networked systems.

In this section, we analyze a model that interpolates between Erdös-Rényi and scale-free networks as far as the degree distribution is concerned through a tunable parameter. The novel feature of the model is that, by construction, new links are not always established with nodes previouly incorporated to the network and thus allowing to interpolate with the classical ER graphs. We explore analytically and numerically the time behavior of nodes attachment as well as of the degree evolution. We find that, depending on the interplay between uniform and preferential linking, the transition from an ER like network to an SF one is smooth or more abrupt. The present model is useful as it provides a unique recipe to go progressively from homogeneous to heterogeneous topologies as well as for exploring the interplay between them. 


\subsubsection{The model}

The model introduced in this work generates a one-parameter family of complex networks. This parameter, $\alpha \in[0,1]$, measures the degree of heterogeneity of the final networks. Let us assume the final size of the network to be $N$. The network is generated in the following way:

(i) Start from a fully connected network of $m_{0}$ nodes and a set $\mathcal{U}(0)$ of $\left(N-m_{0}\right)$ unconnected nodes.

(ii) At each time step choose a new node $j$ from $\mathcal{U}(0)$.

(iii) This node makes a link in two ways:

(a) With probability $\alpha$ it links to any other node $i$ of the whole set of $N-1$ nodes with uniform probability

$$
\Pi_{i}^{\text {uniform }}=(N-1)^{-1} .
$$

(b) With probability $(1-\alpha)$ establish a link following a preferential attachment strategy, that is, the probability for any other node $i$ to attach to node $j$ is a function of its connectivity as,

$$
\Pi_{i}^{\mathrm{PA}}=\mathbf{F}\left(k_{i}\right),
$$

where different choices for the functional form of $\mathbf{F}(x)$ are analyzed below.

(iv) Repeat $m$ times step (iii) for the same node $j$.

(v) Repeat $\mathcal{U}(0)=\left(N-m_{0}\right)$ times steps (ii) to (iv).

A schematic plot of the linking procedure at step (iii) is shown in figure 5.15. The above rules allow for the coexistence of two classes of nodes. On one hand, there are nodes with at least one link. This set will be referred to henceforth as the connected set $\Omega(t)^{6}$. On the other hand, there is another set $\mathcal{U}(t)$ of isolated nodes such that its size is $N-\Omega(t)$. At variance with other models in which there are only nodes with connectivity different from zero and thus the connected component grows linearly with time, the above rules allows the addition of more than one node to the set $\Omega(t)$ as a result of random linking. Therefore, we expect the time dependency of $\Omega(t)$ to be highly non trivial.

\footnotetext{
${ }^{6}$ We recall on the possibility that the connected set is temporarily composed by more than one connected component. This is the case for the initial stage of the network growth when $\alpha \rightarrow 1$. However, when $N$ is high enough the final network is composed by a unique connected component.
} 


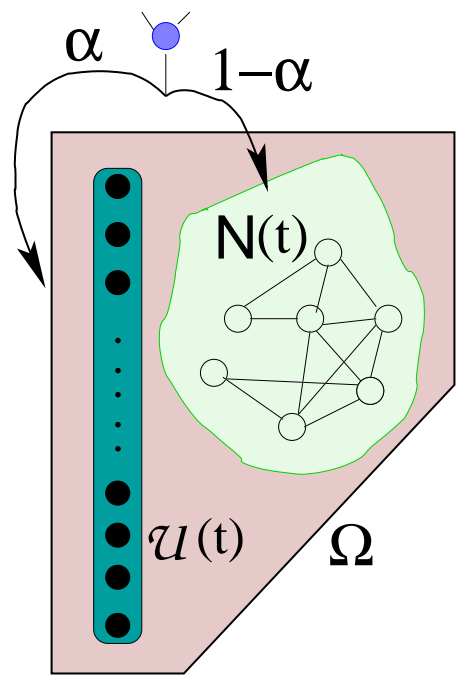

Figure 5.15: Schematic representation of the general procedure for generating the networks. With probability $\alpha$ one of the $m$ links can be made with any of the nodes (and with the same uniform probability) that will take part in the final network. On the other hand, with probability $(1-\alpha)$ the link will be made only with those nodes that form the connected set at that time because the node will choose a preferential linking strategy.

\subsubsection{Network growth and degree evolution}

In order to describe the evolution of the nodes degree, one has to consider the functional form of $\mathbf{F}(x)$ for the preferential attachment probability (5.30). However, we can take into account some previous considerations that do not depend on the particular form of $\mathbf{F}(x)$.

First of all, it is useful to consider two kind of links in order to analyze the model. Namely, the ones that arise from a uniform random choice, $k^{\mathrm{u}}$, and the remaining, $k^{\mathrm{pa}}$, corresponding to the implementation of the preferential attachment rule. The dynamics of $k^{\mathrm{u}}$ is completely independent of the dynamics of the PA links, $k^{\mathrm{pa}}$, but the opposite is not necessarily true. From this, it follows that the probability that one node has $k^{\mathrm{u}}$ uniform links, $P^{\mathrm{u}}\left(k^{\mathrm{u}}\right)$, is a Poisson distribution with $\left\langle k^{\mathrm{u}}\right\rangle=2 \alpha m$.

$$
P^{\mathrm{u}}\left(k^{\mathrm{u}}\right)=\frac{(2 \alpha m)^{k^{\mathrm{u}}} \mathrm{e}^{-2 \alpha m}}{k^{\mathrm{u}} !}
$$

As a consequence, we will concentrate on analyzing the growth dynamics of the PA links for the studied models.

It is particularly interesting to study at this point how uniform random linking affects the evolution of the connected set since this is completely independent on the specific PA rule considered. This feature represents one of the main differences between the studied model and other previous mechanisms used to generate growing networks. That is, in our model nodes are not incorporated to the connected set at a constant rate (like e.g. in the standard Barabási-Albert model) due to the possibility of adding new nodes from $\mathcal{U}(t)$ by applying uniform linking at time $t$ and therefore the set $\mathcal{U}(t) \neq \mathcal{U}(0)-t$. 
We can easily derive the evolution of the connected set size, $\Omega(t)=N-\mathcal{U}(t)$, for any value of the parameter $\alpha$. For this, we consider the growth of the connected set at each time step, i.e. when a new node of $\mathcal{U}(0)$ throws its $m$ links

$$
\Omega(t+1)=\Omega(t)+\frac{N-\Omega(t)}{N-\left(t+m_{0}\right)}+\alpha m\left(1-\frac{\Omega(t)}{N}\right) .
$$

In the above equation the second term on the right accounts for the probability that the new node (which is throwing its $m$ links) of $\mathcal{U}(0)$ does not belong already to the connected set at time $t$ (due to the possible uniform links obtained from previous nodes of $\mathcal{U}(0)$ already connected to the connected set $\Omega(t)$ ). Besides, the third term on the right describes the probability that any uniform link thrown by the node is directed to a node belonging to $\mathcal{U}(t)$. These two terms account for the growth rate of the connected set. We can consider that both time and $\Omega(t)$ are continuous variables and make the time step small enough in order to obtain the corresponding ODE associated to eq. (5.32), whose solution is given by

$$
\Omega(t)=N+\left(t+m_{0}-N\right) \mathrm{e}^{-\alpha m t / N} .
$$

The agreement between this calculation and Monte Carlo simulations is shown in figure 5.16 for different values of $\alpha$ and a preferential attachment as described in what follows (model A). It is worth noting the highly nonlinear behavior of $\Omega(t)$, at variance with models in which its size changes at a constant rate.

We formulate below two different ways to implement the preferential attachment rule, which give rise to different behaviors. In both models we will consider that the PA probability of a node $j$ depends only on the PA links of the node, $k_{j}^{\text {pa }}$. This new separation between PA links and uniform ones introduces a higher differentiation between the two simultaneous kinds of link dynamics implemented here allowing us to manipulate (as shown below) the degree of correlation between them. The two models interpolate between scale-free and Erdös-Rényi topologies but the structural transition is quite different (as we will show in section 5.4.3).

\section{MODEL A}

In this first model we shall study a preferential attachment rule strongly correlated with the simultaneous uniform random linking. First, we consider that the PA probability of a node $i$ is linear with the incoming PA degree of the node, $\hat{k}_{i}^{\mathrm{pa}}$, that is, those links received by $i$ when other node launches (in average) $(1-\alpha) m$ links following the PA rule. This particularity of the PA rule was already considered by Dorogovtsev et al [186]. Besides, we consider that when a node is introduced in the connected component (because either it is chosen 


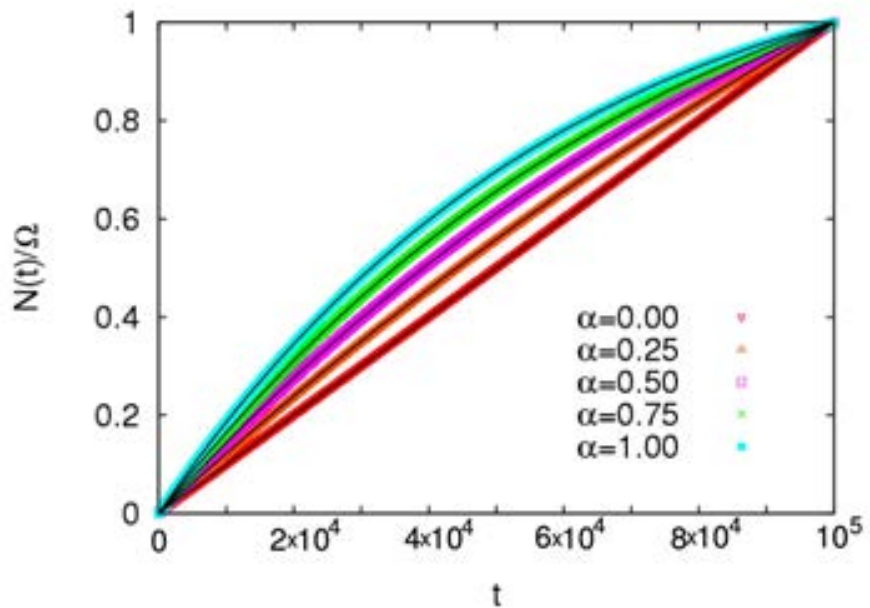

Figure 5.16: Size of the connected set $\Omega(t)$ as a function of time. Solid lines correspond to the analytical results (eq. (5.33)) and points are the Monte Carlo results of network construction (employing model A (sec. 5.4.2)). The comparison is made for $N=10^{5}$ and several values of $\alpha$. The parameters of the model are set to $A=m=m_{0}=1$.

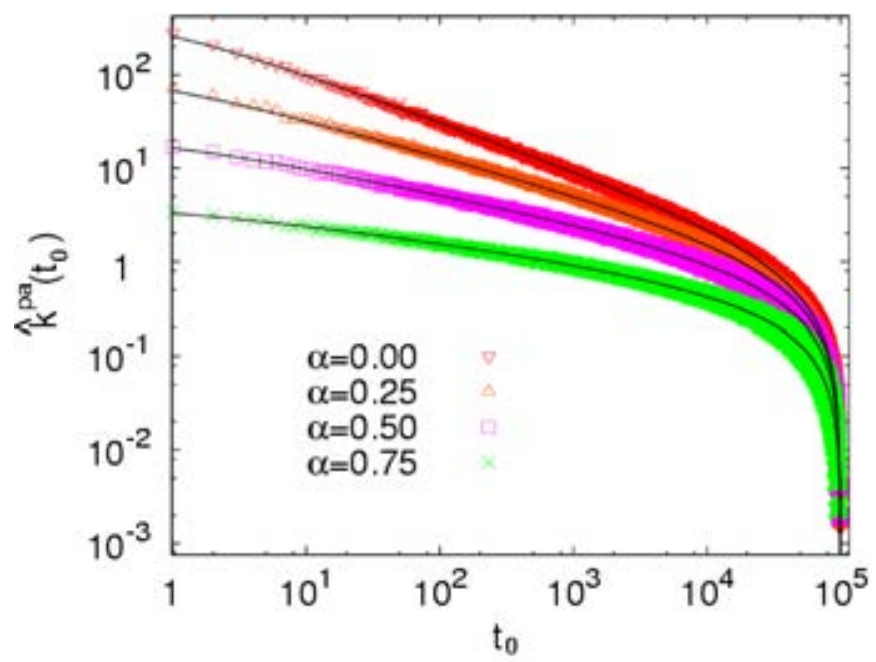

Figure 5.17: Model A. Monte Carlo simulation (points) versus mean field (lines) results for $\hat{k}^{\text {pa }}(t=N)$ as a function of the birth time $t_{0}$ for different values of $\alpha$. The parameters of the model were $N=10^{5}$ and $A=m=m_{0}=1$. The statistics of the Monte Carlo simulations were performed using $10^{4}$ networks for each value of $\alpha$. 
at random by any node or it is launching its $m$ outgoing links over the rest of nodes) it has an initial attractiveness (or fitness) $A$. In other words, each node has an associated parameter $A_{i}$ that is zero if the node $i$ is not in the connected set and is $A_{i}=A$ if $i$ is linked to other nodes (i.e., it belongs to $\Omega(t)$ ). We further consider that the attractiveness $A_{i}$ enters linearly in the preferential linking probability of node $i$. With these two ingredients, the expression for $\Pi_{j}^{\mathrm{PA}}$ is given by

$$
\Pi_{i}^{\mathrm{PA}}=\frac{\hat{k}_{i}^{\mathrm{pa}}+A_{i}}{\sum_{j \in N}\left(\hat{k}_{j}^{\mathrm{pa}}+A_{j}\right)},
$$

The introduction of the fitness $A$ correlates the PA rule with the uniform linking in the sense that the more links are established uniformly (the higher $\alpha$ ), the more new nodes with $\hat{k}_{i}^{\text {pa }}=0$ are incorporated to the connected set from $\mathcal{U}(t)$ and hence (by the presence of $A$ in the PA probability) the more candidates to obtain PA links are available. This can be observed from the evolution of the connected set $\Omega(t)$, when $\alpha$ is high there are a lot of nodes added into $\Omega(t)$ at the early stage of the network construction so that the potential growth of the PA degree of the former members of the connected set is strongly weakened. In order to confirm these heuristic considerations we derive the mean field evolution for the incoming PA degree of a node $i, \hat{k}_{i}^{\text {pa }}$

$$
\frac{\mathrm{d} \hat{k}_{i}^{\mathrm{pa}}}{\mathrm{d} t}=(1-\alpha) m \frac{\hat{k}_{i}^{\mathrm{pa}}+A}{(1-\alpha) m t+A \Omega(t)},
$$

(with the initial condition $\hat{k}_{i}^{\mathrm{pa}}\left(t_{0}^{i}\right)=0$ ). Obviously, in the limit $\alpha=0$ we recover the mean field equation for the Generalized Dorogovtsev model [186] (which, when $A=m$, describes the Barabási-Albert model). For $\alpha \neq 0$ the influence of the uniform random linking is evident from the presence of $\Omega(t)$. The number of nodes that start to have the above dynamics at some time $t_{0}$ is $\mathrm{d} \Omega(t) / \mathrm{d} t$ evaluated at time $t=t_{0}$ which for $\alpha \neq 0$ is not constant as we have seen in the previous calculation of $\Omega(t)$. The solution of (5.35) is then given by

$$
\frac{\hat{k}_{i}^{\mathrm{pa}}(t=N)}{A}=-1+\exp \left[(1-\alpha) m \int_{t_{0}^{i}}^{N} \frac{\mathrm{d} t}{(1-\alpha) m t+A \Omega(t)}\right] .
$$

We have solved numerically eq. (5.36) in order to obtain $\hat{k}_{i}^{\mathrm{pa}}(t=N)$ (or $\left.k_{i}^{\mathrm{pa}}(t=N)=\hat{k}_{i}^{\mathrm{pa}}(t=N)+\alpha m\right)$ as a function of $t_{0}^{i}$. This function, along with the number of nodes which are incorporated to the connected set at time $t_{0}^{i}=t_{0}$, gives the degree distribution of the PA links. We have compared the results given by eq. (5.36) for different values of $\alpha$ with the corresponding ones obtained by performing Monte Carlo simulations of the model (averaging over $10^{4}$ networks for each value of $\alpha$ ). The results, plotted in figure 5.17, show 
a very good agreement for the mean field model and the numerical network construction. As expected, the sooner a node is incorporated to the connected set the higher its final PA degree. However, as discussed above, one can observe that this gain of the oldest nodes becomes less important when the value of $\alpha$ grows due to the combination of two effects: (i) the application of the PA rule becomes less frequent and (ii) the fast growth of the connected set tends to make more homogeneous the PA probability of the nodes.

\section{MODEL B}

In the second proposal the two different linking processes are completely independent. For this, we consider that $\Pi_{i}^{\mathrm{PA}}$ is a linear function of the (incoming and outgoing) links that appear as a product of the application of the PA rule. Then, $k_{i}^{\text {pa }}$ will be zero until it launches its $\alpha m$ PA links over the rest of the nodes, i.e. regardless of $k_{i}^{\mathrm{u}}$. Then, the mean field equation for the evolution of $k_{i}^{\mathrm{pa}}$ is given by

$$
\frac{\mathrm{d} k_{i}^{\mathrm{pa}}}{\mathrm{d} t}=(1-\alpha) m \frac{k_{i}^{\mathrm{pa}}}{2(1-\alpha) m t+m_{0}}
$$

with the initial condition $k_{i}^{\mathrm{pa}}\left(t_{0}^{i}\right)=(1-\alpha) m$ and $t_{0}^{i}$ being the time when node $i$ launches its $m$ links. Solving the above equation yields

$$
k_{i}^{\mathrm{pa}}(t)=(1-\alpha) m\left[\frac{t}{t_{0}^{i}}\right]^{1 / 2} .
$$

Because the nodes launch their links at a constant rate (one node per time step), it is easy to obtain the degree distribution $P\left(k^{\mathrm{pa}}\right)$

$$
P\left(k^{\mathrm{pa}}\right)=2(1-\alpha)^{2} m^{2}\left(k^{\mathrm{pa}}\right)^{-3},
$$

which is simply a power law distribution with a Barabási-Albert exponent regardless of the value of $\alpha$. On the other hand, the relative weight of the power law with respect to the Poisson distribution in the total degree distribution $P(k)$ will be obviously affected by $\alpha$ (as the prefactor in the above equation suggests).

\subsubsection{Network properties}

In this section we discuss the transition from SF to ER networks in terms of the global topological features of the networks. We have performed Monte Carlo simulations of the two models and compared how the relevant topological measures evolve as a function of $\alpha$. We are interested in obtaining how the 

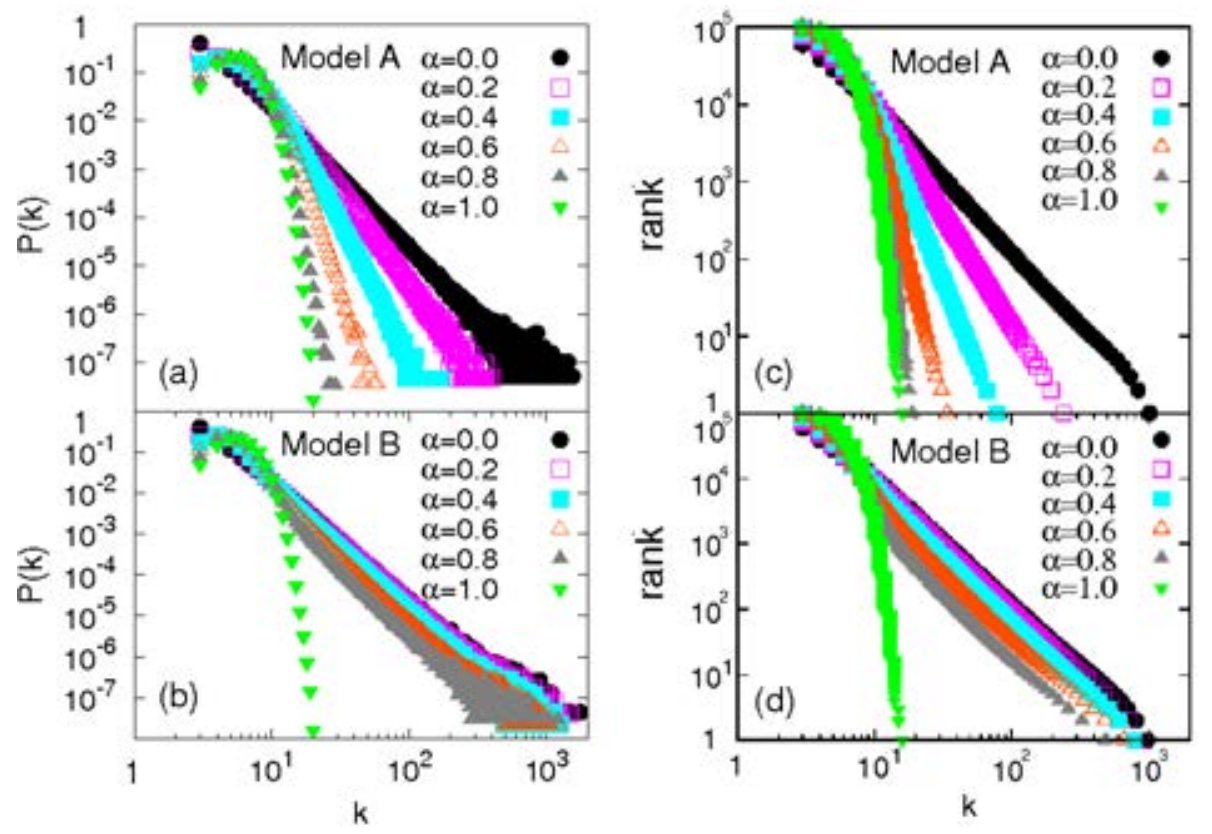

Figure 5.18: Monte Carlo results for the degree distribution $P(k)$ and rank-degree relation for several values of $\alpha$. (a) and (c) show the results for model A revealing a progressive increase of the tails decaying rate when $\alpha \rightarrow 1$. The results for model $\mathrm{B}$ $((\mathbf{b})$ and $(\mathbf{d}))$ show how the decaying rate is not affected by $\alpha$. The networks were generated with the following parameters $N=10^{5}$ and $m=m_{0}=3$ ( $A=3$ for model A).

different correlations between the uniform and PA linking rules affect several structural measures. To do this, we have studied the behavior of three magnitudes that behave very different in the two known limiting cases (SF and ER networks), namely: the degree distribution $P(k)$, the average shortest path length $\langle L\rangle$ and the second moment of the degree distribution $\left\langle k^{2}\right\rangle$.

Degree distribution - The degree distribution evolution is clearly different for the two models. In figure 5.18 we have plotted the degree distribution and the rank-degree relation for both models. The rank-degree relation provides a useful tool for analysing the degree heterogeneity of the networks [204] and thus it is helpful when looking at the transition between ER and SF networks. As can be observed from figures 5.18(a) and 5.18(c) the correlated model A shows a smooth transition from the power law $(\alpha=0)$ to the Poisson distribution $(\alpha=1)$. The decay of the tails $(k>>1)$ of the degree distribution and the rank-degree relation becomes progressively faster as $\alpha$ grows revealing the decrease of the exponent of $P^{\mathrm{pa}}\left(k^{\mathrm{pa}}\right)$ as expected from the results obtained by the analytical insights developed for model A. For model B the transition 


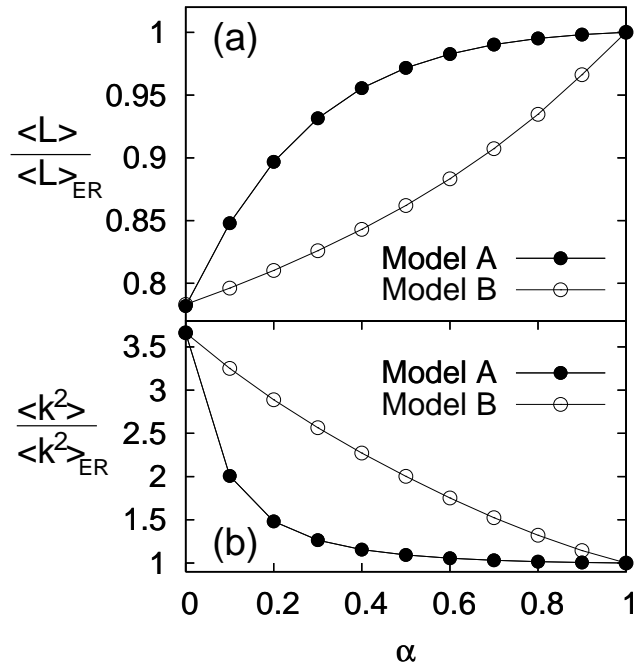

Figure 5.19: Average path length (a) and second moment of the degree distribution (b) as a function of $\alpha$. Both quantities are represented normalized by their respective values in the ER limit. The results clearly manifest the two different transitions of the models regarding the heterogeneity evolution along the interpolating path. The averaged networks had the following parameters $N=10^{4}$ and $m=m_{0}=3$ ( $A=3$ for model $\left.\mathrm{A}\right)$.

is completely different as it is shown in figures 5.18(b) and 5.18(d). In both representations the decaying rate of the tails is independent of $\alpha$ and the transition to the Poisson distribution is much more apparent for low values of $k$. In this sense one can conclude that highly connected nodes persist along the transition of model $\mathrm{B}$ while for model $\mathrm{A}$ the heterogeneity is progressively lost.

Average shortest path length - The different evolution of the degree distributions observed above suggests to look at how the average shortest path length behaves along the two paths of interpolation. It is well known that the existence of high degree nodes makes the network more compact due to the possibility of finding shortcuts between nodes going through the hubs. Hence, the persistence of highly connected nodes determines the small diameter of the scale-free network. The results obtained are shown in figure 5.19(a). As expected, the average shortest path length as a function of $\alpha$ grows slower for model B because the probability of finding hubs is higher than for the networks generated using model $\mathrm{A}$ for the same value of $\alpha$.

Second moment of $P(k)$ - In order to obtain a quantitative measure of the evolution of the degree heterogeneity for the two models it is convenient to measure the second moment of the degree distribution, $\left\langle k^{2}\right\rangle$. This magnitude diverges (in the thermodynamic limit $N \rightarrow \infty$ ) for scale-free networks with exponents between 2 and 3 . So, we expect a decrease of the heterogeneity on the path to ER graphs. As can be observed from figure 5.19(b), model A shows a faster decrease of $\left\langle k^{2}\right\rangle$ as expected from the study of the degree distribution while for model $\mathrm{B}$ the transition is much smoother revealing again 
the persistence of highly connected nodes along the path to the ER limit.

As for other properties like the clustering coefficient and degree-degree correlations we have checked that they remain unchanged irrespective of the value $\alpha$ and wheter model $\mathrm{A}$ or $\mathrm{B}$ is implemented.

The present model provides a useful tool to study the influence of the degree of heterogeneity in dynamical processes of different kinds just as the WattsStrogatz model have proved to do so in the transition from regular to random structures. In particular, there exist open questions in phenomena such as the synchronization of coupled oscillators [205] where this kind of model could be particularly relevant to explore the system's behavior in the region where homogeneous and heterogeneous architectures coexist. This question will be deeply analysed in section 8.3. 



\section{Chapter 6}

\section{Propagation through Complex Networks}

The better a simulation is for its own purposes, by the inclusion of all relevant details, the more difficult it is to generalize its conclusions for other species. For the discovery of general ideas in ecology, therefore, different kinds of mathematical descriptions, which may be called models, are called for. Whereas a good simulation should include as much detail as possible, a good model should includes as little as possible.

— J. Maynard Smith in Models in Ecology [206].

In this chapter we will focus on two of the main dynamical processes studied on top of complex networks, namely, the analysis of Epidemic spreading and Information dynamics. The interest of studying these problems is twofold. First, the simplicity of the description of the two procceses allow for analytical results, heuristic insights and extensive numerical simulations in order to explain the role that the underlying topology has on the dynamics. Then, one of the advantages of studying these dynamics is that the simple formulation of the models (usually expressed by means of linear rules) used for their description does not mask the effects of the topological complexity. Besides, one can realize by looking at the literature that a great number of the networks whose characterization is available (mostly due to the simplicity for unveiling the links between their components) can be regarded as either technological or logistic networks. Then, the study of epidemics and information propagation is justified for practical purposes. 


\subsection{Epidemic spreading and Immunization}

The history of the studies on epidemic spreading starts with the first works by epidemiologist at the beginning of the 20th century [207]. However, the burst in the mathematical modeling of disease transmission took place in the middle of the 20th century by the formulation of a large variety of models (interesting books on the matter are [208-211]) aimed at reproducing the evolution patterns of the number of casualties and infected people during epidemic periods (see figure 6.1). Recently, the attention has been redirected to the spreading of informatic viruses. The interest in this field has been coupled to the availability of data about potential transmission networks (like the internet or peer-to-peer networks). The development and deployment of a digital immune system to prevent technological networks from the spreading of viruses and to minimize the damage produced by intentional attacks are in the root of recent research efforts [161, 212-222].

In this section we will first introduce two general models (SIR and SIS) that describe the spread of epidemics on homogeneous systems. Then, we will turn our attention to the disease transmission in heterogenous substrates and the performance of different immunization strategies will be compared. Finally we will report on a new immunization strategy based on the covering problem of complex networks. The performance of this new algorithm depends on the local structure of the network. We will implement this strategy along with the afore mentioned in order to compare their results when deployed on top of real networks.

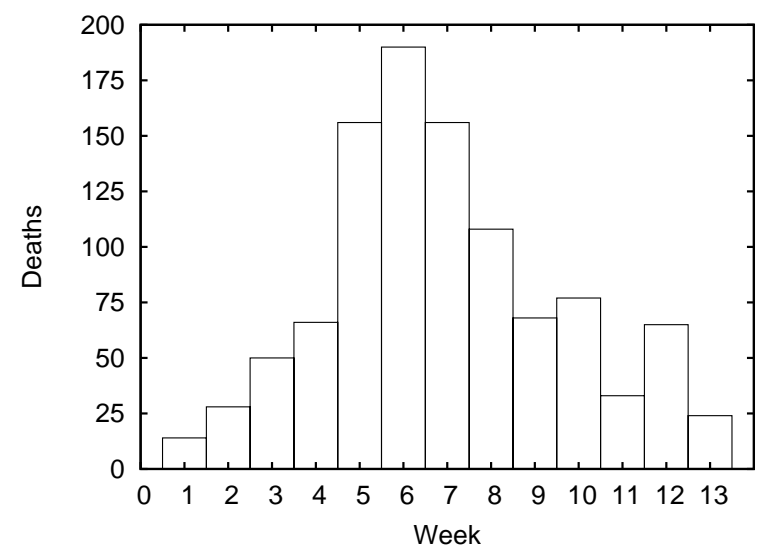

Figure 6.1: Histogram of the number of deaths due to the influenza-pneumonia epidemic during the 1968-1969 winter in New York. This extremely damaging influenza was named the "Hong Kong $f l u$ " due to the place where it started. 


\subsubsection{Modeling epidemic spreading}

There are many different models to describe the epidemic transmission problem. However, nearly all of them are variations of some general and coarse grained models like the SIR (Susceptible-Infected-Removed) and SIS (SusceptibleInfected-Susceptible). The different variations have to do with an increased complication of the models to study particular diseases. In order to focus on the importance that the topology of the network has on the spreading of a disease we will deal with the most simplified descriptions of the epidemics dynamics.

\section{The SIR model}

The SIR model was introduced by Kermack and Mc Kendrick in 1927 [207] to explain the rapid rise and fall in the number of infected patients observed in epidemics such as the plague (London 1665-1666, Bombay 1906) and cholera (London 1865). This model was recovered by the work of Anderson and May [223] after being oversought for decades. The SIR model is a typical example of the so-called compartmental models. In this class of models the elements are viewed as parts of several groups (or compartments) so that the evolution equations are referred to the number of elements of each group. The SIR model describes the spreading of infectious diseases in which each individual can be either immunized or dead after the contagion. Following this assumption we can classify the population into three different groups:

- Susceptible: Those healthy people who have not been infected and thus are likely to contract the disease in the future.

- Infected: People who has been contagied and are currently suffering the effects of the disease. They can infect Susceptible people in the course of their disease.

- Recovered and Removed: Composed by people that finally died due to the disease or recovered and got immunized.

Then, individuals can change their state by means of the jumps between the three compartments, $S \rightarrow I \rightarrow R$. The dynamical rules accounting for the flux among the three states determine a set of differential equations for the densities of the population groups $s(t)=S(t) / N, i(t)=I(t) / N$ and $r(t)=R(t) / N$.

The change rate for susceptible elements is always negative and proportional to the number of contacts among infected and susceptible elements. 
We call $\lambda$ the probability that one susceptible individual gets infected in one contact, then we can write

$$
\frac{\mathrm{d} s}{\mathrm{~d} t}=-\lambda\langle k\rangle s(t) i(t)
$$

The evolution of the proportion of infected individuals, $i(t)$, has two contributions, one positive $-\dot{s}(t)$ and one negative accounting for the recovering (or death) rate of the infected individuals

$$
\frac{\mathrm{d} i}{\mathrm{~d} t}=\lambda\langle k\rangle s(t) i(t)-\mu i(t),
$$

where $\mu$ is the recovering (or death) rate that corresponds to the inverse of the average disease time for an individual. Taking into account that $r(t)=$ $1-s(t)-i(t)$ the last evolution equation for the recovered density is

$$
\frac{\mathrm{d} r}{\mathrm{~d} t}=\mu i(t) .
$$

The above formulation of the model equations assumes the homogeneous mixing hypothesis that considers that the set of susceptible people with whom an infected individual establishes contacts is taken at random within the whole population. This is manifested in the constant value for the number of contacts $\langle k\rangle$ so that the approach is only valid for homogeneous networked systems. Along with this assumption we have considered homogeneity in the agent characteristics so that $\lambda$ and $\mu$ (although seen as averages) are meaningful. This model is seen as a mean field approximation to the epidemic spreading problem. One can modify the SIR model by adding more compartments (like e.g. in models for VIH propagation where a set of people suffering an incubation period, or more technically a latency period, should be distinguished from those who have the disease already diagnosed) or by considering that the time scale involved is slow enough so that additional terms accounting for natural birth and death rates should be incorporated. We can rescale conveniently the above equations $(\mu \rightarrow 1, t \rightarrow \mu t, \lambda \rightarrow \lambda / \mu)$ in order to have a single control parameter $\lambda$ to study the behaviour of the model.

The question to answer in the SIR model has to do with the conditions under which a small infectious seed leads to a significant fraction of individuals at the recovered state when the steady state $(i(t)=0)$ is reached for the whole system. One can translate this question in terms of a general bond percolation problem since an epidemic is set when there is a large enough fraction of "occupied" bonds (those that the epidemy used to spread) to lead to the formation of a network component whose size scales with the size of the graph (signaling an epidemic percolation). In fact, there exist an exact 


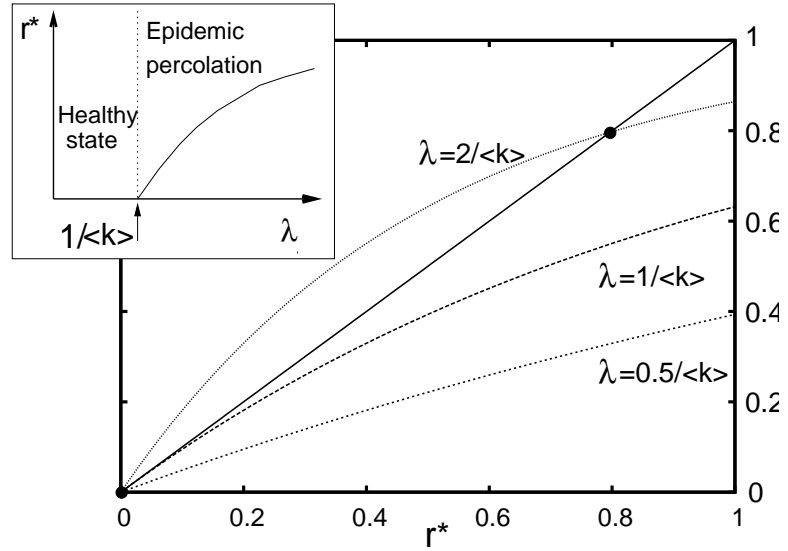

Figure 6.2: Graphical solution of $r^{\star}$ given by last expression in eq. (6.4). It can be observed the emergence of the second solution (corresponding to the epidemic percolation) for $\lambda / \mu>\langle k\rangle^{-1}$. The inset shows a qualitative plot of the phase transition for the SIR model.

mapping between both problems $[224,225]$ that can be used as a powerful tool for solving the epidemic spreading across general networks [226, 227]. In order to answer this question we consider the initial conditions $s(0)=1 / N$, $i(0)=r(0)=0$ and we look for the value $r^{\star}=\lim _{t \rightarrow \infty} r(t)$ It is easy to notice that $r^{\star}=1-s^{\star}$ because $i^{\star}$ is necessarily null. Then, dividing eq. (6.1) by eq. (6.3) to get rid of $i(t)$ we have

$$
\frac{\mathrm{d} s}{\mathrm{~d} r}=-\lambda\langle k\rangle s(t) \rightarrow s(t)=\exp (-\lambda\langle k\rangle r(t)) \rightarrow r^{\star}=1-\exp \left(-\lambda\langle k\rangle r^{\star}\right) .
$$

Last equation has always $r^{\star}=0$ as a solution (no epidemic percolation) and if $R_{0}=\lambda\langle k\rangle>1$ there is a second one with $r^{\star} \neq 0$ corresponding to a significative spread of the disease. $R_{0}$ is usually termed as the effective reproductive rate and its physical meaning is clear: it corresponds to the average secondary infections produced when a single infected individual is introduced in a healthy population. If this number is greater than one the disease reaches a non null fraction of the population. From the phase transitions point of view one would speak about $r^{\star}$ as the appropriate order parameter and $\lambda$ as the control parameter. It can be obtained that at the critical point, $\lambda=\langle k\rangle^{-1}, r^{\star}$ behaves as $r^{\star} \sim\left(\lambda-\langle k\rangle^{-1}\right)$ so that the critical exponent is 1 as expected from a mean field treatment.

\section{The SIS model}

The SIS model was originally introduced by Hethcote and Yorke [228] in 1984 for describing the propagation of gonorrhea and has been largely used for studying the transmission of tuberculosis. These diseases have several common attributes that make it different from other infections. The most important difference is that the infection does not confer immunity to recovered subjects 
so that the SIR model is no longer valid. The SIS model considers only two compartments composed of susceptible and infected nodes so that a continuous flux between both compartments is allowed, $S \leftrightarrow I$. Then, the relevant equation for the SIS model is

$$
\frac{\mathrm{d} i}{\mathrm{~d} t}=\lambda\langle k\rangle[1-i(t)] i(t)-\mu i(t),
$$

where we have assumed $s(t)=1-i(t)$ (i.e. no deaths associated to the disease are considered). Again we can rescale the equation in order to obtain $\mu=1$.

The SIS model is analogous to the SIR model in what refers to the existence of a epidemic transition. However in the SIS model the two regimes are differentiated by whether the disease persist indefinitely in the population (due to the fact that subjects can be reinfected many times) or not. Then, imposing $\mathrm{d} i / \mathrm{d} t=0$ in (6.5) we obtain two different steady states, one with $i(t)=0$ and the second one, for the case $\lambda>\langle k\rangle^{-1}$, with $i(t)=(\lambda-1 /\langle k\rangle) / \lambda$ corresponding to the an endemic state.

Due to the manifest analogy between SIR and SIS models we will focus on the SIR formalism in the forthcoming discussions about the behaviour of epidemic spreading on heterogenous networks. It has been shown that the same qualitative results are also obtained for both models when studying more complex topologies.

\subsubsection{Epidemic spreading in general complex networks}

The homogeneous mixing hypothesis assumed in the previous discussion cannot be applied to many real systems like technological networks where their components do not interact with a similar number of network elements. Then, it is necessary to incorporate the ingredient of heterogeneity to the problem of epidemic spreading. In the SIR model (and analogously for the SIS formulation) the different compartments $S(t), I(t)$ and $R(t)$ are now characterized by the subsets $\left\{S_{k}(t)\right\},\left\{I_{k}(t)\right\}$ and $\left\{R_{k}(t)\right\}$ labeled by the connectivity $k$ of their components $[212-214,216,227]$. These variables are normalized so that $S_{k}(t)+I_{k}(t)+R_{k}(t)=1$, then

$$
\frac{S(t)}{N}=\sum_{k} P(k) S_{k}(t), \quad \frac{I(t)}{N}=\sum_{k} P(k) I_{k}(t) ;, \quad \frac{R(t)}{N}=\sum_{k} P(k) R_{k}(t) .
$$

This second classification of the compartment elements into degree classes allows to take into account the heterogeneous character of real networks.

At a mean field description the evolution of these new magnitudes satisfy 
the following set of coupled differential equations

$$
\begin{aligned}
\frac{\mathrm{d} S_{k}}{\mathrm{~d} t} & =-\lambda k S_{k}(t) \Theta(t), \\
\frac{\mathrm{d} I_{k}}{\mathrm{~d} t} & =\lambda k S_{k}(t) \Theta(t)-I_{k}(t), \\
\frac{\mathrm{d} R_{k}}{\mathrm{~d} t} & =I_{k}(t),
\end{aligned}
$$

where we have already set $\mu=1$. The quantity $\Theta(t)$ represents the probability that an element is linked to an infected node. This probability is given by

$$
\Theta(t)=\frac{\sum_{k} k P(k) I_{k}(t)}{\langle k\rangle} .
$$

Note that we are considering here that the degree correlations are absent in the network with the assumption that $\Theta(t)$ is the same for any set $S_{k}$ regardless of the connectivity of its components.

Considering initial conditions consisting on a infinitesimal fraction of infected nodes distributed homogeneously over the connectivity sets, $I_{k}(0)=$ $i_{0} \ll 1$, we can consider $S_{k}(0) \simeq 1$ and integrate eq. (6.7) to obtain $S_{k}(t)=$ $\exp [-\lambda k \phi(t)]$, where

$$
\phi(t)=\frac{\sum_{k} k P(k) \int_{0}^{t} I_{k}(\tau) \mathrm{d} \tau}{\langle k\rangle}=\frac{\sum_{k} k P(k) R_{k}(t)}{\langle k\rangle} .
$$

In order to obtain a closed relation for $R_{k}(\infty)$ we compute the time derivative of $\phi(t)$

$$
\frac{\mathrm{d} \phi}{\mathrm{d} t}=1-\phi(t)-\frac{\sum_{k} k P(k) \exp [-\lambda k \phi(t)]}{\langle k\rangle} .
$$

Solving the above differential equation we can obtain $R_{k}^{\star}=R_{k}(\infty)=1-$ $S_{k}(\infty)=1-\exp [-\lambda k \phi(\infty)]$ and compute the total epidemic prevalence $R^{\star}$. The solution $\phi(t)$ to eq. (6.12) is not available for a general degree distribution $P(k)$. However, we are interested in the behaviour of $\phi^{\star}=\phi(t \rightarrow \infty)$ to obtain information about $R^{\star}$. In this limit $\dot{\phi(t)}=0$ so that eq. (6.12) transform into a consistent equation for $\phi^{\star}$

$$
\phi^{\star}=1-\frac{\sum_{k} k P(k) \exp \left(-\lambda k \phi^{\star}\right)}{\langle k\rangle} .
$$

The above equation has always the solution $\phi^{\star}=0$ (implying $R_{k}^{\star}=0 \forall k$, $\left.R^{\star}=0\right)$ and when

$$
\frac{\sum_{k} \lambda k^{2} P(k)}{\langle k\rangle}>1
$$


it has a second non trivial solution $\phi^{\star}>0$ that yields $R^{\star}>0$ indicating a significative epidemic prevalence. Then, the epidemic threshold is given by

$$
\lambda_{c}=\frac{\langle k\rangle}{\left\langle k^{2}\right\rangle} \text {. }
$$

This result is very relevant since it points out that complex networks with heterogeneous degree distributions (like SF networks with $2<\gamma \leq 3$ ) show a vanishing epidemic threshold (since $\left\langle k^{2}\right\rangle \rightarrow \infty$ as $N \rightarrow \infty$ ) and become extremely fragile under possible infections. An identical result for the threshold value is found when analyzing the SIS model [212, 214]. In this case the results are explained by a persistence of low levels of infected individuals for low values of $\lambda$. This interpretation is coherent with the long term prevalence found for real informatic viruses and in principle can only be understood in the framework of homogeneous networks by the (unrealistic) existence of a global tuning of the parameters in order to work close enough to the epidemic threshold. However, the bad news about the absence of epidemic threshold are somehow played down when one looks at the behaviour of $R^{\star}$ when $\lambda \ll 1$. Considering the BA model $\left(P(k)=2 m^{2} k^{-3}\right)$ we can compute this behaviour $[212,214,216]$ finding

$$
R^{\star} \sim \exp \left(-\frac{1}{m \lambda}\right)
$$

so that the epidemic prevalence approaches smoothly to 0 at $\lambda_{c}=0$.

\section{Correlated Complex Networks}

The above results have been obtained assuming that degree correlations between pairs of nodes are absent. However correlations are present in most real networks and therefore they cannot be neglected in the expression for $\Theta$,eq. $6.10,[215,218]$. The equations for the SIR model (6.7), (6.8) and (6.9) are then modified to

$$
\begin{aligned}
\frac{\mathrm{d} S_{k}}{\mathrm{~d} t} & =-\lambda k S_{k}(t) \sum_{k^{\prime}} P\left(k^{\prime} \mid k\right) I_{k^{\prime}}(t), \\
\frac{\mathrm{d} I_{k}}{\mathrm{~d} t} & =\lambda k S_{k}(t) \sum_{k^{\prime}} P\left(k^{\prime} \mid k\right) I_{k^{\prime}}(t)-I_{k}(t), \\
\frac{\mathrm{d} R_{k}}{\mathrm{~d} t} & =I_{k}(t) .
\end{aligned}
$$

In order to have some insight above the behaviour of the system for a general form of $P\left(k^{\prime} \mid k\right)$ one consider again the initial conditions $S_{k} \simeq 1$. Then eq. (6.18) becomes uncoupled and can be written as

$$
\frac{\mathrm{d} I_{k}}{\mathrm{~d} t}=\sum_{k^{\prime}}\left[\lambda k P\left(k^{\prime} \mid k\right)-\mu \delta_{k^{\prime}, k}\right] I_{k^{\prime}}(t) \equiv \sum_{k^{\prime}} L_{k, k^{\prime}} I_{k^{\prime}}
$$


The stability of the initial state corresponds to the situation when there is no epidemic prevalence and it is then characterized by the sign of the eigenvalues of the Jacobian matrix $\mathbf{L}$. Since the matrix $\mathbf{C}$ whose elements are $C_{k^{\prime}, k}=$ $k P\left(k^{\prime} \mid k\right)$ has the same eigenvalues as its transposed (see eq. (5.10)) all its eigenvalues are real. If $\Lambda$ is the maximum eigenvalue of $\mathbf{C}$ we obtain that the solution $I_{k}(t)=R_{k}(t)=0 \forall t$ is stable if $-\mu+\lambda \Lambda<0$ and therefore the epidemic threshold is defined as

$$
\lambda_{c}=\frac{\mu}{\Lambda} .
$$

Then, the existence of an epidemic threshold is completely determined by the matrix $\mathbf{C}$ and hence a non zero $\lambda_{c}$ can be recovered depending on the nature of the correlations. It has been shown numerically [218] that for assortative networks the threshold remains zero and the behaviour is qualitative the same as for uncorrelated networks.

\subsubsection{Immunization strategies}

The above study of epidemic spreading suggests the incorporation of mechanisms or strategies for stopping the advance of the infections across the network. From the SIR point of view this means to incorporate some degree of immunity to a fraction $g$ of elements so that they cannot be infected and thus they would locally stop the possible subsequent infections. The question then is where to locate this subset of immune nodes. Obviously, the answer will depend on the topological characteristics of the underlying network so that an efficient immunization design would differ depending on what kind of network we are dealing with.

In the following we will characterize the most common ways for performing such immunizations in order to compare these mechanisms with a new immunization proposal described in the next section.

\section{Random Immunization}

Random immunization consists of choosing a fraction $g$ of the nodes with uniform probability and confer them immunity to the epidemy. This is the simplest way for placing immune elements on a complex network since the choice of the nodes is completely independent of any attribute or characteristic of the set. The presence of a fraction $g$ of immune nodes simply rescale the transmission probability in the above discussed mean field approach, $\lambda^{r}=$ $(1-g) \lambda$. Then, for homogenous networks and for a constant value of $\lambda$ we obtain a critical fraction $g_{c}=1-1 /(\lambda\langle k\rangle)$ so that $r=0$ if $g \leq g_{c}$. On the 


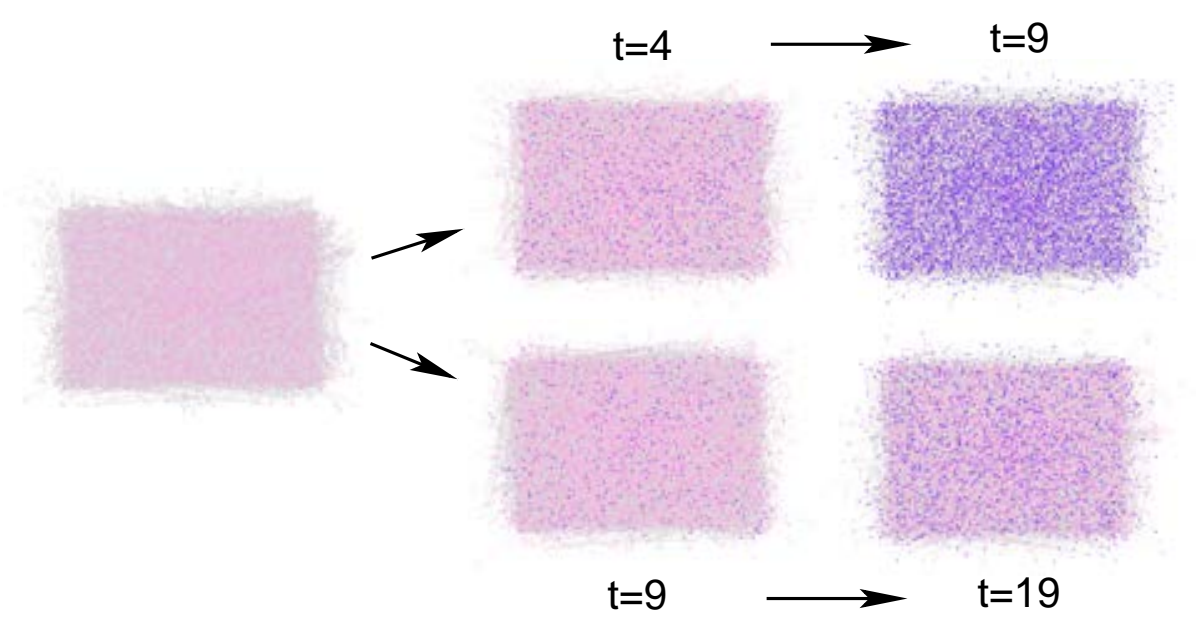

Figure 6.3: Time evolution of a SIR epidemic in the Internet Autonomous Systems representation when $\lambda=0.8$ and $1 \%$ of infected nodes at $t=0$. Pink and blue denote susceptible and recovered nodes respectively. (a) Corresponds to the case when no immunization is applied and (b) when targeted immunization is applied $(g=0.01)$.

other hand, for the same situation in heterogeneous non-correlated networks we obtain $g_{c}=1-\langle k\rangle /\left(\lambda\left\langle k^{2}\right\rangle\right)$ so that $g_{c}=1$ regardless of the value of $\lambda$ for SF networks with $\left\langle k^{2}\right\rangle \rightarrow \infty$ and thus random immunization of heterogeneous networks is completely inefficient.

\section{Targeted Immunization}

The failure of random immunization when applied to SF networks can be understood by the well known results on random failures in these networks. SF networks are very robust (it keeps the global cohesion) under random removal of nodes. Then, in terms of epidemic spreading, no matter the amount of immunized nodes the infection will always find a path to arrive to any susceptible node. On the other hand, SF networks are extremely weak when intentional attacks are performed, i.e. when a selective removal of those highly connected nodes is applied. It is then interesting to apply a targeted immunization strategy [217] just collecting those nodes with the highest connectivity and confer them immunity.

In order to make an estimation of the effects that targeted immunization has on SF networks we take the nodes with $k>k_{t}$ and immunize them ${ }^{1}$. Then

\footnotetext{
${ }^{1}$ Note, however, that in order to do so we should have complete knowledge of the network
} 
we have that the fraction of immune nodes corresponds to

$$
g=\sum_{k \leq k_{t}} P(k)
$$

This implies that those nodes, and the links that pointed to them, are removed from the remaining effective network available for the spread of the epidemy. Then, if we call $p(g)$ the probability that any link of the original network point to an immune node we obtain

$$
p(g)=\frac{\sum_{k \leq k_{t}} k P(k)}{\langle k\rangle},
$$

so that the degree distribution of the new network after removing those immunized nodes is

$$
P_{t}(k)=\sum_{q \leq k}^{k_{t}} P(q)\left(\begin{array}{c}
q \\
k
\end{array}\right)[1-p(g)]^{k} p(g)^{q} .
$$

With this new distribution we can compute its first and second moments so that we can compute the new $\lambda_{c}$ from eq. (6.15). These moments can be expressed in terms of $p(g)$ and the old moments of the original network as $\langle k\rangle_{t}=\langle k\rangle[1-p(g)]$ and $\left\langle k^{2}\right\rangle_{t}=\left\langle k^{2}\right\rangle[1-p(g)]^{2}+\langle k\rangle p[1-p(g)]$ [217]. Applying these results we obtain for the BA network a critical value $g_{c}$ as a function of $\lambda$ so that if $g<g_{c}$ the epidemic prevalence is zero

$$
g_{c} \simeq \exp (-2 / m \lambda) .
$$

The above equation clearly shows that critical immunization is exponentially small for a range of low values of $\lambda$. Although the above estimations are only valid for homogeneous random networks, we illustrate the effects of this type of immunization in SF networks in figure 6.3. The evolution of the SIR model on top of the Internet map at the Autonomous System level when there is no immune nodes (figure 6.3.a) and when targeted immunization is applied (figure 6.3.b). Besides, in figure 6.4 we show the results on both the AS and Router levels for three cases of targeted immunization and the case with zero immune nodes.

\section{Single Acquaintance Immunization}

Single acquaintance immunization (SAI) was introduced in [219] as the first local algorithm to immunize complex networks. In this strategy a fraction $p$ of the network nodes is selected at random and one of their corresponding neighbours is chosen at random and immunized. Then, only a knowledge of 


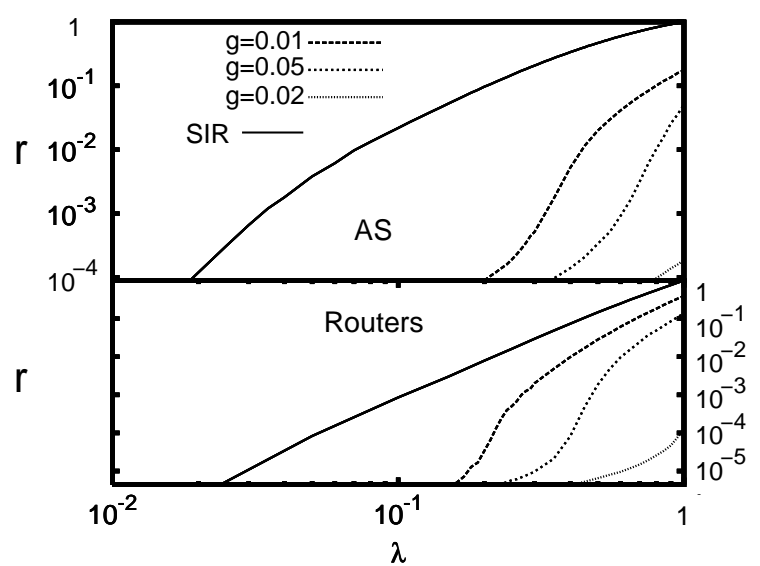

Figure 6.4: Final fraction of infected nodes for the SIR model and SIR with targeted immunization with different number of immunized nodes for the AS (a) and router (b) map representations of the Internet. Simulations were carried out starting from a single infected node at $t=0$ in all cases. The plots are in a $\log$-log scale for a better visualization.

the node neighbourhood is assumed and even the degree of its components is not needed for the performance of the SAI immunization. This is not the same scenario as for random immunization since the probability that a node with connectivity $k$ is chosen by one of its neighbours is proportional to $k P(k)$ and thus immunization of highly connected nodes is favored compared to the purely random situation [229].

The calculation of the critical fraction of nodes $g_{c}$ is performed looking at the percolation threshold of the network (considering again that immunized nodes and their links are removed from the network) so that the infection would become arrested in one part of the network. This calculation [219] yields a value

$$
g_{c}=1-\sum_{k} P(k)\left(1-\frac{1}{N k}\right)^{N k p_{c}},
$$

where $p_{c}$ is numerically computed from

$$
\sum_{k} \frac{P(k) k(k-1)}{\langle k\rangle}\left(1-\frac{1}{N k}\right)^{N(k-2) p_{c}} \cdot \exp \left(\frac{-2 p_{c}}{k}\right)=1,
$$

for every type of network depending on the degree distribution $P(k)$.

Single acquaintance immunization is a useful choice when only minimal network knowledge is available. However, this case is not so usual in real problems when neither the complete knowledge of the network (as targeted immunization assumes) nor the ultra-short horizon of nodes (where SAI proposal is applied) are present. An intermediate situation between targeted and SAI immunization is described in the next section. 


\subsubsection{Covering based Immunization}

The immunization strategy reported here has to do with a general class of problems in the context of graph theory: the problem of identifying the minimal subset of nodes that fulfills certain prescriptions. In particular, we report here on a heuristic method that allows to find near-optimal solutions to the covering problem in networks. The covering problem consists of obtaining the minimum set of covered vertices such that every vertex is covered or has at least one covered vertex at a distance at most $d$ ( $d$-covering problem). The introduction of the distance constrain leads to a wide applicability of the solutions found that can be considered as sets of servers in technological networks or large storing facilities in logistic networks as well as immunized nodes in contact or technological networks. Then, the algorithm implemented here will serve us not only to conveniently place those immune nodes that would help to stop the spread of the disease, but to consider more general problems. In fact, the allocation of network resources to satisfy a given service with the least use of resources is a frequent problem in communication networks. For the immunization problem, we would like to have a robust system in front of a disease or virus spreading process while saving resources, that is, using the minimum number of immune nodes.

For this purpose, we will make use of a heuristic algorithm that targets high degree vertices and computes an upper bound to the minimum fraction of servers needed to cover the network. We will apply the method to three real networks: the AS and Router level graph representations of the Internet and the Gnutella peer to peer network. As exposed in section 5.1.3 these graphs are qualitatively identical in what concerns the degree distribution and the smallworld property but, however, they differ from the point of view of the degree correlations between nearest neighbor vertices: the AS and Gnutella networks exhibit disassortative degree correlations whereas for the Router networks assortative degree correlations are displayed (see figure 5.3). The results shown below point out that the solution to the $d$-covering problem strongly depends on the degree of similarity between the connected vertices. As a consequence, we show that when designing networked systems, whether a centralized or distributed allocation of these immune/serving/storing nodes (henceforth called covered nodes for generality) is to be used relies upon the network properties at a local level. Therefore, the interest of applying this heuristic strategy to correlated real networks is twofold. First we will obtain a nearly optimal covering distribution and secondly we asses the impact of correlations on the design of networked systems.

Before explaining the heuristic method and the results obtained it is worth making a deeper analysis of the real nets considered here. In the $d$-covering 

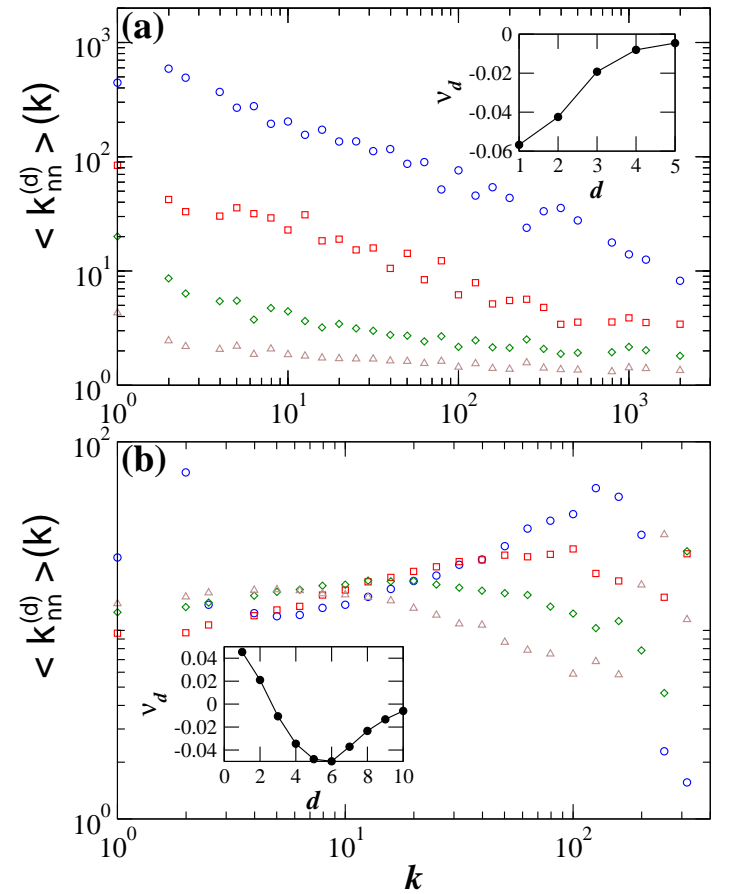

Figure 6.5: Average degree of the distance- $d$ neighbors of a vertex with degree $k,<k_{n n}^{(d)}>(k)$, for $d=1$ (circles), $d=2$ (squares), $d=3$ (diamonds) and $d=4$ (triangles). Note that the average neighbour degree, $\left\langle k_{n n}\right\rangle(k)$ introduced in section 5.1.3 corresponds with $<k_{n n}^{(1)}>(k)$. (a) $<k_{n n}^{(d)}>$ (k) vs $k$ for the AS graph. The inset shows the exponent $\nu_{d}$ obtained from the fit to the power law form $\left\langle k_{n n}^{(d)}>(k)=A k^{\nu_{d}}\right.$ in the range $k>1$. Similar results are obtained for the Gnutella graph, but with more fluctuations due to its small size. $(\mathrm{b})<k_{n n}^{(d)}>$ $(k)$ vs $k$ for the Router graph. The inset shows the exponent $\nu_{d}$ obtained from the best fit to the power law $<k_{n n}^{(d)}>(k)=A k^{\nu_{d}}$ in the range $10 \leq k \leq 100$.

problem one is interested in distances beyond $d=1$, therefore we also analyze the degree correlations for $d>1$ (see figure 6.5). For the disassortative graphs, the average degree of distance- $d$ neighbors $\left\langle k_{n n}^{(d)}>(k)\right.$, restricted to root vertices with degree $k$, follows the same trend as $\left\langle k_{n n}^{(1)}\right\rangle(k)$, tending to be less correlated for larger $d$ (figure 6.5.a). For the assortative graph, however, the degree correlations are assortative up to $d=2$, becoming disassortative for $d>2$ (figure 6.5.b). Finally, for $d>6$ the degree correlations in the originally assortative graph show a similar trend than in the disassortative graphs.

We propose the following heuristic algorithm to obtain an upper bound to the $d$-covering problem. Local algorithm: For every vertex in the graph, cover the highest degree vertex at a distance at most $d$ from the vertex. In case there is more than one vertex with the highest degree, one of them is selected at random and covered. To test this algorithm we first consider the case $d=1$, known as the dominating set problem [230]. In this case we can use a leaf-removal algorithm as a reference method, which yields a nearly optimal solution together with an error estimate. The leaf-removal algorithm is defined as follows. To each vertex $i$ we assign two state variables $x_{i}$ and $y_{i}$, where $x_{i}=0\left(x_{i}=1\right)$ if the vertex is uncovered (covered) and $y_{i}=0$ $\left(y_{i}=1\right)$ if the vertex is undominated (dominated). Here a vertex is said to be 
dominated if it has at least one neighbour covered. Starting with all vertices uncovered and undominated $\left(x_{i}=y_{i}=0\right.$ for all $\left.i\right)$, iteratively, $(i)$ select a vertex with degree one (leaf). If it is not dominated, cover its neighbour, set dominated its second neighbours, and then remove the leaf, its neighbour, and all their incident edges. ( $i i$ ) If no vertex with degree one is found, then cover the vertex with the larger degree (hub), set dominated its neighbours, and then remove the hub and all its incident edges. Finally, if some vertices with degree zero remain, they are covered if they are not dominated, and removed from the graph. Since step (i) always provides an optimal solution, the error in computing the average fraction of covered vertices $\langle x\rangle=\sum_{i=1}^{N} x_{i} / N$ is less than or equal to the fraction of vertices covered applying step (ii).

The comparison between the local and leaf-removal algorithms is shown in figure 6.6. First, notice that the solutions obtained with the leaf-removal algorithm are almost exact for the networks considered here and $d=1$. The local algorithm yields satisfactory, though non-optimal, solutions to the covering problem, with some differences depending on correlations between connected vertices. For the AS and the Gnutella graphs, which exhibit disassortative degree correlations, the local algorithm gives a good estimate, quite close to the optimal one for the AS graph. In contrast, for the Router graph we observe a larger deviation from the optimal solution. The origin of this difference is due to the fact that the local algorithm exploits the degree fluctuations among connected vertices. Indeed, these fluctuations are bigger in disassortative graphs as connected vertices likely have different degrees. In contrast, in assortative graphs, although there may be high degree fluctuations between two vertices selected at random, connected vertices tend to have similar degrees, resulting in poorer solutions. These results indicate that the general belief that heuristic algorithms targeting the hubs may be sufficient to solve computa-

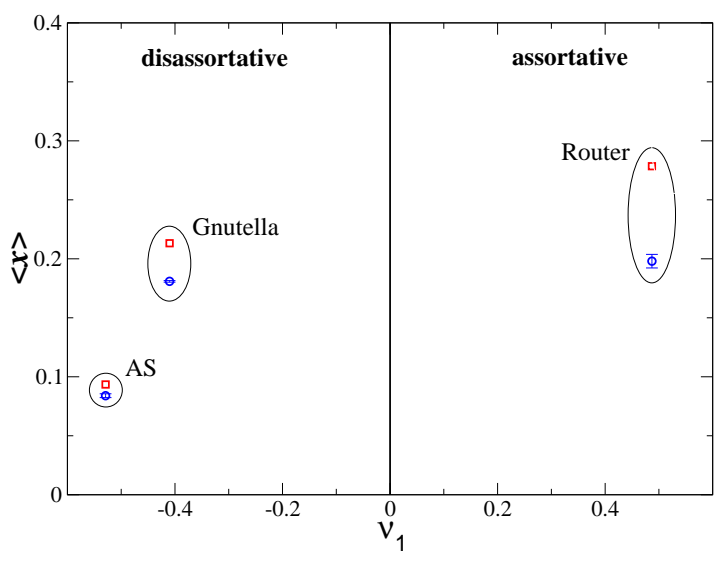

Figure 6.6: Average fraction of covered nodes $\langle x\rangle$ needed to cover a graph under the constraint that a vertex should have a server at most at a distance $d=1$, using the leaf-removal (circles) and local (squares) algorithms, as a function of the exponent $\nu_{1}$ defined in figure 6.5 , with negative and positive values corresponding to disassortative and assortative graphs, respectively. 

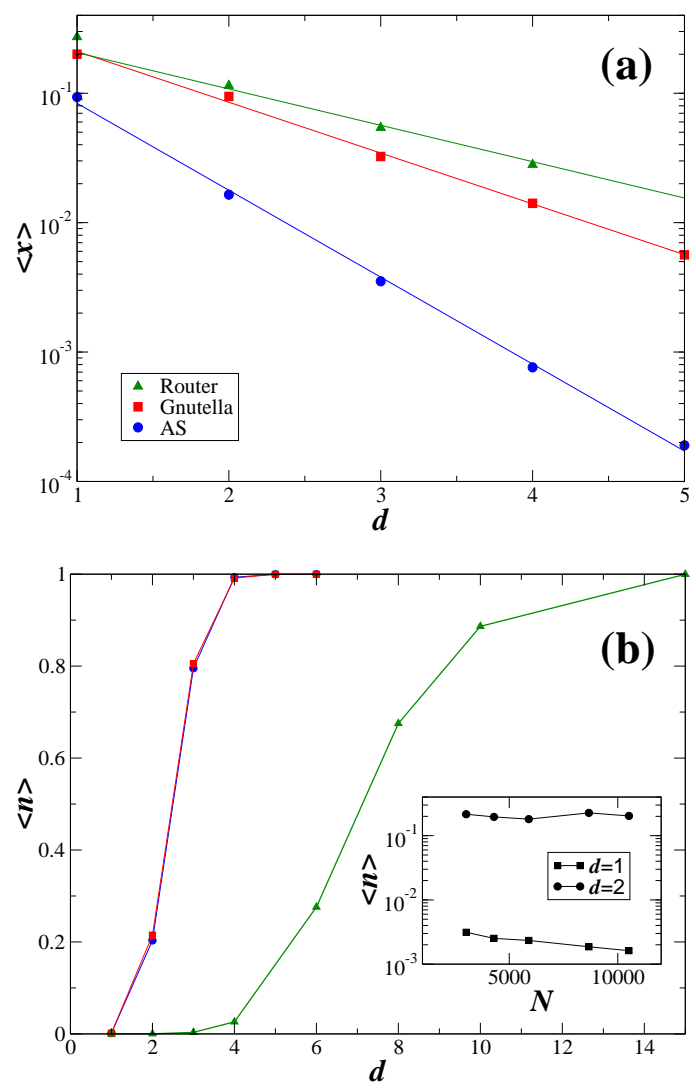

Figure 6.7: (a) Average fraction of servers $\langle x\rangle$ covering the graph for different values of $d$. The continuous lines are the best fits to an exponential decay. (b) Average fraction of vertices $\langle n\rangle$ served by a server for different values of $d$. The inset shows the graph size dependence of $\langle n\rangle$ for the AS graph and $d=1,2$.

tional problems on graphs with wide degree fluctuations may not be the case for assortative graphs.

The $d=1$ covering problem results in a distributed architecture because a finite fraction of the vertices is covered. Let us now extend the method and discuss the results obtained with the local algorithm for the more general and complex problem $d>1$. In figure 6.7.a we show that, with increasing $d$, the average fraction of covered nodes decays exponentially fast, indicating that if we allow the covers to be more distant, a substantial decrease in the number of required covers is obtained. This exponential decay is a consequence of the small-world property of these networks. The decrease in $\langle x\rangle$ is, however, achieved at the expense of an increase in the average fraction of vertices $\langle n\rangle$ served by a covered node (figure 6.7.b). This is a key metric as it marks the trade-off between the number of covers needed and their capacity. 

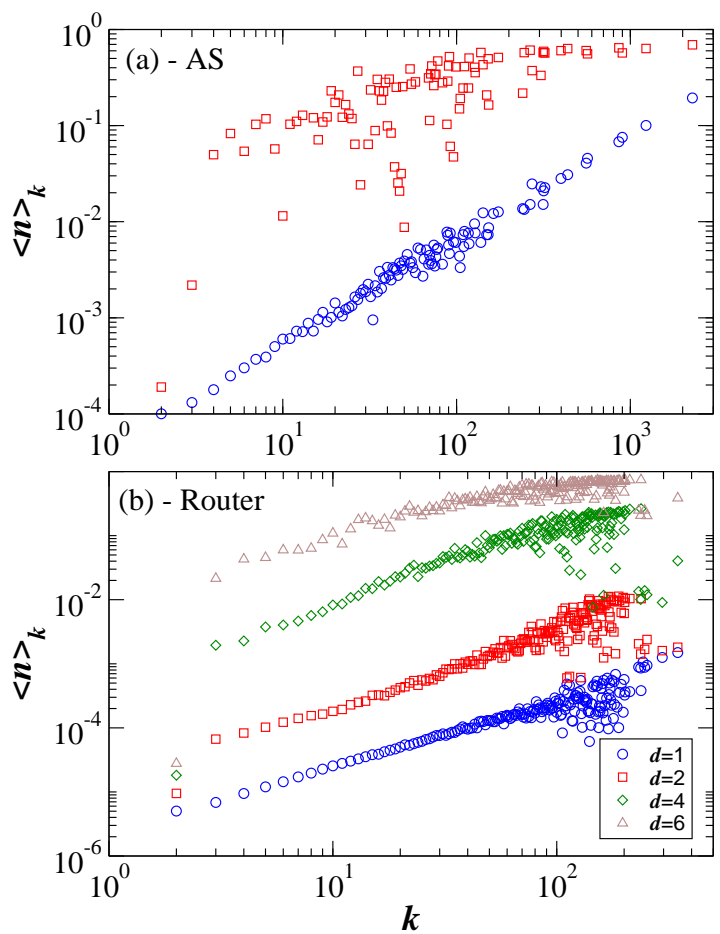

Figure 6.8: Average number of covered vertices $\langle n\rangle_{k}$ restricted to vertices with the same degree $k$ for several values of $d$. The figures show that for disassortative graphs (a), the covers should have a large capacity to serve a finite fraction of the graph even for small to moderate values of $d$. On the contrary, for assortative graphs (b), the fraction of covers is a negligible fraction of $N$ up to large values of $d$.

Again, a remarkable difference depending on the graph assortativities is appreciated. For the Gnutella and AS graphs, with disassortative correlations, $\langle n\rangle$ increases significantly from $d=1$ to $d=2$. Indeed, a finite size study for the AS graph, with a growing tendency from 1997 to 2002 [231], reveals that $\langle n\rangle$ decreases to zero with increasing the graph size for $d=1$, while it remains almost constant for $d=2$ or larger (see inset of figure 6.7.b). On the other hand, in the Router graph, with assortative correlations, $\langle n\rangle$ increases much slower with increasing $d$, being almost zero up to $d=3$ (figure 6.7.b). These results are the signature of a phase transition. There is a threshold distance $d_{c}$ such that the average fraction of vertices served by a covered vertex is very small for $d \leq d_{c}$, going to zero with increasing $N$, while it is finite for $d>d_{c}$. For disassortative graphs $d_{c}=1$ while for assortative ones $d_{c}>1$. Note that the value $d_{c} \approx 3$ for the Router graph coincides with the distance where the degree correlations become disassortative, indicating that the phase transition is determined by the change in the degree correlations. Furthermore, this transition gives a practical measure to get the desired trade-off between $\langle x\rangle$ and $\langle n\rangle$.

Since the graphs considered here are characterized by wide fluctuations in the vertex degrees, one can also compute the average number of covered vertices $\langle n\rangle_{k}$, restricted to vertices with the same degree $k$. In all cases an increasing 
tendency of $\langle n\rangle_{k}$ with $k$ is observed, as it is expected from the definition of the local algorithm, which targets high degree vertices. Two distinct behaviors are once again observed depending on the degree correlations. In the disassortative graphs, $\langle n\rangle_{k}$ is already as large as $10 \%$ of the vertices for $d=2$ and $k>10$ (figure 6.8.a). In contrast, in the assortative graphs, only beyond $d=4$, one observes that large value of $\langle n\rangle_{k}$.

The striking differences between disassortative and assortative correlations have important consequences form the practical point of view, for example, regarding how resources for immune response or fast recovering are allocated. For disassortative graphs, except for the case $d=1$, one would need covers with a vast capacity, serving a large fraction of vertices. The most efficient strategy is, therefore, the allocation of resources in a few covers with a large capacity. The scalability of the cover system would in this case be determined by the single cover capacities, which should be increased as the graph size grows. In the assortative case, we have a different scenario. The decrease of the number of covers with increasing $d$ is not as dramatic as for the disassortative graphs. In compensation, each cover serves a small fraction of vertices. Hence, the most efficient strategy is to allocate the resources in a large number of covers with a limited capacity. The scalability of the system would be driven by the number of required covers, which augments with increasing the graph size. In turn, regarding the design of communication networks, we can decide between disassortative or assortative topologies depending on the available resources. A disassortative topology will be more appropriate for a centralized design, with a few covers having a large capacity, while an assortative network will be best suited for a distributed design, when a large number of covers have a limited capacity.

\section{SIR with immune covers}

In order to apply the covered sets found to the problem of SIR epidemic spreading we will consider covered vertices as immune nodes to the spreading of a disease or virus. For instance, in a technological network, they could be thought of as being special devices devoted to filtering out any virus or attack. This would imply that the spreading process stops when it arrives to such nodes. This is of course the ideal situation. However, it happens more often that immune nodes can not catch the epidemic, but they are not able to stop spreading it through other nodes - as when you have an up-to-date anti-virus. Therefore, we study the worse scenario and consider that immunized nodes just repel the virus cutting the path to infection spreading.

We will consider the different $d$-cover sets labeled by the corresponding 


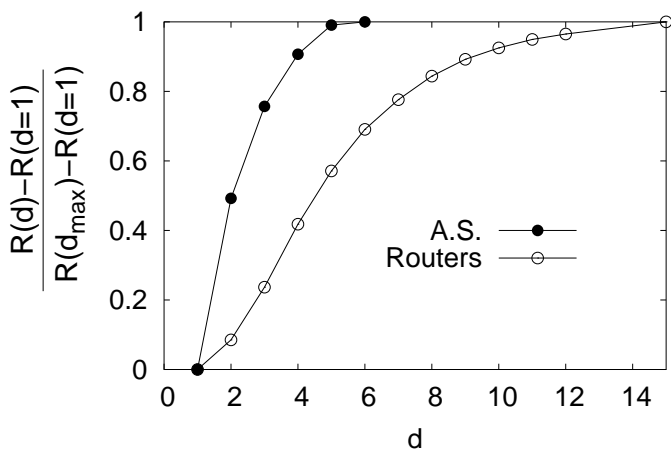

Figure 6.9: Relative difference of the epidemic incidence for different values of $d, R(d)$, with respect to that at $d=1(\lambda=1)$. The behaviour observed in the figure is determined by the number of susceptible nodes each immune vertex has to "protect".

distance $d$ used to solve the $d$-covering problem. For each distance we compute by Monte Carlo simulations the epidemic incidence, $R(d)$, in both the Router and AS representation of the Internet for a constant value of the epidemic transmission rate, $\lambda=1$, when their corresponding $d$-covers are considered as immune nodes. We will then focus on the influence of degree correlations on the final size of the outbreak ${ }^{2}$.

Figures 6.9 and 6.10 reflect the differences in the algorithm's performance for the AS and the Router maps of Internet. Figure 6.9 illustrates the relative difference of the epidemic incidence as a function of $d$, taking as a reference the size of the outbreak at $d=1$. The behaviour depicted in the figure is quite similar to the dependency of the number of nodes covered by each immune node, $\langle n\rangle$, when $d$ is increased (figure 6.7.b). For the AS network, the fraction of infected nodes at the end of the epidemic spreading process rapidly increases. In contrast, the increase in the epidemic incidence for the router network takes place at larger values of $d$. This indicates that for the same $d>1$, the immunization strategy works better at the router level as confirmed in figure 6.10, top panel. The reason of this behavior becomes apparent by noticing that for the router level $\langle x\rangle$ is bigger than for the AS, but the number $\langle n\rangle$ of nodes served on average by each immune node is smaller. The combination of the two factors leads to a more efficient immunization at the router level, however, at the cost of more resources. Both strategies tend to be closer as $d$ is increased because at the router level the correlations change beyond $d \geq 3$.

The previous result has to be carefully interpreted and should not be misunderstood. A closer look at the influence of the correlations reveals that, although in general they determine $\langle x\rangle$ and $\langle n\rangle$ for each map, these two quan-

\footnotetext{
${ }^{2}$ It should be noticed that a number of other topological features such as clustering and hierarchy properties may also be at the root of the different behaviors. Our guess is mainly based on the performance of the local algorithm that we will use below.
} 
tities alone do not suffice to explain all the differences observed. Indeed, the local structure of the network turns out to be at the root of the immunization efficiency and the optimal trade-off between the size of the outbreak and the least use of resources. To see this, we have analyzed the situation in which both $\langle x\rangle$ (though the d's are different) and $\langle n\rangle$ are almost the same in the two representations. This case is represented in the bottom panel of figure 6.10. As can be seen from the figure, in the latter case, the immunization scheme for the AS outperforms that for the router level. This behaviour is due to the fact that in the AS network, the immune nodes are more distributed throughout the network because highly connected vertices alternate with poorly connected ones. On the contrary, at the router level, the hubs are topologically closer to each other (the correlations are positive) and thus some of the immune nodes are not highly connected resulting in a less efficient protection in front of an epidemic.

In principle, one may think that as we are immunizing covers (highly connected nodes), the use of the (global) targeted immunization strategy would produce the same set of immune nodes. Obviously, this is not the case since the covering operates at shorter distances than targeted immunization (which operates at $d=D$, the diameter of network). In fact, a direct comparison of what the immune nodes are in both algorithms shows that no more than $50 \%$ of them are the same and both sets are equal only when $d$ reaches the diameter of the network. Moreover, as a further evidence of the influence of the graph
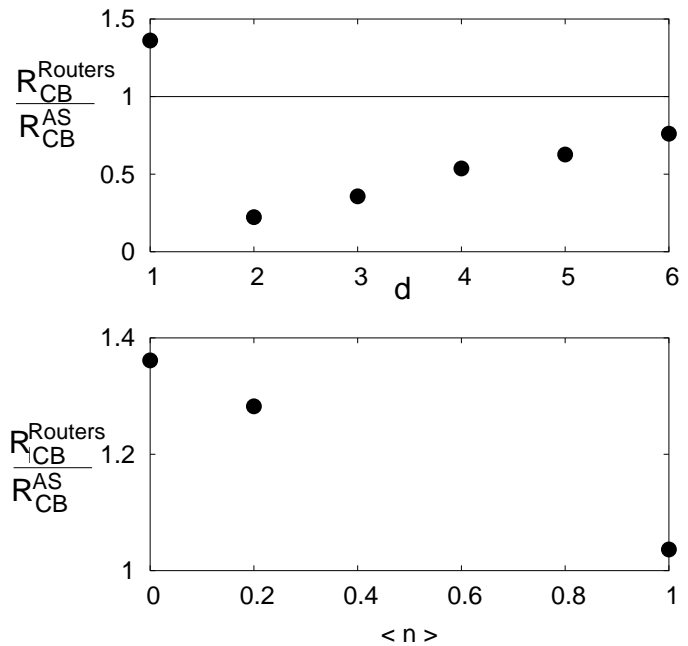

Figure 6.10: Comparison of the covering based immunization (CB) performance on the AS and router representations of the internet. Top: Phase transition is revealed by the best performance in Routers when $\langle n\rangle$ is bigger for $\operatorname{AS}(d>1)$. Bottom: On the contrary, when the nodes served by each immune vertex $\langle n\rangle$ is (roughly) the same, immunization works better in $\mathrm{AS}(\langle n\rangle=0$ for $d=1 ;\langle n\rangle \simeq 0.2$ for $d=2$ in AS and $d=5$ in Routers; $\langle n\rangle=1$ for $d=6$ in $\mathrm{AS}$ and $d=15$ in Routers). The results were obtained starting from a randomly chosen infected node and setting $\lambda=1$. 
representation in the performance of immunization schemes, it is found that for the router level the percentage above can increase up to $70 \%$.

\section{Comparing immunization strategies}

The next step is to compare the performance of all the immunization strategies presented in this chapter when SIR epidemic modeling is employed. It is worth stressing that the heuristic covering-based immunization proposed above is based on a tuned local knowledge of the network (only requiring information about the graph topology up to a distance $d$ ), a key property of utmost importance for most real applications. Indeed, all the graphs considered here are incomplete representations of the systems they are aimed to represent, as it generally happens in graph representations of large systems. An added value of the method developed here is that the covering-based strategy does not only deal with the degree of the immune nodes, as targeted immunization does, but naturally introduces the practical constraint of having limited resources to be distributed in the system on top of which the epidemics is spreading.

Before comparing the performance of the different algorithms let us illustrate the importance of the local properties of the network on the performance of targeted immunization. The results depicted in figure 6.4 suggest that again the degree correlations is one of the main factors influencing the performance of the immunization policy. We see that even for small percentages of immune nodes, targeted immunization performs better in the AS graph. This may be due to the compact distribution of hubs (which play a key role in targeted immunization) in the router map whereas for the AS representation they are distributed throughout the whole network. Therefore, in the AS representation, targeted immunization works better because immune nodes are more efficient in cutting the paths leading to poorly connected nodes, the more abundant.

We now focus on the implementation of the SIR epidemiological model on top of the Internet maps at the AS and router levels and compare with the results obtained by using targeted, random, SAI immunization as well as the heuristic covering-based immunization strategy described above. The results indicate that the local algorithm performs quite well and is near the optimal one. We have performed Monte Carlo simulations of the SIR model on top of the Internet maps starting from an initial state in which a randomly chosen set of nodes corresponding to a $1 \%$ of the network is infected. The results have been averaged over at least 1000 realizations corresponding to different initially infected nodes. We have performed extensive numerical simulations making use of the four different immunization schemes. The immunization obtained following the covering algorithm fixes the fraction, $g_{c}=\langle x\rangle$, of immune 


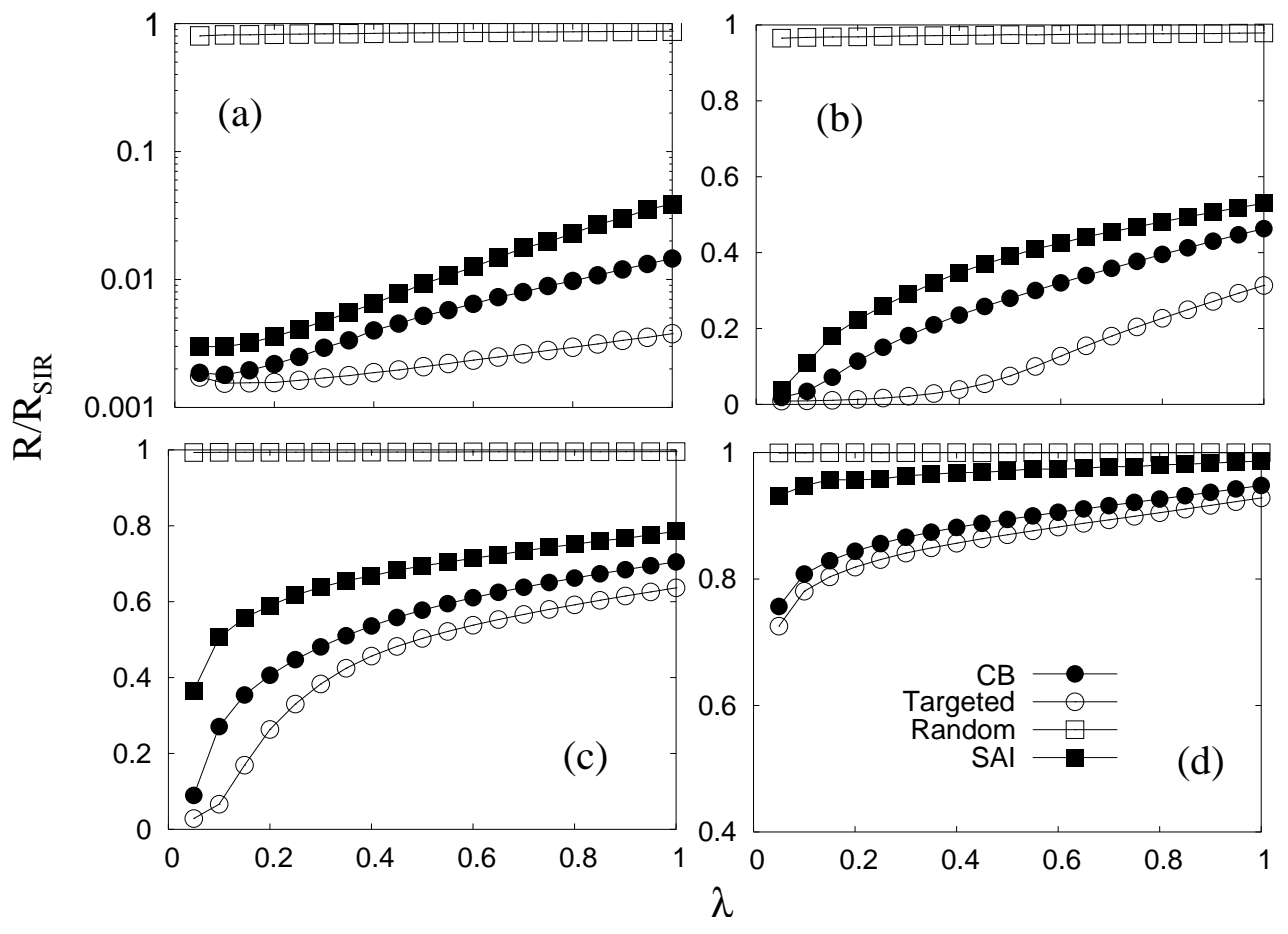

Figure 6.11: Comparison of the immunization strategies for the Internet AS map. In the figure, we have represented the ratio between the epidemic incidence of the four immunization strategies considered $(R)$ and that of the original system without immunization $\left(R_{S I R}\right)$ for different values of $\langle x\rangle$. The legend refers to the following immunization strategies: the one based on the covering problem $(\mathrm{CB})$, targeted immunization (Targeted), random immunization (Random) and single acquaintance immunization (SAI). In this case, $1 \%$ of the non-immune nodes were initially infected at random. See the text for further details. The distances considered in the local algorithm are: (a) $d=1$, (b) $d=2$, (c) $d=3$, (d) $d=5$.

nodes in the whole network for each value of $d$. Then, we cannot compare the performance of the four immunization mechanisms as a function of the fraction of immune nodes $g_{c}$ in a continuous way. Instead, we have to fix this quantity $g_{c}$ for random, targeted and SAI immunization to a discrete set of values $\left\{g_{c}(d)\right\}=\left\{\langle x\rangle_{d}\right\}$ prescribed by the corresponding solutions of the $d$-covering problem of each graph. Then, Random immunization means that a fraction $g_{c}(d)=\langle x\rangle_{d}$ of immune nodes is randomly placed on the networks. Targeted immunization looks for the $\langle x\rangle_{d} N$ highly connected nodes and immunizes them and, finally, the single acquaintance immunization algorithm proposed is run taking $p \geq\langle x\rangle_{d}$ and ensuring that the total number of immune nodes is the same in all the schemes. In all cases, the results are again averaged over many realizations for each value of $\lambda$ and $\langle x\rangle$. The results are displayed in figure 6.11 


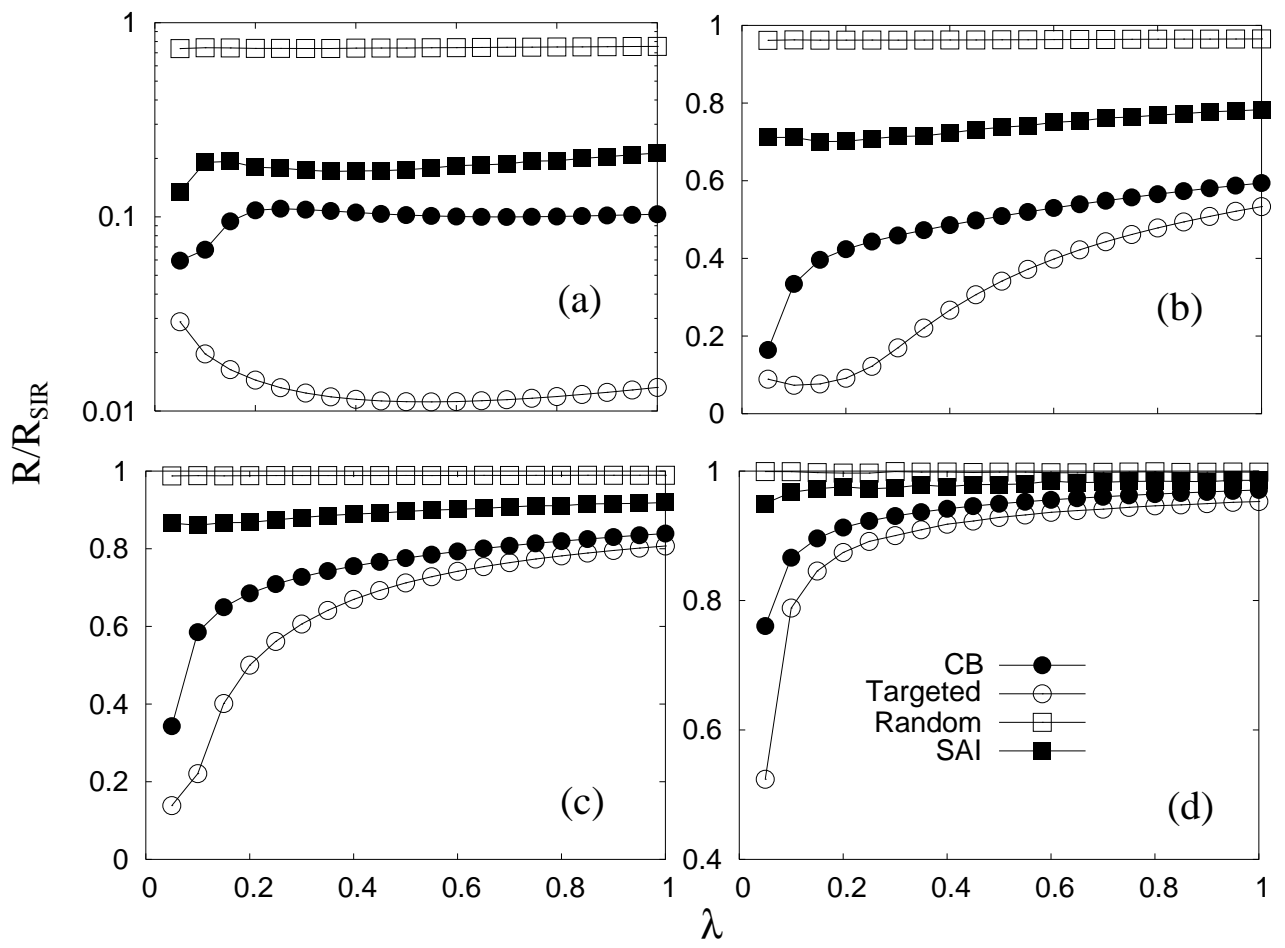

Figure 6.12: Same as previous figure but for the Internet router map. The distances considered in the local algorithm are in this case: (a) $d=2$, (b) $d=5$, (c) $d=7$, (d) $d=10$.

and figure 6.12.

As expected, targeted immunization produces the best results for both topologies. Note that, as discussed in the previous section, the performance of the algorithm depends on the specific topology and produces different results for AS and router maps. On the other extreme we find random immunization, whose performance is not affected by the structure of the underlying networks. Turning our attention to local algorithms, it is found that the immunization scheme based on the covering algorithm performs better than the SAI, even for small values of $d$, where it is truly local. In fact, it is outperformed only by the targeted procedure and for all values of the parameters $d$ and $\lambda$ it lies between the most efficient and the SAI scheme. Additionally, from a practical point of view, the covering strategy could be a good policy since it balances the degree of local knowledge and the efficiency of the vaccination. Moreover, as all network topologies are not neither completely known nor completely unknown, the covering allows to fine-tune the value of $d$ on a case-by-case base (that is, according to the degree of local knowledge of the network) and thus it 
is more flexible than other immunization strategies (recall that it is the result of an optimization).

The strategy introduced has been shown to perform better than all previous methods irrespective of the degree of local knowledge, except for the case of targeted immunization. We finally remark that the introduced covering-based immunization could be more appropriate when more complex immunization devices are placed in the network nodes since the distance constrain allows for a fast communication in order to stop the spread by a possible response mechanism departed from covered nodes.

\subsection{Information transmission and Jamming}

The problem of epidemic propagation shares a common feature with other dynamics like rumour diffusion [232]: the set up of the problem is the spreading across the network of some signal (epidemy, rumour, information,...) launched from a small set of nodes (spreaders) and the study is focused on whether a significative fraction of the network is finally affected (or reached) by this signal. Here, we will address a different class of propagation problems in networks where, as well as the source nodes, the destination of the information is well defined and unique for a particular signal. In this case, the information dynamics between any given source and the destination will end when it arrives to the target. Besides, the information between every pair of nodes follows a single path and it is not bifurcated during its trip as occurs in spreading processes. In this context we find interesting applications to information dynamics between agents in real networks like the internet. The interesting phenomenon found in this class of problems has to do with the existence of overloads and failures due to the excess of information carried on top of the network. This is usually known as jamming. We are concerned in whether or not a network architecture or a routing policy for the information packets is more favorable for handling a large amount of traffic in the network. In principle, the finding of SF character in information networks like the Internet is naively attributed to the compact architecture achieved with this design. However, although the existence of hubs in these networks allows the fast distribution of the information between pairs of nodes, these highly connected nodes are exposed to a large amount of data and thus will be easily congested. Therefore, a detailed analysis of the interplay between both the topology and the routing strategies is needed. We begin this chapter with a review on the more interesting works on the subject and then report two studies on the influence of these two important ingredients (topology and routing) on the functioning of information dynamics. 


\subsubsection{Information dynamics on Networks}

Real data analysis of traffic levels on real computer networks has provided the characterization of information flow [233-235]. This characterization allows for accurate modeling of information dynamics on networks. Among these reported observations and measures are:

- Long-range correlations and self-similarity are observed in the time evolution patterns of the number of data packets, $A(t)$.

- Power law behaviour in the power spectrum of the time activity of the network load,

$$
S(f)=\left|\int_{-\infty}^{\infty} A(t) \mathrm{e}^{\mathrm{i} f t} d t\right|^{2} \sim f^{-\xi},
$$

where $\xi$, called the Hurst exponent, was found to be $\xi \sim 1$.

These findings were attributed to the existence of a dynamical phase transition due to jamming in the system. The explanation of this critical behaviour of the system has been explored by means of models where this large scale organization was reproduced. However, the reasonable doubt about the influence that the substrate network has on the critical behaviour makes essential the incorporation of such ingredient in order to obtain a complete description of the system's dynamics. The availability of the topology of these type of communication systems allows for a detailed description incorporating both the complex dynamical rules and the topology of interactions among the constituents.

Computer network traffic can be modeled in diverse ways. However, the general picture of a traffic model consists in a constant (in average) injection of $p$ packets per unit time in the network. Each packet, which is created with a source node (sender) and a target one (recipient) assigned, is delivered from a sender to a recipient by hops between adjacent nodes and when the packet arrives to its destination it dissapears. The main magnitude used to describe the state of the system is the total amount of traffic in the network which is usually defined as the number of active packets that are currently searching their destination at time $t, A(t)$. The balance between delivered packets and incoming ones governs the behaviour of the system. In the free flow state the balance is reached after a transient time and the network is able to deliver packets at the same rate as new ones are introduced $(A(t)$ is constant in average). This is obviously found for low values of $p$ but, on the other hand, when $p$ is high enough the network is unable to handle the load of information and it gets jammed yielding an unbounded growing of $A(t)$. This scenario is common for a variety of models although they are different concerning particular details. These peculiarities can be summarized as follows: 
- Each node of the the network can be modeled as either a router or a host. A router node simply store and distributes among its neighbours all the traffic packets that it receives from them. On the other hand, host nodes are, at the same time, senders and recipients of the packets and hence the generation and death of information packets occurs at them. Although this node classification was considered in [236-238], in most models host and routers are not distinguished so that all nodes are senders, routers and recipients of the packets at the same time.

- The strategy employed by routers to decide which neighbour is the most convenient for a packet to move in depends on the particular model we use. This is frequently based on the router knowledge, local or global, on the placement of the destination node of the packet. It is frequently assumed that a router knows its relative position to every node in the network and therefore the packet follows a shortest path between its sender and its destination [239]. However, this is not a realistic assumption and randomness is usually incorporated to the routing protocol when the destination node is not found on the router horizon [240-243].

- The relation between the number of packets a router can deliver per unit time (the routing rate), $r$, and $p$ is also meaningful. It is always assumed that a router can only deliver one packet per unit time $r=1$ and then no rescaling is needed. This routing limitation introduces the concept of router queues which are composed by the packets allocated in a node waiting for being delivered. The maximum amount of packets a router can hold is called the buffer capacity, $H$. The general assumption is that buffer capacity is infinite but there are models where buffer size is limited. In these models, packets moving into a node with a full buffer are lost and never reach their destinations. Therefore, in these models the jamming picture described above is not valid anymore since the network has a limited capacity of active processes, $\max [A(t)]=N \cdot H$, and another quantity accounting for lost packets is then necessary.

- There are several ways for routers to manage the packets allocated in their buffers. One can consider that the router picks up the packet which is at the head of the queue at each time step, this is the so-called First-InFirst-Out (FIFO) queue. On the contrary in Last-in-First-Out (LIFO) queues it is the last packet in the queue the one chosen by the router. Queues where packets are chosen at random at each time step are also considered.

The different models reported below belongs to the above described general picture. The efforts are always directed to capture the jamming phenomena 
deduced by the experimental observations and, at the same time, to obtain the simplest and the most tractable modellization of the system.

\section{The Ohira-Sawatari model}

One of the first attempts to reproduce the jamming transition on computer networks was proposed by Ohira and Sawatari [239]. This model distinguish two classes of nodes: hosts and routers (which are capable of queuing an unlimited number of packets). In this work the routing strategy was based on shortest paths (thus assuming that routers have global knowledge of the network architecture). In general more than one neighbour can be chosen due to degeneracy in the shortest path from the router $i$ to the destination node $k$ of the information packet. Then, the router has to decide what neighbour is the best choice among the set of neighbours that are in the way of a geodesic to $k, L(i, k)$. Ohira and Sawatari used the following probability for each possible neighbour to catch the packet

$$
P_{j}^{k}=\frac{\exp \left(-\beta X_{j}\right)}{\sum_{l \in L(i, k)} \exp \left(-\beta X_{l}\right)}
$$

where $X_{j}$ is the number of packets routed by $i$ towards $j$ in the past. Besides, $\beta$ has the role of an inverse temperature in order to have an interpolating parameter from the complete deterministic routing when $\beta \rightarrow \infty$ (with long time routers memory) and the random $\beta \rightarrow 0$ routing (where packets are distributed at random among the possible shortest paths). Between these two limits the probabilistic routing can be explored. The model has been studied on simple network architectures as 2D lattices with hosts on the boundaries. As a function of $p$, it shows a sudden transition to a congestion state. The congestion was measured in terms of the average travel time of packets. The relevant result is that the phase transition point, $p_{c}$, depends on the routing strategy adopted $(\beta)$. In particular, a high degree in randomness $(\beta \simeq 0.01)$ in the path choice is found as the optimal routing policy achieving the maximum shift of the onset of traffic congestion.

\section{Cyclic search routing}

The use of shortest path routing is linked to the unrealistic assumption of global knowledge. Besides, this is not the best routing strategy when dealing with SF networks because of the fast congestion of highly central nodes (which usually are the hubs) that lead to a global congestion in the network. To avoid these problems Tadić et al [240-243] have developed the so-called cyclic search routing. This strategy consist of employing a global random routing 
joined to a local shortest path strategy. The implementation of such strategy is as follows: every router has a finite horizon of radius $d$ so that if the target of a packet is inside this horizon the packet is directly moved towards the target following the shortest path, otherwise it is randomly sent to one of the router neighbours. The simulations of this cyclic search algorithm have been performed on top of a variety of network topologies including SF networks. Besides, the jamming transition for high values of $p$ the dynamical behaviour in the free flow phase was explored. They study the patterns of the time evolution of the network load, $A(t)$, as a function of $p$. In the free flow regime, well below the critical point, the power spectrum of the network activity followed a power law $S(f) \sim f^{-\xi}$ with exponent $\xi=1.2$ for the so-called "Web graph"

3 . In this regime the queue effects are negligible and thus the topology is governing the system behaviour. When the system approach the transition, $p \rightarrow p_{c}$, the $A(t)$ patterns manifest crisis-like activity with sudden growths of the load for relative large windows and the power spectrum of $A(t)$ lose its temporal correlations manifested by an increase of the Hurst exponent, $\xi \simeq 2$. The waiting times (the time a packet spends in routers queue during its trip) distributions were also investigated. The results for the free flow regime showed a power law distribution compatible with those obtained empirically in [233] for the Internet dynamics. However, when the critical point was approached (and hence crisis in $A(t)$ were found) this distribution turned into a Cauchy-type showing long queue times.

\section{Self-regulated traffic}

The findings using cyclic search algorithms about the fluctuations on the load activity in the free flow regime are in agreement with the results when analyzing real time series of data traffic in real networks. Besides, the Internet data analyzed does not (or rarely) manifest a behaviour similar to that of the jammed regime when the activity grows over large times. On the other hand, the whole system efficiency is achieved for $p$ values near the critical point when the average constant activity $A(t)$ is maximum. Valverde and Solé suggested [236] that there is a feedback between users and the system activity so that users demand enhances the congestion of the system but as congestion increases users tend to slow down their requests and tend to leave the network. This feedback has the overall result of set the system operation point near the onset of congestion. This self organization of the system was proposed in a model where router and host nodes where differentiated [237]. They proposed a mean field model for the evolution of the density of information packets

\footnotetext{
${ }^{3}$ This is a directed graph displaying SF behaviour and high clustering and degree correlations [244]
} 
$\Gamma(t)=A(t) / N$

$$
\frac{\mathrm{d} \Gamma}{\mathrm{d} t}=p R-\tau^{-1}\langle k\rangle \Gamma(\Gamma-1)
$$

where $R$ is the density of host and $\tau$ is the mean life time of information packets ( $\tau \simeq\langle L\rangle$ in the free flow state). The fixed point solution guarantees that freeflow is achieved. When this solution is lost the jamming regime is reached. This occurs for eq. (6.30) at $p_{c}=\langle k\rangle /(4 \tau R)$. Assuming the feedback between the density of hosts and the delivery rate $p_{c}$ in order to reach a constant activity $\Gamma^{\star}$ the model predicts a scaling between these two quantities of the form $p \sim R^{-1}$. In [238] the authors have studied an improved mean field model including $p$ as a dynamical variable.

\section{Search and Congestion}

Shortest path routing lead to the problem of the fast congestion of hubs. Another possible alternative to the cyclic search was proposed in [245-247] where the routing mechanism studied takes into account node congestion. In these works packets follow paths of minimum length from their origin $s$ to their destination $l$. At each time step, all the packets allocated in the nodes try to move from its current position $i$ to the next node $j$ in their path with a probability $q_{i j}$ which is called the quality of the channel. This magnitude is defined in terms of the capabilities of the two nodes, $q_{i}$ and $q_{j}$ as $q_{i j}=\sqrt{q_{i} q_{j}}$ so that, when one of the nodes has capability 0 , the channel is disabled. High qualities $\left(q_{i j} \simeq 1\right)$ imply that packets move easily, while low qualities $\left(q_{i j} \simeq 0\right)$ imply that it takes a long time for a packet to jump from one node to the next. It is assumed that $q_{i}=f\left(n_{i}\right)$, i.e. the capability of a node $i$ is a function of the number of packets $n_{i}$, currently at node $i$. The general function $f(n)=1$ for $n=0$ and $f(n)=n^{-\gamma}$ for $n=1,2,3, \ldots$, with $\gamma \geq 0$, has been considered. For $\xi>1(\xi<1)$ the number of delivered packets from $i$ to its neighbour $j$, $i \rightarrow j$, is proportional to $n_{i}^{1-\gamma}$. Then, the transmission between two adjacent nodes decreases (increases) with the number of accumulated packets. For the special case $\gamma=1$ the number of delivered packets is independent of the number of accumulated packets. This routing policy has been studied in 1D, 2D lattices and Cayley trees. To characterize the jamming transition the authors employed an order parameter, $\rho$, defined as

$$
\rho=\lim _{t \rightarrow \infty} \frac{A(t+\delta t)-A(t)}{\delta t \cdot p N} .
$$

A smooth critical transition to congestion is found only for $\gamma=1$, while for $\gamma>1$ the transition to congestion is discontinuous and jumps from $\rho=0$ to $\rho=1$ at $p_{c}$. This is due to the progressive deterioration of the transmission channels as congestion grows in the network leading to a state where no packets 
are transmitted and thus $\rho=1$ at the end. This final state is reached by the emergence of a congestion nuclei. The behaviour of the spectra of $A(t)$ for $p<p_{c}$ was also analyzed showing a power law behaviour $S(f) \sim f^{-\xi}$ with $\xi \simeq 2$.

The choice of an specific routing policy (shortest path, random, cyclic search, etc...) is equivalent to define an effective distance matrix between every pair of nodes (we will return to this picture in section 6.2.3). Obviously, when the shortest path algorithm is chosen this effective distance will be the same as the topological one. In [248] the authors developed a general formulation of the model of traffic dynamics by making use of the probability that a packet located at a node $i$ and whose destination is a node $k$ will move into node $j$ in the next hoop, $p_{i j}^{k}$. The construction of these probabilities depends on the particular routing algorithm employed and several magnitudes can be expressed in terms of them. For example, the probability for a packet with target $k$ to travel from $i$ to $j$ in $n$ steps

$$
P_{i j}^{k}(n)=\sum_{l_{1}, \ldots, l_{n-1}} p_{i l_{1}}^{k} \cdot \ldots \cdot p_{l_{n-1} j}^{k} .
$$

The above probability $P_{i j}^{k}(n)$ allows to calculate the average number of steps between $i$ and $j$ for a packet traveling to $k, d_{i j}^{k}=\sum_{n} n P_{i j}^{n}$, which defines the matrix $\mathbf{d}^{k}$. Then, the element $d_{i k}^{k}$ is the effective distance from $i$ to $k$, i.e. the main topological magnitude governing the flow of data traffic at a given node when shortest path routing is implemented. For this general situation the effective betweenes of the nodes can be also calculated as $B_{j}=\sum_{i} \sum_{k<i} \sum_{n} P_{i j}^{k}(n)$. Hence, for a general routing strategy when $p$ packets per unit time and node are sent and the routing rate is $r$ a node $j$ will be congested when $p B_{j} /(N-1)>r$. For the whole network one can establish a lower bound for the critical point at $p_{c}=r(N-1) / B^{\star}$, where $B^{\star}$ is the maximum node betweenes. The general formulation of the traffic problem performed in this work is of great interest since it allows avoiding extensive numerical simulations of the hard problem of looking for optimal topologies when a given routing strategy is prescribed. In fact the authors found that for a random routing strategy a dramatic change in the optimal network topology is obtained when $p$ is increased jumping from a highly centralized star-like topology at low values of $p$ to a homogeneous one when $p$ grows.

\subsubsection{Shortest path routing}

After the above brief summary on the modeling of information dynamics on networks we focus now on the influence of network structure on the data flow efficiency. Here we will consider a simple routing mechanism based on shortest 
path routing so that we assume global knowledge of routers. In order to discuss how the local topological properties influence the efficiency of a given routing protocol, we use the network studied in section 5.3. Let us recall that in this model, the network is generated by considering the Barabási-Albert procedure [145] (section 5.2.3) but introducing an affinity variable $f_{i}$ and a tolerance $\mu$, which determine the peers $j$ a new node can attach to. This is done by requiring that $f_{j} \in\left(f_{i} \pm \mu\right)$. This network shows the same global properties of the BA graph, like the SF degree distribution, regardless of the tolerance. However, depending on the value of $\mu$, other local properties, such as the clustering coefficient and correlations, differ from the original BA network. The clustering coefficient showed the major deviation compared to the values at the BA limit (where clustering effects are negligible) and grows as $\mu$ decreases. Besides, the average path length $L$ remain nearly constant for a wide range of $\mu$ values but shows a sudden increase when the tolerance approaches $\mu=0$. These two quantities, clustering and average path length, are of importance for the information disemination on networks as we will show below.

Let us now define the set up of the problem. We will assume that routers deliver data packets by ensuring that all routers converge to a best estimate of the path leading to each destination address. In other words, the routing process takes place following the criterion of the shortest available path length from a given source to its destination. We will consider a situation consisting of an initial amount $p$ of information packets to be transmitted across the network. That is, we will not consider the situation before where the network is subjected to a constant flux of processes like in the works described but, instead, we will study how the system relax to its "ground state" $(A(t)=0)$ when an initial perturbation is performed. Then, in our simulations $p$ packets are created at the beginning and both their destinations and the sources are chosen at random. In subsequent time steps, each node $i$ holding a packet sends it to its destination $j$ following the shortest path length between node $i$ and $j$ until all packets reach their destinations. That is, each packet is diverted in such a way that the distance $d_{i j}$, measured as the number of nodes one needs to pass by between $i$ and $j$, is minimized. In the case that there are more than one possible path, the choice is made at random. Besides, we consider that routers deliver $r=1$ packet per unit time and that the router buffer size is infinite, $H \rightarrow \infty$.

The above procedure is repeated many times for a number of processes ranging from $p=1$ to at least $p=500$. Different realizations of the dynamics and the network substrate for the same $p$ are performed in order to average the relevant quantities. As a measure of the efficiency of the process, we have monitored the relaxation time, $\left\langle T_{\text {relax }}\right\rangle$, by computing the maximum time it takes for a packet to travel from its source to its destination, averaged over 


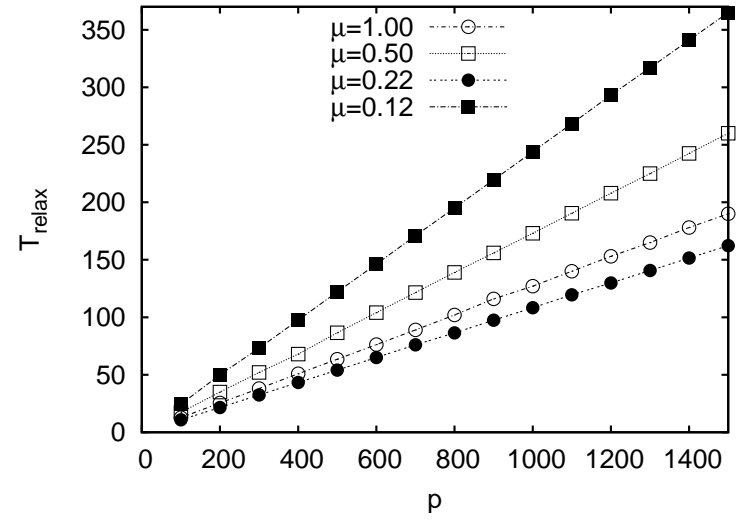

Figure 6.13: Averaged relaxation time, $T_{\text {relax }}$, as a function of the initial number of packets, $p$, for several network topologies (using the model described in section 5.3) corresponding to $\mu=1.0$ (BA limit), $0.5,0.22$ and 0.12 . As can be observed $T_{\text {relax }}$ scales linearly with $p$ for all the networks.

different realizations ${ }^{4}$. Since the SF topology is shared by all the networks studied here, we expect that the packet dissemination follow the same trend for any value of $\mu$. That is all the packets will tend to concentrate into the hubs queues at first instance. In these queues packets will spend most of their life times so that the influence of other topological factors would be captured by the convergence and scape times of the trips into and from the central hubs core.

The numerical results show that this magnitude scales linearly with the number of processes as can be expected from the simple shortest path routing protocol. An example is shown in figure 6.13 where the linear scaling is shown for several values of $\mu$. Therefore, the derivative of $T_{\text {relax }}(p)$ is a proper parameter to characterize the routing performance. figure 6.14 shows the slopes of the straight lines as a function of the control parameter $\mu$ which determines the local properties of the network. It is clear from the figure that the algorithm's outcome depends on the topological details of the network. For the family of networks labeled by $\mu$ the average shortest path length $L$ is roughly the same as that of the BA network up to a value around $\mu \simeq 0.2$. This fact somehow breaks the entanglement between the influence of the characteristic length and other local properties of the network on the packet dynamics allowing to study them separately. As shown in figure 6.14, the efficiency has a well defined maximum and a minimum in the range $\mu$ values $0.2<\mu<1$ where $L(\mu)$ remains almost constant. This implies that local properties are responsible for the behavior observed, namely the clustering coefficient $c$.

We have distinguished four $\mu$-ranges in the figure depending on the performance of the packet dynamics relative to that of the BA network. In region I, $0.8<\mu<1$, we find that the performance is almost the same as in the BA

\footnotetext{
${ }^{4}$ Note that the choice of $\left\langle T_{\text {relax }}\right\rangle$ is arbitrary. One can also use $\left\langle T_{a v g}\right\rangle$ or $\left\langle T_{r m s}\right\rangle$, which leads to the same behaviours.
} 


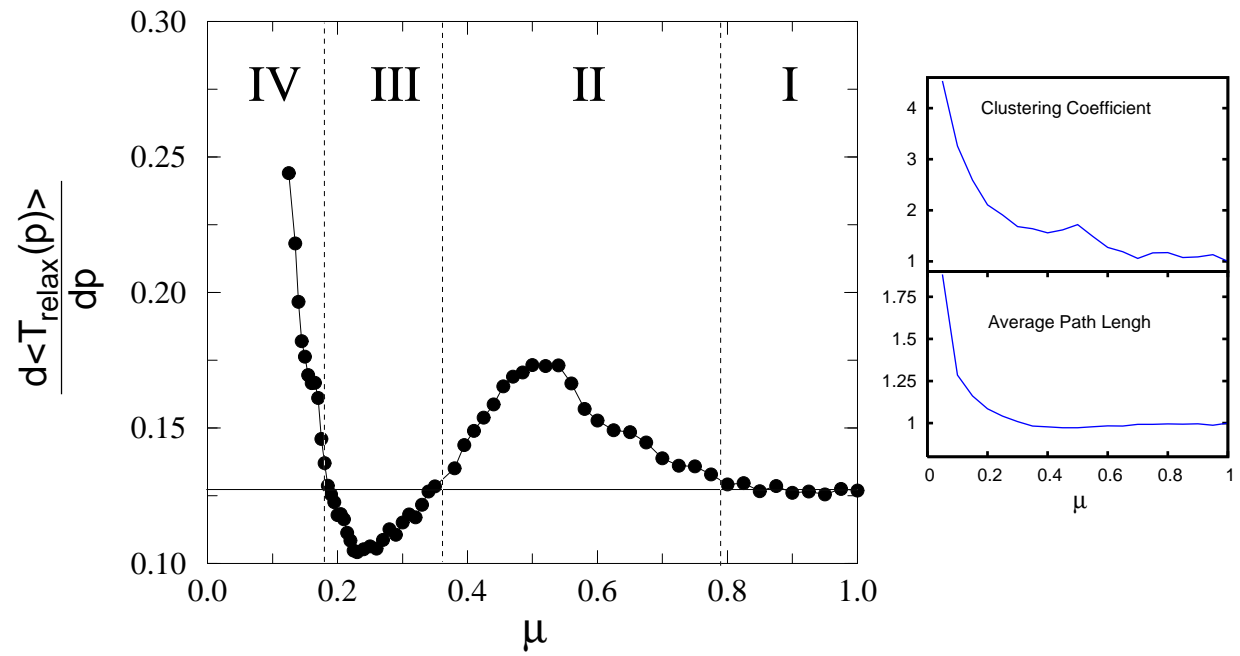

Figure 6.14: $\partial\left\langle T_{\max }\right\rangle / \partial p$, as a function of the network parameter $\mu$. The figure illustrates the dependency of the standard routing protocol on the local properties of the network. The right panels show the variation of $c$ with $\mu$. The size of the network is $N=10^{4}$ nodes and $m_{o}=m=3$. The degree distribution is a power law with exponent equal to 3 . Note that the BA limit corresponds to $\mu=1$. See the text for further details.

limit. In this range both clustering and $L$ show the same values as the BA network. Region II and III, $0.35<\mu<0.8$ and $0.2<\mu<0.35$ respectively, show a different performance with respect to the BA topology. In region II the relaxing times increase significantly reaching a maximum at $\mu \simeq 0.5$. On the other hand, in region III an enhancement in packet diffusion is manifested revealing an optimal topology for packet difussion at $\mu \simeq 0.22$. In both regions II and III the deviation from the BA performance can be only atributted to the clustering growth since $L$ is almost constant in the whole $\mu$-range. The result is apparently contradictory since the growing behaviour of $c$ as $\mu$ decreases is held for the two regions. However, it can be explained in terms of the clustering evolution. Since the number of links is constant for all the networks explored here (due to the growth mechanism employed in section 5.3) an initial growth of the clustering yields to the appearance of loops of length 3, triangles. These structures are useless for the shortest path routing, as figure 6.15.b shows, in the sense that those links used to link neighbours of a given node $i$ does not contribute anymore to any shortest path from $i$ to other nodes in the network. However, if the clustering is further increased the probability of forming loops of length 4 is incremented, see figure 6.15.c, and then the degeneracy in the shortest path from pairs of nodes is incremented. This fact is very important for shortest path routing with congestion since the queue times are decreased 


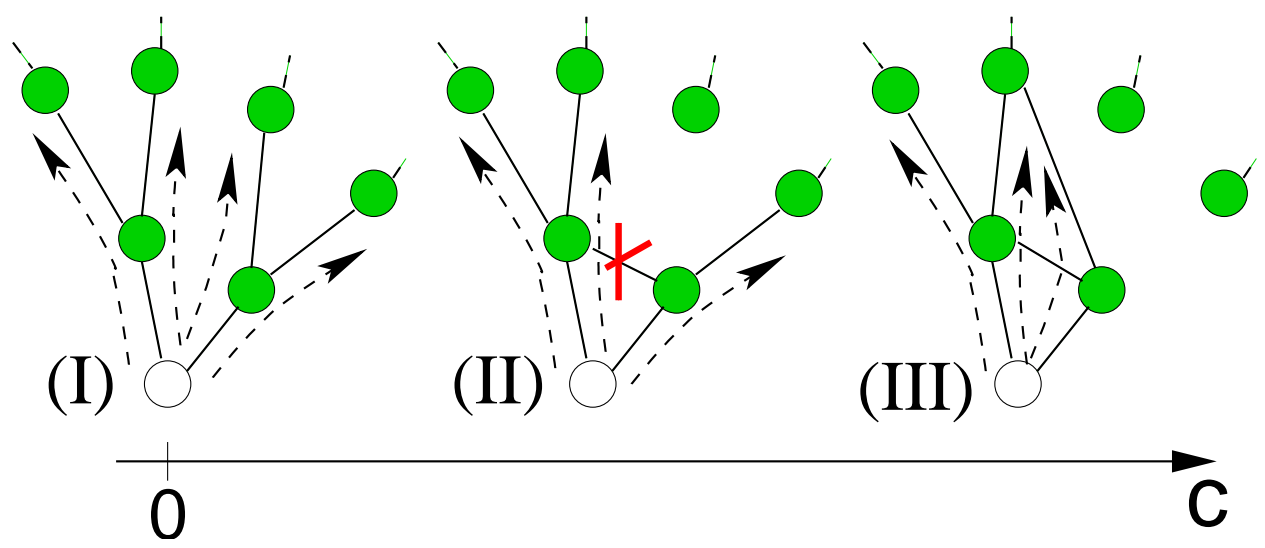

Figure 6.15: Schematic representation of the different structures found in the neighborhood of a particular node (white coloured in the figures) when clustering is increased as happens when going from region I to region III in figure 6.14. When clustering is small (I) the tree-like structure in the neighbourhood of nodes makes the packets to choose a particular branch depending on their destination. When clustering increases (II) the links employed by our model for connecting its neighbours are useless and decrease the number of choices. However, if clustering is even larger (III) the formation of closed loops of length 4 diversify the possible shortest path to be followed by a single packet delivered from the white coloured node.

when packets going to similar parts of the networks are distributed in several shortest paths. Now, packets can circumvent more easily congested nodes, thus making the shortest path protocol more efficient. The routing enhancement provided by the large clustering is lost when length effects appears at low values of $\mu$. For very small $\mu, L$ diverges (see inset in figure 6.14) leading to a bad performance of the protocol, although the clustering continues to grow for these $\mu$ values, since the algorithm works on a shortest-path-delivery basis. The crossover from the minimum to the divergence of $\partial\left\langle T_{\max }\right\rangle / \partial p$ is achieved in the parameter region IV where the interplay between $c$ and $L$ breaks down and the contribution from $L$ to the routing performance prevails.

The above results indicate the strong dependence of the routing efficiency on the underlying topology. Let us finally take a look at the queue times distribution, i.e. the probability that a packet spends a time $T_{\text {queue }}$ waiting in the queues of the nodes visited in its trip to the destination node, when several $\mu$ values are employed for the network substrate. We have plotted in figure 6.16 these distributions for $\mu=1$ (BA limit), $\mu=0.5$ (corresponding to the local maximum in figure $6.14, \mu=0.22$ (corresponding to the optimal topology as explained above) and for the Autonomous System network. For all the networks generated by our model we find a power law behaviour truncated at long queue times where the differences between the topologies are revealed. 


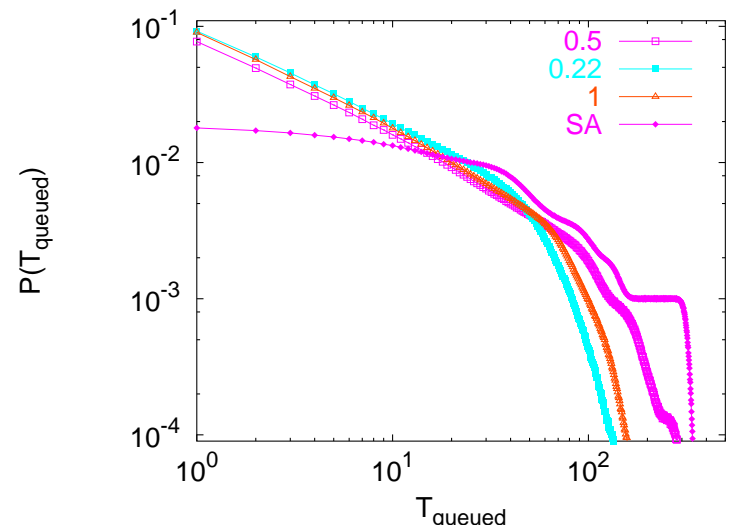

Figure 6.16: Queue time distribution for a set of networks with $\mu=1,0.5,0.22$ and the AS representation of the internet. Truncated power law behaviour is well appreciated for the networks generated using our model. These networks were grown up to a similar size of the AS map $N=11174$.

The distribution corresponding to the AS network shows however a different trend and the power law behaviour is not recovered. In this case, (as we explained in previous chapters), although the SF charater is also preserved, local properties such as degree correlations are very different from those in the modified BA model employed here confirming the importance of these local ingredients on the packet dynamics.

\subsubsection{Congestion-aware routing}

The preceding analysis shows that the routing protocol may be very sensitive to local details of the network on top of which the spreading process is taking place. It is then advisable the use of real nets in order to obtain reliable results. To this end, we will use the Internet Autonomous System map [165], which is a SF network with $\gamma=2.2$ and $N=11174$ nodes. It is worth stressing that each AS groups many routers together and the traffic carried by a node is the aggregation of the traffic generated at the internal routers and on individual end-host flows between the ASs.

Our aim here is to explore routing mechanisms more sophisticated than purely random or shortest path strategies. The first modification of the routing scheme is introduced by noting that the shortest path procedure does not take into account the traffic on the network. Specifically, a routing policy based on the shortest path between two given nodes neglects the queue in overloaded nodes which makes the process slower as the queue lengths become larger. That is, it may be more efficient to divert a packet through a larger but less congested path. Let us hence assume that a node $l$ is holding a packet that should be sent to a node $j$ and define an effective distance $d_{i j}^{\text {eff }}$ from a neighboring node 

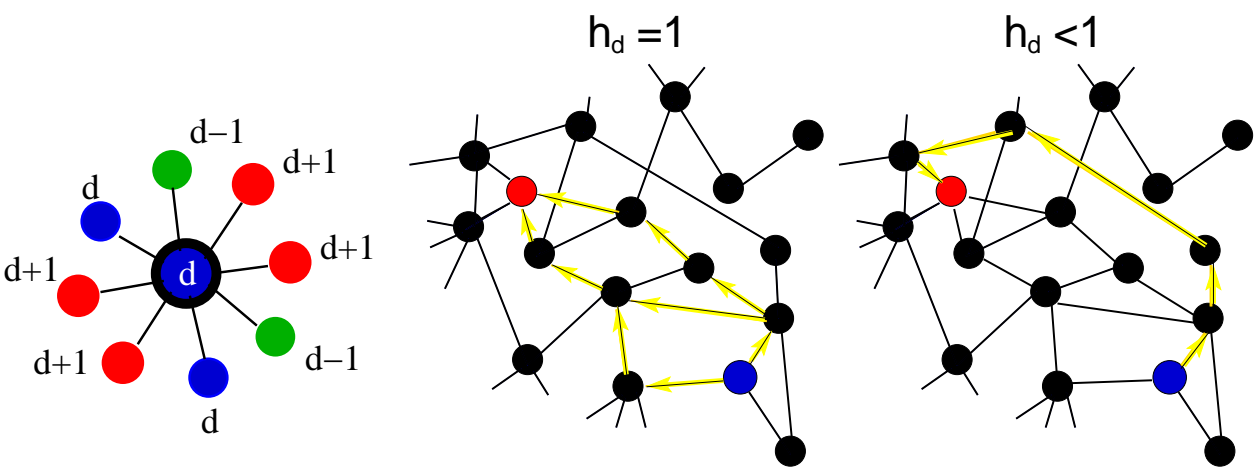

Figure 6.17: Schematic representation of the neighbour choice for a packet transmission. The neighbours of a given node can be at distance $d-1, d$ or $d+1$ to the packet target (where $d$ is the distance from the actual packet position to the target). When $h=1$ only those neighbours at $d-1$ account for the delivery as only geodesics from the node source to the node destination are to be considered. However, for $h<1$, a larger but less congested path could be the choice for the packet route.

$i$ of $l$ to the destination $j$ as

$$
d_{i j}^{e f f}=d_{i j}+c_{i}
$$

where $d_{i j}$ is the shortest path between node $i$ and $j$ and $c_{i}$ is the number of processes (or packets) in the queue of $i$. The above definition, however, does not allow us a direct comparison with the other standard procedures. It is then convenient to redefine the effective distance as

$$
d_{i j}^{e f f}=h_{d} d_{i j}+h_{c} c_{i}
$$

so that the limit $h_{c}=0$ contains the shortest path protocol. Furthermore, without loss of generality, we take $h_{d}+h_{c}=1$. This algorithm will be called deterministic protocol henceforth. The procedure for $0<h_{d}<1$ combines knowledge of the structural properties of the network and its current dynamical state at a local scale, consequently, a trade-off associated to packets' transit times is naturally and dynamically incorporated (see figure 6.17).

Taking into account the above effective distance, $d_{e f f}^{i j}$, we first study a similar situation to the one presented in the previous section. Starting from $p$ packets in the network, at each time step, the remaining packets are delivered in such a way that the neighbour chosen when a packet towards $j$ departs from a node $i$ is that which minimizes $d_{e f f}^{i j}$.

A first look at the dynamics shows that a protocol implemented in this way is more efficient than taking into account only the shortest path criterion. In fact, $\left\langle T_{\text {relax }}\right\rangle$ departs from the linear behavior previously observed and is well 


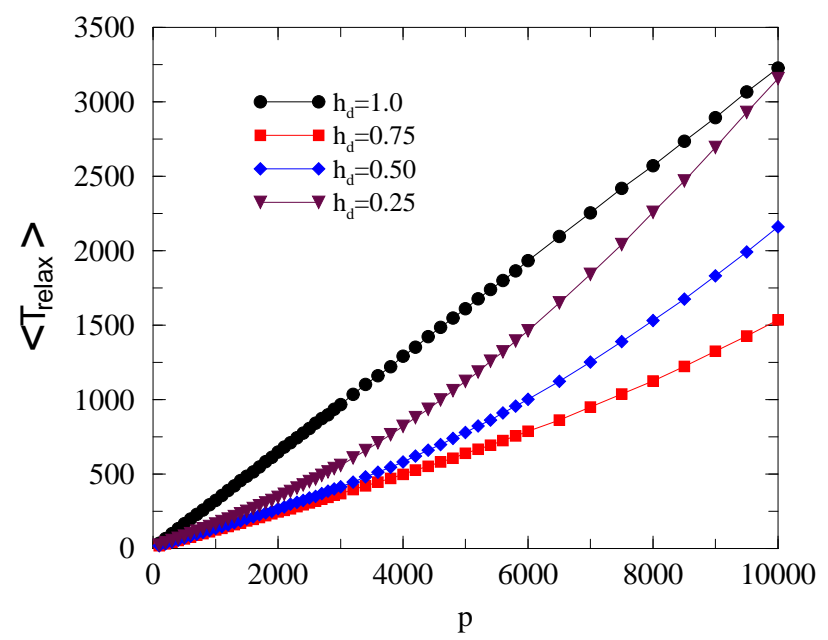

Figure 6.18: Dependency of $\left\langle T_{\text {relax }}\right\rangle$ on the number of initial packets $p$ in the deterministic limit of the model $(\beta=20)$ ran on top of an AS Internet map made up of around 11000 nodes. Each point is an average over at least 200 realizations. The standard protocol corresponds to the limit $h_{d}=1$. Note that although the tendency of the curves is to cross the straight line as $p$ increases, there is an optimal value of $h_{d}$ such that the interception would take place in the limit of very heavy traffic.

below the straight line up to a high $p$. This behaviour clearly depends on $h_{d}$, since it is straightforward to realize that if $h_{d}$ is zero, the packets are diverted following the less loaded node regardless of the path length which results in an uncontrolled increase in the distance traveled by the packets from the sending nodes when $p$ grows.

The above algorithm can be further generalized by including a probabilistic view. In other words, once we have determined the $d_{i j}^{e f f}$ for all pairs $(i, j)$, we can allow for a stochastic choice of the paths. Hence, our second algorithm, referred to as stochastic protocol considers a score function or "energy" $H_{i j}=$ $h_{d} d_{i j}+\left(1-h_{d}\right) c_{i}$ and that the probability $\Pi_{i j}$ that a packet with destination $j$ is sent precisely through node $i$ is given by,

$$
\Pi_{i j}=\frac{\exp \left(-\beta H_{i j}\right)}{\sum_{\{l \mid(l, i) \in V\}} \exp \left(-\beta H_{l j}\right)},
$$

where $\beta$ is the inverse of the temperature. In the limit $\beta \rightarrow \infty$ (at zero temperature) we recover the deterministic protocol.

Figure 6.18 shows the dependency of $\left\langle T_{\text {relax }}\right\rangle$ on the number of packets $p$ for several values of $h_{d}$ in the deterministic limit of the model, which we found to be fulfilled for $\beta=20$. A dynamics which does not take into account the amount of traffic handled by the neighbors of a sender node - straight line in 


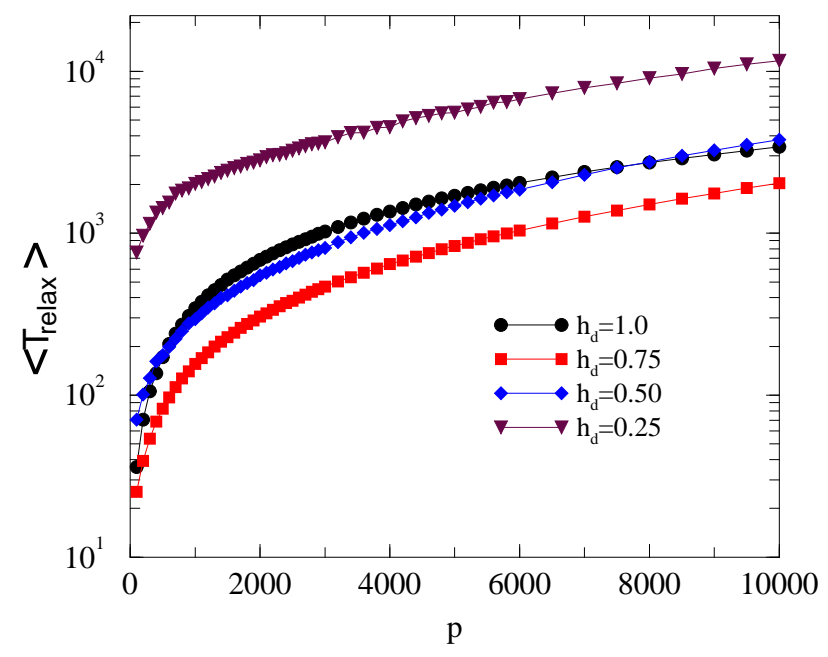

Figure 6.19: Dependency of $\left\langle T_{\text {relax }}\right\rangle$ on the number of packets, $p$, for a middle $\beta=5$ value. The network parameters are as in figure 6.18. In this case, the $h_{d}$ range in which the stochastic strategy performs better than the standard one is reduced.

figure 6.18 - performs worse than the one which integrates both ingredients. However, this depends on both the specific weight of each metric in $H_{i j}$ and $p$. In the regime where the traffic is not heavy (small $p$ values) all curves are below the shortest path protocol performance, but as the amount of traffic handled by the network increases, the deterministic protocol starts performing worse for a range of $h_{d}$ values. From the results, it seems that eventually, when the traffic increases too much, the curves cross the straight line indicating that at those limits the shortest path strategy is best suited. Note, however, that for $h_{d}=0.75$ the convergence of the two algorithms occurs for a very heavy load. Consequently, we can assert that there is an $h_{d}$ region where the combination of the two ingredients gives rise to the best performance. On the other hand, the existence of an optimal $h_{d}$ value distinct from zero can be understood by noting that a mechanism lacking some degree of path length information between the source and destination nodes of the packets performs badly because the packets travel along too large paths that do not compensate the time they would loose trapped in the queues of congested nodes.

The completely stochastic limit of the model corresponds to $\beta=0$. The performance of the protocol in this limit is however very bad. In fact, for an infinite temperature, all neighboring nodes of a given sender have the same probability to receive the message regardless either their congestion level or their distance to the targets. Then, the dynamics becomes a random walk process. With no topological information about what are the destinations of the packets, they arrive to the receiver at longer times and the algorithm is the 


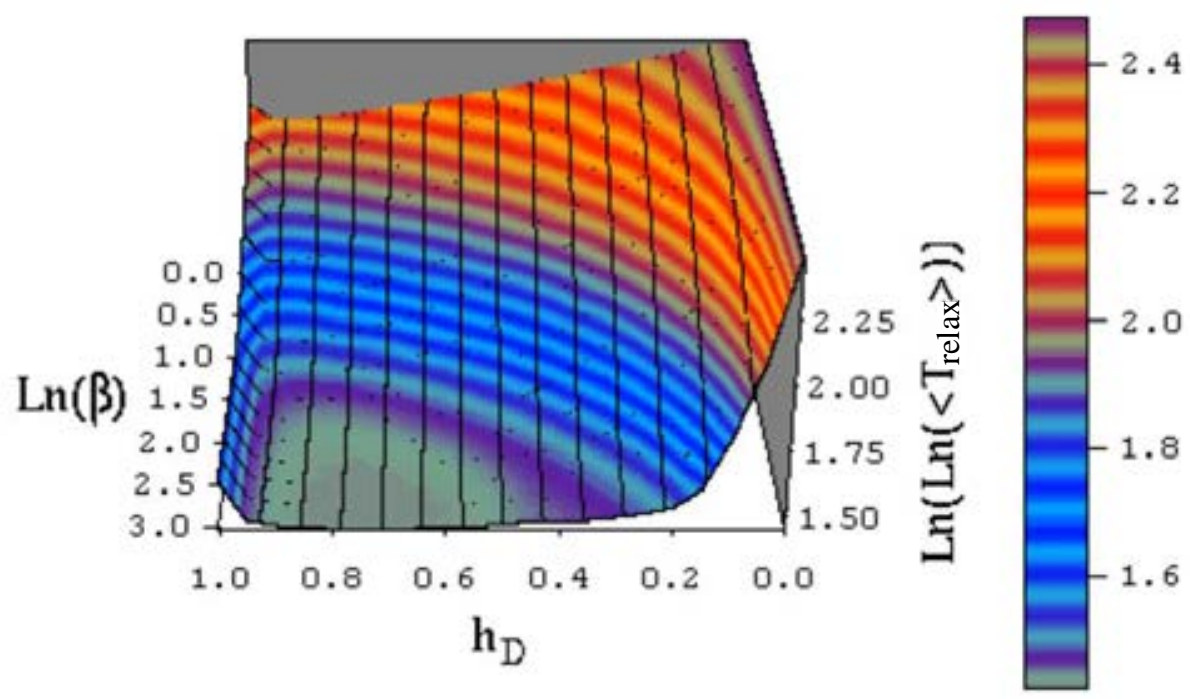

Figure 6.20: Phase diagram of the system's dynamics. The network parameters are as in figure 6.18. The number of processes is $p=500$. Calculations for higher $p$ show that the minimum of $\left\langle T_{\text {relax }}\right\rangle$ is also attained around $h_{d}=0.8 \pm 0.1$.

worst. For intermediate values of $\beta$, we have an stochastic dynamics in which topological and traffic information coexist with some degree of randomness in the choice. This is the case depicted in figure 6.19 for the same values of $h_{d}$ used in figure 6.18. As can be noted from the figure, the stochastic protocol increases $\left\langle T_{\text {relax }}\right\rangle$ by at least one order of magnitude as compared to the deterministic limit $(\beta=\infty)$. Moreover, the shortest path routing seems to be the best choice for a wider range of $h_{d}$ values, although $h_{d}=0.75$ still performs better.

Figure 6.20 summarizes our results for different values of the control parameters $\beta$ and $h_{d}$. It turns out from the study of the whole phase diagram that the best algorithm is one which includes information about both path lengths and congestion at a local scale. Besides, the deterministic limit with $h_{d}=0.75$ gives the best results for $\left\langle T_{\text {relax }}\right\rangle$. It would be worth noticing at this point that, although the figure 6.20 was obtained in not too heavy traffic conditions, the results are consistent for larger values of $p$. Different tests allow us to conclude that the optimal value is $h_{d}=0.8 \pm 0.1$. In any case, this confirms that it would be possible to device more elaborated protocols with the aim of diminishing the time needed for a packet to spread through the network. In light of the present results, such an strategy may be implemented by also taking into account the amount of traffic handled by a local area of the network. 
As suggested by figure 6.20, the best protocol is the deterministic one, which, on the other hand, should be easier to implement in practice. The microscopic dynamics of the routing process in this limit reveals that it is desirable that the routing process incorporates some knowledge of the node's queue lengths. However, the contribution in the score function of the second term in eq. (6.34) should not weigh in excess. For small values of $h_{d}$, say 0.25 , the algorithm performs better that shortest path routing for moderate values of $p$ because the packets do not pass by the hubs of the network, which are likely to be in the shortest path route to any node. Instead, they go around the hubs and $\left\langle T_{\text {relax }}\right\rangle$ is smaller. If $p$ is increased, the neighbors of the hubs also get congested. This leads to a situation in which the packets around a hub get trapped in its neighborhood, getting in and out from it, but without finding their routes to their destinations. We will analyze in depth this situation below.

\section{Jamming transitions at constant information flux}

To analyze in more detail the performance of the congestion-aware protocols we turn our attention to the more realistic situation where the network is subjected to a constant flux of newly created packets. Starting from an unloaded network, at each time step $p$ new information packets are created. As before, the source and destination of each packet are chosen at random among all the nodes and each node sends only one packet at each time step, $r=1$. As an appropriate measure of the efficiency of the process, we monitor the aggregation of packets in the network, given by the number of packets that have not reached their destinations at each time step $t, A(t)$. Figure 6.21 shows the results obtained for different values of $p$ and $h_{d}$. As it can be seen, when the external driving is applied at low rates (i.e., small $p$ ), both protocols allow for a stationary state where $A(t)>0$ is constant. In this state, the system is able to balance the in-flow of packets with the flow of packets that reach their destinations. This stationary state, where no macroscopic signs of congestion is observed, corresponds, as already introduced in previous sections, to the so-called free flow phase. The situation changes when the rate at which new packets are introduced increases. As we will see below, there is a critical value $p_{c}$ beyond which a congested phase shows up. Let us now note that for the shortest path protocol $\left(h_{d}=1\right)$ (figure 6.21.a, dotted line), when $p>p_{c}, A(t)$ grows linearly in time $\forall t$ as expected from earlier works where this routing was implemented [239]. On the contrary, for the traffic-aware algorithm, $h_{d}<1$, we observe that $A(t)$ grows slowly at short times and then becomes steeper as time goes on with a constant slope (figure 6.21.c).

In order to characterize the phase transition from a free phase to a con- 

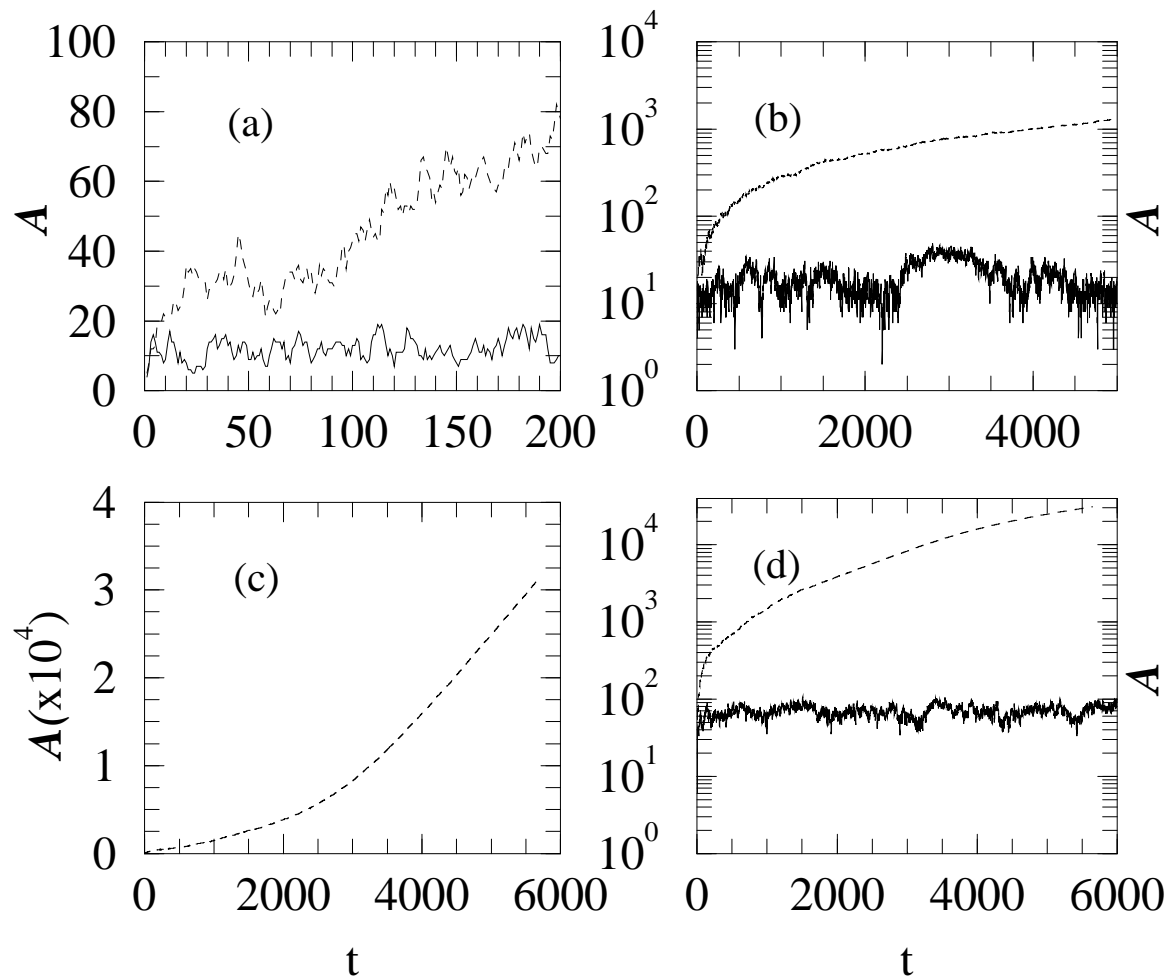

Figure 6.21: Total number of active packets as a function of time steps. Figures (a) and (b) correspond to the shortest path protocol, $h_{d}=1$ while (c) and (d) have been obtained for the traffic-aware routing with $h_{d}=0.85$. In each figure, the continuous line stand for subcritical values of $p((\mathbf{a})$ and $(\mathbf{b}) p=3.0$, (d) $p=8.0)$ and the dotted line corresponds to $p>p_{c}((\mathbf{a})$ and $(\mathbf{b}) p=4.0,(\mathbf{c})$ and $(\mathbf{d}) p=13.0)$.

gested one, we use the order parameter introduced in [245], eq. (6.31)

$$
\rho=\lim _{t \rightarrow \infty} \frac{A(t+\tau)-A(t)}{\tau p}
$$

where $\tau$, the observation time, was typically fixed to 200 time steps. We recall that the limit in the above expression is introduced only to ensure that the system is not in a temporary regime, for our purpose it ranged from few thousands to $10^{4}$ time steps depending on the system behaviour. The order parameter $\rho$ hence measures the ratio between the outflow and the inflow of packets during a time window $\tau$. Then, $\rho$ equals 1 when the congestion is maximal (no packet reaches its destination) and 0 when an equilibrium is established, i.e., in the stationary state.

For the shortest path routing the computation of $\rho$ is simple because of the linear behaviour observed for both the free-flow and the congested phases. 

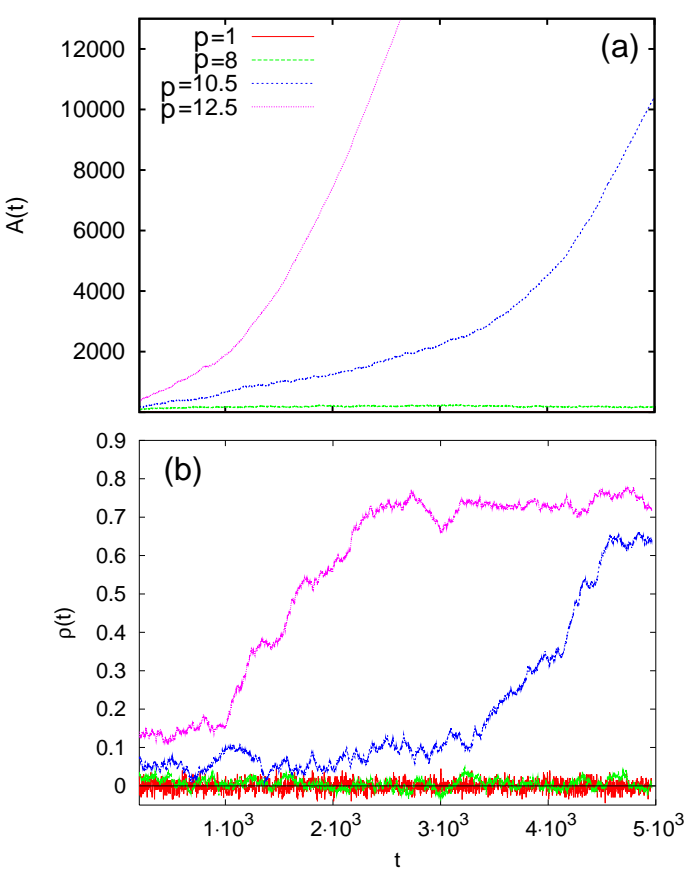

Figure 6.22: (a) Time evolution of the number of active packets in the network for the congestionaware routing $h_{d}=0.75$. The values $p=1$ and $p=8$ correspond to the free-flow phase whereas $p=$ 10.5 and $p=12.5$ lie on the congested regime. The evolution of $A(t)$ shows two different regimes for these two last values: a large transient time (where a nearly linear behaviour for $A(t)$ is observed) and the equilibrium one (where again a linear behaviour is reached with a larger slope). (b) Instant computation of $\rho(t)$. For the values of $p$ where $p<p_{c} \rho(t)$ fluctuates around 0 , whereas when $p>p_{c}$, the two aforementioned regimes are reflected by the regions where $\rho(t)$ is constant.

On the other hand, for the routing aware protocols at the congested phase we have to be careful about the measures of $\rho$. The first transient regime observed in figure 6.21.c can be regarded as a linear regime for the neighbourhood of the corresponding value $p_{c}$. In fact, as observed from figure 6.22.a for values of $p$ so that $p_{c} \lesssim p$ this transient regime could be of the order of several thousands of time steps. If one monitorizes the evolution of $\rho(t)$, figure 6.22.b, this quasilinear behaviour is reflected as a nearly constant low values of $\rho(t)$ for initial times. This behaviour is interrupted by a suddenly growth of $\rho(t)$ to a larger value, where it remains constant signaling that the system have reached its dynamical equilibrium. At first look it seems that, at the beginning, the system is being charged by the unbalanced load of traffic and when the dynamics reaches a critical number of active packets it explodes yielding to the rapid increase of $A(t)$ shown by the large slope of the final state. Although we will analyze in more details this process below, it is important to stress here that no matter how slow the charge rate of the transient regime is, the system will experience the final dramatic increase in $A(t)$. Obviously, as we approach $p_{c}$ from the right, the time needed by the system to reach the final equilibrium diverges and hence the estimation of the critical point $p_{c}$ is computationally hard.

Figure 6.23 depicts the system's phase diagram. The dynamics of the 


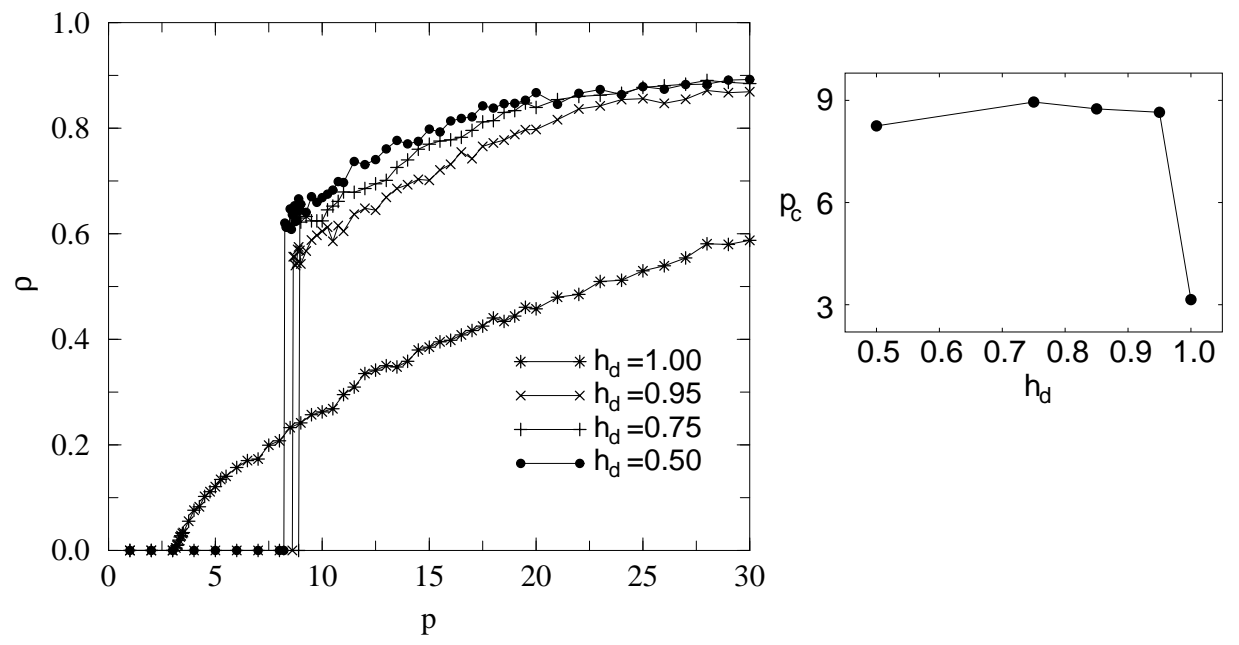

Figure 6.23: Jamming transitions as a function of $p$. The order parameter $\rho$ is given by eq. (6.31). Note that $h_{d}=1$ corresponds to the standard strategy in which traffic awareness is absent. As soon as traffic conditions are taken into account, the jamming transition is reminiscent of a first-order phase transition and the critical point shifts rightward (see right inset).

system is characterized in both shortest path and congestion-aware protocols by a critical point beyond which a macroscopic congestion arises. However, as expected from the previous observations, there are two radically different behaviors for the onset of traffic jams. In the standard protocol $\left(h_{d}=1\right)$, the critical point is small, $p_{c} \simeq 3$ and the jamming transition is reminiscent of a second order phase transition. On the contrary, when $h_{d} \neq 1$, the critical point $p_{c} \simeq 9$ is distinctly larger than for $h_{d}=1$, but the appearance of a congested phase turns out to be consistent with a first order phase transition, with a sharp jump of $\rho$ at the transition point. Moreover, the order of the transition for the latter protocol is independent of $h_{d}$ provided that $h \neq 1$.

The two different types of transitions depending on whether or not trafficawareness is incorporated in the protocol at work, poses an interesting issue. Which of the two protocols will be best suited to handle traffic? It depends on the system. While for the standard protocol we get a smaller critical point, the jammed phase does not appear suddenly. Hence, if we would like to have a system in which traffic jams appear and grow smoothly, the standard algorithm is the best choice. On the contrary, we could implement a sort of traffic-aware protocol if we are interested in delaying the appearance of congestion, however at the cost of a sudden jump to a highly jammed phase due to the lack of previous warnings. 


\section{Microscopic origin of the critical behaviour}

In order to provide more insights into the nature of the phase transitions, we now focus on the microscopic details of the system's dynamics that lead to the macroscopic results shown previously. We have inspected how the nodes get congested. As both classes (shortest path and congestion-aware) of protocols incorporate a shortest path delivery strategy, a suitable description can be obtained by monitoring the number of active packets at each node as a function of the betweenness of the nodes. As explained in section 5.1.2, the betweenness or load of a node $i$ gives the total number of shortest paths among all pairs of nodes in the network that pass through $i[161,169,249]$. It is then a measure of the centrality of a node in the network so that it becomes a relevant quantity in traffic flow modeling. In particular, for our system, AS representation of the Internet, the betweenness of a node scales with its connectivity $k$ [161]. Figure 6.24 clearly illustrates the distribution of congested vertices for the two protocols analyzed. The shortest paths connecting the sources and the destinations of any active packet always tend to visit first the more connected nodes and then go down to the less connected ones. This is a consequence of the hierarchy of the network and is called up-down strategy [161]. For $h_{d}=1$, the protocol only works on a shortest path delivery basis. Then, the hubs become congested early in the process causing the packets to get trapped in a few nodes as shown in figure 6.24. When traffic conditions are taken into account by the routing mechanisms, the same up-down strategy applies up to the hubs. Then, instead of getting trapped in them, the packets circumvent highly jammed nodes and the load is distributed to nodes other than the hubs, provoking the aggregation of traffic in neighborhoods of overcrowded nodes. As it is shown in the figure, when $h_{d}<1$ the congestion gets first localized in the most central nodes, the hubs, and it is progressively distributed among the rest of the nodes following the centrality hierarchy, from the most centrals nodes to the less ones. At the long time limit, the congestion is spread through the network instead of getting trapped in the hubs as happens for $h_{d}=1$.

It is possible to get deeper into what is going on in the system for $h_{d} \neq 1$ by analyzing the redistribution processes that make the packet follow the less congested paths. Let us suppose that a node $i$ is holding a packet to be sent to $j$ through one of its $k_{i}$ neighbours. Among all the neighbours of $i$, there is one node with the lowest load $c_{\min }$. Now, assume the extreme situations in which by going through a given neighbour $l$ the packet is one hop closer to its destination, but taking the path for which the congestion is minimum, it is one hop farther from $j$. Thus it follows that whenever the relation

$$
c_{l}-c_{\min }>\frac{2 h_{d}}{1-h_{d}}
$$




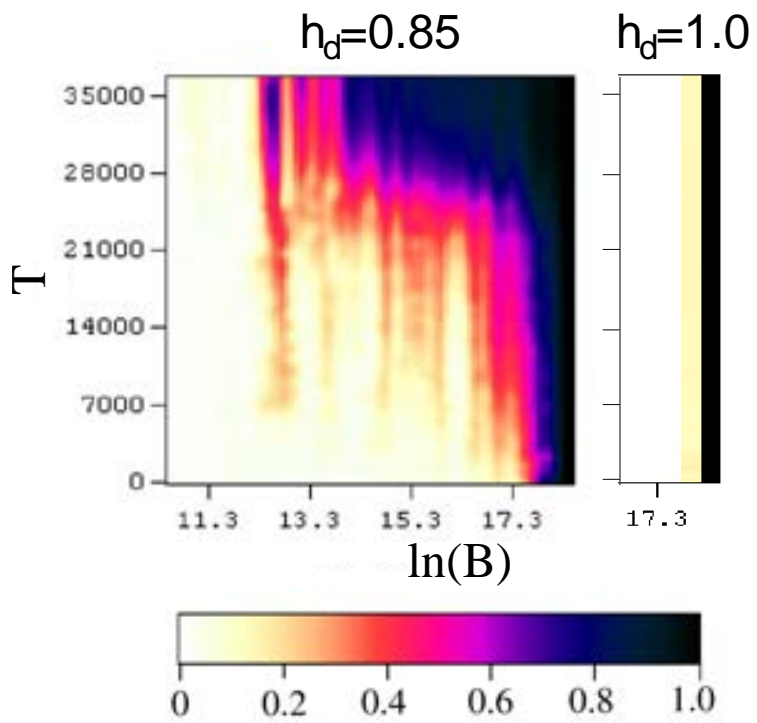

Figure 6.24: Congestion levels as a function of time and nodes' betweenness. At each time step, the color-coded scale is normalized by the number of packets $c_{i}$ in the queue of the node with the largest congestion. Two radically distinct behaviors are obtained for the shortest path routing $\left(h_{d}=1, p=4>p_{c}=3\right.$, right panel) and for the trafficaware protocol $\left(h_{d}=0.85, p=\right.$ $13>p_{c}=9$, left panel).

is verified, no packets (regardless of their destination) will be sent through $l$. This node $l$ is impenetrable for $i$. Then, if a node is impenetrable for all its neighbors, we call it just impermeable, since it does not participate in traffic delivery.

Following this picture, as congestion spreads throughout the network, the number of impermeable nodes would increase up to a constant value since the definition of impermeable nodes is relative to their neighbourhood (it is impossible to obtain a network composed only by impermeable nodes). Then, regardless the total load of the network (that increases with time), the number of impermeable nodes remain nearly constant. Besides, its distribution would change dynamically since an impermeable node does not admit new packets in their queues but it does continue sending packets to their neighbours so that its level of congestion relative to that of their neighbours can only decrease while its neighbours receive its packets. Therefore a dynamical backbone made up

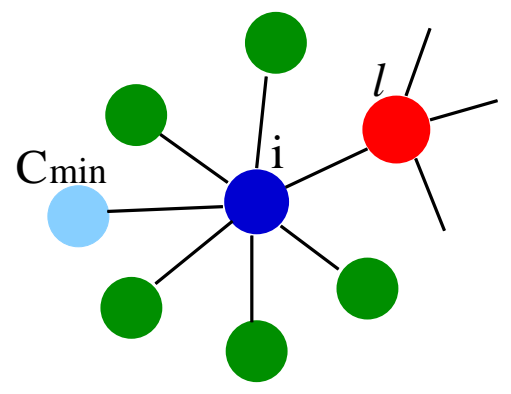

Figure 6.25: A node $l$ is called impermeable to its neighbour $i$ when eq. (6.36) is fulfilled. 


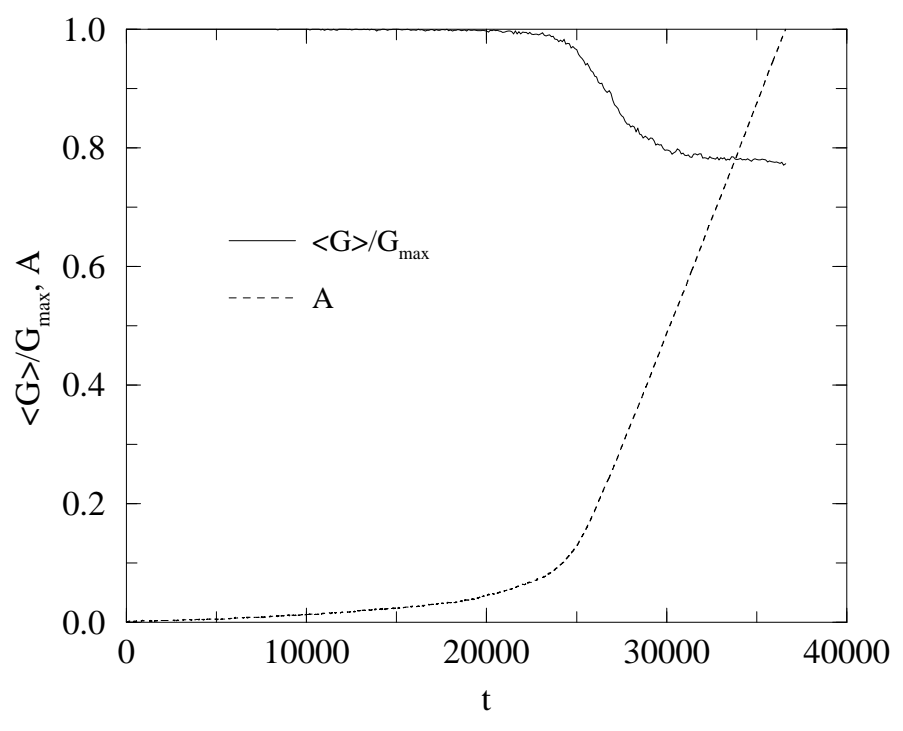

Figure 6.26: Time dependence of the total number of packets in the system and average size of the clusters formed by non impermeable nodes. Note that $A(t)$ becomes steeper just when the inflection of $\langle G\rangle / G_{\max }(t)$ changes. $h_{d}=0.85$ and $p=13$. See the text for further details.

of all nodes that are able to transmit the packets comes up. This picture is similar to the percolation of a fluid through a porous media. Here, packets can flow only through non impermeable nodes as a fluid can only flow through the pore channels.

The existence of impermeable nodes provokes the appearance of both small network components in the form of impenetrable regions, and clusters of allowed paths. By identifying those impermeable nodes at some time $t$ following the criterion (6.36), it is possible to find those dynamically unconnected network components whose boundaries are composed by impermeable nodes. Figure 6.26 depicts the time dependence of the average cluster size (normalized by the largest cluster size) of allowed regions. Starting from $t=0$, as time goes on, the total number of packets in the network increases and there is only one cluster of the size of the network. When signs of congestion first appear, $\langle G\rangle / G_{\max }(t)$ decreases departing from unity signaling that impermeable nodes start to appear. At longer times, traffic jams reach more nodes (see, figure 6.24, for $t>21000$ ) causing the congestion to be more distributed in the network and hence the growth of the total amount of impermeable nodes. Finally, the flow of packets in the network reaches the regime in which $A(t)$ increases linearly in time and $\rho(t)$ saturates to its stationary value (figure 6.22). In this state, marked by an inflection point in the $\langle G\rangle / G_{\max }(t)$ curve beyond which 

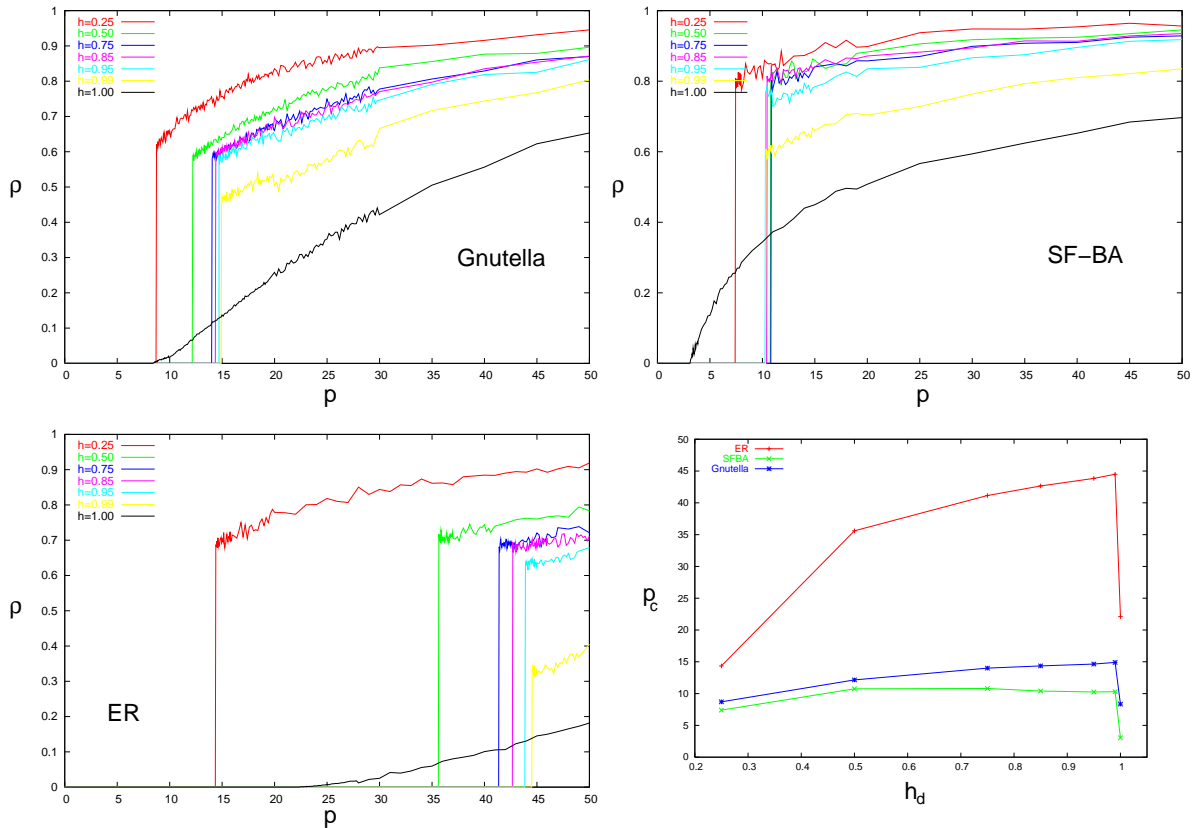

Figure 6.27: Jamming transitions for the Gnutella, Barabási-Albert and Erdös-Rényi networks. The bottom right plot shows the estimation of the critical point, $p_{c}$, for these topologies. Synthetic networks (ER and SF-BA) have the same size as the Gnutella peer-to-peer network $N=711$.

the average cluster size of allowed regions stabilizes, the system seems to have self-organized the distribution of jammed nodes.

This self-organization phenomenon nicely explains why one can not go smoothly from these results to those corresponding to the use of the shortest path protocol by making $h_{d} \rightarrow 1$, as it can seem from eq. (6.34). The discontinuity at $h_{d}=1$ is therefore due to the lack of alternative paths in the standard protocol. Even for $h_{d}$ very close to 1 , the system will self-organize itself into a state in which congested nodes are distributed and not limited to the very hubs of the network. The only dependence with $h_{d}$ is manifested in the time needed for self-organization, that becomes very large and eventually diverges when $h_{d} \rightarrow 1$.

The results found here are reproduced when using more general network topologies as the traffic substrate. We have inspected the case of synthetic networks like Erdös-Rényi and Barabási-Albert graphs as well as other real maps like the Gnutella peer-to-peer network (see figure 6.27). The results are qualitative the same as those shown above: a little knowledge of the local congestion levels enhances the performance of the information dynamics in 
what refers to the shift of the critical point $p_{c}$, but on the other hand, the transition to the congested regime changes from a smooth to a sudden one due to the dynamical fragmentation of the network explained above. Our results demonstrate that whether or not a given protocol is best suited for traffic handling depends on a delicate trade-off between the system's performance and traffic capabilities (how large $p_{c}$ is) and how congestion arises (smoothly or suddenly).

\subsection{Conclusions}

We have explored in section 6.1 and 6.2 two propagation processes in complex networks, namely: epidemic spreading and information routing. Assuming that the networked substrates are scale-free, several features have been studied in detail. As we introduced at the beginning of this second part of the Thesis, the main purpose concerning the study of network dynamics here, is to know how the heterogeneous topology and other structural properties affect the development and efficiency of the dynamics.

The first set of results, explained in section 6.1, concerns the immunization strategy of complex heterogeneous networks. Taken into account that hubs play a central role in these kind of processes it is clear that the best choice for stopping the epidemic spreading is to confer immunity to these most connected nodes. However, taking into account that knowledge of network structure is not complete in most situations, we have proposed a new immunization strategy based on a heuristic solution to the covering problem. This new immunization scheme is neither local nor global, and hence, we have explored several situations corresponding to different knowledge horizons (from local to global knowledge). The immunization strategy, based on the covering solution, takes advantage of the heterogeneous structure of networks and it has been shown to perform better than all previous methods irrespective of the degree of local knowledge, except for the case of targeted immunization. We have also shown that the solution found for the immune set of nodes, i.e. the covering solution, and therefore the output of a SIR epidemics strongly depend on the nature of the degree-degree correlations of the network. In this sense, it is worth mentioning that the traditional belief that by targeting hubs one can efficiently solve most problems on networks with a power-law degree distribution is not completely true if the degree-degree correlations are assortative, where a distributed covering-based immunization is desirable.

We have also analyzed the problem of information routing in complex networks in section 6.2. We have studied different strategies for traffic delivery in complex heterogeneous networks. The results showed that the performance 
of the standard approach, based on routing through minimal distance (between sender and recipient) paths, is sensitive to local topological changes. Specifically, the clustering properties may play a key role in message delivery. Besides, we have studied alternative strategies to the shortest path routing policy. In particular, we have studied algorithms which integrate topological and traffic information. This congestion-aware algorithms have been shown to perform better that the standard protocol when relaxation processes occur. At the same time, we have characterized the jamming transitions that take place when a constant flux of information is introduced in the network. The results have shown that when traffic awareness is incorporated into the routing protocol, new cooperative effects arise and the jamming scenario is totally changed. The use of congestion-aware routing is seen to imply a better performance, for what concerns the onset of jamming, i.e the network allows a higher load of data traffic at the free flow phase. On the contrary, when jamming occur there is a sudden increase of the congestion levels and an abrupt transition is observed in contrast to the well known second order phase transition found for the standard shortest path routing.

The two main strategies introduced in this second part of the Thesis, covering-based immunization and congestion-aware routing, are oriented to take advantage of the scale-free nature of the substrates where the associated dynamics, epidemic spreading and information traffic, take place. In the forthcoming part we will care about the two way relation between structure and dynamics. 



\section{Part III}

\section{Nonlinear Dynamics of Complex Networks}





\section{Presentation of Part III}

I

In the two preceeding parts of the Thesis we have dealt separately with both dynamical and structural complexity. In part I we have been concerned with dynamical systems coupled in a simple regular way conforming perfect nonlinear lattices. These geometries of the arrays of dynamical systems are, in most cases, realistic when concerning applications in fields as solid state physics, optics, etc... and they are currently the subject of a considerable multidisplinary interest [23]. However, there are yet another kind of problems, most of them associated with biological systems, where these simplistic assumptions for the topology of interactions are not valid and, therefore, structural complexity is linked to nonlinear dynamics.

Although our analytical tendency is to separate these two sources of complication, an unitary approach is needed in order to explain how structural and dynamical complexity influence each other [250]. This direction of study is deeply related with the growing interest in the understanding of the many aspects of the correlation between Structure and Function in systems made up of discretely many nonlinearly interacting components. The main assumption behind these studies is that the structure of the network of interactions is the result of a selective process that yields an advantageous topology for the system functioning. The common scale-free character found for many real networks support these speculations and many efforts are currently being made in order to find dynamically based optimization principles for modeling network growth and evolution.

The most interesting problems on the relation between structure and function arise in the context of biological systems such as biomolecular (proteinprotein interaction, gene regulation, cell metabolism), cortical brain and ecological (trophic networks, mutualism) networks. There exist many dynamical models accounting for the interactions among the elements of such systems and, at the same time, the advances in experimental techniques allow for having the topology of these interactions mapped into a large amount of biological networks. The scientific basis are thus well established and the research is now being oriented to the study of the mutual influence between dynamics and topology, attracting the attention of scientist from diverse fields to this interesting problem.

The most relevant results would eventually come from those mentioned network models that, instead of defining network growth in terms of structural properties, incorporate the efficiency of the dynamics (function) of the network as the leading principle for network evolution. However, most of studies up 
to now have concentrated in analyzing how structure affects the emergence of dynamical effects like synchronization patterns, self-sustained dynamical activity, etc... These first approaches to the problem provide useful insights for more ambitious projects where optimization of dynamical properties like synchronizability, dynamical adaptability and stability, etc... will be explicitly employed in network design.

Our main concern in this last part of the Thesis is on the line of these first approaches to the Structure-Function relation problem. We will use the concepts applied in the two former parts to study the dynamical patterns that appear when two different dynamical systems are placed on top of complex geometries. We will characterize both dynamically and structurally these patterns in order to unveil the interplay between topology and dynamics. These patterns are seen as the product of the two coupled sources of complexity and, as we will see, lead to the emergence of new properties that cannot be obtained from a separate study of dynamical and structural complexity.

In chapter 7 we will study a class of complex networks where there exists a competition of inhibitory and activatory interactions between elements. These kind of systems are related with gene regulatory networks and metabolic reactions chains. The description of the system functioning is performed via a Michaelis-Menten equation, widely used for describing the reaction kinetics of catalytic processes. We will first study in detail the phase portrait of the system as a function of the ratio between activatory and inhibitory interactions of the network and characterize the diverse bifurcations found. This dynamical characterization of the system constitutes a generalization of those studies performed in random Boolean networks. A second purpose of this study is to characterize topologically the substructures of self-sustained dynamics that are observed. In this regard, we analyze the emergent dynamical clusters by means of the statistical measures introduced in the second part of the Thesis, chapter 5, and observe that new structural properties absent in the network substrate show up.

Chapter 8 is devoted to the study of synchronization in networks of Kuramoto phase oscillators. Here we will characterize the synchronization transition in several network topologies. In particular, we will describe the evolution of the system towards synchronization paying attention to the emergence of dynamically coherent clusters of nodes as the coupling between network nodes is increased. These studies aim to highlight that the route to synchronization depends strongly on the underlying topology, not only for what concerns the quantitative values for the onset of synchronization, but in the qualitatively different organizational principles that lead to the formation of a macroscopic synchronized cluster. 
These two structural studies of the dynamical patterns, that emerge when coupling dynamical systems in a networked substrate, will help to understand what kind of topologies are best fitted for systems function. 



\section{Chapter 7}

\section{Activatory-Inhibitory interactions in Complex Networks}

How would we express in terms of the statistical theory the marvelous faculty of a living organism, by which it delays the decay into thermodynamical equilibrium (death)?... the device by which an organism maintains itself stationary at a fairly high level of orderliness... really consists in continually sucking orderliness from its environment.

— Erwin Schrödinger in What is life? The physical aspect of theliving cell [251].

How does the interplay between complex structures and nonlinear dynamics may shed new light on what is going on at the cellular and molecular levels of organization of biological systems? As in other natural systems, on one hand, scientists have begun to look for patterns of interactions in biological systems. The idea behind this approach is that we can not completely understand the functioning of the cell by studying its components separately. The next step consists of taking into account the dynamics governing the unraveled interactions. This is certainly not an easy task as one has to deal with two sources of complexity: one coming from the unraveled structural patterns and the other from a dynamics in which analytical insights are difficult to take.

In this chapter we address the problem of networks of agents that regulate their activity by means of activatory and inhibitory interactions. This kind of systems constitute the coarse grained description of regulatory networks of genes at the cellular level. We will first describe in section 7.1 the current trends in modeling biological networks with special interest in gene networks. 
The results obtained when a simple case of this class of systems is analyzed are described in section 7.2.

\subsection{Modeling biological networks}

In 1999, Hartwell and collaborators published an influential paper discussing the new challenges of modern biology [252]. The authors pointed out that an issue of utmost importance is to develop a general framework in which biological functions could be understood as part of a complex modular organization of molecules or cell's constituents. In other words, modern biology should explain not only the functioning of individual cellular components, but also how these components are interconnected through a complex web of interactions leading to the function of a living cell. It is then natural to ask what these biological networks at the cell organization level look like, and how their structure couples to the dynamics.

Cells are life's fundamental units of structure and function. It was expected that, once the complete instructions encoded in DNA would have been interpreted, one could map a gene (the basic information unit in the DNA) into a specific activity or function, with all the consequent potential applications such as targeted drug development [253]. On the contrary, although today the complete knowledge on the genes of several organisms is available, yet the relationship between blueprints in DNA and functional activities of the cell is not fully understood. For instance, the p53 gene and protein (having the function of controlling cell's life and death) are known as tumor-suppressor, since it was found that the p53 protein does not function correctly in most human cancers. However, despite the many studies performed on p53 gene and protein, the way on how effectively suppressing the growth of cancer cells is missing at a genetic level. Recently, it has been proposed that the understanding of such cancer cell growth mechanism would be gathered not only from the study of the p53 gene and protein, but taking into account the whole network interacting with them [254]. That is, the function of the gene should be analyzed through a network in which the gene participates. Similarly to p53 network case, several other observations prove that some functional activities of the cell emerge from interactions between different cell's components through complex webs. Moreover, it is expected that the large-scale network approach may lead to new insights on various longstanding questions on life, such as robustness to external perturbations, adaptation to external circumstances, and even hidden underlying design principles of evolution.

In what follows, we discuss the last advances in the characterization of some biological networks from two points of view: their structural organization and 
their functioning. The main point here is how to uncover the relationship between the two sources of complexity intimately linked (dynamics and structure) as both play a key role in the functioning of the system. We stress here that our main intention is to provide a brief overview of the current state in the field, and that many works may be overlooked due to space constraints. We invite the interested reader to follow the specialized literature.

\subsubsection{Structure}

In the second part of the Thesis we introduced a variety of types of networks that have been analyzed in order to unveil their complex topologies. Among these different network classes biological networks are of special interest since they have emerged following a natural evolving process. A plenty of cellular and molecular networks have been unraveled in the last several years. We here refer to those that have been more used in subsequent studies or because they are considered to be essential for the cell's life.

The first of these complex biological networks is that formed by metabolic reactions: the metabolic network. Jeong et al have considered the metabolic reactions of 43 different organisms, representing the three domains of life, and have constructed directed graphs whose nodes are the metabolites and edges represent biochemical reactions [146]. A node receives an incoming edge when the corresponding metabolite is produced, and receives an outgoing edge when the metabolite is educed. Enzymes are not included in the graph. The total number of connections (edges) of a node is called the degree of the node. If the edges have a direction (incident to or going out from the node), the degree of a node is divided in in-degree and out-degree, respectively. For all investigated organisms, the resulting graphs for metabolic reactions exhibit scale-free properties for both incoming and outgoing degree distributions similarly to many other real world networks.

The above-mentioned property was found universally, irrespective of metabolic pathway databases and of the methods used to construct graphs from biochemical reactions. For example, instead of assuming virtual intermediate complexes, Wagner and Fell built up two networks (the metabolite and the reaction networks) from the metabolic pathways of Escherichia coli [255]. The metabolite network consists of nodes representing metabolites and bidirectional links between educt and product of a metabolic reaction. On the other hand, the reaction network is the network where the nodes correspond to metabolic reactions and two nodes are linked when the two reactions share a metabolite. In metabolite networks, scale-free properties are detected, while the reaction network does not show power-law degree distributions. Small-world properties 
and relatively high clustering (i.e, how probable it is that two nodes with a common neighbor are also connected together) are found in both networks. Other studies with different ways of obtaining graphs show almost identical results [256-259].

Another class of well-studied cellular networks is that of protein-protein and protein-gene interaction networks. This is due to the increasing availability of data sets and new experimental techniques that allows a more detailed study of the interactions at the cellular level. On the other hand, interactions among proteins have a crucial role in several functional activities, such as signal transduction. According to the demand of understanding the protein interaction map, several high-throughput experiments have been performed. They provide evidence of a partial interaction map between proteins. In the graph representation, a node corresponds to a protein and two proteins are linked when they physically interact. The least two-hybrid screen method has been applied for revealing protein-protein interactions by Uetz et al [260] and by Ito et al [261]. Similarly to metabolic networks, scale-free properties, highclustering and small-world properties have been found. Besides, the studies performed have allowed to address other questions such as the robustness of these networks against random and directed failures [262]. It should be noticed that the databases used in the analysis show very small overlap, while the individual networks obtained from each database show a very similar structure. In particular, it has been argued that the biological functional organization and the spatial cellular organization are correlated significantly with the topology of the network, by comparing the connectivity structure with that of randomized networks.

Finally, we note that networks constructed from gene expression data are currently under exploration [263, 264]. For instance, Agrawal [264] have studied networks from gene expression of cancer data. By analyzing individual gene expression level at different samples, networks in which the degree distribution of the nodes shows a power-law behavior in the tails with an exponent 1 can be constructed. Stuart et al have further shown that co-expressed gene networks of humans, flies, worms, and yeast have scale-free properties [263].

In summary, biological networks seems to share many topological properties. What do these properties mean in a biological system? And what basic principles in biology give rise to such universal features? Many steps toward the answers to these questions have been certainly given in the last several years. However, the majority of the issues addressed are based mainly on analyzing the structure of these networks without taking into account their dynamics, i.e., the fact that the structure correlates with the functioning of the underlying system. For instance, from a topological point of view, it has been 
argued that the nodes with a high degree (the hubs, those contributing to the tail of the degree distribution) are critical for the robustness of the system to intentional removal of them. On the other hand, the hubs have been shown to radically change the behaviour of the system in front of several dynamical processes such as epidemic spreading $[163,216]$. It is yet to see whether or not the same results hold when nonlinear dynamics coexists with complex topological structure. We next describe two promising approaches in this direction.

\subsubsection{Dynamics}

During the last several years a wealth of experimental data, obtained with technological advances such as cDNA microarrays, have allowed the dynamical characterization of several biological processes both on a genome-wide and on a multi-gene scales and with fine time resolution. From a theoretical side, compelling models on the dynamics governing metabolic and genetic processes are hard to build as these biological phenomena are highly nonlinear and with many degrees of freedom. However, scientists have certainly advanced towards a comprehensive global understanding of, for instance, gene regulation through genetic engineering that require a thorough understanding of the general principles that can guide the design process. It is impossible here to provide an exhaustive review of the subject. However, it is important to provide at least some ideas about the research lines that relate the structure and the function of biological systems.

Concepts such as operon, regulator gene and transcriptional repression were first introduced in the literature by Jacob and Monod [265]. Their model has served as the basis for more elaborated models as different regulatory mechanisms have been discovered [266]. Recent theoretical studies capitalize on these kind of models in order to elucidate what are the system constituents, their properties and how they interact in order to give rise to the collective behavior of the system. The final goal is to understand the relationship between structure and function as determined by the biological environment. In this sense, different gene circuit designs should be compared to determine which of them confers selective advantage in an ecological context and thus one should be able to advance what the functional consequences of different designs are. This is usually done by exploring the parameter space and looking for performance criteria such as the ability of a system to return to a steady state after a perturbation (called stability) or its responsiveness, that can be measured as the recovery time of the system after an environmental change (a change in an independent variable).

The results obtained for elementary gene circuits certainly provide answers 
to intriguing questions about how gene circuits could be organized, but at the same time pose new ones. With the recent advances in the characterization of the structure of gene networks, it is clear that genome-wide approaches will allow to discover new higher-order patterns. Therefore, more efforts in modeling the dynamics of increasingly complex gene circuits are expected in the near future. Some steps in this direction have been given.

The basic process of single gene expression is depicted in figure 7.1. The chain reaction starts when a protein binds to a particular DNA region. The binded protein is known as transcription factor and the set of DNA sites to which the transcription factor is attached is called promoter gene. The binding stage yields to the activation of a given gene manifested by the transcription of the genetic information located at this gene into messenger RNA. Finally the mRNA is translated into a protein at the ribosomes. The protein product of this reaction chain can be either used as a transcription factor for starting another reaction or modified for taking part or protein complexes like enzymes involved in cellular physiology. Since the resulting protein is a direct product of the activated gene one can say that a given gene regulates the activity of another one when the product of the former act as the transcription factor of the latter. In this case the regulation is seen produce an activating effect but proteins products can as well act as repressors or inhibitors of other gene activity. We will now focus in the modeling of these interaction dynamics between different genes and neglect more sophisticated details about single gene expression.

\section{Boolean modeling of regulatory networks}

The first attempt to describe the functioning of genetic regulatory networks was performed by S.A. Kauffman [267]. This pioneering work settled the basis for modeling the complex nature of dynamics and interactions between genes and their products. In his work, each gene, $i$, and its product, $I$, were abstracted as a node of a random network having a fixed number, $k$, of neighbors that regulate its activity level, $g_{i}$. This level of activation can be viewed as the concentration of the transcribed mRNA and/or the protein $I$ encoded. The boolean character of the formulation done by Kauffman implies a qualitative description of whether a gene is activated $\left(g_{i}=1\right)$ or not $\left(g_{i}=0\right)$. Besides, time is considered as a discrete variable so that the dynamical behavior of the gene ensemble is described by the temporal series of their activity levels. At each time step the activity level of a single gene is updated considering the state of its $k$ neighbors

$$
g_{i}(t+\tau)=f_{i}\left(g_{j_{1}}(t), \ldots, g_{j_{k}}(t)\right) .
$$



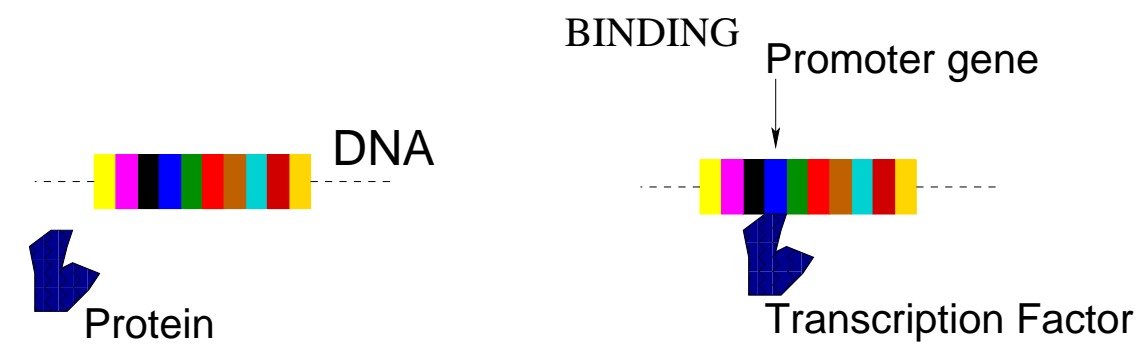

\section{TRANSCRIPTION}

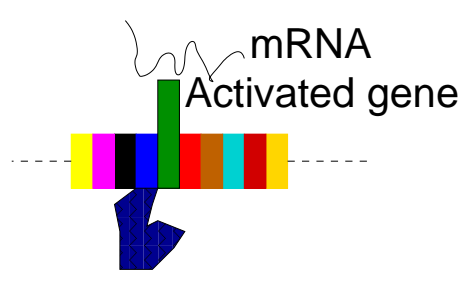

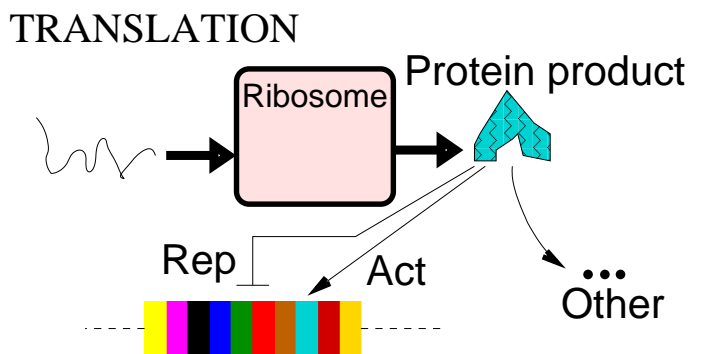

Figure 7.1: Stages of the gene expression. The basic ingredients are the genetic segments in the DNA and proteins. At the first stage a specific protein binds to a part of the DNA sequence called the promoter, the protein is known as the transcription factor since it starts the transcription of the genetic information encoded at the specific gene that the complex promoter + transcription factor regulates. After the genetic information is transcribed into the messenger RNA polymerase it is subsequently translated into proteins at the ribosomes. The protein product that emerges after this process can act either as another transcription factor for the expression of other genes or as a repressor of other genes activity stopping the creation of their protein products. Another possibility is that this protein product participates in the physiological processes of the cell and form protein complexes as enzymes.

This is performed by means of booleans functions, $f_{i}$, that make use of the basic "AND", "OR" and "NOT" logical functions so that the results can be either 1 if the statement is true or 0 if it is false. The construction of each boolean function depends on the particular interactions that a gene shares with its regulators and has to be carefully analyzed with the help of biochemical data. On the other hand, the work by Kauffman was performed from a general point of view and considered a random assignment of the boolean functions that governs the dynamical evolution of the gene's activity. The main result of the work is the existence of a phase transition on the number and length of the dynamical attractors. In particular, for $k>2$ the number of cycles scales with the number of genes, $N$, and its length scales exponentially with $N$. On the other hand, for the case $k=2$ these two quantities scale as $\sqrt{N}$. The above findings are biologically relevant if one considers that different genetic dynamics can be regarded as biologically differentiate cells. Taking into account that the 
cell diversity of a living organism scales approximately with the square root of the genetic population Kauffman suggested that gene regulatory networks should operate just on the border of the dynamically ordered region.

The above findings represented the starting point of a lot of research on the so-called subject of "Kauffman networks" during the last 25 years. These works mainly focus on the search of a full description of the dynamically different regions as well as the characterization of the phase transition (recent work on the matter can be found in [268-273]). On the other hand, "Kauffman networks" have served as a framework for performing a coarse-grained description of real gene regulatory networks. The availability of real regulatory networks inferred from DNA microarray data joined with the easy implementation and management of the boolean dynamics provides a useful tool for understanding the interplay between the topology and the function of biological networks.

The use of boolean dynamics to characterize real genetic regulatory networks has been recently applied to the case of the segment polarity genes in the Drosophila Melanogaster [274]. In this case the whole map of interactions between genes is known and Boolean dynamics is seen to reproduce the patterns of gene expression that appear in the wild type. Besides, it has been tested when mutations are present confirming the validity of the model. The application of this method can help to determine the effects of new mutations and constitute a test for the question of whether the topological features of the interaction network or the kinetic details play the key role in the functioning of biological networks. The success of the use of Boolean modeling points out that it is the former which is the relevant ingredient. Another recent application of Boolean dynamics to a real gene circuit is found in [275] where the yeast transcriptional network is considered. In this case the point of view is drastically different because neither the nature of the interactions between genes nor any dynamical state of the system is available. The starting point is simply a set of connected genes and the authors apply a Boolean modeling of the interactions for determining what set of (Boolean) interaction rules lead to a stable dynamics of the whole system. The authors also study the effect of rewiring links of the network and conclude that dynamical states on top of the original network is more stable than on the perturbed ones. The above two examples show how the coarse-grained Boolean modeling can help to analyze the large amount of available experimental data and answer the question on where the biological stability observed has its roots.

Finally, let us remark that the boolean modeling can be reformulated in order to incorporate realistic features of real regulatory networks. Perhaps, the most important ingredient is to reproduce the effects of noise (which is a substantial characteristic of a biological system). This is usually incorporated 
on the form of a non synchronous update rule, assigning a time delay to each variable of the Boolean functions, $f_{i}$. Another interesting extension of the formulation is considering multi-levels for the gene activity so that the model incorporates some quantitative description on how much the gene is activated.

\section{Continuous time modeling of dynamics}

Now we turn our attention to the ingredients of the continuous time modeling of genetic regulation. We will address three important issues on this matter, namely: (i) the saturable character of the dynamics, (ii) the advances performed when synthetic regulatory networks are used to understand the kinetic of the processes and finally (iii) the application of the knowledge gained with these predesigned networks when real ones are analyzed.

Saturable Dynamics.- The continuous time formulation of a gene response to the activity of its regulating genes is made incorporating the saturable character. There is a wide variety of situations in which the system response to an external action saturates. Perhaps the most familiar example of saturable behavior known to physicists is the adsorption of gas molecules on a solid surface: At thermodynamical equilibrium, the fraction (coverage ratio) $\theta$ of surface interstitials occupied by adsorbed molecules depends on the gas pressure $P$ as [276]

$$
\theta=\frac{P}{P_{0}(T)+P},
$$

where the temperature-dependent constant $P_{0}(T)$ is the pressure value at which the coverage ratio reaches half of its possible maximal value $\theta=1$. While for small values of $P$, compared to $P_{0}(T), \theta$ increases linearly with $P$, for values of the pressure larger than $P_{0}(T)$ the coverage ratio becomes insensitive to pressure variations. Saturable behaviours of this type [277] (and of a more general form; see below) have been introduced by Drossel and McKane in [157] for the modeling of interactions among species in ecological systems, where (most notably) they effectively provide robustness to the limit-cycle behaviour often observed in these systems [278, 279]. In the realm of Social Sciences, saturated response functions have been also used to model some type of social interactions like e.g. the effects of community investments in police pressure and/or educational programs on the street-gang growth phenomena [279].

Biological reaction rates are often saturable; while at small concentrations of a new chemical introduced in a cell, this responds sensitively, the response should not keep growing indefinitely as the new chemical concentration grows. The archetypal example of saturation in biological systems is the MichaelisMenten equation [280, 281] governing the concentration evolution of a product 
catalyzed from a substrate by an enzyme which binds to it. If $x$ and $y$ denote the concentrations of product and substrate respectively, then the reaction rate is given by

$$
\frac{d x}{d t}=\frac{V_{\max } y}{K_{M}+y}
$$

where $K_{M}$ is called the Michaelis constant and $V_{\max }$ is the value at which the rate saturates for high substrate concentrations. This saturation behaviour can be understood from the usual chemical kinetics (law of mass-action) in an intuitive way: when the enzyme molecules are mostly bound to substrate molecules, adding more substrate cannot speed up the reaction [282]. If $n$, instead of only one, substrate molecules bind to the enzyme, the reaction rate takes a more general functional form of saturation, often called Hill equation

$$
\frac{d x}{d t}=\frac{V_{\max } y^{n}}{K_{M}+y^{n}}
$$

showing a sudden increase of the reaction rate towards saturation around $y=$ $K_{M}$. The Hill parameter $n$ often takes on non-integer values. Both MichaelisMenten and Hill equations are often used in models of biological reactions, even when the explicit mechanisms generating them are unknown in many cases.

Synthetic genetic networks.- In cells, the proteins, RNA and DNA form a complex network of interacting chemical reactions governing all cellular functional activities like metabolism, response to stimuli, reproduction, ... While the understanding of the structure of these networks is growing rapidly, the current understanding of their dynamics is still rather limited. In this regard, an interesting body of research is currently addressed to synthetic genetic networks, which offer an alternative approach aimed at providing a controlled test bed for the detailed characterization of some isolated functions of natural gene networks, and also pave the way to engineering of new cellular behaviour.

An example of synthetic gene regulatory network, termed the "repressilator", is becoming one of the best studied model systems of this kind. The repressilator is a network of three genes, whose products (proteins) inhibit the transcription of each other in a cyclic way; they were added to the bacterium E. coli, so periodically inducing the synthesis of green fluorescent protein as a readout of the network state [283]. The authors of the work first argue that the represilator can show temporal fluctuations in the concentration of each of its components, by analyzing a system of six ODE's (which, in turn, were obtained by a process of integration-out or coarse-grain away of the promoter states involved in the regulation, and rescaling of the variables) modeling the network. If $p_{i}(i=1,2,3)$ denote the three repressor-protein concentrations (in 


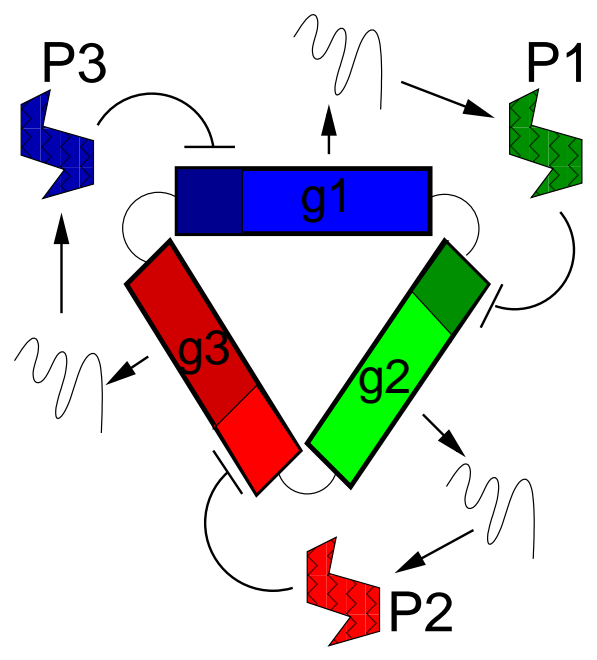

Figure 7.2: Schematic representation of the so-called repressilator. The repressilator is small network composed of three genes $g_{i}(i=1,2,3)$ each of one inhibits the activity of the subsequent. That is, the protein product of gene $g_{i}$ act as the repressor of the activity of gene $g_{i+1}\left(g_{3}\right.$ inhibits $\left.g_{1}\right)$.

units of the Michaelis constant $K_{M}$ ), and $m_{i}$ their corresponding mRNA concentrations (appropriately rescaled), the repressilator equations are (assuming the symmetrical case in which all three repressors are identical except for their DNA-binding specificities):

$$
\begin{aligned}
\frac{d m_{i}}{d t} & =-m_{i}+\frac{\alpha}{1+p_{i}^{n}}+\alpha_{0} \\
\frac{d p_{i}}{d t} & =-\beta\left(p_{i}-m_{i}\right)
\end{aligned}
$$

where $i=1,2,3$ and $j=3,1,2 ; \alpha_{0}\left(\alpha+\alpha_{0}\right)$ is the number of protein copies produced from a given promoter type in the presence (absence) of saturating amounts of repressor, $\beta$ is the ratio of the protein decay rate to the mRNA decay rate, and time is rescaled in units of the mRNA lifetime. This system of equations has a unique steady state which can be stable or unstable depending on the parameter values. In the unstable region of parameter space, the three protein concentrations fluctuate periodically. Experiments show temporal oscillations of fluorescence, which were checked to be due to the repressilator. Though admittedly oversimplified, the model of ODE's guided the experimental design, for it served to identify possible classes of dynamic behaviour and to determine which experimental parameters should be adjusted in order to obtain sustained oscillations.

Not surprisingly, the repressilator called attention from experts on (biological) synchronization, for it offers good prospectives for further insights into the nature of biological rythms, whose mechanisms remain to be understood. In this respect, in reference [284] the authors propose a simple modular addi- 
tion (of two proteins) to the repressilator original design, which allows for a mechanism of coupling between cells containing the repressilator networks.

Modules.- As seen in the previous subsection, even a very small gene network, like the repressilator, requires some simplifying assumptions for an analysis of its dynamic behaviour in terms of ordinary differential equations. With large networks involving thousands of regulatory genes, this approach would require a huge number of differential equations and, what is even more problematic, an exploding number of dimensions of the parameter space (decay rates, production rates, interaction strengths, etc. ). Thus an important issue concerns the right level of description when constructing quantitative models of large genetic networks [285].

In this regard, several works (e.g. [286-288]) have focused on the identification of general building blocks (motifs) in genetic networks, showing robust or "reliable" behaviour. These include small modules of a few genes, such as autoregulatory excitatory feedback loops, inhibitory feedback loops, feed-forward loops and dual positive-feedback loops, which represent different kinds of robust switching elements, whose occurrence as subgraphs in real networks is significantly higher than in their randomized versions. These works provide support to discrete models in which genes are modeled as switch like dynamic elements that are either "on" or "off", of the Boolean type described in the previous section, and point toward strong correlations between structural and functional properties of genetic regulation networks.

The robustness of slightly larger modules, like the segment polarity genes of the fruit fly Drosophila (a subgraph of the segment determination network, responsible for the embryonic development of the insect body segments), has been convincingly tested with a realistic dynamical model [289] supporting the view that segmentation is modular, with each module autonomously expressing a characteristic intrinsic behaviour in response to transient stimuli. A connectionist model for the segment determination system of Drosophila, including cell-cell interaction via one-dimensional diffusion [290, 291] has been thoroughly characterized (along with its continuum limit (PDE) equations [292]). These generalized reaction-diffusion models inspired further work in [157, 293, 294] which identified minimal gene networks associated to different segmentation patterns; also, extensive computer simulation of randomly generated networks showed that combinations of spatial patterns can be mapped into combinations of the basic modules.

The resistance of modules to variations (proxy for mutations of small effect) in the kinetic constants and various parameters that govern its dynamical behaviour, may suggest that evolution could rearrange inputs to modules without 
changing their intrinsic behaviour, or as conjectured in [157, 293, 294], that the target of selection would operate not only on single-gene level structures, but also on the available structures in the high-dimensional parameter space of the model equations.

In summary, it is a major challenge the discovery of how biological entities interact to perform specific biological processes and tasks, as well as how their functioning is so robust under variations of internal and external parameters. Such an achievement is only possible by merging the new knowledge gained from network analysis with nonlinear dynamics models relevant in biological processes such as the genetic regulation. This is what is driving current theoretical efforts, in which new mathematical models and methods borrowed from nonlinear dynamics are being studied on top of the real architecture of biological networks. Besides, the confluent interest of several scientific disciplines in the many aspects of the problem of Structure-Function correlations in systems made up of discretely many nonlinearly interacting components (of which gene regulatory networks are but a particular example), reccomends to pay some attention to general abstract models. These models should be both conceptually simple and universal in their perceptions.

In next section we will consider the essential ingredients of both topology and dynamics of gene regulatory network, namely saturability of the interactions and scale-free character of the patterns of interconnections among constituents. As a result, we face a problem where nodes in a scale-free network self-regulate their dynamics through either activatory or inhibitory interactions in a fashion that resembles the regulation among genes in cells.

\subsection{Regulatory dynamics in scale-free networks}

The model that we analyze in this section tries to capture the general ingredients of the entangled topological and dynamical complexity of genetic regulatory networks introduced in the previous section. For this we employ a relevant kind of nonlinear dynamics: Activation/Inhibition (AI) competing interactions with a "saturated response" rule for the rate of activation (see figure 7.3) of Michaelis-Menten type (introduced previously in section 7.1.2). Besides, the interacting units (genes) sit on a lattice which is both small-world and scale-free. For this we use the Barabási- Albert network (section 5.2.3). We have to remark that neither real genetic networks are explored nor a detailed description of the nonlinear A/I dynamics is incorporated. However, this coarse-grained representation of genetic regulatory networks presents features that are revealed when dynamics and topology from experimental data 
are analyzed.

We will first present in detail the AI dynamics in section 7.2.1. Here, some basic general features of the model are discussed, namely the network fragmentation in subclusters (or islands) of collective dynamics, and the generic types of asymptotic behaviours coexisting in the phase space of collective dynamics (steady, periodic and chaotic states) as well as the observed bifurcations in phase portrait upon parameter variations. These basic consequences of the AI competition on the complex network are prevalent for a range of values of the AI ratio as we will see below. Finally, the bifurcations found are explained in terms of the Floquet analysis of the solutions.

Once the main dynamical regimes of the model are characterized we will turn our attention on the statistical characterization of both the dynamical behaviours observed and the structural characterization of the dynamical islands in section 7.2.2. We perform an extensive exploration of the parameter space, employing different initial conditions and substrate network realizations, in order to find the conditions for the existence of chaotic and periodic behavior as well as to fully characterize the main topological characteristics of the dynamical islands.

We will end this study in section 7.2.3 looking for those substructures of the dynamical islands that are relevant for the dynamical evolution of the system.

\subsubsection{The model: basic dynamical features}

As stated above, we introduce here a model of Activatory/Inhibitory interactions regulating the activity $g_{i}(t)(i=1, . ., N)$, of $N$ constituents (e.g. genes, agents, substrates), with $N$ generally being a large number. The real functions of time $g_{i}(t)$ are each one attached to a node of a graph with adjacency matrix $C_{i j}(N \times N)$. Then, in terms of the dynamics, the matrix element is non-zero, $C_{i j} \neq 0$, only if the rate of variation of the $i$-th node activity, $g_{i}(t)$, depends on the activity $g_{j}$ of the $j$-th node (interaction $i \leftarrow j$ ). Different realizations of the $C_{i j}$ matrix are constructed using the method of Barabási and Albert (section 5.2.3) for $m=3(\langle k\rangle=6)$ in order to have a scale-free network with exponent $\gamma=3\left(P(k) \sim k^{-3}\right)$.

The interaction $(i \leftarrow j)$ can be either activatory (excitatory) or inhibitory; correspondingly we define the interaction matrix element $W_{i j}$ to be +1 or -1 , respectively (and $W_{i j}=0$ whenever $C_{i j}=0$ ), and call $p$ the fraction, among non-zero elements, of negative signs (note that while $C_{i j}$ is a symmetric matrix, $W_{i j}$ is not in general). Moreover, the sign distribution of elements is taken uniform in the set of (approx. $\langle k\rangle N / 2$ ) links of the network realization. 


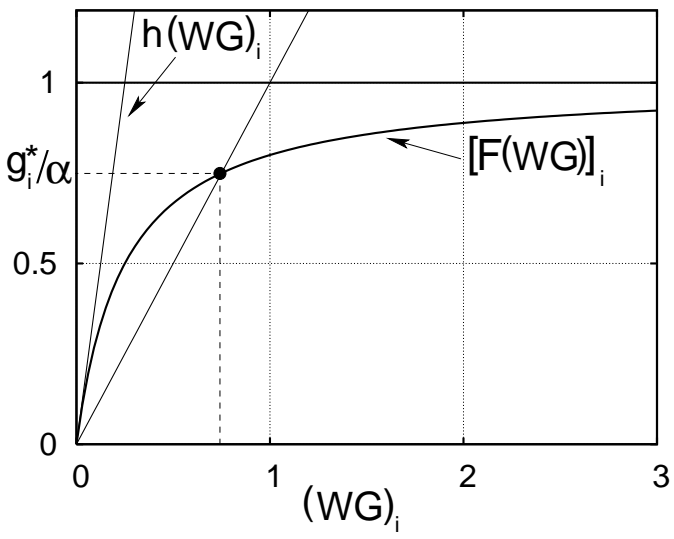

Figure 7.3: Saturated functional response $(h=4)$.

The dynamics of the nodes activity vector $\mathbf{G}(t)=\left\{g_{i}(t)\right\}$ (with $i=$ $1, \ldots, N)$ that we consider is such that only in the presence of excitatory neighbours activity could $g_{i}$ possibly be non null, otherwise $g_{i}$ decays to zero with an exponential rate:

$$
\frac{d \mathbf{G}(t)}{d t}=-\mathbf{G}(t)+\alpha \mathbf{F}[\mathbf{W G}(t)]
$$

where $\mathbf{F}$ is a nonlinear vector function whose argument is the product of the interaction matrix $\mathbf{W}$ and the activity vector $\mathbf{G}$, and $\alpha(>0)$ accounts for the strength of the interaction. The function $\mathbf{F}$ is a saturated response function (see figure 7.3), defined as:

$$
\mathbf{F}(\mathbf{z})=\left\{\frac{\Phi\left(z_{i}\right)}{h^{-1}+\Phi\left(z_{i}\right)}\right\},
$$

where $\Phi(x)$ is a function defined as

$$
\Phi(x)= \begin{cases}0 & \text { if } x \leq 0 \\ x & \text { if } x>0\end{cases}
$$

The dynamics of the system defined as before is determined by only two parameters, $h$ and $p$. One controls the degree of nonlinearity and the other the topological properties of the network, respectively. In our numerical studies of the model we have fixed the value of the parameter $\alpha=3$, and varied the parameters $0 \leq p \leq 1$ and $0 \leq h \leq 10$. One can easily realize that the solutions for non-negative initial conditions remain bounded for all $t \geq 0$. Indeed, as the nonlinear term in eq. (7.7) is bounded above by $\alpha$, one obtains that $\dot{g}_{i}<0$ whenever $g_{i}>\alpha$. Also, if $g_{i}=0$ then $F_{i}(\mathbf{W G}) \geq 0$, so that the activities cannot become negative. 

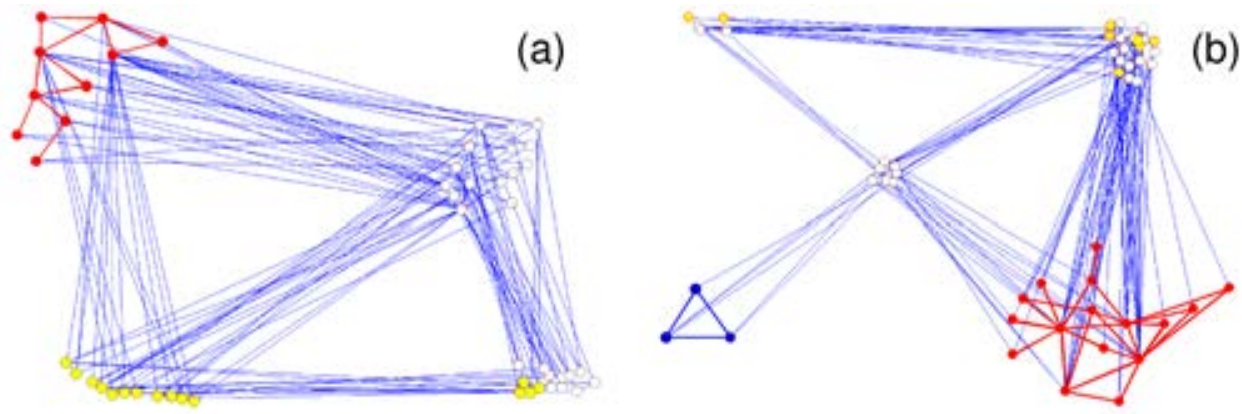

Figure 7.4: Two examples of network fragmentation. The nodes of the networks are classified in: $(i)$ dynamical nodes (red), ( $i i)$ stationary nodes with nonzero activity (blue), (iii) stationary nodes with zero activity belonging to $\mathcal{D}_{0} \oplus \mathcal{D}_{1}$ (yellow) and (iv) remaining nodes with zero activity (white). Note that the white central nodes in (b) act as the frontier between the dynamical island and the steady nonzero activity one.

The above dynamics can be regarded, e. g., as a generalization of the simplified and coarse-grained genetic models, Random Boolean Networks, introduced in section 7.1.2. Equation (7.7) incorporates the experimental observation of a continuous range of activity levels [294]. It is worth mentioning that while continuous time linear models have been successful for the reconstruction of the interaction networks from experimental data [295], nonlinear models like eq. (7.7) are expected to be more appropriate for a quantitative description of the dynamics.

The dynamics (7.7) of a two-agent (dimer) model has been considered in reference [294], in the context of virus-cell interactions in bacteria and general gene regulatory activity models, where a rich repertoire of behaviours, like multi-stability (multiple attractors in phase space) was reported. In the forthcoming paragraphs, we will report some remarkable general features of the network dynamics.

\section{Activation and Inhibition interplay: Fragmentation}

A brief look at equation (7.7) easily reveals that for any value of the parameters $p$ and $h$ the state of inactivity, $\mathbf{G}=0$, is always a solution. As a matter of fact, for $h=0$, or $h \neq 0$ but $p=1$, this is the unique asymptotic solution (global attractor in the phase space) for all possible non-negative initial conditions. However, for $h \neq 0$ and $p \neq 1$ other asymptotic solutions, with islands of positive activity, generically coexist with the rest state. The term islands denotes here subnetworks that are interconnected through nodes which have evolved to null activity, so that their dynamics are effectively disconnected. 
The fragmentation of the network dynamics into disconnected islands is a generic feature of AI interactions, as the following considerations suggest. Let us call $\mathcal{D}$ the set of nodes whose activities, for a given initial condition $\mathbf{G}(t=0)$, asymptotically vanish. It is easy to see that, irrespective of the initial condition, this set is generically non-empty.

Indeed, if a node $i$ is such that $W_{i j}=-1$ or 0 for all $j$, then its activity $g_{i}(t)$ will tend to zero. Let us call $\mathcal{D}_{0}$ the set of these nodes, and note that its measure $\left(\sum_{k} P(k) p^{k}\right)$ is a non-zero increasing function of $p$. Now, call $\mathcal{D}_{1}$ the set of nodes $l$ such that their positive $W_{l j}$ occur for $j$ 's in $\mathcal{D}_{0}$, and so on ... Due to the small-world property, there are in fact very few relevant $\mathcal{D}_{n}$ $(n=0,1, \ldots)$ sets. Its union $\mathcal{D}^{*}=\bigcup \mathcal{D}_{n}$ is easily seen to have a non-zero measure which increases with $p$.

The nodes of $\mathcal{D}^{*}$ are structurally (i.e. irrespective of initial conditions) inactive. Depending on the initial condition, the set $\mathcal{D}$ may include other nodes not contained in $\mathcal{D}^{*}$, namely those nodes that evolve to inactivity due to the initial condition (dynamically, instead of structurally, inactive): See e.g. the white nodes in figure 7.4, where we show two small networks of $N=50$ nodes to allow a simple visualization of the sets $\mathcal{D}^{*}$ and $\mathcal{D}$. In other words, the measure of $\mathcal{D}$ may in general be (much) larger than the measure of the "structurally dead" nodes $\mathcal{D}^{*}$.

From the previous considerations, whether or not the set $\mathcal{D}$ percolates the network realization, leaving out islands of disconnected activity, is an event that clearly depends on both the parameter $p$ and the initial conditions. But also the discussion correctly suggests that fragmentation of the network into subclusters with independent temporal evolution is a generic (non-zero measure) feature. Our numerics, which are extensive in the sense of (both, network realizations and initial conditions) large sampling, convincingly corroborate this assertion. Figures 7.5 and 7.6 show two islands of periodic and chaotic activity, respectively, as well as the temporal evolution of $g_{i}(t)$ for some of their constituent's nodes (see the next section for a more detailed discussion of the figures).

\section{Temporal fluctuations of asymptotic solutions}

The dynamics of the system turns out to be very rich and, depending on the values of $p$ and $h$, three different asymptotic dynamical regimes are observed, characterized by stationary, periodic and chaotic attractors. Here we characterize these different dynamical regimes and the transitions between the different states when $h$ is varied. For this purpose we have performed extensive computations following this scheme: 

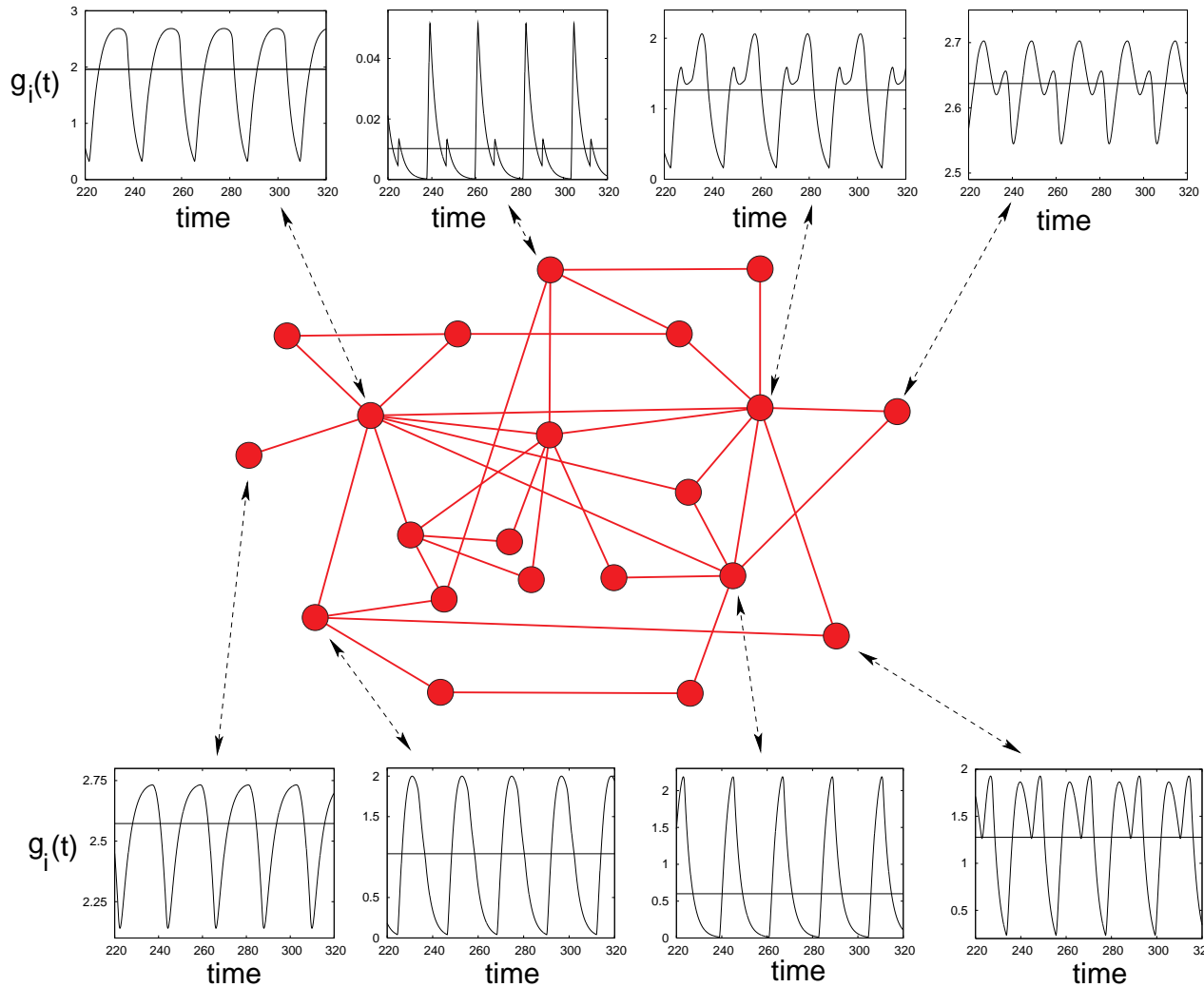

time

Figure 7.5: Example of a cluster of 21 nodes displaying periodic dynamics. The insets show the dynamical patterns of each node (see text for the interpretation). The maximum Lyapunov exponent is $\lambda=-0.00034$ and the dynamical parameters are $h=4$ and $p=0.7$. The cluster is embedded in a substrate network of $N=50$.

( $i$ For a given value of the parameters $h$ and $p$ and network realization the initial values of $g_{i}$ are taken from a uniform distribution in the interval $(0, \alpha)$.

(ii) First integration of the equations is performed using a $4^{\text {th }}$ order RungeKutta scheme. The total integration time is large compared with the transient.

(iii) Check the dynamical state of the network.

(iv) Check the connectivity between the dynamical (if any) nodes in order to obtain the dynamical islands.

$(v)$ In order to determine the state of the system we perform a second integration for computing the largest Lyapunov exponent $\lambda$ (See Appendix B). If $\lambda>5 \cdot 10^{-3}$ the dynamics is considered chaotic. If $\lambda<5 \cdot 10^{-3}$ we look at the frequency of the periodic motion. 


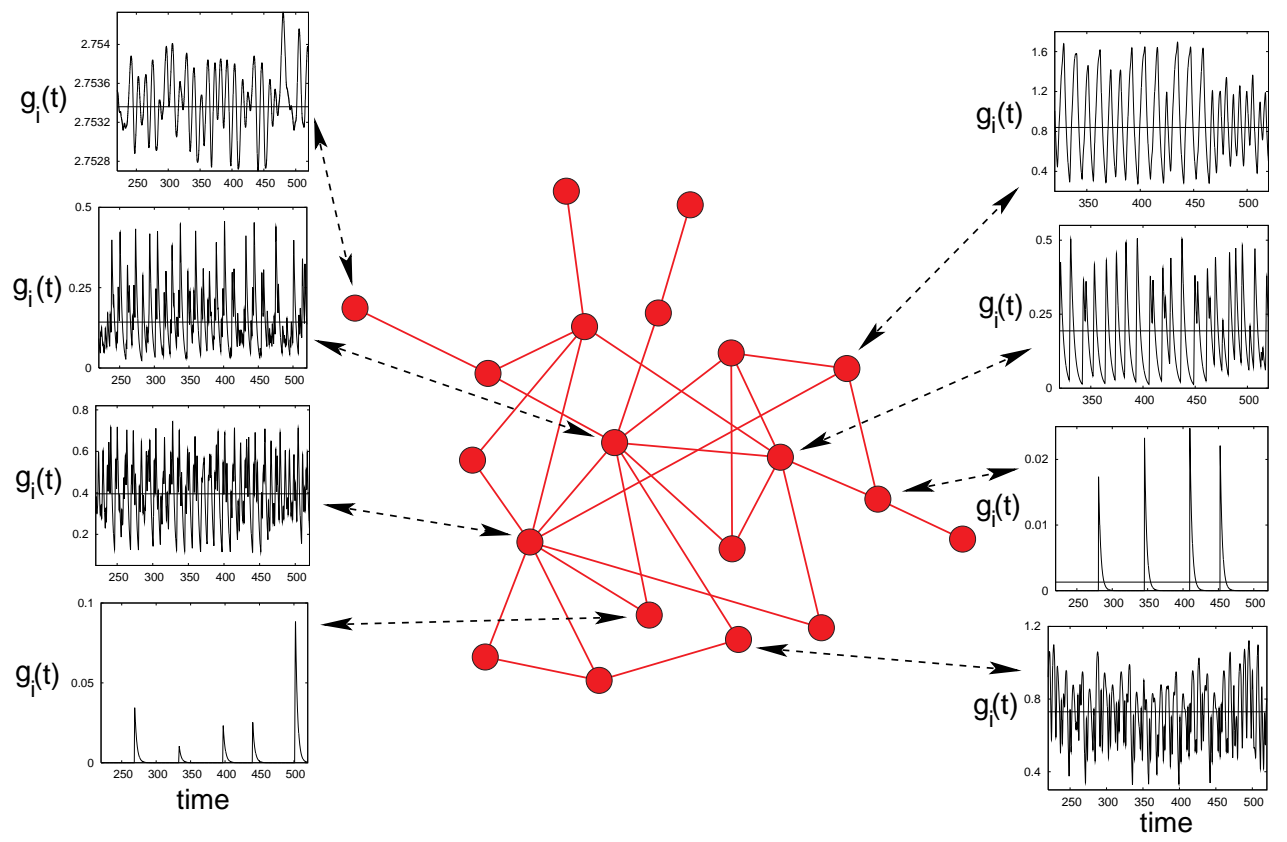

Figure 7.6: Example of a cluster of 19 nodes displaying chaotic dynamics. The insets show the dynamical patterns of each node (see text for the interpretation). The maximum Lyapunov exponent is $\lambda=0.4716$ and the dynamical parameters are $h=4$ and $p=0.7$. The cluster is embedded in a substrate network of $N=50$.

(vi) Change adiabatically the value of $h, h^{\prime}=h+\delta h$ with $|\delta h| \ll 1$, and repeat stages $(i i)-(v)$.

Following this procedure one can monitorize the evolution of the system as a function of the nonlinear parameter $h$.

Steady states.- Let us first focus on the steady state solution to eq. (7.7). As already noted above steady states of zero and non-zero (for $h \neq 0$ and $p \neq 1$ ) activity can be found as asymptotic solutions. Let us first focus on the transition between both steady states. This transition is just the genesis (considering the evolution as the parameter $h$ is increased) of the dynamical islands presented above.

Depending on the specific network realization (i.e. the matrix $W_{i j}$ ), the rest state can become unstable when the value of $h$ is increased from zero. This will occur for the value $h=\tilde{h}$ at which the largest eigenvalue (among those associated to eigenvectors such that all their components have the same sign ${ }^{1}$ ) of the matrix $-\delta_{i j}+\alpha h W_{i j}$ becomes positive. Then $\tilde{h}$ is determined

\footnotetext{
${ }^{1}$ Note that $\mathbf{F}(\mathbf{z})$ has a jump discontinuity in first partial derivatives at the rest state. Thus we consider the matrix of right-handed partial derivatives, and then pay only attention
} 


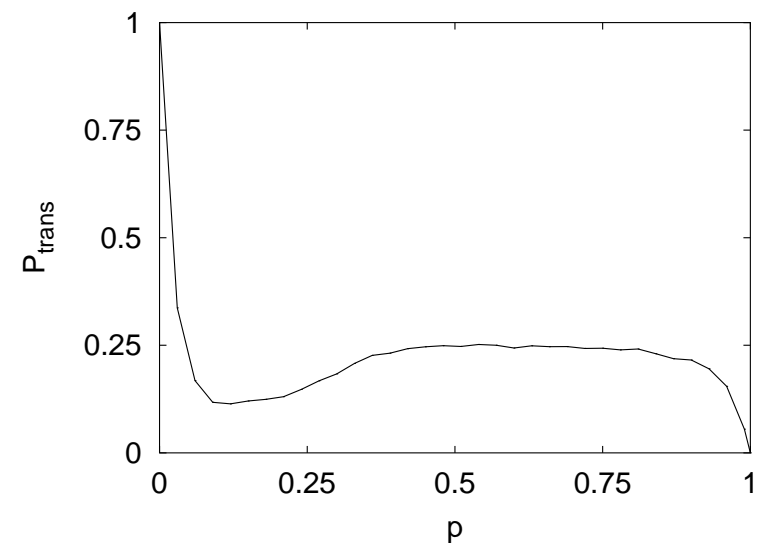

Figure 7.7: Probability $P_{\text {trans }}$ that the uniform state of gene inactivity becomes unstable for some value of $h$, as a function of the parameter $p .10^{4}$ different realizations have been used for each value of $p$.

as $1 /\left(\alpha \lambda_{\max }\right)$, where $\lambda_{\max }$ is the largest eigenvalue of $W_{i j}$, provided $\lambda_{\max }>0$ (no instability of the rest state will occur if $\lambda_{\max } \leq 0$ ). In figure 7.7 we show the probability $P_{\text {trans }}$ that the rest state become unstable for some value of $h$, as a function of the parameter $p$. This probability has been estimated from the computation of $\lambda_{\max }$ for $10^{4}$ different realizations of $W_{i j}$ for each value of $p$. Though for most values of $p$ the rest state remain stable at all values of $h$ in $75 \%$ (or more) of the realizations, it coexists in phase space with other attractors, so that only a basin of initial conditions evolve to this state.

The rest state typically destabilizes through a transcritical bifurcation [296], where an unstable branch of stationary solutions exchanges stability with the rest branch (see figure 7.8.a. The computed largest Lyapunov exponent shows then a variation with $h$ as in figure 7.8.b near $h \simeq 0.33$ : it approaches zero (from negative values) at the bifurcation parameter value, and then decreases indicating that now the attractor belongs to the new stable stationary branch, in which the nodes of a cluster display non-zero constant activity $g_{i}(t)=g_{i}^{*}$ (let us say chemostasis regime). As shown in figure 7.8.a, the activity of these nodes typically increases with $h$. Eventually, this state becomes unstable for larger values of $h$, and evolves to a periodic state in which the activities oscillate (figure 7.8.c) regularly in time.

Periodic states.- The presence of inhibitory interactions makes possible the existence of instabilities in the fixed point chemostatic solutions of evolution eq. (7.7). Using linear stability analysis techniques, these "typical" instabilities are characterized as Hopf bifurcations (either direct or often inverse), where attractors of exactly periodic collective activities, $g_{i}(t)=g_{i}(t+T)$, are born out from the unstable fixed points. To illustrate the aspect of typical periodic fluctua-

to tangent space vectors which do not bring the system into the region of negative activities. 

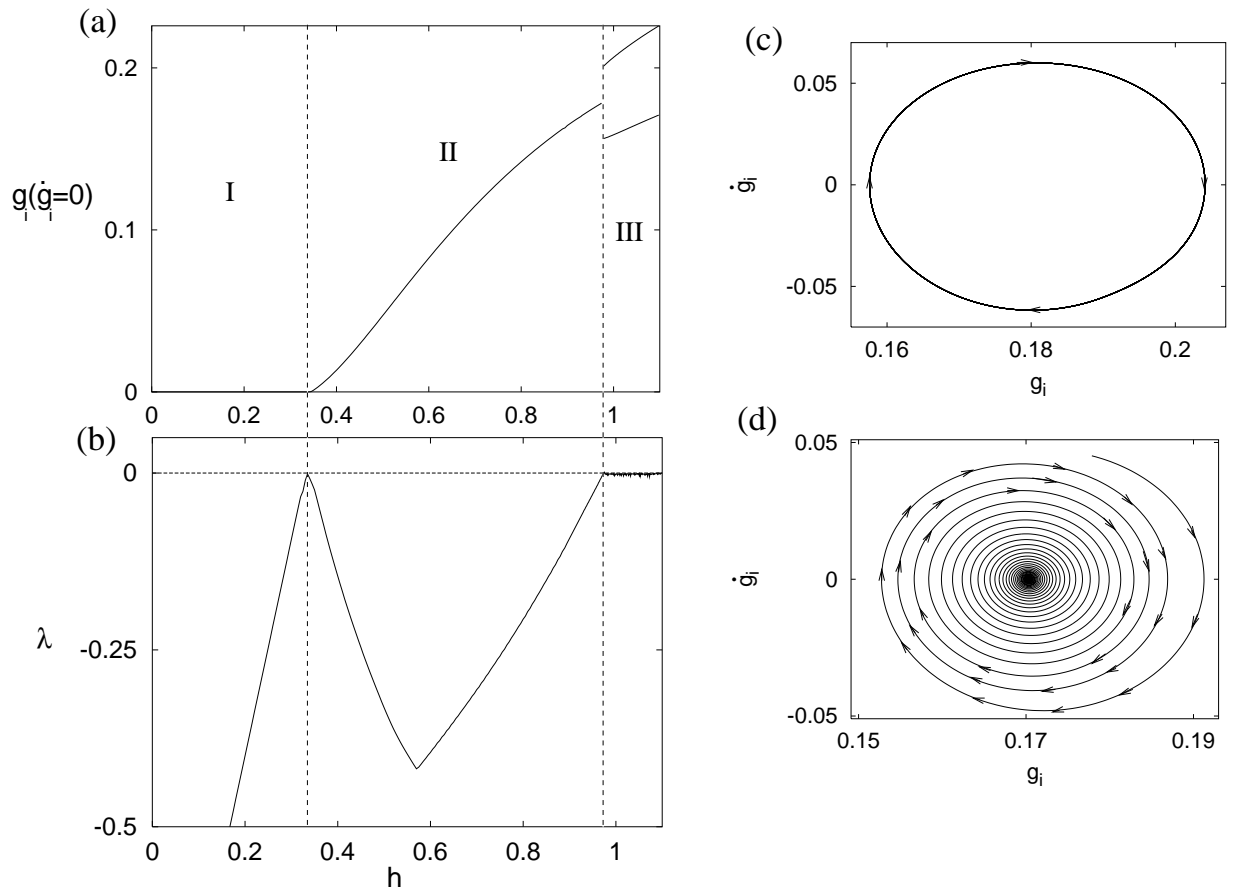

(d)

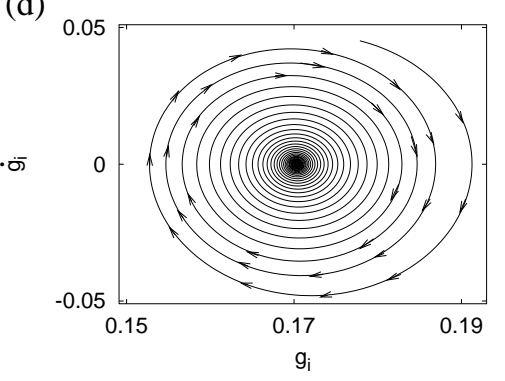

Figure 7.8: (a) Dependence of the quantity $g_{i}\left[\dot{g}_{i}=0\right]$ (activity level when the its first derivative is zero) of a single gene with the parameter $h$. This gene belongs to a cluster which undergoes two bifurcations when increasing the value of $h$ starting from the rest state configuration of the whole network at $h=0$. In the first bifurcation $(h \simeq 0.345)$ a cluster of nodes in a stationary state with non-zero activity level merges. When $h \simeq 0.976$ the genes of this cluster end in a periodic attractor. The evolution of the Largest Lyapunov exponent of the network as $h$ is increased is plotted in (b) showing the two bifurcations. (c) Periodic trajectory in the portion of the phase space corresponding to the gene of figure (a), the value of $h$ is 0.98 . (d) Decay of the activity level of the same gene to the stable fixed point for $h=0.97$ (just before the second bifurcation) when the initial condition of the network is the periodic solution shown in (c).

tions we turn our attention to figure 7.5 where some examples of the temporal activity $g_{i}(t)$ of different nodes inside an island of synchronized activity from a representative system are represented. Note that the abundance of out of phase oscillations of neighbours activity is a natural consequence of inhibitory interactions: the growth of the activity of a gene $j$ inhibiting gene $i\left(W_{i j}=-1\right)$ leads eventually to a null value of $F_{i}(\mathbf{W G}(t))$, thus to an exponential (free) decaying of the activity of gene $i$, until its activity is triggered again (due to the decay of the activity of inhibitory genes and/or the increase of the excitatory genes activity). Horizontal lines in insets of figure 7.5 indicate the average level $\bar{g}_{i}$ of node activity. We see that in some of the island nodes the amplitude 


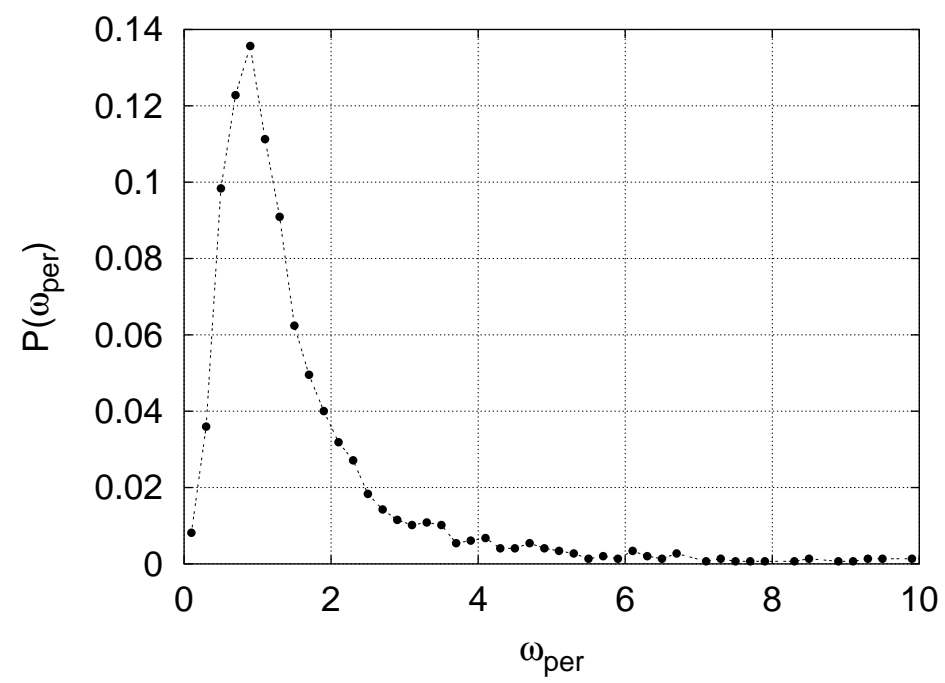

Figure 7.9: Probability that a node with periodic dynamics converges to an orbit of angular frequency $\omega_{\text {per }}$ (in arbitrary units). The results are average over different network realizations and at least 100 different initial conditions for a network of $N=100$ nodes and $p=0.7$. $h$ has been fixed to 4 .

of the oscillation is small compared to $\bar{g}_{i}$ (see e.g. top rightmost and bottom leftmost insets); while in others they are of comparable size, even to the point that lowest levels of activity can reach a null value, before activity is triggered again after inhibiting neighbors activity decreases enough. The inverse period (frequency) $\omega=1 / T$ of a periodic attractor changes with parameter and is naturally dependent on each specific island realization. A sampling over different initial conditions and network realizations allows to compute the frequency distribution for a periodic state $P(\omega)$. For this purpose we identify those realizations in which $\lambda<5 \cdot 10^{-3}$. Then, we focus on the nodes for which $d g / d t \neq 0$. Once identified, a vector $\mathbf{T}_{n}^{i}=\left\{t_{1}^{i}, t_{2}^{i}, \ldots, t_{n}^{i}\right\}$ is constructed and stored for every periodic dynamics $g_{i}$. The $t_{j}^{i}$ 's stand for the times fulfilling the conditions $g_{i}\left(t_{1}^{i}\right)=g_{i}\left(t_{2}^{i}\right)=\ldots=g_{i}\left(t_{n}^{i}\right)$ and $\left.d g_{i}\left(t_{1}^{i}\right) / d t=d g_{i}\left(t_{2}^{i}\right) / d t\right)=\ldots=d g_{i}\left(t_{n}^{i}\right) / d t$ 2. In this way, after verifying that $t_{j}^{i}-t_{j-1}^{i}$ is constant, the period of the corresponding $i$-orbit is given by this constant. As the free decay of a gene activity, that occurs when its inhibitory inputs prevail over activatory ones, has an associated time scale of order unity, one should expect values of this order for the period of oscillations. This expectation is confirmed looking at the probability that a periodic cycle has an angular frequency $\omega_{p e r}, P\left(\omega_{p e r}\right)$

\footnotetext{
${ }^{2}$ Since the integration is done at fixed time intervals, a further numerical check is imposed. Namely, $\left|g_{i}(t)-g_{i}(0)\right|<\frac{d g_{i}(0) / d t}{2}$ and $\left|\frac{d g_{i}(t)}{d t}-\frac{d g_{i}(0)}{d t}\right|<\frac{d^{2} g_{i}(0) / d t^{2}}{2}$.
} 
shown in figure 7.9. As shown in this figure, it is very likely that the frequency of the activity of a periodic island lies around $\omega_{p e r}=1$. It is also of interest that $P\left(\omega_{\text {per }}\right)$ is not symmetric, but biased towards larger frequency values. It is difficult to figure out an explanation to this behavior. It may probably has to do with the spatial distribution of the nodes and the specific value of $p$ which controls the average number of input and output connections a node has.

Chaotic States.- When $h$ is further increased one observes that these periodic attractors, in turn, typically experience also period doubling instabilities, and through the well-known universal scenario of (successive) period doubling bifurcation cascade, the onset of chaotic attractors takes place in the phase portrait of the network dynamics. To help visualization of the generic types of asymptotic network dynamics, we represent in figure 7.10 the bifurcation diagram for a typical attractor. At different values of the (Michaelis-Menten) parameter $h$, and constant values of $\alpha=3, p=0.7$, we plot the activity of an individual node at the instant when its time derivative vanishes. Thus, a single branch in the figure indicates stationary activity, two branches indicate a periodic attractor, etc. We also plot in figure 7.10 the largest Lyapunov exponent $\lambda$ on the attractor, so to allow discerning between chaotic (positive $\lambda$ ) and eventual regular quasiperiodic evolutions $(\lambda=0)$. Figure 7.11 shows the phase space diagrams for the activity of one node (belonging to a different dynamical island) as $h$ is increased. For small values of $h$, the gene is in a periodic cycle, which doubles its period successively until it reaches the chaotic phase.

A similar bifurcation diagram for a different network realization is shown in figure 7.12, where one can appreciate (see inset) a commonly found bifurcation (though it appears much less often than period doubling), namely period tripling bifurcation. Its characterization will be made below in the next subsection where the Floquet analysis of periodic attractors is presented.

A visualization of chaotic temporal fluctuations of the activities in a cluster is shown in figure 7.6. Here again we see nodes (e.g. top left inset) where the size of activity fluctuations is less than 1 per cent of the average level $\bar{g}_{i}$. Most remarkable, there are nodes (like the one in bottom left inset) which remain inactive most of the time intermittently experiencing spikes of short duration activity. This amazing variability of individual node temporal activity on the chaotic attractors is a generic feature of the network dynamics. The existence of spike behaviour of individual nodes activity suggests correctly that eventual variations of parameters like $h$ may lead to permanent inactivity of some particular nodes, so providing a straightaway decreasing of the dynamical cluster size or, the other way around, the activation of inactive nodes in the frontier. 


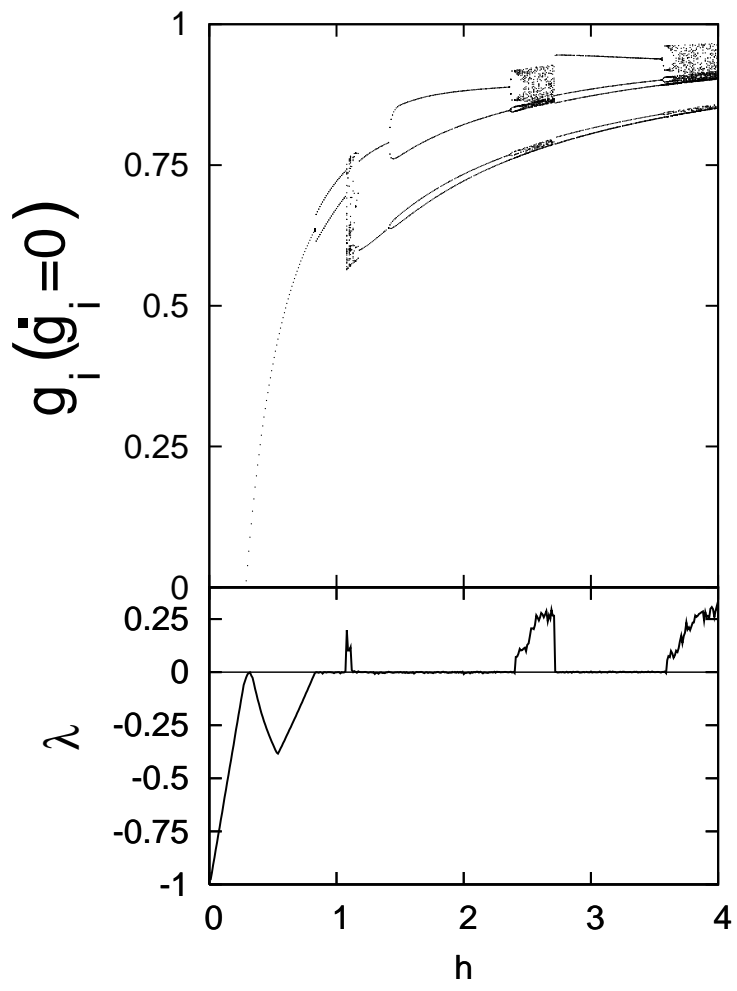

Figure 7.10: Example of bifurcation diagram $(N=60$; island size: $14 ; p=0.8$. One can appreciate an inverse Hopf bifurcation and several (direct and inverse) period doubling bifurcation cascades. The maximum Lyapunov exponent $\lambda$ is plotted in the lower part.
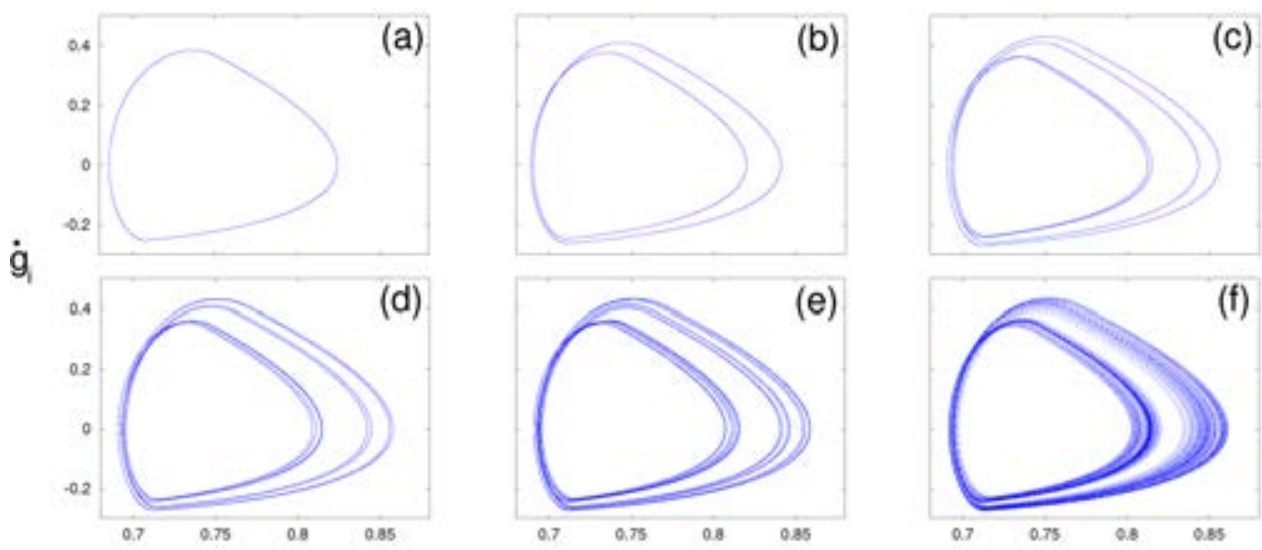

(c)
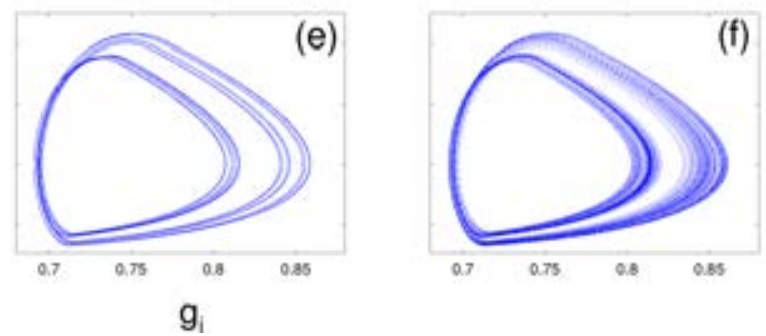

Figure 7.11: Phase space of a node ending up in a chaotic state as the value of $h$ is increased. Successive period doublings starting from a periodic cycle can be appreciated. The values of $h$ (from (a) to (f)) are: 5.30, 5.50, 5.63, 5.65, 5.66, 5.68, respectively. The parameters are set to $p=0.7$ and $N=100$. 


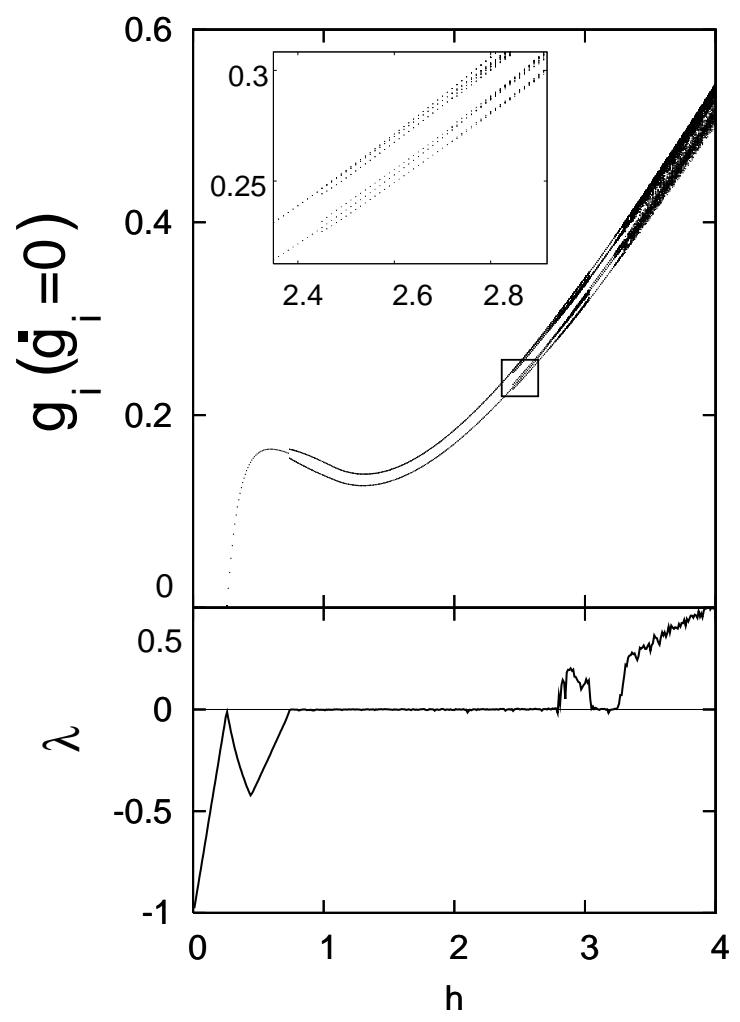

Figure 7.12: Example of bifurcation diagram $(N=60$; island size: $12 ; p=0.8)$ showing (see inset) a period tripling bifurcation. The maximum Lyapunov exponent $\lambda$ is plotted in the lower part.

It is important to note that, for a fixed set of parameter values and a given network realization, there are generally several different attractors coexisting in the phase space portrait of the network dynamics, each one having its own basin (of attraction) of initial conditions. Multi-stability appears as a generic consequence of the excitatory/inhibitory interplay. Importantly also, there can be very many unstable periodic trajectories (often entangled) flowing in between basins of attractions. The excitatory/inhibitory competition is also responsible for the appearance of temporally complex (positive Lyapunov exponent) aperiodic evolutions, associated to the bifurcation cascade scenario. As we will show in section 7.2.2 the manifestation of fluctuating (either periodic or chaotic) temporal behaviours takes importance when inhibitory links predominate, though not too much, over excitatory ones.

\section{Floquet analysis of the periodic attractors}

As shown in the bifurcation diagrams of figures 7.10 and 7.12, periodic solutions of the network dynamics often become unstable under variations of the model parameters. In order to characterize these instabilities in a precise manner, 


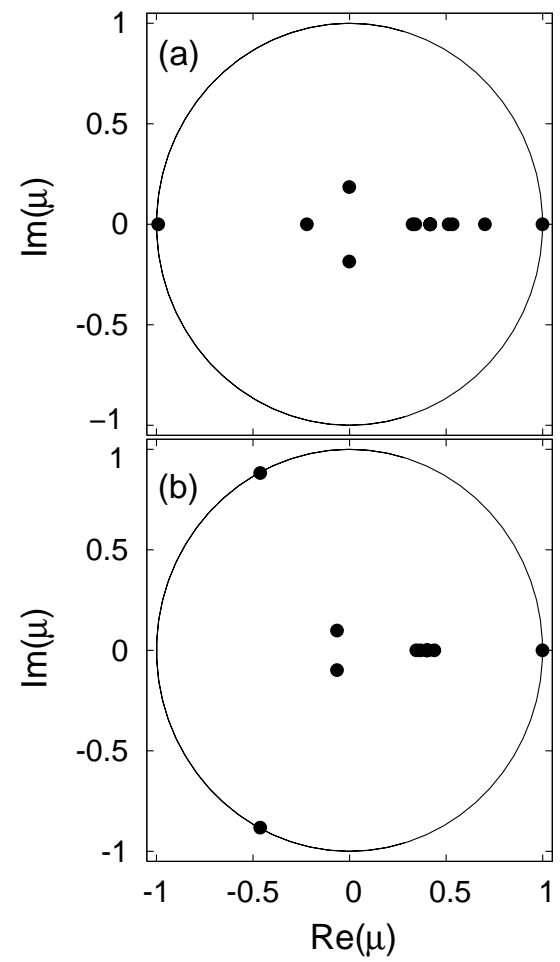

Figure 7.13: Floquet spectra. (a) Period doubling bifurcation in an island of size 14 at $h=1.57$. (b) NaimarkSacker bifurcation (at rational Floquet angle $\theta= \pm 2 \pi / 3)$ in an island of size 12 at $\mathrm{h}=2.44$ (the same as used in the diagram of figure 7.12). For both $N=60$ and $p=0.8$.

one may perform the linear stability analysis of the periodic orbits (see, e.g. [296]) near the bifurcation points.

For this we consider small perturbations of the dynamical variables, $\delta \vec{g}\left(t_{0}\right)=$ $\left\{\delta g_{i}\left(t_{0}\right)\right\}$, and compute their evolution over the period $T$ of the periodic orbit. The evolution of these small perturbations (vectors in tangent space) follows the (linear) dynamics obtained by linearizing equation (7.7) around the periodic orbit $\left\{\hat{g}_{i}(t)\right\}=\left\{\hat{g}_{i}(t+T)\right\}$, i.e.,

$$
\frac{d \delta \vec{g}(t)}{d t}=-\delta \vec{g}+\alpha \cdot \mathcal{A} \delta \vec{g}
$$

where the matrix $\mathcal{A}$ is obtained as

$$
\mathcal{A}_{i, j}=\frac{\Theta\left[\sum_{k} W_{i, k} g_{k}\right]}{\left(1+h^{-1} \Phi\left[\sum_{k} W_{i, k} g_{k}\right]\right)^{2}} \cdot W_{i, j}
$$

and $\Theta[x]$ denotes the (Heaviside) step function. Note that the above equation is only valid when the sum of the inputs (activatories and inhibitories) which receives a node from its neighbours is nonzero. Hence, the Floquet analysis is performed for each dynamical cluster found and not for the whole network.

As introduced in the first part of this thesis when discrete breather solutions were studied the so-called Floquet (or monodromy) matrix $\mathcal{F}$ of the periodic 
solution $\left\{\hat{g}_{i}(t)\right\}$ is defined as the linear operator in tangent space that maps the initial perturbation at $t_{0}, \delta \vec{g}\left(t_{0}\right)$, onto the perturbation at $t_{0}+T$

$$
\delta \vec{g}\left(t_{0}+T\right)=\mathcal{F} \delta \vec{g}\left(t_{0}\right)
$$

The Floquet matrix $\mathcal{F}$ is obtained here by numerical integration of the linearized eq. (7.10) over a period $T$ for a basis of initial conditions in the tangent space. The spectrum of eigenvalues of this matrix provides the information on the linear stability of the periodic solution. Note that because $\mathcal{F}$ is a real matrix, if a Floquet eigenvalue $\mu$ is a complex number, then its complex conjugate $\bar{\mu}$ also belongs to the Floquet spectrum. Also, because solutions of autonomous differential equations can be shifted in the time $t$ direction, their Floquet matrix always has unity as an eigenvalue, say $\mu^{1}=1$, with associated eigenvector $\left\{\dot{\hat{g}}_{i}\left(t_{0}\right)\right\}$. The solution is linearly stable if all the other eigenvalues $\mu^{j}=\left|\mu^{j}\right| \exp \left(\mathrm{i} \theta^{j}\right)$ are in the interior of the unit circle of the complex plane, i.e. $\left|\mu^{j}\right|<1$ for $j \neq 1$. A periodic solution becomes unstable when a Floquet eigenvalue (or a pair of complex conjugate eigenvalues) crosses the unit circle. The associated Floquet eigenvector indicates the direction in tangent space where perturbations will grow exponentially away from the solution.

In figure 7.13.a we plot the Floquet spectrum of a periodic attractor at a period doubling bifurcation. As seen in the figure, a Floquet eigenvalue crosses the unit circle at -1 . In figure 7.13 .b we plot the Floquet spectrum of the periodic attractor of figure 7.12 at $h=2.44$, where the inset suggested that a period tripling bifurcation may occur. We see a complex conjugate pair of Floquet eigenvalues exiting the unit circle at angles $\theta= \pm 2 \pi / 3$. In general, for generic irrational values of $\theta / \pi$ this type of bifurcation (called Naimark-Sacker or generalized Hopf bifurcation) gives rise to a quasiperiodic attractor whose trajectories fill densely a two-frequency torus. However, as a generic feature of our model, the two frequencies of the new attractor are in a commensurate ratio $(2: 3)$, so that the new stable trajectory has a period of $3 \mathrm{~T}$.

In terms of how often different types of bifurcation occur in the network dynamics, as inferred from our (non-exhaustive, but significant at the scales considered) sampling of initial conditions and network realizations, one may say that period doubling cascades and, less often, commensurate Naimark-Sacker bifurcations have been generically found by varying the Michaelis-Menten parameter $h$. But, besides the formal characterization of the dynamical instabilities observed, the Floquet analysis allows also to give an answer on a more general question, namely how temporal instabilities correlate with networking connectivity characteristics. Are there characteristic features discernible in the structure of instabilities? This point will be discussed further below in the next subsection. 


\subsubsection{Statistical characterization of island's dynamics and structure}

As noted before, the dynamics of the system is determined by only two parameters, $h$ and $p$. The behaviour of the system described by equation (7.7) on the underlying network is very rich and one can have steady, periodic or chaotic states as well as fragmentation. In this section, we analyze in more details the system's phase diagram as well as how the dynamical regimes couple to the local structural properties of the underlying network and dynamical islands.

\section{Density distribution functions of dynamical regimes}

The previously reported existence of chaotic states have very interesting implications for the stability of the system under external perturbations or internal variations of the working parameters and points to the central problem of robustness of biological networks studied by Kauffman for the design of Random Boolean networks (see section ??). For this reason it is important to complement the study of the dynamical regimes with the study of the phase diagram in the $(p, h)$-space where chaos appears. The scheme of the computations performed in order to characterize the phase diagram of the system dynamics is slightly modified with respect to that used in the previous section:

(i) For a given value of the parameters $h$ and $p$ and network realization the initial values of $g_{i}$ are taken from a uniform distribution in the interval $(0, \alpha)$.

(ii) First integration of the equations is performed using a $4^{\text {th }}$ order RungeKutta scheme. The total integration time is large compared with the transient.

(iii) Check the dynamical state of the network. If all the nodes are in a steady state we try another initial configuration; if there are dynamical nodes go to the next stage.

(iv) Check the connectivity between the dynamical nodes in order to obtain the dynamical subnetworks (islands).

(v) Second integration for calculating the Largest Lyapunov exponent $\lambda$. If $\lambda>5 \cdot 10^{-3}$ the dynamics is considered chaotic. If $\lambda<5 \cdot 10^{-3}$ we look at the frequency of the periodic motion.

(vi) Repeat stages $(i)-(v)$ for different initial conditions and realizations of the network. 

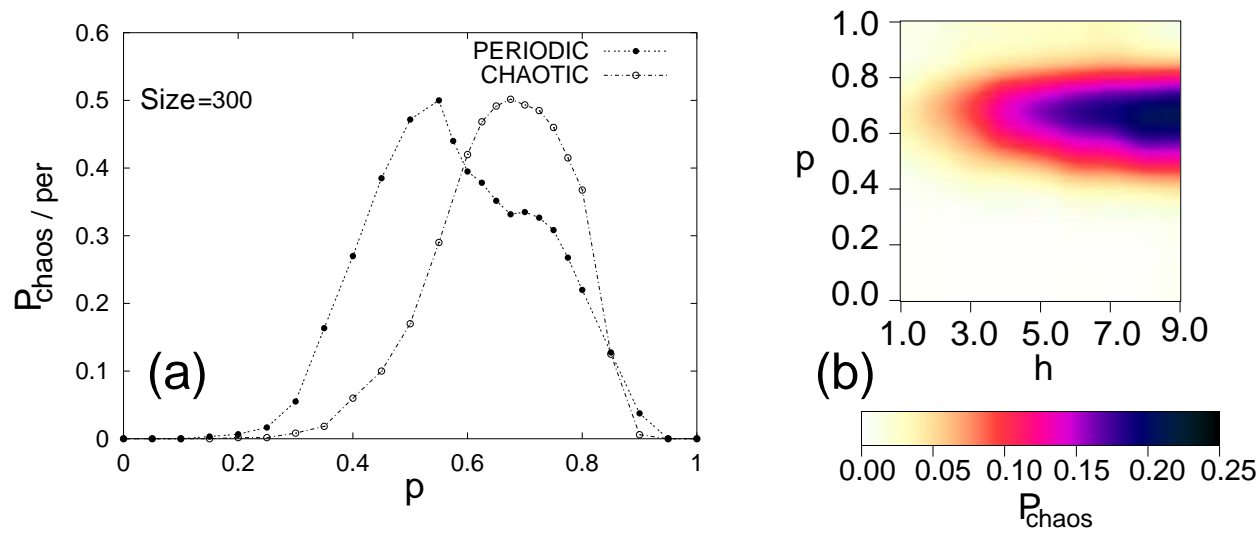

Figure 7.14: (a) Probability, $P_{\text {chaos }}\left(P_{\text {per }}\right)$, that the system evolves to a chaotic (periodic) regime as a function of the probability of inhibitory interactions, $p$, for $h=4$ and $N=300$. (b) Phase diagram in the $(p, h)$-parameter space of the chaotic dynamics of the system. Color code indicate the values of $P_{\text {chaos }}(N=300)$.

In figure 7.14.a, we have represented the probability, $P_{\text {chaos }}$, of ending up in a chaotic regime as a function of $p$ for a network of $N=300$ nodes and $h=4$. This probability is given by the fraction of the total number of realizations (typically $10^{3}$ different initial conditions over different network realizations for each value of $p$ and $h$ were used) in which at least one chaotic dynamics is observed. The figure also shows the corresponding probability, $P_{p e r}$, for periodic orbits. As figure 7.14. a clearly shows, there is a threshold value $p=p_{t h}$ beyond which the network dynamics is not robust under variations of the initial values of the $g_{i}$ 's and constitutes the onset of the chaotic phase ${ }^{3}$. For values of $p$ above $p_{t h} \approx 0.25(5)$, two randomly chosen initial conditions can lead the system to disparate asymptotic regimes. The value $p_{t h}^{c h} \sim 0.25(5)$ Besides, the size of the system affects the value of $P_{\text {chaos }}$, but the onset - and the end- of the chaotic phase seems to be $N$ independent (see figure 7.15).

Figures 7.14.a constitutes a quantitative illustration of how the prevalence of fluctuating asymptotic regimes over chemo-stasis ones depends on the model parameter $p$. The sum of both functions, $P_{p e r}(p)+P_{\text {chaos }}(p)$, gives the probability that the asymptotic state shows temporal variations of the activity vector (either regular or chaotic) as a function of $p$. These results give that in the range of values $0.5 \leq p \leq 0.8$ regimes of temporal fluctuations occur more often than constant activity regimes. This measure is maximized by val-

\footnotetext{
${ }^{3}$ Note that there is a second threshold for $p \sim 1$ which avoids chaotic behaviour. This is a consequence of the dynamics (7.7). Remind that in this region most of the interactions are inhibitory and the dynamics of the genes die out due to the damping term in eq. (7.7). Thus, the nontrivial threshold is the lower one.
} 


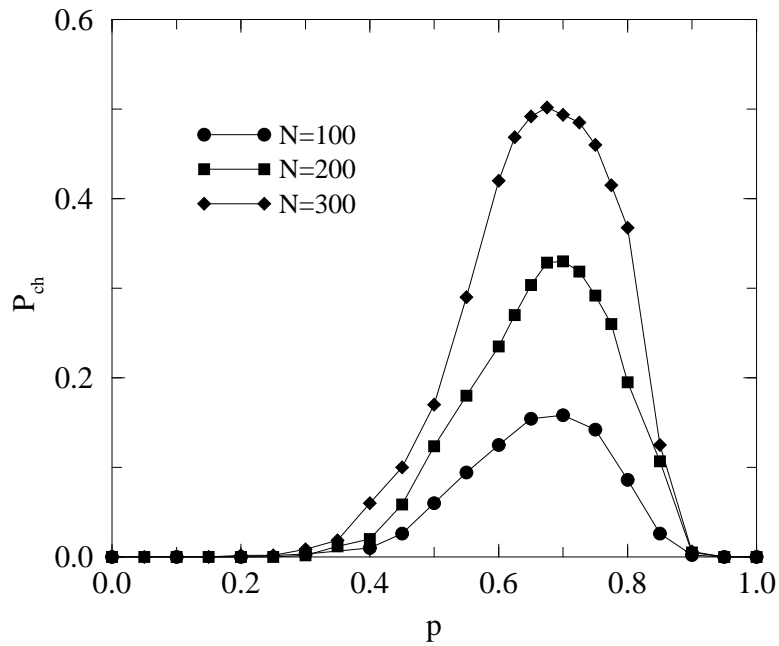

Figure 7.15: Probability, $P_{c h}$, that the system evolves to a chaotic regime as a function of the probability of inhibitory interactions, $p$, for three network sizes. The results shown correspond to a middle value of $h=4$.

ues around $p \simeq 2 / 3$ and, quite naturally, it increases with the value of the Michaelis-Menten parameter $h$, i.e. the slope at the origin of the saturated response function (see figure 7.3). Note that even larger values of $p$ means overabundance of inhibitory interactions, which leads to the predominance of the asymptotic rest state, while smaller values of $p$ favour chemostatic equilibria.

The quantities $P_{\text {chaos }}$ and $p_{t h}$ depend on $h$. As we move to larger values of $h$, the strength of the interactions increases and hence it is expected that slight perturbations produce a behavior in which the fraction of nodes whose dynamical patterns are easily disturbed grows. This is indeed the case, as illustrated in figure 7.14.b. The color-coded figure shows that as $h$ is increased, the probability of having a chaotic phase grows, and that the onset of such chaotic patterns shifts to smaller values of $p$. This drift of $p_{t h}$ is however bounded. For small enough values of $p$ (even for very large $h$ ), most of the elements activate each other $\left(W_{i j}=1\right.$ for a large fraction of pairs $i j$ and $j i$ ) and hence the resulting dynamics is steady. In other words, the onset of chaotic regimes is always located at a nonzero value of $p_{t h}$ (the same applies to the right (decaying) part of $P_{c h}(p)$, but in this case the activity falls down to zero).

Although the model and the underlying topology are very different, the existence of a nonzero value of $p_{t h}$, no matter the value of $h$, points to the original suggestion by Kauffman (explained in section 7.1.2) that there is a relevant (input) connectivity $\kappa_{c}$ for the regulatory gene network of an organism below which the dynamical behavior of the system is frozen and is not easily changed by perturbations [267]. Around $\kappa_{c}$, the behavior is neither chaotic (not robust and then biologically not desiderable) nor frozen (biologically un- 


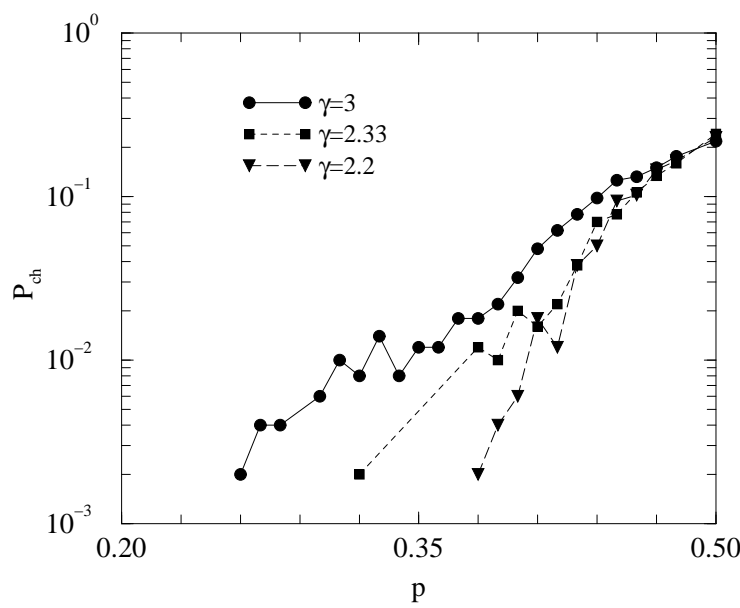

Figure 7.16: Onset of chaotic regime as a function of $\mathrm{p}$ for three different values of the exponent of the degree distribution $P(k) \simeq k^{-\gamma}(\gamma=3,2.33$ and 2.2). The results correspond to a network of $N=300$ and the nonlinear parameter is set to $h=4$ and.

realistic), but exhibits a rich behavioral repertoire. The same picture applies to $p_{t h}$ in our model. In this direction, the quantity $P_{p e r}(p)-P_{\text {chaos }}(p)$ allows to determine the regions in the parameter space where regular dynamics prevails over chaos. As pointed above the range of $p$ values where $P_{p e r}(p)>P_{\text {chaos }}(p)$ can be regarded as dynamically robust and then compatible with the operation points for real biological networks.

We have also performed the computation of the phase diagram for the existence of chaotic dynamics for networks with different degree of heterogeneity. For this purpose, we have varied the exponent $\gamma$ of the connectivity distribution $P(k) \sim k^{-\gamma}$ to values lower than 3 using the so-called generalized BarabásiAlbert model reported in [297]. These networks are even more heterogeneous having a higher number of highly connected genes. These computations allow to gain information on the importance of highly connected genes on the existence of chaotic behavior. The results shown in figure 7.16 manifest that when $\gamma$ decreases the threshold value for displaying chaotic behavior $p_{t h}(\gamma)$ slightly grows, giving a narrower chaotic region but, on the other hand, the probability $P_{c h}$ in the chaotic region takes higher values for the same values of $p$ as $\gamma$ decreases. In table 7.1 we show the thresholds $p_{t h}(\gamma)$ and the values $I(\gamma)$ given by

$$
I(\gamma)=\int_{0}^{1} P_{c h}(\gamma, p) d p,
$$

that accounts for the strength of the chaotic behavior in the whole range of the parameter $p$. When periodic behaviour is also considered one realizes by looking (Figure) at the aforementioned substraction $P_{p e r}(p)-P_{c h}(p)$ that 

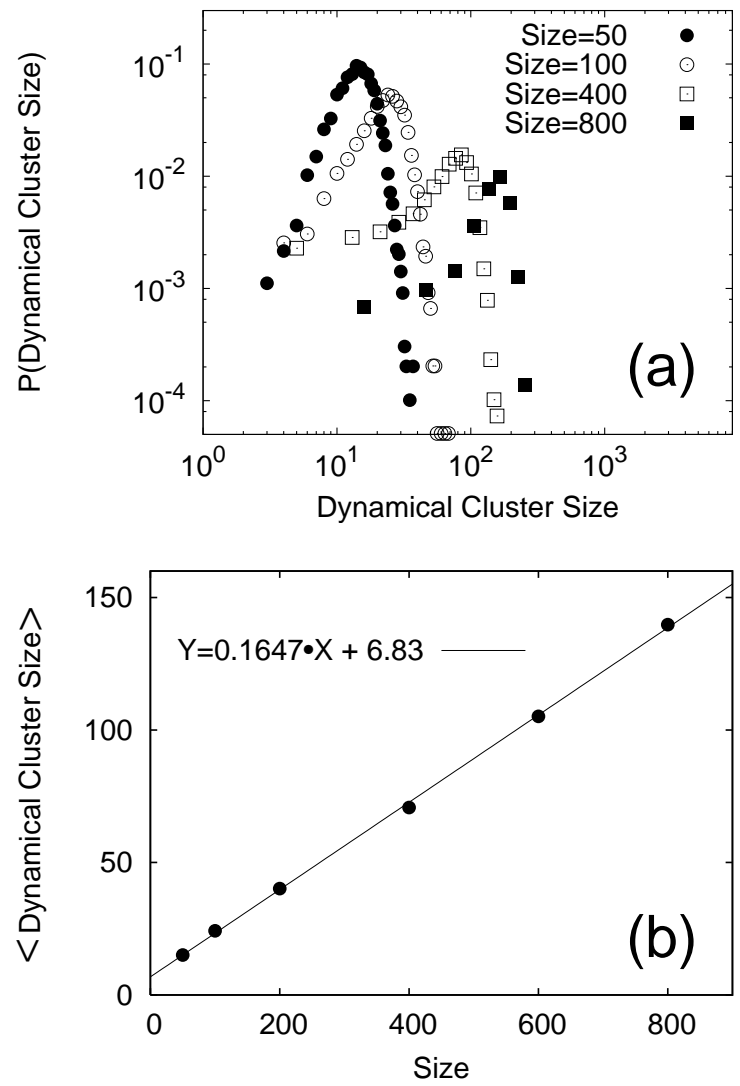

Figure 7.17: (a) Probability that a connected cluster of nodes displaying either chaotic or periodic behavior has a given size (in number of nodes forming the cluster). (b) Scaling of the mean cluster size with $N$. The parameters have been set to $h=4$ and $p=0.7$.

although the onset of chaotic behaviour $p_{t h}$ is lower for the less heterogeneous case $\gamma=3$ it corresponds to the more robust case as it presents the larger region of periodic prevalence. These quantitative results have to be carefully considered since it is clear that, as a matter of fact, regardless of the value of $\gamma$ employed, the dynamical robustness of the system is mainly determined by the topological properties given by $p$. We remark again that this conclusion is in agreement with the findings when Random Boolean Networks are considered.

\section{Dynamical island structure}

We next focus on the topological characterization of islands of dynamical units. For this we will take into account only the nodes belonging to those connected components of the whole network which share common dynamical patterns, 


\begin{tabular}{|c|c|c|c|}
\hline$\gamma$ & 3 & 2.33 & 2.2 \\
\hline$p_{\text {th }}(\gamma)$ & 0.25 & 0.32 & 0.38 \\
\hline$I(\gamma)$ & 0.1581 & 0.1962 & 0.2 \\
\hline
\end{tabular}

Table 7.1: The table shows the values of the probability (of inhibitory interactions) threshold, $p_{t h}$ for having chaotic behavior and the values of the integral 7.13 for the three values of $\gamma=3,2.33$ and 2.2 .

the islands, and only those links that connect two dynamical nodes of the same islands. That is we will not consider those links that connect island's nodes with the rest of the substrate network. In this sense, the islands can be viewed as those modules introduced in section 7.1.2 that display independent dynamics but are located nested in large network of interactions. We expect to find differences between the topological features of the islands and those of the scale-free substrate as a result of the nonlinear dynamics that defines the islands.

We first analyze how the cluster size distribution of islands of nodes displaying either periodic or chaotic activity scales with the system size. Figure 7.17.a represents the probability that an island has a given size for several networks made up of a number of nodes ranging from 50 to 800 . Clearly, the size distribution shows an average value that changes as $N$ grows. A closer look at the figure (see figure 7.17.b) reveals that the mean cluster size scales with $N$ and that about $17 \%$ of the nodes, in average, exhibits nonzero activity. This confirms what we have discussed in section 7.2.1 about the measures of the sets $\mathcal{D}^{*}$ and $\mathcal{D}$, namely, that the fragmentation of the network into islands of independent dynamics appears as one of the most characteristic features of the model.

As we stated above, it is interesting to elucidate how the topological properties of the islands correlate with those of the underlying (original) network. To this end, we have further scrutinized the structure of the clusters and measured two topological quantities of interest. Figure 7.18 shows the degree distribution of nodes belonging to dynamical islands for several system sizes. This property can be regarded as a global one and indicates that within the islands, the probability that a node has $k$ links pointing to other nodes of the same dynamical island also presents a slow decay with $k$, though with a more pronounced cut-off and a (slightly) different value for the exponent $\gamma$ than that of the substrate network. More striking is the result depicted in figure 7.19, where the average clustering coefficient $\langle c\rangle$ of the substrate (original) network and of the islands is plotted as a function of $N$. While for the BA network the clustering is vanishing as the network size grows, as reported in section 


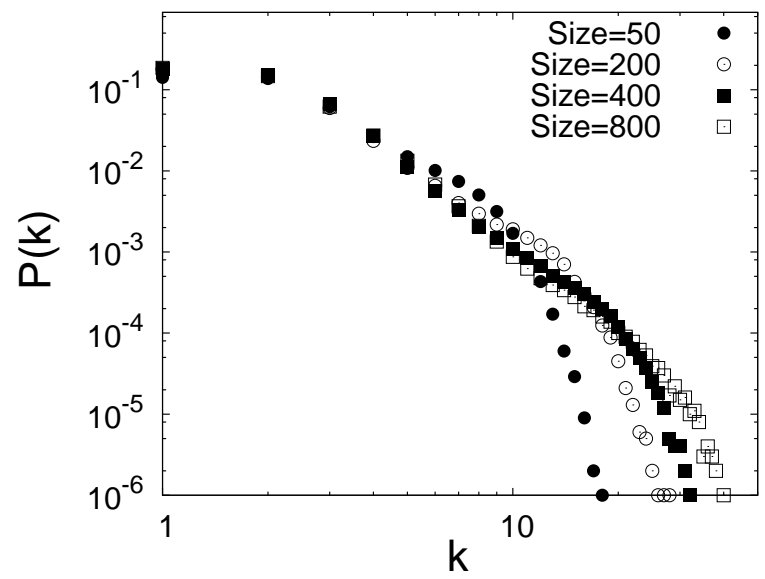

Figure 7.18: Probability that a node belonging to a dynamical island interacts with $k$ other nodes of the island. Parameters were set to $h=4$ and $p=0.7$.

5.2.3, it seems that for dynamical islands its value saturates. This is quite interesting because, on the other hand, the value of the clustering coefficient is very large and comparable to measures of real systems where the kind of dynamics explored here applies, for instance, biological networks [298].

That is, the structure of dynamical islands correctly reproduces several of the most important topological features observed in biological networks and not captured by current network models. Namely, the heterogeneous distribution

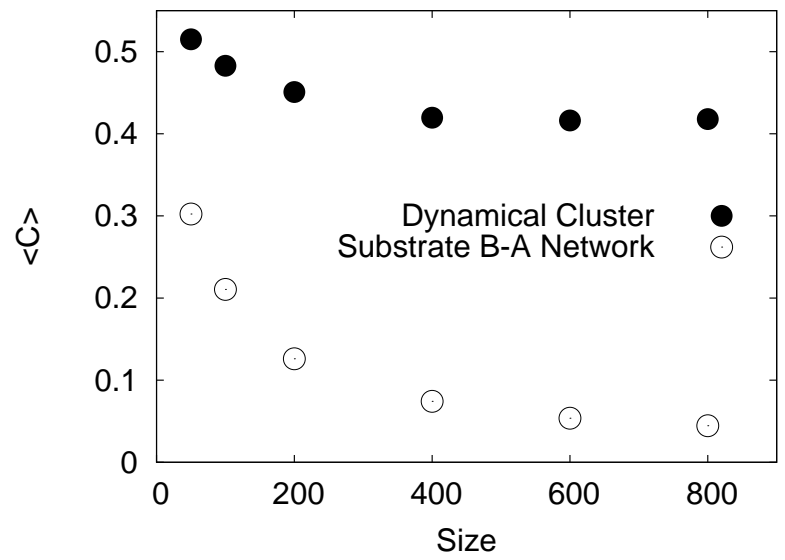

Figure 7.19: Average clustering coefficient $\langle c\rangle$ as a function of the network size for the BA original network and the dynamical cluster. Note that while $\langle c\rangle$ in the BA network continuously decreases, for the dynamical island it saturates. See more details in the text. The results have been obtained using $h=4$ and $p=0.7$. 
of connections, a high average clustering and the independence of $\langle c\rangle$ with respect to the system size. This result points to the conjecture that several topological properties observed in systems driven by AI interactions where nodes are themselves (nonlinear) dynamical units may be biassed by their own dynamics. In other words, what we actually see is the result of the activity showed up by a smaller "dynamical" network whose local topological properties greatly differ from those of a larger substrate network that we don't "see" because many of its components are simply off. This, in fact, may be the case of biological systems where structure and dynamics are indissoluble linked [298].

\subsubsection{Structure inside dynamical islands}

The above findings on new (dynamically) emergent characteristics of the islands structure motivate the question of whether these clusters have an internal organization or hierarchy among its constituents. It is widely known that when one deals with problems where the network topology (scale-free) is the only degree of complexity of the problem the answer to this question is usually based on the presence of highly conected nodes (the hubs). This is the case when linear evolution equations are studied on top of complex networks like epidemic or rumour spreading, traffic and communication problems (chapter 6 ). However, our case is not so simple and the nonlinear excitatory/inhibitory dynamics between the elements of the network plays a crucial role in determining which nodes are governing the evolution of the system. Moreover, the high clustering found for the dynamical clusters points out that this leading role is not played by isolated nodes but by small substructures inside the dynamical islands. This concept is not new, the problem of finding small relevant substructures inside large networks, usually called "motifs" (see section 5.1.4), has been studied in different ways in the field of biological networks.

It is indeed very revealing to pay attention to the networked structure of the unstable manifold, which is given in the linear regime of small perturbations by the Floquet unstable eigenvectors. For this purpose, we look at the behaviour of the components of the dynamical islands when a bifurcation (either period doubling or Naimark-Sacker type) occurs. In these critical points, it is possible to get a deeper insight into what is going on in the dynamical islands by looking at the Floquet eigenvector responsible for the bifurcation, $\overrightarrow{\delta g}^{\star}\left(t_{0}\right)=\left\{\delta g_{i}^{\star}\left(t_{0}\right)\right\}$, corresponding to the Floquet eigenvalue which reaches the unit circle. In particular, integrating equation (7.10) for the initial condition $\overrightarrow{\delta g}^{\star}\left(t_{0}\right)$ we can compute the following vector

$$
\left\langle\delta \vec{g}^{\star}\right\rangle=\left\{\left\langle\delta g_{i}^{\star}\right\rangle\right\}=\left\{\frac{1}{T} \int_{0}^{T}\left|\delta g_{i}^{\star}\right| d t\right\} .
$$



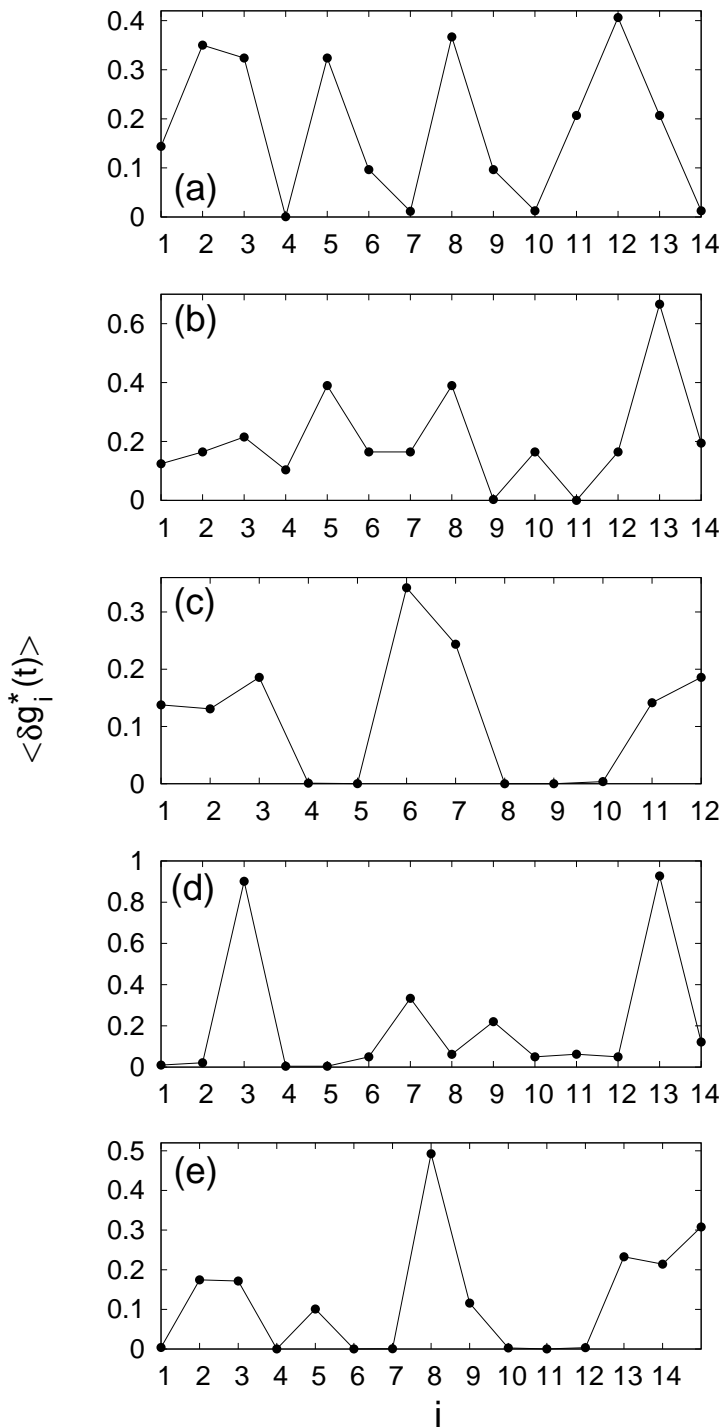

Figure 7.20: The figure shows the components of the vector $\left\langle\overrightarrow{\delta g}^{\star}\right\rangle$ (see text) for five dynamical islands at the critical point of either a period doubling bifurcation $[(\mathbf{a}),(\mathbf{b}),(\mathbf{d})$ and $(\mathbf{e})]$ or a Naimark-Sacker one (c).

The components of this vector measure, for each node, the average (over a period $T$ of the old solution) distance of the new solution after the bifurcation point from the old periodic solution. Note that a zero component of this vector at a node $k$, means orthogonality of the single-site perturbations at that node with respect to the unstable direction in tangent space. In other words, by looking at the components of the vector (7.14) we can identify those nodes that are more affected by the perturbation that leads the system to instability. In figure 7.20 we show this quantity for several dynamical islands (relatively small, but still representative) corresponding to values of $h$ where a bifurcation occurs. In particular, figures 7.20.c and 7.20.e corresponds to those 
islands whose Floquet spectra are given in figure 7.13, one 7.21.c corresponding to a Naimark-Sacker bifurcation and the other 7.21.e to a period doubling bifurcation.

As it can be seen from the figures, the vectors $\left\langle\overrightarrow{\delta g^{\star}}\right\rangle$ have several null components. The unstable perturbation $\overrightarrow{\delta g}^{\star}\left(t_{0}\right)$ that change the island's attractor at the critical point does not perturb the motion of these nodes. For the three latter cases (corresponding to figures 7.20.c, 7.20.d and 7.20.e) there is a representative group of nodes where this measure vanish. The structural profiles reveal for these three cases (see the islands plotted in figure 7.21), apparently irrespective of the type of instability, that the set $\mathcal{S}$ of nodes in the island which are alien to instability (white regions), that is, the set of those nodes $j$ such that $\left\langle\delta g_{j}\right\rangle=0$, is a non-zero measure set; it is sometimes even larger than the complementary set (green area) $\mathcal{U}=I-\mathcal{S}$ of participating nodes on the unstable eigenvector evolution during a period. We observe here that the fragmentation tendency (see discussion on islands of disconnected dynamics made above) operates also at the level of the tangent space, in the sense that a binary partition of the island nodes is well defined at the bifurcation (critical) point. Namely, the instability introduces a partition of the island $I=\mathcal{U} \oplus \mathcal{S}$ into (a) the set $\mathcal{U}$ of nodes that do participate in the instability evolution in the linear regime, and (b) the complementary set $\mathcal{S}$, of nodes such that single-node perturbations are orthogonal to the unstable linear manifold. This drastic, generic fragmentation of the island of periodic activity at the linear description level of the bifurcation, is also clearly the consequence of the AI competition on the network of interactions, and we have not seen any deviation from this observation in the computations performed (of which only five cases are illustrated). In summary, one could say that inside the dynamical islands there are compact substructures (and not single nodes) governing the dynamical changes of the whole cluster of nodes.

The behavior described above suggest the following numerical experiment: we have explored the responses of the different nodes to an external perturbation when the system is in a periodic state near a bifurcation point. In particular, we force a single node $i$ by adding an aditional term to eq. 7.7 of the form

$$
\frac{d \mathbf{G}(t)}{d t}=-\mathbf{G}(t)+\alpha \mathbf{F}[\mathbf{W G}(t)]+\mathbf{e}_{\mathbf{i}} \cdot[A \sin (\omega t)],
$$

where $\mathbf{e}_{\mathbf{i}}$ is a vector whose componets are $\left\{\mathbf{e}_{\mathbf{i}}\right\}_{\mathbf{j}}=\delta_{\mathbf{i j}}$. The forcing frequency is set to $\omega=2 \pi / T$ where $T$ the period of the unperturbed system. Then we compute, as a function of the forcing amplitude $A$, the evolution of the Floquet eigenvalue $\mu^{\star}$ responsible for the forthcoming bifurcation in the unperturbed system. The effects of such a perturbation strongly depend on whether the perturbed node belongs to the subset of those identified as leaders, i.e the 

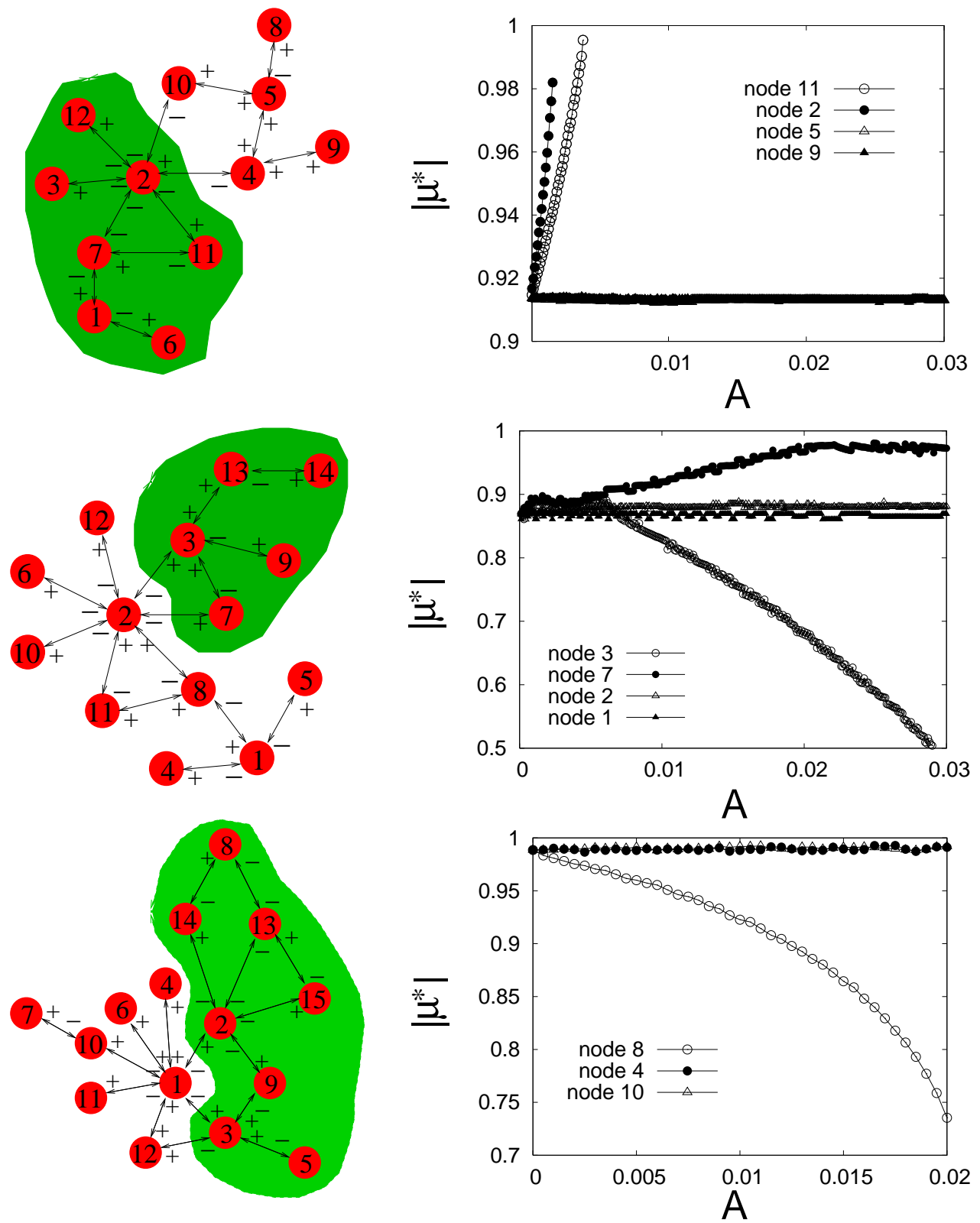

Figure 7.21: In the left side of the figure the dynamical islands corresponding to figures 7.20.c, 7.20.d and 7.20.e are represented. The islands show the distribution (green region) of the nodes with non null component of $\left\langle\overrightarrow{\delta g}^{\star}\right\rangle$ in figure 7.20 respectively. In the right side, the plots show the evolution of the Floquet eigenvalue $\mu^{\star}$ (see text) as a function of the forcing amplitude $A$ applied to different nodes of the left dynamical islands respectively. For all the islands the susbstrate network was of $N=60$ nodes whith a fraction of $80 \%$ of inhibitory interactions $(p=0.8)$. 
ones with non null component in $\left\langle\overrightarrow{\delta g^{\star}}\right\rangle$ (represented inside the green area in figure 7.21). The results obtained for the dynamical islands plotted in figure 7.21 are shown in the right plots of the figure. When the nodes inside the green area are perturbed the Floquet eigenvalue $\mu^{\star}$ significantly deviate (either increase or decrease, we have not been able to elucidate when a given change is expected) from the values of the unperturbed system. On the other hand, the perturbation of the nodes located outside the green region does not imply any change to linear stability of the whole system. These results illustrate the relevant role played by the substructures found above by the computation of $\left\langle\delta \vec{g} \vec{g}^{\star}\right\rangle$.

\subsection{Conclusions}

In this chapter we have analyzed the interplay between complex topologies and activatory-inhibitory interactions driven by a saturated response dynamics of the Michaelis-Menten type. The dynamics of the system is very rich and exhibits steady, periodic and chaotic regimes that in turn lead to the fragmentation of the original substrate network into a smaller cluster of dynamically active nodes. We have fully characterized these states by means of the Lyapunov exponent and the Floquet analyses as well as the topological features of active islands. The reach behavioral repertoire observed is thus a consequence of the entangled complexity of the system temporal behavior and the heterogeneous structure of the underlying network.

The emerging dynamics characterized in this work could plausible describe at least two relevant scenarios in biological systems. On one hand, the dynamics expressed in eq. (7.7) has been proposed as a way to characterize theoretically the individual dynamics of gene expression [294]. In fact, eq. (7.7) is the generalization of the successful Random Boolean models widely used to model gene expression. In this context, two nodes at the ends of a link are considered to be transcriptional units which include a regulatory gene. One of these end-nodes can be thought of as being the source of an interaction (the output of a transcriptional unit). The second node represents the target binding site and at the same time the input of a second transcriptional unit. By studying simplified models as the one implemented here - the intrinsic complexity of the problem does not allow for a complete and detailed description of real gene dynamics -, one can infer the region of the parameter space (i.e. $(p, h))$ that better describes gene networks. The latter seems possible due to latest developments in microarray technologies, biocomputational tools, and data collection software.

A second scenario where the results obtained apply is reaction kinetics in 
metabolic networks. In metabolic systems, a very rich behavioral repertoire is well documented [210], as for instance, the oscillations observed in the concentration of certain chemicals in biochemical reactions such as glycolysis. The system of differential equations, Eqs. (7.7), represents one of the most basic biochemical reactions, where substrates and enzymes are involved in a reaction that produces a given product. In this context, there are several important issues as how fast the equilibrium is reached, how the concentration of substrates and enzymes compare, etc. Besides, it is known that in a large number of situations, some of the enzymes involved show periodic increments in their activity during division, and these reflect periodic changes in the rate of enzyme synthesis. This is achieved by regulatory mechanisms that necessarily require some kind of feedback control as that emerging in our model. The interesting point here is that the real topological features of the underlying metabolic network [146] have not been taken into account in studies performed so far. As this work shows, they have important bearings in the correlation between structure and the observed dynamics.

Finally, on more general theoretical grounds, we anticipate several features of interest such as the fragmentation of the original network according to the dynamical states of the nodes, multistability and different routes to chaotic behavior within the same system. The first of these points is particularly relevant since it may indicate that in networks of dynamical units, the topology observed can be the result of a given network state hiding a larger substrate whose topological properties are completely different at a local level. Of particular interest is also the result gathered in the last part of the work, namely, the existence of an additional substructure inside dynamical islands determined by the different responses of nodes to external perturbations. This points to the central issue in many biological processes of what subset of nodes are the most important in order to sustain (or break) the system's robust functioning. As a conclusion, the characterization of models where nonlinearity and spatial complexity coexist yields new results missed when only one of these ingredients is present and opens the path to a better comprehension of biological processes and the dynamics of networks of nonlinear dynamical units. 


\title{
Chapter 8
}

\section{Synchronization on Complex Networks}

\begin{abstract}
While I was in forced to stay in bed for a few days and made observations on my two clocks of the new workshop, I noticed a wonderful effect that nobody could have thought of before. The two clocks, while hanging [on the wall] side by side with a distance of one or two feet between, kept in pace relative to each other with a precision so high that the two pendulums always swung together, and never varied. While I admired it for some time, I finally found that this happened due to a sort of sympathy: when I made the pendulums swing at different paces, I found that half an hour later, they always return to synchronism and kept it constantly afterwards, as long as I let them go.
\end{abstract}

Discovery of synchronization by Christian Huygens (Extracted from a letter to his father, 26 February 1665) [299, 300].

The understanding of emergent collective phenomena in natural and social systems has driven the interest of scientists from different disciplines during decades. One example of these phenomena is the emergence of localized structures in extended nonlinear lattices like those studied in part I. Besides, the study of synchronization of a set of interacting individuals or units occupies a privileged position among these coherent phenomena because its ubiquity in the natural world. In this chapter, we show how the emergence of local patterns of synchronization behaves differently depending on the properties of the underlying networked structure, driving the process towards a certain global synchronization degree following different paths. The dependence of the dynamics on the coupling strength and on the topology is studied in this chapter 
in an effort to provide a new perspective and tools to understand this emergent phenomena.

We will focus on the synchronization of coupled oscillators, in particular on the paradigmatic Kuramoto model, because of its validity as an approximation for a large number of nonlinear equations and its ubiquity in the nonlinear literature. In section 8.1 we will review the main characteristics of this model and briefly summarize the "state of the art" concerning synchronization in complex networks. Sections 8.2, 8.3 and 8.4 are devoted to the study of the relationship between network topology and synchronization dynamics. For this we will consider a variety of networks whose topological properties (such as clustering, average path length, degree distribution, etc...) can be tuned and study how topological variations affect the emergence of the collective synchronization.

\subsection{The Kuramoto model}

The concept of synchronization studied in this chapter refers to the state of a macroscopic system of limit-cycle oscillators whose frequencies are locked to a common value despite of the differences of their natural frequencies of individual oscillations. However, the very concept of synchronization of $n$ dynamical systems implies that there exist a smooth and invertible map that relate the trajectories of any pair of these systems. Then, synchronization of any type of dynamical behaviour, such as chaos [301], can be studied. Depending on the characteristics of the maps that connect two given evolutions different types of synchronization are considered such as complete synchronization [302], phase synchronization [303], lag synchronization [304], etc... This rich repertoire makes the general problem of synchronization an outstanding field for nonlinear physics. Besides, the synchronization of non-identical interacting units occupies a privileged position among emergent collective phenomena because of its various applications in interdisciplinary fields like Neuroscience, Ecology, Earth Science, among others [300, 305-308].

Let us now focus on the problem of complete synchronization of an extended set of limit-cycle oscillators where the Kuramoto model has been object of intensive research during the last decades [309]. In 1967 Winfree [310] faced the problem of synchronization with the following two simplifications: $(i)$ the coupling between the oscillators is weak and (ii) they are nearly identical (similar natural frequencies). Subjected to these assumptions one can perform a time scales separation. From one hand, at a fast time scale, the oscillators relax to their natural limiting cycles so that they are described by the rotation angle of their phases. At a slow time scale these phases evolve according to the 
weak interaction with their neighbours and the small differences between the frequency of the oscillators. This approach allows to face the problem similarly to a classical mean field model when one assumes that every oscillator is coupled to the entire ensemble of oscillators and hence to the overall rithm generated by the whole population. Winfree expressed the model by means of the following general equations

$$
\dot{\theta}_{i}=\omega_{i}+\left[\sum_{j=1}^{N} X\left(\theta_{j}\right)\right] Z\left(\theta_{i}\right) \quad(i=1, \ldots, N),
$$

where $\theta_{i}$ is the phase of oscillator $i, \omega_{i}$ is its natural frequency, $X\left(\theta_{j}\right)$ accounts for the influence that a oscillator $j$ makes over the rest of the elements and finally $Z\left(\theta_{i}\right)$ denotes the response of the oscillator $i$ to the overall coupling. Winfree found that when the width of the natural frequency distribution $g(\omega)$ is large compared to the coupling strength the system behaves incoherently and each oscillator evolves according to its natural frequency. On the other hand, when the distribution gets narrower the incoherence persists up to a threshold below which a small subset of oscillator gets into synchrony.

After the work of Winfree lots of works tried to understand the mechanism of the synchronization transition. One of the most successful attempts to understand it is due to Kuramoto [311, 312]. By means of perturbative methods Kuramoto proved that for any system of nearly identical weakly coupled limit-cycle oscillators, the long time dynamics is expressed by equations for the phase evolution of the form

$$
\dot{\theta}_{i}=\omega_{i}+\sum_{j=1}^{N} \Gamma_{i j}\left(\theta_{i}-\theta_{j}\right) \quad(i=1, \ldots, N),
$$

where the interaction functions $\Gamma_{i j}$ can be calculated as integrals containing model-dependent terms. These functions can be composed of a (arbitrarily) large number of Fourier harmonics, and besides, one has to provide them including information about the coupling topology. Kuramoto analyzed a mean field case corresponding to an uniform, all-to-all and sinusoidal coupling

$$
\Gamma_{i j}\left(\theta_{i}-\theta_{j}\right)=\frac{K}{N} \sin \left(\theta_{i}-\theta_{j}\right),
$$

where the factor $1 / N$ is incorporated in order to ensure a good behaviour of the model in the thermodynamic limit, $N \rightarrow \infty$. Besides, he assumed that the frequencies $\omega_{i}$ are distributed following a density distribution $g(\omega)$ unimodal and symmetric with respect to an average frequency $\Omega, g(\Omega-\omega)=g(\Omega+\omega)$. One can then take $\Omega=0$ by changing to a rotating frame of frequency $\Omega$ so 
that the phases are redefined according to $\theta_{i} \rightarrow \theta_{i}+\Omega t$, the distribution $g(\omega)$ is even and unimodal and the equations of motion are

$$
\dot{\theta}_{i}=\omega_{i}+\frac{K}{N} \sum_{j=1}^{N} \sin \left(\theta_{i}-\theta_{j}\right) \quad(i=1, \ldots, N) .
$$

This is the so-called Kuramoto model. It is convenient to define the complex order parameter in order to describe properly the synchronization transition

$$
r \exp (\mathrm{i} \phi)=\frac{1}{N} \sum_{j=1}^{N} \exp \left(\mathrm{i} \theta_{j}\right),
$$

where the modulus $r$ measures the phase coherence and $\phi(t)$ is the average phase. For example, let us suppose that the oscillators dynamics is such that the phases move grouped around the unit circle, then $r \simeq 1$ and the system behaves as a macroscopic oscillator. On the other hand, if phases fill densely the unit circle then $r \simeq 0$, and the oscillators behave incoherently and no macroscopic rithm is observed. By multiplying eq. (8.5) by $\exp \left(-\mathrm{i} \theta_{j}\right)$ we obtain for the imaginary part: $r \sin \left(\phi-\theta_{i}\right)=1 / N \sum_{j} \sin \left(\theta_{j}-\theta_{i}\right)$, so that one can write eq. (8.4) as

$$
\dot{\theta}_{i}=\omega_{i}+K r \sin \left(\phi-\theta_{i}\right) \quad(i=1, \ldots, N),
$$

where it is easily realized the mean field character of the model. Each oscillator interacts with the remaining ones through average quantities $(r$ and $\phi)$. Then, the individual phases $\theta_{i}$ are attracted to the average phase $\phi$ and the intensity of this attraction is proportional to the overall coherence of the system $r$. This establishes a positive feedback between coupling and coherence: the more coherent the collective motion the larger is $r$ and so is the tendency for recruiting oscillators into the synchronized cluster.

The numerical integration of eq. (8.4) (using a Gaussian or similar distribution, with infinite tails, for $g\left(\omega_{i}\right)$ ) show that for low enough values of $K$ the oscillators seem uncoupled, i.e for arbitrary initial conditions the phases $\theta_{i}$ tend to distribute uniformly across the unit circle. Then $r(t)$ decays to values that fluctuate around zero $\left(O\left(N^{-1 / 2}\right)\right)$. When $K$ exceeds some threshold value, $K_{c}$, the incoherent state becomes unstable and $r(t)$ grows exponentially up to a nearly constant value $1>r>0$, showing the emergence of a small cluster of synchronized oscillators (see figure 8.1).

\subsubsection{Solution to the Kuramoto model}

It is possible to obtain an analytical estimation of the critical point for the synchronization transition. For this purpose Kuramoto looked for stationary 

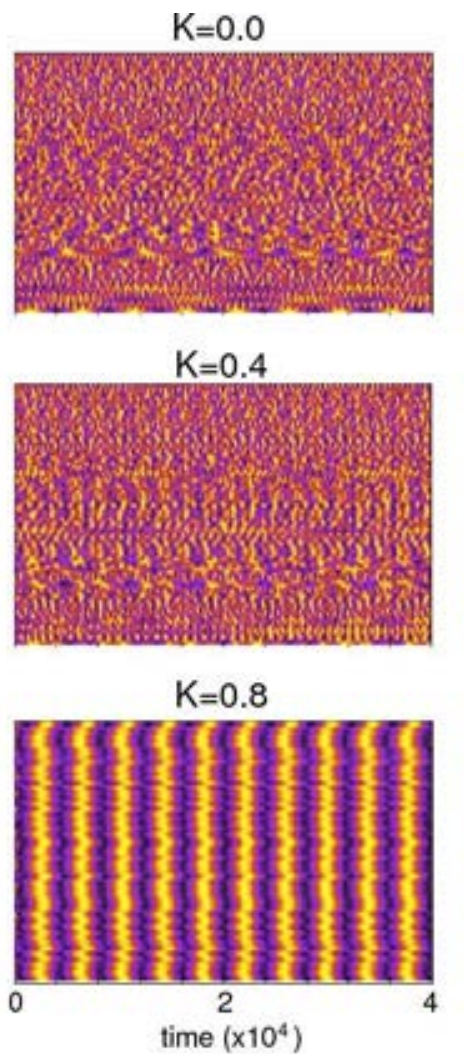

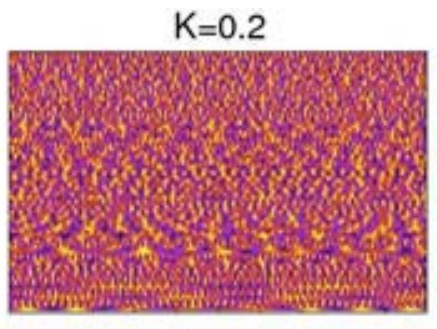

$\mathrm{K}=0.6$
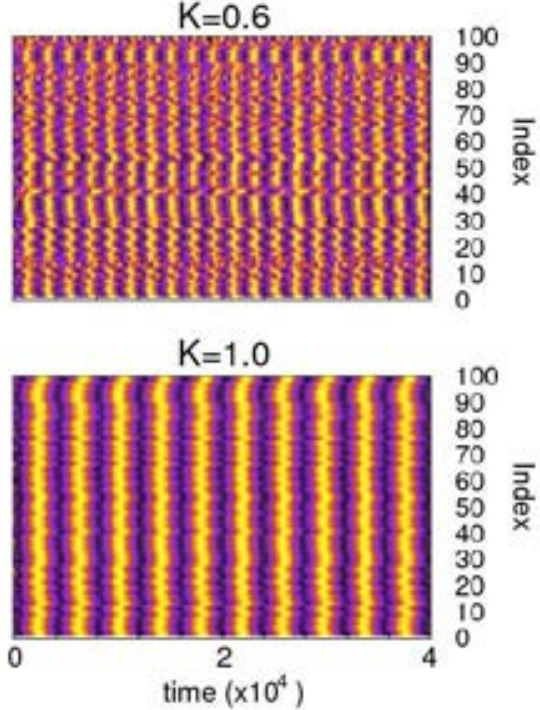

\section{$\overline{\frac{9}{2}}$}
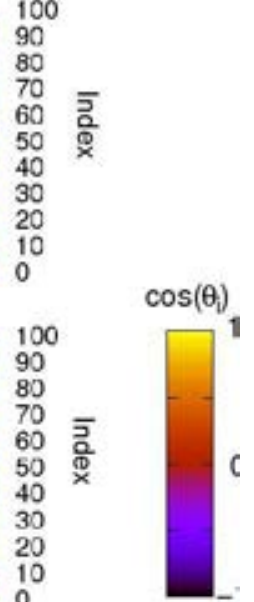

Figure 8.1: Dynamical patterns of a set of $N=100$ globally coupled oscillators. A random set of initial conditions were considered lying on the unit circle, $\theta_{i}(0) \in$ $[-\pi, \pi]$, and the natural frequencies are uniformly distributed between $\omega_{i} \in[-0.5,0.5]$. Starting from an uncoupled system $K=0(r=0.089)$ the coupling is adiabatically increased letting the system reach the equilibrium for every value of $K$ where $r$ is computed. For every pattern we have $K=0.2(r=0.109), K=0.4(r=0.240), K=$ $0.6(r=0.648)$ and $K=1.0(r=0.962)$ where the system reaches the synchronized state (note that the frequency of this synchronized state is seen to be $\omega_{\text {sync }} \simeq 2 \pi \cdot 10^{-3}$, i.e. nearly zero as the mean value for the natural frequency distribution considered).

solutions where $r(t)$ is constant and $\phi(t)$ rotates uniformly around the unit circle with frequency $\Omega$. Then it is possible to take a rotating frame of frequency $\Omega$ and fix the phases origin so that $\phi=0$. With these assumptions eq. (8.6) take the form

$$
\dot{\theta}_{i}=\omega_{i}-K r \sin \theta_{i} \quad(i=1, \ldots, N) .
$$

In the above expression it is clear that the oscillators are completely independent, although their dynamics should be consistent with the prescribed values of $r$ and $\phi$. Equation (8.7) admits two solutions depending on the relative size of the natural frequencies $\left|\omega_{i}\right|$ with respect to the prefactor $K r$. Those 
oscillators such that $\left|\omega_{i}\right| \leq K r$ evolve to a stable fixed point so that

$$
\omega_{i}=K r \sin \theta_{i},
$$

with $\left|\theta_{i}\right| \leq \pi / 2$ to ensure stability. These oscillators are called locked since their phases evolve coherently following a well defined frequency $\Omega$ in the original reference system. On the other hand if $\left|\omega_{i}\right|>K r$ the corresponding oscillators are drifting and rotate around the unit circle in a non-regular fashion. The existence of these oscillators seems to contradict the assumptions of stationary character of $r(t)$ and the regular motion of $\phi$. However, it can be found certain conditions so that the compatibility is fulfilled [311]. In particular, it is enough to assume that the oscillators phase distribution is stationary in order to the assure the stationary state conditions.

Now we compute the value of $r$. Taking into account that $\phi=0$ one obtains $r \cdot \exp (\mathrm{i} \phi)=r=1 / N \sum_{j} \exp \left(\mathrm{i} \theta_{i}\right)=\langle\exp (\mathrm{i} \theta)\rangle$. The latter average can be decomposed into the following two contributions

$$
r=\langle\exp (\mathrm{i} \theta)\rangle_{\text {lock }}+\langle\exp (\mathrm{i} \theta)\rangle_{\text {drift }},
$$

from the locked and drifting oscillators. For the first average one obtains that $\langle\exp (\mathrm{i} \theta)\rangle_{\text {lock }}=\langle\cos \theta\rangle_{\text {lock }}$ since for the locked solutions relation (8.8) holds and hence the contributions for the oscillators with natural frequency $\omega$ cancels with the corresponding to those with $-\omega$ due to the prescribed symmetry $g(\omega)=g(-\omega)$. Then,

$$
\langle\exp (\mathrm{i} \theta)\rangle_{\text {lock }}=\langle\cos \theta\rangle_{\text {lock }}=\int_{-K r}^{K r} \cos [\theta(\omega)] g(\omega) d \omega,
$$

where $\theta(\omega)$ is implicitly defined by eq. (8.8). Changing the variable $\omega$ to $\theta$ using eq. (8.8) in the above expression one finally obtains

$$
\langle\exp (\mathrm{i} \theta)\rangle_{\mathrm{lock}}=\int_{-\pi / 2}^{\pi / 2} \cos ^{2}(\theta) g(K r \sin \theta) d \theta .
$$

The contribution to $r$ from the drifting oscillators, $\langle\exp (\mathrm{i} \theta)\rangle_{\mathrm{drift}}$, is seen to vanish invoking symmetry arguments [311] and hence the control parameter $r$ is equal to the right side of eq. (8.11)

$$
r=K r \int_{-\pi / 2}^{\pi / 2} \cos ^{2}(\theta) g(K r \sin \theta) d \theta .
$$

This equation has always $r=0$ as a solution (which corresponds to the incoherent system). A second solution for $r \neq 0$ is possible if the following relation holds

$$
1=K \int_{-\pi / 2}^{\pi / 2} \cos ^{2}(\theta) g(K r \sin \theta) d \theta .
$$


This will occur for $K>K_{c}$ so that the threshold value $K_{c}$ corresponds to the critical point where coherence appears, $r \rightarrow 0^{+}$. Then one finds

$$
K_{c}=\frac{2}{\pi g(0)}
$$

for the critical coupling. The behaviour of the system's coherence near the critical point $r \ll 1$ can be obtained expanding $g(K r \sin \theta)$ up to second order yielding

$$
r \simeq\left(\sqrt{\frac{16}{-\pi g^{\prime \prime}(0) K_{c}^{4}}}\right)\left(K-K_{c}\right)^{\frac{1}{2}},
$$

where the mean field character of the model becomes evident.

\subsubsection{Synchronization in complex networks}

The Kuramoto mean-field approach to synchronization was a great breakthrough for the understanding of the emergence of synchronization in large populations of oscillators. However, we are aware that a large amount of real systems do not show a homogeneous pattern of interconnections among their parts. That is, the underlying structure is not compatible with the original assumption of the Kuramoto model. Moreover, it is not even well described by random patterns of interconnections in the vast majority of systems. Therefore, the mean-field approach requires of several constraints that are not usually fulfilled in real systems like natural[262, 313], social [148] and technological $[143,314]$ ones. The study of processes taking place on top of complex networks with scale-free character has led to reconsider classical results obtained for regular lattices or random graphs due to the radical changes of the system's dynamics when the heterogeneity of complex networks can not be neglected. It is then natural to investigate how synchronization phenomena in real systems are affected by the complex topological patterns of interaction. This is not an easy task, as one has to deal with two sources of complexity, the nonlinear character of the dynamics and highly non trivial complex structures, which are usually presented to us in an entangled way. In fact, in 1998 Watts and Strogatz in an effort for understanding the synchronization of cricket chirps, which show a high degree of coordination over long distances as though the insects where "invisibly" connected, end up with a seminal paper about the smallworld effect that was the seed of the modern theory of complex networks [155]. Nevertheless, the understanding of the synchronization dynamics in complex networks remains a challenge.

Let us focus again on the paradigmatic Kuramoto model. In order to manage with the KM on top of complex topologies we reformulate eq. (8.4) to 
the form

$$
\frac{d \theta_{i}}{d t}=\omega_{i}+\sum_{j} \Lambda_{i j} A_{i j} \sin \left(\theta_{j}-\theta_{i}\right) \quad(i=1, \ldots, N)
$$

where $\omega_{i}$ stands for its natural frequency, $\Lambda_{i j}$ is the coupling strength between pairs of connected oscillators and $A_{i j}$ is the connectivity matrix $\left(A_{i j}=1\right.$ if $i$ is linked to $j$ and 0 otherwise). The original Kuramoto model studied above assumed mean-field interactions so that $A_{i j}=1, \forall i \neq j$ (all-to-all) and $\Lambda_{i j}=K / N, \forall i, j$.

The first problem when dealing with the KM in complex networks is the definition of the dynamics. In the seminal paper by Kuramoto [311], eq. (8.4), the coupling term in the right hand side of eq. (8.16) is an intensive magnitude. The dependence on the number of oscillators $N$ is avoided by choosing $\Lambda_{i j}=\frac{K}{N}$. This prescription turns out to be essential for the analysis of the system in the thermodynamic limit $N \rightarrow \infty$. However, choosing $\Lambda_{i j}=\frac{K}{N}$ the dynamics of the KM in a complex network becomes dependent on $N$. Therefore, in the thermodynamic limit, the coupling term tends to zero except for those nodes with a degree that scales with $N^{1}$. A second prescription consists of taking $\Lambda_{i j}=\frac{K}{k_{i}}$ (where $k_{i}$ is the degree of node $i$ ) so that $\Lambda_{i j}$ is a weighted interaction factor that also makes intensive the right hand side of eq. (8.16). This form has been used to solve the paradox of heterogeneity that states that the heterogeneity in the degree distribution, which often reduces the average distance between nodes, may suppress synchronization in networks of oscillators coupled symmetrically with uniform coupling strength [316]. One should consider this result carefully because it refers to the stability of the fully synchronized state (see below) not to the whole evolution of synchronization in the network. More important, the inclusion of weights in the interaction strongly affects the original KM dynamics in complex networks because it imposes a dynamic homogeneity that mask the topological heterogeneity of the network. Finally, the prescription $\Lambda_{i j}=K[315,317,318]$, which may seem more appropriate, also presents some conceptual problems because the sum in the right hand side of eq. (8.16) could eventually diverge in the thermodynamic limit if synchronization is achieved.

To our understand, the most accurate interpretation of the KM dynamics in complex networks should preserve the essential fact of treating the heterogeneity of the network independently of the interaction dynamics, and at the same time, should remain calculable in the thermodynamic limit. Taking into account these factors, the interaction $\Lambda_{i j}$ in complex networks should be inversely

\footnotetext{
${ }^{1}$ Note that this is only possible in networks with power-law degree distributions, but with a very small probability as $P(k) \sim k^{-\gamma}$ with $\gamma>0$. In these cases, mean-field solutions independent on $N$ are recovered, with slight differences in the onset of synchronization of all-to-all and scale-free networks [315].
} 
proportional to the largest degree of the system $\Lambda_{i j}=\frac{K}{k_{m a x}}=\lambda$ keeping in this way the original formulation of the KM valid in the thermodynamic limit (in SF networks $k_{\max } \sim N^{1 /(\gamma-1)}$ ). In addition, the same order parameter, eq. (8.5), can be used to describe the coherence of the synchronized state. Since $k_{\max }$ is constant for a given network, the physical meaning of this prescription is a re-scaling of the time units involved in the dynamics. Note, however, that for a proper comparison of the synchronizability of different complex networks, the global and local measures of coherence should be represented according to their respective time scales. Therefore, given two complex networks A and B with $k_{\max }=k_{A}$ and $k_{\max }=k_{B}$ respectively, the comparison between observables must be done for the same effective coupling $\frac{K_{A}}{k_{A}}=\frac{K_{B}}{k_{B}}=\lambda$. With this formulation in mind eq. (8.16) reduces to

$$
\frac{d \theta_{i}}{d t}=\omega_{i}+\lambda \sum_{j} A_{i j} \sin \left(\theta_{j}-\theta_{i}\right) \quad(i=1, \ldots, N),
$$

independently of the specific topology of the network. This allow us to study the dynamics of eq. (8.17) over different topologies in order to compare the results and report properly the interplay between topology and dynamics for what concerns to synchronization.

In recent years, scientists have addressed the problem of synchronization on complex networks capitalizing on the Master Stability Function (MSF) formalism [319] which allows to study the stability of the fully synchronized state [316, 320-326]. The MSF is simply the result of a linear stability analysis for a completely synchronized system. Although we are not going to make use of the MSF along the forthcoming sections, let us briefly summarize the basis of this technique for the sake of completeness.

Consider a general system of $N$ coupled dynamical systems for the $n$ dimensional variables $\left\{\mathbf{x}_{i}\right\}(i=1, \ldots, N)$ of the form

$$
\dot{\mathbf{x}}_{i}=\mathbf{F}\left(\mathbf{x}_{i}\right)+\lambda \sum_{j=1}^{N} L_{i j} \mathbf{H}\left(\mathbf{x}_{j}\right) \quad(i=1, \ldots, N),
$$

where $\mathbf{F}: R^{n} \rightarrow R^{n}$ is the isolated $n$-dimensional dynamical system, $\lambda$ is the coupling strength, $L_{i j}$ is the $N \times N$ Laplacian matrix fulfilling $\sum_{j} L_{i j}=0$ and $\mathbf{H}: R^{n} \rightarrow R^{n}$ is an output function that accounts for the mutual influence of the dynamical states of two coupled dynamical systems. The above equations can be written in a more compact form

$$
\dot{\mathbf{x}}=\left[\mathbf{I}_{N} \otimes \mathbf{F}+\lambda \cdot \mathbf{L} \otimes \mathbf{H}\right](\mathbf{x}),
$$

where $\mathbf{I}_{N}$ is the $N \times N$ identity matrix and $\otimes$ is the direct product. The evolution of a small perturbation around any solution of the above system 
will be governed by the linearized equations around the corresponding solution from which one can compute the Floquet and Lyapunov exponents (see Appendix B). However, if the whole system displays synchronous dynamics one has $\mathbf{x}_{i}=\mathbf{x}_{j} \forall i, j$ and it implies some simplifications about the linear stability analysis. Since the Jacobians $D \mathbf{F}$ and $D \mathbf{H}$ that appear in the linearized equations are the same for every $n$-dimensional tangent subspace corresponding to perturbations of single $n$-dimensional variables $\mathbf{x}_{i}(i=1, \ldots, N)$ one can effectively decouple the stability analysis of the $N$ dynamical system. For this purpose diagonalizing the $N \times N$ Laplacian matrix, and calling $\gamma_{i}(i=1, \ldots, N)$ its eigenvalues, one arrives to a set of $N n$-dimensional systems of equations

$$
\dot{\xi}_{i}=\left[D \mathbf{F}+\lambda \gamma_{i} D \mathbf{H}\right] \xi_{i} \quad(i=1, \ldots, N)
$$

with the same functional form. The similarity of these $n$-dimensional systems of equations, derived from the the symmetry of the synchronized solution, leads to formulate the general problem of finding the maximum eigenvalue, $\Lambda$ of the generic equation

$$
\dot{\mathbf{y}}=[D \mathbf{F}+(a+\mathrm{i} b) D \mathbf{H}] \mathbf{y} \quad(i=1, \ldots, N)
$$

as a function of $a$ and $b$. The above equation is the so-called Master Stability Equation and the surface, $\Lambda(a, b)$ generated by the solutions correspond to the Master Stability Function. By means of this surface, that only depends on the particular equations for the isolated dynamical systems and the form of the coupling between them, one can compute the maximum Lyapunov exponents for each Laplacian eigenvector $\gamma_{i}(i=1, \ldots, N)$, that would depend on the underlying topology employed, and hence obtain the stability of the synchronized state over the complex network structure.

While the MSF approach is useful to get a first insight into what is going on in the system as far as the stability of the synchronized state is concerned, it tells nothing about how synchronization is attained and whether or not the system under study exhibits a critical point similar to the original KM. To this end, one must rely on numerical calculations and explore the entire phase diagram. Surprisingly, there are only a few works that have dealt with the study of the whole synchronization dynamics in specific scenarios $[317,327-$ 329] as compared with those where the MSF is used, given that the onset of synchronization is reacher in its behavioral repertoire than the state of complete synchronization. In the following sections we will study this point using the KM model on top of different substrate topologies in order to get insight about the role of the structural properties on the route towards complete synchronization. 


\subsection{Synchronization in local scale-free networks}

In this section, we take a further step in the detailed characterization of the phase diagram and specifically, in the description of the dynamical behavior at the onset of synchronization in SF networks. By performing a standard finite size scaling analysis, we show that the local topology affects the critical properties of the dynamics, though it is less pronounced that what one may expect a priori. We capitalize on the network model reported in section 5.3 that keeps the power-law exponent fixed while varying the clustering coefficient and the average path length. This model was already used in section 6.2.2 for studying the role of the local topology when studying traffic dissemination.

Let us first review the main features of the model. Roughly speaking, the model mimics the situation in which new nodes are attached to an existing core or network but without having knowledge of the whole topology. The model generates a one parameter family of networks labeled by $\mu \in[0,1]$ that measures the degree of knowledge that is applied when preferential attachment is performed during the network growth. Then, the limit $\mu \rightarrow 1$ assumes global knowledge an thus it recovers the BA network. On the other hand, $\mu \rightarrow 0$ implies extremely local knowledge and the resulting networks while displaying a scale-free structure (the exponent $\gamma$ of the power-law degree distribution is the same, i.e. $\gamma=3$, regardless of the value of $\mu$ ) are very large (large values of $\langle L\rangle$ ) and highly clustered compared to BA networks. Both magnitudes, clustering coefficient $\langle c\rangle$ and average path length $\langle L\rangle$, increase as $\mu$ decreases from 1 to 0 (see figures 7.4 and ??). Remind that the larger variations correspond to the clustering coefficient (a factor greater than 4 as compared to a factor close to 2 for $\langle L\rangle)$ and that it is the first property that deviates from the BA limit while $\langle L\rangle$ holds up close to similar values to that of the BA limit up to small values of $\mu$, where $\langle L\rangle$ raises at a higher rate than $\langle c\rangle$.

We will consider the Kuramoto model (8.17) discussed in the last section and employ the control parameter $r$ introduced above to measure the degree of synchronization as a function of the coupling strength $\lambda$ and the topology parameter $\mu$.

In order to inspect how the dynamics of the $N$ oscillators depends on the underlying topology, we have performed extensive numerical simulations of the model. Starting from $\lambda=0$, we increase at small intervals its value. The natural frequencies $\omega_{i}$ and the initial values of $\theta_{i}$ are randomly drawn from a uniform distribution in the interval $(-1 / 2,1 / 2)$ and $(-\pi, \pi)$, respectively. Then, we integrate the equations of motion eq. (8.17) using a $4^{\text {th }}$ order Runge-Kutta method over a sufficiently large period of time to ensure that the system reaches the stationary state, where the order parameter $r$ is computed. 


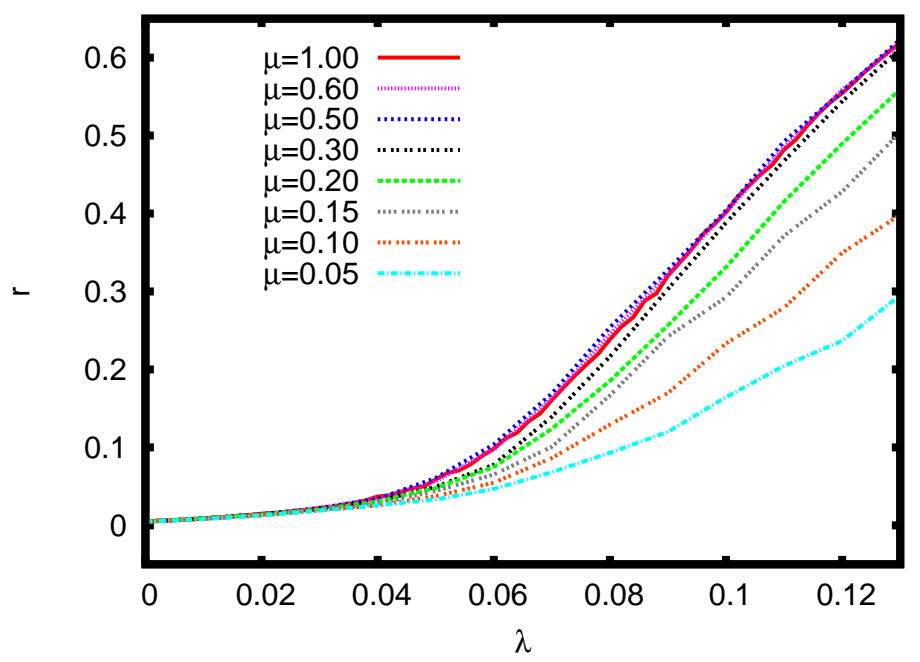

Figure 8.2: Order parameter $r$ as a function of $\lambda$ for different values of $\mu$ as indicated. The network parameters are $N=1000$ and $\langle k\rangle=6$.

The procedure is repeated gradually increasing $\lambda$ for every network realization labeled by $\mu$. All the results are averaged over at least 100 realizations.

The results for $r$ versus the control parameter $\lambda$ are shown in figure 8.2 for several networks characterized by different values of $\mu$. For all values of $\mu$, when the coupling is increased from small values, the incoherent solution prevails and macroscopic synchronization is not attained. This behavior persists until a certain critical value $\lambda_{c}(\mu)$ is crossed. At this point some elements lock their relative phase and synchronized clusters of nodes appear. This constitutes the onset of synchronization. Beyond this value, the population of oscillators splits into a partially synchronized state contributing to $r$ and a group of nodes whose natural frequencies are too spread as to be part of the coherent pack. Finally, after further increasing the value of $\lambda$, more and more nodes get entrained around the mean phase and the system settles down in a completely synchronized state where $r \approx 1$.

A comparison between the results for different values of $\mu$ (and thus different $\langle c\rangle$ and $\langle L\rangle$ values) indicate several interesting features of the synchronization process. First, it is remarkable that when the clustering coefficient increases, the system reaches complete synchronization at higher values of the coupling. This result agrees with the results reported in [328], where a different network model able to generate topologies with a tunable clustering coefficient was implemented.

At this point, one may ask whether the effects are only due to the influence 
of $\langle c\rangle$ or to the increase of the average path length $\langle L\rangle$ (note that the model implemented in [328] does not explore this possibility). Unfortunately, the two factors are generally linked together so they can not be considered separately. However, a closer look at figures 7.4 and ?? reveals that there is a region of the parameter $\mu$ where the clustering coefficient grows while the average shortest path length remains almost constant. This corresponds to the interval $0.4 \leq \mu \leq 1.0$ approximately. Going back to figure 8.2, the behaviour of $r$ in this interval of $\mu$ reveals that synchronization is almost unaffected. In fact, the $r(\lambda)$ curves lie slightly above that corresponding to the BA limit. Therefore, though the above comparison is not conclusive, it seems that the delayed transition to complete synchronization is mainly due to the effect of the increase in $\langle L\rangle$ at smaller values of $\mu$ rather than to the increase in $\langle c\rangle$. This conclusion is further supported by a direct comparison of the results in figure 8.2 with those reported in [328], where the authors explored a region with higher values of $\langle c\rangle$ (up to 0.7 ) and the profile of $r(\lambda)$ is almost the same as ours.

The second region of interest is the onset of synchronization. From figure 8.2 , it is difficult to elucidate how the critical point for the BA limit compares with those at values of $\mu<1$. At first glance, it seems that $\lambda_{c}(\mu)$ shifts rightward as the parameter $\mu$ is decreased below 1 . However, a more detailed analysis shows that it is indeed the contrary. To this end, we have performed a finite size scaling analysis that allows to determine the critical points $\lambda_{c}(\mu)$. We assume a scaling relation of the form

$$
r=N^{-\alpha} f\left[N^{\beta}\left(\lambda-\lambda_{c}\right)\right]
$$

where $f(x)$ is a universal scaling function bounded as $x \rightarrow \pm \infty$ and $\alpha$ and $\beta$ are critical exponents to be determined. The estimation of $\lambda_{c}$ can then be done by plotting $N^{\alpha} r$ as a function of $\lambda$ and tuning $\alpha$ for several system sizes $N$ until the curves cross at a single point, the critical one.

The results of the FSS analysis are shown in figure 8.3 for different values of $\mu$ (from top to bottom and from left to right $\mu=0.05,0.15,0.50,0.60$ ). The insets show a blow-up around the critical points $\lambda_{c}(\mu)$. Although the differences in the critical points at different values of $\mu$ are small, they are certainly distinguishable. In fact, the higher the value of $\mu$, the higher the critical point. That is, when the clustering coefficient and the average path length grow with respect to the BA network, the onset of synchronization is anticipated. Moreover, taking into account that the increase in $\langle L\rangle$ is likely to inhibit synchronization, one may hypothesize that the effects of the clustering coefficient prevail in this region of the parameter $\lambda$. To check this hypothesis, we have also included in figure 8.3 the analysis performed for $\mu=0.50$ and $\mu=$ 0.60 . As pointed out before, for these values, the differences can only arise from 

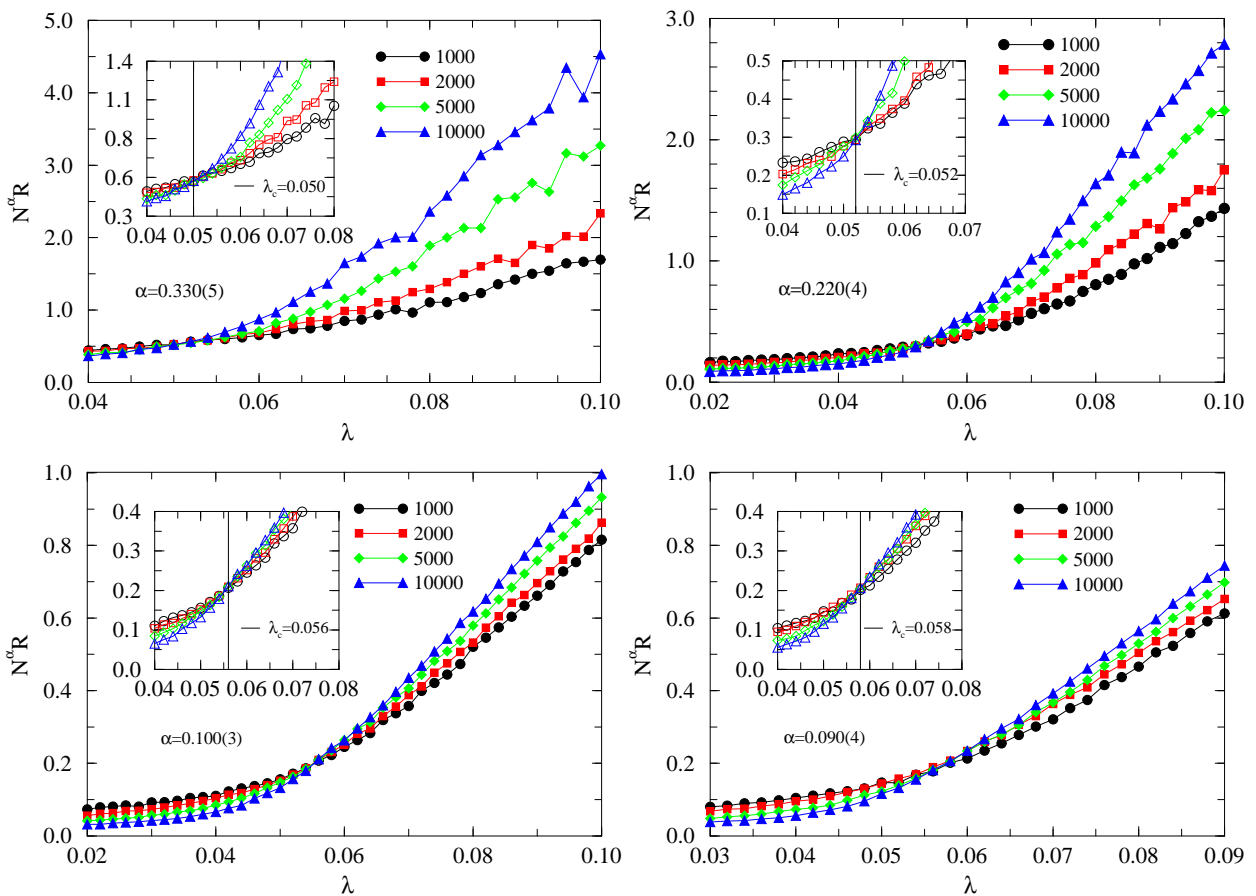

Figure 8.3: Finite size scaling analysis for several values of $\mu$. From top to bottom and from left to right the values of $\mu$ are: $0.05,0.15,0.50$ and 0.60 . In each panel, it is represented the rescaled order parameter against the control parameter $\lambda$. The insets are a zoom to the regions around the critical points $\lambda_{c}(\mu)$. The data are averaged over at least 100 realizations for each value of $\lambda$. The sizes of the networks, the critical points $\lambda_{c}(\mu)$ at which the onset of synchronization takes place, as well as the values of the critical exponents $\alpha$ are those indicated in the plots. See the main text for more details.

the variations of the clustering coefficient as the average path length remains constant in this region of the parameter $\mu$. The critical points, although very close to each other, are clearly different. Therefore, the main contribution to the onset of synchronization at low values of $\lambda$ comes from the raising of the clustering coefficient.

Rounding off, the results point to a nontrivial dependence between the clustering coefficient and the average path length, and the synchronization patterns of phase oscillators. Separately, the onset of synchronization seems to be mainly determined by $\langle c\rangle$, promoting synchronization at low values of the coupling strength with respect to networks not showing high levels of structural clustering. On the other hand, when the coupling is increased beyond the critical point, the effect of $\langle L\rangle$ dominates and the phase diagram is smoothed out (a sort of stretching), delaying the appearance of the fully synchronized 
state. These results confirm and complement those anticipated in [328] and show that general statements about synchronizability using the MSF are misleading. Whether or not a system is more or less synchronizable than others showing distinct structural properties is depends on the region of the phase diagram in which the system operates.

With this first study we have shown that synchronizability of complex networks is dependent on the effective coupling $\lambda$ among oscillators, and on the properties of the underlying network: For small values of $\lambda$, the incoherent solution $r=0$ first destabilizes as the clustering coefficient is higher, while the coherent solution $r=1$ is promoted when both the structural clustering and the average path length are small.

\subsection{Homogeneous versus heterogeneous topologies}

The results obtained in the above section shed light about the influence of the interactions topology on the route to synchronization. However, in this study at least two parameters (clustering and average path length) vary along the studied family of networks. This paired evolution, although yielding a interesting interplay between the two topological parameters, made difficult to distinguish what effects were due to one or other factors. The family of networks used in the present section are comparable in their clustering, average distance and correlations so that the only difference relies on the degree distribution, ranging from a Poissonian type to a scale-free distribution. In this sense, the obtained results are as far reaching as the highly acclaimed ones obtained for percolation and epidemic spreading on top of homogeneous or heterogeneous graphs, where the radical differences are rooted in the topology of the underlying networks.

The main goal of this section is to scrutinize and compare the synchronization patterns in Erdös-Rényi (ER) and scale-free (SF) networks. These kinds of synchronization patterns have been observed in the all-to-all KM model for broadly heterogeneous (in natural frequencies) populations of oscillators[330], however, those reported in this section are shown to be intrinsically related to the underlying topological structure and hence of importance for the structurefunction problem. For this purpose we make use of the model presented in section 5.4 that allows a smooth interpolation between these two important topologies. Besides, we introduce a new parameter for characterizing the synchronization paths in order to unravel their differences. The results reveal that the synchronizability of these networks does depend on the coupling between units, and hence, that general statements about their synchronizability are eventually misleading. Moreover, we show that even in the incoherent solu- 
tion, $r=0$, the system is self-organizing towards synchronization. We will analyze in detail how this self-organization is attained.

The first studies about the onset of synchronization in SF networks [317] revealed some important differences from the behaviour observed from purely random networks as ER graphs. The main difference relied on the great tendency of SF networks to synchronizability, which is revealed by a non-zero but very small critical value $\lambda_{c}$. Besides, it was observed that at the synchronized state, $r=1$, hubs are extremely robust to perturbations since the recovery time of a node as a function of its degree follows a power law with exponent -1 . These findings point out that the special architecture of SF networks enhances the synchronizability both at its onset and at the coherent regime. In this sense, it is interesting to characterize the roots of this different behaviour comparing it to that observed for ER graphs.

We first concentrate in global synchronization for the Kuramoto model (8.17). For this we follow the evolution of the order parameter $r$, eq. (8.5), as $\lambda$ increases, to capture the global coherence of the synchronization in networks. We will perform this analysis on the family of networks generated with the model introduced in section 5.4. This model generates a one-parameter family of networks labeled by $\alpha \in[0,1]$. The parameter $\alpha$ measures the degree of heterogeneity of the final networks so that $\alpha=0$ correspond to the heterogeneous BA network and $\alpha=1$ to homogeneous ER graphs. For intermediate values of $\alpha$ one obtains networks that has been grown combining both preferential attachment and homogeneous random linking so that each mechanism is chosen with probabilities $(1-\alpha)$ and $\alpha$ respectively. It is worth stressing that the growth mechanism preserves the total number of links, $N_{l}$, and nodes, $N$, for a proper comparison between different values of $\alpha$. We will consider the variant $\mathrm{A}$ of the model since with this formulation the interpolation in terms of the degree distribution is seen to be smoother than in the second variant of the model (see figure 5.18).

The curves $r(\lambda)$ for several network topologies ranging from ER to SF are shown in figure 8.4. We have performed extensive numerical simulations of eq. (8.17) for each network substrate starting from $\lambda=0$ and increasing it up to $\lambda=0.4$ with $\delta \lambda=0.02$. A large number (at least 500) of different network realizations and initial conditions were considered for every value of $\lambda$ in order to obtain an accurate phase diagram of the synchronization onset. As in the previous section the natural frequencies $\omega_{i}$ and the initial values of $\theta_{i}$ were randomly drawn from a uniform distribution in the interval $(-1 / 2,1 / 2)$ and $(-\pi, \pi)$, respectively.

Figure 8.4 reveals the differences in the critical behaviour as a function of the substrate heterogeneity. The global coherence of the synchronized state, 


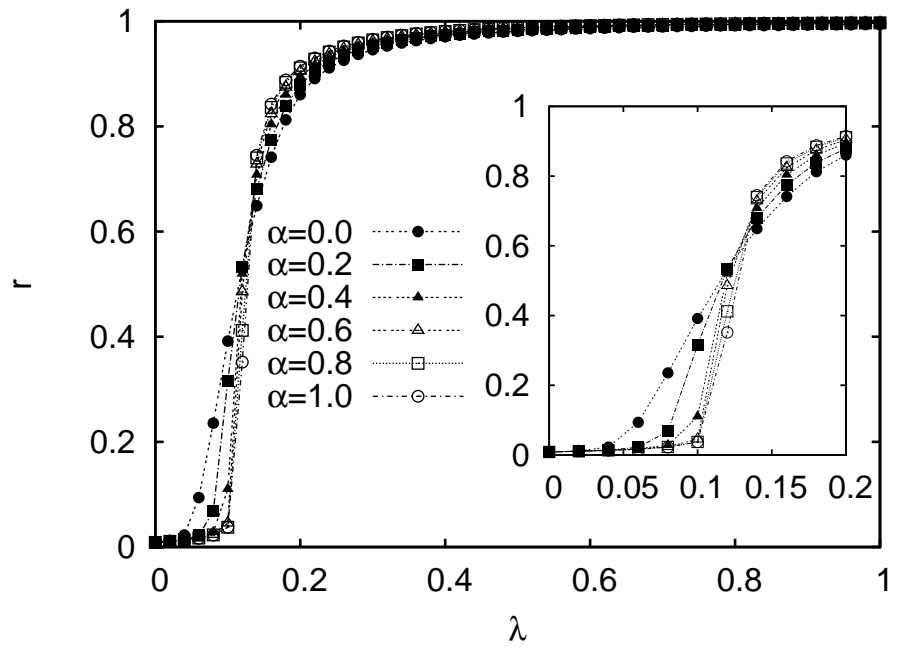

Figure 8.4: Global synchronization curves $r(\lambda)$ for different network topologies labeled by $\alpha$ ( $\alpha=0$ corresponds to the BA limit and $\alpha=1$ to ER graphs). The network sizes are $N=10^{4}$ and $\langle k\rangle=6\left(N_{l}=3 \cdot 10^{4}\right)$.

represented by $r$, shows that the onset of synchronization first occurs for SF networks. As the network substrate becomes more homogeneous the critical point $\lambda_{c}$ shifts to larger values and the system seems to be less synchronizable. On the other hand, it is also clear that the route to complete synchronization, $r=1$, is faster for homogeneous networks. That is, when $\lambda>\lambda_{c}(\alpha)$ the rate growth of $r$ grows with $\alpha$. In order to inspect in more details the critical parameters of the system dynamics we proceed as in the previous section by following a finite size scaling procedure. This allows to determine with precision the curve $\lambda_{c}(\alpha)$ and study the critical behaviour near the synchronization transition. A detailed analysis performed for both SF and ER topologies shows that the critical value of the effective coupling, $\lambda_{c}$, corresponds in scale-free networks to $\lambda_{c}^{S F}=0.05(1)$, and in random networks to $\lambda_{c}^{E R}=0.122(2)$, accordingly with figure 8.4. In both cases, the transition strongly recalls the classical transition of the original KM [311] with a critical exponent near 0.5 (0.46(2) for the SF network [317]).

The differences between ER and SF topologies observed when looking at global patterns of synchronization motivate a more detailed study of the synchronization onset for both topologies. The original work by Kuramoto pointed out that at the onset of synchronization small clusters of locked oscillators emerge and that the recruitment of more oscillators into these clusters as the coupling is increased makes larger the global coherence $r$ of the system. Obviously the emergence of these clusters would depend on the underlying topology 
which drives the possible configurations that locked oscillators would eventually form. In order to inspect how this initial coherence is achieved we propose a new order parameter, $r_{l i n k}$. This parameter measures the local construction of the synchronization patterns and allows for the exploration of how global synchronization is achieved. Then we define

$$
r_{\text {link }}=\frac{1}{2 N_{l}} \sum_{i} \sum_{j \in \Gamma_{i}}\left|\lim _{\Delta t \rightarrow \infty} \frac{1}{\Delta t} \int_{t_{r}}^{t_{r}+\Delta t} e^{i\left[\theta_{i}(t)-\theta_{j}(t)\right]} d t\right|,
$$

being $\Gamma_{i}$ the set of neighbours of node $i$. The parameter $r_{\text {link }}$ measures the fraction of all possible links that are synchronized in the network. The averaging time $\Delta t$ should be taken large enough in order to obtain good measures of the degree of coherence between each pair of physically connected nodes. Besides, $r_{\text {link }}$ is computed after the system relaxes at some large time $t_{r}$.

In figure 8.5 we represent the evolution of both order parameters, $r$ and $r_{\text {link }}$, as a function of the coupling strength $\lambda$ for both ER and SF networks. The behaviour of $r_{\text {link }}$ shows a change in synchronizability between ER and $\mathrm{SF}$ and provides additional information to that reported by $r$. Interestingly, the nonzero values of $r_{l i n k}$ for $\lambda \leq \lambda_{c}$ indicate the existence of some local synchronization patterns even in the regime of global incoherence $(r \approx 0)$. Right at the onset of synchronization for the SF network, its $r_{l i n k}$ value deviates from that of the ER recovering the known result about the synchronization of SF networks for lower values of the coupling. In this region, while the synchronization patterns continue to grow for the ER network at the same rate, the formation of locally synchronized structures occurs at a faster rate in the SF network. Finally, when the incoherent solution in the ER network destabilizes, the growing in its synchronization pattern increases drastically up to values of $r_{\text {link }}$ comparable to those obtained in SF networks and even higher.

The results in terms of $r_{\text {link }}$ show again that statements about synchronizability are dependent on the coupling strength value. Additionally, the previous discussion suggests that synchronization is attained following two different paths that depend on the underlying topology. To shed new light on this phenomenon, we have studied the characteristics of the synchronization patterns along the evolution of $r_{\text {link }}$. Following the usual picture synchronization patterns are formed by pairs of oscillators, physically connected, whose phase difference in the stationary state tends to zero. In order to determine which pairs of nodes are truly synchronized we have to determine the coherence of their dynamics. Note that eq.(8.23) is the average dynamical coherence between every pair of linked nodes and then the synchronization degree of every 


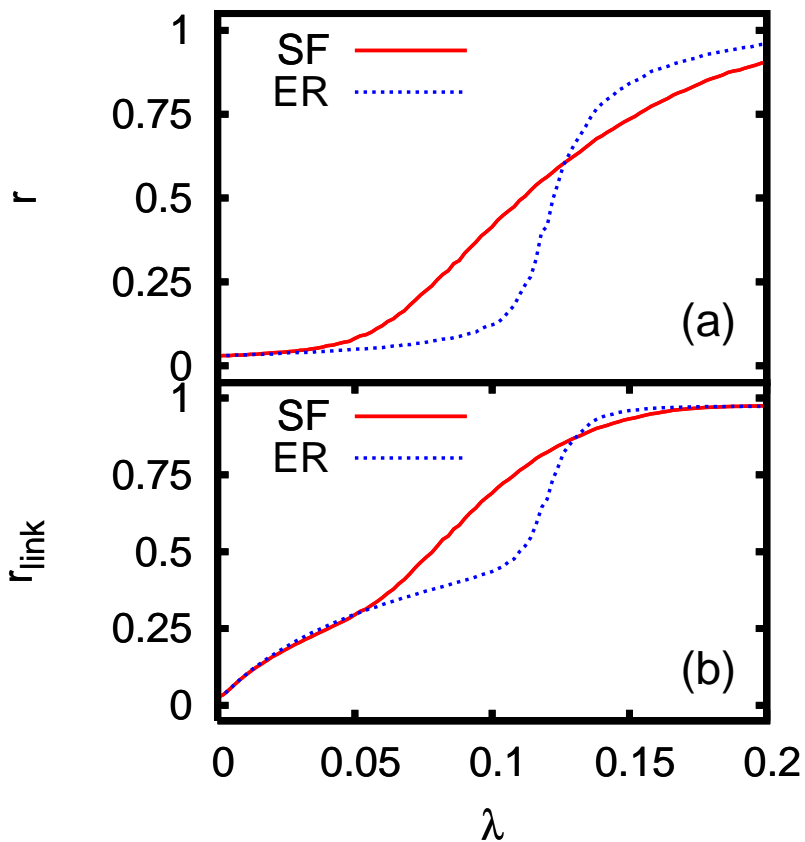

Figure 8.5: Evolution of (a), the KM order parameter defined in eq. (8.5), and (b) the fraction of synchronized links $r_{l i n k}$, eq. (8.23), as a function of $\lambda$. The curves separate when the incoherent solution for SF networks destabilizes. The figure clearly illustrates that the synchronizability of the networks does depend on the value of the coupling strength. Both plots are represented for Erdös-Rényi (ER) and scale-free (SF) networks as indicated. The size of the networks is $N=10^{3}$ and their average degree is $\langle k\rangle=6$. The exponent of the SF network is $\gamma=-3$.

pair of connected oscillators can be written in terms of a symmetric matrix

$$
\mathcal{D}_{i j}=A_{i j}\left|\lim _{\Delta t \rightarrow \infty} \frac{1}{\Delta t} \int_{t_{r}}^{t_{r}+\Delta t} e^{i\left[\theta_{i}(t)-\theta_{j}(t)\right]} d t\right| .
$$

Then one has to analyze each matrix term $D_{i j}$ in order to label a link $(i, j)$ as synchronized or not. As introduced above, from the computation of $r_{\text {link }}$ one determines the fraction of physical links that are synchronized so that one would expect that $2 r_{\text {link }} \cdot N_{l}$ elements of the matrix $\mathcal{D}$ are $D_{i j}=1$, while the remaining elements are $D_{i j}=0$. However, this is not the real situation since the network dynamics is not well defined in terms of a fully synchronized cluster and a set of completely incoherent oscillators. On the other hand the worst scenario would be found if there were $2 N_{l}$ elements of matrix $\mathcal{D}$ so that $D_{i j}=r_{\text {link }}$, implying that all the physically connected pairs are equally synchronized and hence the parameter $r_{l i n k}$ could not be interpreted as the fraction of links that are dynamically coherent and no information about the 


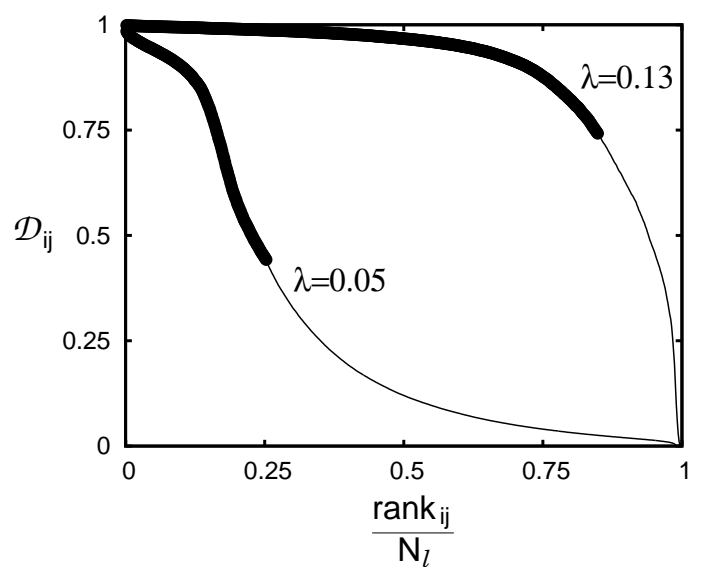

Figure 8.6: Degree of synchronization, $\mathcal{D}_{i j}$ between pairs $(i, j)$ of connected nodes. These elements of the matrix $\mathcal{D}$ are ordered from largest $r a n k_{i j}=1$ to the lowest $\operatorname{rank}_{i j}=N_{l}$. The network is SF with $N=10^{3}$ and $\langle k\rangle=6$. The two curves correspond to $\lambda=0.05$ (onset of synchrony) and $\lambda=0.13$ with $r_{\text {link }} \simeq 0.25$ and 0.82 respectively for these two particular realizations. The thick regions of the curves correspond to the links chosen in order to reconstruct the synchronized cluster.

topological patterns of synchronization could be extracted from matrix $\mathcal{D}$. The situation found is not as simple as the former possibility and not so dramatic as the latter. In figure 8.6 the contributions $D_{i j}$ of the $N_{l}$ elements of matrix $\mathcal{D}$ that corresponds to physical links are plotted ordered from the highest to the lowest one. The two situations plotted correspond to the onset of synchronization $(\lambda=0.05)$ and when high global coherence $(\lambda=0.13)$ is observed for a SF network. As can be observed for the onset of synchronization, a subset of nearly $20 \%$ of links displaying coherent dynamics with high degree of synchronization, $\mathcal{D}_{i j}>0.8$, is well separated from the behaviour of the remaining links as the dramatic decrease of $D_{i j}$ reveals for the remaining $80 \%$ of links. In this sense, it is clear that the dynamics of a $20 \%$ of the possible pairs can be regarded as synchronized which is in agreement with the obtained value $r_{\text {link }}=0.25$ for $\lambda=0.05$ and hence supporting that although macroscopic coherence is not observed ( $r \simeq 0$ at this point) the system is seen to walk towards it. For the curve corresponding to $\lambda=0.13$ a plateau of nearly $75 \%$ of links is observed, thus revealing the high degree of global coherence, $r \simeq 0.7$, at this point. As a conclusion, the shape of both curves allows to interpret $r_{l i n k}$ as the fraction of synchronized links and thus to obtain information about synchronized patterns from $\mathcal{D}$.

In order to determine exactly which pairs of nodes are regarded as synchronized, matrix $\mathcal{D}$ is thus filtered using a threshold $T$ such that the fraction of synchronized pairs equals $r_{l i n k}$. In this way $T$ is a moving threshold so that if $\mathcal{D}_{i j}>T$ oscillators $i$ and $j$ are considered synchronized. The value of $T$ depends on the particular realization and is determined by means of an iterative scheme starting from $T=1$ and decreasing it with $\delta T=0.01$ one computes 

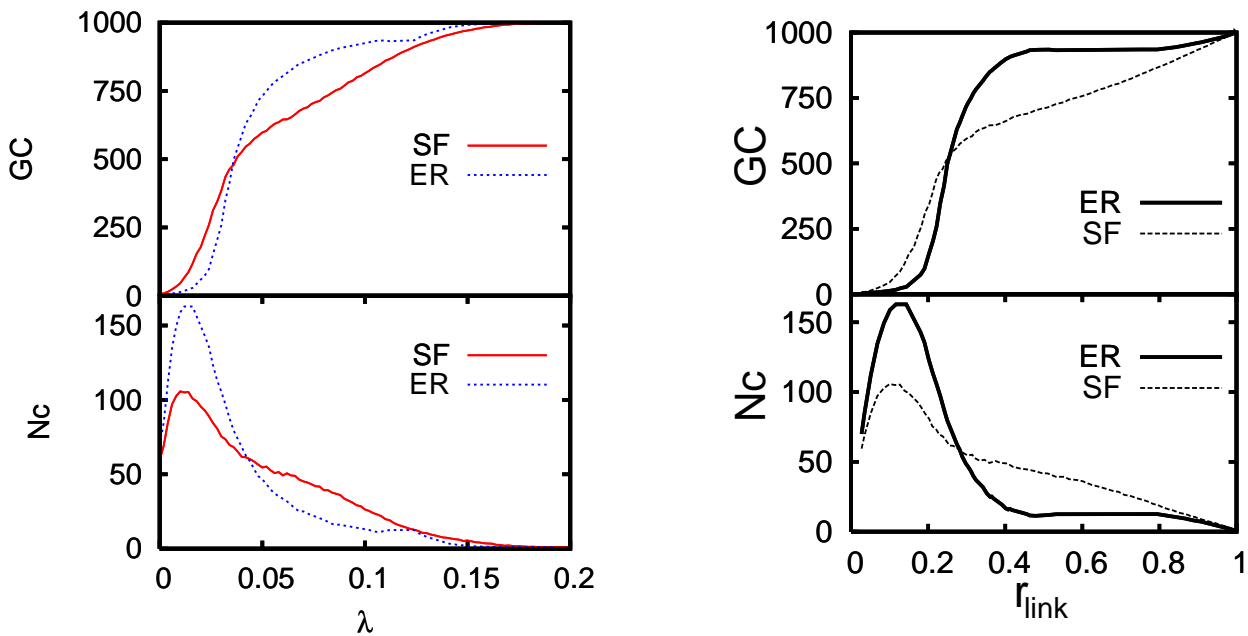

Figure 8.7: Size of largest synchronized connected component $(G C)$ and number of synchronized connected components $(N c)$, as a function of $\lambda$ (left) and $r_{l i n k}$ (right) for the different topologies considered. Small values of $r_{l i n k}$ correspond to values of $\lambda$ for which $r \approx 0$. Despite $r$ being vanishing and hence no global synchronization is yet attained, a significant number of clusters show up. This indicates that for any $\lambda>0$ the system self-organizes towards macroscopic synchronization. The network parameters are as in figure 8.5

the amount of links that fulfills the condition. Decreasing progressively the value of $T$ more pairs of oscillators are chosen and the process is stopped when the value of $T$ is such that the fraction of chosen links is equal to the desired value $r_{\text {link }}$ previously computed from $\mathcal{D}$. When the synchronized links are identified the clusters of synchronized nodes are reconstructed. In figure 8.6 the chosen links according to the corresponding values of $r_{l i n k}$ are those lying in the thicker part of both curves.

In figure 8.7 we represent the number of synchronized clusters and the size of the largest one (GC) as a function of $\lambda$ and $r_{l i n k}$ for ER and SF networks. The local information extracted from it unveils an astonishing and novel feature of the synchronization process that can not be derived from figures 8.4 and 8.5 , and that in some sense is counterintuitive. The emergence of clusters of synchronized pairs of oscillators (links) in the networks shows that for values of $\lambda \leq \lambda_{c}^{S F}$, i.e., still in the incoherent solution $r=0$, both kind of networks have developed a largest cluster of synchronized pairs of oscillators involving $50 \%$ of the nodes of the network, and an equal number of smaller synchronization clusters. From this point on, in the SF network the GC grows and the number of smaller clusters goes down, whereas for the ER network the growth explodes. These results indicate that although SF networks present more coherence in 
terms of $r$ and $r_{l i n k}$, the microscopic evolution of the synchronization patterns is faster in ER networks, being these networks far more locally synchronizable than the heterogeneous ones.

The observed differences in the behaviour at a local scale are rooted in the growth of the GC. It turns out that for the ER networks, many different clusters of synchronized pairs of oscillators (note in figure 8.7 the large number of clusters formed when a $15 \%$ of the links are synchronized) merge together to form a GC when the effective coupling is increased. The coalescence of many small clusters leads to a giant component of synchronized pairs that is almost the size of the system once the incoherent state destabilizes. On the other hand, this is not anymore the case for SF networks, where oscillators are incorporated slowly to the GC practically one-by-one (forming new pairs) in terms of $\lambda$ (or $r_{\text {link }}$ ), but starting from a core made up of half the nodes of the network. As a conclusion, while for ER network the collapse at $\lambda_{c}$ of the small clusters of synchronized nodes that have been grown when $r=0$ is the root of the fast increase of the global coherence, for SF the process is described as a slow and progressive growth as the coupling is increased of an initial core of synchronized nodes.

The above picture is confirmed in figure 8.8 , where we have represented the evolution of local synchronization patterns in ER and SF networks for several values of $\lambda$. It is clear that when $r \simeq 0$ the two networks follow different

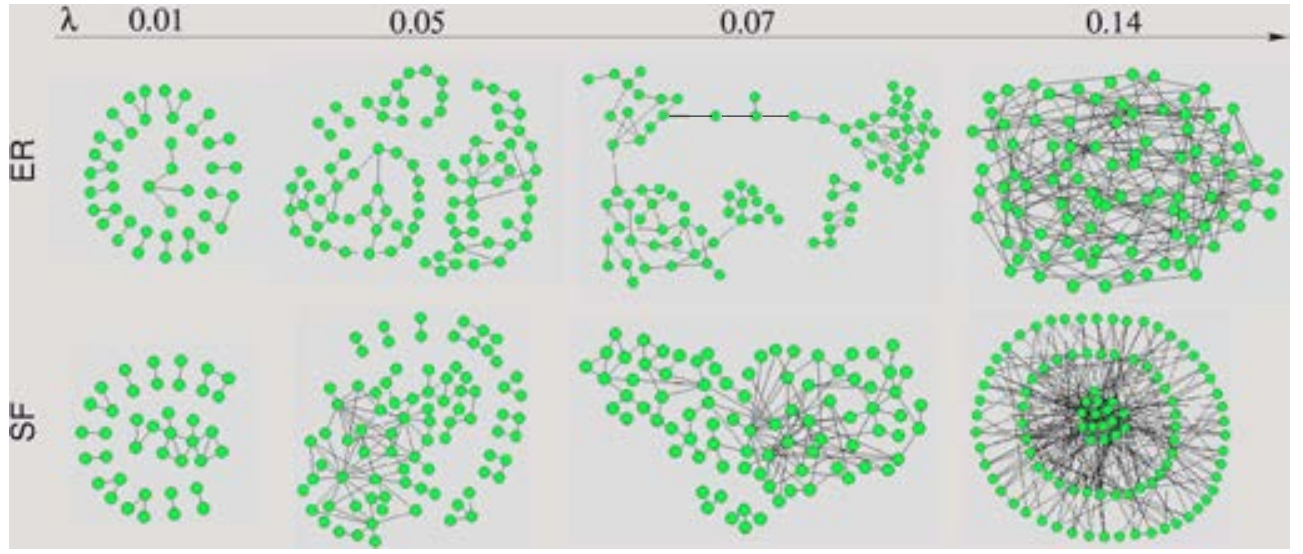

Figure 8.8: Synchronized clusters for several values of $\lambda$ for the two different topologies studied (ER and SF). These networks are made up of 100 nodes, in order to have a sizeable picture of the system. The evolution of local synchronization patterns is always agglomerative, however, it follows two different routes. In the ER case, the growth of the GC proceeds by aggregation of small clusters of synchronized nodes, while for the SF network the central core groups the smaller clusters around it. 

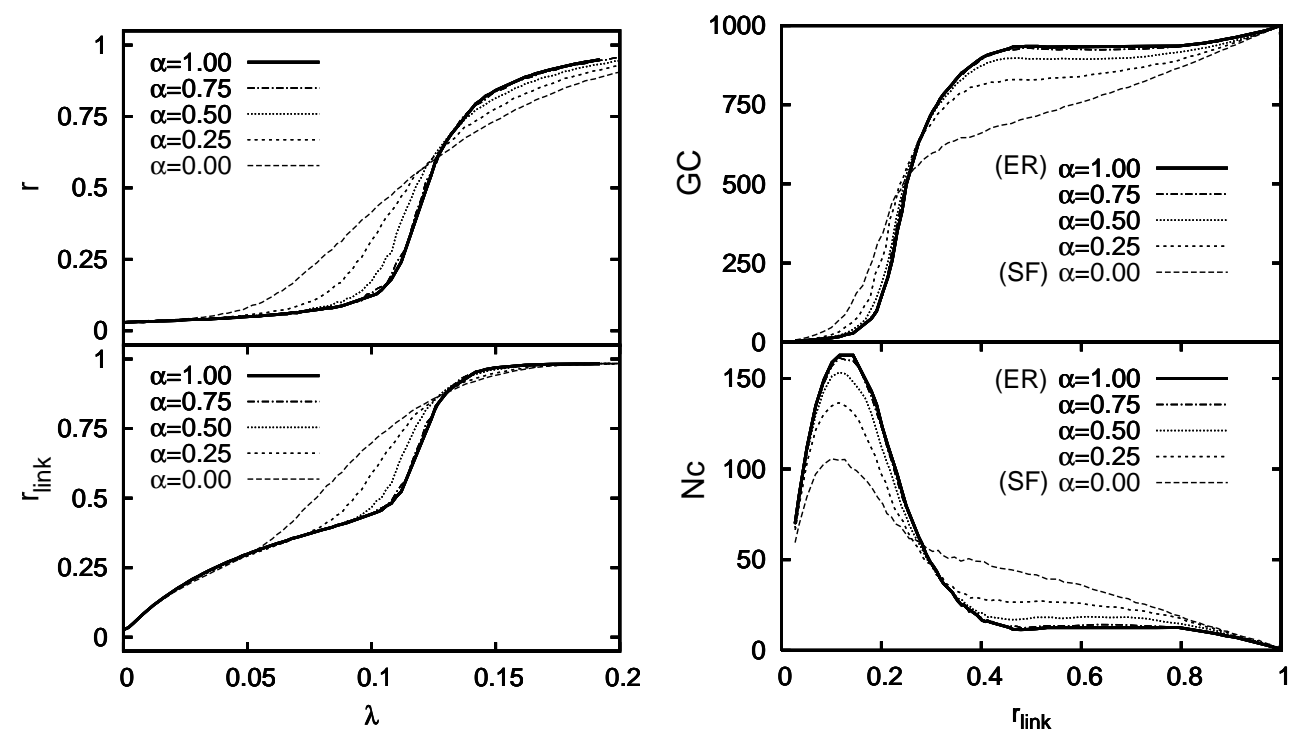

Figure 8.9: (Left) Evolution of the control parameters $r$ and $r_{\text {link }}$ as a function of the coupling strength for networks generated with the model introduced in section () corresponding to $\alpha=0.0,0.25,0.5,0.75$ and 1.0. (Right) Evolution of the number of synchronized clusters $N_{c}$ and the synchronized giant component size $G C$ as a function of $r_{\text {link }}$ for the same topologies. The network parameters are the same as in figure 8.5 .

paths toward synchronization as can be observed for $\lambda=0.05$ where for the ER network three clusters of synchronized nodes of similar size appear while for the SF network a unique large cluster is present. The coalescence of the synchronized patterns for the ER is clear at $\lambda=0.07^{2}$ and corresponds to the emergence of the global coherence of the system. On the other hand, for the SF network the unique cluster suffers a smooth growth by adding new synchronized nodes to the giant cluster.

This study about the patterns of self-organization towards synchronization reveals that the quantitative difference about the macroscopic behaviour, shown by the computation of the evolution of the global coherence $r$ for ER and SF networks, has its roots on a qualitatively different route at the microscopic level of description. The use of the new parameter $r_{\text {link }}$ which involves the computation of the degree of coherence between each pair of linked nodes is a useful tool for describing such differences.

We have repeated analogous computations using those networks that interpolate between ER and SF topologies. As expected the behaviour of relevant

\footnotetext{
${ }^{2}$ Note that the size of the network is relatively small $(N=100)$ and thus the critical point is shifted to lower values $\left(\lambda_{c}^{E R} \simeq 0.07\right.$ in this case) than that found using a FSS analysis.
} 
magnitudes such as $r_{\text {link }}$, the number of synchronized clusters and the size of the giant synchronized cluster lies between the two limiting cases studied above. In figure 8.9 we have plotted the evolution of these magnitudes for these interpolating network topologies labeled with $\alpha$. The results suggest that the degree of heterogeneity of the network is the key ingredient to explain the two different routes observed.

The technique developed for extracting the synchronization patterns allows the analysis of the topological features of such clusters of nodes. Considering as we did in last chapter 7 the emergent clusters of synchronized nodes and links, we can compute the average measures of relevant quantities such as the clustering coefficient or the degree distribution, and see how these magnitudes evolve from the uncoupled limit, where no synchronization occurs, to the coherent regime where the synchronized network coincides with the underlying substrate. It is then relevant to explore the regions where the onset of synchronization takes place and characterize topologically these emergent synchronized clusters.

In figure 8.10 the evolution of the clustering coefficient, $\langle c\rangle$, of the giant synchronized cluster is plotted as a function of $\lambda$ for the topologies corresponding to SF to ER networks. The results are illustrative, for these topologies the clustering decreases as the coupling is increased or, in other words, as the giant component grows by the addition of new synchronized nodes. However,

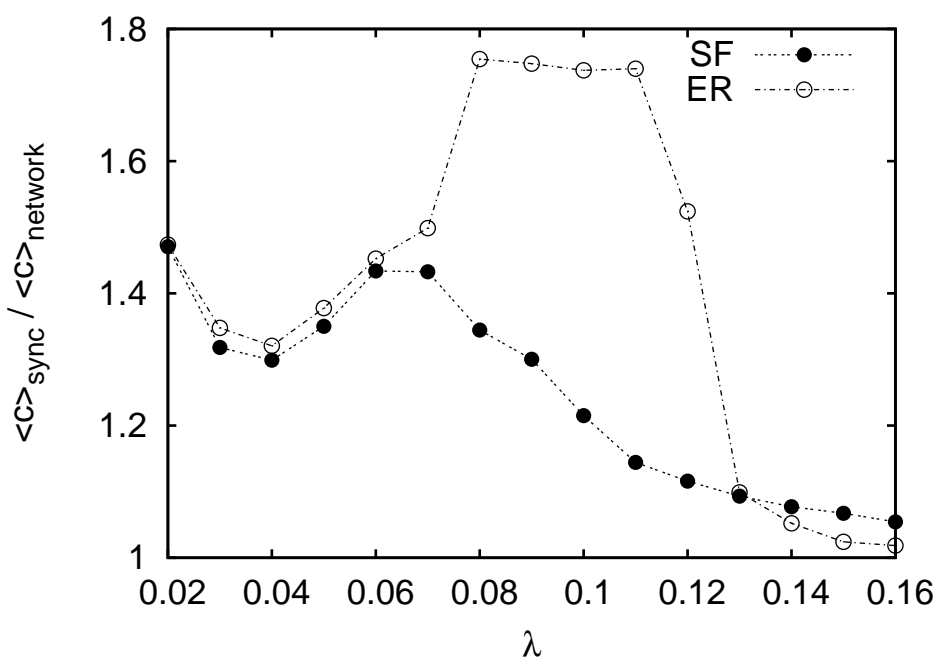

Figure 8.10: Evolution of the clustering coefficient of the giant synchronized cluster, $\langle c\rangle$, as a function of $\lambda$ for ER and SF network topologies $(\alpha=0$, and 1). Network parameters are those used in figure 8.5. 
the effects of the two different routes to complete synchronization observed for $\mathrm{ER}$ and SF are well appreciated from the results. For SF networks there is a smooth decrease of the clustering coefficient and the effects of the emergence of global coherence for $\lambda>\lambda_{c}^{S F}=0.05(1)$ are not dramatic in what refers to the behaviour of $\langle c\rangle$. That is, it takes too long in terms of $\lambda$ to arrive to values of the clustering similar to those of the substrate network. On the other hand, for ER graphs the slow decay rate observed for $\lambda<\lambda_{c}^{E R}=0.122(2)$ when no macroscopic coherence is observed is interrupted by a sudden jump near their critical value. In fact, for $\lambda>\lambda_{c}$ the clustering of the synchronized cluster is similar to that found for the substrate network. This effect becomes clear if one has in mind the coalescence of small clusters, which happens around the critical point for ER graphs. In fact, taking into account the giant synchronized component on ER for $\lambda<\lambda_{c}^{E R}$, implies to consider one of the several disjoint synchronized clusters of similar size that are in this region. Then, when these clusters collapse into a much larger one the topological features change dramatically as observed from the evolution of the clustering coefficient. This is not anymore the case in SF where the topological characteristics of the giant component change smoothly as new nodes are dynamically attached to the component. The remaining curves corresponding to the interpolating networks connect these two different behaviours.

All the results reported above point out that the ultimate reason behind the two different routes to complete synchronization is the heterogeneous character of the SF network and the role played by the hubs. The natural cohesion that hubs provide to SF network prevents the existence of independent macroscopic clusters of synchrony as occurs for ER networks. It is then interesting to study how these hubs participate in the formation of the final synchronized state. For this, we first study the evolution with $\lambda$ of the composition of the synchronized cluster in terms of the degree of its components. In figure 8.11, we have plotted the probability that a node with degree $k$ belongs to the giant synchronized cluster as a function of its degree $k$ and the coupling $\lambda$ for the SF network. This probability turns out to be an increasing function of $k$ for every value of the coupling $\lambda$. Hence one can state that the more connected a node is, the more likely it takes part in the cluster of synchronized links. In particular, the results confirm the hypothesis made above that the hubs participate from the very beginning on the formation of the synchronized cluster. Recently [331], Zhou and Kurths have reported the study of hierarchical organization in complex networks, using the MSF and a mean-field approach in the weak coupling limit. Our results thus substantiate and generalize those about the role of hubs in the synchronization process presented in [331].

The above characterization of the synchronized cluster in terms of the degree of its component should be completed studying their effective degree, $k_{i n t}$. 


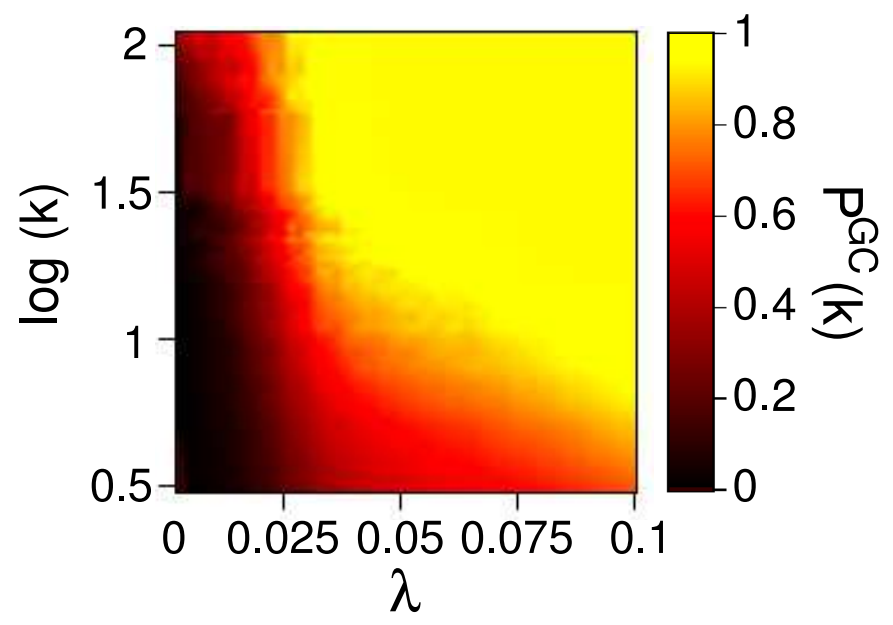

Figure 8.11: The plot shows the correlation between the likelihood that a node belongs to the GC of pairs of synchronized oscillators and its degree $k$ as a function of the coupling strength $\lambda$. This probability, $P^{G C}(k)$, is color-coded as indicated in the right panel. The figure convincingly demonstrates that highly connected nodes are those that recruit poorly connected nodes as the GC grows. The network is $\mathrm{SF}$ and its parameters are those used in figure 8.5.

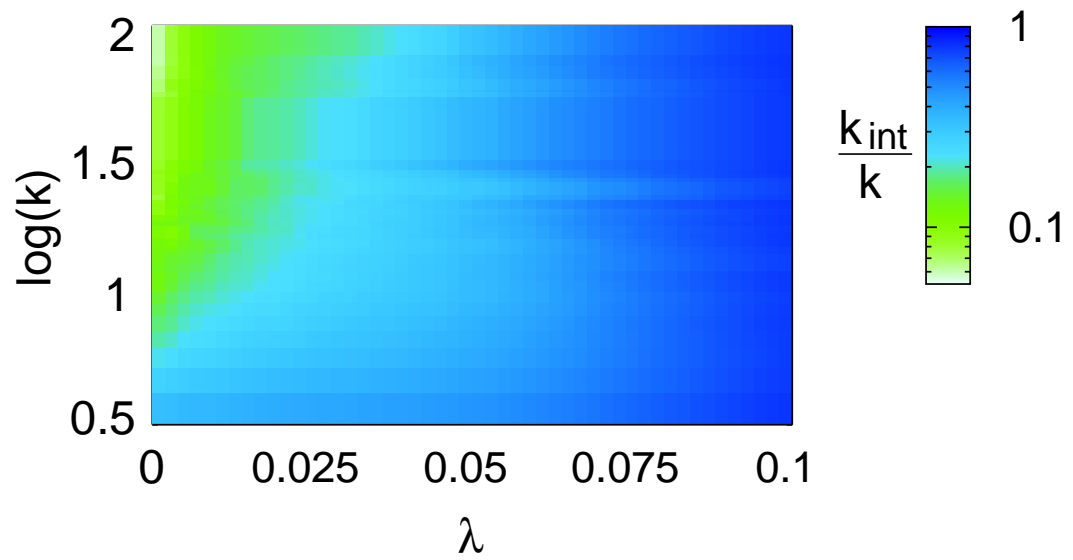

Figure 8.12: The plot shows the fraction of links that a node with degree $k$ belonging to the synchronized cluster shares with other nodes of the same synchronized cluster. This fraction $k_{i n t} / k$ is plotted as a function of $k$ and $\lambda$. The figure shows how the hubs progressively incorporate their neighbours to the synchronized component as $\lambda$ grows. The network is SF with parameters as those used in figure 8.5. 
The effective degree of a synchronized node is the number of links it shares with other nodes belonging to the same synchronized cluster. Obviously, at the complete synchronized regime a node with degree $k$ will have $k_{i n t}=k$. We have plotted in figure 8.12 the quantity $k_{i n t} / k$ (the fraction of links that a node has with synchronized neighbours) as a function of $\lambda$ and the degree $k$ of the nodes. The results reveal that although hubs are the first to take part of the synchronized cluster, their neighbours are progressively incorporated to the cluster as $\lambda$ grows. Besides, if a node with small $k$ is synchronized the probability that its neighbours are also synchronized grows very fast with $\lambda$ which is an effect of the network topology (this nodes in the BA network are physically connected to hubs with high probability). These results further support the statement about the essential role played by hubs in the path to synchronization in SF networks.

The results of this section clearly show that synchronizability of complex networks is dependent on the effective coupling $\lambda$ among oscillators. For small values of $\lambda$, SF networks outperform ER topologies, but the tendency is reverted for intermediate to large values of the coupling. On the other hand, the detailed analysis of evolution of patterns of synchronization showed that there are two radically different mechanisms to attain synchronization. In the presence of hubs, a giant component of synchronized pairs of oscillators forms and grows by recruiting nodes linked to them. On the contrary, in homogeneous structures, many small clusters first appear and then group together through a sharp merging process. These results are in the same direction of those found for percolation and epidemic spreading (see section 6.1.2) on top of homogeneous or heterogeneous graphs, where the radical differences of the system's dynamics are found depending on the heterogeneity of the underlying networks, demonstrating that the same behavior may hold for nonlinear dynamical systems coupled to complex structures. More importantly, the fact that the route to complete synchronization is radically different in homogeneous and heterogeneous networks, raises the question of its motivation in nature and shed light on the structure-function interplay. Besides, the results open new paths to clarify how synchronization is attained in complex topologies and give new tools to analyze this ubiquitous phenomenon.

\subsection{Synchronization in structured networks}

In the light of the results of the above section we have extended the study beyond unstructured networks to structured or modular networks. This is a limiting situation in which the local structure may greatly affect dynamics, irrespective of whether or not we deal with homogeneous or heterogeneous 
networks and then they constitute a perfect framework for testing the new order parameter $r_{\text {link }}$ introduced in the last section.

Many complex networks in nature are modular, i.e. composed of certain subgraphs with differentiated internal and external connectivity that form communities (see section 5.1.4). The use of modular networks where a proper comparison in synchronizability can be performed (same number of nodes and links) restricts us to the consideration of synthetic structured networks. Then we make use of a common benchmark of random network with community structure, first proposed by Newman[332] considering one hierarchical level and later extended to two hierarchical levels[329].

The modular network structure we build is as follows: in a set of $N$ nodes, we prescribe $n$ compartments that will represent our first community organizational level, and $m$ compartments, each one embedding four different compartments of the first level, that define the second organizational level of the network. The internal degree of nodes at the first level $z_{i n_{1}}$ and the internal degree of nodes at the second level $z_{i n_{2}}$ keep an average degree $z_{i n_{1}}+z_{i n_{2}}+z_{\text {out }}=\langle k\rangle$ so that these networks are strictly homogeneous in the sense of the degree distribution, $P(k)=\delta(k-\langle k\rangle)$. Networks with two hierarchical levels are labeled as $z_{i n_{1}}-z_{i n_{2}}$, e.g. a network with $i$-j means $i$ links with the nodes of its first hierarchical community level (more internal), $j$ links with the rest of communities that form the second hierarchical level (more external) and $(\langle k\rangle-i-j)$ links with any community of the rest of the network.

Synchronization processes on top of modular networks of this type has been recently studied as a mechanism for community detection [329]. Starting from a set of homogeneous (in terms of the natural frequencies) Kuramoto oscillators with different initial conditions the system evolves after a transient of time to the synchronized state. It has been shown that the community structure is progressively unveiled at the same time the system's dynamics evolve toward the coherent state and the synchronization is attained. In particular, the nodes belonging to the first community level are the first to get synchronized, subsequently the second level nodes achieve the frequency entrainment and finally the whole system shows global synchronization.

Here we adopt a different perspective since we will consider as previously a set of non-identical Kuramoto oscillators with random assignment of natural frequencies and hence the final degree of system's synchronization will depend on the strength of the coupling. It is then interesting to study how the degree of synchronization evolves as a function of $\lambda$ and whether the coherence between nodes is progressively distributed following the hierarchy imposed by the underlying topology. For this, we make use of the order parameters $r$, eq. (8.5), and $r_{\text {link }}$, eq (8.23), to characterize the synchronization transition on two 


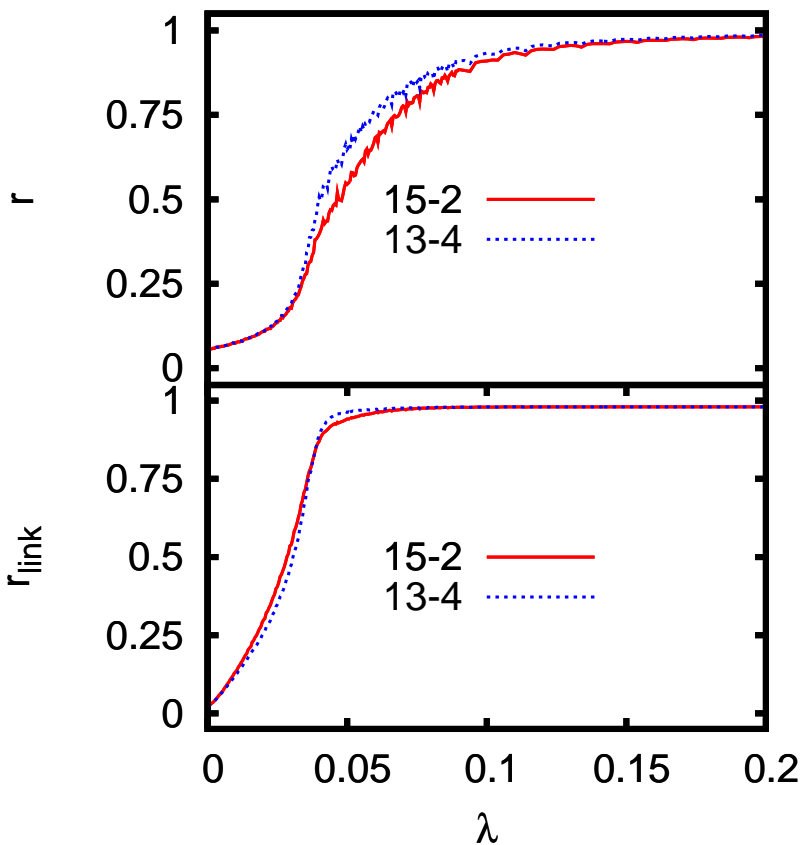

Figure 8.13: Global and local synchronization in modular networks. Evolution of (a), $r$ and (b), $r_{\text {link }}$ as a function of $\lambda$ for structured modular networks. The networks are synthetically built with an a priori community structure. The network size is 256 nodes and the number of links is 4608 . We prescribe 16 compartments that will represent our first community organizational level, and four compartments each one embedding four different compartments of the above first level, that define the second organizational level of the network. Each node has 18 links distributed between its first community level, the second, and the whole network at random. The network 134 has 13 internal connections in its first hierarchical level, 4 external connections in its second hierarchical level, and 1 connection with any other community in the network. The generation of the 15-2 structure is equivalent. The curves show that although 13-4 has always a better global synchronization, 15-2 has better local synchronization as shown by $r_{l i n k}$.

slightly different modular networks with two well defined hierarchical levels, $13-4$ and $15-2$, being this difference the cohesion of the internal community core, 13 links out of 15 possible neighbors or 15 links (i.e., all-to-all) at the most internal level. Both networks have $N=256$ and $\langle k\rangle=18$. Figure 8.13 show the results for both kinds of networks revealing that the path towards synchronization as a function of the interaction is again affected by the structure. They also show that the information provided by $r_{l i n k}$ is essential to unveil the synchronization process. While the global synchronization parameter $r$ is reflecting that the $13-4$ structure globally synchronizes always better, $r_{\text {link }}$ tells us again about the local synchronization. It shows that local synchronization 

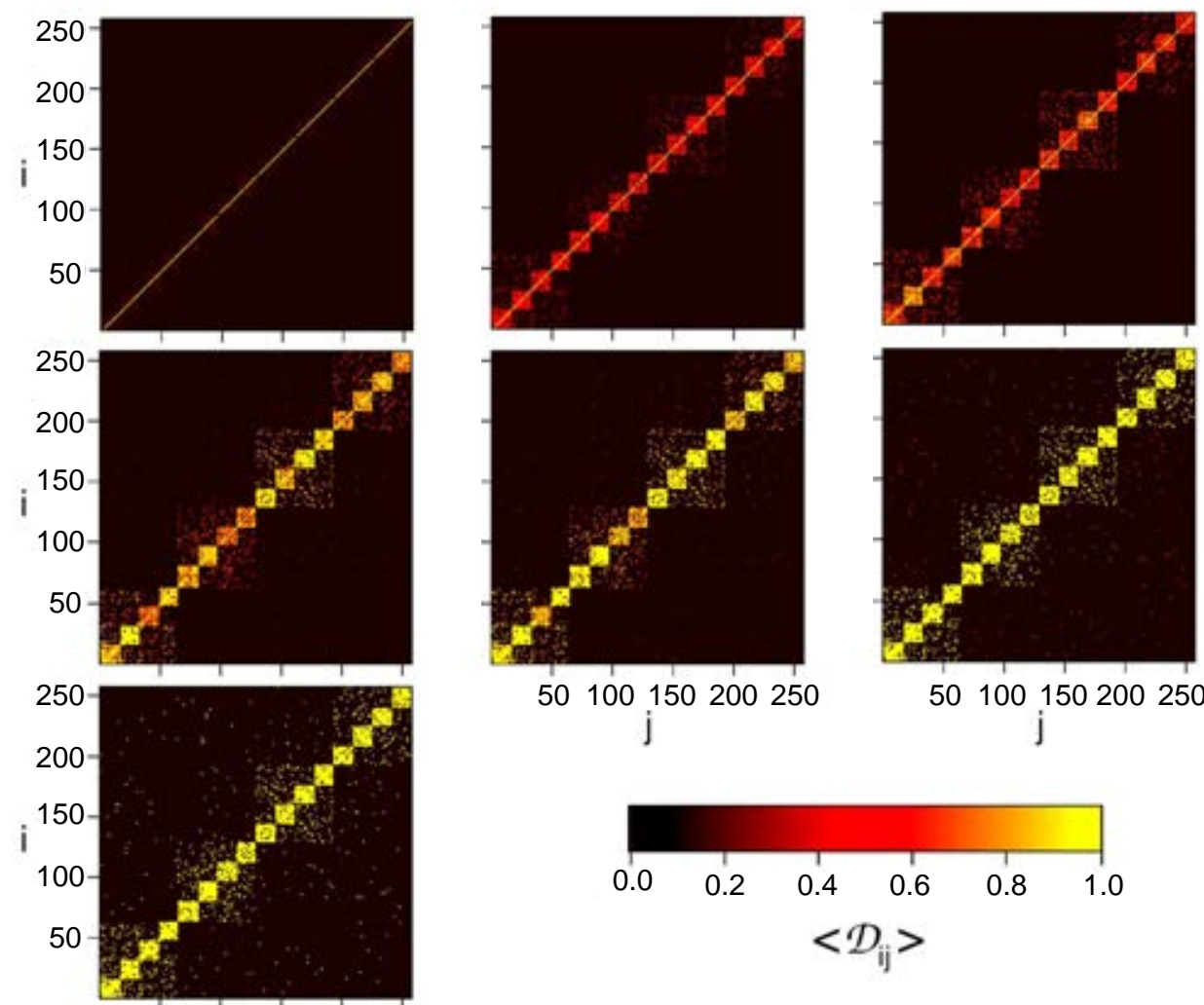

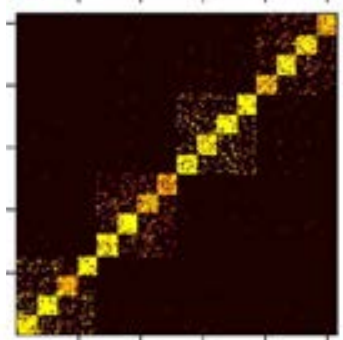

50100150200250 j

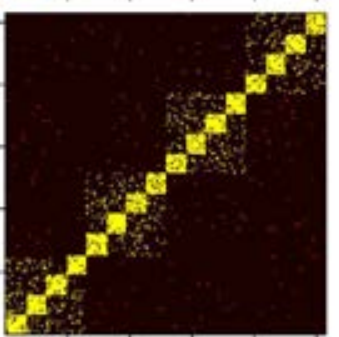

50100,150200250

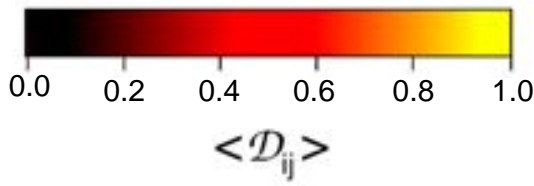

50100,150200250

Figure 8.14: Synchronization patterns in a 15-2 modular network. We represent the degree of synchronization between pairs of connected nodes for several values of the coupling $\lambda$ in a 15-2 modular network (with two organizational levels) of $N=256$ nodes. The color code denotes the value of the averaged (over different initial conditions) filtered matrix $\left\langle\mathcal{D}_{i j}\right\rangle \in[0,1]$. The values of the coupling are (from left to right and top to bottom) $\lambda=0.0110,0.0210,0.0275,0.0305,0.0360,0.0440$ and 0.210 (corresponding to full synchronization). The pictures show that the order of synchronization is given by the organizational levels. The first community level is the first one to get synchronized, subsequently, second level nodes attain synchronization for a larger value of $\lambda$ and finally the full synchronized state is reached when outer links have $\left\langle D_{i j}\right\rangle=1$. 
is indeed favored in the $15-2$ structure since $r_{\text {link }}$ is larger for this topology for small values of $\lambda$ where the system is locally forming synchronized clusters. This result, not captured by the macroscopic indicator $r$, is expected since the internal cohesion of communities at the first hierarchical level is larger for the 15-2 than for the 13-4. The evolution of $r_{\text {link }}$ shows that when the coupling $\lambda$ is increased the number of links synchronized in the $13-4$ network becomes larger than in the $15-2$ structure revealing that complete synchronization is then favoured by the presence of more external links connecting the first level communities.

The inspection of the synchronization path in modular networks can be easily visualized by the representation of the filtered matrix $\mathcal{D}$. It implies to reassign the values of matrix $\mathcal{D}$ so that $\mathcal{D}_{i j}=1$ if $\mathcal{D}_{i j}>T \mathcal{D}_{i j}=0$ otherwise. Plotting this filtered matrix for different values of the coupling $\lambda$ one can easily determine which links are the first to synchronize since the form of the adjacency matrix $\mathcal{A}$ (that includes all the physical links between nodes) is also easy to interpret because of its nested structure. Figure 8.14 shows how the community structure determines the internal organization of the system in the route towards full synchronization for the $15-2$ network. For this study we have computed the value of the filtered matrix $\mathcal{D}$ for a number of initial conditions and then took its average value so that $\left\langle\mathcal{D}_{i j}\right\rangle \in[0,1]$ accounts for the synchronization strength of the network link $(i, j)$. The results point out that link synchronization depends on the organizational level they belong to. Those connecting nodes belonging to the same first level community are the fastest (in terms of the coupling strength $\lambda$ ) to reach full synchronization. For larger values of $\lambda$ full synchronization is attained progressively for the subsequent organizational levels. Then, one can conclude that the inner the link is the faster it gets synchronized in agreement with previous studies reported above [329].

As a conclusion, the framework of structured networks has provided a useful benchmark for testing the validity of the new parameter $r_{l i n k}$ and the information obtained from the computation of matrix $\mathcal{D}$. The results obtained by means of these quantities allow to conclude that for modular networks synchronization is first locally attained at the most internal level of organization and, as the coupling is increased, it progressively evolve toward outer shells of the network. Besides, we have obtained evidences that a high cohesion at the first level communities produce a high degree of local synchronization although it delays the global coherent state. 


\subsection{Conclusions}

In this chapter we have explored several issues about synchronization in complex networks of Kuramoto phase oscillators. Our main concern has been the study of the synchronization patterns that emerge as the coupling between non-identical oscillators increases. As in the previous chapter, nonlinear dynamics on top of complex networks lead to the formation of activity patterns. These dynamical patterns (synchronized clusters here) are the byproduct of the dynamical and structural complexity of the problem.

First, in section 8.2 we have focused on scale-free networks. In this section we have explored the macroscopic behaviour of synchronization when both clustering and average path length are continuously varied making use of the network model defined in section 5.3. The results show that the onset of synchronization is favoured as the clustering coefficient grows but, on the other hand, as the average path length increases the path toward full synchronization becomes larger. Section 8.3 accounts for the main concern of this third part of the Thesis, the analysis of the emergence of dynamical patterns. For this purpose we compute the degree of synchronization between each pair of connected oscillators. This technique allows to reconstruct the synchronization clusters from the dynamical data. We have studied how the underlying topology (ranging from homogeneous to heterogeneous structures) affects the evolution of synchronization with the help of the network model introduced in section 5.4. The results reveal that the route towards full synchronization depends strongly on whether one deals with homogeneous or heterogenous topologies. In particular, it has been shown that the synchronization cluster in heterogeneous networks grows from a unique core formed by those highly connected nodes (hubs) whereas for homogeneous networks several synchronization clusters of similar size can coexist. In the latter case, a coalescence of these clusters is observed in the synchronization path which is macroscopically manifested by the sudden growth of global coherence typically observed for Erdös-Rényi networks. The main result of this section is that systems are seen to organize towards synchronization even when no macroscopic signal of global coherence is observed. The difference between these two observed organizational behaviours influences the eventual onset of synchronization. Finally, in section 8.4, we have applied the new technique for unveiling the local synchronization patterns to the analysis of the emergence of synchronization in structured networks. In this case, the technique allows to observe how synchronization is progressively attained from the most internal communities until coherence of the whole network shows up. 


\section{Chapter 9}

\section{Conclusions}

We want to conclude with a brief summary of the most relevant results obtained along the three parts of this Thesis. We want to stress here their relevance as well as some prospective research that arises in the light of these results.

In the first part we have studied the issue of intrinsic localization (discrete breathers) in nonlinear Schrödinger anharmonic lattices (described by the Salerno model). The major achievement of these studies is the computation of exact mobile localized modes. For these computations, it was important to develop a generalized continuation method, that can be thought of, as the natural extension of those employed for computing standard (pinned) discrete breathers. The generalized continuation method allows to obtain, in a highly systematic way, families of mobile, oscillating and vortical discrete breathers (as well as pinned ones).

The problem on the existence of mobile discrete breathers has been extensively discussed after the theory for pinned localized modes was successfully developed. The use of collective variable methods and numerical simulations of perturbed pinned solutions lacks the precision required to obtain general arguments about the possibility of having mobile localized states in nonlinear lattices. However, the computation of mobile discrete breathers in this Thesis is neither unbiased (i.e. based on a priori ansatzes) neither suffers from low numerical accuracy. On the contrary, our continuation procedure computes mobile discrete breather solutions as exact fixed points solutions of a map and, therefore, the unique requirement is that the Jacobian of the map is invertible so that the iterative method converges to the desired solution.

Concerning mobile breathers, our results point out that, except for integrable and other exceptional (e.g. vanishing Peierls-Nabarro barrier) situa- 
tions, mobile localized states in nonlinear Schrödinger lattices are described by a localized part (the core) and an asymptotically extended background composed of plane waves. We have obtained numerical evidences of the importance of this extended background in the core mobility. In particular, we have shown how the Peierls-Nabarro barriers that a mobile breather experiences periodically in its motion across the lattice are surpassed with the help of the energy balance core-background. In this sense, we have observed that the higher is the Peierls-Nabarro barrier, the higher is the energy flux between core and background and the higher is the amplitude of the extended background. These observations reveal the essential role of the background in core mobility pointing out that collective variables approaches are incomplete when considering only those degrees of freedom relative to the core.

The study of the whole Salerno lattice, both in its one- and two-dimensional versions, has provided a detailed account of the existence and properties of discrete breather solutions and, at the same time, it has proved the versatility of the continuation method.

Several questions remain open after these studies. In particular, it would be desirable to perform a deep analysis on the mobility of two-dimensional discrete breathers and more exotic solutions like trains of discrete breathers or vortical states. It would be also interesting to apply the continuation methods to other important nonlinear lattices such as Klein-Gordon or Peyrard-BishopDauxois models. Finally, it is also interesting to develop a collective variable theory accounting for the relevant ingredients of the mobile solutions found.

In the second part we have studied the structure of complex networks and the performance of propagation dynamics on top of them. Several results have been obtained for each of these two issues.

We have first presented two models of network construction that provide two network families where only a few topological characteristic vary significantly among the members of these families. The purpose of these models is to provide a useful tool for analyzing the role that these changing structural properties have on the performance of different network dynamics. In fact, these models have been used for this purpose along the Thesis. Whereas one model preserves the scale-free character of the degree distribution while the clustering coefficient and average characteristic path length are varied, the second one allows the degree distribution to interpolate between the scale-free and Poisson distributions while other magnitudes remain comparable. This latter model would be very useful to shed new light on the roots of the different phenomena found when dealing with homogeneous and heterogeneous topologies.

The studies on the propagation dynamics on networks have been focused on two important dynamics, namely, the SIR model for epidemic spreading 
and the analysis of coarse-grained information routing models. The main objective of these two studies is to analyze the efficiency of different routing and immunization algorithms depending on the substrate topology.

For the studies performed on epidemic spreading the main results concern the development of a new immunization method based on the $d$-covering problem. We have implemented an heuristic method for finding the nearly smallest subset of network's elements that should be covered so that every node in the network has at least one covered node at a distance less than or equal to $d$. The results show that, depending on the degree-degree correlations of the network, the obtained solution is very different. Besides we have shown that the obtained solution of the $d$-covering problem, when thought of as immune nodes to a SIR epidemics, yields a very efficient algorithm compared to those already existing in the literature. The efficiency of the $d$-covering subset of immune nodes also depends strongly on the correlations of the network when a SIR-like epidemics is studied.

The study of information routing dynamics also yields interesting results. In particular, the main result concerns the study of a congestion-aware strategy for the routing of information packets across the network. The use of shortestpath strategies in scale-free networks lead to a fast congestion of highly connected nodes and hence to the development of jamming for low levels of injected information. We have obtained a more robust routing policy making use of an effective distance that takes into account the congestion level of the network at the local scale. However, the shift of the onset of jamming is achieved at the expenses of a sudden growth of the congestion levels at the jammed phase. We have explained the microscopic origins of this first order like phase transition as a consequence of the effective fragmentation of the network. This fragmentation is due to the formation of dynamic walls composed of those nodes that do not allow to receive data packets from their neighbors, due to their high level of local congestion.

We have seen in this second part several examples on the relation of topological characteristics like the clustering coefficient, the average path length and the degree-degree correlations on the development of two simple model dynamics of importance for scale-free networks. Besides, the modeled algorithms for immunization and data routing have been designed for a nearly optimal deployment when applied on top of highly heterogeneous networks. The nature of the simple dynamics studied here and their application to human-made real systems allows our models to be reliable in these kind of networks. This is not anymore the case of real biological networks where both topology and (nonlinear) dynamics are imposed to the system. This extreme has been analyzed in the third part of the Thesis. 
The third part of this thesis is devoted to the study of nonlinear dynamics on top of complex networks. It is thought of as the confluence of the above two parts because it applies the tools obtained from the studies on nonlinear localization in homogeneous lattices and the analysis of complex networks structure. Along with the results obtained in this part, the mixed use of these tools constitutes a, somewhat, novel feature since the study of nonlinear complex networks is still in its infancy.

We have studied two different nonlinear systems: a Michaelis-Menten regulatory dynamics (where activatory and inhibitory terms compete) and the paradigmatic Kuramoto model of coupled phase oscillators. In these two problems, related to diverse natural systems, the main purpose is to unveil the relation between the networked structure of the systems and the function they fulfill. The search for this "Structure-Function" connection is based on the assumption that the evolution of the real networks is the result of a kind of optimization for the performance of their function. Then, a first step is to analyze coarse-grained synthetic systems modeled by relevant nonlinear dynamics.

The study of activatory-inhibitory regulation, modeled by means of a general Michaelis-Menten dynamics between the network nodes, allows to approach the problem of gene-gene regulation. In this sense, some important results are related to the experimental observations of this kind of systems. The first important result concerns the fragmentation of the network into independent dynamical clusters while the rest of the network remains at the rest (zero activity) state. The dynamics of these dynamical islands show a very rich dynamical behavior: steady, periodic and chaotic states. When these emergent dynamical clusters of self-sustained non-zero activity are considered as networks defined by its nodes and the links among them, new topological features, different from those of the underlying network, are obtained. In this regard, the most important finding is a clustering coefficient for the dynamical islands much higher than that of the substrate network (a Barabási-Albert scale-free network). A second important result is obtained when looking at the observed bifurcations. Periodic clusters display either period doubling or tripling bifurcations on their route to chaos. Analyzing the shape of the Floquet eigenvectors associated to these bifurcations it is possible to determine which nodes are responsible for the transition from the old (period 1) attractor to the new (period 2 or 3 ) one. This method allows us to observe that, differently from other processes on networks, nodes' substructures and not single nodes are responsible for the evolution of the dynamical clusters.

The second objective of this third part is the study of the synchronization paths in networks of Kuramoto phase oscillators. This study is performed on a variety of networked substrates, namely, the two networks families introduced 
in the first part of the Thesis and structured networks. For this purpose, we have introduced a new order parameter that allows to unveil the local patterns of the synchronized clusters that emerge as the coupling strength is increased. In this sense, the main result is obtained when comparing the synchronization paths in homogeneous and heterogeneous networks. The results point out that the emergence of the giant synchronized cluster for Erdös-Rényi networks is the result of the coalescence of multiple small synchronized clusters. This simultaneous clusters' collapse is thus produced in a narrow region for the coupling parameter so that the degree of global synchronization is rapidly increased from zero near the synchronization onset. On the other hand, for scale-free networks the process is described by a gradual growth of the giant synchronization cluster. This synchronization cluster, organized around the central hubs of the networks, grows by incorporating more and more synchronized nodes as the coupling is increased. As a result, the onset of global synchronization occurs earlier (in terms of the coupling strength) than in the Erdös-Rényi case. However, the global coherence in scale-free networks grows, far from the synchronization onset, at a much slower rate than in the case of Erdös-Rényi graphs due to the (one-by-one) additive growth for the synchronized cluster.

These two works constitute interesting examples on the interplay between Function and Structure. In the first study it is clear that nonlinear dynamics shows up an emergent structure with new topological characteristics. For the second work it is shown that, depending on the underlying structure, radically different patterns of synchronization are obtained. Therefore, the importance of tackling the combined study of both structural complexity and nonlinear dynamics is clear, since a separate analysis would be incomplete. The mutual influence observed thus prevent from going from one to the other or vice versa. The continuation of the presented work would be carried following different directions. Perhaps, the most ambitious direction is to make one step further into the analysis of real networks. For example, the availability of gene expression data (although one must be careful and selective with the large amount of experimental data sets) motivates the study of real gene regulatory networks in order to apply the tools and results found in this part. At the same time, other kind of relevant nonlinear dynamics, like models of neural activity (Hopfield, integrate and fire, etc...), could be also studied by means of similar techniques in order to obtain more examples on the interplay of structure and dynamics.

The results presented in this Thesis are intended to analyze and understand several phenomena displayed when two essential ingredients of complexity are present (both separated and combined). It would take still a long time before the understanding of simple dynamics and models allows to go one step farther and attack the unification of these two ingredients in order to have a framework within which one can solve the "Structure-Function" problem. In this sense, the 
present work provides some examples and tools about how the problem could be first tackled. As all research work that does not suffice to fully unravel the features of a given problem, our work also motivates further studies on this interesting question that we intend to pursue in the years to come. 


\section{Appendices}

We want to add two appendices about the computation and stability characterization of periodic orbits since these solutions have extensively appeared throughout this Thesis. Although for particular types of periodic solutions and dynamical systems, the results reported here can be further extended we have tried to briefly summarize the essential features about these two issues.

\section{A Computation of Periodic Orbits}

The computation of periodic solutions to a set of $N$ coupled nonlinear differential equations

$$
\frac{\partial \vec{x}}{\partial z}=\vec{F}_{\vec{\xi}}[\vec{x}]
$$

where $\vec{x}$ are the variables of the system and $\vec{\xi}$ denote the parameters of the particular equations, can be formulated as a problem of finding the solution of a system of $N$ nonlinear equations, with $N$ variables $x_{i}(i=1, \ldots, N)$ and a set of $m$ parameters $\xi_{i}(i=1, \ldots, m)$, of the form

$$
\vec{G}(\vec{x} ; \vec{\xi})=0 .
$$

As we introduced in section 2.2, let us consider a periodic solution $\vec{x}_{\vec{\xi}}$ as a fixed point solution of a $N$-dimensional map $\mathcal{M}$

$$
\mathcal{M} \vec{y}^{n}=\vec{y}^{n+1},
$$

where the map $\mathcal{M}$ can be constructed using the $z$-evolution operator ( $z$ is usually time or space) given by equations (A.1) over a (time or space) period $T$ when we are looking for $z$-periodic solutions

$$
\mathcal{M}=\mathcal{T}_{\vec{\xi}, T}
$$

or a combination of an index (lattice) displacement and a $z$-evolution operators when looking for combined periodicities as in $(p, q)$-resonant states for time and 
lattice displacement, eq. (2.24),

$$
\mathcal{M}=\mathcal{L}^{p} \mathcal{T}_{\vec{\xi}, T}^{q} .
$$

Given the particular definition of the map $\mathcal{M}$, the desired periodic solution will satisfy eq. (A.2) in the form

$$
\vec{G}(\vec{x} ; \vec{\xi})=\mathcal{M} \vec{x}_{\vec{\xi}}-\vec{x}_{\vec{\xi}}=0 .
$$

One is typically interested in a particular solution corresponding to a special choice of the parameters $\vec{\xi}$ but, on the other hand, the only available solution corresponds to a simplified version of the system corresponding to $\vec{\xi}^{0}$. In these cases the solution can be found by means of a homotopy procedure [296]: given a known solution, $\vec{x}_{\vec{\xi}^{0}}$, to some special choice of parameters, $\vec{\xi}^{0}$, the solution, $\vec{x}_{\vec{\xi}}$, is computed via the computation of intermediate solutions to a chain of equations with parameters, $\vec{\xi}^{0} \rightarrow \vec{\xi}^{1} \rightarrow \ldots \rightarrow \vec{\xi}^{n-1} \rightarrow \vec{\xi}^{n}=\vec{\xi}$. The latter path in parameter space is conveniently used so that every intermediate solution can be found. There are several methods used for solving each step in the chain of equations and nearly all of them make use of the solution found for the latter system as the ansatz for the analytical or numerical methods used at each step.

The homotopy strategy is based on the implicit function theorem that assures the existence of an unique solution $\vec{x}_{\vec{\xi}^{n}}$, so that $\vec{G}\left(\vec{x}_{\vec{\xi}^{n}} ; \vec{\xi}^{n}\right)=0$, when there exist a solution $\vec{x}_{\vec{\xi}^{n-1}}\left(\vec{G}\left(\vec{x}_{\vec{\xi}^{n-1}} ; \vec{\xi}^{n-1}\right)=0\right)$ and $\vec{\xi}^{n}$ belongs to an open set centered at $\vec{\xi}^{n-1}$. The conditions that must be fulfilled are:

(i) $\vec{G}(\vec{x} ; \vec{\xi})$ is continuous on an open set centered at $\left(\vec{x}_{\vec{\xi}^{n-1}}, \vec{\xi}^{n-1}\right)$.

(ii) The Jacobian determinant of $\vec{G}(\vec{x} ; \vec{\xi})$ evaluated at $\left(\vec{x}_{\vec{\xi}}^{n-1}, \vec{\xi}^{n-1}\right)$ is nonnull,

$$
\operatorname{Det}\left\{\left[D_{\vec{x}} G\left(\vec{x}_{\vec{\xi}^{n-1}} ; \vec{\xi}^{n-1}\right)\right]_{i j}\right\}=\operatorname{Det}\left[\left.\frac{\partial G_{i}(\vec{x} ; \vec{\xi})}{\partial x_{j}}\right|_{\left(\vec{x}_{\vec{\xi}^{n-1}}, \vec{\xi}^{n-1}\right)}\right] \neq 0 .
$$

In order to satisfy the local convergence conditions of the theorem, the homotopic computation must be carried by dividing the path toward the desired solution into as much intermediate steps as necessary. In this way, the solution for $\vec{x}_{\vec{\xi}^{n}}$ would not differ very much to that for $\vec{x}_{\vec{\xi}^{n-1}}$ so that expressing the new solution as $\vec{x}_{\vec{\xi}^{n}}=\vec{x}_{\vec{\xi}^{n-1}}+\vec{\Delta}$ one would write

$$
\vec{G}\left(\vec{x}_{\vec{\xi}^{n-1}}+\vec{\Delta} ; \vec{\xi}^{n}\right)=0=\vec{G}\left(\vec{x}_{\vec{\xi}^{n-1}} ; \vec{\xi}^{n}\right)+D_{\vec{x}} G\left(\vec{x}_{\vec{\xi}^{n-1}} ; \vec{\xi}^{n}\right) \vec{\Delta}+\ldots
$$




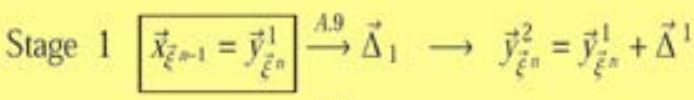

Stage $2 \quad \vec{y}_{\vec{k} n}^{2} \stackrel{A .9}{\rightarrow} \vec{\Delta}_{2} \rightarrow \vec{y}_{\dot{z}^{n}}^{3}=\vec{y}_{\dot{\varepsilon}^{n}}^{2}+\vec{\Delta}^{2}$

Stage $m \quad \vec{y}_{\vec{\xi}^{n}}^{m} \stackrel{A .9}{\rightarrow} \vec{\Delta}_{m} \rightarrow \vec{y}_{\vec{\xi}^{n}}^{m+1}=\vec{y}_{\vec{\xi}^{n}}^{m}+\vec{\Delta}^{m}$

Stop when $\left|\vec{G}\left(\vec{y}_{\vec{\xi}^{n}}^{i}, \vec{\xi}^{n}\right)\right|<\epsilon \rightarrow \vec{x}_{\vec{\xi}^{n}}=\vec{y}_{\vec{\xi}^{n}}^{i}$.

Figure A.1: Schematic representation of the iterative process of homotopic continuation.

neglecting those higher order terms in $\vec{\Delta}$. From the above expression one can obtain the difference $\vec{\Delta}$ between the old and the desired solution by just computing the inverse of the Jacobian matrix $D_{\vec{x}} G$

$$
\vec{\Delta}=-\left[D_{\vec{x}} G\left(\vec{x}_{\vec{\xi}^{n-1}} ; \vec{\xi}^{n}\right)\right]^{-1} \cdot \vec{G}\left(\vec{x}_{\vec{\xi}^{n-1}} ; \vec{\xi}^{n}\right) .
$$

Due to the error at the truncation in Taylor expansion (A.8), one must iterate this procedure until the desired convergence (bounded by machine precision) is reached, $\left|\vec{G}\left(\vec{x}_{\vec{\xi}^{n}} ; \vec{\xi}^{n}\right)\right|<\epsilon$. For this purpose, one uses as the new trial solution the one obtained by the last computation of $\vec{\Delta}$. Calling $\vec{y}_{\xi}^{i}$ the trial function used at the $i^{\text {th }}$ stage of the iterative computation of solution $\vec{x}_{\xi^{n}}$ and $\vec{\Delta}^{i}$ the obtained solution of eq. (A.9) at this stage, a schematic picture of the whole process for computing $\vec{x}_{\vec{\xi}^{n}}$ from the initial ansatz $\vec{x}_{\vec{\xi}^{n-1}}$ (the solution of the before equation in the homotopy chain) is reflected in figure A.1. The convenience of using the above iterative process relies on its quadratic convergence but, on the other hand, one must posses a good ansatz for the initial trial function (since no global convergence is assured) and hence a homotopic continuation to the desired solution is required.

For the particular situation when one is interested in the computation of purely $z$-periodic solutions (such as discrete breathers for the case of time periodic solutions), the equation to solve would be written as

$$
G\left(\vec{x}_{\vec{\xi}}\left(z_{0}\right) ; \vec{\xi}\right)=\mathcal{T}_{\vec{\xi}, T}\left(\vec{x}_{\vec{\xi}}\left(z_{0}\right)\right)-\vec{x}_{\vec{\xi}}\left(z_{0}\right)=0,
$$


where $z_{0}$ stands for the $z$-origin of integration. Therefore, eq. A.9 will take the form

$$
\vec{\Delta}=-\left\{\left[D_{\vec{x}} \mathcal{T}_{\vec{\xi}^{n}, T}\left(\vec{x}_{\vec{\xi}^{n-1}}\left(z_{0}\right)\right)\right]-\mathcal{I}\right\}^{-1} \cdot G\left(\vec{x}_{\vec{\xi}^{n-1}}\left(z_{0}\right) ; \vec{\xi}^{n}\right),
$$

where the matrix $D_{\vec{x}} \mathcal{T}_{\vec{\xi}, h}\left(\vec{x}\left(z_{0}\right)\right)$ is computed integrating from $z_{0}$ to $z_{0}+h$ the equations that are obtained by deriving eq. (A.1) respect to the initial conditions, $\vec{x}\left(z_{0}\right)$,

$$
\begin{aligned}
{\left[\frac{\partial D_{\vec{x}} \mathcal{T}_{\vec{\xi}, z}\left(\vec{x}\left(z_{0}\right)\right)}{\partial z}\right]_{i j}=} & \frac{\partial^{2} x_{i}(z)}{\partial z \partial x_{j}\left(z_{0}\right)}=\sum_{k=1}^{N} \frac{\partial\left[F_{\vec{\xi}}(\vec{x}(z))\right]_{i}}{\partial x_{k}(z)} \frac{\partial x_{k}(z)}{\partial x_{j}\left(z_{0}\right)}= \\
& =\sum_{k=1}^{N} \mathcal{A}_{i k} \frac{\partial x_{k}}{\partial x_{j}\left(z_{0}\right)}=\left[\mathcal{A} \cdot D_{\vec{x}} \mathcal{T}_{\vec{\xi}, z}\left(\vec{x}\left(z_{0}\right)\right)\right]_{i j}
\end{aligned}
$$

with the initial condition $D_{\vec{x}} \mathcal{T}_{\vec{\xi}, z_{0}}\left(\vec{x}\left(z_{0}\right)\right)=\mathcal{I}$, i.e. integrating the linearized equations around the solution, $\vec{x}_{\vec{\xi}}(z)$.

The matrix $D_{\vec{x}} \mathcal{T}_{\vec{\xi}, h}\left(\vec{x}_{\vec{\xi}}\left(z_{0}\right)\right)$ provides a map between an initial perturbation of the solution, $\overrightarrow{\delta x_{\vec{\xi}}}\left(z_{0}\right)$, and its evolution up to $z_{0}+h$,

$$
\overrightarrow{\delta x}_{\vec{\xi}}\left(z_{0}+h\right)=\left[D_{\vec{x}} \mathcal{T}_{\vec{\xi}, h}\left(\vec{x}_{\vec{\xi}}\left(z_{0}\right)\right)\right] \cdot \overrightarrow{\delta x}_{\vec{\xi}}\left(z_{0}\right) .
$$

For a $z$-periodic solution the elements of matrix $\mathcal{A}$ in eq. (A.12) are $z$-periodic functions with the same period $T$ and hence

$$
D_{\vec{x}} \mathcal{T}_{\vec{\xi}, q T}\left(\vec{x}_{\vec{\xi}}\left(z_{0}\right)\right)=\left[D_{\vec{x}} \mathcal{T}_{\vec{\xi}, T}\left(\vec{x}_{\vec{\xi}}\left(z_{0}\right)\right)\right]^{q},
$$

with $q$ integer. This implies that it is enough to integrate the evolution of the linearized equations over a period $T$ and obtain $D_{\vec{x}} \mathcal{T}_{\vec{\xi}, T}\left(\vec{x}_{\vec{\xi}}\left(z_{0}\right)\right)$ for characterizing the time evolution of the perturbations after an integer number of periods. For a time periodic solution the so-called Floquet matrix, $\mathcal{F}=D_{\vec{x}} \mathcal{T}_{\vec{\xi}, T}\left(\vec{x}_{\vec{\xi}}\left(t_{0}\right)\right)$, contains all the information about the linear stability of a periodic solution (we will discuss it later in Appendix B).

As the implicit function theorem states, the key point for being able to compute the solutions to eq. (A.10), is that the Jacobian is invertible so that eq. (A.11) can be solved. In the particular case of time periodic solutions this implies that the eigenvalues of the Floquet matrix, $\mathcal{F}$, must be different from +1 . In other words, if the spectra of the Floquet matrix contains perturbations that are also time periodic with the same period of the solution, $T$, their associated eigenvalue will be +1 and therefore we could not solve eq. (A.11). The existence of such perturbations causes the degeneracy of the linear problem 
since given any solution of period $T$ one can construct another one by adding any combination of such perturbations and therefore the solution is not unique.

Although the existence of degeneracies depends on the particular dynamical system, when we are dealing with autonomous dynamical systems, such as eq. (A.1), there always exist one Floquet eigenvalue +1 . The associated eigenvector is related with the time translation invariance of the solutions, $\overrightarrow{\delta x}_{\vec{\xi}}(t)=\dot{\vec{x}}_{\vec{\xi}}(t)$, and acts translating the instant solution across its path in the phase space. However, the non invertibility of the Jacobian does not prevent us from implementing the continuation scheme. The common way used to solve this problem is to restrict the rank of the Jacobian matrix to a subspace orthogonal to its kernel. This restriction does not influence the efficency of the method since the kernel vectors correspond to directions in tangent space that convert the solution into itself.

There exist several ways for restricting the Jacobian rank that depend on the particular properties of the periodic solution. For example, in the case of time-reversible orbits, i.e. those which are invariant under the transformation $\mathcal{R}(\vec{q}, \vec{p}, t) \rightarrow(\vec{q},-\vec{p},-t)$ (where $\vec{q}$ and $\vec{p}$ denote the two sets of canonically conjugated variables of the system), one can fix the time origin without loss of generality setting $\vec{p}=0$ [81]. With this restriction we prevent from perturbations inside the same periodic manifold and, as a plus, we have reduced the degrees of freedom to the half, $N / 2$. However, this tricky method does not allow to compute other kind of periodical orbits which are not time-reversible (as mobile discrete breathers) and the use of other methods such as the Singular Value Decomposition $[82,83]$ is required.

\section{B Linear Stability of Periodic Orbits}

Before analysing the stability analysis for periodic solutions let us briefly focus on the stability characterization of general orbits of dynamical systems. The linear system of equations defined in (A.12) defines the most general tool for characterizing the stability of dynamical systems solutions: the Lyapunov exponents which are the eigenvalues, $\left\{\mu_{j}\right\}(j=1, \ldots, N)$, of matrix $\mathcal{A}$ that define the system of linear differential equations for the evolution of linear perturbations. In particular, the general definition of Lyapunov exponents can be expressed in terms of the eigenvalues of matrix $D_{\vec{x}} \mathcal{T}_{\vec{\xi}, t}\left(\vec{x}_{\vec{\xi}}\left(t_{0}\right)\right.$ ) (which for period $T$ solutions and $t=T$ is the Floquet operator), $\left\{\lambda_{j}(t)\right\}$, as

$$
\mu_{j}=\lim _{t \rightarrow \infty} \frac{1}{t} \ln \left|\lambda_{j}(t)\right|
$$




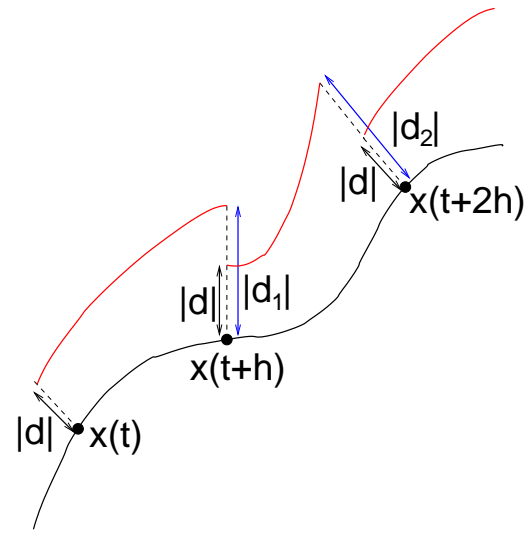

Figure B.1: Schematic representation of the rescaling procedure for the computation of the largest Lyapunov exponent $\mu_{1}$.

Although there are several techniques for computing Lyapunov exponents, it is somehow a hard task since it implies large integration times in order to get accurate values independent of the time origin choice. However, one is most of the times interested in the value of the largest Lyapunov exponent (say $\left.\mu_{1}\right)$ which is the easiest to calculate due to the tendency of any perturbation to grow towards the direction associated to the largest Lyapunov exponent (see $[296,333])$. The largest Lyapunov exponent, $\mu_{1}$, tell us whether the solution repeals nearby orbits (perturbations), $\mu_{1}>0$, and thus the solution is regarded as chaotic. In the case of stable periodic orbits of autonomous dynamical systems the maximum Lyapunov exponent is always 0 , this corresponds to the Floquet eigenvalue +1 associated to the time translational invariance. The largest Lyapunov exponent can be expressed as

$$
\mu_{1}=\lim _{t \rightarrow \infty} \frac{1}{t} \ln \left[\frac{\left|D_{\vec{x}} \mathcal{T}_{\vec{\xi}, t}\left(\vec{x}_{\vec{\xi}}\left(t_{0}\right)\right) \overrightarrow{\delta x}_{\vec{\xi}}\left(t_{0}\right)\right|}{\left|\overrightarrow{\delta x_{\vec{\xi}}}\left(t_{0}\right)\right|}\right] .
$$

This expression, which makes uses of the ratio of separation of an initial perturbation $\overrightarrow{\delta x}_{\vec{\xi}}\left(t_{0}\right)$ after large times, turns out to be helpful for computing $\mu_{1}$. The computational method consists in making a perturbation of the solution with a tangent vector of arbitrary direction and modulus $|d|$, and follow the evolution of the perturbed orbit for a time interval, $h$. Then, the distance, $\left|d_{1}\right|$ between the original and the perturbed orbits is measured. At the same time $h$, the perturbed solution is varied by preserving its direction but being rescaled to $|d|$. This process is iterated for a number of times (see figure B.1) so that a set of distances $\left\{\left|d_{i}\right|\right\}$ with $(i=1, \ldots, n)$ is collected. Finally, averaging these measures one obtains the largest Lyapunov exponent

$$
\mu_{1}=\frac{1}{n h} \sum_{i=1}^{n} \ln \frac{\left|d_{i}\right|}{|d|}
$$


As stated above, this method turns out to be helpful since the contribution of the largest Lyapunov exponent to the dynamics of the perturbed orbits dominates for large times.

Turning our attention to periodic orbits it is then convenient to look at the properties of the Floquet matrix. An unstable periodic orbit is manifested by the presence of Floquet eigenvalues with $\left|\lambda_{i}\right|>1$, which implies that the modulus of the perturbations of the fixed point solution of map (A.4) (the periodic orbit) along the eigenvector associated to this eigenvalue will grow at a rate $\left|\lambda_{i}\right|$.

Since the Floquet matrix, $\mathcal{F}$, is real the Floquet eigenvalues come in pairs $\left(\lambda_{i}, \overline{\lambda_{i}}\right)$. For Hamiltonian systems, one can say a little bit more about the structure of the Floquet spectra. In particular, when the nonlinear evolution is Hamiltonian, so that we have an even set of dynamical variables, $N=2 n$ $\left(\vec{x}=(\vec{q}, \vec{p})^{t}\right)$, and

$$
\dot{\vec{x}}=(\dot{\vec{q}}, \dot{\vec{p}})^{t}=\left(\begin{array}{cc}
0 & \mathcal{I}_{n} \\
-\mathcal{I}_{n} & 0
\end{array}\right)\left(\frac{\partial \mathcal{H}}{\partial q_{1}}, \ldots, \frac{\partial \mathcal{H}}{\partial q_{n}}, \frac{\partial \mathcal{H}}{\partial p_{1}}, \ldots, \frac{\partial \mathcal{H}}{\partial p_{n}}\right)^{t}=\mathcal{J} \cdot \nabla_{\vec{x}} \mathcal{H}
$$

the map defined by the Floquet operator () is symplectic, i.e. given an orthogonal and skew-symmetric matrix such as $\mathcal{J}$ in ()$, \mathcal{F}$ obeys the relation

$$
\mathcal{F} \mathcal{J F}^{t}=\mathcal{J}
$$

From this property it can be shown that if $\lambda$ is an eigenvalue of $\mathcal{F}$ so is $1 / \lambda$ by proving that the characteristic polynomial, $p(\lambda)$, of $\mathcal{F}$ is reflexive (i.e. the coefficients $a_{i}$ of degree $i$ in $\lambda$ satisfy $a_{i}=a_{N-i}$ with $\left.i=0, \ldots, N\right)$

$$
\begin{aligned}
p(\lambda) & =\operatorname{det}(\mathcal{F}-\lambda \mathcal{I})=\operatorname{det}\left(\mathcal{J F} \mathcal{J}^{t}-\lambda \mathcal{I}\right)=\operatorname{det}\left(\left(\mathcal{F}^{-1}\right)^{t}-\lambda \mathcal{I}\right)= \\
& =\operatorname{det}\left(\mathcal{F}^{-1}-\lambda \mathcal{I}\right)=\operatorname{det}(\mathcal{F}) \operatorname{det}\left(\mathcal{F}^{-1}-\lambda \mathcal{I}\right)=\operatorname{det}(\mathcal{I}-\lambda \mathcal{F})= \\
& =(-\lambda)^{N} \operatorname{det}\left(\mathcal{F}-\lambda^{-1} \mathcal{I}\right)=(\lambda)^{N} p\left(\lambda^{-1}\right) .
\end{aligned}
$$

Therefore, for Hamiltonian dynamical systems the Floquet eigenvalues for a periodic orbit come in quadruplets $(\lambda, \bar{\lambda}, 1 / \lambda, 1 / \bar{\lambda})$. This result implies that the Floquet multipliers of a stable periodic solution will lie on the unit circle of the complex plane so that, these solutions are marginally stable since any perturbed solution will not decay into the original one, i.e. the cycle is not an attractor of surrounding trajectories. Besides, this result implies that in order to a pair (or a single if it is at +1 or -1 ) of Floquet eigenvalues leave the unit circle it is necessary that they (it) collide with another pair.

By computing the periodic solutions for a chain of equations corresponding to different parameters $\vec{\xi}$ one can study how the stability of the periodic solution changes across the continuation path. In this regard, stability changes of 
the periodic solution can occur for certain values $\vec{\xi}_{c}$ manifested by the existence of Floquet eigenvalues leaving the unit circle. There are several mechanisms of loosing stability and one can identify which type of bifurcation occurs (differenced by the new stable solutions that emerge when the original periodic orbit loss its stability) at $\vec{\xi}_{c}$ by looking how the associated Floquet eigenvalue, $\lambda_{j}$, leaves the unit circle. One can distinguish three types of bifurcations corresponding to:

(a) $\lambda_{j}=1$,

(b) $\operatorname{Im}\left(\lambda_{j}\right) \neq 0$,

(c) $\lambda_{j}=-1$.

These three types of bifurcations are schematically plotted in figure B.2. In the first one (figure B.2.a) a Floquet eigenvalue leaves the unit circle at +1 and therefore the perturbation responsible for this instability, $\overrightarrow{\delta x}_{\vec{\xi}_{c}}^{*}(t)$, is time periodic with the same period as the original solution. It is thus an harmonic bifurcation or also called stationary bifurcation since the new solution, $\vec{x}_{\vec{\xi}_{c}}(t)+\overrightarrow{\delta x_{\xi_{c}}} \vec{*}^{*}$ ), is also a fixed point of the original map (eq. (A.4)). The most common scenario is depicted in figure B.2.a at bottom, where two new orbits of identical period, $T$, emerge passed $\vec{\xi}_{c}$. For this case a pitchfork (supercritical) bifurcation has occurred. Pitchfork bifurcations are often associated with a symmetry breaking since, after the bifurcation, the system dynamics can occupy either stable periodic orbit. This latter situation was reported in section (3.1.3). Other possible scenarios are saddle-node and transcritical bifurcations. In these two cases there are two solutions, one stable and another unstable, meet at $\vec{\xi}_{c}$, while in the former situation no solutions are available after the bifurcation point, for the latter there is an interchange of stability between the two solutions.

Case (b) (see figure B.2.b) corresponds to an oscillatory or generalized Hopf bifurcation or also a Naimark-Sacker bifurcation. In this case the frequency of the new solution, $\vec{x}_{\vec{\xi}_{c}}(t)+\overrightarrow{\delta x}_{\vec{\xi}_{c}}^{*}(t)$, can be incommensurable with the period of the map if the angle where the Floquet eigenvalue) leaves the unit circle is an irrational multiple of $2 \pi, \theta \neq p 2 \pi / q$ with $p$ an $q$ integers. In this case the quasiperiodic movement of the emergent solution moves over a Torus defined by the frequency of the Map (the frequency of the original solution) $\omega=2 \pi / T$ and the angle where the eigenvalue leaves the unit circle (the frequency of the unstable perturbation) $\omega^{*}=\theta / T$. For the cases where these two frequencies are commensurate the new orbit is periodic (see section 7.2.1).

Finally, case (c) (see figure B.2.c) constitutes the fingerprint of a perioddoubling bifurcation since the angle where the unstable Floquet eigenvalue is 
(a)
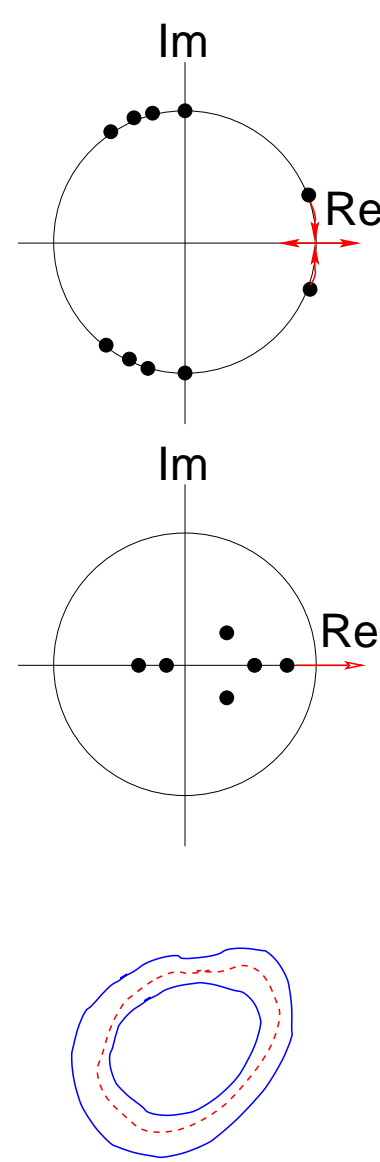

(b)
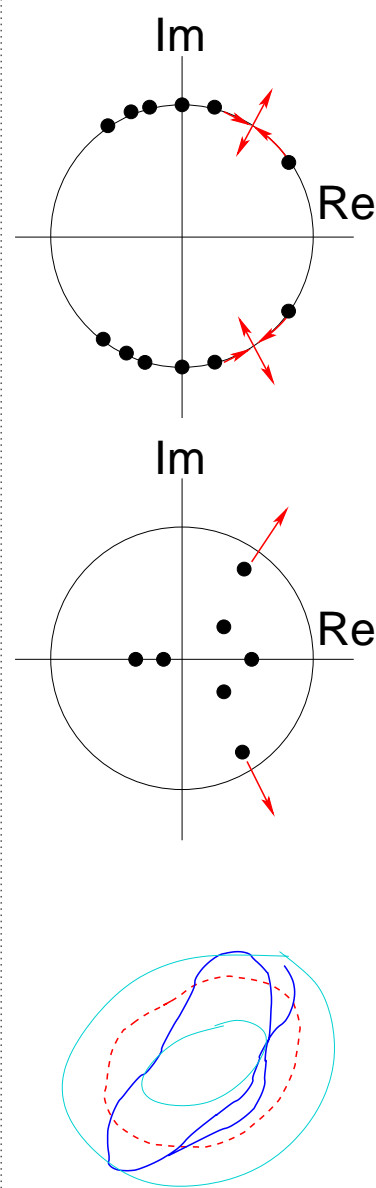

(c)

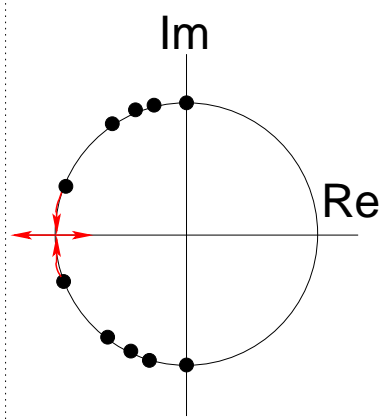

Im
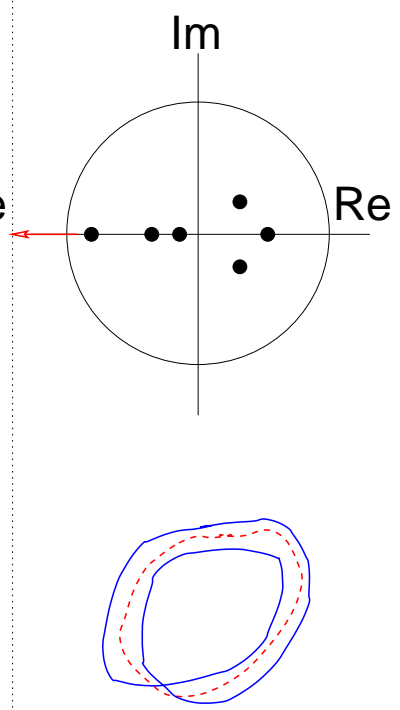

Figure B.2: Schematic plot of the three types of bifurcations and their corresponding Floquet behaviour (the upper plot correspond to a Hamiltonian System and the one below for a non-Hamiltonian one). The lowest plots are the representation of the unstable (dashed and red) original orbit and the stable (solid and blue) emergent solution after the bifurcation.

located at $\theta=\pi$ and, therefore, the period of the unstable eigenvector, $\overrightarrow{\delta x}_{\vec{\xi}_{c}}^{*}(t)$, will be $T^{*}=2 \pi / \omega=2 \pi T / \theta=2 T$. Therefore, the new solution $\vec{x}_{\vec{\xi}_{c}}(t)+\overrightarrow{\delta x_{\xi_{c}}^{*}}(t)$, will be periodic with period $2 T$ (see section 7.2.1).

The Floquet analysis of the periodic solutions is threfore useful for identifying the nature of the bifurcations found. Besides, the inspection of the eigenvectors associated with the Floquet eigenvalues that leave the unit circle help us to investigate how the unstable solution evolves. 



\section{Bibliography}

[1] E. O. Wilson, Consilience (Knopf, (New York, US), 1998).

[2] P. W. Anderson, Science 177, 393 (1972).

[3] K. R. Popper, Objective Knowledge: An evolutionary approach (Clarendon Press, (Oxford, UK), 1972).

[4] L. D. Landau, Phys. Z. Soviet 11, 26 (1937).

[5] L. D. Landau, Phys. Z. Soviet 11, 545 (1937).

[6] L. Neel, Ann. Phys. 17, 1932 (1933).

[7] L. D. Landau, Phys. Z. Soviet 4, 675 (1933).

[8] O. Penrose, Philos. Mag. 42, 1373 (1951).

[9] C. N. Yang, Rev. Mod. Phys. 34, 694 (1962).

[10] J. Bardeen and L. N. Cooper and J. R. Schrieffer, Phys. Rev. 108, 1175 (1957).

[11] C. S. Gardner, J. M. Greene, M. D. Kruskal, and R. M. Miura, Phys. Rev. Lett. 19, 1095 (1967).

[12] E. Fermi, J. R. Pasta, S. M. Ulam, and M. Tsingou, Los Alamos Report LA-1940 (1955).

[13] P. Bak, C. Tang, and K. Wiesenfeld, Phys. Rev. Lett. 59, 381 (1987).

[14] P. Bak, C. Tang, and K. Wiesenfeld, Phys. Rev. A 38, 364 (1988).

[15] N. Eldredge and S. J. Gould in (T. J. M. Shopf ed.), Models in Paleobiology (Freeman, Cooper and co., (San Francisco, USA), 1972).

[16] P. W. Anderson, Physics Today July, 9 (1991).

[17] P. W. Anderson, Phys. Rev. 109, 1492 (1958). 
[18] A. J. Sievers and S. Takeno, Phys. Rev. Lett. 61, 970 (1988).

[19] S. Takeno, K. Kisoda, and A. J. Sievers, Prog. Theor. Phys. 94, 242 (1988).

[20] D. K. Campbell, S. Flach, and Y. S. Kivshar, Physics Today 57, 43 (2004).

[21] N. Zabusky and M. Kruskal, Phys. Rev. Lett. 15, 240 (1965).

[22] R. S. Mackay and S. Aubry, Nonlinearity 7, 1623 (1994).

[23] A. Scott, Nonlinear Science. Emergence and Dynamics of Coherent Structures (Oxford Univeristy Press, Oxford, 1999).

[24] M. Peyrard and A. R. Bishop, Phys. Rev. Lett. 62, 2755 (1989).

[25] M. Peyrard, Nonlinearity 17, R1 (2004).

[26] S. F. Mingaleev, Y. B. Gaididei, P. L. Christiansen, and Y. S. Kivshar, Europhys. Lett. 59, 403 (2002).

[27] A. R. McGurn, Chaos 13, 754 (2003).

[28] Y. S. Kivshar and G. P. Agrawal, Optical Solitons: From Fibers to Photonic Crystals (Academic, San Diego, 2003).

[29] D. N. Christodoulides and R. I. Joseph, Opt. Lett. 13, 794 (1998).

[30] A. J. Legget, Rev. Mod. Phys. 73, 307 (2001).

[31] E. Trías, J. J. Mazo, and T. Orlando, Phys. Rev. Lett. 84, 741 (2000).

[32] P. Binder, D. Abraimov, A. V. Ustinov, S. Flach, and Y. Zolotaryuk, PRL 84, 745 (2000).

[33] F. S. Cataliotti, S. Burger, C. Fort, P. Maddaloni, F. Minardi, A. Trombettoni, A. Smerzi, and M. Inguscio, Science 293, 843 (2001).

[34] K. E. Strecker, G. B. Partridge, A. G. Truscott, and R. G. Hulet, Nature 417, 150 (2002).

[35] A. Smerzi, A. Trombettoni, P. G. Kevrekidis, and A. R. Bishop, Phys. Rev. Lett. 89, 170402 (2002).

[36] M. Sato, B. E. Hubbard, A. J. Sievers, B. Ilic, D. A. Czaplewski, and H. G. Craighead, Phys. Rev. Lett. 90, 044102 (2003).

[37] M. Sato, B. E. Hubbard, A. J. Sievers, B. Ilic, and H. G. Craighead, Europhys. Lett. 66, 318 (2004). 
[38] M. Sato, B. E. Hubbard, and A. J. Sievers, Rev. Mod. Phys. 78, 137 (2006).

[39] S. Aubry, Physica D 103, 201 (1997).

[40] J. A. Sepulchre and R. S. MacKay, Nonlinearity 10, 679 (1997).

[41] S. Flach and C. R. Willis, Phys. Rep. 295, 181 (1998).

[42] Y. Kivshar and S. Flach (eds.), Chaos 13, 586 (2003).

[43] S. Aubry, Physica D 216, 1 (2006).

[44] J. Scott Russel, 14th Meeting of the British Association for the Advancement of Science p. 311 (1844).

[45] S. A. Kisilev and V. I. Rupasov, Phys. Lett. A 148, 355 (1990).

[46] V. M. Burlakov, S. A. Kisilev, and V. N. Pyrkov, Phys. Rev. B 42, 4921 (1990).

[47] M. Peyrard, T. Dauxois, and C. R. Willis, Physica D 57, 267 (1992).

[48] T. Dauxois and M. Peyrard, Phys. Rev. Lett. 70, 3935 (1993).

[49] S. Aubry in (C. Godréche ed.), Structures et Instabilitiés (Les Ullis, Editions de Physique, France, 1985).

[50] S. Aubry, Physica D 71, 196 (1994).

[51] K. O. Rasmussen, S. Aubry, A. R. Bishop, and G. P. Tsironis, Eur. Phys. J. B 15, 169 (2000).

[52] D. Chen, S. Aubry, and G. P. Tsironis, Phys. Rev. Lett. 77, 4776 (1996).

[53] S. Aubry and T. Cretegny, Physica D 119, 34 (1998).

[54] J. Cuevas and F. Palmero and J. F. R. Archilla and F. R. Romero, Phys. Lett. A 299, 221 (2002).

[55] M. Oster and M. Johansson and A. Eriksson, Phys. Rev. E 67, 056606 (2003).

[56] A. A. Vakhnenko and Y. B. Gaididei, Theor. Math. Phys. 68, 873 (1987).

[57] C. Claude, Y. S. Kivshar, O. Kluth, and K. H. Spatschek, Phys. Rev. B 47, 14228 (1993).

[58] D. Cai and A. R. Bishop and N. Gronbech-Jensen, Phys. Rev. Lett. 72, 591 (1994). 
[59] D. Cai and A. R. Bishop and N. Gronbech-Jensen, Phys. Rev. E 53, 4131 (1996).

[60] R. S. Mackay and J. A. Sepulchre, J. Phys. A 35, 3985 (2002).

[61] M. Salerno, Phys. Rev. A 46, 6856 (1992).

[62] R. K. Bullough and P. J. Caudrey (eds), Solitons (Springer, (Berlin, Ge), 1980).

[63] G. Eilemberger, Solitons (Springer, (Berlin, Ge), 1981).

[64] V. E. Zakharov and A. B. Shabat, Sov. Phys. JETP 34, 62 (1972).

[65] A. Smerzi, S. Fantoni, S. Giovanazzi, and S. R. Shenoy, Phys. Rev. Lett. 79, 4950 (1997).

[66] F. Dalfovo, S. Giorgini, L. P. Pitaevskii, and S. Stringari, Rev. Mod. Phys. 71, 463 (1999).

[67] J. Denschlag, J. E. Simsarian, D. L. Feder, Charles W. Clark, L. A. Collins, J. Cubizolles, L. Deng, E. W. Hagley, K. Helmerson, W. P. Reinhardt, et al., Science 287, 97 (2000).

[68] A. Trombettoni and A. Smerzi, Phys. Rev. Lett. 86, 2353 (2001).

[69] H. S. Eisenberg, Y. Silberberg, R. Morandotti, A. R. Boyd, and J. S. Aitchison, Phys. Rev. Lett. 81, 3383 (1998).

[70] R. Morandotti, U. Peschel, J. S. Aitchison, H. S. Eisenberg, and Y. Silberberg, Phys. Rev. Lett. 83, 2726 (1999).

[71] R. Morandotti, H. S. Eisenberg, Y. Silberberg, M. Sorel, and J. S. Aitchison, Phys. Rev. Lett. 86, 3296 (2001).

[72] J. W. Fleisher, M. Segev, N. K. Efremidis, and D. N. Christodoulides, Nature 422, 147 (2002).

[73] M. Ablowitz and J. Ladik, Stud. Appl. Math. 55, 213 (1976).

[74] M. Ablowitz and J. Ladik, J. Math. Phys. 17, 1011 (1976).

[75] K. M. Case and M. Kac, J. Math. Phys. 14, 594 (1973).

[76] K. M. Case, J. Math. Phys. 14, 916 (1973).

[77] M. Salerno, Phys. Rev. A 46, 6856 (1992).

[78] Z. D. Li, P. B. He, L. Li, J. Q. Liang, and W. M. Liu, Phys. Rev. A 71, $053611(2005)$. 
[79] T. Cretegny and S. Aubry, Phys. Rev. B 55, R11929 (1997).

[80] S. Flach and K. Kladko, Physica D 127, 61 (1999).

[81] J. L. Marín and S. Aubry, Nonlinearity 9, 1501 (1996).

[82] G. Strang, Linear Algebra (Academic Press, New York, 1980).

[83] H. Press, S. A. Teukolsky, W. T. Vetterling, and B. P. Flannery, Numerical Recipes (Cambridge University Press, New York, 1992).

[84] J. L. Marín, S. Aubry, and L. M. Floría, Physica D 113, 283 (1998).

[85] Y. S. Kivshar and M. Peyrard, Phys. Rev. A 46, 3198 (1992).

[86] Y. S. Kivshar and M. Salerno, Phys. Rev. E 49, 3543 (1994).

[87] M. Johansson, S. Aubry, Y. B. Gaididei, P. L. Christiansen, and K. O. . Rasmussen, Physica D 119, 115 (1998).

[88] P. G. Kevrekidis, K. O. Rasmussen, and A. R. Bishop, Int. Journal of Modern Physics B 15, 2833 (2001).

[89] D. Henning, N. G. Sun, H. Gabriel, and G. P. Tsironis, Phys. Rev. E 52, 255 (1995).

[90] D. Henning and G. P. Tsironis, Phys. Rep. 307, 333 (1999).

[91] J. L. Marín and F. Falo and P. J. Martínez and L. M. Floría, Phys. Rev. E 63, 66603 (2001).

[92] P. J. Martínez and M. Meister and L. M. Floría and F. Falo, Chaos 13, 610 (2003).

[93] D. Zueco, P. J. Martínez, L. M. Floría, and F. Falo, Phys. Rev. E 71, $036613(2005)$.

[94] S. V. Dmitriev, P. G. Kevrekidis, B. A. Malomed, and D. J. Frantzeskakis, Phys. Rev. E 68, 56603 (2003).

[95] D. B. Duncan, J. C. Eilbeck, H. Feddersen, and J. A. D. Wattis, Physica D 68, 1 (1993).

[96] S. Flach, Y. Zolotaryuk, and K. Kladko, Phys. Rev. E 59, 6105 (1999).

[97] J. L. Marín and S. Aubry, Physica D 119, 163 (1998).

[98] K. Kundu, Phys. Rev. E 61, 5839 (2000).

[99] Y. S. Kivshar and D. K. Campbell, Phys. Rev. E 48, 3077 (1993). 
[100] R. Boesch and M. Peyrard, Phys. Rev. B 43, 8491 (1991).

[101] T. Dauxois and M. Peyrard, Physics of Solitons (Cambridge University Press, (Cambridge, UK), 2006).

[102] K. I. Pushkarov, D. I. Pushkarov, and I. V. Tomov, Opt. Quant. Electr. 11, 471 (1979).

[103] M. G. Vakhitov and A. A. Kolokolov, Sov. J. Radiophys. Quant. Electr. 16, 783 (1973).

[104] I. M. Merhasin, B. V. Gisin, R. Driben, and B. A. Malomed, Phys. Rev. E 71, 016613 (2005).

[105] T. Kapitula, P. G. Kevrekidis, and B. A. Malomed, Phys. Rev. E 63, 036604 (2001).

[106] D. E. Pelinovsky, P. G. Kevrekidis, and D. J. Frantzeskakis, Physica D 212, 1 (2005).

[107] M. J. Ablowitz, Z. H. Musslimani, and G. Biondini, Phys. Rev. E 65, 026602 (2002).

[108] H. Feddersen in (M. Remoissenet and M. Peyrard eds.), Nonlinear coherent structures in physics and biology (Springer, (Berlin, Ge), 1991).

[109] Y. Sire and G. James, Physica D 204, 15 (2005).

[110] G. James and Y. Sire, Commun. Math. Phys. 257, 51 (2005).

[111] B. Sánchez-Brey and M. Johansson, Phys. Rev. E 71, 036627 (2005).

[112] T. R. O. Melvin and A. R. Champneys and P. G. Kevrekidis and J. Cuevas, Phys. Rev. Lett. 97, 124101 (2006).

[113] D. Cai and A. R. Bishop and N. Gronbech-Jensen and M. Salerno, Phys. Rev. Lett. 74, 1186 (1995).

[114] D. K. Campbell, Nature 432, 455 (2004).

[115] P. G. Kevrekidis, K. O. Rasmussen, and A. R. Bishop, Phys. Rev. E 61, 2006 (2000).

[116] P. G. Kevrekidis, K. O. Rasmussen, and A. R. Bishop, Phys. Rev. E 61, 4652 (2000).

[117] B. A. Malomed and P. G. Kevrekidis, Phys. Rev. E 64, 026601 (2001).

[118] S. Flach, K. Kladko, and R. S. MacKay, Phys. Rev. Lett. 78, 1207 (1997). 
[119] M. I. Weinstein, Nonlinearity 12, 673 (1999).

[120] M. Kastner, Phys. Rev. Lett. 92, 104301 (2004).

[121] M. Kastner, Nonlinearity 17, 1923 (2004).

[122] G. Kalosakas, K. O. Rasmussen, and A. R. Bishop, Phys. Rev. Lett. 89, $030402(2002)$.

[123] V. K. Mezentsev, S. L. Musher, I. V. Ryzhenkova, and S. K. Turitsyn, JETP Lett. 60, 829 (1994).

[124] E. W. Laedke, K. H. Spatschek, and S. K. Turitsyn, Phys. Rev. Lett. 73, 1055 (1994).

[125] E. W. Laedke, K. H. Spatschek, V. K. Mezentsev, S. L. Musher, I. V. Ryzhenkova, and S. K. Turitsyn, JETP Lett. 62, 677 (1995).

[126] J. J. Rasmussen and K. Rypdal, Phys. Scr. 33, 481 (1986).

[127] P. L. Christiansen, Y. B. Gaididei, and K. O. Rasmussen, Phys. Rev. B 54, 900 (1996).

[128] P. L. Christiansen, Y. B. Gaididei, V. K. Mezentsev, S. L. Musher, K. O. Rasmussen, J. J. Rasmussen, I. V. Ryzhenkova, and S. K. Turitsyn, Phys. Scr. 67, 160 (1996).

[129] N. K. Efremidis, S. Sears, D. N. Christodoulides, J. W. Fleischer, and M. Segev, Phys. Rev. E 66, 046602 (2002).

[130] J. Fleisher, T. Carmon, M. Segev, N. K. Efremidis, and D. N. Christodoulides, PRL 90, 023902 (2003).

[131] J. C. Eilbeck and M. Johansson in (L. Vázquez, R. S. MacKay, and M. P. Zorzano ed.), Proceedings of Localization and Energy Transfer in Nonlinear Systems (World Scientific, Singapore, 2003).

[132] D. Cheskis, S. Bar-Ad, R. Morandotti, J. S. Aitchison, H. S. Eisenberg, Y. Silberberg, and D. Ross, HAIT J. Sci. Eng. 1, 363 (2004).

[133] T. Peschel and F. Lederer in (D. B. Duncan and J. C. Eilbeck ed.), Proceedings of the Confererence on Nonlinear Coherent Structures in Physics and Biology (WWW: http://www.ma.hw.ac.uk/solitons/procs/).

[134] P. G. Kevrekidis, D. J. Frantzeskakis, R. M. Carretero-Gonzalez, B. A. Malomed, and A. R. Bishop, Phys. Rev. E 72, 046613 (2005).

[135] J. C. van der Meer, Nonlinearity 3, 1041 (1990). 
[136] D. N. Christodoulides and E. D. Eugenieva, Phys. Rev. Lett. 87, 233901 (2001).

[137] A. L. Barabási, Nature Physics 1, 68 (2005).

[138] B. Bollobas, Random Graphs (Academic Press, London, 1985).

[139] C. Berge, The Theory of Graphs (Dover, New York, 2001).

[140] P. Erdös and A. Rényi, Pub. Math. Debrecen 6, 290 (1959).

[141] P. Erdös and A. Rényi, Pub. Math. Inst. Hung. Acad. Sci. 5, 17 (1960).

[142] S. Redner, Eur. Phys. J. B 4, 131 (1998).

[143] M. Faloutsos, P. Faloutsos, and C. Faloutsos, Comput. Commun. Rev. 29, 251 (1999).

[144] R. Albert and H. Jeong and A. L. Barabási, Nature 401, 130 (1999).

[145] A. L. Barabási and R. Albert, Science 286, 509 (1999).

[146] H. Jeong, B. Tombor, R. Albert, Z. N. Oltvai, and A. L. Barabási, Nature 407, 651 (2000).

[147] L. A. N. Amaral, A. Scala, M. Barthelemy, and H. Stanley, Proc. Nat. Acad. Sci. 97, 11149 (2000).

[148] M. E. J. Newman, Proc. Nat. Acad. Sci. 98, 404 (2001).

[149] A. Vazquez and R. Pastor-Satorras and A. Vespignani, Phys. Rev. E 65, 066130 (2002).

[150] M. E. J. Newman, S. Forrest, and J. Balthrop, Phys. Rev. E 66, 035101 (2002).

[151] H. Ebel and L. I. Mielsch and S. Bornholdt, Phys. Rev. E 66, 035103 (2002).

[152] P. Sen, S. Dasgupta, A. Chatterjee, P. A. Sreeram, and G. Mukherjee, Phys. Rev. E (2003).

[153] R. Guimerá, L. Danon, A. Diaz-Guilera, F. Giralt, and A. Arenas, Phys. Rev. E 68, 065103 (2003).

[154] R. Guimerá and S. Mossa and A. Turtschi and L. A. N Amaral, Proc. Nat. Acad. Sci. 102, 7794 (2005).

[155] D. J. Watts and S. H. Strogatz, Nature 393, 440 (1998). 
[156] R. Albert and A. L. Barabási, Rev. Mod. Phys. 74, 47 (2002).

[157] S. Bornholdt and H. G. Schuster (eds.), Handbook of Graphs and Networks: From the Genome to the Internet (Wiley-VCH, Berlin, 2002).

[158] S. N. Dorogotsev and J. F. F. Mendes, Adv. Phys. 51, 1079 (2002).

[159] M. E. J. Newman, SIAM Review 45, 167 (2003).

[160] S. N. Dorogotsev and J. F. F. Mendes, Evolution of Networks (Oxford University Press, Oxford, 2003).

[161] R. Pastor-Satorras and A. Vespignani, Evolution and Structure of the Internet (Cambridge University Press, Cambridge, U.K., 2004).

[162] E. Ben-Naim and H. Frauenfelder and Z. Toroczkai (eds.), Complex Networks (Springer, (Berlin, Ge), 2004).

[163] S. Boccaletti, V. Latora, Y. Moreno, M. Chavez, and D.-U. Hwang, Physics Reports 424, 175 (2006).

[164] Map of peer-2-peer Gnutella network, Provided by Clip2 Distributed Search Solutions (http://www.gnutellahosts.com/).

[165] Autonomous System level representation of the Internet as of April 16th 2001, Provided by the National laboratory for applied networ research (NLANR), National Science Foundation (http://moat.nlanlr.net/).

[166] Router level graph representation of the Internet. Mapping the internet within the scan project, Information Sciences Institute (http://www.isi.edu/div7/scan/).

[167] S. Milgram, Psycol. Today 2, 60 (1967).

[168] P. S. Dodds, R. Muhamad, and D. J. Watts, Science 301, 827 (2003).

[169] M. E. J. Newman, Phys. Rev. E 64, 016131 (2001).

[170] M. Ripeanu, I. Foster, and A. Iamnitchi, IEEE Internet Computing 6, 50 (2002).

[171] S. Wasserman and K. Faust, Social Networks Analysis (Cambridge University Press, Cambridge, 1994).

[172] F. Radicchi, C. Castellano, F. Cecconi, V. Loreto, and D. Parisi, Proc. Nat. Acad. Sci. 101, 2658 (2004).

[173] S. Shen-Orr, R. Milo, S. Mangan, and U. Alon, Nature Genetics 31, 64 (2002). 
[174] R. Milo, S. Shen-Orr, S. Itzkovitz, N. Kashan, D. Chklovskii, and U. Alon, Science 298, 824 (2002).

[175] S. Mangan and U. Alon, Proc. Nat. Acad. Sci. 100, 11980 (2003).

[176] R. Milo, S. Itzkovitz, N. Kashtan, R. Levitt, S. Shen-Orr, I. Ayzenshtat, M. Sheffer, and U. Alon, Science 303, 1538 (2004).

[177] N. Kashtan, S. Itzkovitz, R. Milo, and U. Alon, Bioinformatics 20, 1746 (2004).

[178] J. J. Binney, N. J. Dowrick, A. J. Fisher, and M. E. J. Newman, The Theory of Critical Phenomena. An Introduction to the Renormalization Group (Clarendon Press, Oxford, UK, 1992).

[179] M. E. J. Newman and D. J. Watts, Phys. Lett. A 263, 341 (1999).

[180] M. Barthelemy and L. A. N. Amaral, Phys. Rev. Lett. 82, 3180 (1999).

[181] A. Barrat and M. Weigt, Eur. Phys. J. B 13, 547 (2000).

[182] M. E. J. Newman, C. Moore, and D. J. Watts, Phys. Rev. Lett. 84, 3201 (2000).

[183] M. E. J. Newman and D. J. Watts, Phys. Rev. E 60, 7332 (1999).

[184] P. L. Krapivsky, S. Redner, and F. Leyvraz, Phys. Rev. Lett. 85, 4629 (2000).

[185] P. L. Krapivsky, G. J. Rodgers, and S. Redner, Phys. Rev. Lett. 86, 5401 (2001).

[186] S. N. Dorogotsev, J. F. F. Mendes, and A. N. Samukhin, Phys. Rev. Lett. 85, 4633 (2000).

[187] A. L. Barabási and R. Albert, Physica A 272, 173 (1999).

[188] B. Bollobás and O. Riordan, Preprint. Department of Mathematical Sciences, University of Memphis (2002).

[189] R. Albert and A. L. Barabási, Phys. Rev. Lett. 85, 5234 (2000).

[190] G. Bianconi and A. L. Barabási, Europhys. Lett. 54, 436 (2001).

[191] G. Bianconi and A. L. Barabási, Phys. Rev. Lett. 86, 5632 (2001).

[192] E. M. Jin, M. Girvan, and M. E. J. Newman, Phys. Rev. E 64, 046132 (2001). 
[193] P. Holme and B. J. Kim, Phys. Rev. E 65, 026107 (2002).

[194] K. Klemm and V. M. Eguíluz, Phys. Rev. E 65, 036123 (2002).

[195] S. N. Dorogovtsev, A. V. Goltsev, and J. F. F. Mendes, Phys. Rev. E 65, $06122(2002)$.

[196] G. Szabó, M. Alava, and J. Kertész, Phys. Rev. E 67, 056102 (2003).

[197] E. Ravasz and A. L. Barabási, Phys. Rev. E 67, 026112 (2003).

[198] A. Vázquez, M. Boguña, Y. Moreno, R. Pastor-Satorras, and A. Vespignani, Phys. Rev. E 67, 046111 (2003).

[199] M. A. Serrano, M. Boguña, and A. Díaz-Guilera, Phys. Rev. Lett. 94, 038701 (2005).

[200] S. Mossa, M. Barthelemy, H. E. Stanley, and L. A. N. Amaral, Phys. Rev. Lett. 88, 138701 (2002).

[201] P. L. Krapivsky and S. Redner, Phys. Rev. E 63, 066123 (2001).

[202] G. Caldarelli, A. Capocci, P. D. L. Ríos, and M. A. Muñoz, Phys. Rev. Lett. 89, 258702 (2002).

[203] Z. Liu, Y. C. Lai, N. Ye, and P. Dasgupta, Phys. Lett. A 303, 337 (2002).

[204] E. F. Keller, BioEssays 27, 1060 (2005).

[205] G. Grinstein and R. Linsker, Proc. Nat. Acad. Sci. 102, 9948 (2005).

[206] J. Maynard Smith, Models in Ecology (Cambridge University Press, Cambridge (UK), 1974).

[207] W. O. Kermack and A. G. McKendrick, Proc. Roy. Soc. Lond. A 115, 700 (1927).

[208] N. T. J. Bailey, The Mathematical Theory of Infectious Diseases and its Applications (Hafner Press, New York, 1975).

[209] R. M. Anderson and R. M. May, Infectious Diseases in Humans (Oxford University Press, Oxford, 1992).

[210] J. D. Murray, Mathematical Biology (Springer, (Berlin, Ge), 1993).

[211] O. Diekmann and J. Heesterbeek, Mathematical Epidemiology of Infectious Diseases: Model Building, Analysis and Interpretation (Wiley, New York, 2000). 
[212] R. Pastor-Satorras and A. Vespignani, Phys. Rev. Lett. 86, 3200 (2001).

[213] R. M. May and A. L. Lloyd, Phys. Rev. E 64, 066112 (2001).

[214] R. Pastor-Satorras and A. Vespignani, Phys. Rev. E 63, 066117 (2001).

[215] M. Boguña and R. Pastor-Satorras, Phys. Rev. E 66, 047104 (2002).

[216] Y. Moreno, R. Pastor-Satorras, and A. Vespignani, Eur. Phys. J. B 26, 521 (2002).

[217] R. Pastor-Satorras and A. Vespignani, Phys. Rev. E 65, 036104 (2002).

[218] Y. Moreno, J. B. Gómez, and A. F. Pacheco, Phys. Rev. E 68, 035103(R) (2003).

[219] R. Cohen, S. Havlin, and D. ben-Avraham, Phys. Rev. Lett. 91, 247901 (2003).

[220] P. Holme, Europhys. Lett. 68, 908 (2004).

[221] P. Echenique, J. Gómez-Gardeñes, and Y. Moreno, Phys. Rev. E 71, 035102(R) (2005).

[222] J. Gómez-Gardeñes, P. Echenique, and Y. Moreno, Eur. Phys. J. B 49, 259 (2006).

[223] R. M. Anderson and R. M. May, Nature 280, 361 (1979).

[224] P. Grassberger, Math. Biosci. 63, 157 (1983).

[225] L. M. Sander, C. P. Warren, I. M. Sokolov, C. Simon, and J. Koopman, Math. Biosci. 180, 293 (2002).

[226] C.Moore and M. E. J. Newman, Phys. Rev. E 61, 5678 (2000).

[227] M. E. J. Newman, Phys. Rev. E 64, 016128 (2002).

[228] H. W. Hethcote and J. A. Yorke, Lecture Notes in Biomathematics 56 (1984).

[229] M. E. J. Newman, Soc. Netw. 25, 83 (2003).

[230] M. Garey and D. Johnson, Computers and Intractability: A Guide to the Theory of NP-completeness (Freeman, San Francisco, 1979).

[231] R. Pastor-Satorras, A. Vázquez, and A. Vespignani, Phys. Rev. Lett. 87, 258701 (2001). 
[232] Y. Moreno, M. Nekovee, and A. F. Pacheco, Phys. Rev. E 69, 066130 (2004).

[233] M. Takayasu, H. Takayasu, and T. Sato, Physica A 233, 824 (1996).

[234] M. Takayasu, H. Takayasu, and K. Fukuda, Physica A 277, 248 (2000).

[235] M. Argollo de Menezes and A. L. Barabási, Phys. Rev. Lett. 92, 028701 (2004).

[236] R. V.Solé and S. Valverde, Physica A 289, 595 (2001).

[237] S. Valverde and R. V. Solé, Physica A 312, 636 (2002).

[238] S. Valverde and R. V. Solé, Eur. Phys. J. B 38, 245 (2004).

[239] T. Ohira and R. Sawatari, Phys. Rev. E 58, 193 (1998).

[240] B. Tadić and G. J. Rodgers, Adv. in Complex Systems 5, 445 (2002).

[241] B. Tadić, S. Thurner, and G. J. Rodgers, Phys. Rev. E 69, 036102 (2004).

[242] B. Tadić and S. Thurner, Physica A 332, 566 (2004).

[243] B. Tadić and S. Thurner, Physica A 346, 183 (2005).

[244] B. Tadić, Physica A 293, 273 (2001).

[245] A. Arenas, A. Díaz-Guilera, and R. Guimerá, Phys. Rev. Lett. 86, 3196 (2001).

[246] R. Guimerá, A. Arenas, and A. Díaz-Guilera, Physica A 299, 247 (2001).

[247] R. Guimerá, A. Arenas, A. Díaz-Guilera, and F. Giralt, Phys. Rev. E 66, 026704 (2002).

[248] R. Guimerá, A. Díaz-Guilera, F. Vega-Redondo, A. Cabrales, and A. Arenas, Phys. Rev. Lett. 89, 248701 (2002).

[249] K. Goh, B. Kahng, and D. Kim, Phys. Rev. Lett. 87, 278701 (2001).

[250] S. H. Strogatz, Nature 410, 268 (2001).

[251] E. Schrödinger, What is life? The physical aspect of the living cell (Macmillan, (London, UK), 1944).

[252] L. H. Hartwell, J. J. Hopfield, S. Leibler, and A. W. Murray, Nature 402, C47 (1999).

[253] D. B. Searls, Nature Reviews 4, 45 (2005). 
[254] B. Vogelstein, D. Lane, and A. J. Levine, Nature 408, 307 (2000).

[255] S. A. Wagner and D. A. Fell, Proc. R. Soc. London B 268, 1803 (2001).

[256] H. Ma and A.-P. Zeng, Bioinformatics 19, 270 (2003).

[257] H. Ma and A.-P. Zeng, Bioinformatics 19, 1423 (2003).

[258] M. Arita, Proc. Nat. Acad. Sci. 101, 1543 (2004).

[259] R. Tanaka, Phys. Rev. Lett. 94, 168101 (2005).

[260] P. Uetz, L. Glot, G. Cagney, T. A. Mansfield, and E. Al., Nature 403, 623 (2000).

[261] T. Ito, T. Chiba, R. Ozawa, M. Yoshida, M. Hattori, and Y. Sakaki, Proc. Nat. Acad. Sci. 98, 4569 (2001).

[262] H. Jeong, S. P. Mason, A. L. Barabási, and Z. N. Oltvai, Nature 411, 41 (2001).

[263] J. M. Stuart, E. Segal, D. Koller, and S. K. Kim, Science 302, 249 (2003).

[264] H. Agrawal, Phys. Rev. Lett. 89, 268702 (2002).

[265] F. Jacob and J. Monod, J. Mol. Bio. 3, 318 (1961).

[266] M. E. Wall, W. S. Hiavacek, and M. A. Savageau, Nature Review Genetics 5, 34 (2004).

[267] S. A. Kauffman, J. Theor. Biol. 22, 437 (1969).

[268] U. Bastolla and G. Parisi, Physica D 115, 203 (1998).

[269] U. Bastolla and G. Parisi, Physica D 15, 219 (1998).

[270] J. E. S. Socolar and S. A. Kauffman, Phys. Rev. Lett. 90, 68702 (2003).

[271] B. Samuelsson and C. Troein, Phys. Rev. Lett. 90, 98701 (2003).

[272] B. Drossel, T. Mihaljev, and F. Greil, Phys. Rev. Lett. 94, 88701 (2005).

[273] B. Drossel, Phys. Rev. E 72, 16110 (2005).

[274] R. Albert and H. G. Oltmer, J. Theor. Biol. 223, 1 (2003).

[275] S. Kauffman, C. Peterson, B. Samuelsson, and C. Troein, Proc. Nat. Acad. Sci. 100, 14796 (2003).

[276] I. Langmuir, J. Chem. Soc. 40, 1361 (1918). 
[277] C. S. Holling, Mem. Ent. Soc. Can. 45, 1 (1965).

[278] J. Hofbauer and K. Sigmund, Evolutionary Games and Population Dynamics (Cambridge University Press, (Cambridge, UK), 1998).

[279] N. Boccara, Modeling Complex Systems (Springer, (New York, US), 2004).

[280] L. Michaelis and L. M. Menten, Biochem. Z. 49, 333 (1913).

[281] L. A. Segel, Modeling Dynamic Phenomena in Molecular and Cellular Biology (Cambridge University Press, (Cambridge, UK), 1984).

[282] J. P. Sethna, Statistical Mechanics: Entropy, Order Parameters, and Complexity (Oxford University Press, (Oxford, UK), 2006).

[283] M. B. Elowitz and S. Leibler, Nature 403, 335 (2000).

[284] J. García-Ojalvo, M. B. Elowitz, and S. H. Strogatz, Proc. Nat. Acad. Sci. 101, 10955 (2004).

[285] S. Bornholdt, Science 310, 449 (2005).

[286] K. Klemm and S. Bornholdt, Proc. Nat. Acad. Sci. 102, 18414 (2005).

[287] O. Bradman, J. E. Ferrel Jr., R. Li, and T. Meyer, Science 310, 496 (2005).

[288] A. Ma'ayan, S. L. Jenkins, S. Neves, A. Hasseldine, E. Grace, B. DubinThaler, N. J. Eungdamrong, G. Weng, P. T. Ram, J. J. Rice, et al., Science 309, 1078 (2005).

[289] G. von Dassow, E. Meir, E. M. Munro, and G. M. Odell, Nature 406, 188 (2000).

[290] E. Mjolsness, D. H. Sharp, and J. Reitnitz, J. Theor. Biol. 152, 429 (1991).

[291] J. Reitnitz, E. Mjolsness, and D. H. Sharp, J. Exp. Zoology 271, 47 (1992).

[292] V. V. Gursky, J. Reitnitz, and A. M. Samsonov, Chaos 11, 132 (2001).

[293] I. Salazar, J. García-Fernández, and R. Solé, J. Theor. Biol. 205, 587 (2000).

[294] R. V. Solé, I. Salazar-Ciudad, and J. García-Fernández, Physica A 305, 640 (2002). 
[295] T. S. Gardner, D. di Bernardo, D. Lorenz, and J. J. Collins, Science 301, 102 (2003).

[296] R. Seydel, Practical Bifurcation and Stability Analysis (Springer, (New York, US), 1994).

[297] S. N. Dorogovtsev and J. F. F. Mendes, Evolution of Networks. From Biological Nets to the Internet and the $W W W$ (Oxford University Press, (Oxford, UK), 2003).

[298] A. L. Barabási and Z. N. Oltvai, Nat. Rev. Gen. 5, 101 (2004).

[299] Ch. Huygens, Horologium Oscillatorium (Apud F. Muguet, (Parisiis, France), 1673).

[300] A. Pikovsky, M. Rosenblum, and J. Kurths, Synchronization. A universal concept in nonlinear systems (Cambridge University Press, (Cambridge, UK), 2001).

[301] S. Boccaletti, J. Kurths, D. L. Valladares, G. Osipov, and C. Zhou, Phys. Rep. 366, 1 (2002).

[302] L. M. Pecora and T. L. Carroll, Phys. Rev. Lett. 64, 821 (1990).

[303] M. G. Rosemblum, A. S. Pikovsky, and J. Kurths, Phys. Rev. Lett. 76, 1804 (1996).

[304] M. G. Rosemblum, A. S. Pikovsky, and J. Kurths, Phys. Rev. Lett. 78, 4193 (1997).

[305] A. T. Winfree, The geometry of biological time (Springer-Verlag, (New York, US), 1990).

[306] S. H. Strogatz, Sync: The Emerging Science of Spontaneous Order (Hyperion, (New York, US), 2003).

[307] S. C. Manrubia, A. S. Mikhailov, and D. H. Zanette, Emergence of Dynamical Order. Synchronization Phenomena in Complex Systems (World Scientific, (Singapore, I), 2004).

[308] J. A. Acebron, L. L. Bonilla, C. J. P. Vicente, F. Ritort, and R. Spigler, Rev. Mod. Phys. 77, 137 (2005).

[309] S. H. Strogatz, Physica D 143, 1 (2000).

[310] A. T. Winfree, J. Theor. Biol. 16, 15 (1967).

[311] Y. Kuramoto, Lect. Notes in Physics 30, 420 (1975). 
[312] Y. Kuramoto, Chemical oscillations, waves, and turbulence (SpringerVerlag, (New York, US), 1984).

[313] R. V. Solé and J. M. Montoya, Proc. R. Soc. London B 268, 2039 (2001).

[314] F. Wang, Y. Moreno, and Y. Sun, Phys. Rev. E 73, 036123 (2006).

[315] J. Restrepo, E. Ott, and B. R. Hunt, Phys. Rev. E 71, 036151 (2005).

[316] A. E. Motter, C. Zhou, and J. Kurths, Phys. Rev. E 71, 016116 (2005).

[317] Y. Moreno and A. Fernández-Pacheco, Europhys. Lett. 68, 603 (2004).

[318] T. Ichinomiya, Phys. Rev. E 70, 026116 (2004).

[319] L. M. Pecora and T. L. Carroll, Phys. Rev. Lett. 80, 2109 (1998).

[320] M. Barahona and L. M. Pecora, Phys. Rev. Lett. 89, 05101 (2002).

[321] T. Nishikawa, A. E. Motter, Y. -C. Lai, and F. C. Hoppensteadt, Phys. Rev. Lett. 91, 014101 (2003).

[322] H. Hong, B. J. Kim, M. Y. Choi, and H. Park, Phys. Rev. E 69, 067105 (2004).

[323] M. Chavez, D.-U. Hwang, A. Amann, H. G. E. Hentschel, and S. Boccaletti, Phys. Rev. Lett. 94, 218701 (2005).

[324] D.-S. Lee, Phys. Rev. E 72, 026208 (2005).

[325] L. Donetti, P. I. Hurtado, and M. A. Muñoz, Phys. Rev. Lett. 95, 188701 (2005).

[326] C. Zhou, A. E. Motter, and J. Kurths, Phys. Rev. Lett. 96, 034101 (2006).

[327] E. Oh, K. Rho, H. Hong, and B. Kahng, Phys. Rev. E 72, 047101 (2005).

[328] P. N. McGraw and M. Menzinger, Phys. Rev. E 72, 015101(R) (2005).

[329] A. Arenas, A. Díaz-Guilera, and C. J. Pérez-Vicente, Phys. Rev. Lett. 96, $114102(2006)$.

[330] A. S. Mikhailov, D. H. Zanette, Y. M. Zhai, I. Z. Kiss, and J. L. Hudson, Proc. Nat. Acad. Sci. 101, 10890 (2004).

[331] C. Zhou and J. Kurths, Chaos 16, 015104 (2006).

[332] M. E. J. Newman, Phys. Rev. E 69, 066133 (2004).

[333] H. G. Schuster, Deterministic Chaos: An Introduction (Wiley-VCH, Berlin, 1995). 


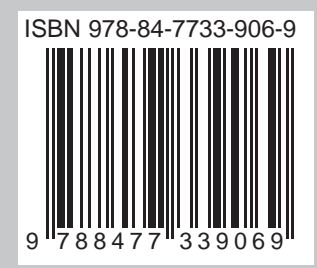

\section{Prest}

Prensas Universitarias de Zaragoza

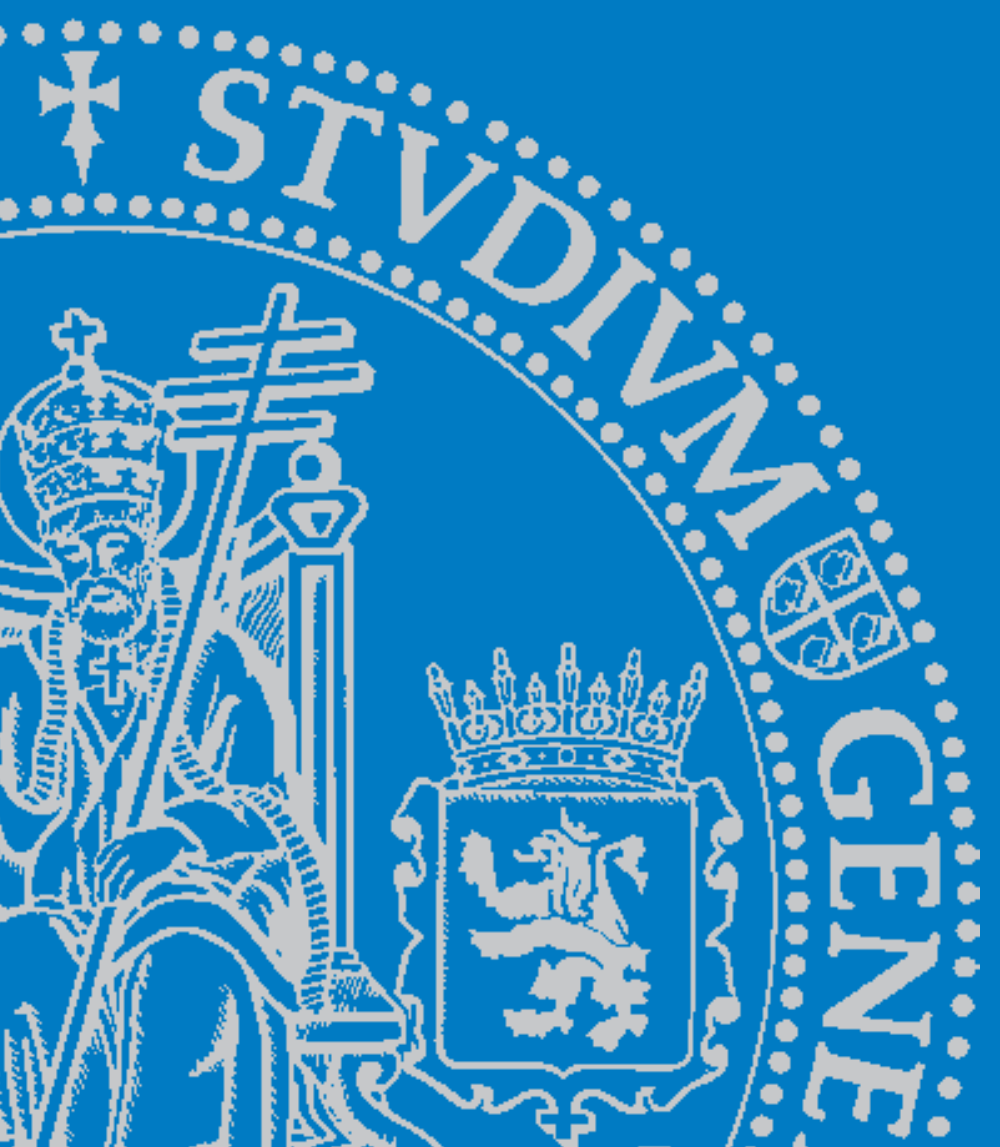

